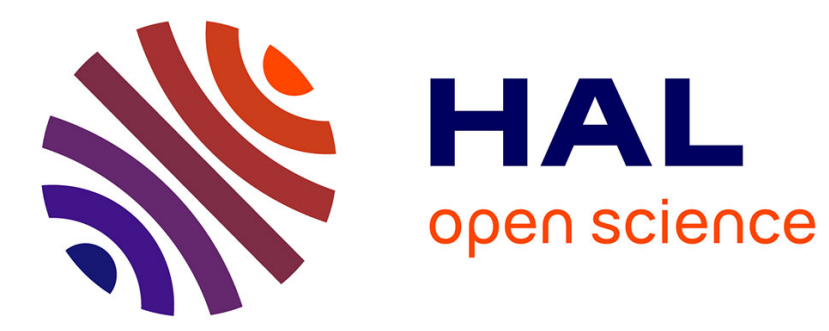

\title{
Dynamique et bilan de masse des glaciers de montagne (Alpes, Islande, Himalaya) : contribution de l'imagerie satellitaire
}

\author{
Etienne Berthier
}

\section{- To cite this version:}

Etienne Berthier. Dynamique et bilan de masse des glaciers de montagne (Alpes, Islande, Himalaya): contribution de l'imagerie satellitaire. domain_other. Université Paul Sabatier - Toulouse III, 2005.

Français. NNT: . tel-00011256

HAL Id: tel-00011256

https://theses.hal.science/tel-00011256

Submitted on 22 Dec 2005

HAL is a multi-disciplinary open access archive for the deposit and dissemination of scientific research documents, whether they are published or not. The documents may come from teaching and research institutions in France or abroad, or from public or private research centers.
L'archive ouverte pluridisciplinaire HAL, est destinée au dépôt et à la diffusion de documents scientifiques de niveau recherche, publiés ou non, émanant des établissements d'enseignement et de recherche français ou étrangers, des laboratoires publics ou privés. 
UNIVERSITÉ TOULOUSE III

-PAUL SABATIER-

ECOLE DOCTORALE DES SCIENCES DE L'UNIVERS

Formation doctorale Océan Atmosphère Environnement

\section{THĖSE}

pour obtenir le grade de

DOCTEUR DE L'UNIVERSITÉ TOULOUSE III

Discipline : Glaciologie \& Télédetection

présentée par

ETIENNE BERTHIER

le 16 septembre 2005

\section{DYNAMIQUE ET BILAN DE MASSE DES GLACIERS DE MONTAGNE (ALPES, ISLANDE, HIMALAYA) : CONTRIBUTION DE L'IMAGERIE SATELLITAIRE}

\begin{tabular}{lll} 
& \multicolumn{2}{c}{ Composition du jury : } \\
Michel RABINOWICZ & Professeur, Université de Toulouse III & Président du Jury \\
Michel FILY & Professeur, Université de Grenoble & Rapporteur \\
Jean-Philippe AVOUAC & Professeur, CALTECH & Rapporteur \\
Helgi BJÖRNSSON & Professeur, Université d'Islande & Examinateur \\
Christian VINCENT & IR CNRS, LGGE & Invité \\
Frédérique RÉMY & DR CNRS, LEGOS & Directrice de thèse \\
Yves ARNAUD & CR IRD, LGGE & Co-directeur de thèse
\end{tabular}

Laboratoire d'Étude Géophysique et d'Océanographie Spatiale, Observatoire Midi Pyrénées, 14, avenue E. Belin, TOULOUSE 



\section{Résumé court}

Face au déclin récent des glaciers de montagne, un suivi régulier à l'échelle globale est nécessaire mais n'est pas réalisable par des campagnes de terrain. Nous montrons dans cette thèse que l'imagerie satellitaire haute résolution est une solution pour observer l'évolution dynamique et volumétrique des glaciers.

Les vitesses de surface des glaciers du Mont-Blanc montrent des fluctuations à court terme que nous relions à l'intensité de la fonte et à l'hydrologie sous-glaciaire. Sur le long terme, un ralentissement important (30 à 40\%) suggère une réponse dynamique des glaciers aux changements climatiques.

Pour les glaciers alpins, les pertes de glace dans les zones basses s'accélèrent lors des dix dernières années alors qu'à haute altitude l'épaisseur glaciaire ne varie presque pas. Un fort amincissement à basse altitude est aussi observé en Islande et en Himalaya entre 1998-9 et 2004. Aussi, la fonte de ces glaciers expliquerait 5\% de l'élévation récente du niveau marin.

Mots clés : glaciers de montagne, dynamique, bilan de masse, indicateurs climatiques, montée niveau marin, SPOT5, satellite, MNT, corrélation d'images 



\section{Résumé long}

Le déclin rapide des glaciers de montagne implique un suivi régulier de leur évolution. Ce recul a des conséquences à l'échelle globale sur l'élévation du niveau marin mais aussi localement, les glaciers jouant un rôle régulateur des débits des rivières donc de la ressource en eau. L'état de santé des glaciers de montagne, présents à toutes les latitudes, constitue de plus un excellent indicateur climatique.

L'utilisation des techniques satellitaires pour l'étude de ces glaciers est rendue difficile par la petite taille des objets étudiés et la forte topographie de ces zones. Cette thèse montre que des images optiques haute résolution (SPOT5 notamment) et des méthodologies adaptées permettent de mesurer deux paramètres clefs des glaciers : l'écoulement de surface et leurs variations d'épaisseur. Le massif du Mont-Blanc a été choisi comme site test car les glaciers y sont bien documentés par les campagnes de terrain. Après validation, nos techniques sont appliquées à des régions plus difficiles d'accès comme la calotte glaciaire du Vatnajökull (Islande) ou les glaciers de l'Himalaya indienne.

Nous déterminons les vitesses de surface des glaciers en corrélant deux images SPOT5 acquises à quelques semaines d'intervalle et bien superposées. Des comparaisons avec des relevés GPS indiquent une précision de $\pm 50 \mathrm{~cm}$ sur les déplacements (soit 0.2 pixel d'une image SPOT5). Une accélération estivale est mise en relation avec la canicule d'août 2003 et illustre un contrôle hydrologique de la dynamique glaciaire. Sur le long terme, la comparaison des mesures satellitaires à des relevés in situ indique un ralentissement de 30 à $40 \%$ de la langue de la Mer de Glace. Par ailleurs, et de façon inattendue, nous montrons que les variations de vitesse du court terme (saisonnier) au long terme sont loin d'être homogènes spatialement.

Des topographies précises, déduites d'images SPOT pour des années différentes, sont comparées pour estimer les variations d'épaisseur des glaciers. Nos mesures sur les glaciers du MontBlanc, couvrant les périodes 1979-1994, 1994-2000 et 2000-2003, sont comparées aux relevés in situ, indiquant une précision de \pm 1 à $2 \mathrm{~m}$. Une accélération de l'amincissement à basse altitude est observée lors des 10 dernières années alors qu'aucune tendance significative n'apparaît au dessus de $2500 \mathrm{~m}$. Des fortes pertes d'épaisseur à basse altitude sont également observés pour les glaciers himalayens et islandais depuis 5 à 6 ans. Par leur bilan de masse négatifs, ces deux régions contribueraient ainsi de façon significative à l'élévation actuelle du niveau marin.

Deux aspects de la dynamique de la calotte glaciaire du Vatnajökull (Islande) sont éclairés par nos travaux. Sur le long terme, nous quantifions le rôle des crues glaciaires (surges) dans l'évolution de la topographie du glacier. Un trait marquant est un rapide épaississement du bassin d'accumulation dans la phase post-surge. La corrélation d'images SPOT5 acquises avec des incidences similaires et obliques détecte le soulèvement d'une plateforme de glace qui flotte au-dessus d'un lac sous-glaciaire (Grímsvötn) au cœur du Vatnajökull. Grâce à la carte du soulèvement, nous délimitons l'extension du lac sous-glaciaire et estimons l'activité géothermique de cette zone quelques semaines avant une éruption volcanique. 



\section{Abstract}

In a context of general glacier retreat, a continuous and global monitoring of mountain glaciers is required but cannot be achieved thanks to ground measurements. In this thesis, we demonstrate that high resolution satellite optical images permit to observe the dynamic and volumetric evolutions of glaciers.

Surface velocities of Mont Blanc glaciers (Alps) show short term (summer) fluctuations that are connected to the intensity of surface melting and subglacial hydrological network. A significant slow down (30 to $40 \%$ in 20 years) of the Mer de Glace suggests that glacier dynamics play an important role in their response to climate change.

For the Mont Blanc glaciers, thinning is enhanced in the ablation area during the last 10 years whereas, at higher elevation, no significant thickness change is detected. A similar signal is also observed in Himalaya and Iceland. Melting of glaciers in these 2 regions may have risen global sea level by up to $0.13 \mathrm{~mm} /$ year in the last 5 years.

Keyword : mountain glaciers, dynamics, mass balance, climate indicator, sea level rise, SPOT5, satellite, DEM, images correlation 



\section{Remerciements}

Je tiens avant tout à remercier pour leur soutien mes deux directeurs de thèse, Frédérique Rémy et Yves Arnaud. Frédérique m'a acceuilli dans son équipe "Glacio" acceptant mon sujet éloigné des "pôles" d'intérêt de l'équipe cryosphère satellitaire. Merci Frédérique pour ta disponibilité sans faille et de ne jamais sourciller lors de nos intrusions dans ton bureau. La même disponibilité te caractérise Yves : plus réactif que toi au mail tu meures! Les missions de terrain, notamment les siestes, en pleine acquisition DGPS dans les vertes prairies alpines ont renforcé les liens amicaux que nous avions déjà tissés sur les glaciers boliviens. J'espère qu'avec tous les deux nous partagerons d'autres bouts de chemins scientifiques et humains dans le futur.

Sans David Baratoux, Kurt Feigl et Hélène Vadon cette thèse n'aurait été que l'ombre d'elle même. Merci David pour ton enthousiasme scientifique sans borne (?). Kurt, j'admire ton art de stimuler la collaboration scientifique. J'ai beaucoup appris de ta rigueur. Hélène, j'attends le jour où je reussirai à travailler aussi efficacement que toi. Merci pour ta gentillesse. Les discussions et les observations de terrain fournis par Christian Vincent (LGGE) ont été indispensables à ce travail. Christophe (Delacourt) quand tes glissements de terrain (sous-marins?) bougeront aussi vite que mes glaciers je veux bien re-travailler avec toi!

Trois missions en Islande ont créé des liens solides et amicaux avec Helgi Björnsson, Finnur Palsonn et Sverrir Gudmundsson. Certes, cela m'a demandé de gros efforts orthographiques mais la découverte des glaciers islandais et de leurs gardiens scientifiques en valaient la chandelle. Takk! Bless!

Heureusement qu'il y a d'autres thésards embarqués dans la même galère. Merci tout parti-

culièrement à Agathe, co-bureau pendant cette thèse. J'espère que l'avenir te sourira. Les pauses gouters-Prince avec Arnaud et Noémie m'ont bien manqué la dernière année ainsi que les sursauts provoqués par "Grand" alias Mr Barbecue. Merci à l'ensemble des thésards du LEGOS et du DTP, notamment Sébastien (Mr "un voyage tous les midis"), Pascal, Raymond, Stéphane et Loïc. La détente pendant la thèse ce sont aussi les pots récurrents au sein de l'équipe glacio : tous les prextextes sont bons. Continuez c'est cool.

Merci à Patrick Monfray, directeur du LEGOS de m'avoir acceuilli au sein de son laboratoire. Merci à l'équipe informatique pour son aide notamment Christian lors de la préparation de la soutenance. Les "drôles de dames" font tourner la maison tout en lui insufflant de la bonne 
humeur. Un merci tout particulier à Martine et Nadine. Je rêve de vous embêter à nouveau dans quelques années pour me trouver un coin de bureau ou pour partir en mission...

Merci aux différents enseignants de l'UPS qui m'ont accompagné pour la durée du monitorat, notamment Anne Nédélec, Raoul Vehil et la sympathique équipe d'Aérologie ainsi que les géologues/géophysiciens du LMTG. Certains enseignants ou encadrants dans les années pré-thèses ont beaucoup compté. Je pense en particulier à Paul Duval, Pierre Ribstein, Bruce Raup. Je tiens à rendre hommage et dédier ce travail à Gérard Mégie, professeur en DEA, qui même après avoir accédé à de lourdes responsabilités est toujours resté à l'écoute de ses étudiants.

Merci à ceux qui ont facilité le financement de ce travail de thèse : Yves grâce à son ACI Observation de la Terre, Frédérique et le LEGOS, Hervé Jeanjean et le programme ISIS du CNES, Helgi Björnsson (Science Institute) et l'ambassade de France en Islande.

Riquet a eu la bonne idée de construire le canal du midi. Le vélo au quotidien le long du canal est le meilleur moyen de rester en adéquation avec ces convictions environnementales, de réfléchir à la journée à venir ou d'évacuer tout son fiel de 12 heures de rédaction. Avis aux amateurs!

Merci à mes parents de m'avoir laissé une grande liberté de choix au cours des études et de leur soutien jusqu'au pot de thèse. Et toute la familia aussi !

Merci à Fleur, qui rend la vie si belle. 


\section{Avant propos}

\section{De la glaciologie de terrain à l'observation spatiale}

"Si un observateur pouvait être transporté à une assez grande hauteur au-dessus des Alpes, pour embrasser d'un coup d'œil celles de la Suisse, de la Savoie et du Dauphiné, il verrait cette chaîne de montagnes, sillonnée par de nombreuses vallées, et composée de plusieurs chaînes parallèles, la plus haute au milieu, et les autres décroissant graduellement, à mesure qu'elles s'en éloignent" écrivait Horace Benedict de Saussure en 1803 dans son livre Premières ascensions au Mont-Blanc. Ce précurseur des mesures et observations in situ, avait peut-être déjà deviné l'immense intérêt de la télédétection dans l'étude des chaînes de montagne en général et des glaciers en particulier.

Après 18 mois à arpenter les glaciers andins, une "mise en orbite" m'a été proposée par Frédérique Rémy et Yves Arnaud afin d'observer les glaciers depuis $800 \mathrm{~km}$ d'altitude. Comme de Saussure, tous les deux sont (et m'ont) convaincu(s) de l'intérêt de l'observation depuis l'espace. Mais, pour que les satellites fournissent une vision régionale de l'évolution récente des glaciers, cela implique le développement et la validation de méthodologies adaptées à ces objets, souvent de petites tailles et nichés au cœur d'une topographie abrupte. La confrontation aux mesures in situ est donc indispensable, car elle permet de détecter certains biais que ne révèlent pas la (parfois très bonne) cohérence interne de la mesure satellitaire...

\section{Organisation de la thèse}

La thèse débute par 3 chapitres bibliographiques. Nous montrons en premier lieu la place des glaciers de montagne au sein de la cryosphère et l'importance de leur étude dans un contexte de changement climatique (chapitre 1) avant de décrire leur fonctionnement (chapitre 2). Ces deux chapitres nous conduisent aux objectifs scientifiques de ce travail. Puis, en dressant un état de lieux de l'observation satellitaire des glaciers de montagne, nous détaillons les objectifs méthodologiques de la thèse (chapitre 3 ). 
Dans les 3 chapitres suivants, nous présentons les trois grands chantiers qui ont été au cœur de cette étude. Un premier chantier consiste en la mesure des vitesses d'écoulement des glaciers alpins à partir d'images SPOT (chapitre 4). Les bilans de masse des glaciers alpins et himalayens sont ensuite estimés en comparant des topographies déduites d'images SPOT ou d'autres origines (chapitre 5). Enfin, nos observations satellitaires contribuent à mieux comprendre certains aspects de la dynamique d'une calotte glaciaire islandaise (chapitre 6).

Dans une synthèse (chapitre 7), nous proposons un regroupement thématique des principaux résultats de ce travail et détaillons les perspectives, en regard notamment des missions satellitaires futures.

Plutôt que de les insérer dans le texte, il a été choisi de placer en annexe (A à D) les articles en anglais publiés ou soumis au cours de ces trois années. L'objectif est d'assurer une cohérence d'ensemble au manuscrit et, si possible, de prendre du recul par rapport aux résultats de ces publications. Trois autres annexes sont ajoutées, les deux premières ( $\mathrm{E}$ et $\mathrm{F}$ ) pour décrire des applications non glaciologiques, la dernière (annexe $\mathrm{G}$ ) pour présenter un développement méthodologique prometteur qui n'a pu être mise en œuvre pour le moment, faute d'images adaptées.

\section{Bonne lecture!}




\section{Table des matières}

1 Introduction : Glaciers \& Climat $\quad 17$

1.1 Les zones englacées à la surface du globe . . . . . . . . . . . . . . . . . . . 18

1.1.1 L'eau sur Terre : cycles interne et externe . . . . . . . . . . . . . . 18

1.1.2 L'eau sous forme de glace : la cryosphère . . . . . . . . . . . . . . . . . . . 19

1.1.3 Les glaciers et leur répartition géographique . . . . . . . . . . . . . . 21

1.2 Bref historique de l'étude des glaciers de montagne . . . . . . . . . . . . . . 22

1.3 Un recul marqué et quasi généralisé au $\mathrm{XX}^{\text {eme }}$ siècle . . . . . . . . . . . . . . . . . . . . . . . . . . . . . .

1.3.1 Un exemple : évolution des glaciers alpins . . . . . . . . . . . . 25

1.3.2 Variations régionales autour du thème du recul des glaciers . . . . . . . . 27

1.4 Conséquences du recul des glaciers . . . . . . . . . . . . . . . . . . 29

1.4.1 Contribution à la hausse du niveau marin . . . . . . . . . . . . . . . . 29

1.4.2 Impact hydrologique : perturbation de la ressource en eau . . . . . . . . 31

1.4.3 L'accroissement du risque glaciaire . . . . . . . . . . . . . . . . . . 32

1.4.4 Impacts économique et touristique . . . . . . . . . . . . . . 34

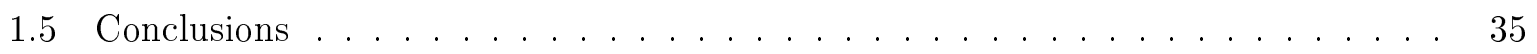

2 Naissance, vie \& mort d'un glacier de montagne $\quad 37$

2.1 Naissance \& mort de la glace : accumulation vs ablation . . . . . . . . . . . . 38

2.1 .1 L'accumulation et la formation de la glace . . . . . . . . . . . . 38

2.1 .2 Les processus d'ablation . . . . . . . . . . . . . . . . 39

2.1.3 Le bilan de masse : indicateur de l'état de santé du glacier . . . . . . . . . 40

2.2 La vie de la glace ? Mouvementée... . . . . . . . . . . . . . . . . . . . . . . . 47

2.2.1 Mesure de l'écoulement glaciaire . . . . . . . . . . . . . . . 47

2.2.2 La déformation interne de la glace . . . . . . . . . . . . . . . . . 49

2.2 .3 Le glissement ou dérapage basal . . . . . . . . . . . . . . . . . . . 51

2.2.4 Variation saisonnière et inter-annuelle de l'écoulement . . . . . . . . . . . 53

2.3 Conclusions et objectifs scientifiques . . . . . . . . . . . . . . . 55

3 Observation satellitaire des glaciers de montagne $\quad 57$

3.1 Difficultés \& nécessité de l'observation satellitaire . . . . . . . . . . . . . . 58 
3.1 .1 Une observation difficile... . . . . . . . . . . . . . . . 58

$3.1 .2 \quad \ldots$ mais nécessaire car les glaciers... . . . . . . . . . . . . . 62

3.2 Les différents capteurs satellitaires . . . . . . . . . . . . . . . . 64

3.2 .1 Imagerie optique . . . . . . . . . . . . . . . . . . . . 64

3.2 .2 Imagerie RADAR . . . . . . . . . . . . . . . . . . 67

3.2 .3 Altimétrie Radar et Laser . . . . . . . . . . . . . . . . . . . . . 68

3.3 Observation satellitaire des glaciers : état des lieux . . . . . . . . . . . . 69

3.3.1 État de surface des glaciers . . . . . . . . . . . . . . . . 69

3.3 .2 Variations de longueur et superficie glaciaire . . . . . . . . . . . . 70

3.3 .3 Vitesses d'écoulement des glaciers . . . . . . . . . . . . . . . 71

3.4 Conclusions et objectifs méthodologiques . . . . . . . . . . . . . . . . 74

4 Satellite \& écoulement des glaciers alpins $\quad \mathbf{7 5}$

4.1 La corrélation d'images optiques : état des lieux . . . . . . . . . . . 76

4.2 Géométrie du problème . . . . . . . . . . . . . . . . . . 78

4.2 .1 Cas général . . . . . . . . . . . . . . . . . . 78

4.2 .2 Mesure des déplacements horizontaux . . . . . . . . . . . . . . 80

4.2 .3 Mesure des déplacements verticaux . . . . . . . . . . . . . . 80

4.3 Notre chaîne de traitement : ME DECOIS PAS . . . . . . . . . . . . . . . 81

4.3 .1 Orientation absolue . . . . . . . . . . . . . . . . 82

4.3 .2 Orientation relative . . . . . . . . . . . . . . 83

4.3 .3 Ré-échantillonnage de l'image esclave . . . . . . . . . . . . . . 84

4.3 .4 Corrélation . . . . . . . . . . . . . . . . . 84

4.3.5 De la géométrie image à la géométrie terrain . . . . . . . . . . . . . . . 84

4.4 Vitesses des glaciers du Mont Blanc durant l'été $2003 \ldots$. . . . . . . . . . . 86

4.4.1 Les glaciers du massif du Mont-Blanc . . . . . . . . . . . . . . . 86

4.4.2 Caractéristiques géométrique et radiométrique des images SPOT5 . . . . 87

4.4 .3 Champs de déplacements pour l'été $2003 \ldots \ldots$. . . . . . . . . 89

4.4.4 Précision et exactitude de notre mesure des vitesses . . . . . . . . . . . . 91

4.4.5 Apports et limites de la méthodologie proposée . . . . . . . . . . . . . . 99

4.5 Variations à court terme de l'écoulement . . . . . . . . . . . . . . . . . 100

4.5.1 Cartographie des variations de vitesse . . . . . . . . . . . . . 100

4.5.2 Une interprétation de ces variations de vitesse . . . . . . . . . . . . . 103

4.5.3 Discussion de ces variations de vitesse . . . . . . . . . . . . . . . . 104

4.6 Variabilité interannuelle de l'écoulement . . . . . . . . . . . . . 106

4.6.1 Comparaison des vitesses estivales . . . . . . . . . . . . . 106

4.6.2 Comparaison des vitesses annuelles . . . . . . . . . . . . . . . 109

4.7 Conclusions . . . . . . . . . . . . . . . . . . . . 111 
5 Satellite \& bilan de masse des glaciers 113

5.1 Variations d'épaisseur des glaciers alpins . . . . . . . . . . . . . . . . . 114

5.1.1 Construction de MNT des glaciers à partir d'images satellites . . . . . . . 114

5.1 .2 Ajustements spatio-temporels des MNT . . . . . . . . . . . . . 116

5.1 .3 Extraction des variations moyennes d'épaisseur . . . . . . . . . . . . 119

5.1 .4 Précision de la mesure satellitaire . . . . . . . . . . . . . . . . 120

5.1.5 Evolution des variations d'épaisseur lors des 25 dernières années . . . . . . 123

5.2 Vers l'estimation satellitaire du bilan de masse glaciaire . . . . . . . . . . . . . 124

5.2.1 Comparaison des MNT aux profils topographiques . . . . . . . . . . . . 124

5.2.2 Variations d'épaisseur des glaciers en 1979-1999 et 1999-2003 . . . . . . . 128

5.2.3 Bilan de masse des glaciers alpins entre 1979 et 2003 . . . . . . . . . . . . 131

5.3 Bilans de masse de 2 glaciers himalayens . . . . . . . . . . . . . . . . . . . 134

5.3 .1 La zone d'étude : l'Himalaya indienne . . . . . . . . . . . . . . . 134

5.3.2 Génération d'un MNT SPOT5 sans point d'appui . . . . . . . . . . . 135

5.3.3 Déclin récent du Bara Shigri et Chhota Shigri . . . . . . . . . . . . . . 136

5.4 Conclusions . . . . . . . . . . . . . . . . . . . . . . . . . 138

5.4 .1 Conclusions méthodologiques . . . . . . . . . . . . . . . 138

5.4 .2 Conclusions glaciologiques . . . . . . . . . . . . . . . . . . 139

6 Dynamique de la calotte glaciaire du Vatnajökull. $\quad 141$

6.1 La calotte glaciaire du Vatnajökull . . . . . . . . . . . . . . . . . . . . . . 141

6.2 Soulèvement de la plateforme de Grímsvötn . . . . . . . . . . . . . . . . . . . 143

6.2.1 Plateforme, lac sous-glaciaire et volcan de Grímsvötn . . . . . . . . . . . . 144

6.2.2 Cartographie du soulèvement de la plateforme de Grímsvötn . . . . . . . 146

6.2.3 Crue potentielle, bilan énergétique et activité géothermique . . . . . . . . 149

6.2.4 Perspectives et implications de cette mesure . . . . . . . . . . . . . . . 150

6.3 Variations volumétriques du Vatnajökull . . . . . . . . . . . . . . . . . . . . 151

6.3.1 Les topographies disponibles sur la calotte (figure 6.8) . . . . . . . . . 151

6.3 .2 Observation et origine des variations d'épaisseur . . . . . . . . . . . 153

6.4 Conclusions . . . . . . . . . . . . . . . . . . . . . . 157

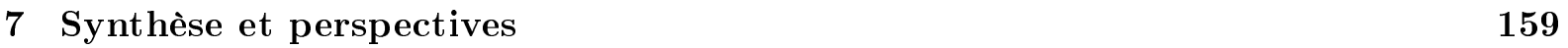

7.1 Synthèse méthodologique . . . . . . . . . . . . . . . . . . 160

7.1 .1 Choix des images satellites . . . . . . . . . . . . . . 160

7.1.2 Mouvements horizontaux des glaciers . . . . . . . . . . . . . . . 160

7.1.3 Variations d'altitude de la surface des glaciers . . . . . . . . . . . . . 161

7.2 Synthèse glaciologique . . . . . . . . . . . . . . . . . . . . . . 162

7.2 .1 Importance de l'hydrologie sous-glaciaire . . . . . . . . . . . . . . . 162

7.2.2 Adaptation dynamique des glaciers aux changements climatiques . . . . 164 
7.2.3 Évolution comparée des glaciers alpins, himalayens et islandais . . . . . . 167

7.3 Perspectives . . . . . . . . . . . . . . . . . . . . . 168

7.3.1 Relation entre l'écoulement et l'hydrologie sous-glaciaire . . . . . . . . . . 169

7.3.2 Réponse dynamique des glaciers au changement climatique . . . . . . . . 169

7.3.3 Bilan de masse et contribution des glaciers à l'élévation du niveau marin . 170

A Vitesse et Bilan de Masse du Glacier MERTZ (Antarctique de l'Est) 171

B Amincissement de la Mer de Glace déduit d'Images Satellitaires $\quad 181$

C SPOT5 mesure 1'Ecoulement des Glaciers de Montagne (Alpes) 187

D Soulèvement de la Plateforme Glaciaire de Grimsvötn (Islande) 203

E "ME DECOIS PAS" \& déformation co-sismique 217

E.1 Séisme d'Izmit : déformation co-sismique . . . . . . . . . . . . . . . . 217

E.1.1 Particularités méthodologiques . . . . . . . . . . . . . . . . . 218

E.1.2 Cartographie de la rupture de surface et valeur du glissement . . . . . . . 221

E.1.3 Amélioration du modèle de rupture de surface . . . . . . . . . . . . . . . . 221

E.2 Séisme d'Al-Hoceima : rupture de surface ? . . . . . . . . . . . . . . . . . 222

E.2.1 Déformations mesurées par SPOT5 . . . . . . . . . . . . . . . . 223

E.2.2 Y'a-t-il eu rupture de surface $\quad \ldots \ldots \ldots \ldots \ldots \ldots$

F "ME DECOIS PAS" \& glissements de terrain $\quad 227$

F.1 Introduction . . . . . . . . . . . . . . . . . . . . 227

F.2 Zones d'étude et images SPOT5 . . . . . . . . . . . . . . . 228

F.3 Orientation absolue et relative des images . . . . . . . . . . . . . . . . . 229

F.4 Détection des glissements de terrain . . . . . . . . . . . . . . . . . 231

G Et l'écoulement 3D des glaciers?

G.1 Fusion InSAR / corrélation d'images optiques . . . . . . . . . . . . . . . . 233

G.2 Corrélation de deux couples d'images optiques . . . . . . . . . . . . . . . 234

G.3 Ces applications sont-elles possibles sur les glaciers? Autrement dit... que mesure-

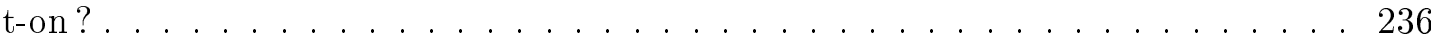

$\begin{array}{ll}\text { Bibliographie } & 237\end{array}$ 


\section{Chapitre 1}

\section{Introduction : Glaciers \& Climat}

\section{Sommaire}

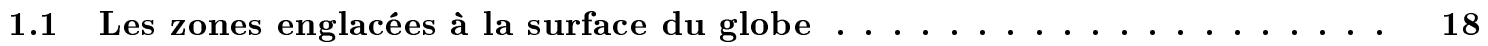

1.1.1 L'eau sur Terre : cycles interne et externe . . . . . . . . . . . . . . . . 18

1.1.2 L'eau sous forme de glace : la cryosphère . . . . . . . . . . . . . . . . . . . 19

1.1.3 Les glaciers et leur répartition géographique . . . . . . . . . . . . . . . . 21

1.2 Bref historique de l'étude des glaciers de montagne . . . . . . . . . . . 22

1.3 Un recul marqué et quasi généralisé au $\mathrm{XX}^{e m e}$ siècle $\ldots \ldots \ldots \ldots$

1.3.1 Un exemple : évolution des glaciers alpins . . . . . . . . . . 25

1.3.2 Variations régionales autour du thème du recul des glaciers . . . . . . . . . . . 27

1.4 Conséquences du recul des glaciers . . . . . . . . . . . . . . . . 29

1.4.1 Contribution à la hausse du niveau marin . . . . . . . . . . . . . . . . 29

1.4.2 Impact hydrologique : perturbation de la ressource en eau . . . . . . . . . . . 31

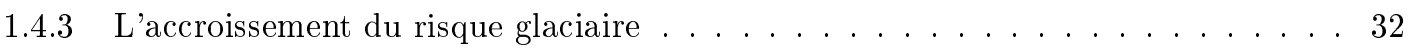

1.4.4 Impacts économique et touristique $\ldots \ldots \ldots \ldots \ldots \ldots$

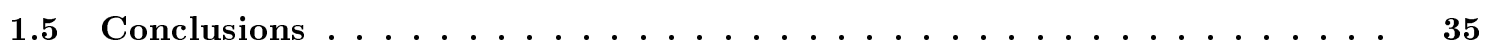

Les glaciers ont toujours suscité un mélange de craintes et d'attirance. Cette relation ambiguë est illustrée par cette procession annuelle qui se déroule au Pérou dans la région de Cusco : chaque année plusieurs dizaines de milliers de pèlerins viennent rendre hommage au "Dieu Glacier" lors de la fête du Coyllur Ritti ${ }^{1}$. A vol d'oiseau nous sommes à quelques centaines de kilomètres de la vallée de Yungay, ville dévastée par une catastrophe glaciaire le 31 mai 1970. Ce jour là, un violent séisme provoque la chute d'un glacier suspendu sur les flancs du Huascarãn (6768 m). L'avalanche de glace se transforme rapidement en une coulée de boue très fluide capable de véhiculer à $150 \mathrm{~m} / \mathrm{s}$ d'énormes bloc rocheux (Lliboutry, 1971). Bilan : plus de 15000 victimes. Le glacier : dieu ou démon?

Leur difficulté d'accès et leur éloignement des zones habitées ont longtemps retardé l'étude des glaciers. Pourtant, la glaciologie occupe désormais une place à part entière au sein des sciences de la Terre et les équipes de glaciologues instrumentent et parcourent les glaciers de toutes les chaînes de montagne pour mieux les comprendre.

\footnotetext{
${ }^{1}$ Coyllur $=$ étoiles et Ritti $=$ neige ou glace soit "étoile des neiges"
} 
Dans cette introduction, nous verrons d'abord où se situent les glaciers et la place qu'ils occupent au sein de la cryosphère ${ }^{2}$ avant de résumer l'historique de leur étude. Nous verrons ensuite que, dans un contexte de réchauffement climatique, les glaciers de montagne sont en fort recul. Ce déclin a des conséquences environnementales importantes que nous développerons dans une dernière partie.

\subsection{Les zones englacées à la surface du globe}

Les glaciers de montagne, qui sont le cœur de notre étude, ne constituent qu'une des entités de la cryosphère. Cette dernière s'intègre et joue un rôle clef dans l'hydrosphère ${ }^{3}$ et plus généralement dans le système climatique terrestre. Mais à l'échelle des temps géologiques, les processus de la terre interne vont aussi avoir une influence sur les processus externes. Dans cette partie, on se propose d'étudier la répartition de l'eau sur Terre en progressant de l'échelle globale jusqu'à celle des glaciers. Cette introduction semble nécessaire, certains auteurs (Van der Veen, 1999) oubliant parfois qu'il existe de l'eau ailleurs que dans l'hydrosphère!

\subsubsection{L'eau sur Terre : cycles interne et externe}

L'existence de l'eau sous ses trois états à la surface de la Terre (liquide, gaz, solide) est l'une de ses caractéristiques. La coexistence de ces trois états a été cruciale pour l'évolution de notre planète. L'eau liquide dans les océans a ainsi été le berceau de la vie sur Terre. La présence d'eau sur Mars est l'une des raisons qui poussent à y rechercher des traces de vie. Souvent notre attention se focalise sur l'eau du cycle externe (océans, atmosphère...), alors qu'une part importante de l'eau se trouve à l'intérieur de la Terre. Elle entre en jeu dans des processus aussi divers que la fusion des magmas dans les zones de subduction, l'hydrothermalisme au niveau des dorsales ou encore dans la déformation des roches crustales et mantelliques en abaissant leur viscosité. Le tableau 1.1 présente les différents réservoirs des cycles interne et externe de l'eau.

TAB. 1.1 - Les différents réservoirs du cycle de l'eau sur Terre. Les temps de résidence (durée moyenne de séjour de l'eau dans un réservoir) sont indiqués, et sont 4 ordres de grandeur supérieurs pour le cycle interne.

\begin{tabular}{llrr}
\hline cycle & Réservoir & Masse $(\mathrm{kg})$ & Temps de résidence $(\mathrm{an})$ \\
\hline \multirow{2}{*}{ interne } & Manteau (non lithosphèrique) & 0,05 à $410^{21}$ & $10^{8}$ à $10^{10}$ \\
& Lithosphère continentale & $410^{20}$ & $210^{8}$ \\
& Lithosphère océanique & $210^{12}$ & $10^{6}$ à $10^{8}$ \\
\hline \multirow{2}{*}{ externe } & Océans & $1,410^{21}$ & $410^{4}$ \\
& Atmosphère & $1,510^{16}$ & $10^{-1}$ \\
& Eaux continentales & $1,510^{19}$ & $210^{3}$ \\
& Cryosphère & $4,310^{19}$ & $210^{4}$ \\
\hline
\end{tabular}

\footnotetext{
${ }^{2}$ ensemble des zones glacées de la Terre

${ }^{3}$ ensemble des eaux à la surface terrestre
} 
Le cycle interne ne peut pas être observé directement ce qui explique les fortes incertitudes notamment sur le contenu total en eau dans le manteau. Selon certaines estimations les roches mantelliques sont, avec les océans, le principal réservoir de l'eau sur notre planète. Les échanges avec le cycle externe se font au niveau des volcans, des zones de subduction et des zones hydrothermales (notamment aux dorsales océaniques). Cette eau "interne" existe depuis la formation de la Terre : notre planète s'est en effet formée par collision et accrétion de météorites qui contenaient de l'eau. Si la présence d'eau à l'extérieur de la Terre est précoce (3,9 milliards d'années) et attestée par l'existence de roches sédimentaires, l'origine de cette eau "externe" reste discutée. Dégazage de l'eau interne par l'activité volcanique ou origine extraterrestre (collision de comètes par exemple) sont les deux hypothèses principales.

En opposition avec le cycle interne, les réservoirs et les flux du cycle externe de l'eau sont mieux contraints. L'océan constitue de loin le plus gros réservoir devant les roches crustales. La cryosphère vient en troisième position. Relativement à toute l'hydrosphère, l'eau à l'état solide ne représente que $2 \%$ (Williams \& Hall, 1993). En revanche, près de $87 \%$ de l'eau douce se trouve sous forme de glace (Van der Veen, 1999; IPCC, 2001). Ce dernier chiffre montre l'importance des zones englacées pour la ressource en eau : seule l'eau douce est utilisable directement pour les besoins humains.

\subsubsection{L'eau sous forme de glace : la cryosphère}

La cryosphère comprend toutes les zones constituées de glace ou de neige à la surface du globe et donc, en plus des glaciers (calottes polaires et glaciers de montagne), la banquise, les régions couvertes de neige, les sols gelés (permafrost) et même la glace des nuages (tableau 1.2 \& figure 1.1). Nous proposons un bref descriptif de chacune de ces entités de la cryosphère.

TAB. 1.2 - Les différents réservoirs de glace terrestre. Pour les deux calottes polaires, seule la glace "posée" est prise en compte ce qui exclut les plateformes de glace (IPCC, 2001).

\begin{tabular}{lcrr}
\hline & Surface $\left(10^{6} \mathrm{~km}^{2}\right)$ & Volume $\left(10^{6} \mathrm{~km}^{3}\right)$ & Niveau marin $(\mathrm{m})$ \\
\hline Antarctique & 12.1 & 29 & 68 \\
Groenland & 1.71 & 2.95 & 7.4 \\
Autres glaciers & 0.68 & $0.18 \pm 0.04$ & $0.5 \pm 0.1$ \\
Banquise & 16 à 25 & & 0 \\
Neiges continentales & 1 à 50 & & 0 \\
\hline
\end{tabular}

Les zones couvertes de neige se situent dans les grandes plaines du Nord (Sibérie ou Canada) ou en altitude dans les chaînes de montagne. Leur extension varie entre 1 et 50 millions $\mathrm{km}^{2}$ selon la saison (tableau 1.2). La présence d'une couverture neigeuse refroidit l'atmosphère localement notamment au moment de sa fonte qui consomme de l'énergie.

La banquise ou glace de mer se forme annuellement quand la température de surface de l'eau de mer descend en dessous de $-1.8^{\circ} \mathrm{C}$ environ (Rémy, 2003). Son extension varie avec les saisons (de 7 à 15 millions de $\mathrm{km}^{2}$ dans l'Arctique; de 1 à 18 millions de $\mathrm{km}^{2}$ autour de l'Antarctique) mais aussi d'une année à l'autre. Une nette tendance à la diminution de surface de la banquise 
arctique émerge pour les dernières années (Stroeve et al., 2005).

Les neiges continentales et la banquise jouent un rôle climatique majeur à cause de leur albédo élevé ${ }^{4}$. Leur extension influence donc considérablement le bilan énergétique de la planète. Actuellement, sous l'effet du réchauffement climatique, neiges et glace de mer arctique sont en recul ce qui tend à diminuer le pouvoir réflecteur de la surface terrestre. Plus d'énergie reste dans le système climatique et le réchauffement risque de s'intensifier : on parle de rétroaction positive.

L'extension des calottes polaires varie beaucoup moins et donc, aux courtes échelles de temps (quelques décennies), les calottes ne modifient presque pas le bilan énergétique de la Terre. En revanche, les variations de leur volume influencent le niveau des mers. Indirectement, elles sont donc également actrices des changements climatiques. Dans les calottes polaires, la glace s'écoule du centre vers la périphérie. L'ablation (par fonte ou sublimation) étant faible, une partie de la glace atteint l'océan où elle forme des plateformes de glace, en équilibre hydrostatique. Seul le bilan de masse de la glace posée sur le socle (non flottante) contribue à l'élévation du niveau marin (tableau 1.2). Mais les plateformes peuvent contribuer indirectement aux variations du niveau de la mer. Leur présence constitue un barrage à l'écoulement des fleuves de glace. Leur disparition sous l'effet d'un réchauffement atmosphérique et/ou océanique (Scambos et al., 2000) peut donc engendrer une augmentation des flux de glace posée vers l'océan au niveau des fleuves de glace. La désintégration récente de la plateforme de Larsen B, l'accélération et l'amincissement consécutifs des glaciers qui l'alimentent sont l'illustration de ce rôle de la dynamique sur le bilan de masse de la calotte (Scambos et al., 2004; Rignot et al., 2004).

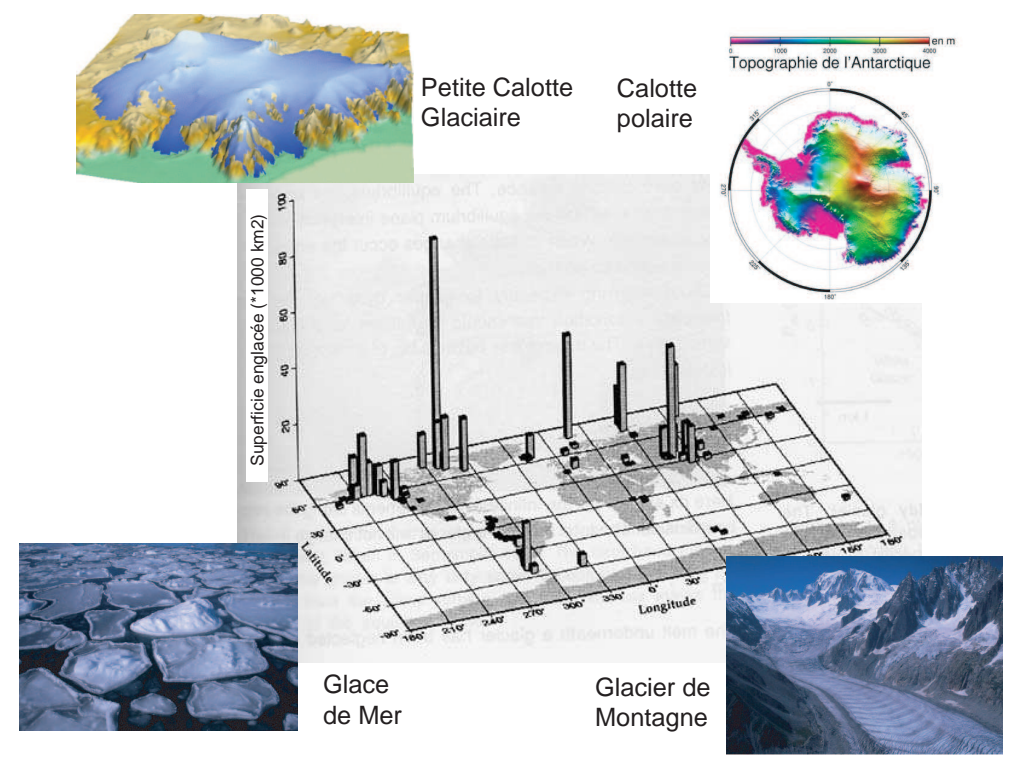

FIG. 1.1 - Les glaciers au sein de la cryosphère. La carte centrale montre la localisation et la surface couverte par les glaciers de montagne (D'après Oerlemans (2001)). Les photos qui accompagnent cette carte illustrent la diversité morphologique des formes prises par la glace à la surface de la Terre.

Les "glaciers" constituent la dernière entité de la cryosphère. On désigne par glacier une masse de glace d'un seul tenant permanente à l'échelle d'une vie humaine (Lliboutry, 1993). Sous cette dénomination, nous regroupons dans cette thèse les petites calottes glaciaires et les

\footnotetext{
${ }^{4}$ L'albédo désigne la proportion de l'énergie solaire qui est réfléchie par une surface.
} 
glaciers de montagne, excluant les deux calottes polaires du Groenland et de l'Antarctique. Cette catégorie correspond à moins de $1 \%$ de la cryosphère : si toute cette glace fondait le niveau de la mer s'élèverait d'un demi mètre (tableau 1.2). Mais nous verrons que les glaciers répondent rapidement aux fluctuations climatiques et qu'aux courtes échelles de temps, ils ont une forte influence sur le niveau marin.

\subsubsection{Les glaciers et leur répartition géographique}

La glace se forme par métamorphose et compaction du manteau neigeux lorsque celui-ci persiste plus d'une année. Pour qu'un glacier apparaisse en un lieu il faut que, localement, le bilan net annuel soit positif : les gains (ou accumulation, essentiellement par les précipitations) doivent être supérieurs aux pertes (ou ablation, essentiellement par fonte). Un glacier ne peut se former que dans des zones froides (ce qui permet des précipitations solides et limite les pertes) où l'accumulation est suffisamment importante. Les facteurs qui vont contrôler l'existence d'un glacier sont nombreux mais comptent l'altitude, la latitude, l'orientation (adrêt/ubac), la circulation atmosphérique et la proximité de sources d'humidité (Lliboutry, 1964). La topographie locale influence la redistribution de la neige par les avalanches ou par le vent, donc l'accumulation.

En conséquence de ces différents facteurs et de la topographie actuelle de notre planète, les glaciers ont une très large répartition géographique (figure 1.1). A basse latitude, les zones englacées sont relativement rares et ne concernent que les sommets les plus élevés des chaînes de montagnes les plus hautes (Cordillère des Andes, Afrique de l'Est, Indonésie). En première approximation, la densité et la taille des glaciers augmentent avec la latitude. Selon les régions, les glaciers montrent une grande diversité de morphologie : petite calotte, glaciers de cirques, de plateau, de vallée ou même champs de neige simplement. Une description complète de cette diversité et des caractéristiques propres aux différentes régions glaciaires pourront être trouvées dans Lliboutry (1964) ou Dyurgerov (2002). Face à cette diversité et au grand nombre de glaciers (plus de 160000 selon Arendt et al. (2002)), l'élaboration d'un inventaire exhaustif s'avère difficile (Braithwaite \& Raper, 2002).

Plutôt qu'une classification morphologique, nous préférons distinguer ici les glaciers à partir de leur propriété thermique. Cela permet de distinguer les glaciers froids (température inférieure à $0^{\circ} \mathrm{C}$ dans toute la masse du glacier) des glaciers tempérés ${ }^{5}$ pour lesquels la température est homogène et de $0^{\circ} \mathrm{C}$ sauf dans la couche de surface qui est sensible aux températures négatives de la saison froide (Lliboutry, 1964). Ce sont ces derniers que nous étudions dans cette thèse. Leurs propriétés thermiques impliquent:

1. une réaction quasi instantanée à des changements climatiques puisque tout apport d'énergie supplémentaire va induire un changement de masse par fusion et/ou sublimation. L'énergie incidente à la surface de la glace n'a pas à réchauffer le glacier avant de le faire changer d'état. Ceci explique que le bilan de masse des glaciers tempérés soit un indicateur quasi instantané des changements climatiques.

2. une dynamique complexe en partie contrôlée par la présence d'eau à la base du glacier. Ces glaciers peuvent glisser sur leur lit rocheux et donc connaître des fluctuations rapides de leur écoulement.

C'est le bilan de masse et la dynamique de l'écoulement de ces glaciers tempérés qui sont au cœur de cette thèse.

\footnotetext{
${ }^{5}$ Attention! un glacier tempéré ne se trouve pas nécessairement sous un climat tempéré. Les glaciers tropicaux, la calotte glaciaire du Vatnajökull en Islande entrent aussi dans cette catégorie.
} 
Non seulement ces glaciers sont sensibles aux fluctuations du climat mais leur large distribution à la surface du globe implique qu'ils subissent des forçages climatiques très différents. Cela complique la tache du glaciologue qui ne peut pas extrapoler simplement les résultats d'un massif glaciaire à un autre. Mais cela satisfait le climatologue qui, à travers la diversité d'évolutions des glaciers, peut étudier un spectre large de fluctuations climatiques.

\subsection{Bref historique de l'étude des glaciers de montagne}

Il ne s'agit pas ici de dresser un historique exhaustif de l'histoire de cette science nommée aujourd'hui glaciologie. Pour plus de détails, le lecteur pourra se référer aux ouvrages de Lliboutry (1964) et Vivian (2001, chap. VI) ainsi qu'à l'article de Bard (2004). Nous nous appuyons également sur un article de synthèse soumis récemment par Rémy \& Testut (2005) qui traite de la progressive découverte des mécanismes de l'écoulement des glaciers.

Ce sont probablement les avancées importantes des glaciers des Alpes au XVIII ${ }^{\text {eme }}$ siècle (au cœur du petit âge de glace) qui poussent les naturalistes à s'intéresser à ces masses de glace. La première question qui se pose est celle de la formation même des glaciers. Dès 1705, les frères Scheuchzer auront la bonne intuition en l'attribuant à l'accumulation des neiges à haute altitude. On s'étonne ensuite de la coexistence, à basse altitude, de vertes prairies et de glace. Cette observation conduit à admettre que le glacier s'écoule depuis les sommets sous l'effet de la gravité. En 1773, André César Bordier admet qu' "il est absolument nécessaire que de la glace nouvelle remplace perpétuellement celle qui a fondu; cette glace doit donc descendre en vertu de la pression des couches supérieures" (Bordier, 1773). Il faudra beaucoup de temps pour comprendre les processus qui gouvernent les déformations de ce matériau aux propriétés mécaniques originales qu'est la glace. Comment un solide peut-il "s'écouler" dans les fonds de vallée comme le suggère l'image même d'un glacier (figure 1.2)?

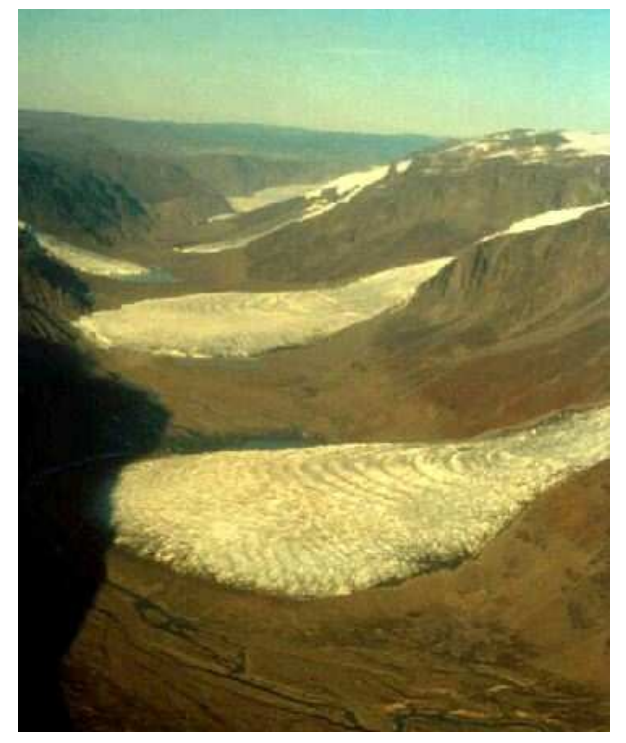

Fig. 1.2 - Photographie de glaciers de piedmont dans la vallée des Petites Roches (Evigedsfjord, Sud-ouest du Groenland). L'étalement de la glace dans la zone de faible pente en fond de vallée suggère que la glace a un comportement plastique. Photo : Louis Reynaud, 1994. 
H. B. de Saussure, scientifique suisse infatigable, second ascensionniste du Mont Blanc en 1787, pense que le glacier glisse d'un seul bloc sur les pentes (de Saussure, 1803). Cette théorie du glissement en masse n'explique pas la forme de ces bandes sombres et claires qui alternent sur la Mer de Glace (figure 1.3) et qui portent aujourd'hui le nom de l'anglais qui les a étudiées (Forbes, 1845). A cette hypothèse du glissement, d'autres scientifiques (de Charpentier, Agassiz, Tyndall) opposent donc une théorie qui fait la part belle aux cycles gel-degel. Pour essayer de trancher, des campagnes de mesure des vitesses sont organisées notamment par Agassiz, Forel et Vallot (figure 1.4).
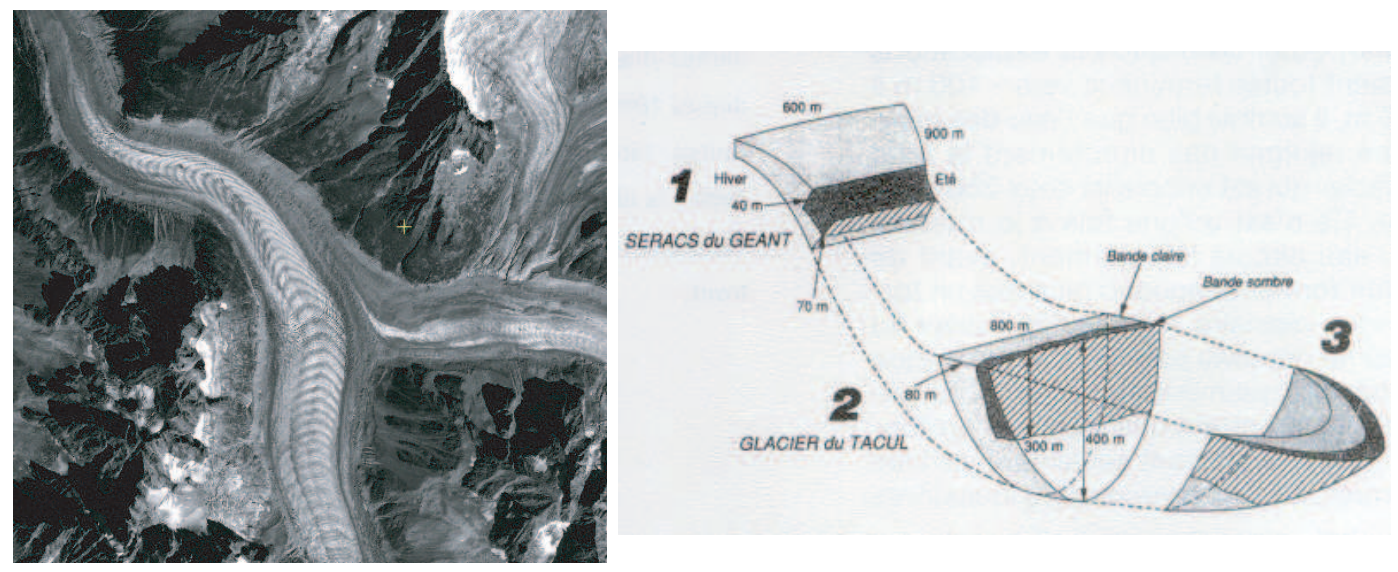

Fig. 1.3 - A gauche : la Mer de Glace observée par le satellite SPOT5 au cours de l'été 2003. On note l'alternance de bandes sombres et claires, ce sont les bandes de Forbes. Leur forme en ogive résulte de l'écoulement plus rapide du glacier au centre que sur les bords. A droite : bloc diagramme montrant la morphologie à 3 dimensions de ces bandes.

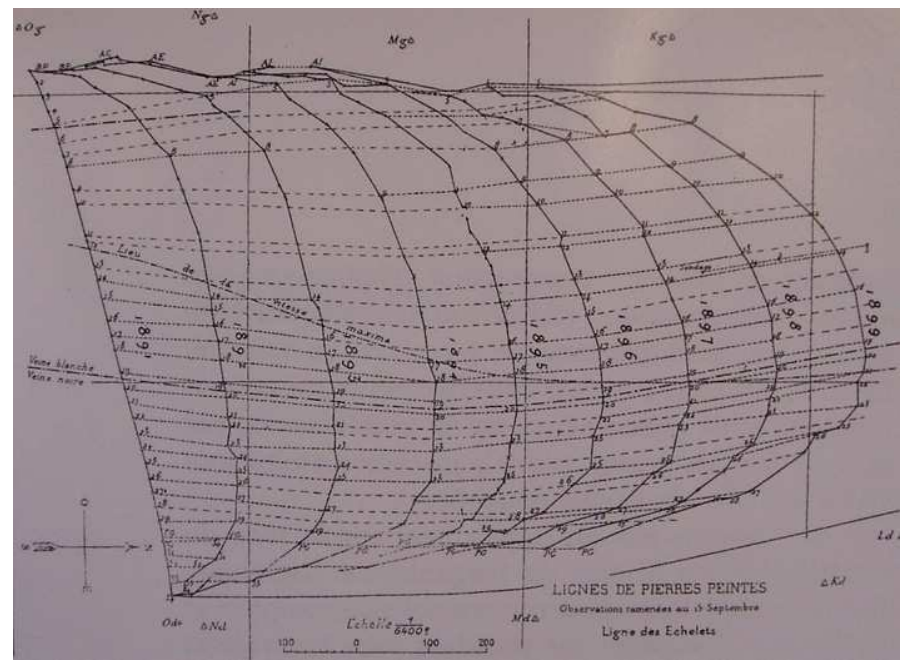

FIG. 1.4 - Premières mesures systématiques des vitesses de surface des glaciers par Vallot entre 1891 et 1899. Des pierres sont disposées à la surface de la Mer de Glace, sous les séracs du Géant, le long d'une droite en 1891. Mais les vitesses sont plus rapides (environ deux fois plus) au centre que sur les bords et l'alignement de pierres prend une forme arquée. Cette figure est extraite de Vivian (2001). 
C'est seulement au $\mathrm{XX}^{\text {eme }}$ siècle que les premières lois d'écoulement des glaciers sont proposées. D'abord, le glacier est considéré comme un fluide visqueux et Weinberg lui applique les équations de Navier \& Stokes. Ensuite, E. Orowan et M. F. Perutz, spécialistes de la physique des solides, proposent eux de traiter la glace comme un matériau plastique. Ce sont les expériences en laboratoire de Glen (Glen, 1955) qui permettront de trancher. Il montre que la glace a un comportement intermédiaire entre le fluide visqueux et le solide plastique. La loi de Glen est, aujourd'hui encore, utilisée par les glaciologues.

En parallèle à la compréhension de la déformation de la glace, dans la première moitié du XIX $^{e m e}$ siècle, les scientifiques découvrent que les glaciers n'ont pas toujours eu la même extension. On doit cette découverte aux discussions entre des guides de montagne, Venetz (ingénieur géologue suisse), de Charpentier (scientifique et directeur de mines de sels) et Agassiz (un jeune scientifique suisse). Mais c'est seulement le nom de ce dernier et sa fameuse publication "Etudes sur les glaciers" (Agassiz, 1840) que l'histoire retiendra. C'est l'observation d'énormes blocs erratiques à grande distance des glaciers (dans le Jura notamment) qui oblige à admettre une extension beaucoup plus grande dans le passé. Ces blocs sont alors protégés, l'un d'entre eux à Chamonix est même désigné comme monument national français! Plus tard, la chronologie de ces avancées glaciaires est précisée par A. Penck et E. Bruckner qui les désignent par des noms de rivières bavaroises : Würm, Riss, Mindel et Günz (Penck \& Brückner, 1909). Il faudra attendre Milankovitch et sa théorie astronomique des climats pour expliquer l'origine climatique de ces fluctuations glaciaires (Milankovitch, 1941; Berger, 1988).

Progressivement, les glaciologues sont donc parvenus à la compréhension du fonctionnement du glacier. Il se forme par accumulation et métamorphose de la neige à haute altitude. Sous son propre poids, la glace s'écoule vers les parties basses où elle disparaît essentiellement par fonte. Le volume du glacier est lié au climat puisque ce dernier détermine l'importance relative des gains et des pertes. L'extension glaciaire est certes liée à son volume mais dépend fortement de la dynamique de l'écoulement glaciaire. Nous décrirons plus en détail au chapitre 2, le cycle de vie de la glace au sein d'un glacier.

Si les méthodes ont beaucoup évolué depuis les début de la glaciologie, certains des objectifs sont toujours les mêmes : comprendre la dynamique de l'écoulement des glaciers, étudier la relation entre leur évolution et le climat. Les glaciologues disposent aujourd'hui de longues séries temporelles grâce à des programmes de mesures régulières sur le terrain qui ont été initiés par le Suisse F. A. Forel (Forel, 1895).

Hoelzle et al. (1998) résument en quatre points les enjeux de la glaciologie moderne :

1. Amélioration continue de la compréhension des processus glaciaires,

2. validation de modèles numériques pour prévoir l'évolution future des masses de glace,

3. analyse de la fonction d'indicateur climatique,

4. appréciation des conséquences directes et indirectes des variations glaciaires sur l'environnement.

Ces objectifs sont notamment motivés par l'observation d'un déclin important et croissant des glaciers de montagne dans les différentes régions de la planète. 


\subsection{Un recul marqué et quasi généralisé au $\mathrm{XX}^{\text {eme }}$ siècle}

Leur grande distribution géographique et leur sensibilité font des glaciers des indicateurs des changements climatiques. L'histoire nous a montré que cette observation n'est pas nouvelle et depuis plus d'un siècle, les glaciologues mesurent et suivent l'évolution des glaciers. Différents paramètres sont utilisés pour caractériser l'évolution d'un glacier donné (Lliboutry, 1964; Haeberli et al., 1998) :

1. l'avancée ou le recul du front glaciaire. Cette mesure ne peut être interprétée en terme de fluctuation climatique que si la série temporelle est suffisamment longue (Oerlemans, 1994; Hoelzle et al., 2003; Oerlemans, 2005) notamment au regard du temps de réponse du glacier. En revanche, les mesures sont nombreuses et permettent de remonter plus d'un siècle dans le passé.

2. les changements de la surface totale du glacier. Cette mesure peut être réalisée à partir de photographies aériennes ou d'images satellites. Les limites pour l'interprétation de changements de superficie sont les mêmes que pour les avancées ou recul du front du glacier.

3. les variations d'altitude de profils transversaux ou longitudinaux. A cause de la saisonnalité, ces profils doivent être réalisés chaque année au même endroit et à la même époque.

4. les évolutions du volume total du glacier ou de sa masse si la densité du matériel gagné ou perdu est connue.

Les Alpes sont le berceau de la glaciologie et c'est donc là que les séries de données sur l'évolution des glaciers sont les plus longues. Ceci permet de replacer les variations récentes dans une perspective à plus long terme et d'aborder une question importante : l'évolution actuelle des glaciers se situe-t-elle dans la gamme des fluctuations naturelles? Nous verrons ensuite si les observations sur les glaciers alpins peuvent être étendues aux autres chaînes de montagnes.

\subsubsection{Un exemple : évolution des glaciers alpins}

Les gravures de la vallée de Chamonix dans la première moitié du $\mathrm{XX}^{\text {eme }}$ siècle et leur comparaison avec des photos actuelles ne laissent place à aucun doute (figure 1.5).

Depuis la fin du petit âge de glace (il y a 150 ans environ) le recul des glaciers du massif du Mont Blanc a été important. Le détail de ce recul au cours du temps peut être précisé en regardant les variations de longueur pour différents glaciers (figure 1.6). Ces longueurs sont mesurées depuis 1870 dans les Alpes mais peuvent être reconstituées pour les périodes antérieures en observant des peintures ou des textes historiques (Vincent et al., 2005a). Entre 1600 et 1850 (non montré, cf. Vincent et al. (2005b)), ces longueurs fluctuent ( $\pm 200 \mathrm{~m}$ par an) autour d'une valeur moyenne supérieure de plus d'un kilomètre à la valeur actuelle. Depuis 1850, la tendance pour tous les glaciers est donc au retrait du front glaciaire.

Ce retrait n'est pas identique pour tous les glaciers. Il est presque linéaire au cours du temps pour les glaciers d'Aletsch $\left(127 \mathrm{~km}^{2}\right)$ et de Saint Sorlin $\left(3 \mathrm{~km}^{2}\right)$ alors que des fluctuations décennales existent pour les glaciers d'Argentière et des Bossons. Pour ces deux derniers glaciers, distants de quelques kilomètres et donc sujets au même climat, les fluctuations ne sont pas synchrones : le glacier des Bossons, plus pentu et plus rapide, réagit avec un temps de réponse plus court que le glacier d'Argentière. Cela illustre la difficile interprétation des changements de longueur sur le court terme, ces derniers étant principalement contrôlés par la dynamique de l'écoulement glaciaire (Jóhannesson et al., 1989; Paterson, 1994). 


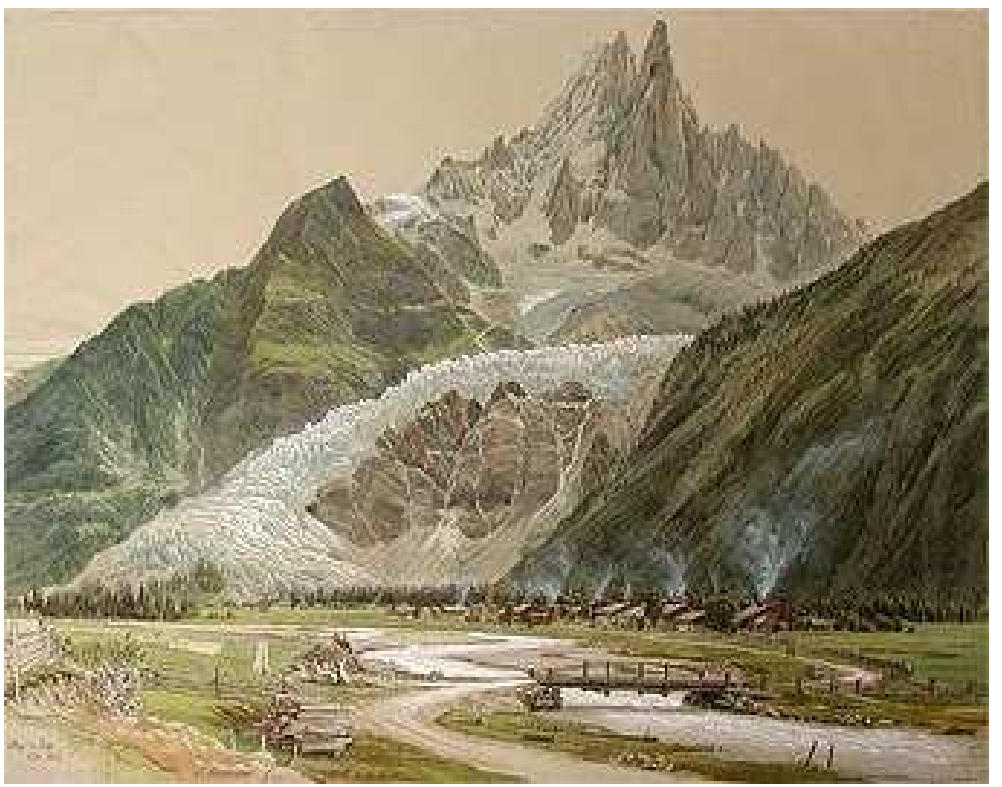

FIG. 1.5 - Lithographie de la vallée de Chamonix en 1823 (J. Dubois). La Mer de Glace, qu'on nommait alors le glacier des Bois, descendait jusque dans la vallée à une altitude de $1100 \mathrm{~m}$ environ. Aujourd'hui, le front de ce glacier se situe bien en amont, à $1550 \mathrm{~m}$.
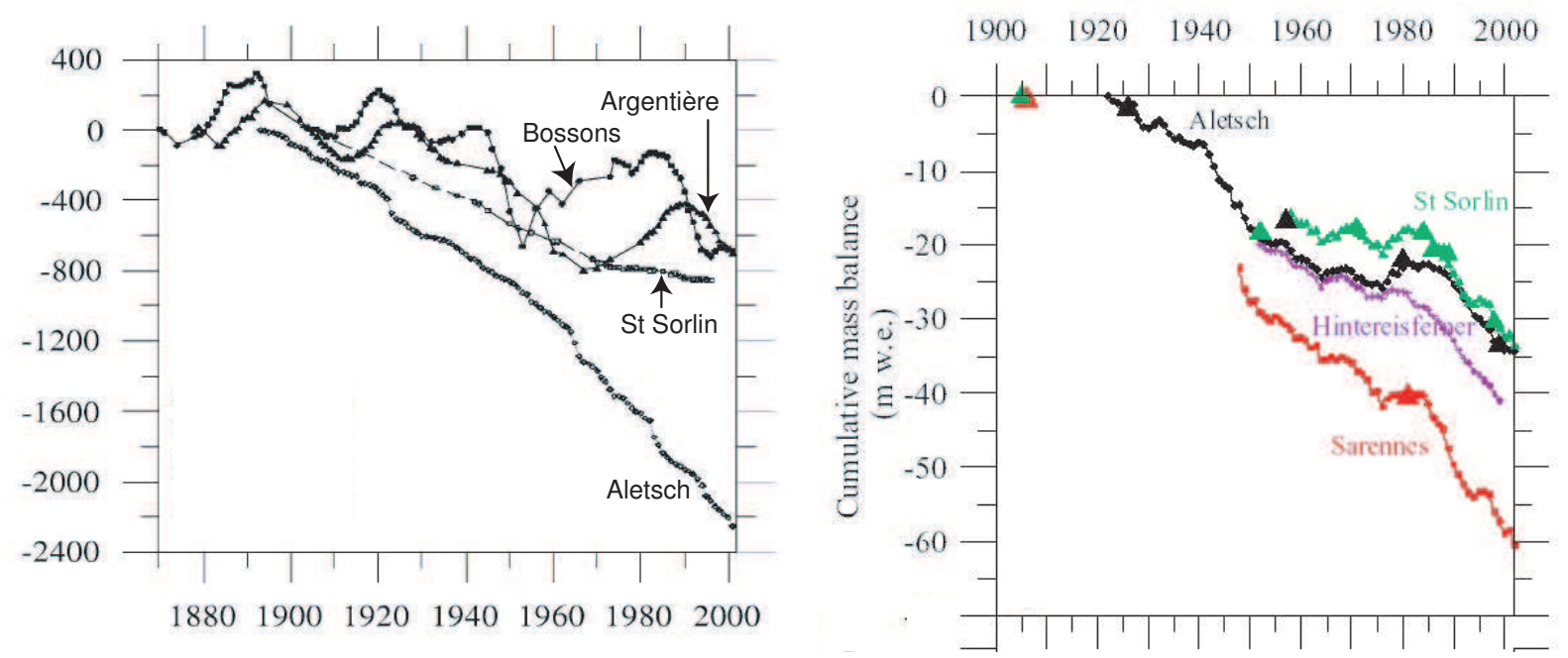

FIG. 1.6 - A gauche : évolution des longueurs de différents glaciers des Alpes françaises et suisses au cours des 130 dernières années. D'après Vincent et al. (2005a). A droite : Bilans de masse cumulés de 4 glaciers des Alpes françaises et suisses. Les triangles indiquent les mesures photogrammétriques (cf. §2.1.3.4) qui permettent de contrôler et recaler les bilans mesurés sur le terrain. D'après Vincent et al. (2004).

Pour extraire un signal commun aux glaciers alpins et le relier aux paramètres climatiques, il faut étudier les séries de bilans de masse. La partie droite de la figure 1.6 permet de comparer les plus longues séries de bilans de masse disponibles dans des Alpes.

Ces séries de bilans cumulés montrent une nette tendance à la diminution de masse. Cette 
tendance est différente d'un glacier à l'autre à cause de facteurs comme l'exposition, et l'hypsométrie $^{6}$. Ainsi, pour la période 1953-1999 le bilan de masse moyen (exprimé sous forme d'une lame d'eau donc en mètres w.e. ${ }^{7}$ ) est de $-0.7 \mathrm{~m}$ w.e./an pour Sarennes, $-0.33 \mathrm{~m}$ w.e./an pour St Sorlin et seulement -0.1 m w.e./an pour Argentière. De plus, une rupture de pente apparaît dans la série des bilans de masse au début des années 1980. Les bilans de masse sont équilibrées (St Sorlin), positifs (Argentière) ou légèrement négatif (Sarennes, Hintereisferner) entre 1954 et 1981, tous fortement négatifs ensuite (Vincent, 2002; Vincent et al., 2004). Cette cohérence entre glaciers démontre qu'ils répondent à un signal climatique commun.

En étudiant séparément le bilan de masse hivernal et estival de deux glaciers éloignés de $150 \mathrm{~km}$ (Sarennes et Claridenfirn), cette rupture de pente à partir de 1981 a pu être expliquée par une augmentation forte du taux d'ablation. Ceci confirme la sensibilité des glaciers alpins aux variations de température (Vincent et al., 2004).

En conclusion, les variations de longueur des glaciers alpins ne sont pas synchrones. Une tendance au recul du front est toutefois sensible depuis la fin du petit âge de glace (1850). Les séries de bilans de masse concordent entre les différents glaciers et permettent d'expliquer leur déclin récent par l'augmentation des températures estivales. Ce signal n'est pas uniquement observé pour les glaciers de l'arc alpin mais peut être généralisé à presque tous les massifs de montagne.

\subsubsection{Variations régionales autour du thème du recul des glaciers}

A partir de l'année géophysique internationale (1957-1959) et surtout depuis le début des années 1960, les mesures glaciologiques se sont développées hors de l'Europe (Dyurgerov, 2002). Les séries de mesures ne sont pas toujours continues, les interruptions ayant parfois des origines politiques comme dans le cas du glacier Abramov (Kyrgyzstan) où les études glaciologiques ont cessé en 1999 suite à l'attaque de la station scientifique par des opposants au gouvernement.

Le suivi de la majorité des glaciers se fonde sur des mesures in situ du bilan de masse à partir de balises dans la partie basse et de carottages dans la zone d'accumulation (Braithwaite, 2002). Mais ce n'est pas la seule possibilité et un panorama détaillé des différentes méthodes sera dressé au chapitre 2.

Quelles que soient les méthodes de mesures, à de rares exceptions près, la tendance est la même dans beaucoup de régions du monde. Les glaciers sont en net recul depuis le petit âge de glace. Cela apparaît souvent nettement dans le paysage sous la forme de larges moraines qui marquent l'avancée maximale des glaciers au milieu du XIX ${ }^{\text {eme }}$ (figure 1.7).

Pour la période récente, ce recul a été confirmé par des observations détaillées dans différentes régions du monde. De plus, lorsque ces études distinguent plusieurs périodes, presque toutes concluent que les pertes de masse des glaciers s'accélèrent avec le temps. C'est le cas pour des régions aussi éloignées que l'Alaska (Arendt et al., 2002), la Patagonie (Rignot et al., 2003), les Andes (Arnaud et al., 2001; Francou et al., 2004), l'Asie centrale (Khromova et al., 2003; Shahgedanova et al., 2005) ou les Alpes (Paul et al., 2004). Dans les régions tropicales, nombre de petits glaciers sont sur le point de disparaître (Francou et al., 2003). A l'échelle globale, cette accélération des pertes se situerait à la fin des années 1980 (Dyurgerov, 2003).

\footnotetext{
${ }^{6}$ L'hypsométrie désigne la répartition des surfaces d'un glacier en fonction de l'altitude.

${ }^{7}$ w.e. est la notation classique pour water equivalent donc équivalent en eau
} 


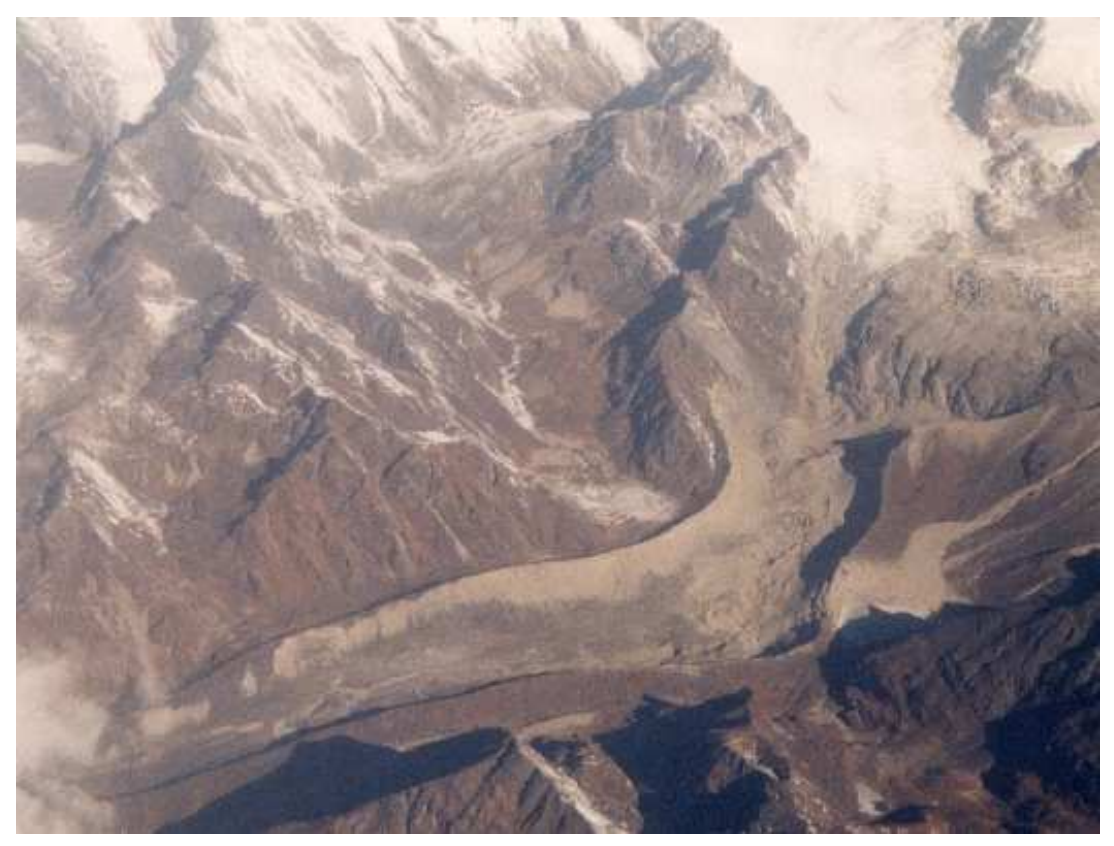

FIG. 1.7 - Moraine du petit âge de glace en Himalaya. Cette moraine est un véritable squelette externe du glacier et permet de reconstituer son volume il y a environ 150 ans. D'après http ://www.glims.org.

Un bémol doit être apporté à ce paysage semble-t-il uniforme (figure 1.8). En Scandinavie et en Nouvelle-Zélande, certains glaciers montrent un bilan de masse positif (Tvede \& Laumann, 1997; Chinn, 1999; IPCC, 2001). Le point commun de ces deux régions est l'influence d'un climat maritime. L'accumulation joue un rôle important pour la détermination du bilan annuel. Des précipitations accrues, en lien avec une intensification du cycle hydrologique, expliquent ces bilans de masse positifs.

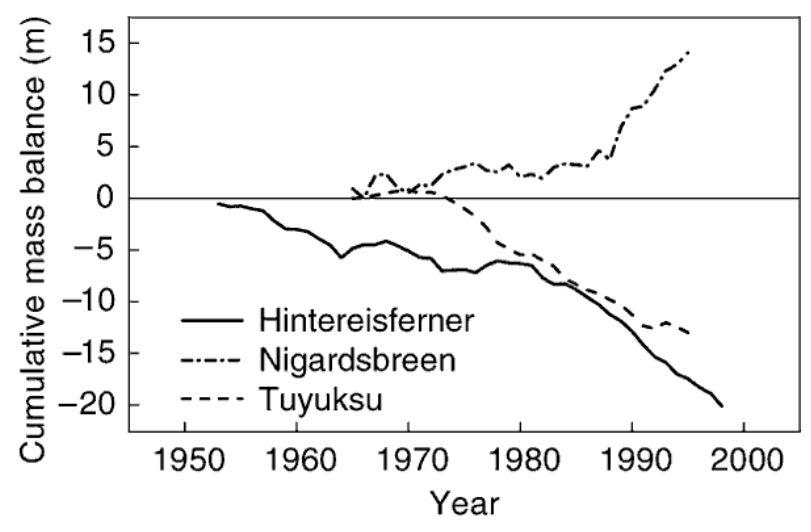

FIG. 1.8 - Bilan de masse cumulé de 3 glaciers situés dans des régions climatiques différentes. Hintereisferner (Alpes autrichiennes), Nigardsbreen (Norvège), Tuyuksu (Tien Shan, Kazakhstan). D’après IPCC (2001).

A l'exception de ces quelques glaciers sous influence océanique, les inventaires à l'échelle de la planète concluent tous à des pertes de masse importantes des glaciers de montagne (IPCC, 
2001). Les glaciers montrent une réaction croissante aux variations climatiques et donc une accélération de leur déclin. Ils témoignent aussi d'une intensification du cycle hydrologique puisqu'à la fois l'accumulation et l'ablation augmentent (Dyurgerov, 2003). Cette évolution rapide a des conséquences importantes aux échelles locales comme globales.

\subsection{Conséquences du recul des glaciers}

Le déclin de l'ensemble des glaciers a des conséquences globales, parce qu'ils contribuent à l'élévation du niveau marin, mais aussi locales car ils constituent une ressource en eau et en énergie importante pour de nombreuses populations.

\subsubsection{Contribution à la hausse du niveau marin}

A l'échelle du quaternaire, ce sont surtout les fluctuations des calottes polaires qui contrôlent l'élévation du niveau marin. L'analyse des isotopes de l'oxygène des carottes sédimentaires a montré qu'en période de maximum glaciaire, la teneur en ${ }^{18} 0$ dans les eaux océaniques est plus élevée qu'actuellement. Ceci s'explique par le stockage d'une grande quantité d'eau aux pôles sous forme de glace appauvrie en ${ }^{18} 0$. La croissance des calottes polaires explique que le niveau marin ait été $120 \mathrm{~m}$ plus bas au dernier maximum glaciaire, il y a 18000 ans environ (Jouzel et al., 2002).

Le niveau des mers monte de $1.5 \pm 0.5 \mathrm{~mm} /$ an environ (IPCC, 2001) depuis le début du siècle (figure 1.9). Cette montée s'accélère pour atteindre près de $3 \mathrm{~mm} /$ an pour la période 1993-2003 (Cazenave \& Nerem, 2004). Jusqu'à peu, certains auteurs (Cabanes et al., 2001) pensaient que la contribution stérique (effet de dilatation des eaux dont la température augmente) expliquait la majorité de cette montée. Miller \& Douglas (2004) puis Lombard et al. (2005) ont revu récemment à la baisse le rôle de la dilatation suggérant que le déclin de la cryosphère est le principal responsable de la hausse du niveau marin. A ces courtes échelles de temps, la contribution des calottes polaires est faible ou du moins discutée (IPCC, 2001; Rémy et al., 2002; Thomas et al., 2004; Davis et al., 2005). En revanche, leur retrait rapide fait des glaciers de montagne l'un des principaux acteurs de l'élévation actuelle.

La détermination de la contribution des glaciers est un exercice difficile. Il s'agit d'estimer l'évolution de la masse de 160000 glaciers répartis sur toute la planète. C'est Meier (1984) qui le premier souligne que les glaciers de montagne et les petites calottes ont un effet significatif sur le niveau des mers. D'après cette étude, ils sont à l'origine de $0.46 \pm 0.26 \mathrm{~mm} /$ an de l'élévation des océans entre 1900 et 1961. Cette estimation, comme celles qui suivront, utilise un inventaire des glaciers à la surface du globe et extrapole les mesures de bilan de masse de quelques glaciers "modèles" (Braithwaite \& Raper, 2002). Une estimation plus récente (Dyurgerov, 2003) révise la contribution des glaciers pour des périodes plus récentes : $0.15 \mathrm{~mm} / \mathrm{an}$ en 1961-1976, 0.24 $\mathrm{mm} /$ an en 1977-1987 et $0.41 \mathrm{~mm} /$ an en 1988-1998 soit 10, 16 et 27\% de l'élévation totale.

Pourtant, plusieurs études récentes suggèrent que la fonte des glaciers pourrait être plus importante. Arendt et al. (2002) ont montré que l'amincissement des glaciers d'Alaska pour la période 1995-2001 est équivalent à $0.27 \mathrm{~mm} /$ an d'élévation du niveau des mers (figure 1.10). La contribution des champs de glaces Nord et Sud de la Patagonie est de $0.1 \mathrm{~mm} /$ an (Rignot et al., 2003 ) et celle des petites calottes de glace de l'Arctique canadien de $0.064 \mathrm{~mm} / \mathrm{an}$ (Abdalati

et al., 2004). La fonte des glaciers de la péninsule antarctique (qui sont séparés de la calotte 
polaire) pourrait expliquer $0.1 \mathrm{~mm} /$ an (Rignot et al., 2005). A elles seules, ces quatre régions contribueraient donc à $0.53 \mathrm{~mm} /$ an ce qui dépasse les $0.41 \mathrm{~mm} /$ an proposés par Dyurgerov (2003). Le décalage temporel entre les différentes études peut expliquer partiellement ces différences. Le tableau 1.3 synthétise les études les plus récentes et détaille les contributions de différentes régions englacées (les calottes polaires ont été incluses). Ce tableau n'est pas exhaustif, certaines régions (l'Himalaya par exemple) n'ayant pas fait l'objet d'inventaires récents.
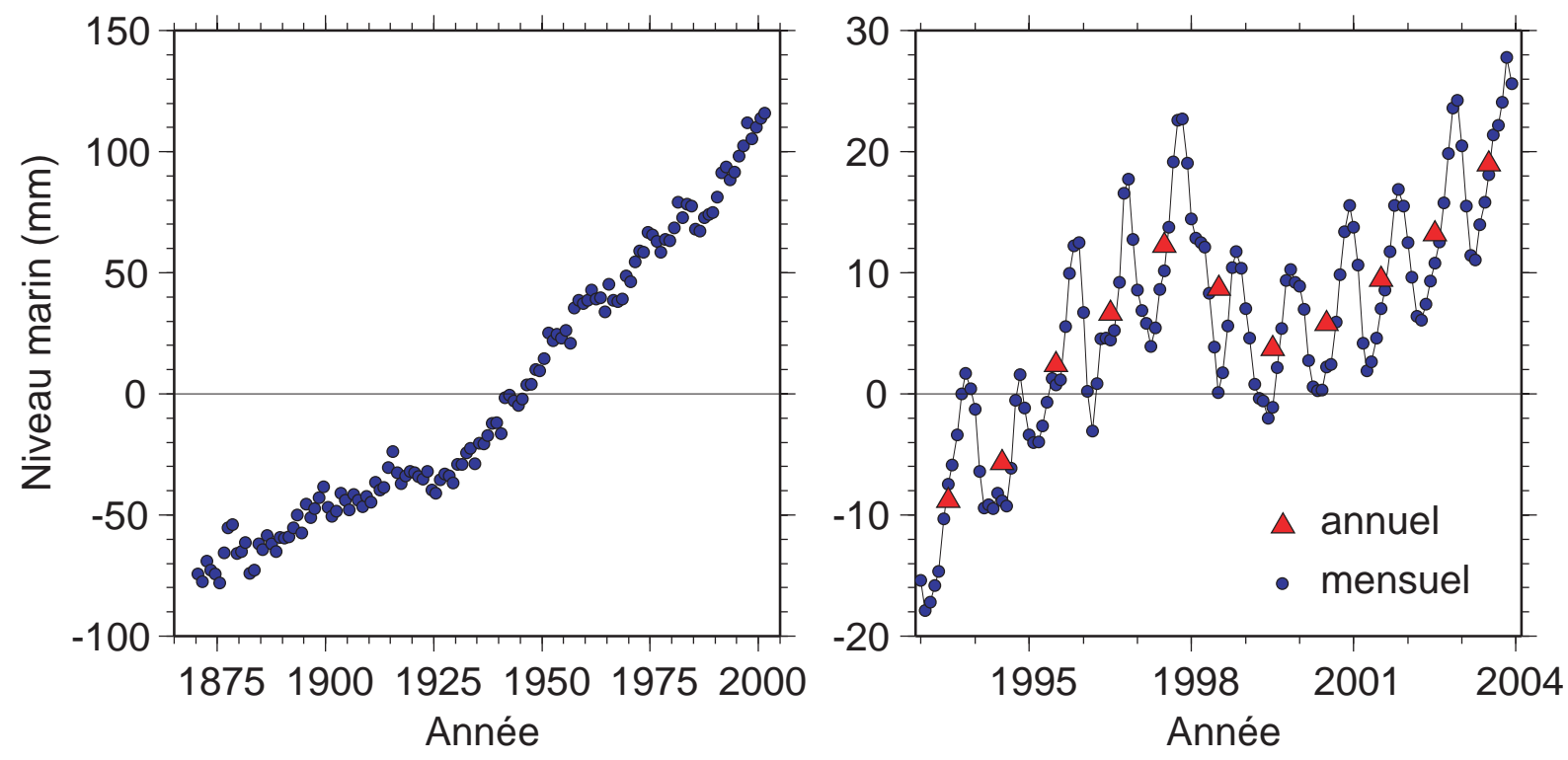

FIG. 1.9 - Élévation du niveau moyen des mers depuis 1880 (à gauche, synthèse des données marégraphiques Church et al. (2004)) et pour la période 1993-2004 (à droite à partir de l'altimétrie satellitaire, Do Minh, communication personnelle).
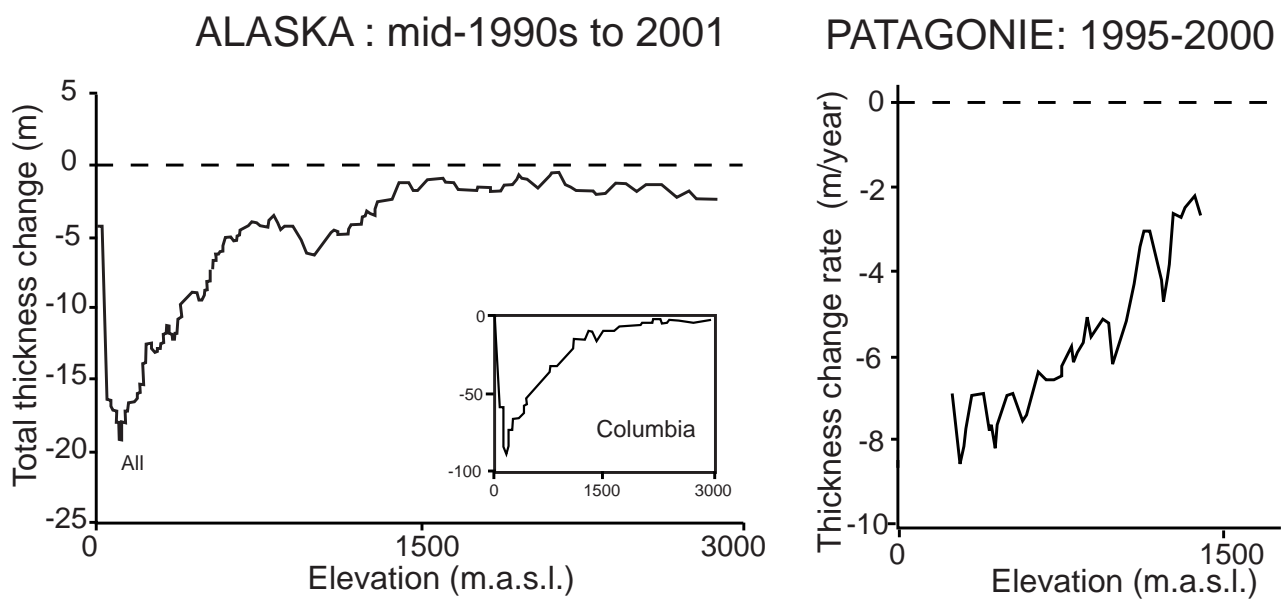

FIG. 1.10 - Variation d'épaisseur des glaciers d'Alaska (à gauche) et du champ de glace Sud de la Patagonie (à droite) en fonction de l'altitude. Ces courbes montrent pour les deux régions un amincissement marqué à basse altitude. Ces figures ont été adaptées de celles publiées par Arendt et al. (2002) et Rignot et al. (2003). Pour l'Alaska c'est le changement d'épaisseur total qui est montré alors que pour la Patagonie c'est le taux annuel de variation d'épaisseur. 
TAB. 1.3 - Contribution de quelques régions englacées à l'élévation du niveau des mers. A désigne l'Antarctique. On notera que les incertitudes sont rarement fournies et, lorsqu'elles existent, nous semblent peu réalistes (à l'exception de la barre d'erreur de Arendt et al. (2002)).

\begin{tabular}{lcccl}
\hline Région & Contribution (m/an) & Période & Incertitude & Référence \\
\hline Alaska & 0.27 & $1995-2001$ & \pm 0.1 & Arendt et al. (2002) \\
Patagonie & 0.1 & $1995-2000$ & \pm 0.01 & Rignot et al. (2003) \\
Arctique canadien & 0.06 & $1995-2000$ & & Abdalati et al. (2004) \\
Péninsule A. & 0.1 & $1995-2004$ & & Rignot et al. (2005) \\
Groenland & 0.2 & $1997-2003$ & & Krabill et al. (2004) \\
A. de l'Ouest & 0.2 & $2002-2003$ & & Thomas et al. (2004) \\
A. de l'Est & -0.12 & $1991-2004$ & \pm 0.02 & Davis et al. (2005) \\
\hline Total Glaciers & 0.53 & & & \\
Total Cryosphère & 0.81 & & & \\
\hline
\end{tabular}

Les projections pour le siècle à venir sont entachées de fortes incertitudes. Elles se fondent sur l'estimation de la sensibilité des glaciers à l'élévation de la température et sur des scénarios de changement climatique. Toutes ces projections prédisent une contribution croissante des glaciers à l'élévation du niveau des mers qui accompagnera la disparition de $1 / 4$ des glaciers d'ici 2050 et de la moitié d'ici 2100 (Kuhn, 1993; Oerlemans et al., 1998).

\subsubsection{Impact hydrologique : perturbation de la ressource en eau}

Un rapport récent, publié par le WWF, dresse un état des lieux de l'évolution des glaciers en Himalaya (WWF, 2005). Une des conclusions les plus alarmantes de ce rapport concerne le déclin de la ressource en eau douce pour cette région du globe. Pendant la saison sèche et l'étiage des rivières, une partie substantielle du débit des rivières provient de la fonte des neiges et des glaciers. Les glaciers jouent un rôle tampon en stockant l'eau sous forme solide en saison humide et en la restituant sous forme liquide en saison sèche. Cette eau de fonte alimente les populations indiennes, chinoises et népalaises et est cruciale pour leur boisson, irrigation et industrie. Le déclin des glaciers devrait, lors des prochaines décennies, engendrer des débits plus importants des rivières, conduisant à un accroissement du nombre d'inondations et de glissements de terrain (IPCC, 2001). En revanche, sur le long terme, la réduction de la taille et du nombre des glaciers empêchera qu'ils jouent leur rôle régulateur des débits. Ainsi, le débit du Gange en Inde pourrait être divisé par trois pour la période juillet-septembre. L'approvisionnement en eau de 500 millions de personnes et d'un tiers des zones irriguées de l'Inde seraient alors perturbés.

Des conclusions similaires peuvent être tirées pour les régions andines, notamment dans la Cordillère Royale bolivienne. Là aussi, les glaciers régulent les débits alimentant la ville de La Paz et les cultures de l'Altiplano entre mai et septembre, la saison sèche (Ribstein et al., 1995).

Plus de la moitié des êtres humains dépendent plus ou moins directement de l'eau qui descend des montagnes. Le déclin actuel et futur des glaciers va donc engendrer de forts déséquilibres 
hydrologiques qui auront des conséquences humanitaires et écologiques majeures. Prévoir ces déséquilibres pour mieux les anticiper (faute de pouvoir les empêcher) semble un objectif prioritaire pour la recherche en glaciologie.

\subsubsection{L'accroissement du risque glaciaire}

Au sein de la famille des risques naturels, le risque glaciaire n'occupe pas une place centrale. Les catastrophes engendrées par les glaciers ont des ampleurs plus faibles que celles occasionnées par les sécheresses, les séismes, les cyclones ou les tsunamis. De plus, elles touchent des régions de haute montagne généralement peu peuplées. Pourtant, le risque glaciaire existe et devrait s'accentuer dans les décennies à venir à cause du retrait des glaciers.

Il ne s'agit pas ici d'établir un inventaire exhaustif des risques liés aux glaciers. Un tel inventaire a été établi récemment pour les Alpes françaises (Buisson et al., 1999). La figure 1.11 est une synthèse illustrant quelques risques liés au glacier. De ce rapport, quatre principaux risques émergent et sont brièvement décrits.

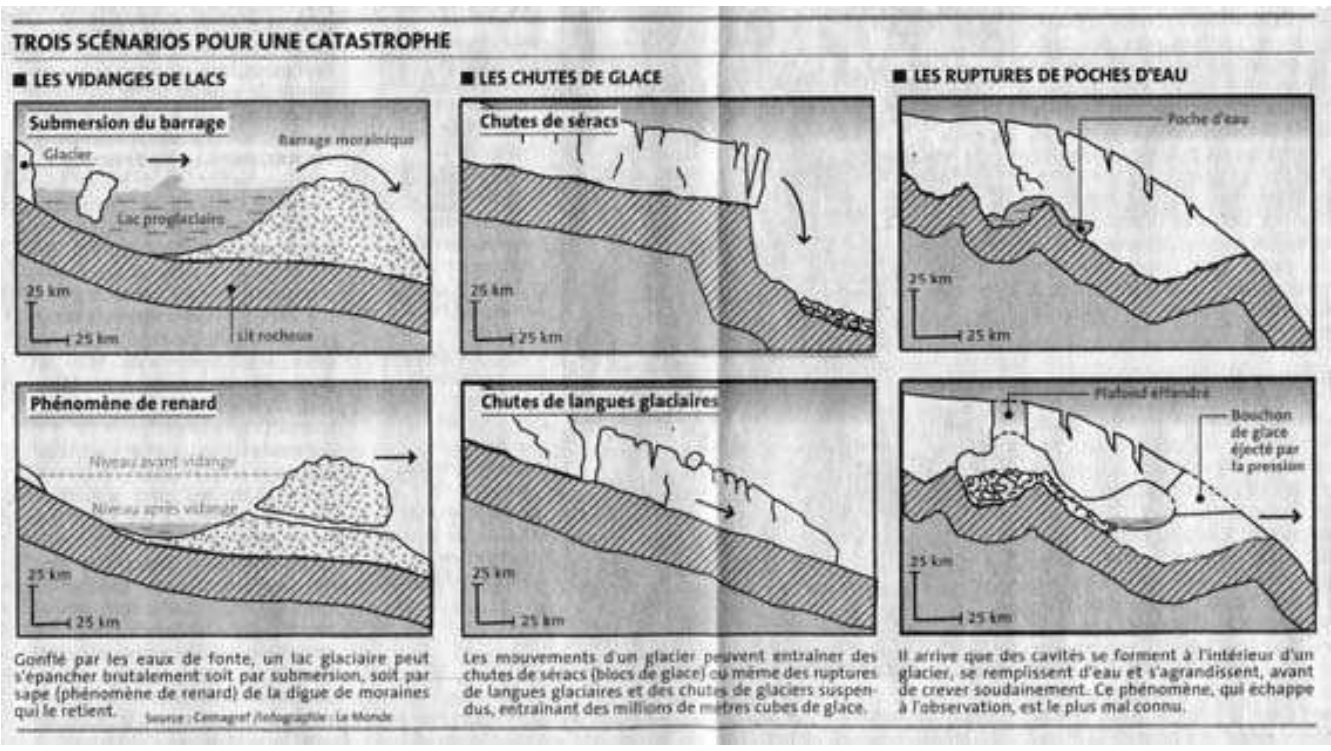

FIG. 1.11 - Les différents risques associés aux glaciers (extrait du "Monde" du 26 juin 2002). Ces différents phénomènes ont été étudiés dans le cadre du projet européen GLACIORISK (http ://glaciorisk.grenoble.cemagref.fr/).

\subsubsection{La vidange de lacs pro- et supra- glaciaires}

En se retirant, les glaciers laissent à leur front des moraines jouant le rôle de barrages naturels. Les eaux de fonte s'accumulent alors dans des lacs à l'avant ou même sur le glacier. Mais la barrière morainique est souvent mal consolidée et son étanchéité est mise à mal lors de la fonte de la glace morte qu'elle peut contenir (Lemarchand, 2002). La figure 1.12 montre un lac proglaciaire en cours de développement au front de la Mer de Glace (massif du Mont Blanc). Des lacs supra-glaciaires sont aussi en croissance comme celui de Rochemelon dans les Alpes.

La rupture de la barrière morainique (fonte de la glace morte, vague créée par la chute d'un bloc de glace) libère une grande partie de l'eau du lac qui s'écoule vers le vallée sous la 
forme de lave torrentielles très rapides et dévastatrices. Le débordement de la lagune Palcacocha (Cordillère Blanche, Pérou) a ainsi détruit une partie de la ville de Huaraz (Pérou) en 1941. 7000 personnes disparurent lors de cette catastrophe.

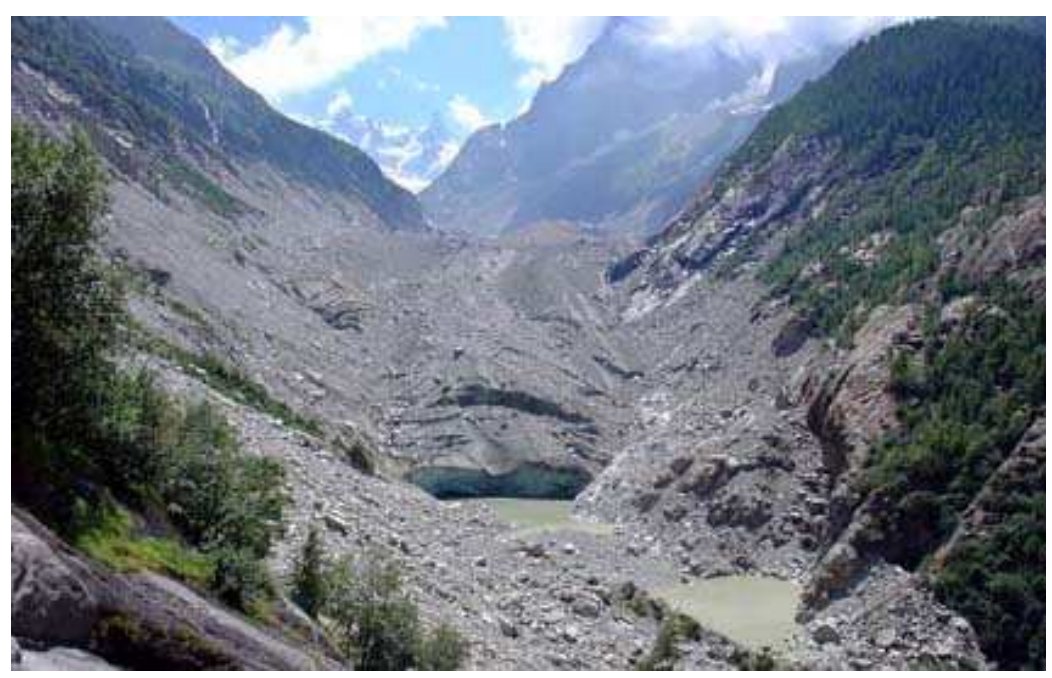

Fig. 1.12 - Lac pro-glaciaire en cours de développement au front de la Mer de Glace. Les eaux des deux lacs sont retenues par des dépôts morainiques récents et mal consolidés. Le lac le plus proche du glacier érode le front glaciaire à sa base ce qui peut provoquer des chutes de blocs de glace. Photo J. Nomade http ://meije.univ-savoie.fr/jnomade/jnoma.htm.

Avec le retrait accéléré des glaciers et la montée des températures, la fréquence de ces évènements devrait augmenter. Une surveillance accrue est donc nécessaire et permet, quand le danger devient trop important, de procéder à des vidanges contrôlées de ces lacs comme dans le cas de Rochemelon lors de l'été 2004 (figure 1.13).

\subsubsection{Vidange de poches d'eau sous-glaciaires}

Un autre risque est la vidange de poches d'eau situées à l'intérieur du glacier. En 1892, une poche d'eau de $200000 \mathrm{~m}^{3}$ d'eau au sein du glacier de Tête-Rousse s'est rompu, emportant près de $100000 \mathrm{~m}^{3}$ de glace et $300000 \mathrm{~m}^{3}$ de roches et débris. La vitesse de la lave torrentielle, qui a détruit une partie du village du Fayet (près de Saint Gervais en Haute-Savoie), a été estimée à $14 \mathrm{~m} / \mathrm{s}$.

Nous détaillerons dans le chapitre 6, le cas particulier du lac sous-glaciaire de Grímsvötn dont la vidange quasi-périodique engendre elle aussi des crues dévastatrices.

\subsubsection{Risque sismique associé au recul des glaciers.}

Une étude récente montre que le retrait des glaciers pourrait provoquer des séismes (Sauber \& Molnia, 2004). Quand le glacier se retire, la charge qu'il exerçait sur les roches sous-jacentes disparaît ce qui facilite le déclenchement de séismes. Un séisme de magnitude 7.2 en 1979 pourrait avoir été déclenché par le rapide retrait des glaciers en Alaska. 


\section{Le réchauffement accroît les risques de vidange accidentelle de lacs glaciaires $\mathfrak{S}_{\mathbb{R}}$ ITtonde.fr}

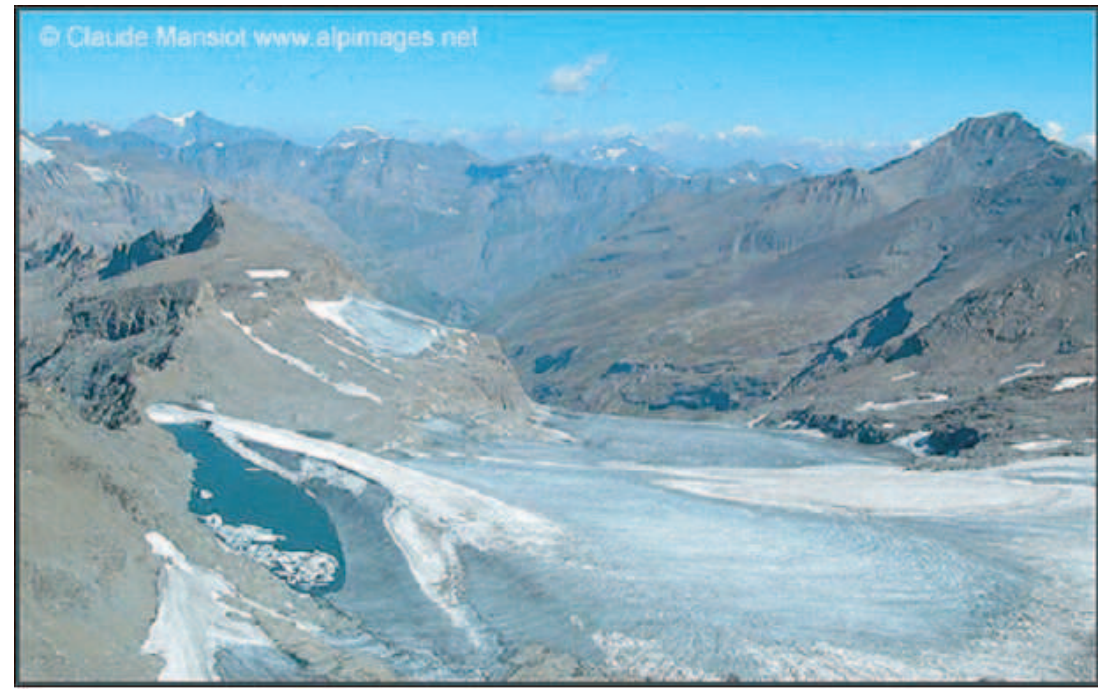

FIG. 1.13 - Extrait du "Monde" du 9 octobre 2004. Le lac supra-glaciaire de Rochemelon (Vanoise) est apparu au milieu des années 1980 et ne cesse de crô̂tre depuis. Les fortes pertes d'épaisseur du glacier au cours des étés 2003 et 2004 ont conduit le lac à des niveaux alarmants. Des vidanges du lac permettent d'éviter son débordement et de protéger les communes situées en aval.

\subsubsection{Chute de séracs}

Enfin, les chutes de séracs ${ }^{8}$ sont un dernier type d'accident glaciaire. Ce processus est parfaitement naturel, résultat de la fracturation du glacier et de son écoulement. Cet aléa se transforme en risque quand les hommes (alpinistes le plus souvent) s'en approchent de trop près ou quand le sérac tombe dans un lac glaciaire. Les chutes de séracs hivernales peuvent déstabiliser le manteau neigeux et déclencher des avalanches.

Dans un contexte de fort déclin, les risques liés aux glaciers sont de plus en plus importants. C'est surtout le rapide développement de fragiles lacs au voisinage des glaciers qui est le plus préoccupant. Nous verrons que l'imagerie satellitaire peut contribuer à la surveillance de ces zones à risques.

\subsubsection{Impacts économique et touristique}

L'énergie hydroélectrique est fondamentale pour l'économie de nombreux pays (Népal, Bolivie, Norvège ou Islande par exemple). La réduction programmée des débits de nombreux cours d'eau risque donc de poser des problèmes énergétiques importants pour des pays en forte croissance économique. L'irrigation des cultures pourrait, elle aussi, souffrir de ces débits plus faibles.

\footnotetext{
${ }^{8}$ Un sérac est un bloc de glace provenant du morcellement du glacier par un réseau de crevasses. Sa taille peut atteindre celle d'un immeuble de 3 ou 4 étages.
} 
Les glaciers attirent et fascinent chaque année de nombreux touristes dans des sites comme le Montenvers au dessus la Mer de Glace. Avec son retrait, le glacier se couvre de roches et poussières et se transforme progressivement en glacier noir, perdant son attractivité. En Bolivie, l'unique station de ski (la plus haute du monde!) sur le glacier de Chacaltaya ne fonctionne plus depuis une dizaine d'années car c'est de la glace vive qui affleure. En Suisse, une station de ski s'apprête même à emballer une partie d'un glacier pour le protéger de la forte fonte estivale! Nos tentatives pour limiter les conséquences du réchauffement climatique souligne notre impuissance à lutter contre les causes...

\subsection{Conclusions}

Ce chapitre nous a permis de présenter les glaciers, les premiers développements de la science qui les étudie et leur place au sein du système climatique. Les glaciers entretiennent une relation étroite avec le climat :

Le glacier est tout d'abord un acteur climatique. A l'échelle locale, cette masse de glace à $0^{\circ} \mathrm{C}$ (au plus) entretient un microclimat froid qui limite la fonte et facilite les précipitations solides. Ceci lui permet de s'auto-entretenir : il crée en effet autour de lui les conditions nécessaires à son maintien. A l'échelle globale, les glaciers peuvent aussi être acteurs de changements climatiques brutaux. Ainsi, un apport accru d'eau douce dans l'Atlantique Nord, lié à la fonte des glaciers des régions arctiques et du Groenland, pourrait ralentir la circulation thermohaline modifiant la distribution des climats à l'échelle planétaire.

Mais les glaciers de montagnes constituent aussi d'excellents indicateurs climatiques car :

- leur bilan de masse répond chaque année aux fluctuations de paramètres climatiques comme les températures ou les précipitations. C'est leur caractère "tempéré" (décrit plus haut) qui explique cette réactivité.

- La morphologie glaciaire répond, elle, en quelques dizaines d'années à des changements du climat. Cette échelle de temps est perceptible par l'homme et voisine de celle des changements climatiques qui affectent la planète depuis un peu plus d'un siècle. Dans certains cas, ces changements morphologiques menacent les hommes ou leurs activités.

Mais avant d'utiliser le glacier comme indicateur climatique ou d'étudier l'adaptation de sa dynamique à une nouvelle morphologie, il convient de comprendre son fonctionnement en régime stationnaire. Le chapitre suivant nous permet donc de présenter les définitions de base concernant un glacier type, l'équilibre entre l'accumulation et l'ablation, les lois qui régissent son écoulement. Une attention particulière sera dédiée aux techniques de mesures in situ. 



\section{Chapitre 2}

\section{Naissance, vie \& mort d'un glacier de montagne}

\section{Sommaire}

2.1 Naissance \& mort de la glace : accumulation $v s$ ablation . . . . . . . . . 38

2.1 .1 L'accumulation et la formation de la glace . . . . . . . . . . . . . 38

2.1 .2 Les processus d'ablation . . . . . . . . . . . . . . . . . 39

2.1.3 Le bilan de masse : indicateur de l'état de santé du glacier . . . . . . . . . . . . . . 40

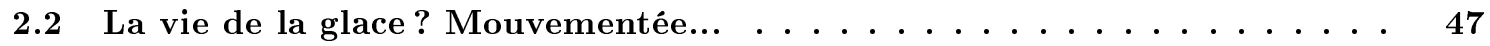

2.2 .1 Mesure de l'écoulement glaciaire . . . . . . . . . . . . . . . . 47

2.2 .2 La déformation interne de la glace . . . . . . . . . . . . . . . . . . 49

2.2 .3 Le glissement ou dérapage basal . . . . . . . . . . . . . . . . . . . 51

2.2.4 Variation saisonnière et inter-annuelle de l'écoulement . . . . . . . . . . . . 53

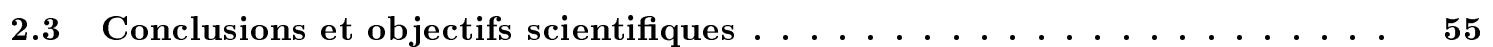

La figure 2.1 définit les principales zones d'un glacier de montagne. Le but de ce chapitre est de détailler les différents processus qui contrôlent le fonctionnement du glacier et les méthodes qui permettent de les étudier. Les méthodes satellitaires seront détaillées au chapitre 3.

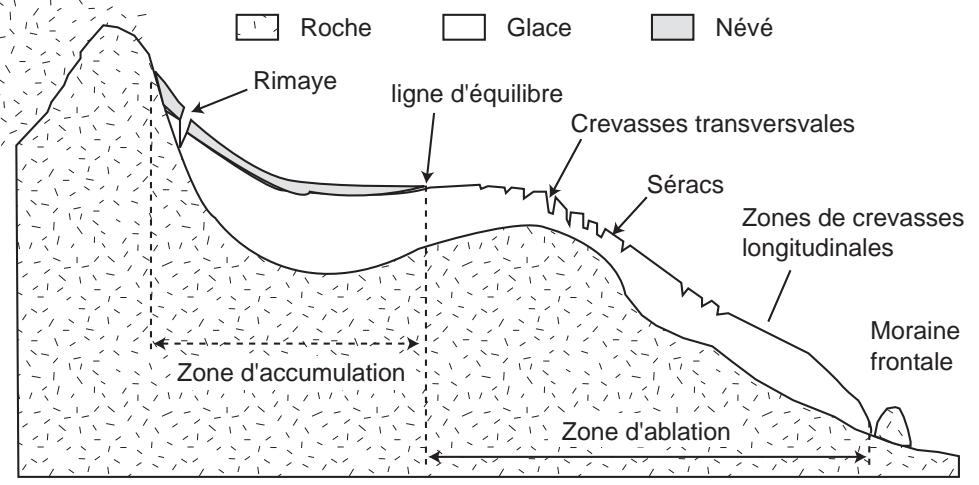

Fig. 2.1 - Coupe longitudinale d'un glacier de montagne fictif. Adapté d'une figure du site internet du Laboratoire de Glaciologie et Géophysique de l'Environnement (LGGE) http ://www-lgge.ujfgrenoble.fr. 


\subsection{Naissance \& mort de la glace : accumulation $v s$ ablation}

Le volume du glacier et son évolution d'une année sur l'autre dépendent d'un équilibre entre l'accumulation de la neige (principalement à haute altitude) et l'ablation de neige et de glace (essentiellement à basse altitude).

\subsubsection{L'accumulation et la formation de la glace}

L'accumulation comprend tous les processus qui apportent de la masse au glacier : chutes de neige, apports des pentes voisines par les avalanches et le vent ou regel de la pluie dans le glacier.

La figure 2.2 illustre la transformation de la neige en glace. La neige se métamorphose sous l'effet de la température, du vent, des cycles fonte-regel et de la compaction. Elle devient alors le névé. Cette transformation est rapide (quelques mois au maximum) pour les glaciers tempérés. Le passage du névé à la glace est plus lent et c'est sa densité (au delà de $830 \mathrm{~kg} \mathrm{~m}^{-3}$ ) et l'absence de circulation d'air qui permettent de distinguer la glace du névé (Paterson, 1994).

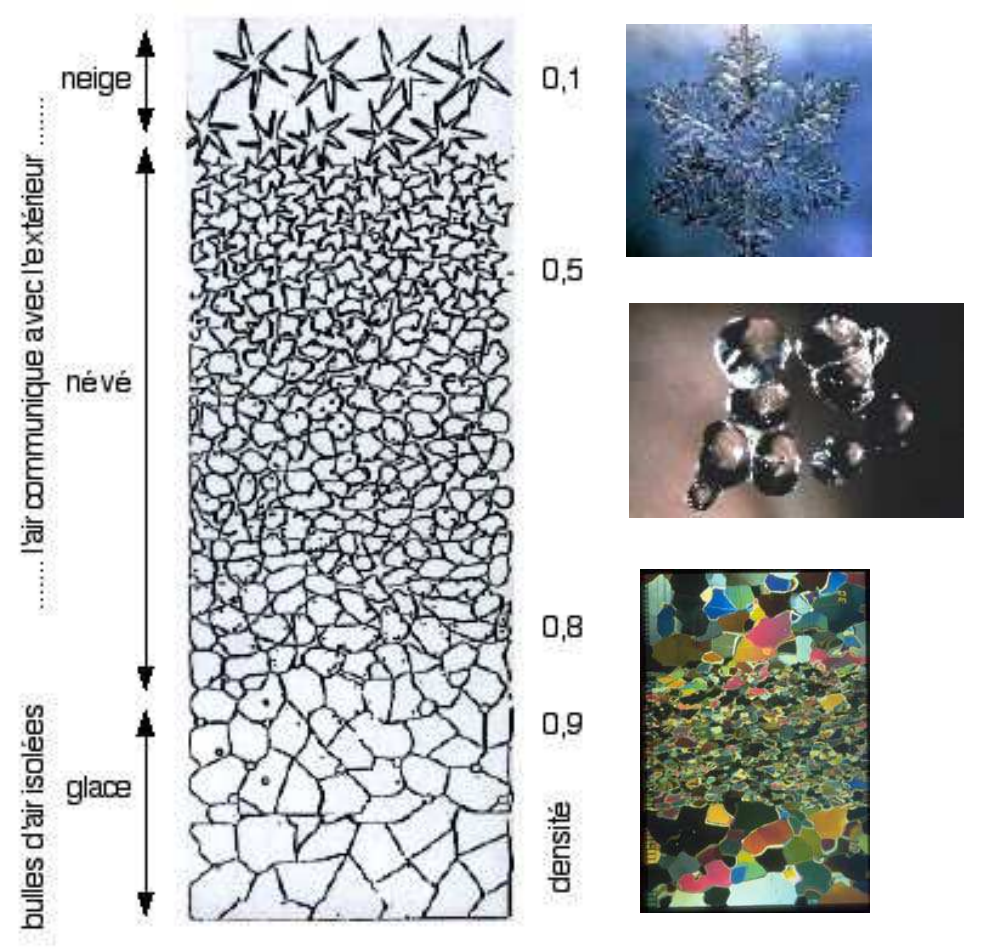

FIG. 2.2 - De la neige à la glace, la transformation du manteau neigeux. Les photographies de droite montrent, du haut vers le bas, un flocon de neige, des grains de neige transformée (névé) et des cristaux de glace en lumière polarisée et analysée. Sources : LGGE, Centre d'Etude de la Neige (Météo France) et Rémy (2003).

La durée de cette transformation de la neige en glace dépend des conditions climatiques (température, taux de précipitation). Elle varie entre quelques années pour les glaciers tempérés ( 5 ans pour le Upper Seward Glacier en Yukon (Alaska), 13 ans pour la zone d'accumulation de la Mer de Glace d'après Vallon et al. (1976)) et atteint plusieurs centaines d'années au centre 
des calottes polaires.

\subsubsection{Les processus d'ablation}

Différents processus sont à l'origine de perte de masse pour un glacier, ce qu'on désigne par ablation.

- la fonte intervient lorsque le bilan énergétique en surface du glacier est positif (le glacier reçoit plus d'énergie qu'il n'en perd) et que sa température est de $0^{\circ} \mathrm{C}$. Les bédières ${ }^{1}$ ou les moulins $^{2}$ sont des manifestations de cette fonte. Plus rarement, la fonte peut se faire à la base du glacier. Cette quantité (de l'ordre de quelques millimètres par an) est négligeable pour la plupart des glaciers (Paterson, 1994). Elle peut être importante pour des glaciers audessus de zones de fort flux géothermique. Nous verrons le rôle de cette fonte géothermique dans le cas d'un lac sous-glaciaire de la calotte du Vatnajökull en Islande (chapitre 6).

- la sublimation (passage directement de l'état solide à la vapeur d'eau) intervient quand le bilan d'énergie est positif mais que l'air atmosphérique est sec (Sicart, 2002, p. 36). Elle nécessite 8 fois plus d'énergie que la fonte (Paterson, 1994, p. 58). Pour une quantité d'énergie donnée, la sublimation s'accompagne donc de pertes de masse 8 fois plus faibles que la fonte. De ce fait, elle joue un rôle important pour le bilan de masse des glaciers où elle intervient comme les glaciers tropicaux (Wagnon et al., 2003).

- Le vent peut contribuer à l'ablation en chassant la neige.

- Les glaciers qui atteignent la mer ou se terminent dans un lac perdent aussi de la masse par vêlage des icebergs. La figure 2.3 montre une photo d'un tel vêlage dans le partie Sud de la calotte du Vatnajökull en Islande. L'intensité du vêlage est déterminée par la vitesse de l'écoulement glaciaire.

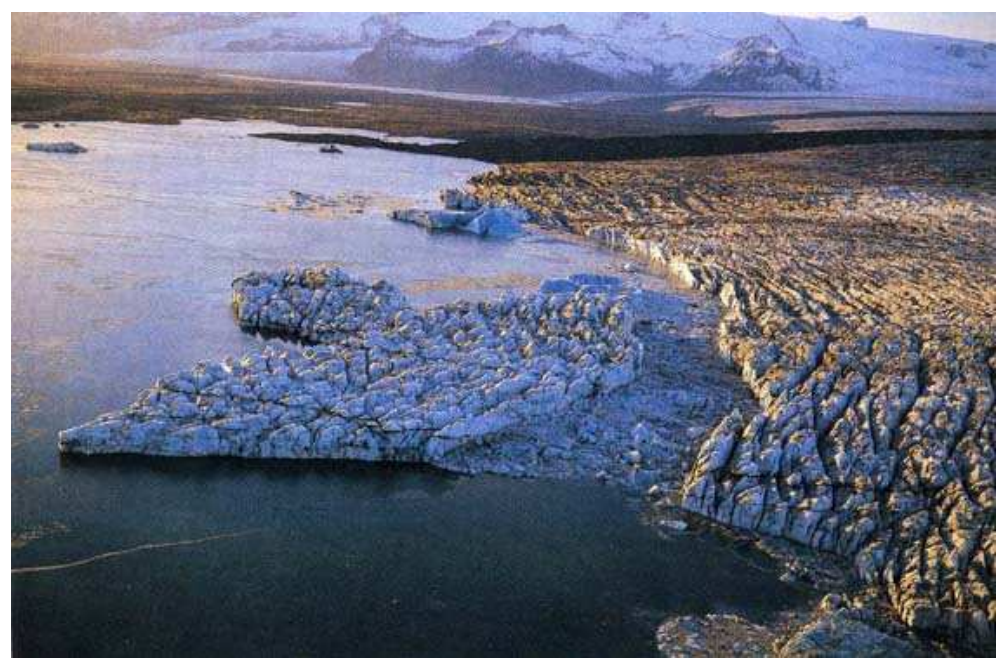

FIG. 2.3 - Vêlage d'icebergs dans le Jökulsárlón, au pied du glacier du Breiðamerkurjökull (Vatnajökull, Islande). Photo H. Björnsson.

Pour la fonte et la sublimation ce sont les flux énergétiques à la surface du glacier qui vont contrôler leur intensité. En effet, pour un glacier tempéré (à la température de fusion), tout

\footnotetext{
${ }^{1}$ torrent en surface des glaciers

${ }^{2}$ chenaux verticaux qui permettent à l'eau de fonte de pénétrer au cœur du glacier
} 
excès d'énergie en surface induit un changement d'état. Le bilan d'énergie à la surface du glacier est contrôlé par les flux radiatifs (courtes et grandes longueurs d'onde) et les flux turbulents (chaleur latente et sensible). La radiation nette à courte longueur d'onde (solaire) est la principale source d'énergie à la surface des glaciers (Sicart, 2002; Vincent et al., 2005a) ce qui explique que l'albédo ${ }^{3}$ soit une variable clef pour le bilan d'énergie. Lorsque le glacier est recouvert de neige fraîche (fort albédo), l'énergie courte longueur d'onde est fortement réfléchie par la surface et le glacier est protégé de l'ablation. En revanche, quand de la glace ou de la neige vieille (riche en poussières) affleurent en surface, une partie importante de l'énergie solaire est absorbée et l'ablation augmente.

La partie du glacier où, en une année, l'accumulation excède l'ablation est nommée zone d'accumulation. Le reste du glacier correspond à la zone d'ablation (figure 2.1). Ces deux zones sont séparées par une limite théorique où le bilan est nul, c'est la ligne d'équilibre. L'altitude de cette ligne varie chaque année pour chaque glacier mais reste homogène au sein d'une région climatique. Elle est en moyenne de $1200 \mathrm{~m}$ en Islande, $2800 \mathrm{~m}$ dans les Alpes et au-dessus de $5000 \mathrm{~m}$ dans les Andes et en Himalaya. L'équilibre entre les processus d'ablation et d'accumulation vont déterminer le bilan de masse du glacier.

\subsubsection{Le bilan de masse : indicateur de l'état de santé du glacier}

L'évolution depuis 1850 des glaciers alpins (chapitre 1) nous a montré que leur longueur (ou leur surface) n'est pas directement un bon indicateur climatique. En effet, pour deux glaciers voisins donc soumis au même forçage climatique, les fluctuations du front sont rarement identiques et synchrones. En revanche, l'évolution du stock de glace contenu dans un glacier (son bilan de masse) est directement liée aux variations de l'accumulation (donc des précipitations) et de l'ablation (donc des flux énergétiques et des températures). Les séries de bilan de masse glaciaire et la distribution spatiale de ces bilans (en fonction de l'altitude notamment) permettent donc de détecter des changements climatiques.

Dans cette partie, nous définirons les différents termes du bilan de masse avant de voir les différentes approchent qui permettent de l'évaluer.

\subsubsection{Bilan de masse : définitions}

En un point i du glacier, le bilan de masse $b_{i}$ s'exprime comme la différence entre l'accumulation et l'ablation :

$$
b_{i}=\text { accumulation }_{i}-\text { ablation }_{i}
$$

Un bilan de masse est le plus souvent ramené à une unité de surface et s'exprime sous forme d'équivalent en eau (noté w.e.). Le bilan de masse est, si possible, calculé chaque année. Son unité est donc le mètre par an (m w.e./an).

La convention est de calculer le bilan de masse à la fin de la saison d'ablation (septembre ou octobre dans les Alpes par exemple). Or, l'ablation ne s'arrête pas au même moment chaque année et en chaque point du glacier. Théoriquement, le bilan devrait donc être calculé chaque année pour une durée différente ne valant pas exactement 365 jours (Paterson, 1994). En pratique,

\footnotetext{
${ }^{3}$ Il varie entre 0.9 (neige fraîche) et 0.2 pour la vieille glace (Sicart, 2002)
} 
la mesure de bilan de masse se fait sensiblement à la même époque en se calquant sur l'année hydrologique ( $1^{\text {er }}$ octobre au 31 septembre dans les Alpes).

Pour certains glaciers, des mesures à la fin de la saison d'accumulation (l'hiver le plus souvent), permettent de distinguer un bilan de masse hivernal et estival. La figure 2.4 représente l'évolution des bilans hivernaux et estivaux au cours d'une année hydrologique.

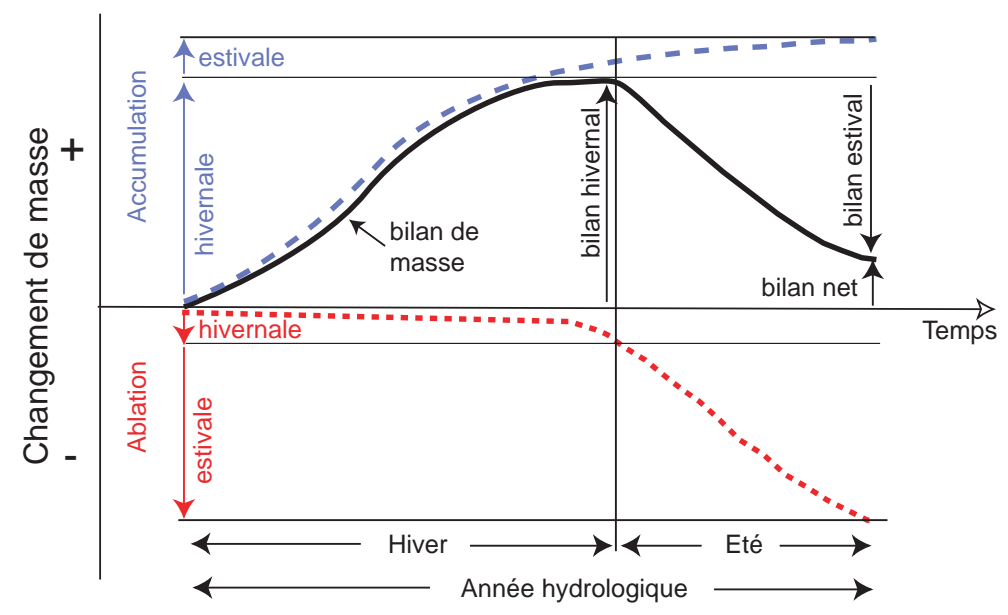

Fig. 2.4 - Définition des principaux termes du bilan de masse et leur évolution au cours d'une année hydrologique. Adapté de Paterson (1994).

Le bilan hivernal est positif et surtout contrôlé par l'accumulation. L'ablation hivernale est limitée car la radiation solaire incidente, déjà faible, est majoritairement réfléchie vers l'espace par la couverture neigeuse à albédo élevé. Le bilan estival est négatif et essentiellement contrôlé par l'ablation. Pour l'année choisie sur la figure 2.4, l'accumulation dépasse l'ablation et le bilan de masse annuel est positif. Ce type de nomenclature, valable pour les glaciers des latitudes tempérées, ne s'applique pas simplement à d'autres glaciers pour lequel le schéma hiver = accumulation, été = ablation ne vaut pas. C'est le cas des glaciers des Andes tropicales où accumulation et ablation se produisent toute l'année. En Bolivie par exemple, l'hiver (juin à septembre) est aussi la saison sèche...

Disposer de bilans de masse hivernaux et estivaux est important pour comprendre les causes des variations du bilan de masse annuel. Ainsi, Vincent et al. (2004) montre que c'est une forte ablation estivale qui explique les bilans de masse négatifs des glaciers alpins.

Pour passer du bilan de masse ponctuel $\left(\mathrm{b}_{i}\right)$ à celui du glacier $(\mathrm{b})$, il suffit d'intégrer l'équation 2.2 pour tout le glacier. Pour conserver une quantité équivalent à une lame d'eau (m w.e.), on divise par la surface totale du glacier (notée $\mathcal{S}$ ) :

$$
b=\frac{1}{\mathcal{S}} \int_{\mathcal{S}} b_{i} d \mathcal{S}_{i}
$$

\subsubsection{Méthode glaciologique}

Cette méthode se fonde sur des mesures in situ de l'accumulation et de l'ablation. En zone d'accumulation, le bilan de masse local est déterminé par des carottages et des mesures de densité. 
En zone basse, des balises de plusieurs mètres sont ancrées dans le glacier et leur émergence (qui augmente chaque année) permet de déterminer la lame de glace qui a été perdue (figure 2.5).

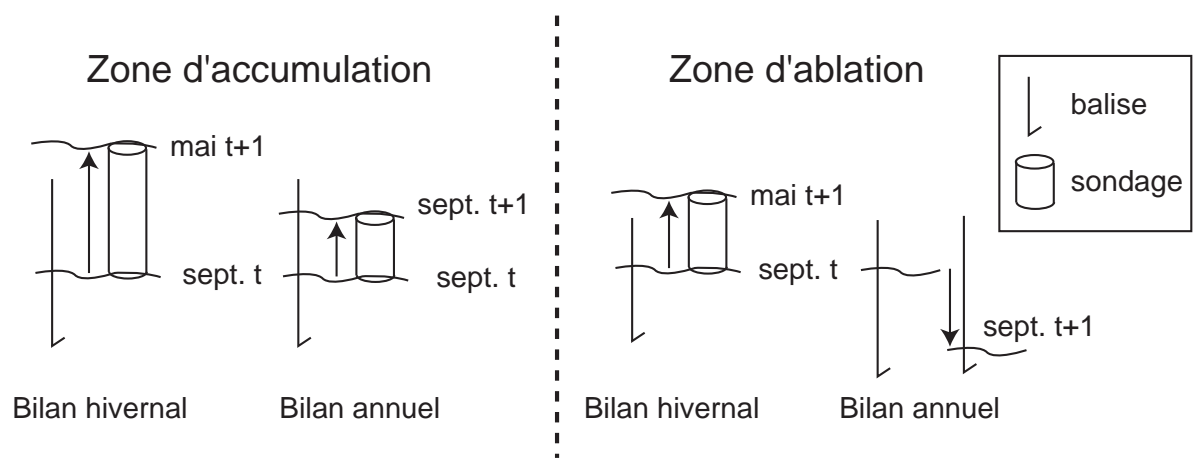

FIG. 2.5 - Méthodes de mesure du bilan de masse en zone d'accumulation (à gauche) et en zone d'ablation (à droite) pour l'hiver et pour toute l'année budgétaire. Le bilan estival s'obtient par la différence des deux. Dans les Alpes, les mesures sont réalisées en septembre d'une année (t) puis en mai et septembre de l'année suivante $(t+1)$. Adapté d'après Six (2000).

Disposant d'un nombre limité d'observations, le bilan de masse (b) s'obtient en discrétisant l'équation 2.2 (Braithwaite, 2002) :

$$
b=\frac{1}{\mathcal{S}} \sum_{i=1}^{n} b_{i} \delta \mathcal{S}_{i}
$$

où $\mathrm{b}_{i}$ est le bilan de masse représentatif de toute la zone $\delta \mathcal{S}_{i}$. Classiquement, $\mathrm{b}_{i}$ est estimé en moyennant les résultats de plusieurs balises ou carottage pour chaque gamme d'altitude. La courbe hypsométrique fournie la surface $\delta \mathcal{S}_{i}$. A basse altitude, les valeurs de $\mathrm{b}_{i}$ peuvent être aussi négatives que $-10 \mathrm{~m}$ w.e./an. La figure 2.6 est une carte des bilans de masse à la surface du glacier Zongo (Bolivie) pour l'année 2000-2001 et illustre le gradient des bilans avec l'altitude.

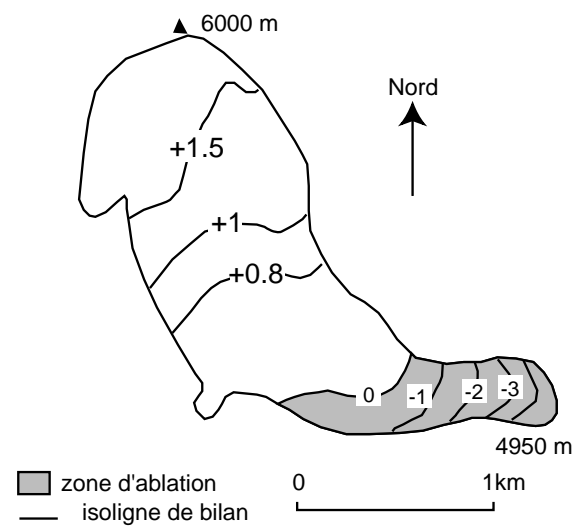

FIG. 2.6 - Distribution des bilans de masse sur le glacier Zongo (Cordillère royale, Bolivie) pour l'année hydrologique 2000/2001. L'intégration pour l'ensemble du glacier conduit à un bilan de masse positif de $0.57 \mathrm{~m}$ w.e pour cette année hydrologique caractérisée par des fortes précipitations (+20\% par rapport à la moyenne). D'après Berthier et al. (2001). 
Les incertitudes sur la mesure glaciologique du bilan de masse sont liées à :

- l'utilisation d'un nombre limité de sites de mesure alors que le bilan varie spatialement;

- la difficulté d'échantillonner certaines zones du glacier : par exemple à haute altitude ou les zones fortement crevassées;

- les erreurs pour chaque point de mesure (notamment dans les carottages d'accumulation).

Les auteurs s'accordent pour attribuer une erreur totale de 0.2 à $0.4 \mathrm{~m}$ (w.e.) à la mesure annuelle des bilans par la méthode glaciologique (Six, 2000; Sicart et al., sous presse).

\subsubsection{Méthode des flux}

La figure 2.7 représente les flux de glace à travers une section donnée du glacier. La conservation de la masse au sein de cette portion du glacier s'écrit (Reynaud et al., 1986; Oerlemans, 2001) :

$$
\mathcal{S}_{i} \frac{\partial h_{i}}{\partial t}=F_{i}-F_{i+1}+\mathcal{S}_{i} \times \frac{b_{i}}{\rho_{i}}
$$

où $\mathcal{S}_{i}$ est la surface du glacier compris en $\mathrm{x}_{i}$ et $\mathrm{x}_{i+1}, \mathrm{~F}_{i}$ est le flux à travers la section transversale $\mathrm{x}_{i}$ du glacier, $\mathrm{h}_{i}$ est l'épaisseur du glacier et $\rho_{i}$ la densité.

Cette équation traduit le fait qu'à flux constant, un bilan de masse positif va provoquer un épaississement du glacier. Le même résultat peut être obtenu à bilan de masse constant par un flux plus fort en amont qu'en aval. Pour un glacier à l'état stationnaire (aucune variation d'épaisseur), la différence des flux est équilibré par le bilan de masse. Ceci explique les bilans très négatifs dans la partie terminale du glacier, zone où l'épaisseur et la vitesse d'écoulement diminuent fortement (donc $\mathrm{F}_{i} \gg \mathrm{F}_{i+1}$ ).

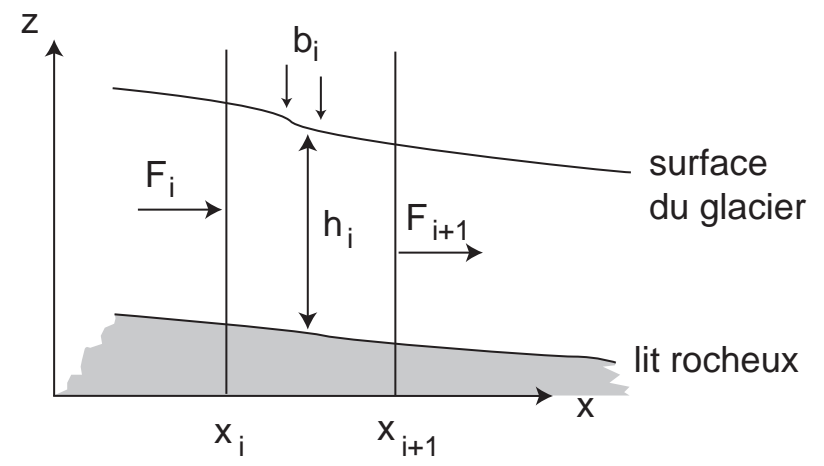

Fig. 2.7 - Bilan de masse $b_{i}$ d'une portion d'un glacier de vallée. L'axe z indique la verticale alors que l'axe des $x$ est dirigé dans le sens d'écoulement du glacier. Voir le texte pour la signification des symboles. D'après Oerlemans (2001).

On peut donc déduire le bilan de masse de cette portion de glacier comme :

$$
b_{i}=\rho_{i}\left(\frac{F_{i+1}-F_{i}}{\mathcal{S}_{i}}+\frac{\partial h_{i}}{\partial t}\right)
$$

Avec cette méthode d'évaluation du bilan de masse, il faut connaître pour deux profils transversaux du glacier les vitesses annuelles en surface, les épaisseurs et leurs variations au cours de 
la période étudiée. Une hypothèse sur le profil de vitesse en profondeur doit aussi être faite pour déterminer les flux à travers une section du glacier. Cette méthode est donc contraignante et les données sont rarement réunies pour les appliquer. Reynaud et al. (1986) ont pu l'appliquer à deux glaciers dans les Alpes (Gébroulaz en France et Unteraargletscher en Suisse) obtenant des résultats cohérents avec des mesures par la méthode glaciologique sur des glaciers voisins.

Plus récemment, cette méthode a été appliquée en utilisant des mesures de télédétection. Des cartes topographiques précises et des vitesses annuelles de surface sont déduites de couples de photographies aériennes et couplées à une topographie du lit rocheux pour déduire, en chaque point du glacier, les termes de droite de l'équation 2.5. Cette approche (Guðmundsson \& Bauder, 1999; Kääb \& Funk, 1999) a été testée pour deux glaciers en Suisse et les résultats sont relativement décevants, notamment parce qu'une hypothèse forte doit être faite sur la distribution de la vitesse verticale. Des résultats plus encourageants ont été obtenus en couplant cette approche à un modèle d'écoulement du Haut Glacier d'Arolla (Hubbard et al., 2000). La limite de cette méthode est qu'elle implique de connaître la topographie sous-glaciaire, information rarement disponible. Elle ne peut donc pas être utilisée pour obtenir le bilan de masse de glaciers de régions retirées, non étudiés in situ.

Cette méthode des flux est en revanche très utilisée pour les immenses glaciers antarctiques (Rignot \& Thomas, 2002; Berthier et al., 2003). Au niveau de la ligne d'échouage, on compare les flux entrants (à partir d'une carte d'accumulation sur le bassin versant) aux flux sortants. Comme le glacier commence à flotter, la vitesse peut être considérée comme constante sur toute l'épaisseur du glacier (pas de frottement à la base).

\subsubsection{Méthode géodétique ou photogrammétrique}

Une attention toute particulière est accordée à cette méthode car elle a été utilisée dans cette thèse pour déterminer le bilan de masse des glaciers du Mont Blanc (Berthier et al. (2004) et chapitre 5). Il s'agit ici de mesurer les changements d'épaisseur pour l'ensemble du glacier. En effet, si l'équation 2.5 est intégrée pour tout le glacier de surface $\mathcal{S}$, les termes de flux disparaissent et le bilan de masse se déduit de :

$$
\int_{\mathcal{S}} b_{i}=\int_{\mathcal{S}} \rho_{i} \frac{\partial h_{i}}{\partial t}
$$

Ainsi, en comparant deux cartes topographiques du glacier établies à une ou plusieurs années d'intervalle, le terme $\frac{\partial h_{i}}{\partial t}$ est mesurée et le bilan de masse net peut être déterminé. On notera que cette approche ne fournit pas la distribution spatiale des bilans de masse $\left(\mathrm{b}_{i}\right)$. Une hypothèse doit être faite sur la densité $\left(\rho_{i}\right)$ de la masse gagnée ou perdue. Si dans la zone d'ablation cette question peut être résolue simplement en utilisant une densité de $900 \mathrm{~kg} \mathrm{~m}^{-3}$, il n'y a pas de solution simple pour la zone d'accumulation. Certains auteurs (Andreassen et al., 2002) proposent d'utiliser également 0.9 argumentant que le matériel gagné ou perdu est de la glace. Cela suppose implicitement un profil de densité constant en fonction de la profondeur (Singh \& Singh, 2001) et semble justifié si l'accumulation varie peu. D'autres auteurs utilisent eux une densité de 0.65 (densité du névé) pour toutes les zones au-dessus de la ligne d'équilibre (Haag et al., sous presse).

L'incertitude associée à cette mesure géodétique du bilan de masse dépend directement de la précision des deux cartes qui sont comparées et de l'erreur sur l'estimation de la densité. La 
précision de cette méthode va donc dépendre de la technique d'élaboration de la topographie de surface du glacier. Cette topographie est le plus souvent représentée sous forme d'un modèle numérique de terrain (noté MNT par la suite) qui est une représentation numérique du relief : à chaque point d'une grille est associée une altitude. Les MNT des glaciers peuvent être établis par :

- Photogrammétrie analogique et maintenant numérique. Cette technique, utilisant des photographies aériennes, est apparue au début du siècle. Deux photos aériennes de la même zone acquises de deux points de vue différents présentent des distorsions, c'est l'effet stéréoscopique. La mesure de ces distorsions (ou parallaxe) permet de reconstituer la topographie. Il s'agit donc d'identifier des points homologues sur le couple d'image ce qui peut être réalisé soit manuellement soit automatiquement par corrélation des images. La précision des MNT est de l'ordre de quelques dixièmes de mètre. Cette erreur est souvent beaucoup plus forte dans la zone d'accumulation à fort albédo à cause du manque de contraste radiométrique.

- Photogrammétrie numérique appliquée aux images satellites optiques. C'est la transposition de la technique précédente à deux images satellites prises de points de vue différents. Toutin (2001) propose une revue complète de la génération de MNT à partir de l'imagerie satellitaire. Jusqu'à notre travail, la comparaison de MNT satellitaires n'a jamais (à notre connaissance) été réalisée pour estimer les variations d'épaisseur des glaciers.

- Interférométrie Radar, InSAR. Nous verrons au chapitre 3 l'application de cette technique à des images espacées d'un jour pour la détermination des vitesses de surface des glaciers. Si la vitesse est connue ou si plusieurs couples d'images sont disponibles, elle permet de déterminer également la topographie des glaciers (Joughin et al., 1996). L'idéal est d'acquérir deux images $\mathrm{SAR}^{4}$ simultanément ce qui élimine la contribution de l'écoulement et permet de déduire directement la topographie (Rabus et al., 2003; Magnússon et al., 2004). Nous utiliserons ce type de topographie sur des glaciers himalayens (topographie SRTM obtenue depuis la navette spatiale) et sur la calotte du Vatnajökull (MNT EMISAR).

- altimétrie satellitaire ou aéroportée. La carte topographique est construite à partir d'un nombre limité de profils altimétriques sur les glaciers (Echelmeyer et al., 1996; Baltsavias et al., 1999). Le plus souvent ce n'est pas une carte qui est construite mais simplement des profils voisins qui sont comparés (notamment en leur point de croisement). L'altimétrie radar, qui est adaptée pour les grandes calottes glaciaires (Rémy \& Legrésy, 1998), ne fonctionne pas sur les zones à fort relief donc les glaciers de montagne. L'altimétrie laser aéroportée (voire satellitaire avec IceSAT (Zwally et al., 2002b)) est la technique la plus adaptée pour le suivi de grand glaciers (Arendt et al., 2002) et excelle dans la zone d'accumulation.

Le comparaison des topographies déduites de photos aériennes est actuellement l'estimation la plus fiable du bilan de masse des glaciers (Paterson, 1994). Elle est d'autant plus précise que la séparation temporelle entre les MNT est importante. Classiquement, les variations d'épaisseur des glaciers sont mesurées à des intervalles de 5 à 10 ans. Cette méthode sert souvent de référence pour tester et corriger les bilans de masse cumulés obtenus par la méthode glaciologique (Funk et al., 1997; Andreassen et al., 2002). Il n'est pas rare que de larges différences soient observées, le plus souvent imputées à des biais ou des problèmes d'échantillonnage de la mesure de terrain (Haag et al., sous presse).

\footnotetext{
${ }^{4}$ Synthetic Aperture Radar pour radar à ouverture synthétique
} 
La méthode géodétique est actuellement la plus prometteuse pour étendre les mesures de bilan de masse à des régions difficiles d'accès. Mais les photographies aériennes offrent une couverture limitée (typiquement $3 \times 3 \mathrm{~km}^{2}$ ) et nécessitent l'acquisition de nombreux points de contrôle au sol. De plus, il n'est pas toujours possible de faire voler un avion au cœur d'une chaîne de montagne. C'est pour cette raison que, dans cette thèse, nous avons choisi d'exploiter des couples d'images satellitaires.

\subsubsection{Méthode hydrologique}

Comme la méthode géodétique, cette méthode mesure seulement le bilan de masse net de l'ensemble du glacier. Elle compare les gains sur le glacier par précipitations $(\mathcal{P})$, aux pertes liées à la fonte (mesurées par le débit $\mathcal{Q}$ ) et à l'évaporation $(\mathcal{E})$ :

$$
b=\mathcal{P}-\mathcal{Q}-\mathcal{E}
$$

Simple par son principe, cette méthode est en pratique délicate à mettre en œuvre. Les difficultés sont liées à :

- la forte variabilité spatiale des précipitations en zone de montagne et donc la difficulté de les mesurer avec quelques pluviomètres (Sicart, 2002).

- l'estimation de l'évaporation est loin d'être simple (Paterson, 1994)

- il est nécessaire de séparer l'écoulement de l'eau de fonte du glacier du débit provenant des surfaces non glaciaires (Sicart, 2002).

Des écarts importants sont constatés entre la méthode hydrologique et les précédentes, près de $100 \%$ d'écart dans l'étude de Haag et al. (sous presse) et $0.6 \mathrm{~m} / \mathrm{a}$ (w.e.) selon Sicart et al. (sous presse). Paterson (1994) souligne d'ailleurs que cette méthode ne devrait être utilisée que lorsqu'on ne peut pas faire autrement.

\subsubsection{Méthode énergétique : intégration du bilan d'énergie}

La méthode énergétique de détermination du bilan d'énergie en est encore à ces balbutiements. L'accumulation est déterminée de la même manière (donc avec les mêmes incertitudes...) que pour la méthode hydrologique. L'ablation est calculée à partir du bilan énergétique à la surface du glacier. Une des difficultés est d'extrapoler à tout le glacier le bilan d'énergie car on ne dispose en général que d'une mesure ponctuelle à une station météorologique (Sicart, 2002).

NB : Nous n'avons pas détaillé ici une dernière méthode qui utilise le suivi de l'altitude la ligne de neige en fin d'année hydrologique. Utilisant des données satellitaires, cette méthode sera présentée au chapitre 3 .

Au sein d'un glacier, la glace naît à haute altitude par densification progressive de la neige. Elle disparaît surtout dans la zone basse par fonte en surface principalement. Il existe donc un déséquilibre spatial entre une zone haute qui devrait constamment s'épaissir et une zone basse s'amincir. C'est l'écoulement de la glace soumise à la gravité qui permet au glacier de "garder la ligne". Entre sa naissance et sa disparition, une particule de glace a donc une vie mouvementée... 


\subsection{La vie de la glace? Mouvementée...}

La figure 2.8 montre l'écoulement schématique d'un glacier sur une coupe longitudinale. Avec une morphologie aussi simpl(ist)e, la vitesse est plus forte à la surface qu'en profondeur et plus rapide à la ligne d'équilibre qu'au sommet ou au front glaciaire. Nous verrons, dans cette partie, quelles sont les méthodes qui ont permis de parvenir à cette connaissance de l'écoulement glaciaire avant de décrire les mécanismes physiques qui expliquent cet écoulement.

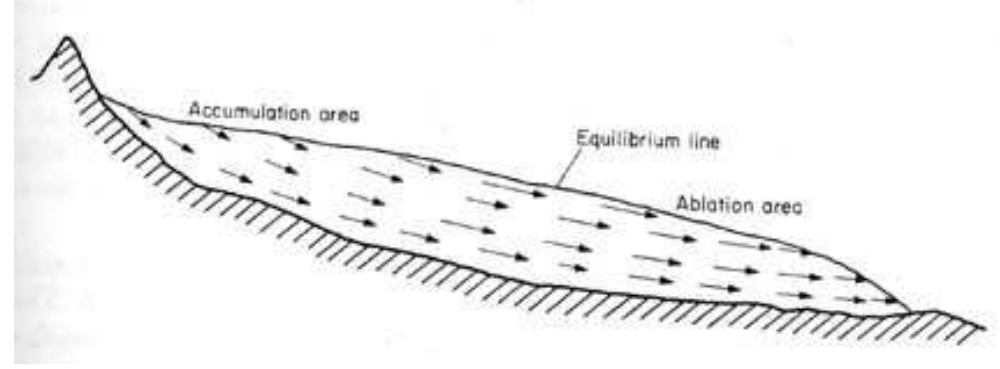

Fig. 2.8 - Profil idéalisé de vitesse dans un glacier de montagne. On remarquera des vitesses plus fortes au centre et près de la zone d'équilibre. D'après Paterson (1994).

\subsubsection{Mesure de l'écoulement glaciaire}

Nous devons à Joseph Vallot les premières mesures in situ systématiques et pluri-annuelles de l'écoulement glaciaire à la fin du siècle précédent (figure 1.4). Vallot place, le long d'un profil transversal, des pierres peintes et revient chaque année les localiser. Il confirme ce qu'avait déjà indiqué L. Agassiz : la vitesse du glacier est plus rapide au centre qu'à sa périphérie.

La technique des pierres peintes est encore utilisée aujourd'hui mais manque de précision puisque les pierres peuvent rouler indépendamment de la glace sous-jacente. De plus, des pierres n'indiquent que la projection du vecteur vitesse parallèlement à la surface du glacier (notée $\left.\overrightarrow{V_{/ /}}\right)$. Aujourd'hui, c'est le positionnement répété de balises d'ablation enfoncées de quelques mètres dans le glacier qui permet de déterminer les vitesses de surface. Le DGPS 5 a remplacé les techniques de positionnements classiques (triangulation). La figure 2.9 illustre comment des balises ancrées dans le glacier permettent de mesurer à la fois les vitesses horizontales et verticales de la glace. En régime stationnaire c'est à dire pour un glacier conservant la même morphologie, les vitesses d'enfouissement (notées $\overrightarrow{V_{\downarrow}}$ ) dans la partie haute doivent compenser l'accumulation, les vitesses d'émergence (notées $\overrightarrow{V_{\uparrow}}$ ) l'ablation dans les parties basses. C'est Reid (1896) qui le premier propose des trajectoires qui s'enfonce dans la zone d'accumulation pour émerger dans la zone d'ablation.

Lorsqu'il y a confluence de deux glaciers, leur glace ne se mélange pas (Lliboutry, 1964), en accord avec un écoulement qui est laminaire. Les glaciers restent séparés par une zone de glace souvent chargée de débris qui constituent une moraine médiane (figure 2.10). Du point de l'écoulement, la situation est différente. Pour Lliboutry (1964, p. 461), les glaces "ne constituent plus qu'un glacier unique au point de vue mouvement". Ceci se vérifie effectivement pour la Mer de Glace en aval de la confluence entre les glaciers du Tacul et de Leschaux. Cependant, pour certains glaciers émissaires de la calotte du Vatnajökull (Breiðamerkurjökull notamment), des

\footnotetext{
${ }^{5}$ Differential Global Positionning System
} 
mesures d'écoulement réalisées pendant cette thèse indiquent que chaque glacier conserve sa dynamique propre (Björnsson et al., 2005).
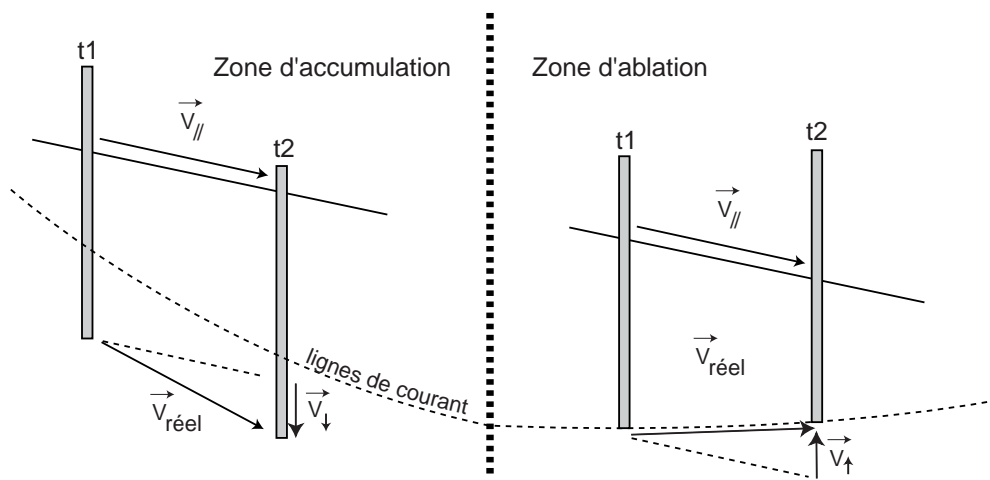

FIG. 2.9 - Mesure des vitesses dans les zones d'accumulation et d'ablation par positionnement successif d'une balise ancrée dans la glace. Cette figure permet de mettre en évidence l'écart de la vitesse réelle par rapport à la vitesse parallèle à la surface. Ceci résulte de l'existence d'une vitesse d'enfouissement dans la zone d'accumulation et d'émergence dans la zone d'ablation.
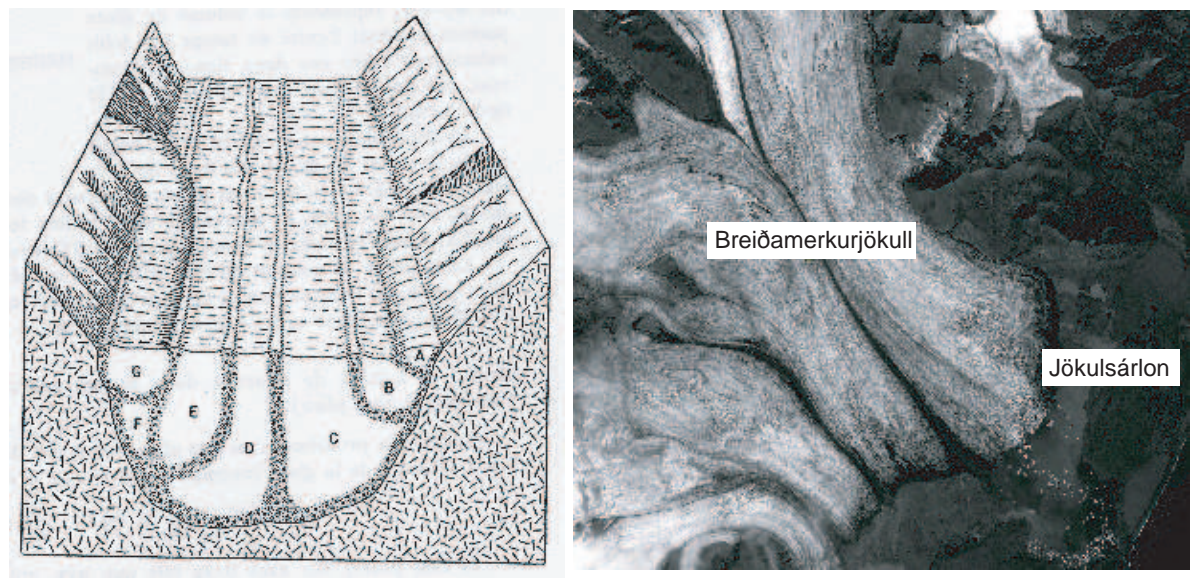

Fig. 2.10 - Bloc diagramme montrant l'organisation relative des glaciers lors de la confluence (Lliboutry, 1964). Les glaciers ne se mélangent pas. A droite, un extrait d'une image SPOT5 au niveau de la confluence des glaciers du Breiðamerkurjökull.

Les vitesses en profondeur dans le glacier peuvent être mesurées en suivant la déformation d'un trou de forage au cours du temps. Raymond (1971) a utilisé une série de 5 trous le long d'un profil transversal au glacier pour déduire la section des vitesses longitudinales de la figure 2.11(a). Cette section révèle les différents processus de l'écoulement d'un glacier. La vitesse n'est pas nulle sur le lit rocheux. Le glacier glisse (ou dérape selon les auteurs) sur son lit. Cette section confirme que la vitesse est plus forte à la surface (au centre) qu'en profondeur (que latéralement) : la glace se déforme par fluage. Un dernier processus qui contribue aux vitesses d'écoulement est la déformation par cisaillement des sédiments ${ }^{6}$ situés sous le glacier. Cette déformation peut jouer un rôle important notamment quand les sédiments sont saturés en eau. L'homogénéité des contours de vitesses proposés par Raymond (1971) est en partie liée à l'interpolation de 5

\footnotetext{
${ }^{6}$ nommés till en anglais
} 
mesures seulement. Une étude plus complète (31 trous de forages sont suivis) et remarquable a permis récemment de cartographier la géométrie à 3 dimensions de l'écoulement au sein d'un glacier (Harper et al., 1998, 2001). Les contour de vitesses longitudinales et transversales sont moins simples que ceux présentées en figure 2.11(a).

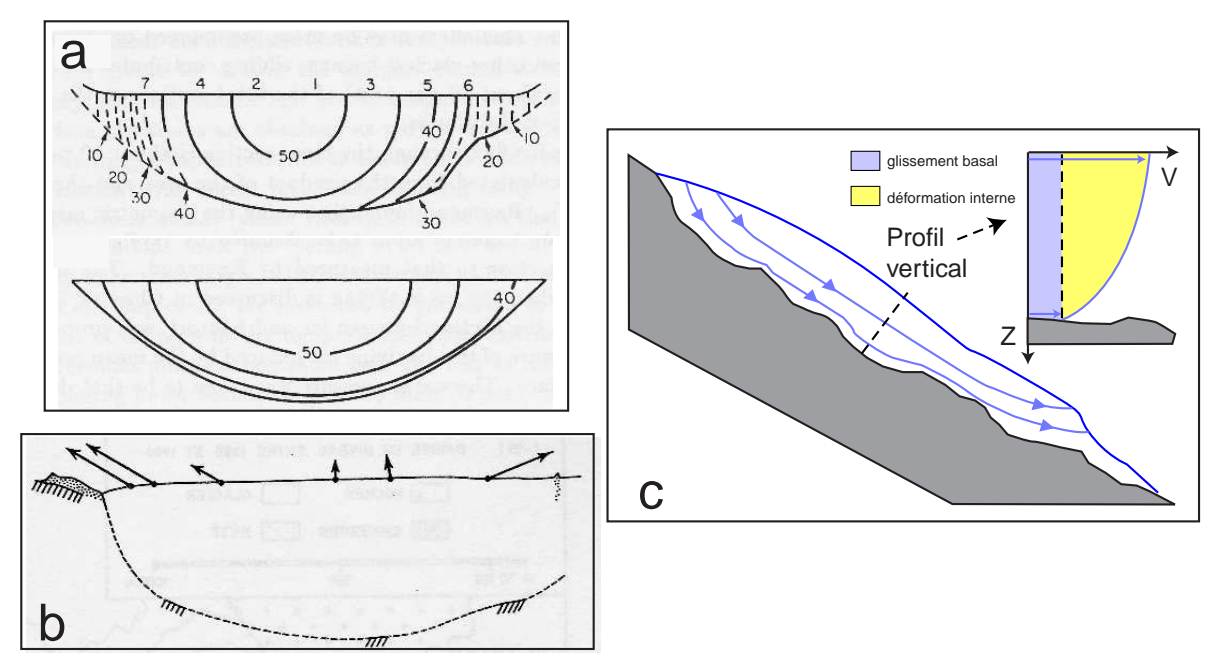

FIG. 2.11 - (a) Distribution des vitesses longitudinales sur une section transversale du glacier Athabasca déduite de 5 trous de forages par Raymond (1971). Elle est comparée aux vitesses calculées pour un écoulement dans un canal parabolique. Figure extraite de Paterson (1994). (b) Projection des vecteurs vitesses dans un plan vertical pour une section transversale de la zone d'ablation d'un glacier de vallée (Saskatchewan). D'après Meier in Lliboutry (1964). (c) Profil vertical théorique des vitesses longitudinales pour un glacier de vallée.

Du fait de l'écoulement plus lent sur les bords qu'au centre, dans la zone d'ablation, le glacier a davantage le temps de fondre sur ses marges. En conséquence, la langue du glacier est bombée. L'écoulement du glacier est alors divergent (dans un plan horizontal) ce qui tend à combler cette dépression latérale. Cette divergence horizontale, combinée aux vitesses d'émergence est à l'origine des vecteurs vitesses de la figure 2.11 (b). La glace remonte donc le long des parois du lit rocheux (Lliboutry, 1964). Des exceptions à cette situation sont les glaciers alimentés latéralement par des avalanches.

La figure 2.11 (c) présente de manière schématique un profil vertical de vitesse à travers un glacier. Ce profil idéalisé est en fait voisin de celui mesuré pour le glacier Athabasca par Savage \& Paterson (1963) in Singh \& Singh (2001, p 472). Il met en évidence nettement deux contributions à la vitesse d'écoulement. La contribution du glissement basal qui est constante dans l'épaisseur du glacier et celle de la déformation interne qui est nulle à la base (frottements) et maximum en surface. Ce sont ces deux contributions que nous allons détailler.

\subsubsection{La déformation interne de la glace}

Soumise à la contrainte, la glace se déforme. Une question qui, aujourd'hui encore, agite la glaciologie est la loi de comportement de la glace. Historiquement, les glaciologues ont longtemps hésité entre deux comportements : visqueux ou plastique. Pour un corps plastique parfait, la déformation n'apparaît qu'à partir d'une certaine contrainte ou seuil de plasticité. Ce seuil atteint, 
le taux de déformation est infini et la contrainte ne peut plus augmenter. Le corps visqueux lui se déforme de façon permanente quelque soit la contrainte appliquée (Rémy, 2003). La figure 2.12 compare les comportements rhéologiques de ces deux corps. Les expériences en laboratoire de Glen mirent en évidence un comportement intermédiaire. Toute contrainte appliquée à la glace induit une déformation mais la relation entre la déformation et la contrainte n'est pas linéaire.

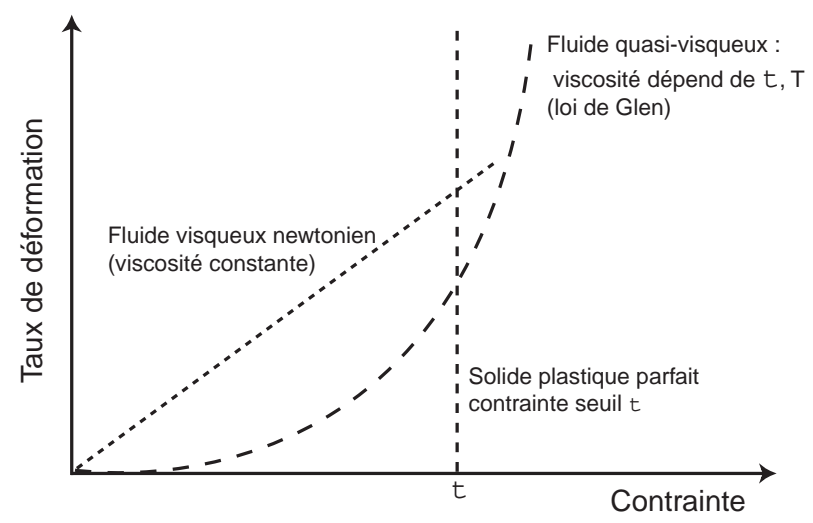

FIG. 2.12 - Relation entre la déformation et la contrainte pour différents comportements rhéologiques. La glace a un comportement intermédiaire entre un fluide visqueux et un solide plastique. D'après Rémy 8 Testut (2005).

La loi d'écoulement de la glace, ou loi de Glen, relie le taux de déformation cisaillant $\left(\dot{\epsilon}_{x y}\right)$ à la contrainte cisaillante $\left(\tau_{x y}\right)$ :

$$
\dot{\epsilon}_{x z}=\mathcal{A} \tau_{x z}^{n}
$$

$\mathcal{A}$ est un paramètre qui dépend de la température, de l'orientation des cristaux de glace ou de la présence d'impuretés. Il va notamment varier avec l'âge de la glace. La dépendance de $\mathcal{A}$ avec la température s'exprime selon une loi d'Arrhenius $\mathcal{A}=\mathcal{A}_{0} \exp (-Q / R T)$ où $\mathcal{A}_{0}$ est indépendant de la température, $\mathrm{T}$ est la température, $\mathrm{R}$ la constante des gaz parfaits et $\mathrm{Q}$ est l'énergie d'activation pour le fluage de la glace. Cette dernière valeur varie considérablement entre 40 et $140 \mathrm{~kJ} / \mathrm{mol}$ (Paterson, 1994). L'exposant n est une constante dont la valeur est discutée (entre 1.5 et 4.2, voir Marshall et al. (2002)) même si Paterson (1994) recommande une valeur de 3. L'axe $\mathrm{z}$ est perpendiculaire à la surface alors que $\mathrm{x}$ est la direction longitudinale (figure 2.13).

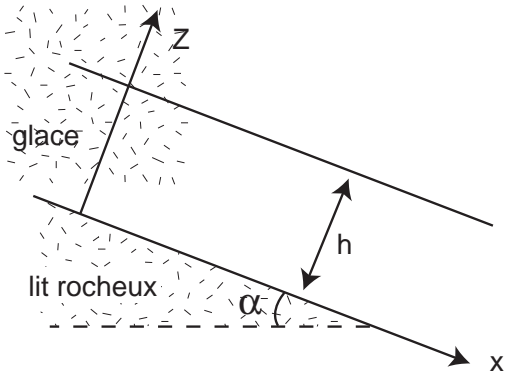

FIG. 2.13 - Système de coordonnées pour dériver la vitesse de déformation au sein d'un bloc de glace soumis à la gravité. L'axe z est perpendiculaire à la surface de la glace et l'axe x (axe longitudinal) est orienté selon l'écoulement. $\alpha$ est la pente à la surface du glacier. 
Avec cette géométrie, à un niveau z donné, la composante du poids de la colonne de glace (d'épaisseur h-z) dirigée selon l'axe des x vaut : $\rho g(h-z) \sin \alpha$. Dans cette équation g est la pesanteur, $\rho$ est la densité de la glace, h est l'épaisseur totale du glacier et $\alpha$ la pente locale dans la direction longitudinale. Cette composante ${ }^{7}$ doit être équilibrée par le frottement basal $^{8}$ (noté $\left.\tau_{x z}\right)$ qui s'écrit donc :

$$
\tau_{x z}=\rho g(h-z) \sin \alpha
$$

Dans le cas simplifié d'un écoulement laminaire (avec u la vitesse longitudinale), pour lequel la composante verticale de la vitesse peut être négligée (Paterson, 1994, p. 251), l'équation 2.8 se réduit à :

$$
\frac{1}{2} \frac{\partial u}{\partial z}=\mathcal{A} \tau_{x y}^{n}
$$

En intégrant, l'équation 2.10 entre un niveau z donné et la surface, on déduit pour ce cas très simplifié le profil de vitesse en fonction de $\mathrm{z}\left(\mathrm{u}_{s}\right.$ est la vitesse en surface) :

$$
u_{s}-u(z)=\frac{2 \mathcal{A}}{n+1}(\rho g \sin \alpha)^{n}(h-z)^{n+1}
$$

Du fait de la valeur de l'exposant $\mathrm{n}$ (voisin de 3), la vitesse augmente rapidement près du lit rocheux, beaucoup moins à la surface du glacier (voir par exemple les profils calculés par Oerlemans (2001, p. 59) pour différentes pentes). Pour $z=0$ (au niveau du lit rocheux), on trouve la relation entre la vitesse en surface et la vitesse basale (notée $\mathrm{u}_{b}$ ) :

$$
u_{s}-u_{b}=\frac{2 \mathcal{A}}{n+1}(\rho g \sin \alpha)^{n} h^{n+1}
$$

Cette formulation simplifiée de la vitesse de surface a permis de modéliser l'écoulement du Breiðamerkurjökull au voisinage de son front (Björnsson et al., 2001a, 2005). Ils introduisent toutefois dans l'expression de la contrainte basale un facteur de forme ${ }^{9}$ comme suggéré par Paterson (1994). Nous utiliserons également cette formulation pour tester l'hypothèse d'un ralentissement de la Mer de Glace entre 1994 et 2000 en réponse à une diminution de la déformation interne.

Dans l'expression de la vitesse de surface (équation 2.12), apparaît la vitesse basale. Elle traduit le glissement en masse du glacier sur son lit rocheux.

\subsubsection{Le glissement ou dérapage basal}

Le glissement de la glace sur le lit rocheux n'apparaît que lorsque la température à la base du glacier atteint le point de fusion. Du fait de la pression de la glace sus-jacente, la glace fond "plus facilement". Dans une calotte polaire de $2000 \mathrm{~m}$ d'épaisseur, la fusion apparaît à $-1.6^{\circ} \mathrm{C}$ contre $0^{\circ} \mathrm{C}$ à pression atmosphérique (Rémy, 2003).

Les mesures des vitesses de glissement sont difficiles sous plusieurs centaines de mètres de glace. Dans des cas particuliers, le glissement peut s'observer directement grâce à des cavités

\footnotetext{
${ }^{7}$ nommée driving stress en anglais car c'est elle qui détermine, au premier ordre, la déformation du glacier

${ }^{8}$ basal shear stress en anglais

${ }^{9}$ shape factor en anglais
} 
entre le lit rocheux et le glacier. L'installation d'une roue de vélo (Moreau \& Vivian, 2000), qui tourne en même temps que le glacier avance, permet de telles mesures en continu sous les séracs de Lognan (glacier d'Argentière). Ce dispositif original a montré que les périodes de fort glissement suivaient les pluies dans cette zone. La mesure du glissement peut aussi se faire en forant jusqu'au lit rocheux grâce à un dispositif nommé drag pool par les anglo-saxons (Singh \& Singh, 2001).

Comme le montre la section transversale en figure 2.11 (a), le glissement n'est pas uniforme spatialement. Dans ce cas précis, il vaut plus de $40 \mathrm{~m} / \mathrm{a}$ au centre du glacier et s'annule sur les bords. Les vitesses de glissement basal sont caractérisées par leur variabilité spatiale. Pour deux points d'un même glacier espacés de $15 \mathrm{~m}$, elles peuvent différer par deux ordres de grandeur (Paterson, 1994, p. 134). Les vitesses de glissement les plus élevées sont observées dans des régions où le glacier est décollé de son lit rocheux.

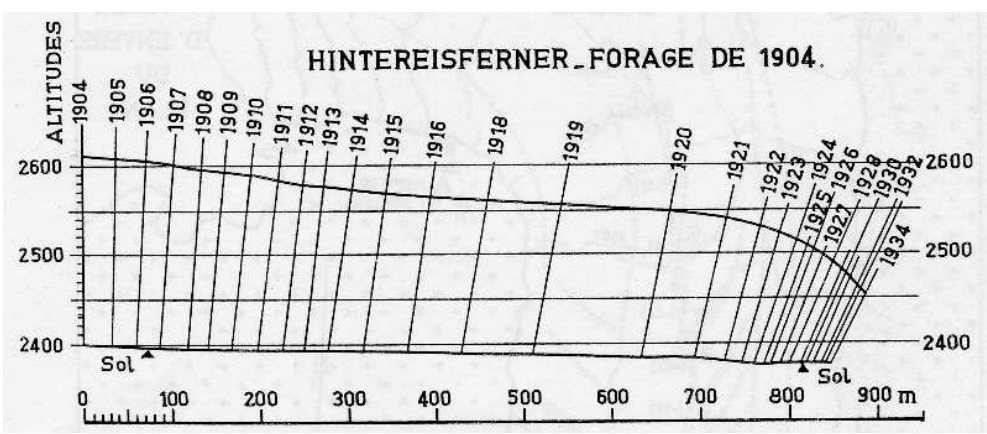

Fig. 2.14 - Position de la tige de forage dans l'Hintereisferner entre 1904 et 1934. D'après Koechlin in Lliboutry (1964).

Une méthode pour déterminer la part relative du glissement et de la déformation interne du glacier est d'étudier l'évolution d'un trou de forage. C'est ce qui a été fait pendant 30 années pour un forage réalisé en 1904 sur l'Hintereisferner (figure 2.14). Dans ce cas, le glissement explique $89 \%$ du déplacement. Mais cette proportion est très variable d'un glacier à l'autre. Le rapport $\mathrm{u}_{b} / \mathrm{u}_{s}$ varie de quelques \% à plus de $90 \%$ (Paterson, 1994). Mais en moyenne, le glissement est responsable de la plus grande partie de l'écoulement.

De nombreuses théories ont été développées pour tenter de représenter le glissement du glacier. Le problème est de comprendre comment la glace se déplace au-dessus des micro-reliefs du lit rocheux. Weertman (1957) propose deux mécanismes explicatifs : la regelation et l'écoulement plastique accru. Le premier mécanisme consiste en la fonte de la glace en amont des bosses (où il y a une surpression) et son regel en aval. Le second est lié à un accroissement des contraintes longitudinales au voisinage d'une rugosité (Paterson, 1994). Lliboutry (1964, p. 640) ajoute la cavitation comme mécanisme possible.

Le glissement basal est donc difficile à étudier directement. Une approche indirecte (que nous utiliserons au chapitre 4) consiste à observer les variations à court terme de l'écoulement de surface du glacier. En effet, pour de courtes périodes de temps, la déformation interne du glacier varie peu alors que le glissement est, lui, susceptible de changer. 


\subsubsection{Variation saisonnière et inter-annuelle de l'écoulement}

Nombreux sont les travaux qui ont permis de mesurer les variations inter-annuelles, saisonnières voire diurnes des vitesses des glaciers de montagne.

A l'échelle pluri-annuelle, les mesures de Vallot et de Finterwalder ont permis d'étudier le passage d'ondes de crues sur la Mer de Glace et le glacier de Vernagtferner. Ces ondes se traduisent par un épaississement (quelques mètres) et une accélération du glacier (25\% pour la Mer de Glace, un facteur 10 pour Vernagtferner). Elles sont la réponse dynamique du glacier à des anomalies du bilan de masse. La vitesse de propagation de cette onde est plusieurs fois supérieure à la vitesse du glacier (Lliboutry, 1964). Lliboutry \& Reynaud (1981) montrent que la théorie des ondes cinématiques (proposée par Weertman et Nye) ne permet pas de rendre compte des observations réalisées sur la Mer de Glace et introduisent la notion de dynamique globale.

Des variations de vitesse sur plusieurs décennies sont aussi reportées pour plusieurs glaciers. Reynaud (1979) a notamment utilisé des photographies aériennes de la Mer de Glace pour déduire sa vitesse depuis un siècle de la morphologie des bandes de Forbes. Ces données complètent les mesures de terrain déjà nombreuses sur ce glacier. La figure 2.15 montre l'évolution des vitesses pour différents secteurs de la Mer de Glace. Leur comparaison avec les variations d'épaisseur du glacier suggère un lien entre ces deux paramètres. Nous reviendrons plus amplement sur cette relation entre variations d'épaisseur et de vitesse annuelle en conclusion de cette thèse.

A l'échelle saisonnière, les vitesses des glaciers fluctuent avec des valeurs estivales plus fortes dans la partie basse, alors qu'en zone d'accumulation les vitesses sont maximales en hiver (Lliboutry, 1964, p. 624). Mais ce schéma n'est pas identique pour tous les glaciers (Willis, 1995). L'étude de Iken et al. (1983) est sans doute l'une des plus détaillées (figure 2.16). Durant une année complète, ils mesurent les déplacements horizontaux et verticaux sur l'Unteraargletscher. Les vitesses sont plus rapides en été, période au début de laquelle ils enregistrent un soulèvement du glacier de $0.4 \mathrm{~m}$. Puis, dans les trois mois qui suivent, le glacier subside pour revenir à son niveau initial. Ils mettent également en évidence de courtes périodes (quelques jours) durant lesquels les vitesses longitudinales augmentent d'un facteur 3 à 6 . Ces accélérations coïncident avec des maximums de vitesses verticales mais aussi des périodes de forte fonte en surface du glacier. Ils proposent donc que l'afflux d'eau à la base du glacier engendre son soulèvement. L'augmentation consécutive de la pression d'eau limiterait le frottement sur le lit rocheux, intensifiant le glissement basal.

Depuis, de nombreuses études ont permis de mettre en relation des variations de vitesses et des changements de pressions d'eau. Une revue détaillée des variations intra-annuelles des vitesses des glaciers est proposée par Willis (1995). Une conclusion importante de cette revue est que les variations rapides de la vitesse sont à relier aux changements du contenu en eau à l'intérieur et à la base du glacier. Cela entraîne des variations non seulement du glissement basal, mais aussi de la déformation des sédiments du lit du glacier. Les changements des pressions d'eau interviennent parfois à l'endroit même de la variation de vitesse mais, dans d'autres cas

à une certaine distance. Dans ce dernier cas, des zones clefs du glacier peuvent contrôler les variations de vitesse à distance (Lliboutry \& Reynaud, 1981; Hanson et al., 1998; Sugiyama \& Guðmundsson, 2003) via des variations des gradients de contraintes longitudinales (Willis, 1995). 

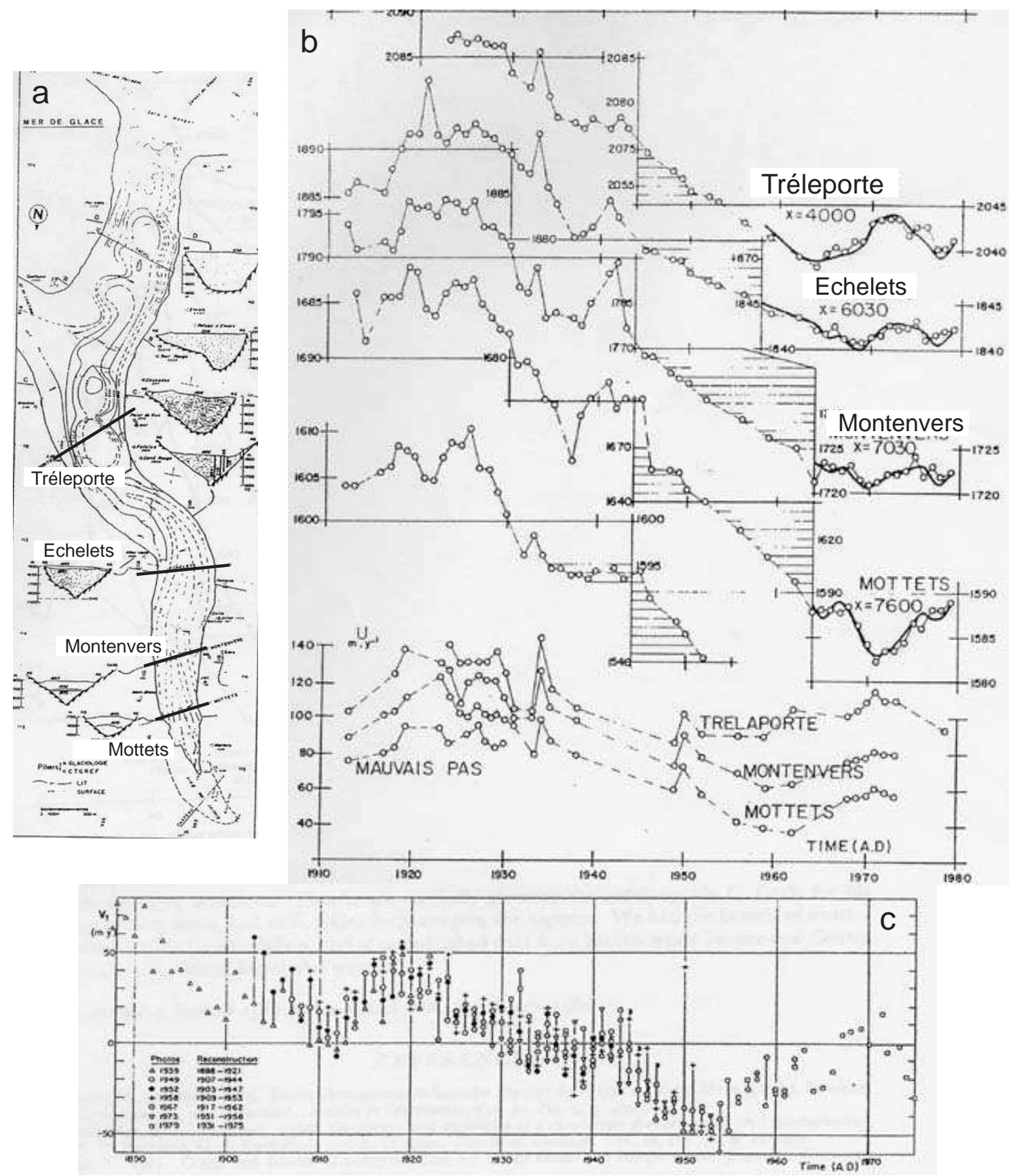

FIG. 2.15 - Variations d'épaisseur et de vitesse pour quelques profils transversaux de la Mer de Glace. (a) Localisation des profils sur une carte des iso-contours de l'altitude du lit rocheux. (b) Mesure in situ des variations de l'altitude (en haut) et de la vitesse de profils transversaux entre 1910 et 1980 (en bas). (c) Reconstruction des fluctuations des vitesses à partir de la morphologie des bandes de Forbes. Cette figure est une compilation des figures 2, 7 et 11 de Lliboutry 63 Reynaud (1981). 

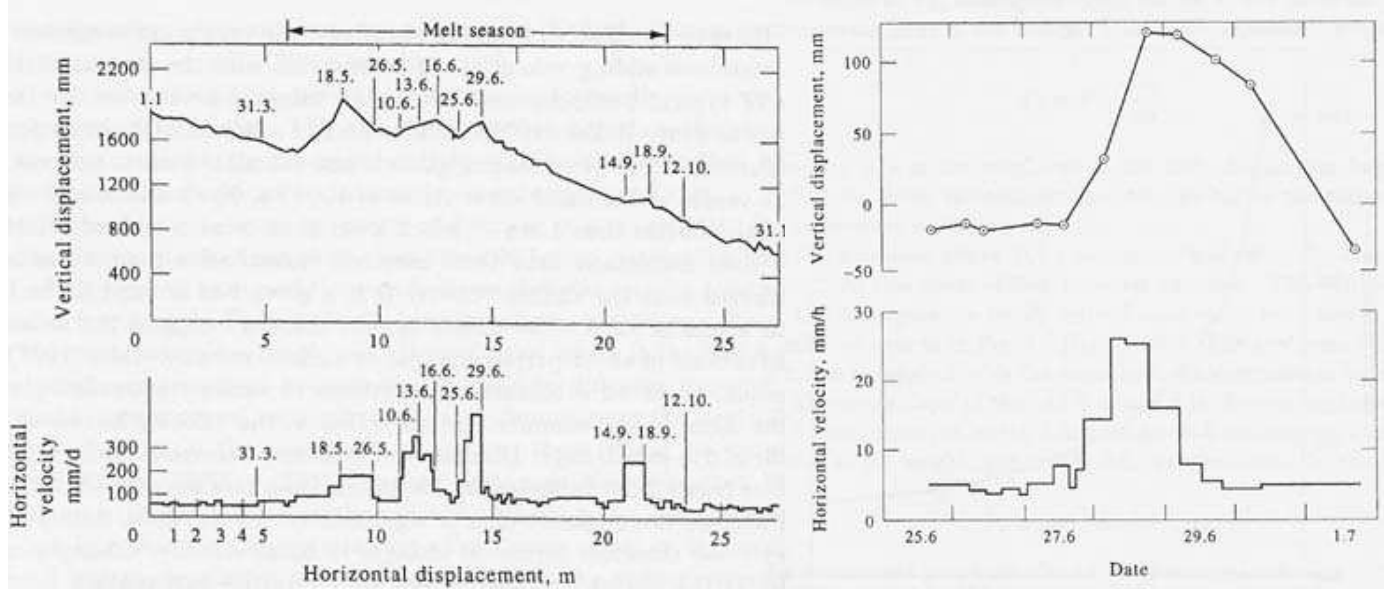

FIG. 2.16 - Evolution des déplacements verticaux et des vitesses horizontales au cours d'une année sur l'Unteraargletscher. In Paterson (1994) d'après Iken et al. (1983). A gauche pour l'ensemble de l'année, à droite pour un évènement précis à la fin du mois du juin.

Un des limites de ces études in situ est que les variations de vitesses ne sont mesurées qu'en quelques points du glacier seulement (Willis, 1995). L'intérêt principal de nos mesures satellitaires de l'écoulement des glaciers du Mont Blanc (chapitre 4) est de fournir une vision d'ensemble des variations de vitesse. Lliboutry (1964) ne soulignait-il pas qu' "il serait plus utile d'obtenir une vue d'ensemble des zones où apparâ̂t le glissement dans un glacier unique"? Il définit ainsi l'un des objectifs de ce travail de thèse.

\subsection{Conclusions et objectifs scientifiques}

Ce chapitre nous a permis de mieux comprendre le cycle de vie de la glace au sein d'un glacier de montagne. Entre sa naissance à haute altitude et sa disparition à basse altitude, la glace s'écoule selon des lois physiques encore mal connues. Le bilan de masse global est une vision quelque peu statique du glacier qui ne rend pas compte de sa dynamique importante mais permet d'évaluer son état de santé. Parce que les processus d'accumulation et d'ablation sont directement contrôlés par les forçages climatiques, le bilan de masse et sa distribution spatiale sont de bons indicateurs des fluctuations des variables météorologiques.

Nous avons également entrevu la difficulté de l'observation des glaciers :

- Leur accès est délicat voire dangereux. Les zones fortement crevassées ou l'intérieur du glacier sont presque toujours inaccessibles. C'est souvent à partir de quelques points de mesure que les glaciologues tentent de percer les mystères de l'intérieur de la glace.

- La dynamique du glacier est par nature complexe et varie à des échelles de temps et d'espace très différentes. Par exemple, la notion d'état stationnaire pour un glacier reste théorique. Il est en effet constamment soumis à des perturbations des forçages. Dans certains cas (les calottes polaires par exemple), la dynamique glaciaire intègre des changements climatiques sur plusieurs milliers d'années.

Nous allons voir comment les capteurs satellitaires ont permis de surmonter certaines de ces difficultés, permettant des avancées significatives dans la compréhension du fonctionnement du glacier. 
Ce chapitre introduit aussi les objectifs scientifiques de cette thèse que l'on peut regrouper en trois points :

1. Étudier les distributions spatiales des variations de vitesse à court et long terme des glaciers afin de mieux les expliquer.

2. Observer les adaptations de la dynamique glaciaire aux changements morphologiques pour mieux prévoir leur évolution future.

3. Mesurer l'évolution récente des épaisseurs (donc des bilans de masse) de glaciers situés dans des régions variées : Alpes, Himalaya et Islande. 


\section{Chapitre 3}

\section{Observation satellitaire des glaciers de montagne}

Sommaire

3.1 Difficultés \& nécessité de l'observation satellitaire . . . . . . . . . 58

3.1 .1 Une observation difficile... . . . . . . . . . . . . . . . . 58

$3.1 .2 \quad \ldots$ mais nécessaire car les glaciers... . . . . . . . . . . . . . 62

3.2 Les différents capteurs satellitaires $\ldots \ldots \ldots \ldots \ldots \ldots$

$3.2 .1 \quad$ Imagerie optique $\ldots \ldots \ldots \ldots \ldots$. . . . . . . . . . . . . . . . .

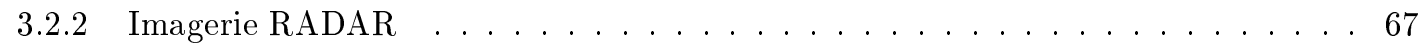

3.2 .3 Altimétrie Radar et Laser . . . . . . . . . . . . . . . . . . . 68

3.3 Observation satellitaire des glaciers : état des lieux . . . . . . . . . 69

3.3 .1 État de surface des glaciers . . . . . . . . . . . . . . . . 69

3.3 .2 Variations de longueur et superficie glaciaire . . . . . . . . . . . . 70

3.3 .3 Vitesses d'écoulement des glaciers . . . . . . . . . . . . . . . . 71

3.4 Conclusions et objectifs méthodologiques . . . . . . . . . . . 74

Le premier satellite, Spoutnik I un satellite russe, a été lancé en 1957. Depuis cette date, les satellites ont été largement utilisés pour l'observation de l'atmosphère, de l'océan ou de la surface terrestre car leur couverture spatiale et temporelle est précieuse. La cryosphère, elle aussi, est étudiée depuis l'espace. Contrairement à la glaciologie de terrain qui a pris son essor sur les glaciers de montagne, ce sont les calottes polaires qui sont le plus observées par les satellites (Bindschadler, 1998). L'utilisation de la télédétection spatiale sur les glaciers de montagne a été longtemps limitée par des difficultés que nous détaillerons dans ce chapitre. Nous verrons pourtant que cette observation est nécessaire pour surmonter certaines déficiences des mesures de terrain. Les principaux capteurs seront présentés avec une attention particulière pour les satellites de la famille SPOT (figure 3.1). Nous verrons enfin les différentes avancées scientifiques qui ont été permises par l'utilisation des satellites et comment se positionne notre étude par rapport à ces travaux antérieurs. 


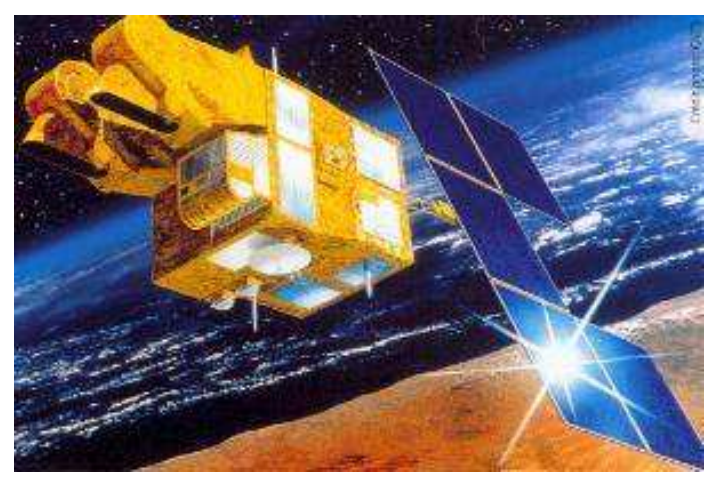

FIG. 3.1 - Le satellite SPOT5.

\subsection{Difficultés \& nécessité de l'observation satellitaire}

\subsubsection{Une observation difficile...}

L'observation par satellite des glaciers de montagne est rendue difficile par leur petite taille, leur situation dans des zones de forte topographie et par la nature même de leur surface, changeante et parfois à fort albédo.

\subsubsection{La relative petite taille des objets étudiés}

Comparé à une calotte polaire qui s'étend sur plusieurs millions de $\mathrm{km}^{2}$ (chapitre 1), un glacier de montagne est de petite taille, en moyenne quelques $\mathrm{km}^{2}$. Plus précisément, la taille des glaciers couvre un spectre large. Les plus longs approchent la centaine de km (glaciers d'Alaska, du Karakorum) couvrant des surfaces de plusieurs centaines de $\mathrm{km}^{2}$ (Lliboutry, 1964). Les plus petits, les champs de neige, couvrent quelques dizaines de $\mathrm{m}^{2}$. Même les glaciers les plus longs, confinés par des parois abruptes, dépassent rarement quelques kilomètres de large. Les petites calottes glaciaires, à la forme plutôt circulaire, font exception.

La taille du pixel ${ }^{1}$ de l'image est donc cruciale. L'image sera presque inutile si le glacier n'est représenté que par quelques pixels. Nous verrons, par exemple, que les techniques de corrélation d'images (nécessaires pour générer des topographies ou estimer des champs de vitesse) utilisent un ensemble de pixels (au moins $15 \times 15$ soit 225 pixels pour mesurer des vitesses). Logiquement, le nombre de mesures indépendantes augmente avec le nombre de pixels dans la largeur du glacier.

Compte tenu de la taille des glaciers, ce sont seulement les capteurs avec des résolutions inférieures à la centaine de mètres qui sont utiles. Nous verrons que ce sont essentiellement des capteurs optiques ou radar avec des résolutions de $30 \mathrm{~m}$ ou moins qui sont utilisés en glaciologie alpine $^{2}$. Pour certaines régions, comme les Andes boliviennes où $80 \%$ des glaciers ont une superficie inférieure à $0.5 \mathrm{~km}^{2}$ (Francou et al., 2003), c'est vers la très haute résolution qu'il faut se tourner (figure 3.2 ).

\footnotetext{
${ }^{1}$ c'est à dire la surface au sol couverte par l'unité élémentaire d'une image

${ }^{2}$ il est classique de nommer glaciers alpins, les glaciers de montagne sans préjuger de leur localisation géographique. Voir Mattar et al. (1998).
} 

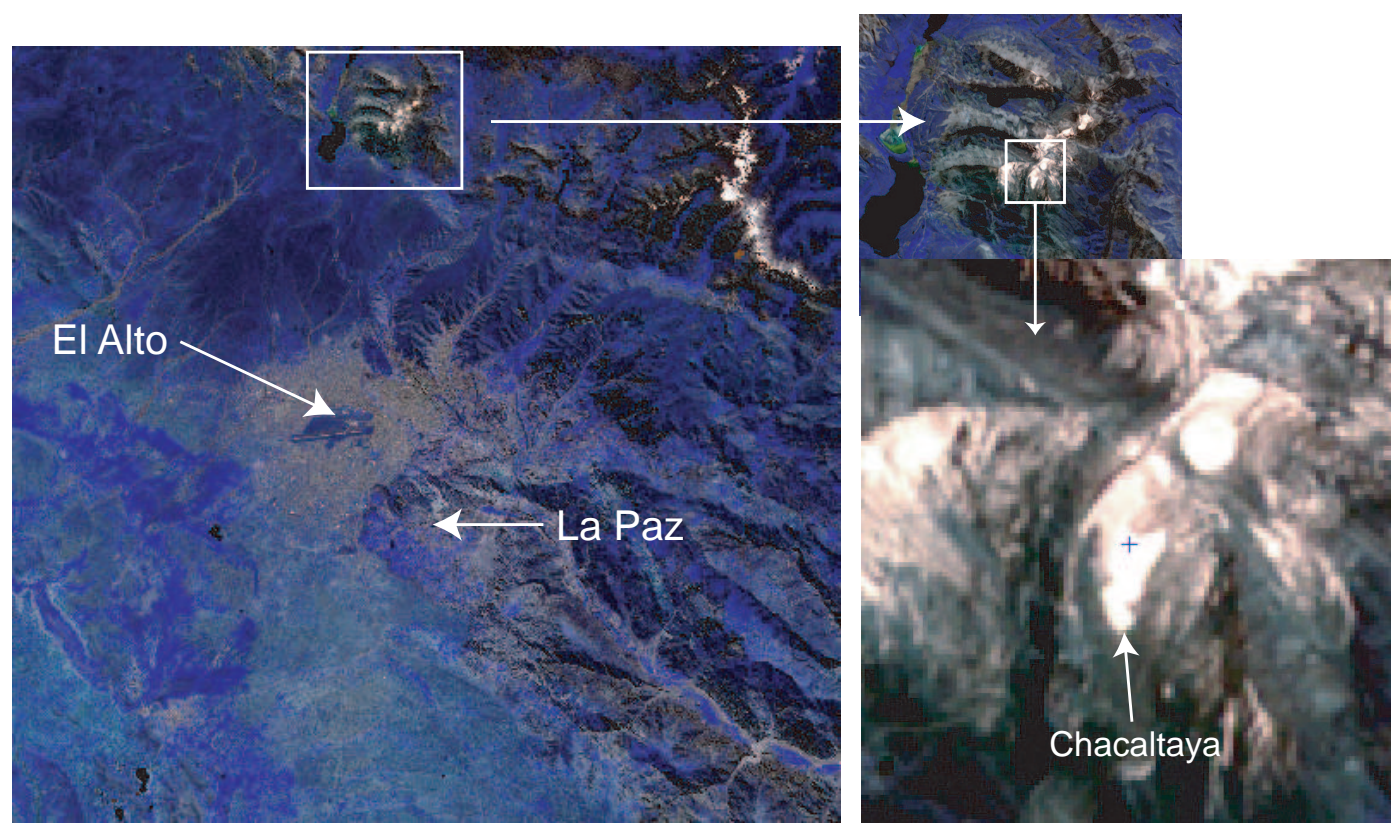

FIG. 3.2 - Le glacier de Chacaltaya (Cordillera Real, Bolivie) observé par le satellite ASTER en mai 2002. L'ensemble de l'image ASTER, à gauche, montre la ville de La Paz et son aéroport installé à El Alto sur l'Alti Plano. Le glacier de Chacaltaya couvre $0.06 \mathrm{~km}^{2}$ soit une très faible proportion de l'image couvrant $3600 \mathrm{~km}^{2}$. En zoomant sur le glacier (à droite), les pixels (225 $m^{2}$ chacun) deviennent visibles et 250 pixels seulement suffisent à couvrir tout le glacier. Une telle résolution permet simplement l'estimation de l'extension glaciaire mais interdit toutes les méthodes (calcul de MNT, corrélation d'images) utilisant des fenêtres de plusieurs pixels.

\subsubsection{La forte topographie qui entoure les glaciers (ombres, pentes)}

Par définition, les glaciers de montagne se trouvent au cœur de zones où la topographie est marquée. Les dénivelés sont importants, généralement plusieurs milliers de mètres et les pentes sont fortes, souvent au-delà de $45^{\circ}$.

Au niveau géométrique, ces fortes pentes provoquent des distorsions importantes des images, notamment lorsque elles sont acquises de points de vue différents. Lorsqu'elles restent modérées, ces distorsions permettent de reconstruire la topographie (Toutin, 2001). L'importance de ces distorsions est illustrée par la variation de la taille du pixel d'une image SPOT5 (mode THR) du massif du Mont-Blanc. Au mieux (par exemple pour une visée au nadir sur une surface plane) elle vaut $2.5 \mathrm{~m}$ mais dépasse $10 \mathrm{~m}$ par endroit. Si l'image n'est pas acquise verticalement (cas des images SAR ou des capteurs optiques avec dépointage comme SPOT), certaines zones seront totalement occultées ${ }^{3}$. Heureusement, les glaciers eux-mêmes présentent généralement des pentes plus modérées (pente moyenne de $17^{\circ}$ par exemple pour la Mer de Glace).

Au niveau radiométrique, les reliefs élevés entourant les glaciers créent des zones d'ombres sur les images optiques. La figure 3.3 montre l'ombre créée par la face Nord des Grandes Jorasses sur le glacier de Leschaux (cf figure 4.7 pour sa localisation géographique). La taille et l'orientation de l'ombre vont dépendre de la topographie mais également de la position du soleil. Ces ombres sont plus faibles si les images sont acquises au voisinage de midi solaire et proche du solstice

\footnotetext{
${ }^{3}$ Cet effet est nommé shadowing en terminologie SAR
} 
d'été (21 juin dans l'hémisphère Nord). Mais les satellites optiques privilégient des observations le matin (moins de nuages) et les glaciologues des images acquises à la fin de la saison d'ablation (en septembre) : les ombres sont donc souvent présentes. Dans le cas des images SAR, des variations radiométriques existent également et dépendent de l'orientation et de la pente.

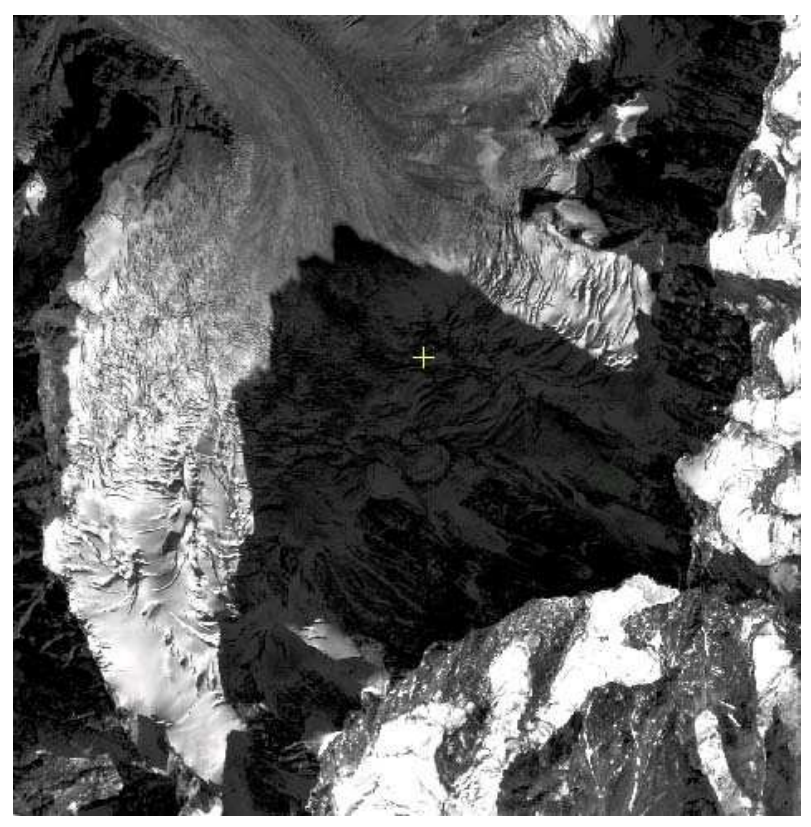

FIG. 3.3 - Ombre de la face Nord des Grandes Jorasses sur le glacier de Leschaux. Cette image SPOT5 a été acquise le 18 septembre 2003 à $10: 51$ (GMT). Le soleil se trouvait à N167. $5^{\circ}$ (azimut) et $45.5^{\circ}$ au dessus de l'horizon. Dans l'ombre, des structures (crevasses) restent visibles.

\subsubsection{Changements rapides de l'état de surface des glaciers}

A cause des processus qui contrôlent l'accumulation et l'ablation (chapitre 2), l'état de surface du glacier évolue rapidement. Les glaciers tempérés sont sujets régulièrement à des chutes de neige et à la fonte. Les deux phénomènes peuvent être séparés dans le temps (glacier alpin) ou simultanés (glaciers andins).

Selon les capteurs satellitaires, la sensibilité à ces changements est différente. Ainsi, un capteur optique est sensible à l'albédo de surface (le rayonnement visible ou proche infrarouge (IR) ne pénètre pas la glace où la neige) qui peut changer drastiquement lors d'une chute de neige. Un capteur radar actif comme le SAR est en revanche insensible à la neige fraîchement tombée puisque l'onde radar pénètre à travers la neige si elle est sèche. Ceci doit être pris en compte dans le choix ou la programmation des images.

\subsubsection{La fréquente couverture nuageuse}

A cause de la barrière orographique, les masses d'air atmosphériques sont contraintes à s'élever pour franchir des chaînes de montagnes. Elles voient donc leur température chuter ce qui provoque la saturation de la vapeur d'eau et la condensation sous forme de nuages. Les zones montagneuses sont donc fréquemment sous les nuages. 
Ceci est un obstacle majeur pour l'observation des glaciers dans le domaine visible ou proche IR. Les images optiques et les données des lasers altimétriques sont inexploitables lorsque des nuages sont présents. Les nuages masquent la surface terrestre et ont une signature spectrale voisine de la neige (König et al., 2001a). L'un des avantages des capteurs actifs comme le SAR est qu'ils sont indépendants de l'éclairement solaire et insensibles aux nuages. Ils fonctionnent donc de nuit et en présence de nuages.

\subsubsection{La forte réflectance de la neige et de la glace}

Dans le domaine visible $(0.4$ à $0.7 \mu \mathrm{m})$, la réflectance de la neige et, à un moindre degré, de la glace sont forts (jusqu'à 0.9 pour la neige fraîche). Le gain des capteurs optiques est généralement adapté à l'observation du sol, des forêts, des zones cultivées aux réflectances plus faibles. Si le gain du capteur n'est pas modifié, les surfaces enneigées et englacées apparaîtront totalement blanches avec des comptes numériques saturés à 255. La partie gauche de la figure 3.4 nous montre le bassin d'accumulation de la Mer de Glace observé fin août 2000 sans modification du gain du capteur (image d'archive). Les zones couvertes de neige sont partout saturées. A droite, la même zone observée par SPOT5 fin août 2003. Pour cette image, acquise suite à notre demande, le gain a été fixé à sa valeur minimale. Aucune zone du glacier n'est saturée et le contraste radiométrique est important. En revanche, les zones hors du glacier sont sombres et peu contrastées avec des comptes numériques couvrant une gamme restreinte entre 5 et 25 .
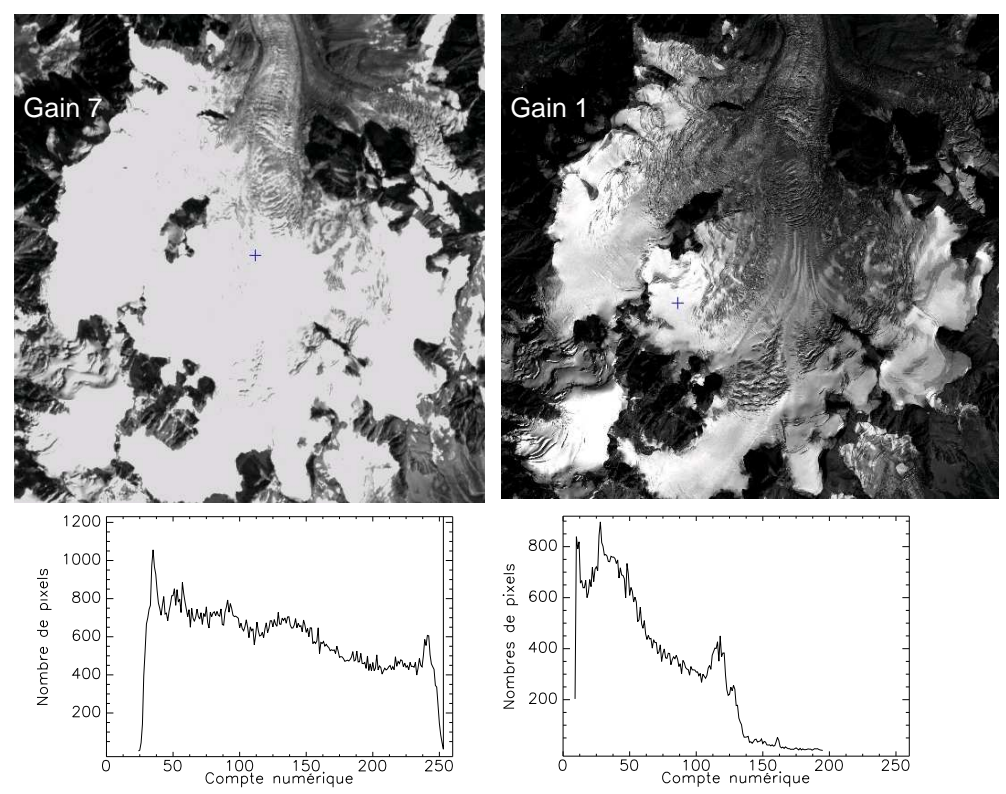

Fig. 3.4 - Observation du bassin d'accumulation de la Mer de Glace à la même période de l'année par les satellites SPOT avec des gains différents. A gauche, l'image de SPOT1 est acquise avec un gain de 7 le 25 août 2000, à droite l'image SPOT5 du 23 août 2003 avec un gain de 1. Pour chaque image, on montre également la distribution des comptes numériques (en bas). Pour l'histogramme de 2000, l'axe des Y a été tronqué, plus de 80000 de pixels ont un compte numérique de 255.

Des images optiques acquises en milieu ou fin de période d'accumulation semblent inutiles (même avec un gain faible) car la couverture neigeuse masque les structures de surface du glacier. La texture radiométrique des images est donc réduite. Des images optiques acquises à la fin de la 
période d'ablation (fin août, début septembre), quand la couverture neigeuse est réduite, doivent être privilégiées. Notons ici qu'à la lumière de nos travaux, nous rediscuterons de ce point au chapitre 5 et en conclusion.

Compte tenu des caractéristiques de l'environnement glaciaire, l'utilisation des capteurs satellitaires est sujette à de fortes restrictions :

- Les capteurs actifs micro-ondes comme les altimètres radars ne sont pas adaptés car leur empreinte au sol est beaucoup trop large (2 à $3 \mathrm{~km}$ pour les satellites ERS) et ils "décrochent" lorsque le relief est trop abrupt.

- Les images SAR permettent de travailler de nuit, en présence de nuages ou de neige fraîche. Les limites à leur utilisation sont géométriques (angle de vue fort) et parfois radiométriques (changement rapide de la surface dès qu'il y a de la fonte).

- Les images optiques présentent l'avantage de se rapprocher de la vision humaine. Elles bénéficient également des progrès technologiques qui permettent des résolutions toujours plus hautes (submétriques aujourd'hui). Elles souffrent de la couverture neigeuse et nuageuse. Un gain faible des images est donc un paramètre crucial pour étudier la zone d'accumulation des glaciers. Une attention toute particulière doit porter sur la géométrie d'acquisition pour accroître ou, au contraire, limiter les distorsions liées au relief accidenté.

\subsection{2 ... mais nécessaire car les glaciers...}

Malgré ces difficultés et limites inhérentes à l'environnement glaciaire, l'utilisation des techniques satellitaires a connu un essor important au cours des années 90 (Bindschadler, 1998; König et al., 2001a). La glaciologie bénéficie ou développe des techniques de pointe pour étudier des objets répartis sur toute la planète et difficiles d'accès.

\subsubsection{1 … ont une large répartition géographique.}

La figure 1.1 nous a montré que les glaciers étaient situés sous toutes les latitudes au sein des chaînes de montagne. Parmi les 300 glaciers qui sont (ou ont été) étudiés in situ, les trois quarts sont situés en Europe ou en Amérique du Nord (Braithwaite, 2002). Ceci s'explique par des raisons historiques (et économiques) puisque c'est sur le "Vieux Continent" puis en Amérique que la glaciologie a émergé. Il existe donc un biais géographique dans l'échantillonnage des glaciers du globe.

\subsubsection{2 $\quad$... sont nombreux et de taille variable.}

Les estimations les plus récentes font état de plus de 160000 glaciers sur Terre (Arendt et al., 2002). Seuls $0,2 \%$ de ces glaciers ont été étudiés (Dyurgerov, 2003) le plus souvent pendant de courtes durées. Les séries temporelles de bilan de masse dépassent 15 ans pour seulement $25 \%$ des glaciers étudiés (Braithwaite, 2002). Grâce à l'imagerie satellitaire ce n'est pas un ou deux glaciers que l'on étudie mais l'ensemble des glaciers d'un massif. Ceci permet d'étudier des effets comme l'orientation, la pente qui vont moduler leur réponse aux changements climatiques.

Aussi, dans le suivi des glaciers à l'échelle globale, plusieurs auteurs notent un biais vers les glaciers de petite taille (Braithwaite, 2002; Dyurgerov, 2002). Les mesures satellitaires sont plus 
faciles à réaliser sur des objets de grande taille et sont donc complémentaires des mesures de terrain.

En combinant les observations in situ et spatiales, on augmente le nombre et la diversité des glaciers étudiés. L'épineux problème de la représentativité d'un glacier seulement (Braithwaite, 2002) est ainsi évité.

\subsubsection{3 ... sont difficiles d'accès et sous échantillonnés par les mesures de terrain}

La morphologie glaciaire complique l'accès aux différentes zones du glacier. Les zones fortement crevassées, les chutes de séracs sont inaccessibles et dangereuses et il est impossible d'y installer des instruments. La figure 3.5 illustre ces difficultés d'échantillonnage dans le cas du glacier Zongo dans les Andes tropicales. Ces difficultés sont décuplées lorsqu'il s'agit d'un glacier de grande taille.

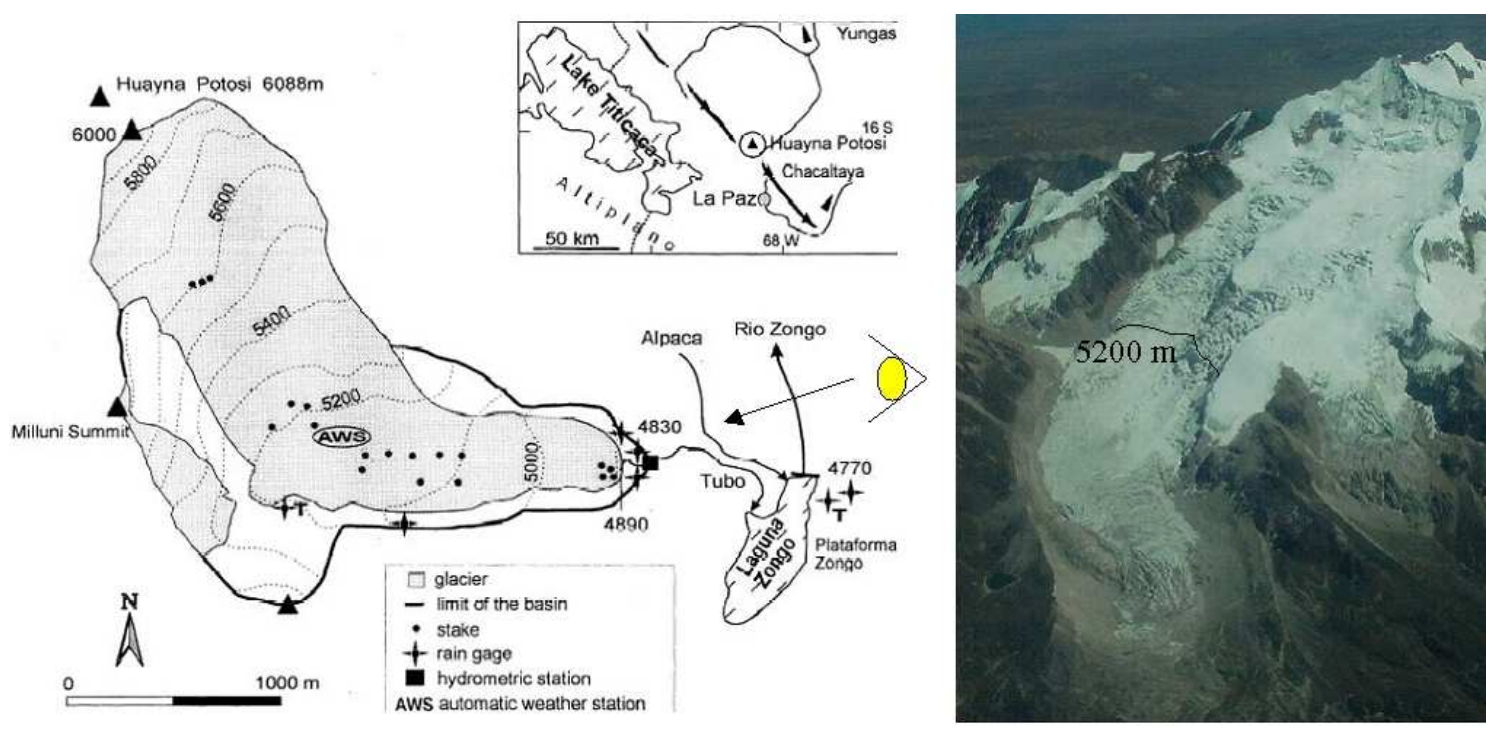

Fig. 3.5 - Dispositif d'évaluation du bilan de masse du Glacier Zongo (Cordillera Real, Bolivie). Même pour ce glacier de petite taille $\left(2.4 \mathrm{~km}^{2}\right)$, certaines zones ne sont pas échantillonnées car leur accès est dangereux ou laborieux (à près de $6000 \mathrm{~m} .$. ). Ainsi, des crevasses rendent inaccessibles les zones autour de $5000 \mathrm{~m}$ et entre 5300 et $5500 \mathrm{~m}$. D'après Berthier et al. (2001). A droite, une photographie aérienne du glacier prise depuis l'Est (Pouyaud, 2001). L'altitude $5200 \mathrm{~m}$ a été localisée.

Disposant d'un temps et d'un budget limité, le nombre de balises et de carottages sont restreints même dans les zones accessibles. Au mieux, c'est le long d'un profil longitudinal et de quelques profils transversaux que l'accumulation, l'ablation, et les vitesses de surface sont mesurées. Le comportement particulier des chutes de séracs, des zones latérales couvertes de débris ou sujettes aux avalanches ou encore la disparité spatiale des précipitations solides (Sicart et al., sous presse) sont difficilement pris en compte par un tel dispositif. L'observation, sans discrimination, de toutes les zones d'un glacier est en revanche possible par satellite étant donné l'extension des images.

Leur large couverture spatiale, l'accès à des zones retirées et la répétitivité de leur passage 
sont des avantages reconnus de l'observation satellitaire. Ils peuvent être exploités pour l'étude des glaciers de montagne mais dans des conditions strictes d'utilisation. Seuls quelques capteurs remplissent ces conditions et seront brièvement décrits dans la partie suivante.

\subsection{Les différents capteurs satellitaires}

L'objectif de cette partie n'est pas de détailler les caractéristiques de chacun des capteurs satellitaires utiles pour les glaciers de montagne. Pour cela, on se tournera vers les différents sites Internet (fournis au fil du texte). Il s'agit plutôt de les résumer en mettant l'accent sur certaines caractéristiques géométriques ou orbitales qui sont importantes pour notre étude. Ceci explique que l'on détaille plus spécialement le capteur HRG de SPOT5.

\subsubsection{Imagerie optique}

L'imagerie satellitaire optique, comme la photographie au sol ou aérienne, utilise la réflexion du rayonnement solaire dans les domaines visibles et IR. Elle fonctionne donc lorsque l'éclairement solaire est suffisant et les nuages absents. Ces images, en particulier celles des satellites SPOT, sont à la base de ce travail.

\subsubsection{Les satellites SPOT}

La famille des satellite SPOT (http ://www.cnes.fr \& http ://www.spotimage.fr) est composée de 5 membres depuis SPOT1 lancé en février 1986 jusqu'à SPOT5 en orbite depuis mai 2002. Actuellement, trois de ces satellites sont en fonctionnement : SPOT2 (depuis janvier 1990), SPOT4 (depuis mars 1998) et SPOT5. SPOT3, lancé en septembre 1993, a cessé de fonctionner en novembre 1996 alors que SPOT1 devient inutilisable en novembre 2003 après 18 années de service. Tous ces satellites partagent une même orbite héliosynchrone à une altitude de $830 \mathrm{~km}$. Le tour de la Terre est accompli en 101 minutes et la durée du cycle orbital ${ }^{4}$ est de 26 jours. L'acquisition des images se fait par un capteur de type "push-broom" : tous les pixels d'une ligne sont acquis simultanément par une série de capteurs CCD, l'avancée du satellite permettant de passer à la ligne suivante (figure 3.6). Les quatre barrettes de 1500 CCD chacune des SPOT1-4 ont été remplacées par une seule barrette de 12000 CCD sur SPOT5 ce qui limite les artefacts géométriques aux limites des barrettes (cf. annexe $\mathrm{E}$ ).

La résolution des images des SPOT1-4 est de $10 \mathrm{~m}$ en mode $\mathrm{P}$ ou panchromatique (longueur d'ondes de 0.49 à $0.69 \mu \mathrm{m}$ ), et $20 \mathrm{~m}$ en mode XS ou couleur (3 ou 4 canaux dans les longueurs d'ondes visibles ou proche IR). Pour SPOT5, la résolution est plus haute, $5 \mathrm{~m}$ en mode $\mathrm{P}$ et $10 \mathrm{~m}$ en mode XS. La taille du pixel peut même descendre à $2.5 \mathrm{~m}$ en combinant deux scènes à $5 \mathrm{~m}$ (figure 3.7 et Fratter et al. (2001); Latry \& Rougé (2002)).

L'une des qualités principales de ce système est qu'une zone peut être observée de façon répétée depuis différents points de vue grâce à une constellation de trois satellites (actuellement) sur une même orbite mais avec un décalage temporel ( 7 jours entre SPOT2 \& SPOT4; 7 jours entre SPOT4 \& SPOT5; 12 jours entre SPOT5 \& SPOT2). Ceci est également lié aux possibilités de dépointage de chaque capteur. En effet, un miroir pivotant permet d'observer au nadir, vers

\footnotetext{
${ }^{4}$ durée entre deux orbites identiques du satellite : après 26 jours, le satellite occupe la même position ce qui minimise les distorsions entre les deux scènes.
} 
la droite ou vers la gauche de la trajectoire (figure 3.6). Ceci permet de définir l'angle d'incidence qui est l'angle, en un point au sol, entre la verticale et la ligne de visée du satellite (CNES, 1996). Il est nul au nadir du satellite et peut atteindre $30^{\circ}$ dans le cas de visées obliques.
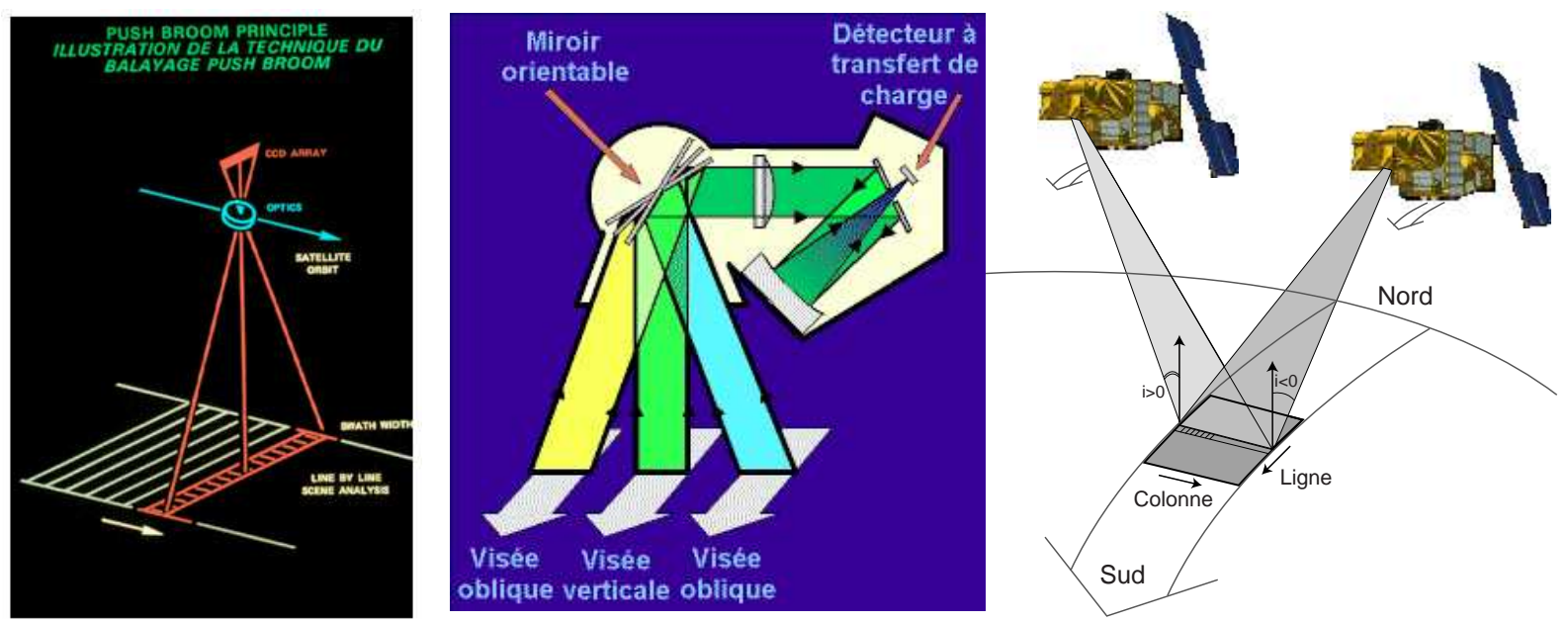

FIG. 3.6 - Acquisition des images par les satellites SPOT. A gauche : le système "push broom". Au centre : miroir orientable du système optique qui permet d'observer des zones situées hors de la trajectoire (www.spotimage.fr). A droite : observation de la même zone depuis deux trajectoires différentes (stéréoscopie latérale) et convention sur les angles d'incidence et les directions des lignes et colonnes.

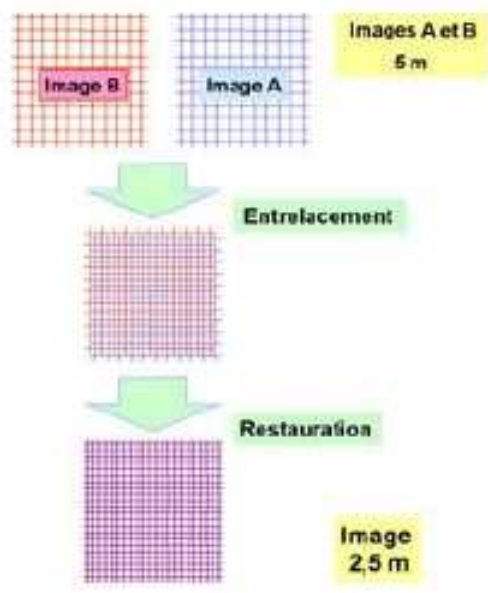

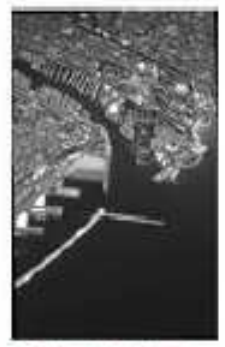

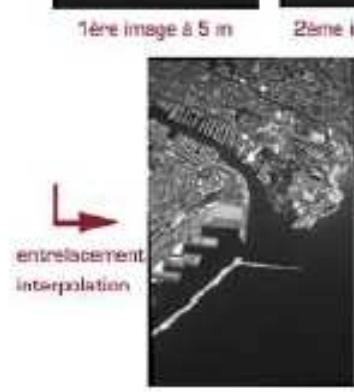

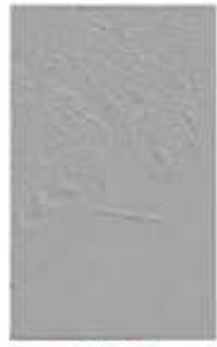

Litférence

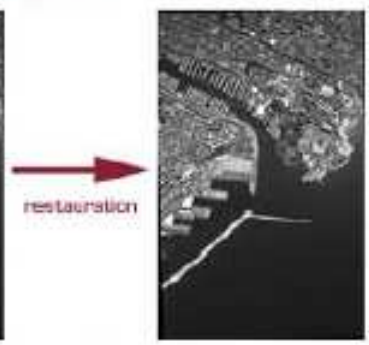

FIG. 3.7 - Principe du supermode. L'entrelacement de deux images SPOT5 panchromatiques (de résolution $5 \mathrm{~m}$ chacune) conduit à une image THR de $2.5 \mathrm{~m}$ de résolution.

Cette géométrie d'acquisition induit des différences importantes entre les lignes et les colonnes de l'image. Contrairement à la direction des colonnes, la direction des lignes de l'image est perpendiculaire à la ligne de visée du satellite. Nous en verrons une conséquence impor- 
tante au chapitre 4. Un autre effet est un pixel qui n'est pas carré. Sa taille est proche de la résolution optimale partout dans l'image dans la direction des lignes (aux effets topographiques près) alors que dans la direction des colonnes sa taille va augmenter avec l'angle d'incidence, proportionnellement à l'inverse du cosinus de l'angle d'incidence.

Les possibilités de dépointage permettent donc d'observer la même scène depuis deux points de vue différents à quelques jours d'intervalle. Les distorsions (essentiellement dans la direction des colonnes) entre les deux images sont liées à la topographie. Les scènes SPOT sont donc classiquement utilisées pour calculer des MNT (voir 2.1.3.4 pour la définition du MNT et le chapitre 5 pour leur construction).

L'une des améliorations avec le lancement du satellite SPOT5 concerne le contrôle de l'orbite du satellite. Roulis, tangage et lacet (voir Van Puymbroeck et al. (2000) pour leur définition) sont mieux maîtrisés et surtout mesurés le long de l'orbite (notamment grâce au système DORIS). Les images SPOT5 sont donc livrées avec des éphémérides détaillées pour chacune des lignes. Même sans point de contrôle au sol mais avec un MNT, une image SPOT5 peut être positionnée au sol avec une incertitude de seulement $\pm 30 \mathrm{~m}$ (intervalle de confiance à $66 \%$ ) contre plusieurs centaines de mètres pour SPOT1-4.

Concernant SPOT5, nous avons jusqu'alors uniquement décrit le capteur HRG ${ }^{5}$. Ce satellite héberge également le capteur $\mathrm{HRS}^{6}$ dédié à la génération de MNT. Ce capteur comprend deux systèmes optiques l'un pour observer la surface à l'avant de la trajectoire, l'autre à l'arrière. Une zone du globe est observée, quasi-simultanément, de $\pm 20^{\circ}$ (figure 3.8). La simultanéité des deux images assure leur ressemblance : les conditions d'éclairement et l'état de surface sont similaires. Malheureusement, les données de HRS ne sont pas disponibles pour la communauté scientifique. Nous verrons en annexe G qu'une combinaison originale de ces images permettraient pourtant la détermination des 3 composantes des déplacements de la surface terrestre.

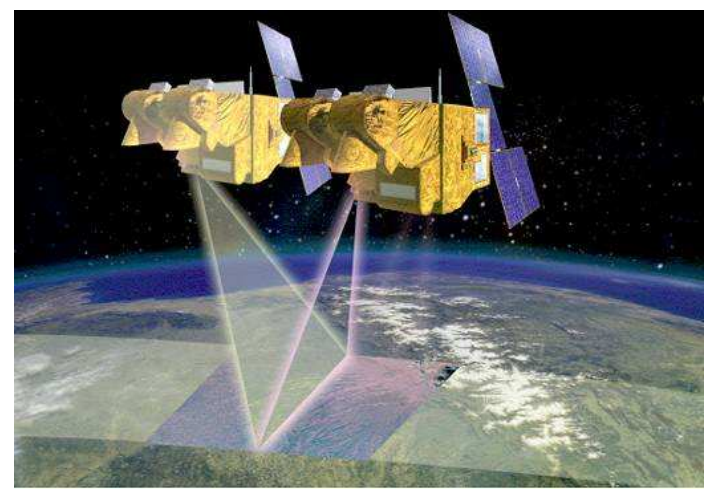

FIG. 3.8 - Stéréoscopie le long de la trajectoire du capteur HRS à bord de SPOT5. La même zone du globe est observée à quelques secondes d'écart ce qui permet la génération d'un MNT (www.spotimage.fr).

\subsubsection{Autres capteurs optiques}

Les autres capteurs optiques utiles en glaciologie alpine sont :

\footnotetext{
${ }^{5}$ Haute Résolution Géométrique

${ }^{6}$ Haute Résolution Stéréoscopique
} 
- Les capteurs TM (Landsat 4 et 5) et ETM+ (Landsat 7) à bord des satellites Landsat. L'utilisation glaciologique de ces images est détaillée par Bindschadler (1998); König et al. (2001a); Bindschadler et al. (2001). Landsat 7 a été lancé en 1999 et dispose d'une résolution de $15 \mathrm{~m}$ (contre $30 \mathrm{~m}$ pour ces prédécesseurs) en mode panchromatique. Il conserve une très large empreinte au sol $\left(185 \times 170 \mathrm{~km}^{2}\right)$ mais pas de possibilité de dépointage. La durée du cycle orbital est de 16 jours. Une description plus complète est disponible à http ://landsat.gsfc.nasa.gov/.

- La capteur ASTER à bord du satellite TERRA lancé en décembre 1999. Ce capteur n'acquiert pas d'images panchromatiques mais dispose d'une résolution de $15 \mathrm{~m}$ dans chacun des trois canaux du visible et proche infrarouge (Kääb, 2002). L'empreinte au sol est de $60 \times 60 \mathrm{~km}^{2}$. Comme le capteur HRS à bord de SPOT5, l'un des avantages de ASTER est de permettre l'acquisition d'images stéréoscopiques simultanées le long de la trajectoire du satellite. En revanche, il ne dispose pas d'un contrôle orbital aussi performant que SPOT5. Les images ASTER ont pu être obtenues gratuitement grâce au projet GLIMS (http ://www.glims.org/). Des compléments d'information sur ce satellite sont disponibles sur http ://asterweb.jpl.nasa.gov/.

\subsubsection{Imagerie RADAR}

Dans le domaine des hyperfréquences, seul le traitement du signal retrodiffusé par synthèse d'ouverture permet d'obtenir des images avec une résolution métrique ou décamétrique compatible avec le suivi des glaciers de montagne. Ce traitement fourni des images $\operatorname{SAR}^{7}$ qui ont le grand avantage d'être indépendantes de la couverture nuageuse et de l'éclairement solaire. La figure 3.9 compare pour le même glacier de l'Antarctique une image SAR et optique. Deux des capteurs SAR les plus utilisés en glaciologie sont ceux des satellites de l'agence spatiale européenne (ESA) ERS-1 (lancé en 1991), ERS-2 (lancé en 1995) et ENVISAT (lancé en 2002) ou canadienne, RADARSAT-1 (lancé en 1995).
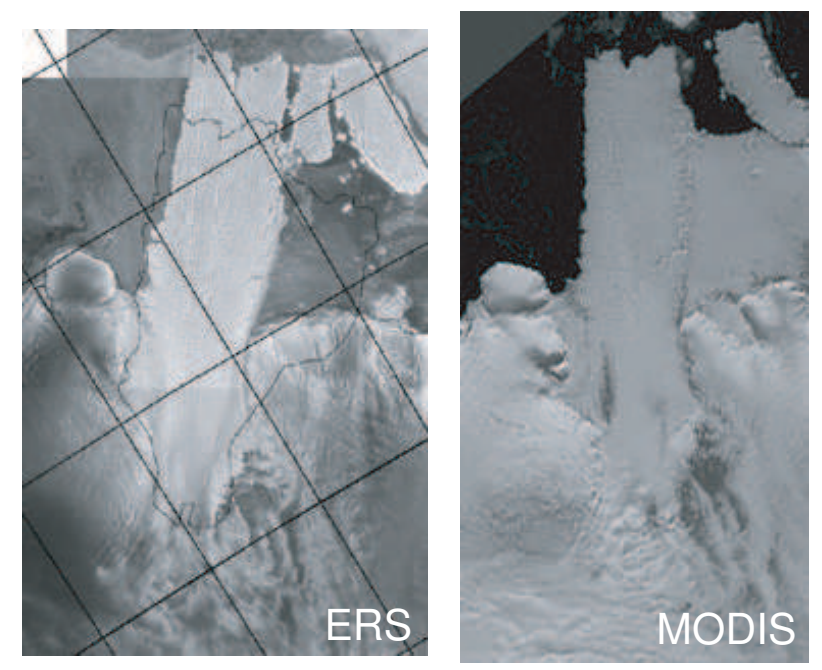

Fig. 3.9 - Le glacier Mertz (Antarctique de l'Est) observé par deux capteurs : image SAR du

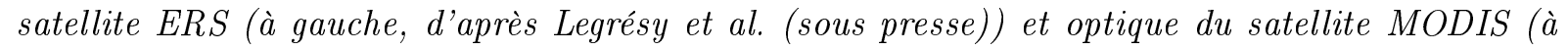
droite, d'après Berthier et al. (2003)).

\footnotetext{
${ }^{7}$ Synthetic Aperture Radar
} 
Les images SAR sont utiles car l'intensité de la rétrodiffusion dépend de la nature de la surface : elles permettent donc de caractériser l'état de surface (présence et quantité d'eau dans la neige par exemple). Outre l'amplitude, c'est aussi la phase du signal rétrodiffusé qui est utile. Quand deux images successives sont cohérentes, la différence de leur phase permet de mesurer les déplacements de la surface du sol et/ou la topographie. Nous verrons l'application de cette technique, l'interférométrie radar (Massonnet \& Feigl, 1998), dans le cas des glaciers de montagne.

\subsubsection{Altimétrie Radar et Laser}

Le principe de la mesure altimétrique est assez simple. Le temps de parcours d'une impulsion émise au nadir du satellite et réfléchie par la surface terrestre est convertie en distance surface-satellite (Testut, 2000). Les altimètres embarqués dans les satellites SEASAT puis ERS $(1 \& 2)$ ont montré leur efficacité pour cartographier les calottes polaires. Mais ils ne sont pas utilisables sur les glaciers de montagne. Deux autres altimètres (ICEsat et CryoSAT) peuvent potentiellement être utiles pour le suivi des plus gros glaciers tempérés.

\subsubsection{ICESat}

L'altimètre GLAS embarqué à bord de ICESat (lancé en janvier 2003) est de type laser : c'est un faisceau de forte puissance, de longueur d'onde 1,06 $\mu \mathrm{m}$, qui est réfléchi par la surface (Zwally et al. (2002b) et http ://icesat.gsfc.nasa.gov/). Cet altimètre est donc sensible à la présence de nuages et permet d'ailleurs d'étudier leur organisation. En l'absence de nuages, GLAS doit permettre de mesurer l'altitude de zones de $60 \mathrm{~m}$ de diamètre, avec une précision inférieure au mètre tous les $172 \mathrm{~m}$ le long de la trajectoire. Ces caractéristiques sont compatibles avec le suivi des glaciers de grande taille ou des petites calottes glaciaires. Des premiers résultats ont été obtenus sur l'Antarctique (Scambos et al., 2004) mais, à notre connaissance, pas sur des glaciers tempérés. Il n'a pas été utilisé dans cette thèse mais un des projets futurs est l'observation avec ICESaT des variations de volume de la calotte du Vatnajökull.

\subsubsection{CryoSAT}

Le lancement du satellite CryoSAT est actuellement programmé pour septembre 2005. Cette mission (http ://www.esa.int/esaLP/cryosat.html) de l'agence spatiale européenne est spécialement dédiée à l'observation des régions polaires : calottes et banquises. CryoSAT est équipé d'un radar altimétrique (SIRAL) qui peut fonctionner en mode standard (pour le centre des calottes) ou en mode interférométrique pour les zones à plus forte pente. C'est ce dernier mode qui pourrait éventuellement être utile pour l'étude des plus gros glaciers de montagne.

Ce rapide panorama des différents satellites qui peuvent ou pourront observer les glaciers de montagne est enthousiasmant pour les années futures. Le nombre et la résolution croissante des capteurs optiques, ainsi que des missions altimétriques de plus en plus adaptées à l'étude de petites surfaces, vont dynamiser l'observation des glaciers depuis l'espace. Mais nous allons voir que les glaciologues n'ont pas attendu les missions qui leur étaient dédiées pour tirer parti des données satellitaires. 


\subsection{Observation satellitaire des glaciers : état des lieux}

Les données satellitaires ont permis des progrès dans trois grands domaines de la glaciologie : suivi de l'état de surface, de la géométrie des glaciers et mesure de leur vitesse d'écoulement.

\subsection{1 État de surface des glaciers}

Les images optiques comme les images SAR permettent de caractériser l'état de surface des glaciers et d'étudier la distribution des différents faciès : neige fraîche à humide, névé aux différents stades d'évolution et glace vive. La distinction entre ces différentes zones n'est pas toujours facile. Par exemple, des zones de neige fraîche peuvent avoir une signature SAR similaire à celle des zones dépourvues de neige à cause de la pénétration des micro-ondes dans la neige froide et sèche (Fily et al., 1995). La détermination des épaisseurs de neige et leur équivalent en eau est une question importante (risques d'avalanche, prévision des débits) mais complexe. On notera juste des résultats encourageants obtenus récemment par interférométrie radar (Guneriussen et al., 2001; Rott et al., 2003). De telles méthodes permettent d'espérer la mesure de l'accumulation (donc du bilan de masse hivernal) par satellite.

Un des objectifs des études en optique est la détermination de l'albédo de surface du glacier. L'albédo est en effet un des paramètres importants contrôlant l'ablation (Sicart, 2002) et sa mesure régulière pourrait permettre d'envisager une méthode énergétique d'évaluation du bilan de masse (chapitre 2). Mais la conversion de la réflectance en albédo n'est pas simple. La réflectance est mesurée dans quelques canaux du visible et proche IR, dans une géométrie unique (mesure dans la direction de visée du satellite d'un rayonnement venant de la position au temps $t$ du soleil) alors que l'albédo est la réflectance intégrée sur toutes les longueurs d'onde (0.4 à $3 \mu \mathrm{m})$ et dans toutes les directions de l'espace (König et al., 2001a). Toutefois, des résultats prometteurs ont été obtenus par De Ruyter de Wildt et al. (2002) sur la calotte glaciaire du Vatnajökull.
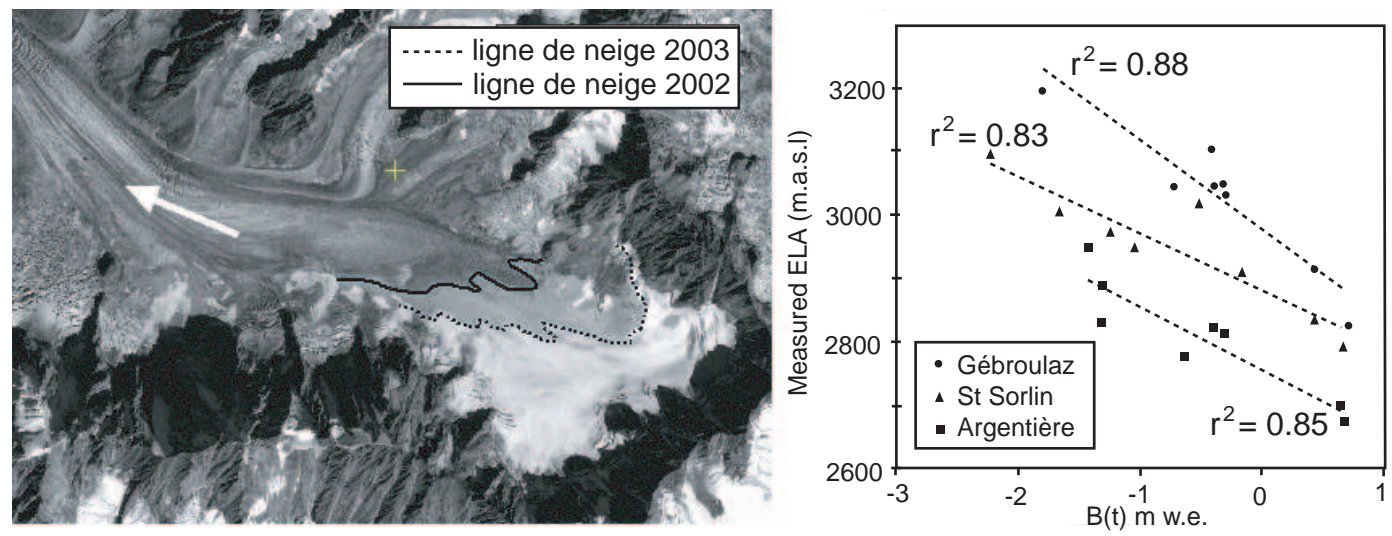

FIG. 3.10 - A gauche : localisation de la ligne de neige (tireté) sur une image SPOT5 du glacier d'Argentière acquise le 23 août 2003. Pour cette année fortement déficitaire, la ligne de neige est remontée à haute altitude ce qui permet d'observer celle de l'année 2002 (trait continu). La flèche indique le sens de l'écoulement. A droite : relation entre le bilan de masse et l'altitude de la ligne d'équilibre pour ce glacier (et deux autres glaciers des Alpes françaises). D'après Rabatel et al. (2005). 
Il est souvent possible de localiser sur des images la ligne de neige (figure 3.10 à gauche), ligne qui sépare la neige de la glace vive ou du névé (Rabatel et al., 2005). En première approximation, cette ligne devrait être confondue avec la ligne d'équilibre (notée $E L A^{8}$ et définie au chapitre 2) à la fin de la saison d'ablation. Or, il existe une relation quasi linéaire entre la $E L A$ et le bilan de masse du glacier (figure 3.10 à droite). L'observation chaque année de la $E L A$ doit donc permettre d'évaluer les fluctuation du bilan de masse du glacier. Pratiquement, la position de cette ligne peut être mesurée soit sur des images optiques soit sur des images SAR. En optique, la difficulté consiste à obtenir une image le plus tard possible dans la saison d'ablation, sans nuages et avant les premières chutes de neige (König et al., 2001a). Pour les images SAR, la trace de cette ligne peut être mesurée sur des images acquises au cœur de l'hiver suivant car l'onde RADAR traverse la neige sèche. Il existe toutefois une incertitude de la mesure SAR. Certains auteurs pensent que plutôt que la ligne de neige ${ }^{9}$, c'est la ligne de névé ${ }^{10}$ qui est observée (Hall et al., 2000; König et al., 2001b). Une fois la ligne de neige positionnée, son altitude est déduite d'un MNT (si possible contemporain) du glacier.

Cette approche est séduisante. Sa principale limite concerne son utilisation sur des glaciers jamais étudiés in situ où la sensibilité du bilan de masse à une variation d'altitude de la ligne d'équilibre $\left(\delta b / \delta z_{E L A}\right)$ n'a pas été établie. Rabatel et al. (2005) soulignent qu'une valeur unique de $\delta b / \delta z_{E L A}$ conduit à des résultats cohérents pour trois glaciers des Alpes françaises d'orientation voisine (Nord). L'extrapolation à des glaciers alpins d'orientations autres ou, pire, situés dans des régions climatiques différentes semble plus problématique. De plus, on évalue ainsi le bilan de masse au voisinage de la ligne d'équilibre, qui n'est pas identique au bilan de masse de l'ensemble du glacier.

Même sans convertir son altitude en bilan de masse, il existe une information climatique dans les fluctuations inter-annuelles de la ligne de neige. Arnaud et al. (2001) ont pu utiliser cette information sur le volcan du Sajama (Bolivie) et la relier avec les alternances Niño-Niña qui contrôlent la variabilité climatique dans cette région. Une évolution temporelle nette émerge également puisque la ligne de neige est remontée de $5200 \mathrm{~m}$ (début des années 1960) à près de 6000 à la fin des années 1990.

Enfin, la réflectance de l'eau étant très faible, les lacs supra glaciaires et leur extension peuvent être détectés grâce à des images satellites. Ainsi, le satellite ASTER a révélé de nombreux lacs potentiellement dangereux en cours de développement sur les langues glaciaires himalayennes (Wessels et al., 2002). Un suivi régulier est important car, dans un contexte de retrait glaciaire, le nombre et la taille de ces lacs augmentent.

\subsubsection{Variations de longueur et superficie glaciaire}

Si l'information radiométrique des images satellites permet de caractériser différents faciès, elle différencie également les zones avec ou sans glace. Il est ainsi possible de délimiter le glacier et donc d'étudier au cours du temps l'évolution de sa géométrie (longueur et superficie). Ces limites sont faciles à déterminer quand le contraste radiométrique avec les zones environnantes est important. Cela devient plus délicat lorsque le glacier est couvert de rochers car il ressemble aux moraines qui le bordent (zone d'ablation, voir figure 1.12) ou lorsque une chute de neige récente est intervenue. Le contour du glacier peut être dessiné manuellement, mais des algorithmes

\footnotetext{
${ }^{8}$ Equilibrium Line Altitude

${ }^{9}$ ligne de neige : limite entre la glace vive et la neige pour l'année hydrologique qui vient de se terminer

${ }^{10}$ ligne de névé : limite entre la glace vive et la neige, moyennée sur plusieurs années (König et al., 2001a)
} 
automatiques sont également utilisés (Paul et al., 2001).

La précision de la délimitation du glacier est étroitement liée à la résolution de l'image. Ainsi, la comparaison avec les mesures de terrain indique le plus souvent une précision voisine de \pm 1 pixel, parfois plus (Hall et al., 2003).

La large couverture des images satellites (notamment LANDSAT) permet de déterminer les extensions glaciaires pour des massifs entiers et de créer des inventaires complets. Parmi ces inventaires, on peut citer le Satellite Image Atlas of Glaciers of the World lancé en 1978 par l'USGS et qui n'est pas encore achevé. Il faut dire que réaliser un inventaire exhaustif est fastidieux. Le programme GLIMS a maintenant pris la relève avec des objectifs similaires. C'est dans ce cadre que des études ont mis en évidence un recul important des glaciers dans les Alpes suisses (Paul et al., 2004) dont la surface a diminué de plus d'1\% par an entre 1985 et 1999 (figure 3.11). Des observations similaires ont été faites dans le Tien Shan (Khromova et al., 2003), dans la Cordillère blanche péruvienne (Silverio \& Jaquet, 2005) ou dans la Péninsule Antarctique (Cook et al., 2005). Les limites actuelles (par satellite) sont souvent comparées aux anciennes cartes topographiques pour obtenir une évolution sur le long terme.

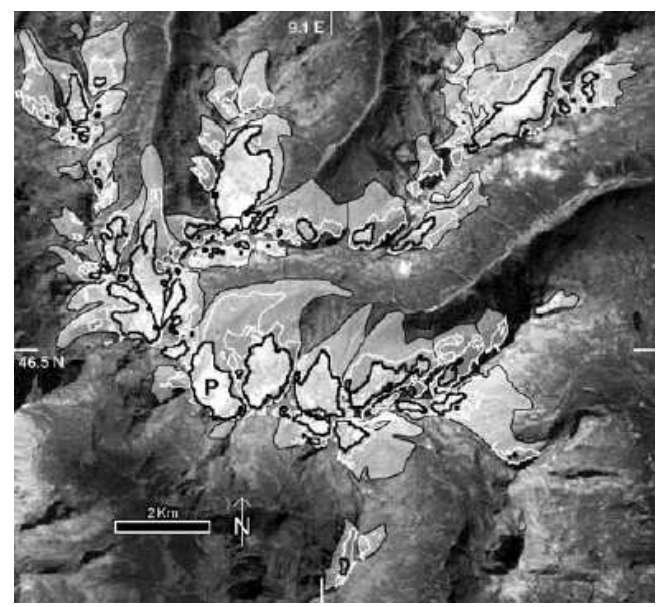

FIG. 3.11 - Limites du glacier dans le Massif du Rheinwald (Alpes suisses) à trois époques différentes : 1850 (limite noire avec un fond grisé), 1973 (limite blanche) et 1998 (limite noire épaisse). D'après Paul et al. (2004).

Si la réalisation d'inventaires globaux et le suivi de l'extension glaciaire sont importants, ils ne sont pas directement liés à des fluctuations climatiques et ne permettent pas non plus de préciser la contribution glaciaire à l'élévation du niveau marin. Arendt et al. (2002) ont montré qu'il n'y avait pas de corrélation entre variations d'épaisseur et de longueur des glaciers en Alaska. Un des objectifs de notre travail est d'ajouter la dimension verticale, autrement dit, de mesurer par satellite les variations volumétriques des glaciers. Les méthodes pour atteindre cet objectif et nos résultats seront présentés au chapitre 5 .

\subsubsection{Vitesses d'écoulement des glaciers}

Le chapitre 2 nous a montré le rôle de l'écoulement dans la redistribution de la masse au sein du glacier. Dans certains cas, la dynamique contrôle même le bilan de masse des glaciers. Une accélération du glacier transporte un surplus de glace dans les zones basses où l'ablation 
est forte (Zwally et al., 2002a). Les mesures in situ souffrent d'un échantillonnage limité et des techniques satellitaires ont été développées pour réaliser des cartes d'écoulement des glaciers. L'interférométrie SAR est la technique la plus utilisée depuis les années 90 . Nous en verrons les avantages mais aussi les limitations dans le cas des glaciers de montagne. Les autres méthodes de mesure des vitesses seront brièvement décrites.

\subsubsection{Interférométrie SAR (InSAR)}

L'InSAR consiste à soustraire les phases de deux images SAR pour mesurer les changements de distance entre le satellite et une cible au sol. La cible doit être suffisamment ressemblante lors des deux passages des satellites, autrement dit les images SAR doivent être cohérentes. La différence des phases conduit à un réseau de franges (à l'intérieur desquelles les valeurs varient entre 0 et $2 \pi$ ) nommé interférogramme. La topographie, la teneur en eau atmosphérique, les écarts entre les deux orbites, et le bruit du capteur contribuent à la différence des phases. Toutes ces contributions doivent être estimées pour déterminer le déplacement de surface du glacier $\Phi_{\text {moti }}$ (Massonnet \& Feigl, 1998; Rabus \& Fatland, 2000) :

$$
\Phi=\Phi_{\text {topo }}+\Phi_{\text {atmo }}+\Phi_{\text {orbi }}+\Phi_{\text {nois }}+\Phi_{\text {moti }}
$$

Ce sont les déplacements générés par un séisme, celui de Landers en 1992, qui ont été cartographiés par satellite pour la première fois avec cette technique (Massonnet et al., 1993). Goldstein et al. (1993) réalisent eux la première mesure interférométrique de l'écoulement d'un glacier, le fleuve de glace de Rutford en Antarctique. Depuis, cette technique a été utilisée régulièrement pour mesurer l'écoulement des glaciers des calottes polaires (König et al., 2001a).

L'InSAR a également permis de mieux contraindre la dynamique des petites calottes glaciaires, notamment des crues glaciaires surges de leurs émissaires (Dowdeswell et al., 1999; Fischer et al., 2003; Murray et al., 2003). Les applications aux glaciers de montagne sont moins nombreuses (Mattar et al., 1998; Rabus \& Fatland, 2000) notamment à cause d'un état de surface qui évolue rapidement. Avant (Legrésy et al., 2002) et pendant cette thèse (avec une meilleure topographie), des interférogrammes ont été réalisés sur les glaciers du Mont-Blanc (figure 3.12).

La comparaison et l'analyse de ces deux interférogrammes ainsi que de nombreuses autres études permettent de dégager certains des avantages et inconvénients de l'InSAR appliquée aux glaciers de montagne.

Les principaux avantages sont :

- La précision de mesure des déplacements. Elle dépend des conditions d'acquisition des images, de la précision du MNT et des perturbations atmosphériques mais est voisine de \pm 1 à $2 \mathrm{~cm}$ (Goldstein et al., 1993; Mattar et al., 1998). Pour des images espacées d'un jour, l'incertitude sur les vitesses est donc inférieure à $\pm 10 \mathrm{~m} / \mathrm{an}$.

- L'indépendance vis à vis de la couverture neigeuse ou nuageuse (l'interférogramme de gauche est déduit d'images du cœur de l'hiver alors que le glacier est couvert de neige).

- Une large couverture spatiale. Un interférogramme (ERS) couvre $100 \times 100 \mathrm{~km}^{2}$.

Les limitations concernent :

- L'absence de mesure dans les zones trop rapides et les zones où le gradient de déplacement est trop important (Massonnet \& Feigl, 1998). C'est une des raisons expliquant que l'interférogramme à 3 jours (figure 3.12 à droite) est bruité sur les glaciers.

- La perte de cohérence rapide sur les glaciers (Strozzi et al., 2002), liée aux changements de 
l'état de surface (effet surtout de la fonte). Les images hivernales sont donc préférables. En bande $\mathrm{C}(\lambda=5.67 \mathrm{~cm})$, seuls les couples interférométriques à 1 jour sont cohérents sur les surfaces des glaciers tempérés. Sur la figure 3.12 à gauche, même pour $\delta \mathrm{t}=1$ jour, il y a perte de cohérence dans la partie haute de la Mer de Glace. Se pose également la question de la signification de ces vitesses journalières, compte tenu de la forte variabilité temporelle de la dynamique glaciaire.

- Une mesure ambiguë, les franges doivent être déroulées ce qui n'est pas toujours possible.

- La mesure des déplacements du glacier projeté dans une seule direction, celle de visée du satellite. Ainsi, la mesure n'est possible que si le glacier s'écoule dans une direction non perpendiculaire à la ligne de visée. Sur le figure 3.12 (à gauche), le glacier de Leschaux est bien orienté contrairement à la Mer de Glace. Pour déduire les déplacements horizontaux, des hypothèses peuvent être faites sur la direction de l'écoulement : dans la ligne de plus grande pente et parallèlement à la surface (Kwok \& Fahnestock, 1996). Si deux interférogrammes peuvent être combinés depuis des orbites ascendantes et descendantes (Mohr et al., 1998; Joughin et al., 1998), deux observations des 3 composantes du vecteur déplacement sont disponibles et une seule hypothèse suffit. Mais cette combinaison suppose la constance de la vitesse du glacier, condition rarement remplie par les glaciers de montagne.

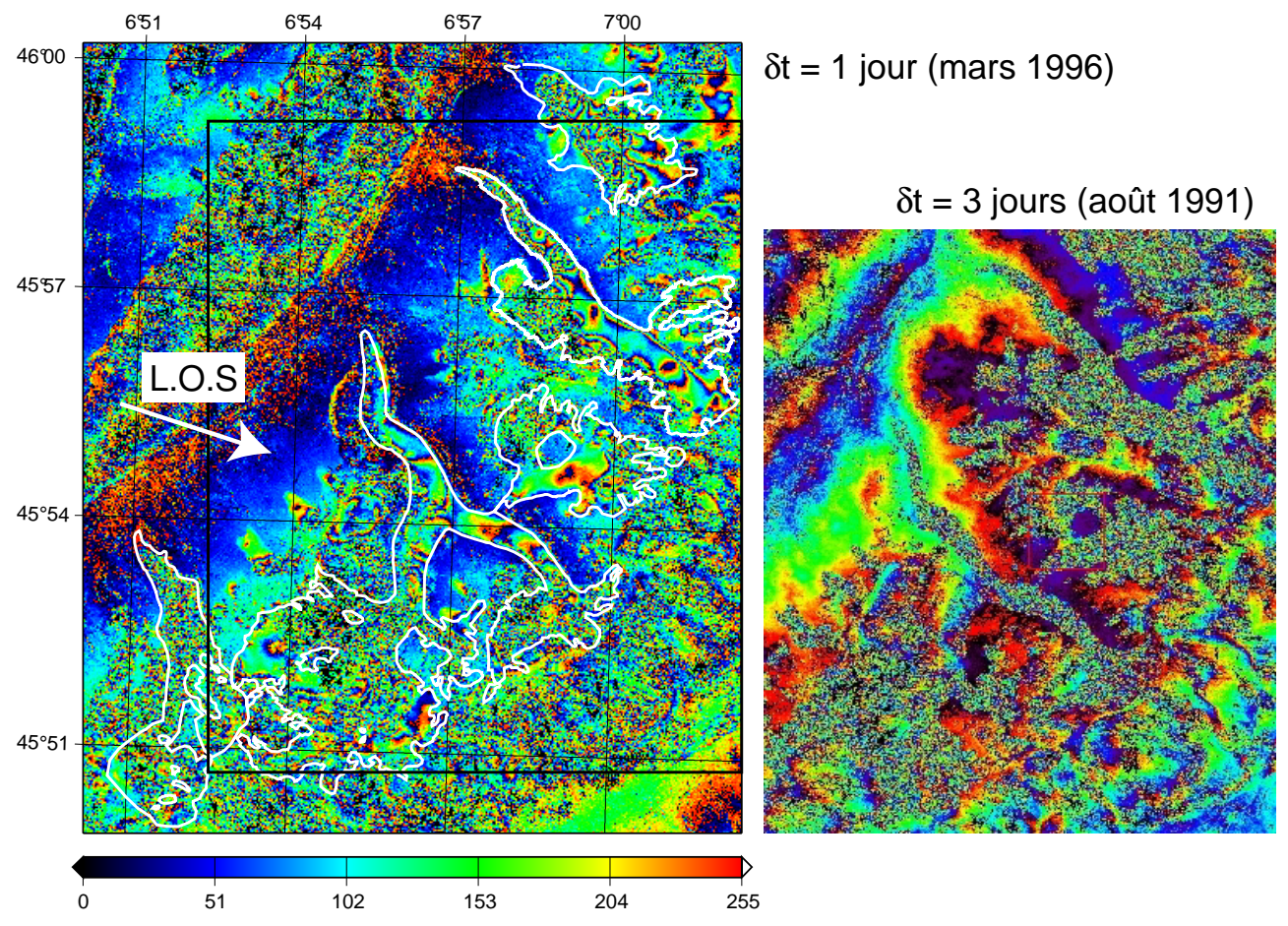

Fig. 3.12 - Interférogrammes du Massif du Mont-Blanc. A gauche, couple d'images de la mission tandem ERS1/ERS2 acquises les 10 et 11 mars $1996(\delta t=1$ jour $)$. En blanc, les limites des glaciers en 2003. A droite, couple d'images ERS1 acquises les 17 et 20 août 1991 ( $\delta t=3$ jour). Une frange représente un déplacement de $28 \mathrm{~mm}$ dans la direction de visée du satellite (l.o.s. indiquée par une flèche).

Actuellement, aucune mission satellitaire ne permet d'obtenir des images SAR en condition interférométrique séparées de un jour seulement. L'application cryosphèrique de l'InSAR est donc limitée aux zones froides des hautes latitudes où l'accumulation est faible, la fonte absente et l'écoulement réduit (Gray et al., 2005). Une des motivations de cette thèse est donc de proposer 
une méthode alternative à l'interférométrie SAR pour la mesure des vitesses des glaciers tempérés. Nous développerons notre méthodologie et les résultats glaciologiques dans le chapitre 4 .

\subsubsection{Alternatives à l'interférométrie SAR}

En s'appuyant également sur des images SAR, les déplacements peuvent être mesurés en suivant par corrélation le déplacement de structures (Strozzi et al., 2002). Lorsque c'est le bruit de phase qui est suivi il s'agit du speckle tracking ${ }^{11}$ (Michel et al., 1999; Michel \& Rignot, 1999; Gray et al., 2001; Joughin, 2002). La corrélation peut également être appliquée aux images d'amplitude (intensity tracking). Ces techniques sont moins précises que l'InSAR mais mesurent deux dimensions du vecteur déplacement et sont moins sensibles aux changements de l'état de surface. Mais l'application de ces techniques aux glaciers de montagne reste délicate (Arnaud, communication personnelle).

La corrélation d'images satellites optiques permet de suivre le déplacement de structures en surface du glacier. Elle procure également une cartographie des vitesses d'écoulement sur de grandes zones. C'est la solution que nous avons choisie pour étudier la dynamique des glaciers de montagne. Le principe de cette technique sera donc détaillé au chapitre 4 . On notera simplement ici que l'application de cette méthode à des photographies terrestres nous semble complémentaire de la méthode satellitaire (Evans, 2000).

Le GPS (Global Positionning System) différentiel ne procure pas une couverture globale des déplacements du glacier mais la position successive de quelques points visités sur le terrain. Le positionnement se fait à partir d'une constellation de satellites (ce qui justifie de le mentionner ici). Deux missions DGPS sur les glaciers de Mont-Blanc nous ont permis d'obtenir une vérité terrain presque simultanément à la mesure satellitaire (cf. figure 4.16).

\subsection{Conclusions et objectifs méthodologiques}

L'imagerie satellitaires a permis de mieux décrire l'évolution des glaciers depuis 2 décennies et de mieux comprendre certains aspects de leur dynamique. C'est surtout les calottes polaires qui ont bénéficié de ces avancées. Les glaciers de montagne, au cœur d'une topographie abrupte, sont plus difficiles à étudier depuis l'espace. Leur suivi suppose des développements méthodologiques et donc, outre les objectifs scientifiques détaillés en conclusion du chapitre 2 , les questions méthodologiques abordées par cette thèse sont les suivantes :

1. Peut on proposer une alternative à l'interférométrie SAR pour la mesure des vitesses de surface des glaciers? Nous verrons au chapitre 4 que la corrélation d'images optiques à haute résolution répond à cette question et apporte une vision quasi globale de l'écoulement des glaciers.

2. Les données satellitaires permettent-elles de mesurer des variations d'épaisseur et donc le bilan de masse des glaciers? Ce problème sera examiné au chapitre 5 en calculant des MNT différentiels.

3. Les méthodes satellitaires précédentes, développées sur les glaciers de Mont-Blanc, sont elles transposables à la calotte glaciaire du Vatnajökull (chapitre 6) ou à des glaciers himalayens $(\S 5.3)$ pour étudier leur bilan de masse et/ou leur dynamique?

\footnotetext{
${ }^{11}$ ou suivi du chatoiement
} 


\section{Chapitre 4}

\section{Satellite \& écoulement des glaciers alpins}

\section{Sommaire}

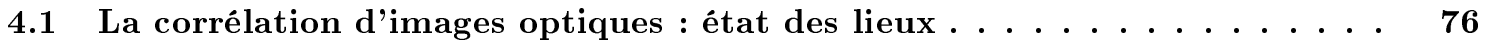

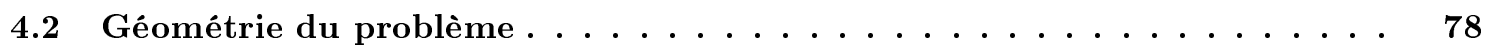

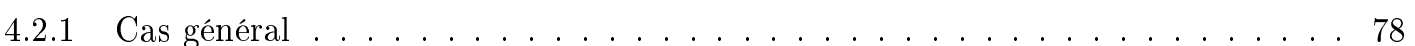

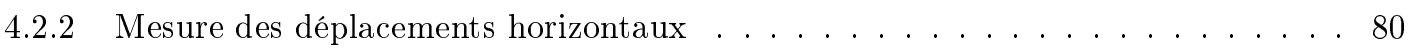

4.2 .3 Mesure des déplacements verticaux . . . . . . . . . . . . . . . 80

4.3 Notre chaîne de traitement : ME DECOIS PAS . . . . . . . . . . . 81

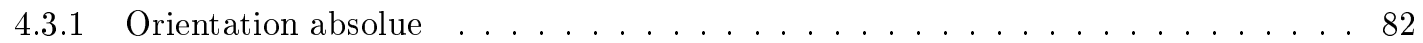

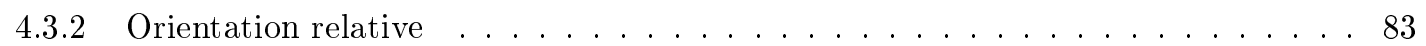

4.3 .3 Ré-échantillonnage de l'image esclave . . . . . . . . . . . . . . . . . . 84

4.3 .4 Corrélation . . . . . . . . . . . . . . . . . . . . . 84

4.3.5 De la géométrie image à la géométrie terrain . . . . . . . . . . . . . . . 84

4.4 Vitesses des glaciers du Mont Blanc durant l'été $2003 \ldots \ldots$. . . . . . . 86

4.4 .1 Les glaciers du massif du Mont-Blanc . . . . . . . . . . . . . . . 86

4.4.2 Caractéristiques géométrique et radiométrique des images SPOT5 . . . . . . 87

4.4 .3 Champs de déplacements pour l'été 2003 . . . . . . . . . . . . . . . . 89

4.4.4 Précision et exactitude de notre mesure des vitesses . . . . . . . . . . . . . . . . 91

4.4.5 Apports et limites de la méthodologie proposée . . . . . . . . . . . . . . . 99

4.5 Variations à court terme de l'écoulement . . . . . . . . . . . . . . . 100

4.5.1 Cartographie des variations de vitesse . . . . . . . . . . . . . . . 100

4.5.2 Une interprétation de ces variations de vitesse . . . . . . . . . . . . . . . . 103

4.5.3 Discussion de ces variations de vitesse . . . . . . . . . . . . . . . . . . . . . 104

4.6 Variabilité interannuelle de l'écoulement f . . . . . . . . . . . . 106

4.6 .1 Comparaison des vitesses estivales . . . . . . . . . . . . . . . 106

4.6 .2 Comparaison des vitesses annuelles . . . . . . . . . . . . . . . . . . 109

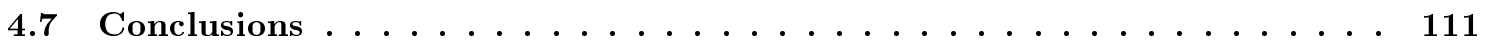

Nous avons vu dans le chapitre 2 qu'en régime stationnaire l'écoulement du glacier permet une redistribution de l'excès de masse de la zone d'accumulation vers la zone d'ablation. En régime perturbé (gain ou perte de masse), la dynamique va déterminer le temps de réponse (Jóhannesson et al., 1989) du glacier : plus un glacier est rapide, plus son temps de réponse sera 
court. Le schéma simplifié de la figure 4.1 illustre ce rôle de filtre de la dynamique. Une bonne compréhension de cette dynamique est donc importante.

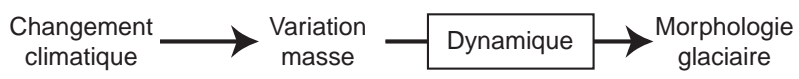

FIG. 4.1 - Effet de filtre de la dynamique du glacier entre des variations du bilan de masse et des changements morphologiques. Adapté de Meier (1962).

L'impossibilité d'utiliser actuellement l'interférométrie radar sur les glaciers tempérés nous a conduit à développer une chaîne de traitement des images SPOT utilisant la corrélation de deux images optiques. Après avoir dressé un panorama des études géophysiques utilisant cette technique (\$4.1), nous présentons les équations qui décrivent la géométrie de notre problème $(\S 4.2)$. Nous détaillons dans les deux parties qui suivent $(\S 4.3 \& \S 4.4)$, les différentes étapes permettant de calculer les déplacements à partir de deux images SPOT avant de présenter les résultats obtenus pour deux couples d'images SPOT5 acquis durant l'été 2003. Ces travaux ont donné lieu à une publication dans le journal Remote Sensing of Environment (Berthier et al., 2005) qui se trouve en intégralité en annexe C. Nous discutons ensuite les variations à court terme de l'écoulement au cours de l'été 2003 (§4.5). Des images d'archives SPOT1-4 permettent d'étudier la variabilité inter-annuelle de la vitesse (§4.6), avant de conclure (§4.7).

\subsection{La corrélation d'images optiques : état des lieux}

La corrélation d'images de capteurs optiques pour déterminer des mouvements géophysiques est apparue dès le début des années 1970. Leese et al. (1971) ont ainsi corrélé des images du satellite ATS-1 (Applications Technology Satellites I) acquises à $24 \mathrm{mn}$ d'intervalles pour déterminer les vitesses de déplacements des nuages. La technologie a ensuite été transposée en océanographie pour mesurer les courants d'advections à la surface de l'océan (Emery et al., 1986). La première application glaciologique de cette méthode a permis la cartographie du mouvement des icebergs à partir d'images du satellite AVHRR (Ninnis et al., 1986).

Le mouvement des glaciers est d'abord déterminé en suivant à l'oeil le déplacement de crevasses visibles sur des images LANDSAT du glacier Bird (Lucchitta \& Ferguson, 1986). La corrélation d'images est introduite par Scambos et al. (1992) et apporte une vue globale de l'écoulement de fleuves de glaces qui drainent la glace de l'Antarctique (Bindschadler \& Scambos, 1991). La figure 4.2 montre un exemple de champ de vitesse de surface obtenu en corrélant deux images LANDSAT acquises à deux ans d'intervalle sur le glacier Mertz. De plus amples détails sur l'obtention de ce champ de vitesse et son utilisation pour déterminer le bilan de masse et la fusion basale de la partie flottante de la langue glaciaire pourront être trouvés dans l'article publié dans Journal of glaciology (Berthier et al., 2003), rédigé au début de cette thèse suite à une visite au National Snow and Ice Data Center. Cet article est présenté en annexe A.

En parallèle, la corrélation d'images optiques a été améliorée par la communauté "Terre solide", sans doute car les mouvements étudiés sont faibles, souvent inférieurs au pixel : une bonne précision est donc indispensable. Crippen (1992) étudie le déplacement (au maximum de $7 \mathrm{~m}$ ) de dunes de sable par corrélation d'images SPOT (résolution $10 \mathrm{~m}$ ) et montre ainsi que des mouvements sous pixellaires peuvent être détectés. Il suggère que cette technique peut mesurer les déplacements liés à des séismes (ou les volcans) mais ne dispose pas d'images pour tester son hypothèse. Il faut attendre 8 ans (Van Puymbroeck et al., 2000; Vadon \& Massonnet, 2000) pour 
que les déformations engendrées par les séismes de Landers (Californie, juin 1992) et d'Izmit (Turquie, août 1999) soient cartographiées par corrélation d'images SPOT. Michel \& Avouac (2002); Feigl et al. (2002) d'un part et Dominguez et al. (2003) d'autre part utilisent la même technique pour cartographier, estimer la rupture de surface et modéliser le fonctionnement des failles responsables des séismes d'Izmit et de Chi-Chi (Taiwan, septembre 1999) respectivement. Récemment, la corrélation d'images SPOT5 a permis d'obtenir des résultats similaires pour le séisme de Bam (Binet \& Bollinger, 2005). La corrélation de photographies aériennes et d'images satellites à très haute résolution (QuickBird) a aussi permis de caractériser les glissements de terrain (Delacourt et al., 2004).

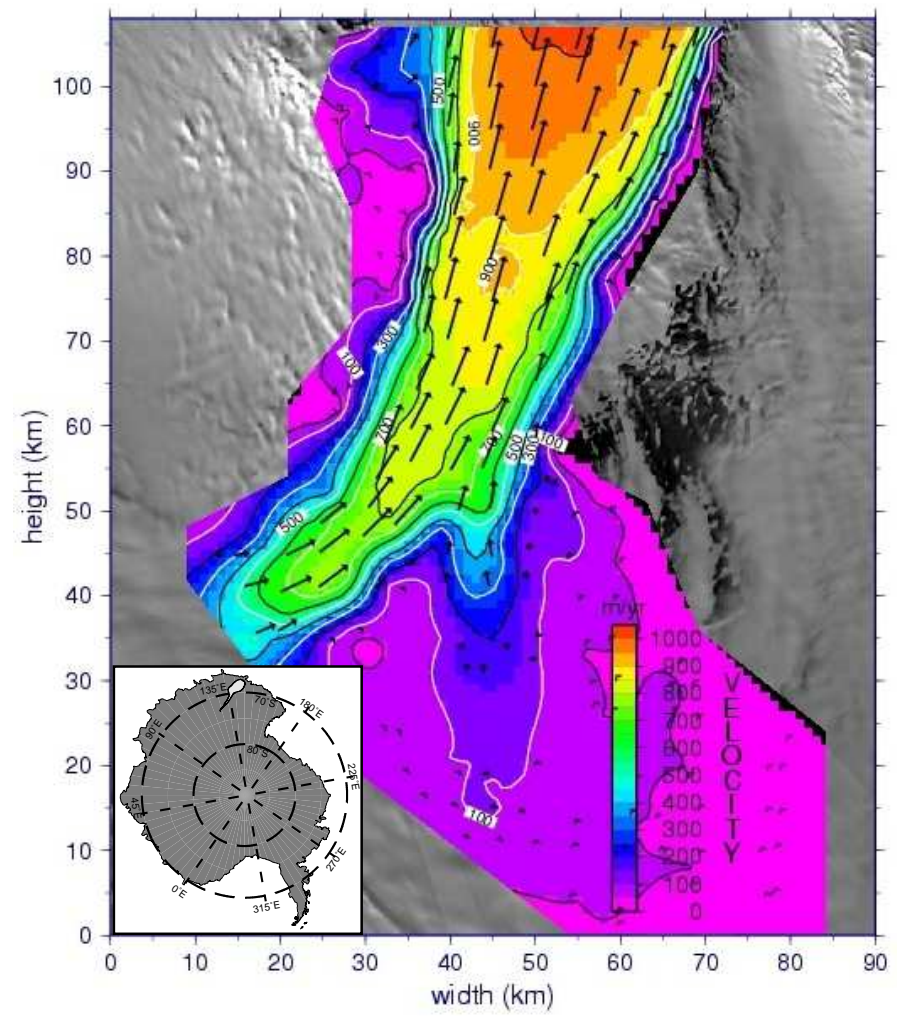

Fig. 4.2 - Vitesse d'écoulement du glacier Mertz déterminée par corrélation de deux images LANDSAT séparées de deux ans. La carte de l'Antarctique, en bas à gauche, permet de localiser le basin versant du Glacier Mertz (en gris clair en haut de la carte).

L'objectif de notre étude est de mesurer les vitesses d'écoulement des glaciers alpins, avec une couverture spatiale, une résolution et une précision permettant (1) d'étudier les mécanismes qui contrôlent leur écoulement et (2) de détecter les adaptations éventuelles de la dynamique dans un contexte de fort retrait glaciaire. Pour atteindre ce but, une chaîne de traitement, qui s’inspire des travaux de Vadon \& Massonnet (2000), a été développée.

Les problèmes spécifiques pour l'application de la corrélation d'images aux glaciers de montagne sont les suivants :

1. les changements sont rapides à la surface des glaciers. Ces changements s'expliquent par les chutes de neige, la redistribution de la neige par le vent et, à un degré moindre, par la fusion de surface et par les déformations liées à l'écoulement (cisaillement par exemple sur les bords 
du glacier). Ces problèmes, communs à toutes les zones glacées du globe, sont toutefois exacerbés à la surface des glaciers de montagne du fait de conditions météorologiques très variables. La séparation temporelle entre les images est donc un facteur clef : il s'agit de trouver un juste compromis entre un déplacement suffisant du glacier et une surface glaciaire bien préservée.

2. Les glaciers de montagne sont localisés dans des zones de forte topographie et peuvent eux-mêmes présenter des pentes importantes (au niveau des chutes de séracs par exemple). Les distorsions entre deux images vont être importantes sur nos sites d'étude. Des images acquises depuis deux positions voisines du satellite permettent de minimiser ces distorsions. Les images acquises en été, lorsque les rayons solaires sont proches de la verticale (milieu de journée), permettent de limiter la taille des ombres.

3. L'albédo élevé des zones glaciaires (proche de 1 quand la neige est encore peu transformée, plus faible sur la neige sale ou la glace) tend à saturer les capteurs optiques (cf. figure 3.4). Nous verrons que les images programmées spécifiquement pour nos études avec le gain le plus faible (1 pour SPOT) permettent d'éviter ces problèmes de saturation.

4. Par rapport aux fleuves de glace des calottes polaires, qui s'écoulent souvent à plus de $500 \mathrm{~m} \mathrm{a}^{-1}$ (même $12600 \mathrm{~m} \mathrm{a}^{-1}$ soit $1.5 \mathrm{~m}$ /heure pour le Jakobshavn Glacier au Groenland), les vitesses typiques des glaciers de montagne sont de l'ordre de $50 \mathrm{~m} \mathrm{a}^{-1}$. En conséquence, et puisque la séparation temporelle des deux images ne doit pas être trop importante, le signal recherché sera beaucoup plus faible que pour les études sur les fleuves de glace. Notre méthodologie doit donc viser une incertitude inférieure au \pm 1 pixel obtenu précédemment (Scambos et al., 1992; Kääb, 2005). Ceci est d'autant plus vrai que les changements temporels de la vitesse (de l'ordre de quelques $\mathrm{m} \mathrm{a}^{-1}$ ) nous intéressent également. Une attention particulière sera apportée à l'évaluation de la précision (écart-type) mais aussi l'exactitude (biais) de nos mesures.

Nous verrons comment le choix des images satellites et une méthodologie adaptée permettent de répondre à ces différents problèmes.

\subsection{Géométrie du problème}

En résumé, la corrélation consiste à mesurer des décalages en colonnes et en lignes entre deux images recalées sur les zones sans mouvement puis à les convertir en déplacement au sol. Notre chaîne de traitement sera détaillée dans la partie suivante, mais avant cela, nous souhaitons détailler le passage de la géométrie image à terrain. Ce passage est simple dans le cas d'images acquises verticalement. On mesure la projection du vecteur déplacement dans le plan focal du satellite qui est parallèle au sol : seules les composantes horizontales sont mesurées. C'est le cas par exemple pour les images LANDSAT. Cette conversion est donc rarement détaillée dans les publications. Pourtant, la géométrie du problème est plus complexe dès que l'angle d'incidence n'est pas vertical, notamment dans le cas des images SPOT. L'objectif de cette partie est de développer les équations qui permettent de convertir les décalages mesurés en géométrie image en déplacement au sol.

\subsubsection{Cas général}

En se plaçant dans un cas général, il est possible comme en InSAR (Massonnet \& Feigl, 1998) de définir des vecteurs de sensibilité au mouvement (figure 4.3$)$. Ces deux vecteurs $\left(\overrightarrow{U_{C O L}}\right.$ 
et $\left.\overrightarrow{U_{L I G}}\right)$ sont parallèles à chacune des directions de l'image. Leurs coordonnées dans le repère terrestre $(\lambda, \phi, \mathrm{z})^{1}$ sont :

$$
\begin{gathered}
\overrightarrow{U_{L I G}}=[-\sin \alpha,-\cos \alpha, 0] \\
\overrightarrow{U_{C O L}}=[\cos \theta \cos \alpha,-\cos \theta \sin \alpha, \sin \theta]
\end{gathered}
$$

où $\alpha$ représente l'angle de la direction des lignes avec le Nord géographique (azimut) et $\theta$ l'angle d'incidence.

Un déplacement de la surface terrestre $\vec{\delta}\left(\delta_{\lambda}, \delta_{\phi}, \delta_{z}\right)$ projeté dans le plan focal de l'image engendrera des déplacements $\delta_{C O L}$ et $\delta_{L I G}$ :

$$
\left\{\begin{array}{l}
\delta_{C O L}=\vec{\delta} \cdot \overrightarrow{U_{C O L}}=\delta_{\lambda} \cos \theta \cos \alpha-\delta_{\phi} \cos \theta \sin \alpha+\delta_{z} \sin \theta \\
\delta_{L I G}=\vec{\delta} \cdot \overrightarrow{U_{L I G}}=-\delta_{\lambda} \sin \alpha-\delta_{\phi} \cos \alpha
\end{array}\right.
$$

$\delta_{C O L}$ et $\delta_{L I G}$ sont mesurés par la corrélation des images. Classiquement, ils permettent d'évaluer des déplacements horizontaux $\left(\delta_{\lambda}, \delta_{\phi}\right)$ mais, si $\theta$ est non nul, ils sont aussi sensibles à des mouvements verticaux $\left(\delta_{z}\right)$.

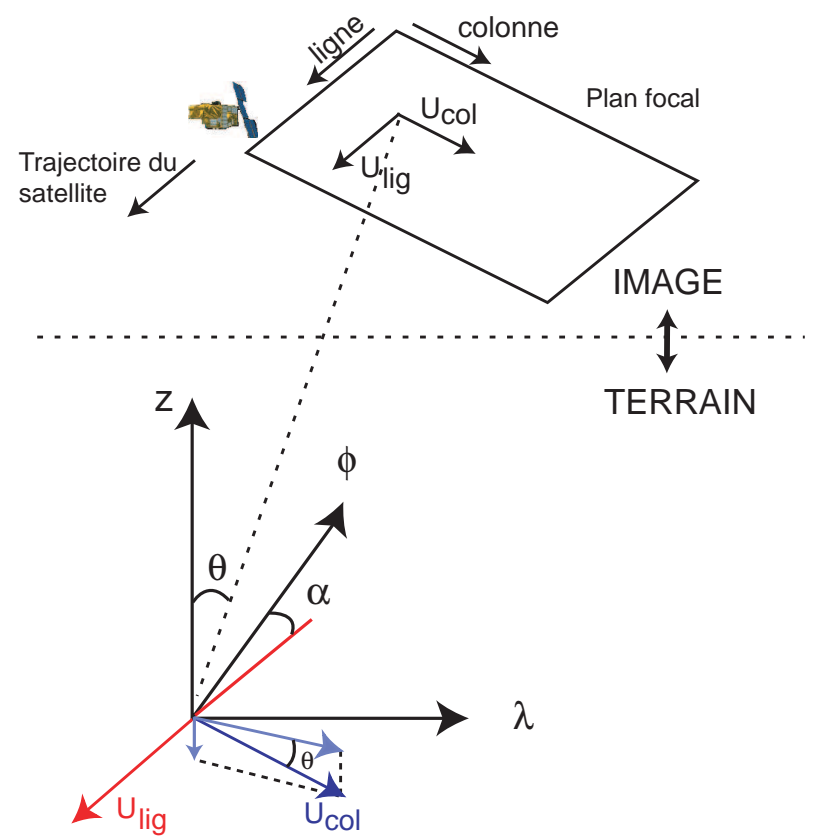

Fig. 4.3 - Représentation des vecteurs unitaires en ligne et colonnes ( $U_{L I G}$ et $U_{C O L}$ ) en géométries image et terrain. L'angle d'incidence $(\theta)$ et l'azimut de la direction des lignes $(\alpha)$ sont aussi définis.

\footnotetext{
${ }^{1} \lambda$ désigne la longitude, $\phi$ la latitude, z l'altitude
} 


\subsubsection{Mesure des déplacements horizontaux}

Cette mesure est possible directement dans le cas où les images sont acquises avec des angles d'incidence verticaux. $\theta$ est nul et donc $\delta_{\lambda}$ et $\delta_{\phi}$ sont les deux inconnues d'un système de deux équations :

$$
\left\{\begin{array}{l}
\delta_{C O L}=\delta_{\lambda} \cos \alpha-\delta_{\phi} \sin \alpha \\
\delta_{L I G}=-\delta_{\lambda} \sin \alpha-\delta_{\phi} \cos \alpha
\end{array}\right.
$$

Dans le cas général, $\theta$ est non nul et les déplacements en colonne sont sensibles aux mouvements verticaux (équation 4.3). Cette sensibilité est proportionnelle à $\sin \theta$ et augmente donc avec l'angle d'incidence. Une information extérieure à la corrélation d'images est requise pour obtenir des déplacements horizontaux non biaisés dans la direction des colonnes. Nous verrons comment cette information est obtenue et utilisée dans le cas de la mesure des vitesses des glaciers alpins.

\subsubsection{Mesure des déplacements verticaux}

D'après le système d'équations 4.3 , puisque $\delta_{C O L}$ dépend de $\delta_{z}$, la mesure du déplacement vertical est possible. Suivant le contexte, deux cas sont à envisager.

\subsubsection{La direction de l'écoulement est connue}

La direction de l'écoulement d'un glacier peut être connue ou estimée d'au moins trois manières :

1. Comme indiqué dans le paragraphe précédent, la corrélation d'images optiques acquises au nadir n'est pas sensible aux mouvements verticaux. Elle fournit donc directement une carte des directions d'écoulement.

2. A partir d'une image optique ou d'une image d'amplitude SAR, il est souvent possible de suivre une ligne d'écoulement ${ }^{2}$ qui indique la direction d'écoulement

3. Si les deux mesures précédentes ne sont pas possibles, elle peut être déduite d'un MNT en faisant l'hypothèse d'un écoulement dans la direction de la ligne de plus grande pente.

Si la direction est connue, $\delta_{L I G}$, obtenu par la corrélation d'images et insensible aux mouvements verticaux, peut être converti en $\delta_{C O L_{t h e}}$, valeur théorique de $\delta_{C O L}$ pour que l'écoulement se fasse dans la bonne direction. Cela correspondrait à la valeur mesurée de $\delta_{C O L}$ si le mouvement vertical était nul $\left(\delta_{z}=0\right)$ :

$$
\delta_{C O L_{\text {the }}}=\delta_{\lambda} \times \cos \theta \times \cos \alpha-\delta_{\phi} \times \cos \theta \times \sin \alpha
$$

L'écart entre la valeur théorique et mesurée de $\delta_{C O L}$ s'explique par le fait que le terme $\delta_{z} \times \sin \theta$ ne peut pas être négligé lorsque les images ont un angle d'incidence important :

$$
\delta_{C O L_{m e s}}-\delta_{C O L_{\text {the }}}=\delta_{z} \times \sin \theta
$$

\footnotetext{
${ }^{2}$ flowline en anglais
} 
Il est alors facile de déduire le déplacement vertical :

$$
\delta_{z}=\frac{\delta_{C O L_{m e s}}-\delta_{C O L_{t h e}}}{\sin \theta}
$$

\subsubsection{Le déplacement horizontal est négligeable}

Si le déplacement horizontal peut être négligé $\left(\delta_{\lambda}=\delta_{\phi}=0\right)$, l'équation 4.3 nous montre que les déplacements en colonne entre deux images sont uniquement liés aux mouvements verticaux. La conversion des décalages en colonne en un mouvement vertical de la surface se fait alors simplement en utilisant :

$$
\delta z=\frac{\delta_{C O L}}{\sin \theta}
$$

De même, si le déplacement en longitude est négligeable $\left(\delta_{\lambda} \sim 0\right)$, le système d'équation 4.3 peut être réécrit :

$$
\left\{\begin{array}{l}
\delta_{C O L}=-\delta_{\phi} \cos \theta \sin \alpha+\delta_{z} \sin \theta \\
\delta_{L I G}=-\delta_{\phi} \cos \alpha
\end{array}\right.
$$

On peut alors déduire le déplacement vertical par :

$$
\delta_{z}=\frac{\delta_{C O L}-\delta_{L I G} \times \cos \theta \times \frac{\sin \alpha}{\cos \alpha}}{\sin \theta}
$$

C'est en utilisant cette équation que nous avons pu mesurer le soulèvement de la plateforme de glace de Grimsvötn (Islande) et le remplissage du lac sous-glaciaire sur lequel elle repose. Cette mesure est présentée au chapitre 6.

Puisqu'on peut mesurer dans des contextes différents les mouvements verticaux et horizontaux, il semble possible d'estimer les 3 composantes du champ de déplacement à la surface du globe. Cette perspective est présentée en annexe G.

\subsection{Notre chaîne de traitement : $M E$ DECOIS PAS}

Cet acronyme pour le moins insolite désigne la MEsure des DEplacements par COrrélation d'Images Spot avec une Précision Assez Sympa ${ }^{3}$.

Pour mesurer les déformations de la surface terrestre, il faut que les deux images corrélées présentent les distorsions ${ }^{4}$ les plus faibles possibles. Le but de notre méthodologie est donc

\footnotetext{
${ }^{3}$ cet acronyme n'a rien d'officiel et n'apparaît pas dans les publications, il est difficilement traduisible...

${ }^{4}$ On désigne par distorsions des différences entre deux images qui ne sont pas liées au phénomène géophysique (écoulement du glacier) ou déformation que l'on étudie.
} 
de reprojeter l'une des images dans la géométrie de l'autre, comme si les deux images étaient acquises exactement du même point de vue. Les distorsions entre les deux images s'expliquent principalement par les effets du relief (effets stéréoscopiques) et les différences de position et d'attitude (roulis, tangage, lacet) du satellite lors de ses deux passages successifs.

Notre chaîne de traitement se compose d'une série de scripts (SHELL) qui font appel à d'autres programmes:

- Programme OUST (spatio-triangulation) gèrant l'orientation absolue et relative des images

- Bibliothèque SP5LIB manipulant la géométrie des images SPOT

- Programme ORION permettant de réechantillonner précisément les images

- Programme MEDICIS réalisant la corrélation des images

- Programmes IDL assurant des calculs numériques sur les matrices

Les scripts SHELL (qui font le lien entre tous les programmes) et IDL ont été écrits pendant cette thèse alors que les autres programmes ont été mis à notre disposition par le CNES. Intégrer ces différents programmes dans une chaîne de calcul n'est pas toujours simple. A titre d'exemple, ces programmes utilisent des conventions souvent différentes pour la désignation du pixel origine (en haut à gauche) d'une image. L'aide d'Hélène Vadon (CNES) a été déterminante pour la réalisation de cette chaîne de traitement ${ }^{5}$.

La figure 4.4 présente les différentes étapes suivies pour reprojeter l'image esclave dans la géométrie de l'image maîtresse.

\subsubsection{Orientation absolue}

Avant cette étape, un point d'une image peut être localisé au sol grâce à la connaissance des paramètres orbitaux du satellite au moment de l'acquisition. Selon les satellites, les erreurs de localisation sont plus ou moins importantes : quelques centaines de mètres pour SPOT1-4, quelques dizaines de mètres pour SPOT5. On appelle par la suite modèle stéréo ${ }^{6}$ l'ensemble des équations qui permettent ce passage de la géométrie image à la géométrie terrain (Toutin, 2002). Au cours de l'orientation absolue (étape 1 sur la figure 4.4), des points de contrôles (notés GCP par la suite) dont la position géographique est connue et qui sont reconnaissables dans l'image, permettent d'améliorer la connaissance des paramètres orbitaux et donc le modèle stéréo.

Concrètement, cette étape utilise le logiciel OUST. Les GCP permettent d'estimer de nouvelles valeurs de l'attitude du satellite (roulis, tangage, lacet) et de distance focale qui minimisent:

$$
\sum_{i=1}^{n}\left\|\overrightarrow{G_{i} G_{i}^{\prime}}\right\|^{2}
$$

où $\mathrm{G}_{i}=(\lambda, \phi, z)_{i}$ est la position connue du i ${ }^{\text {eme }} \mathrm{GCP}$ et $\mathrm{G}_{i}{ }_{i}=\left(\lambda^{\prime}, \phi^{\prime}, z^{\prime}\right)_{i}$ sa position au sol déduite de ces coordonnées images à partir du modèle stéréo. Cette étape n'est pas indispensable mais évite des décalages entre les images et le modèle numérique de terrain (MNT). Si les GCP ne sont disponibles que pour une image, cette dernière doit ensuite être utilisée comme image maîtresse.

\footnotetext{
${ }^{5}$ Le terme chaîne de traitement ne signifie pas ici un procédé entièrement automatique et convivial... De nombreuses étapes dépendent de l'utilisateur et de l'objectif visé.

${ }^{6}$ certains auteurs utilisent modèle géométrique
} 


\subsubsection{Orientation relative}

Cette étape est primordiale pour obtenir deux images qui se superposent à quelques fractions de pixels près (étapes 2 à 4 sur la figure 4.4). L'une des images est choisie comme maîtresse et des points homologues, représentant la même structure dans les deux images, permettent d'affiner le modèle stéréo de l'image esclave. Les points homologues doivent être extraits de zones stables. Parce que la précision du pointage manuel de structures identiques sur deux images n'est pas suffisante ( \pm 1 pixel), nous extrayons ces points homologues en grand nombre (plusieurs centaines) par corrélation des deux images selon une grille assez lâche (tous les 50 ou 100 pixels). Les zones en mouvements (les glaciers pour nous) sont masquées. Un MNT est nécessaire à cette étape. Les points homologues, précis et distribués dans toute la zone commune aux deux images, sont sélectionnés parmi la nappe de corrélation : on découpe l'image en cadrans (typiquement $30 \times 30$ cadrans) et on sélectionne les points pour lesquels le coefficient de corrélation est maximum.

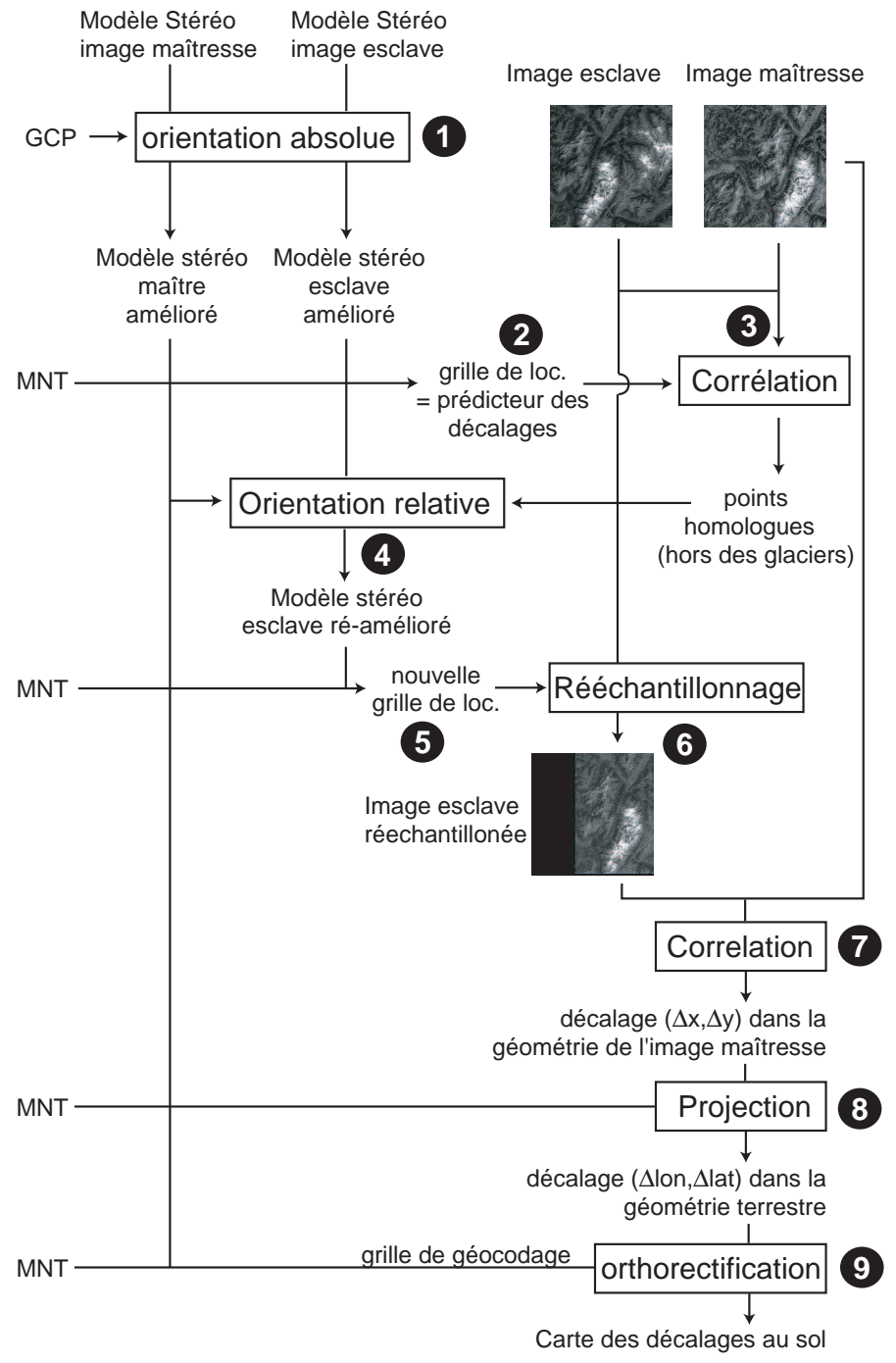

FIG. 4.4 - Diagramme des étapes suivies pour extraire les déplacements de la surface terrestre à partir de deux images SPOT (loc.=localisation). 


\subsubsection{Ré-échantillonnage de l'image esclave}

Les modèles stéréos affinés des deux images et un MNT sont utilisés pour ré-échantillonner l'image secondaire comme si elle avait été acquise du même point de vue que l'image maîtresse (étapes $5 \& 6$ sur la figure 4.4). Le filtre utilisé au cours de ce ré-échantillonnage ne doit pas créer de biais et préserver la radiométrie. Van Puymbroeck et al. (2000) et Vadon \& Massonnet (2000) ont montré qu'un filtre de type sinus cardinal $\left(\frac{\sin x}{x}\right)$ apodisé (c'est à dire multiplié par une gaussienne) respecte ces contraintes. Après cette étape, nous disposons de deux images superposables à quelques fractions de pixels près au niveau des zones stables.

\subsubsection{Corrélation}

Le logiciel MEDICIS (Centre National d'Etude Spatiale, 2002) permet de corréler les deux images pour mesurer leurs décalages en colonnes et en lignes (étape 7 sur la figure 4.4). La corrélation est réalisée sur toute l'image (même si les glaciers n'en occupent qu'une partie) car les zones hors des glaciers apportent des renseignements sur la bonne superposition des scènes. Une grande taille de la fenêtre de corrélation permet de limiter le bruit mais va aussi atténuer les hautes fréquences (spatiales) des champs de déplacements et limiter le nombre de mesures indépendantes. Des fenêtres de $21 \times 21$ pixels (soit $52.5 \times 52.5 \mathrm{~m}$ ) nous ont semblé un bon compromis au vu de la largeur des glaciers étudiés qui est de quelques centaines de mètres. La corrélation est réalisée tous les 10 pixels ce qui permet d'éviter des mesures voisines trop dépendantes, de gagner du temps de calcul tout en échantillonnant suffisamment les variations spatiales de la déformation des glaciers.

La corrélation est calculée entre une imagette principale (extraite de l'image maîtresse) et une imagette secondaire (extraite de l'image esclave). La taille de l'imagette secondaire doit être suffisamment grande pour prendre en compte les décalages importants des zones les plus rapides du glacier (Vadon \& Berthier, 2004). Le déplacement d'une structure entre les deux images est obtenu en positionnant le maximum du pic de corrélation (figure 4.5). Ce maximum peut s'obtenir par interpolation d'un pic de corrélation calculé tous les pixels (Scambos et al., 1992). Mais un résultat plus précis s'obtient en calculant la corrélation entre l'imagette principale et une imagette secondaire ré-échantillonnée pour être décalée (Vadon \& Massonnet, 2000).

La corrélation de deux images SPOT5 $(24000 \times 24000)$, tous les 10 pixels, permet d'obtenir deux grilles de décalage (en colonnes et en lignes) de $(2400 \times 2400)$. A chaque mesure de décalage est associé un coefficient de corrélation et un flag de validité ${ }^{7}$.

\subsubsection{De la géométrie image à la géométrie terrain}

C'est seulement dans cette dernière étape que s'effectue le passage à la géométrie terrain (étapes 8 \& 9 sur la figure 4.4). L'intérêt de travailler en géométrie image (colonne, ligne) plutôt qu'en géométrie sol est de pouvoir utiliser pleinement la description précise (surtout pour SPOT5) de l'attitude du satellite qui est fournie par SPOTIMAGE avec les scènes (Vadon \& Berthier, 2004). Nous verrons de plus que les erreurs de nos mesures sont souvent différentes selon les directions des images et n'ont pu être comprises qu'en travaillant en géométrie image.

\footnotetext{
${ }^{7}$ Ce flag (= drapeau) vaut 1 quand la corrélation est valide. Il prend d'autres valeurs quand la corrélation échoue ce qui permet de connaître la raison de l'échec, par exemple lorsque le pic de corrélation est au bord de l'imagette secondaire.
} 

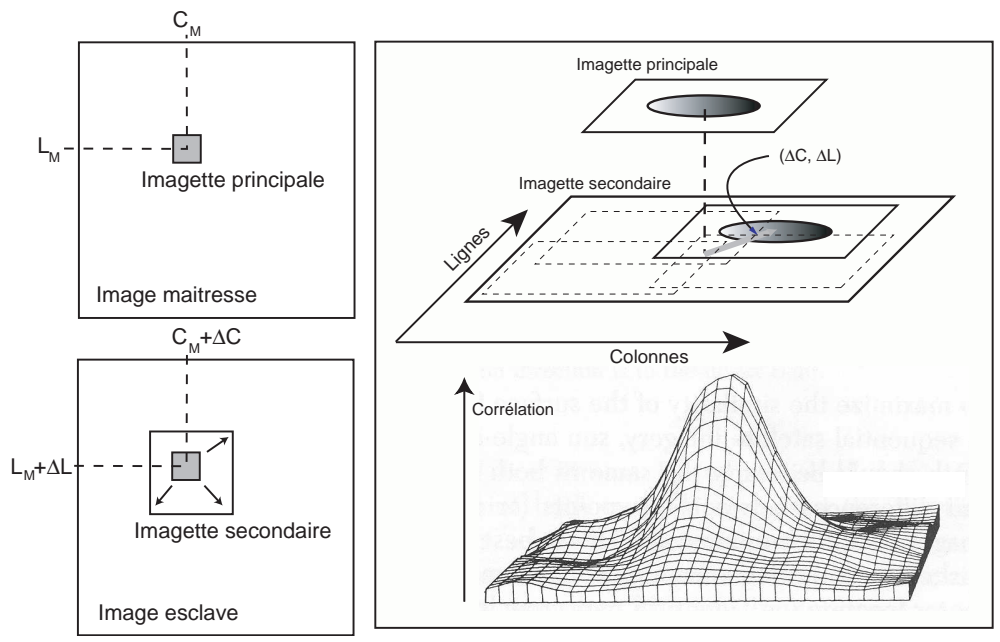

Fig. 4.5 - Principe de la mesure du déplacement par corrélation d'images. Deux imagettes, principale et secondaire, sont extraites des deux images (partie gauche) et la corrélation est calculée pour des positions variables au sein de l'imagette secondaire. Le maximum du pic de corrélation indique la valeur du déplacement (partie droite).

Ce passage est réalisé en deux étapes. Dans un premier temps, les décalages (mesurés en pixels) sont convertis en déplacements (exprimés en mètres) en prenant en compte le MNT et le modèle stéréo de l'image maîtresse. On localise au sol chaque pixel de l'image maîtresse puis le pixel équivalent de l'image esclave. La différence des positions permet d'obtenir les décalages en longitude et latitude (figure 4.6). On mesure aussi un déplacement vertical $\left(\delta_{z}\right)$ reflétant un écoulement parallèle à la surface topographique qui n'est pas nécessairement celui des particules de glace (cf. §G.3 in annexe G).

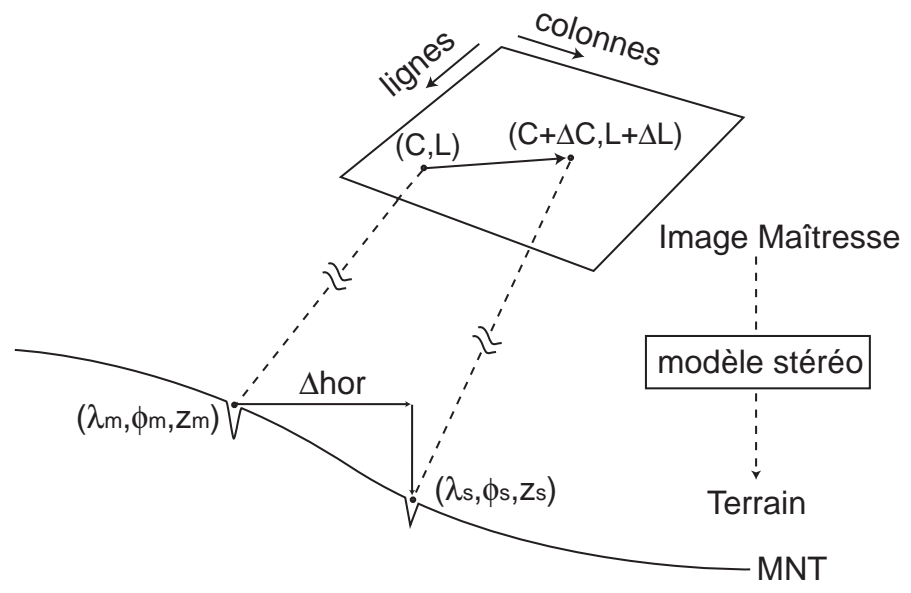

FIG. 4.6 - Conversion des décalages en géométrie image en des déplacements au sol.

Une fois cette conversion réalisée, les grilles de décalages sont ré-échantillonnées pour être cartographiées (cette étape revient à une orthorectification). Ceci permet notamment la comparaison aux mesures de terrain. 


\subsection{Vitesses des glaciers du Mont Blanc durant l'été 2003}

La méthodologie décrite dans la partie précédente est appliquée aux glaciers du massif du Mont Blanc durant l'été 2003. Nous présenterons rapidement ces glaciers et les champs de déplacements que nous avons mesurés. Une attention toute particulière sera accordée à l'estimation des incertitudes sur nos mesures en étudiant à la fois les zones stables hors des glaciers mais aussi en comparant les mesures SPOT5 avec une campagne de terrain.

\subsubsection{Les glaciers du massif du Mont-Blanc}

Les glaciers du Massif du Mont Blanc (figure 4.7) ont été choisis afin de tester notre méthodologie pour plusieurs raisons.

- Ils font partie des glaciers les mieux suivis du globe et ont été le berceau de la glaciologie. Les premières mesures de la dynamique et du bilan de masse glaciaire par Vallot (1900), Forel (1895) et Mougin (1934) ont été réalisées sur la Mer de Glace. Plus proche de nous, les études de Reynaud (1979) et Lliboutry \& Reynaud (1981) sur ce même glacier ont permis des avancées significatives pour la compréhension de la dynamique de l'écoulement des glaciers tempérés.

- La continuité du suivi de la Mer de Glace et du glacier d'Argentière par le Laboratoire de Glaciologie et de Géophysique de l'Environnement (LGGE) permet de replacer la mesure satellitaire au sein d'une série à plus long terme.

- Ces glaciers sont relativement accessibles pour mener des campagnes de terrain de validation de la mesure satellitaire, notamment grâce à la logistique du LGGE.

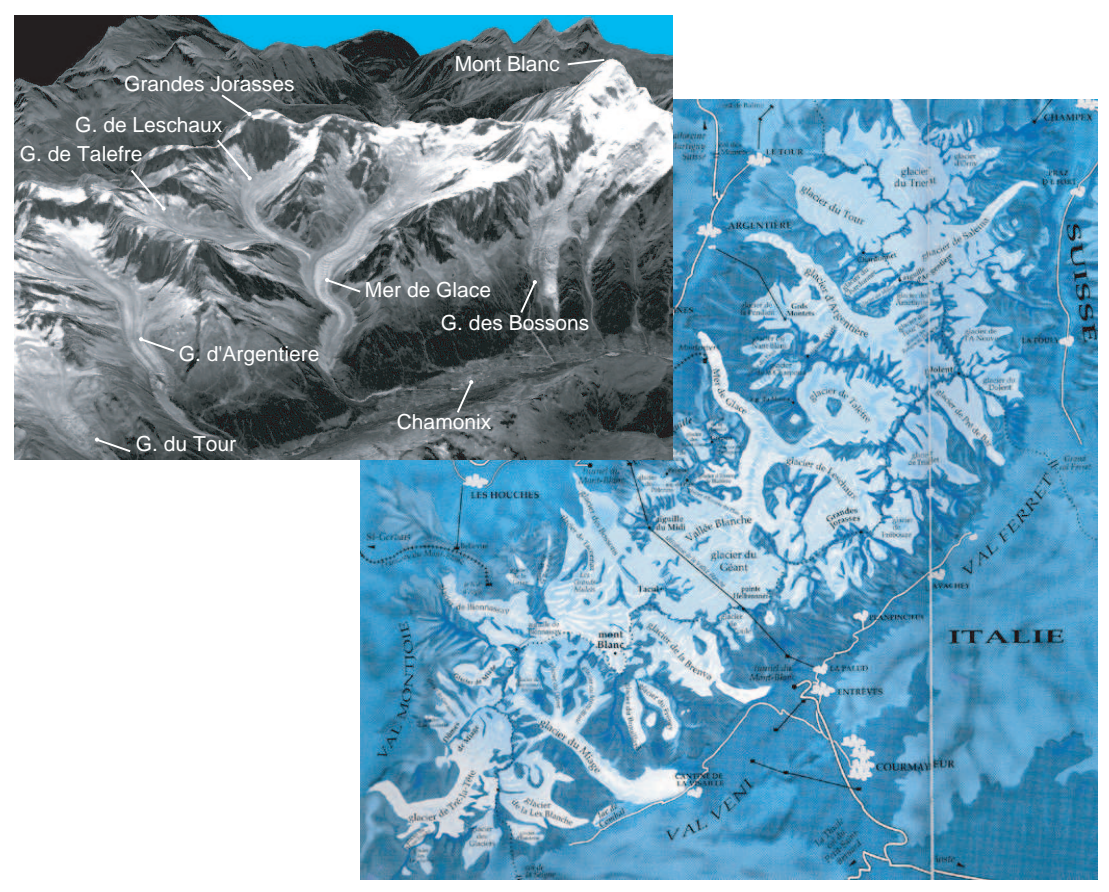

FIG. 4.7 - Les principaux glaciers du massif du Mont-Blanc. A gauche, une image SPOT5 de 2003 a été drapée sur un MNT de la zone (exagération verticale 1). A droite un carte tirée de Moreau É Vivian (2000). 


\subsubsection{Caractéristiques géométrique et radiométrique des images SPOT5}

L'été 2003, marqué en Europe par une forte canicule au mois d'août (Trigo et al., 2005), a été favorable pour l'acquisition d'images SPOT5 (tableau 4.1). Dans le cadre d'une demande ISIS $^{8}$, ces images ont été programmées spécialement pour notre étude et nous avons donc pu choisir le gain le plus faible (1) du capteur HRG sur SPOT5 (cf. figure 3.4 pour l'importance de ce gain faible). La figure 4.8 (a) localise les empreintes au sol des images du couple $\{23$ août/18 septembre\}

TAB. 4.1 - Caractéristiques des 4 images SPOT5 acquises durant l'été 2003 sur le Massif du Mont Blanc. Les couples d'images \{19 juillet/19 août\} et \{23 août/18 septembre\} sont utilisés pour calculer l'écoulement du glacier en 31 et 26 jours respectivement. La paire $\{19$ août/23 août\} est utilisée pour calculer un MNT de la zone. Les angles d'incidence sont ceux au centre des images. Voir le texte pour la signification du rapport $B / H$.

\begin{tabular}{lcrr}
\hline Couple d'images & Angle d'incidence & $\mathrm{B} / \mathrm{H}$ & $\delta \mathrm{t}$ (jours) \\
\hline 19 juillet / 19 août & $-23.6 /-15.2$ & 0.106 & 31 \\
23 août / 18 septembre & $+15.7 /+17.3$ & 0.0006 & 26 \\
19 août / 23 août & $-15.2 /+15.7$ & 0.596 & 4 \\
\hline
\end{tabular}

Le rapport $\mathrm{B} / \mathrm{H}$ présenté dans le tableau 4.1 désigne le rapport entre $\mathrm{B}^{9}$ (la distance entre les deux positions des satellites) et $\mathrm{H}^{10}$ (la hauteur du satellite au dessus de la surface terrestre). Il quantifie les distorsions entre deux images dues au relief (Toutin, 2002). Un B/H fort (forte sensibilité au relief) est nécessaire pour construire un MNT, alors que ce rapport doit être faible dans le cas de la mesure par corrélation des mouvements de la surface terrestre. Dans ce second cas, B/H indique quel est l'effet des erreurs du MNT (Van Puymbroeck et al., 2000). Ainsi, pour le couple $\{23$ août/18 septembre $\}$, une erreur de $100 \mathrm{~m}$ du MNT engendre une erreur de $\sigma_{M N T} \times B / H$ soit $6 \mathrm{~cm}$. Ce couple est donc acquis dans des conditions optimales pour la mesure des déplacements : les deux images sont séparées de 26 jours (un cycle orbital de SPOT5) et sont donc acquises du même point de vue (§3.2). Nous verrons que, dans une telle configuration orbitale, même un MNT très imprécis, comme GTOPO30 (USGS, 1996) de résolution $1 \mathrm{~km}$, est suffisant. Le couple d'images $\{19$ juillet/19 août $\}$ est moins favorable, une erreur de $10 \mathrm{~m}$ du MNT créant une erreur de $1 \mathrm{~m}$ sur la mesure du déplacement. Une topographie précise et contemporaine (car les variations d'épaisseur de ces glaciers sont rapides) est donc essentielle pour utiliser ce couple d'images. Ce MNT est obtenu à partir de la paire $\{19$ août $/ 23$ août $\}$. La construction de ce MNT est détaillée au chapitre 5.

\footnotetext{
${ }^{8}$ Le programme ISIS (http ://medias.obs-mip.fr/isis/) permet aux scientifiques français -et européens grâce à OASIS- l'acquisition d'images SPOT à tarif préférentiel

${ }^{9}$ pour $B$ aseline en anglais

${ }^{10}$ pour $H$ eight en anglais
} 


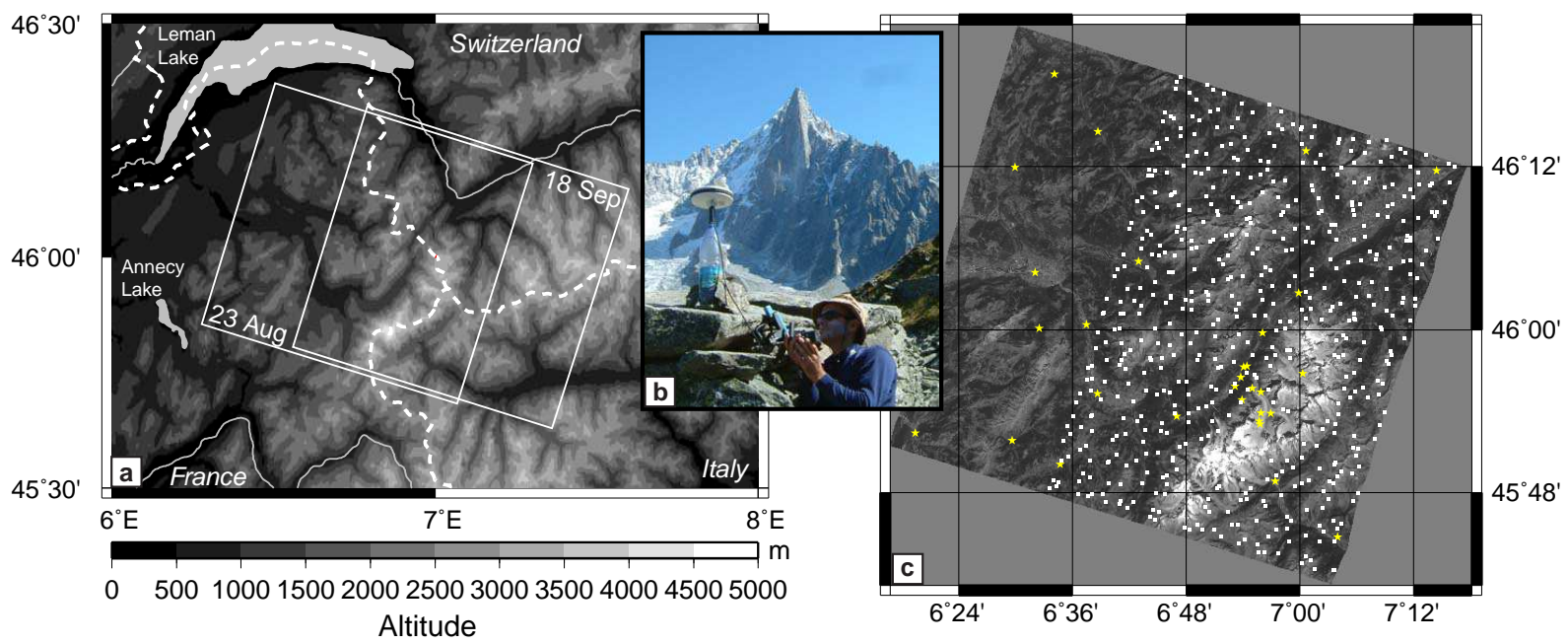

FIG. 4.8 - La zone d'étude : le massif du Mont-Blanc. (a) MNT utilisé dans cette étude et la localisation des empreintes au sol de deux des scènes SPOT5. (b) Acquisition par Yves Arnaud d'un des points de contrôle dans la vallée de Chamonix (en arrière plan les Drus). (c) Image SPOT5 du 23 août 2003 sur laquelle sont positionnés les points de contrôle (30 étoiles jaunes) utilisés pour l'orientation absolue et les points homologues à l'image du 18 septembre (573 carrés blanc) utilisés pour l'orientation relative.

Les deux couples d'images destinés à mesurer le déplacement des glaciers sont traités selon la méthodologie présentée dans la partie précédente $(\S 4.3)$ :

L'orientation absolue, dont les résultats sont présentés dans le tableau 4.2, est réalisée à partir de points de contrôles mesurés lors de deux campagnes DGPS ${ }^{11}$ organisées en septembre et octobre 2003. Des structures bien identifiables sur les images SPOT5 ont pu être mesurées sur le terrain à $\pm 1 \mathrm{~m}^{12}$. La figure 4.8 (b) montre l'acquisition d'un point de contrôle au pied des Drus en vallée de Chamonix alors que la figure 4.8 (c) localise ces points de contrôle (étoiles jaunes) sur l'image du 23 août. Après cette étape, et pour les 4 images, tout point peut être positionné au sol à $\pm 2.5 \mathrm{~m}$ (ou mieux).

Les 573 points homologues utilisés pour l'orientation relative du couple $\{23$ août/18 septembre\} sont localisés sur la figure 4.8 (c). Aucun point n'est pris sur les glaciers. Pour ce couple d'images, l'orientation relative permet d'obtenir une superposition à $\pm 0.65 \mathrm{~m}$ près (tableau 4.2). Un résultat identique est obtenu sans orientation absolue préalable même avec un MNT de résolution faible. Ceci résulte directement du faible B/H de ce couple. Dans une telle configuration satellitaire, des champs de déplacement précis peuvent donc être obtenus sans MNT précis ni points de contrôle, ce qui permet d'appliquer notre méthode à des zones retirées du globe. Pour la paire $\{19$ juillet/19 août\}, l'orientation relative est plus délicate. Pour ce couple, avec un B/H plus élevé, la disponibilité d'un MNT précis et de GCP assurant une bonne localisation des images par rapport au MNT est impérative pour mesurer des déplacements corrects.

\footnotetext{
${ }^{11}$ DGPS : Differential Global Positionning System

${ }^{12}$ Cette précision est suffisante au regard de la précision du pointage sur l'image SPOT5 qui est au mieux de 0.5 pixel, soit $1.25 \mathrm{~m}$
} 
TAB. 4.2 - Ecart type ( $\sigma$ en mètres) des résidus de l'orientation absolue et relative (par définition la moyenne doit être nulle) des différentes images SPOT5. La paire \#1 désigne le couple $\{19$ juillet/19 août\} alors que la paire \#2 correspond au couple \{23 août/18 septembre\}. L'orientation absolue de chaque image est obtenue à partir de points de contrôle (GCP). L'orientation relative des deux images d'une même paire est obtenue à partir de points homologues extraits par corrélation hors des glaciers. Le résidu maximum (en mètres) pour tous les points retenus dans ces orientations est aussi indiqué. Le tableau permet de comparer les résultats obtenus avec ou sans GCP et avec deux MNT de résolution différente, HR désignant le MNT à $20 \mathrm{~m}$ déduit d'images SPOT5 alors que LR désigne le MNT GTOPO30 (1 km).

\begin{tabular}{|c|c|c|c|c|c|}
\hline \multirow[b]{2}{*}{ Paire d'images } & \multirow[b]{2}{*}{ Date (2003) } & \multicolumn{2}{|c|}{ Orientation abs. } & \multicolumn{2}{|c|}{ Orientation rel. } \\
\hline & & $\sigma$ & Résidu Max. & $\sigma$ & Résidu Max. \\
\hline$\# 1$ avec GCP & $19 \mathrm{Jul}$ & 2.55 & 4.3 & 2.84 & 11.1 \\
\hline $\mathrm{MNT}_{H R}$ & 19 Aug & 1.89 & 3.59 & & \\
\hline$\# 1$ sans GCP; $\mathrm{MNT}_{H R}$ & $19 \mathrm{Jul} / 19 \mathrm{Aug}$ & - & - & 5.45 & 31.7 \\
\hline$\# 1$ sans $\mathrm{GCP} ; \mathrm{MNT}_{L R}$ & $19 \mathrm{Jul} / 19 \mathrm{Aug}$ & - & - & 14.5 & 38.8 \\
\hline$\# 2$ avec $\mathrm{GCP}$ & $23 \mathrm{Aug}$ & 1.55 & 2.66 & 0.63 & 1.45 \\
\hline $\mathrm{MNT}_{H R}$ & 18 Sep & 1.82 & 2.97 & & \\
\hline$\# 2$ sans GCP; $\mathrm{MNT}_{H R}$ & $23 \mathrm{Aug} / 18 \mathrm{Sep}$ & - & - & 0.67 & 1.68 \\
\hline$\# 2$ sans GCP $; \mathrm{MNT}_{L R}$ & $23 \mathrm{Aug} / 18 \mathrm{Sep}$ & - & - & 0.67 & 1.68 \\
\hline
\end{tabular}

Les orientations absolues et relatives des images étant réalisées, elles peuvent être corrélées et les décalages sont alors projetés sur la surface terrestre.

\subsubsection{Champs de déplacements pour l'été 2003}

La figure 4.9 montre le champ de vitesse horizontale sur les glaciers du massif du Mont-Blanc déduit du couple $\{23$ août/18 septembre $\}$ et la figure 4.10 le coefficient de corrélation associé à ce champs de vitesse. Pour la Mer de Glace, quelques vecteurs vitesses sont ajoutés. A ce stade, toutes les mesures de la corrélation sont montrées et aucun filtrage n'a été réalisé. La vitesse horizontale cache en fait deux composantes : la vitesse en longitude et latitude. Ce sont bien deux des trois composantes du vecteur vitesse qui sont mesurées. L'homogénéité des champs de vitesse et le fait que les zones hors des glaciers aient un mouvement faible sont des indications de la qualité de notre mesure satellitaire. On note toutefois du bruit à haute altitude (bassin d'accumulation de la Mer de Glace par exemple) et sur les pentes orientées vers l'Ouest. Ce bruit résulte essentiellement des changements de surface (déplacement de la ligne de neige par exemple), des effets des ombres (détaillés plus loin). 

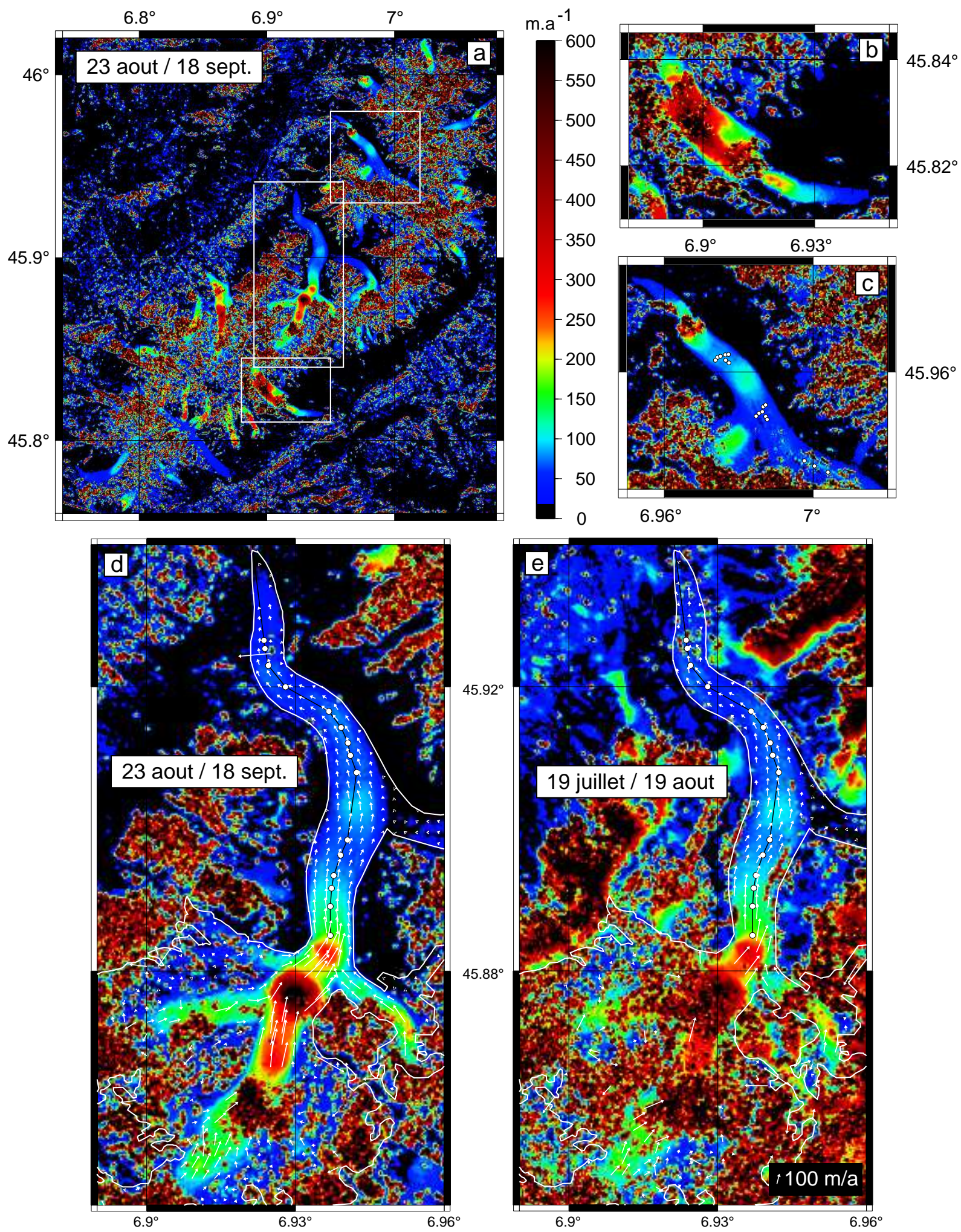

FIG. 4.9 - Vitesse horizontale d'écoulement pour la période du 23 août au 18 septembre pour l'ensemble des glaciers du Massif du Mont-Blanc (a), le glacier de la Brenva (b), d'Argentière (c) et la Mer de Glace (d). En (e), les vitesses sur le Mer de Glace du 19 juillet au 19 août. Les cercles blancs indiquent la position des balises positionnées par DGPS en août et septembre. Des flèches indiquent direction et magnitude de l'écoulement sur la Mer de Glace. 


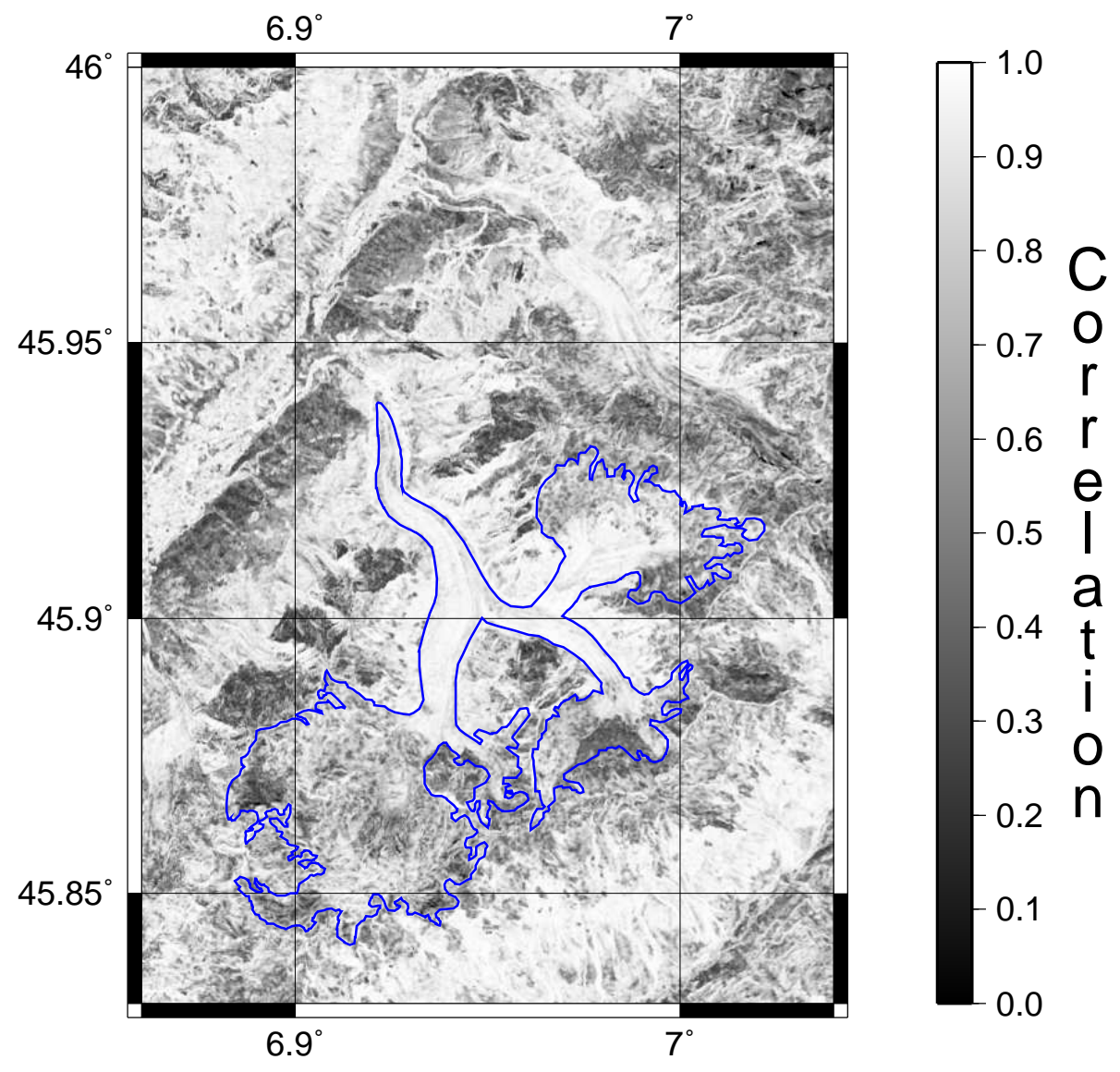

Fig. 4.10 - Coefficent de corrélation pour le couple d'images 23 août - 18 septembre 2003. Ce coefficient, variant entre 0 et 1, indique la ressemblance entre les deux imagettes et dépend aussi de la dynamique radiométrique. Il est plus faible dans la zone d'accumulation que dans la zone d'ablation ainsi que sur les pentes orientées vers l'Ouest (ombres importantes et changeantes).

Les vitesses les plus rapides sont enregistrées au niveau du Glacier de la Brenva et de la chute de sérac des Géants (700 $\mathrm{m} \mathrm{a}^{-1}$ soit plus de $2 \mathrm{~m}$ par jour). De telles vitesses sont légèrement inférieures aux valeurs mesurées sur des photographies aériennes par Reynaud (1973). Pour nombre de ces glaciers, la vitesse d'écoulement n'avait jamais été estimée. Il convient maintenant d'associer une précision à notre mesure.

Le couple d'images $\{19$ juillet/19 août $\}$ est traité de la même manière. Les décalages importants sur les zones stables hors des glaciers (zones de fortes pentes, lignes de crêtes) indiquent que les distorsions entre les deux scènes n'ont pas pu être parfaitement modélisées (figure 4.9 en bas à droite). En revanche, sur les glaciers (où la pente est plus faible), les champs de déplacements restent homogènes et montrent des structures cohérentes.

\subsubsection{Précision et exactitude de notre mesure des vitesses}

La précision peut tout d'abord être évaluée sur les zones stables qui entourent les glaciers. On s'attend à un déplacement nul. Dans un second temps, on comparera nos mesures en quelques points du glacier avec des relevés de terrain. 


\subsubsection{Le déplacement est-il nul hors des glaciers?}

La figure 4.11 montre les histogrammes des décalages hors des glaciers dans les deux directions, lignes et colonnes, pour le couple $\{23$ août $/ 18$ septembre $\}$.

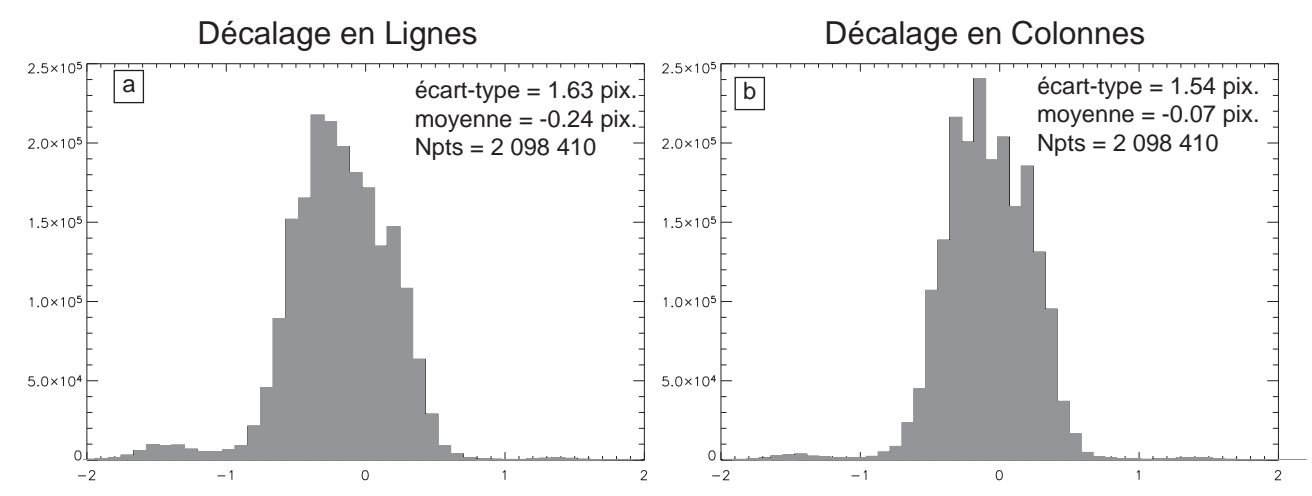

FIG. 4.11 - Distribution des décalages en lignes (a) et colonnes (b) déduits du couple \{23 août/18 septembre\} pour l'ensemble des zones où la corrélation est considérée comme valide et hors des glaciers.

On s'attend à une distribution symétrique et centrée sur 0 ce qui est effectivement le cas pour les décalages en colonne (biais de -0.07 pixel). En revanche, les décalages en lignes présentent un biais négatif (approximativement vers le Nord) de -0.24 pixel. Ceci est surprenant car, étant donné la géométrie d'acquisition des images SPOT (§3.2.1.1), c'est dans la direction des colonnes que l'on s'attend à observer des distorsions entre les images. Ce biais indique que les points homologues sélectionnés pour l'orientation relative ne sont pas représentatifs de l'ensemble des zones stables des images. Pour comprendre cette observation, des histogrammes complémentaires sont tracés pour différents intervalles du taux de corrélation (figure 4.12 \& figure 4.13).
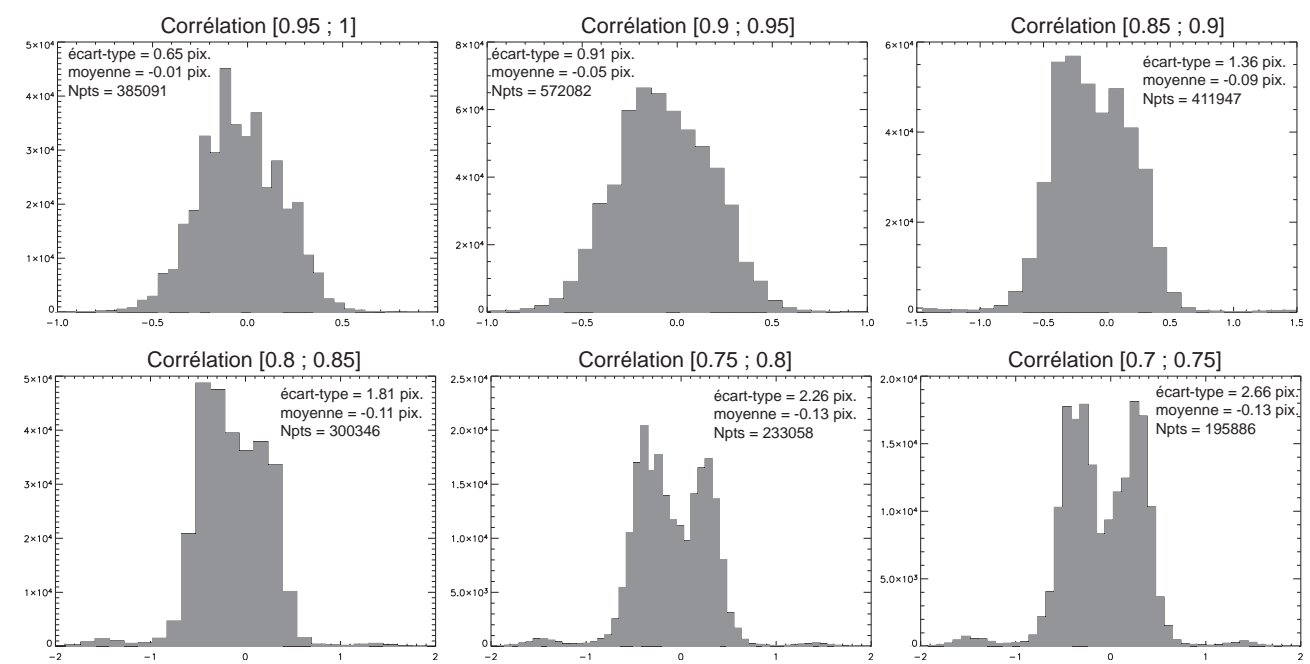

FIG. 4.12 - Distribution des décalages en colonnes déduits du couple \{23 août/18 septembre\} pour l'ensemble des zones hors des glaciers où la corrélation est considérée comme valide et pour différents intervalles du coefficient de corrélation. Quand le coefficient de corrélation est inférieur à 0.7 la corrélation est considérée comme non valide. 


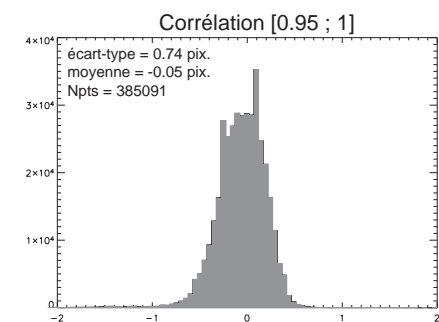

Corrélation $[0.8 ; 0.85]$

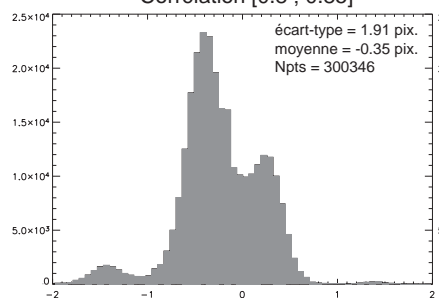

Corrélation $[0.9 ; 0.95]$

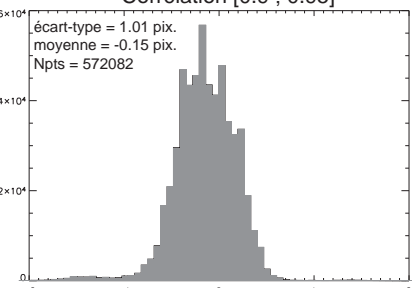

Corrélation $[0.75 ; 0.8]$

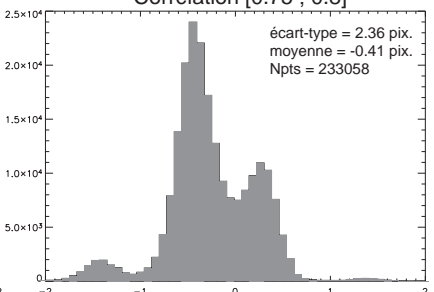

Corrélation $[0.85 ; 0.9]$

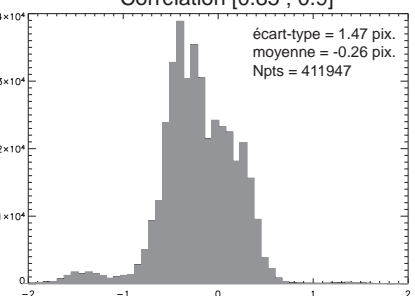

Corrélation $[0.7 ; 0.75]$

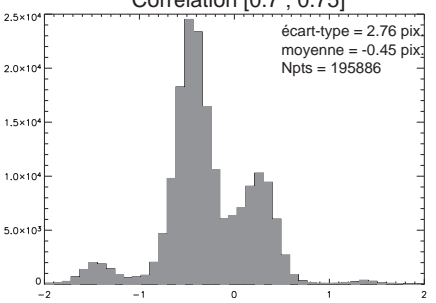

FIG. 4.13 - Idem figure 4.12 pour les décalages en lignes.

Comme attendu, quand le coefficient de corrélation est supérieur à 0.95, les écart-types des distributions sont faibles et elles sont centrées sur 0 en ligne comme en colonne. En effet, les points homologues qui permettent de recaler les deux images sont extraits de cette catégorie. Pour les décalages en colonne, le biais reste faible quand le coefficient de corrélation baisse, l'écart type augmente et la distribution devient bimodale ${ }^{13}$. L'écart type varie de la même manière pour les décalages en lignes mais le biais augmente rapidement : il approche 0.5 pixel quand le coefficient de corrélation est compris entre 0.7 et 0.75 . Nous expliquons cette distribution des décalages en lignes par un changement des conditions d'éclairement solaire. Entre le 23 août et le 18 septembre, l'incidence et l'azimut des rayons solaires ont changé. Ce changement et son effet sur les ombres sont illustrés par la figure 4.14 : les ombres subissent un déplacement essentiellement en ligne.

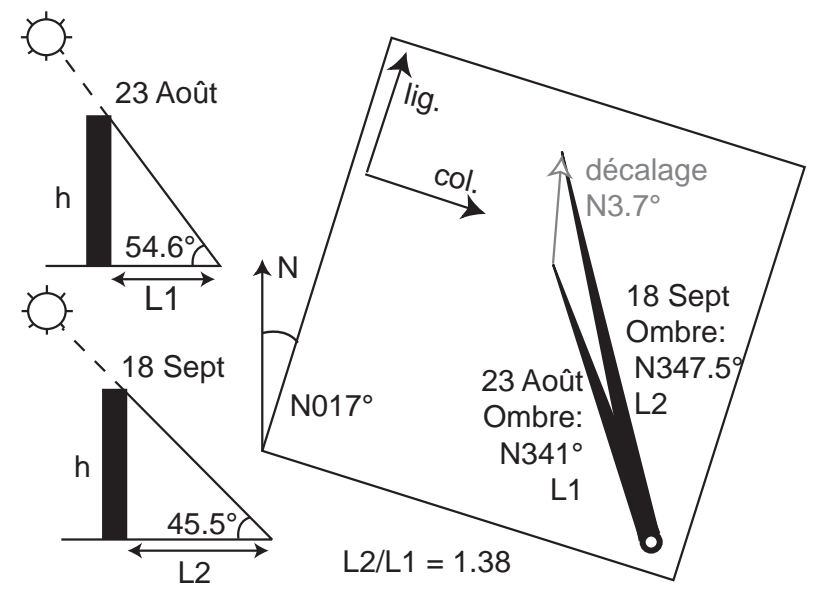

FIG. 4.14 - Explication des biais observés sur les zones stables à partir des changements des conditions d'éclairement entre le 23 août et le 18 septembre.

\footnotetext{
${ }^{13}$ La distribution bimodale pourrait s'expliquer par une dynamique radiométrique limitée hors des glaciers donc un rapport signal sur bruit faible. Le critère de Shannon, qui doit être rempli pour qu'une interpolation ne crée pas de biais, ne serait pas respecté (Vadon, communication personnelle).
} 


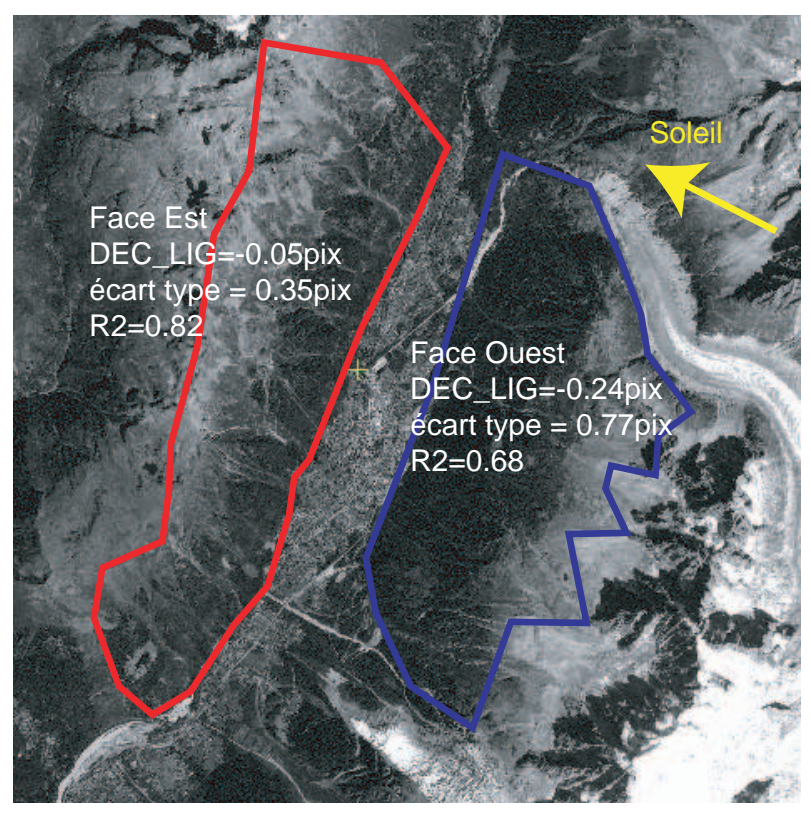

FIG. 4.15 - Comparaison des biais sur les décalages en lignes pour deux flancs de montagne d'orientation opposée autour de la vallée de Chamonix. Le biais et l'écart type sont plus faibles et le taux de corrélation moyen plus élevé pour la pente qui regarde vers l'Est (donc vers le soleil) que pour celle qui regarde vers l'Ouest.

Cette observation explique bien un biais principalement dirigé dans la direction des lignes. Comment expliquer que ce biais dépende du coefficient de corrélation? Les zones où les changements d'ombres sont les plus importants auront un biais en ligne fort et un coefficient de corrélation faible car la ressemblance entre les imagettes sera faible. Si cette interprétation est vérifiée, on doit pouvoir mettre en évidence une relation entre l'orientation des pentes topographiques, le biais en ligne et le coefficient de corrélation : les pentes orientées vers l'Est, face au soleil au moment de l'acquisition des scènes SPOT (à 11h du matin) devraient présenter des biais faibles et des corrélations élevées. Nous l'avons vérifié sur les pentes qui entourent la vallée de Chamonix (figure 4.15).

A notre connaissance c'est la première fois que cet impact du changement d'éclairement solaire sur la mesure des décalages au sol est décrit. Il doit être pris en compte pour toutes les régions où les ombres sont importantes. Dans le cas des glaciers, les ombres sont rares et cet effet a donc peu d'effet sur nos mesures de l'écoulement glaciaire.

\subsubsection{Comparaison aux vitesses mesurées in situ}

Deux campagnes de terrain ont été spécialement conduites pour obtenir une vérité terrain simultanée à l'acquisition des images satellites. Lors de ces campagnes, les positions de balises implantées dans la glace ou de pierres en surface du glacier ont été mesurées en août et septembre 2003 avec un DGPS bi-fréquence. Ces campagnes ont pu se faire grâce à l'aide de Christian Vincent du LGGE. Les dates d'acquisition des images satellites n'étant pas connues à l'avance, les missions de terrain n'ont pas pu être réalisées exactement aux même dates (figure 4.16). Les glaciers pouvant connaître des changements rapides de leur vitesse (Willis, 1995), ces différences de couverture temporelle peuvent expliquer des différences entre mesures satellitaire et in situ. 
La précision intrinsèque du positionnement DGPS est excellente $( \pm 5 \mathrm{~cm})$ mais l'incertitude sur les vitesses est plus grande du fait de la difficulté de placer le mat du DGPS toujours au même endroit par rapport aux balises : elles sont souvent entourées d'un trou d'eau à la fin de la saison d'ablation. Les déplacements des balises sont donc mesurées à $\pm 25 \mathrm{~cm}$ environ. Cette incertitude est plus grande et difficile à quantifier pour les pierres qui peuvent se déplacer/rouler indépendamment de la glace sous-jacente.
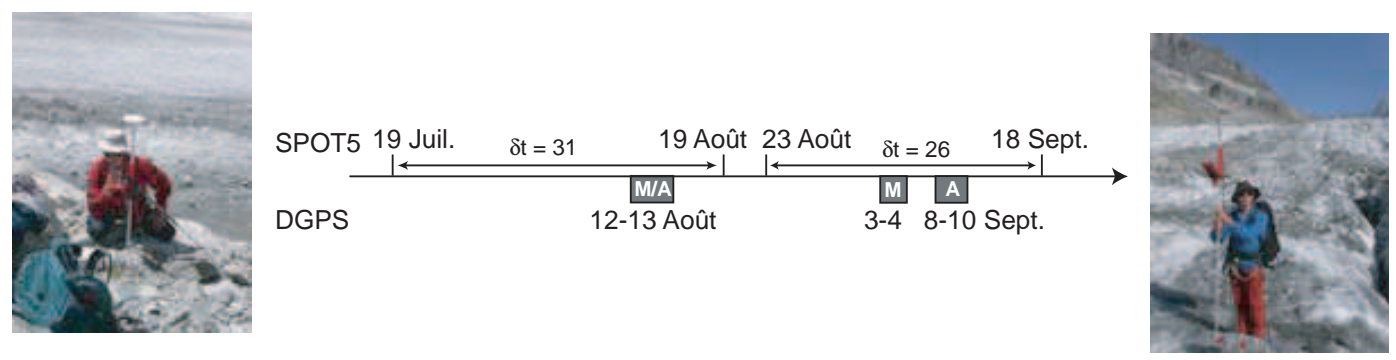

FIG. 4.16 - Comparaison des dates des images satellites et des campagnes DGPS de terrain. Pour ces dernières, le " $M$ " correspond à la Mer de Glace et le "A" au glacier d'Argentière. A gauche, vérification de la précision des mesures DGPS sur un point géodésique de la moraine en rive droite du glacier d'Argentière. A droite, positionnement d'une pierre sur le même glacier.

Sur la figure 4.17 sont représentés les déplacements en colonne et en ligne pour le couple $\{19$ juillet/19 août\} le long d'un profil longitudinal de la Mer de Glace passant par les relevés in situ (figure 4.9). Ce profil met nettement en évidence un écart systématique pour les décalages en colonne, la mesure satellitaire surestimant le déplacement de près d'un mètre. Dans la direction des lignes (direction principale de l'écoulement de la Mer de Glace) un tel biais n'est pas observé. Les mêmes observations peuvent être faites sur le glacier d'Argentière et également pour le couple $\{23$ août/18 septembre $\}$, même si le biais est moins fort.
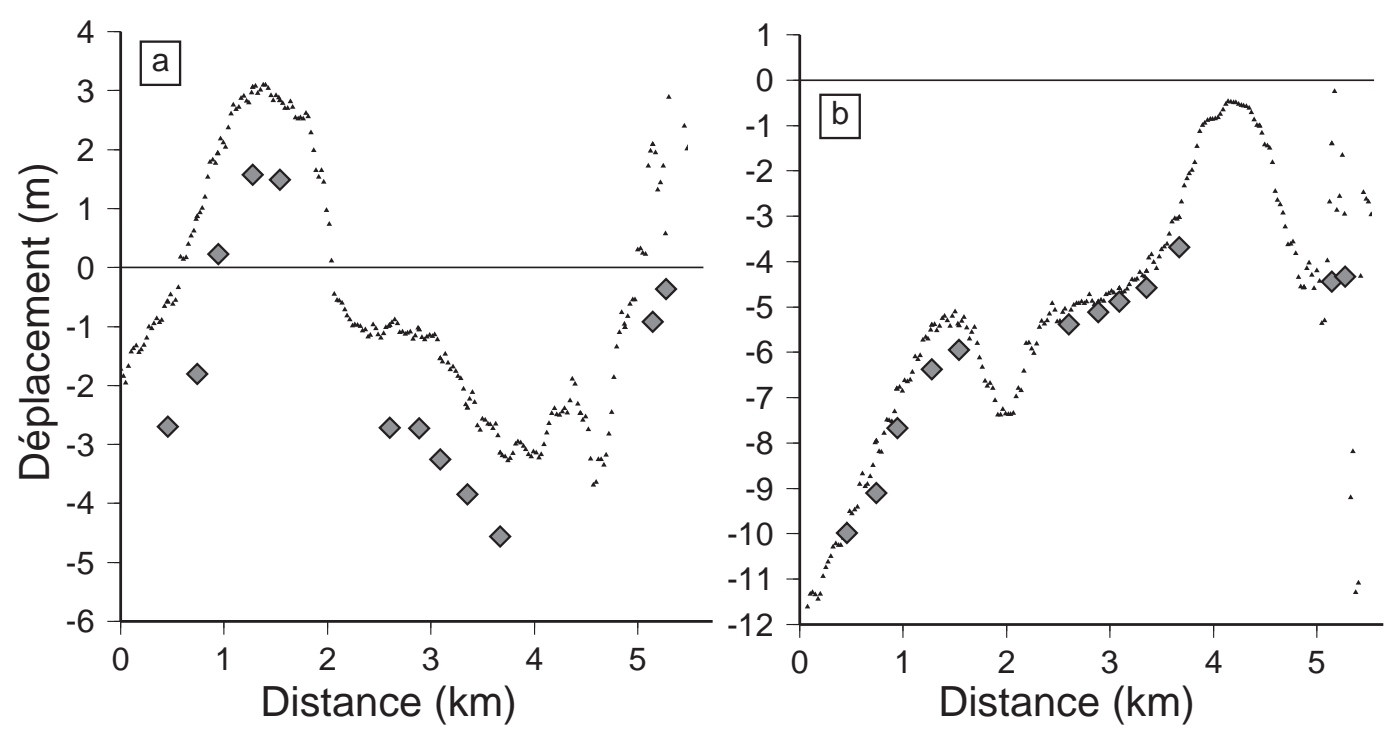

FIG. 4.17 - Déplacements en colonne (a) et en ligne (b) pour un profil longitudinal de la Mer de Glace. Les petits triangles noirs correspondent aux mesures satellitaires -couple $\{19$ juillet/19 août\}-alors que les losanges gris correspondent aux mesures DGPS. 
Ce biais, dans la direction des colonnes uniquement, s'explique par la géométrie de l'acquisition des images SPOT. Nous mesurons en effet la projection du vecteur déplacement au sol dans le plan focal du satellite. A cause d'un angle d'incidence non vertical de nos images (tableau 4.1), le décalage en colonne est aussi sensible à des mouvements verticaux (équation 4.3). L'été est une période de forte ablation sur les glaciers, les décalages en colonnes sont donc affectés par l'amincissement du glacier. La figure 4.18 (partie de gauche) explique cet effet géométrique. Pour obtenir une mesure précise des déplacements horizontaux, l'ablation entre les deux acquisitions a été corrigée grâce à un modèle degré-jour (Vincent, 2002). Ce modèle stipule qu'à une altitude donnée sur le glacier tout degré positif engendre une ablation de $6.6 \mathrm{~mm}$ de glace. En utilisant les températures de la station Météo France de Chamonix et en supposant que la température diminue de $0.6^{\circ} \mathrm{C}$ tous les $100 \mathrm{~m}$, l'ablation peut être estimée et le biais sur les déplacements en colonne corrigé. Notre correction est satisfaisante (figure 4.18.b), même s'il reste des écarts.
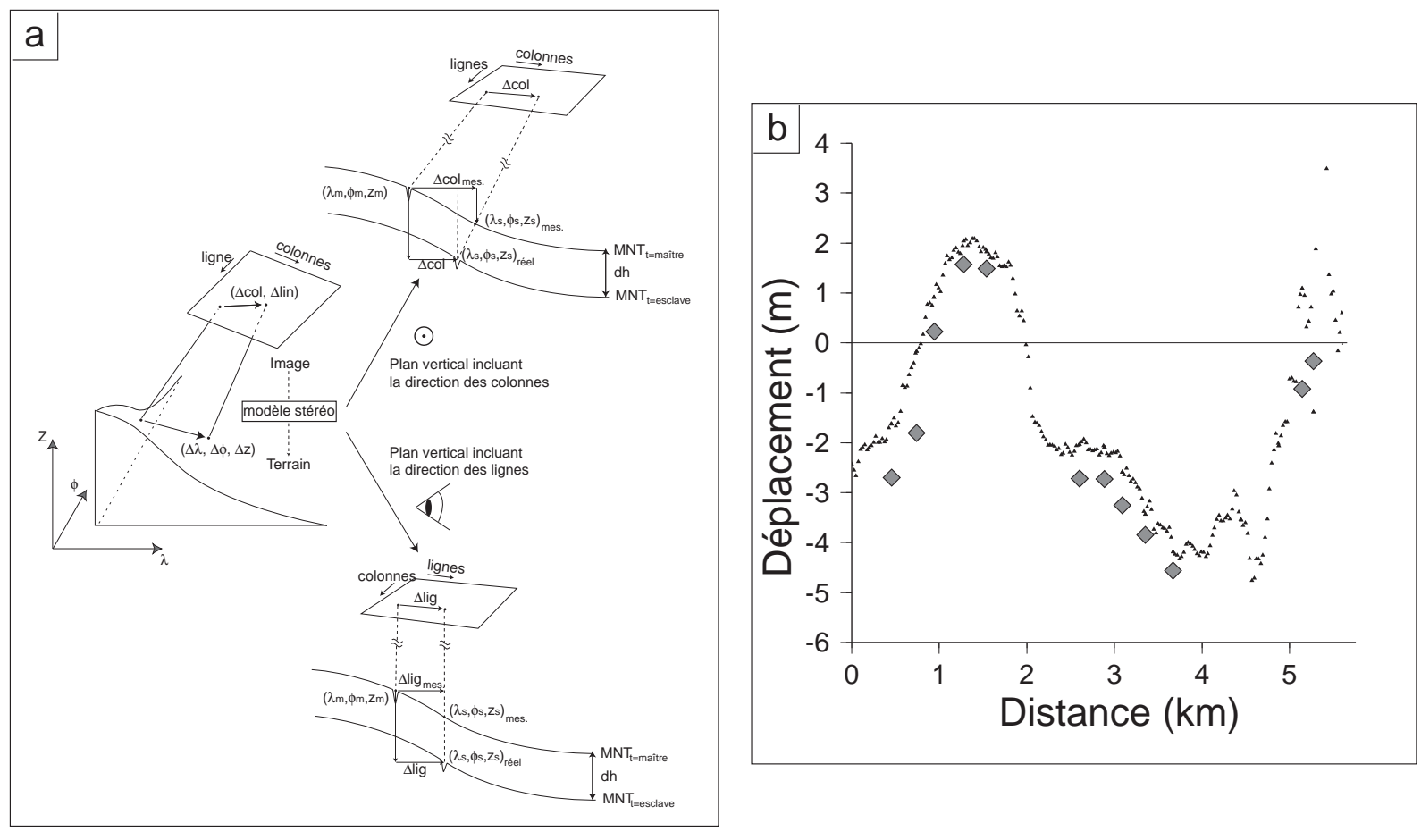

FIG. 4.18 - (a) Schéma expliquant comment l'ablation estivale engendre un biais systématique sur la mesure des déplacements dans la direction des colonnes mais pas des lignes. (b) Effet de la prise en compte de l'ablation estivale grâce à un modèle degrés jour positifs le long du même profil longitudinal que la figure 4.1\%.

Remarque : Si les structures (bandes de Fordes, crevasses), qui sont reconnues dans les deux images par la corrélation, ne sont pas perpendiculaires à la surface, une variation d'épaisseur va créer un biais supplémentaire même si les images sont acquises au nadir. Ce biais a été détecté en observant des vitesses vers l'amont (!) dans la partie frontale du Bruárjökull (Islande). Il est difficile à modéliser car il faudrait connaître le pendage des structures en profondeur.

Pour le couple $\{23$ août/18 septembre $\}$, ce biais est réduit car les angles d'incidence sont plus faibles. Il est corrigé avec la même méthodologie.

Ce biais corrigé, il est alors possible de comparer systématiquement les mesures satellitaires 
et les vitesses in situ. Pour limiter l'effet des mesures aberrantes, les champs de vitesses ont été au préalable filtrés de la manière suivante:

- conservation uniquement des points pour lesquels la corrélation est jugée valide (un coefficient de corrélation minimum de 0.7 est choisi);

- filtrage des champs de décalage en colonnes et en lignes avec un filtre médian qui permet de conserver les changements brutaux de vitesses (marges des glaciers). Ce filtre fait l'hypothèse qu'il existe une continuité spatiale du champ de vitesse. Cette continuité résulte de la propriété de l'écoulement du glacier qui est laminaire c'est à dire que les trajectoires des différents particules de glace ne s'emmêlent pas (Lliboutry, 1964);

- exclusion des vecteurs pour lesquels la direction du déplacement est "trop" différente de la ligne de plus grande pente. L'écart d'angle maximum toléré entre les deux directions est assez important $\left(30^{\circ}\right)$ car un glacier de montagne ne s'écoule pas nécessairement selon la ligne de plus grande pente locale. Les parois latérales, qui confinent le glacier, influencent aussi la direction d'écoulement;

- positionnement des vecteurs restant sur une grille régulière (fonctions blockmean et surface de GMT (Wessel \& Smith, 1998)). Les zones où il n'y a pas de mesure sont masquées.
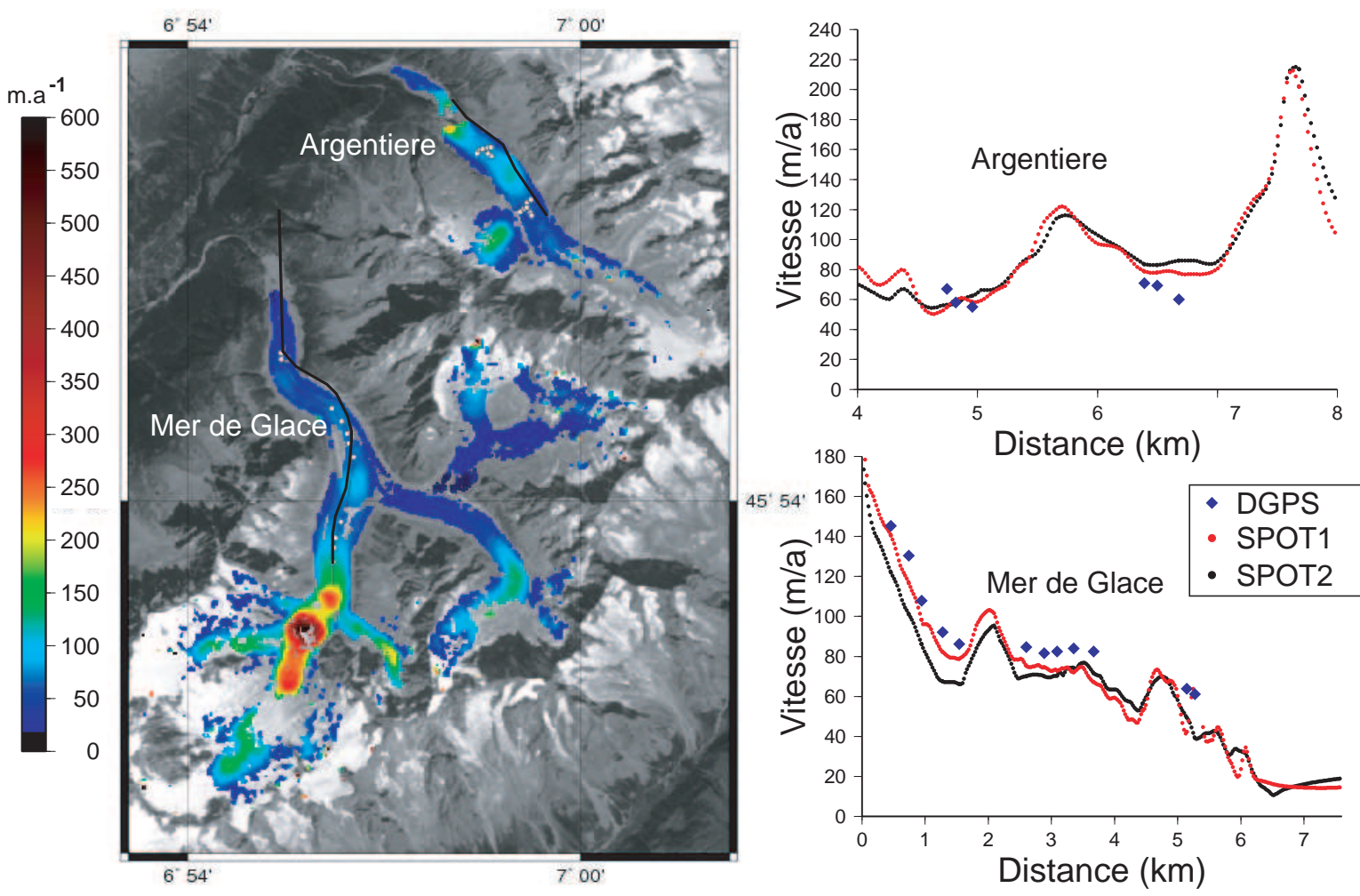

FIG. 4.19 - Champs de vitesse horizontale pour le couple \{23 août/18 septembre\} sur les glaciers d'Argentière et la Mer de Glace après filtrage. Pour les zones hors des glaciers ou sans mesure de vitesse, c'est l'image SPOT5 du 23 août 2003 que l'on voit. La partie droite permet de comparer les vitesses satellitaires et in situ le long des deux profils longitudinaux localisés en blanc sur la carte de gauche $(\# 1=\{19$ juillet $/ 19$ août $\}$ et $\# 2=\{23$ août $/ 18$ septembre $\})$. On remarquera la reproductibilité de la mesure satellitaire. 
Le résultat de ce filtrage pour le groupe de la Mer de Glace ${ }^{14}$ et le glacier d'Argentière est montré sur la figure 4.19. Un profil longitudinal de chacun des glaciers permet de comparer les deux mesures satellitaires et les mesures in situ.

On constate que, après filtrage, la couverture du glacier reste excellente. et que, même dans la zone d'accumulation, nos mesures de vitesses sont cohérentes. C'est un résultat important puisque, jusqu'à présent, la corrélation d'images échouait dans cette partie haute (Kääb, 2005). Pour le couple \#1 (non montré ici) acquis plus tôt dans la saison d'ablation, les résultats sont moins satisfaisants dans cette partie haute. Outre les problèmes géométriques déjà mentionnés, ceci s'explique probablement par une ligne de neige à plus haute altitude et un état de surface plus changeant durant 31 jours incluant la forte canicule de début août 2003.

La figure 4.20 montre, pour les deux couples d'images, les résidus $\mathrm{VIT}_{S P O T}$ - $\mathrm{VIT}_{G P S}$ en longitude, latitude, et pour la vitesse totale.

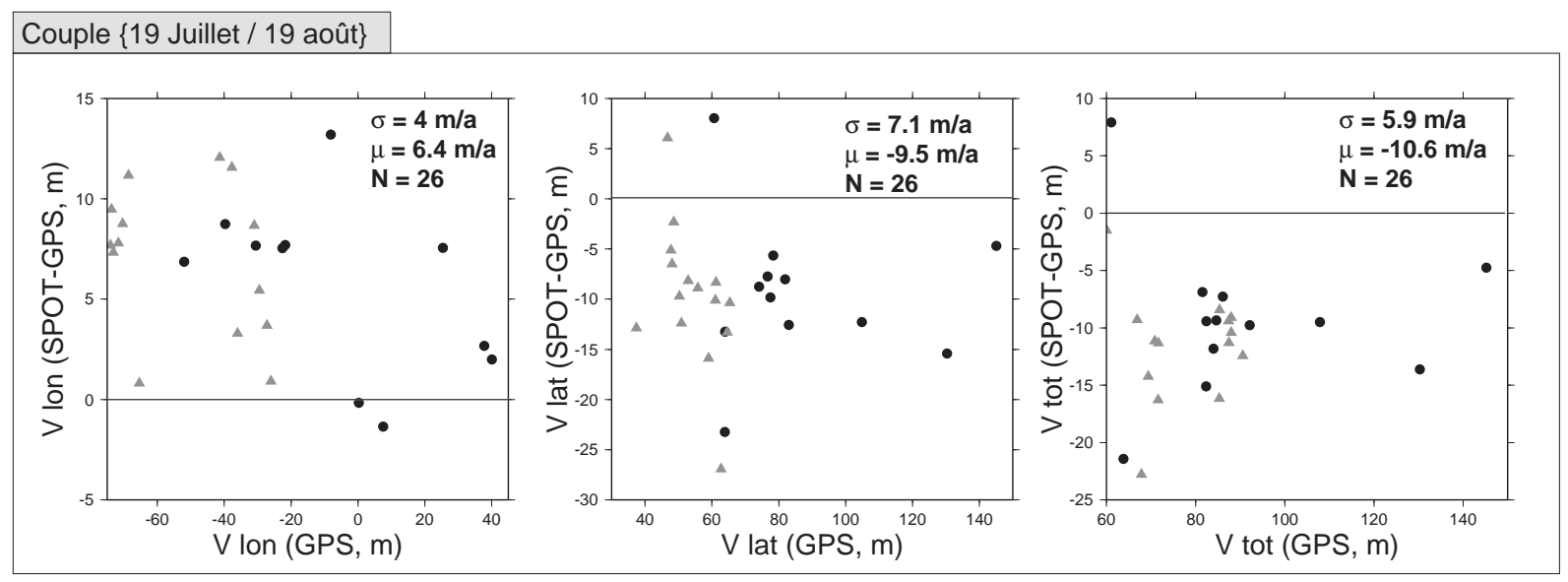

Couple $\{23$ août / 18 septembre $\}$
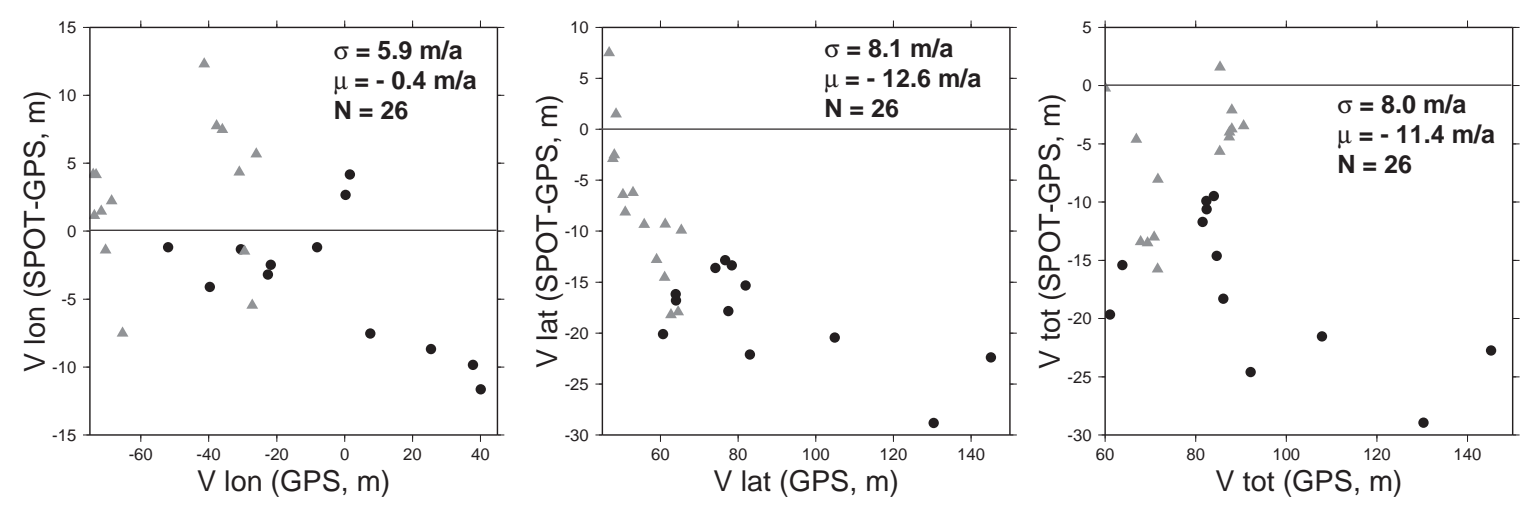

FIG. 4.20 - Comparaison des vitesses déduites des 2 couples d'images satellites à celles mesurées in situ ( $\sigma=$ écart type, $\mu=$ moyenne, $N=$ nombre d'observations). Les figures du haut correspondent au couple $\{19$ juillet/19 août\} et celles du bas au couple \{23 août/18 septembre\}. A gauche, les vitesses dans la direction des longitudes, au centre dans celle des latitudes et à droite la vitesse horizontale totale. Les triangles représentent les points du glacier d'Argentière (altitude variant de 2400 à $2700 \mathrm{~m}$ ), les ronds noirs représentent les points sur la Mer de Glace (altitude variant de 1800 à $2200 \mathrm{~m}$ ).

\footnotetext{
${ }^{14}$ qui désigne la Mer de Glace (sens strict), les glaciers de Talèfre, Leschaux, Géant
} 
Dans un premier temps, regardons les écart-types qui informent sur la précision de nos mesures. Ils varient entre 4 et $8 \mathrm{~m} \mathrm{a}^{-1}$ (soit 0.3 à $0.55 \mathrm{~m}$ de déplacement en 26 jours). On peut s'étonner des écart-types plus faibles en longitude (direction plus affectée par la correction de l'ablation). Globalement, une précision de $6 \mathrm{~m} \mathrm{a}^{-1} \mathrm{ou} \pm 0.2$ pixel peut être avancée.

Il est plus difficile de déterminer l'exactitude (i.e. l'absence de biais) de nos mesures car les vitesses de la Mer de Glace et d'Argentière ont probablement varié au cours de l'été et les différents jeux de mesures ne couvrent pas la même période (figure 4.16). On peut simplement noter que les mesures satellitaire indiquent des vitesses légèrement plus faibles que les mesures in situ. L'observation et la compréhension d'éventuelles variations rapides des vitesses sont traitées dans la partie 4.5 .

\subsubsection{Apports et limites de la méthodologie proposée}

Le traitement des 2 couples d'images SPOT5 sur les Alpes du Nord nous ont permis de mettre en évidence quelques caractéristiques importantes pour le choix des scènes SPOT :

- Les images doivent être acquises près de la fin de la période d'ablation pour que la ligne de neige soit la plus haute possible. Ainsi, le couple \{août/septembre\} présente une couverture plus globale que le couple \{juillet/août\}.

- Un gain faible lors de l'acquisition des images est primordial pour accentuer le contraste radiométrique sur la neige et la glace et, par conséquent, améliorer la corrélation. Ceci suppose la programmation spécifique de scènes pour les études glaciologiques. Nous avons pu le faire grâce au support du programme ISIS du CNES.

- Du point de vue géométrique, les images acquises à un cycle orbital d'intervalle sont les plus adaptées car la distorsion est la plus faible. Dans les autres cas, et du fait de la géométrie du capteur HRG sur SPOT5, les distorsions seront présentes en colonnes mais resteront toujours faibles en lignes. Un MNT de bonne précision permet de limiter les distorsions en colonne.

- Pour les glaciers, les variations d'épaisseur par perte ou gain de masse peuvent biaiser la mesure du déplacement horizontal. Ce biais est évité si les deux images sont acquises avec un angle d'incidence vertical. Sinon, il peut être modélisé et corrigé avec un modèle simple d'ablation. Alternativement, si la direction de l'écoulement est connue (par les mesures in situ ou grâce à un autre champ de vitesse), ce biais pourrait permettre d'estimer les variations d'épaisseur des glaciers pour de courtes périodes de temps.

- Cette étude a mis en évidence que les changements de conditions d'éclairement limitent la qualité de la corrélation et peuvent engendrer des biais.

Par rapport aux études précédentes de la déformation des glaciers, les principales avancées liées à notre méthodologie sont :

- Une couverture globale du glacier. Les études publiées à ce jour (Kääb, 2005) utilisent des images optiques séparées le plus souvent d'une année. Cet important écart temporel permet d'augmenter le signal ce qui est nécessaire lorsque une précision de \pm 1 pixel est obtenue avec des images de résolution 15 m (ASTER, LANDSAT). Mais, après une année, les changements de la surface glaciaire sont importants notamment dans les zones hautes. De plus, se pose aussi le problème de la stationnarité des structures : les crevasses s'ouvrent en amont puis se referment en aval d'une chute de séracs et auront donc une morphologie

et une position voisine d'une année sur l'autre. C'est le risque de mesurer des vitesses 
quasi nulles sur des zones qui se déplacent de plusieurs centaines de mètres par an. Une meilleure précision et l'utilisation d'images à haute résolution nous permettent de réduire la séparation temporelle à quelques semaines. Ceci évite le problème de stationnarité des structures et de trop forts changements à la surface du glacier.

- Un gain d'un facteur 5 sur la précision intrinsèque. La précision de \pm 0.2 pixel obtenue par comparaison aux mesures de terrain est 5 fois meilleure que celles des études précédentes (Scambos et al., 1992; Kääb, 2005). Ce gain s'explique en partie par une méthode de corrélation précise mais surtout par une bonne superposition des deux images. Ceci passe par une bonne prise en compte de la géométrie du capteur.

- Notre précision de $0.5 \mathrm{~m} / 26$ jours équivaut à $2 \mathrm{~cm} /$ jour, valeur du même ordre de grandeur que pour les études en InSAR (images SAR espacées d'un jour (Luckman et al., 2002)). Mais notre méthode permet de mesurer deux des trois composantes du vecteur déplacement alors que l'InSAR se contente de la projection du vecteur déplacement dans la ligne de visée du satellite. Tous les glaciers peuvent être mesurés par notre technique alors qu'un glacier s'écoulant perpendiculairement à la ligne de visée du SAR ne pourra pas être observé. De plus, les capteurs permettant la corrélation d'images optiques sont nombreux, alors que l'InSAR est impossible actuellement sur les glaciers tempérés.

Notre méthode conserve cependant certaines limites :

- La séparation temporelle optimale pour la réalisation de notre mesure satellitaire est de l'ordre d'un mois. Nous verrons dans le paragraphe suivant que des variations de vitesses estivales peuvent ainsi être détectées. En revanche, cet échantillonnage temporel ne permet pas le suivi du cycle diurne ou des accélérations printanières qui durent de quelques heures à quelques jours. Actuellement, seules des mesures continues (en général par DGPS) permettent de telles études (Sugiyama \& Guðmundsson, sous presse).

- Notre méthode n'a été testée que sur des images acquises au cours de l'été au moment où la ligne de neige est la plus élevée. Cependant, des mesures se sont avérées possibles au dessus de cette ligne, dans la zone d'accumulation. Ainsi, des mesures de vitesse au printemps, période importante pour la dynamique glaciaire, semblent possibles mais ceci reste à démontrer.

- Excepté le cas particulier de deux images espacées exactement d'un cycle orbital, l'obtention de résultats satisfaisants est assujetti à la disponibilité d'un MNT précis et donc contemporain à cause des évolutions rapides des morphologies glaciaires.

\subsection{Variations à court terme de l'écoulement}

La comparaison des vitesses satellitaires et in situ (figure 4.19 \& figure 4.20) suggère l'existence de variations des vitesses au cours de l'été 2003, notamment pour la Mer de Glace. Dans ce paragraphe, nous proposons une analyse plus détaillée de la distribution spatiale de ces variations de vitesses qui nous conduit à un mécanisme explicatif possible.

\subsubsection{Cartographie des variations de vitesse}

Les cartes des différences de vitesse entre les deux couples sont présentées sur la figure 4.21 pour 4 glaciers. Seule les différences dans la direction des lignes (de l'image du 19 juillet) sont représentées pour plusieurs raisons. Tout d'abord, les glaciers étudiés ici (notamment la Mer de 
Glace) s'écoulent principalement dans cette direction. D'autre part, nous avons remarqué que, dans la direction des colonnes, les fluctuations de vitesse étaient aberrantes. C'est probablement le résultat des incertitudes sur le MNT et d'une correction imparfaite de l'ablation. Nous observons sur la figure 4.21 les écarts des vitesses en \{août/septembre\} par rapport à celles en \{juillet/août\}. Comme les vitesses dans la direction des lignes sont négatives pour les 4 glaciers considérés, cette différence est positive en cas de ralentissement, négative sinon.

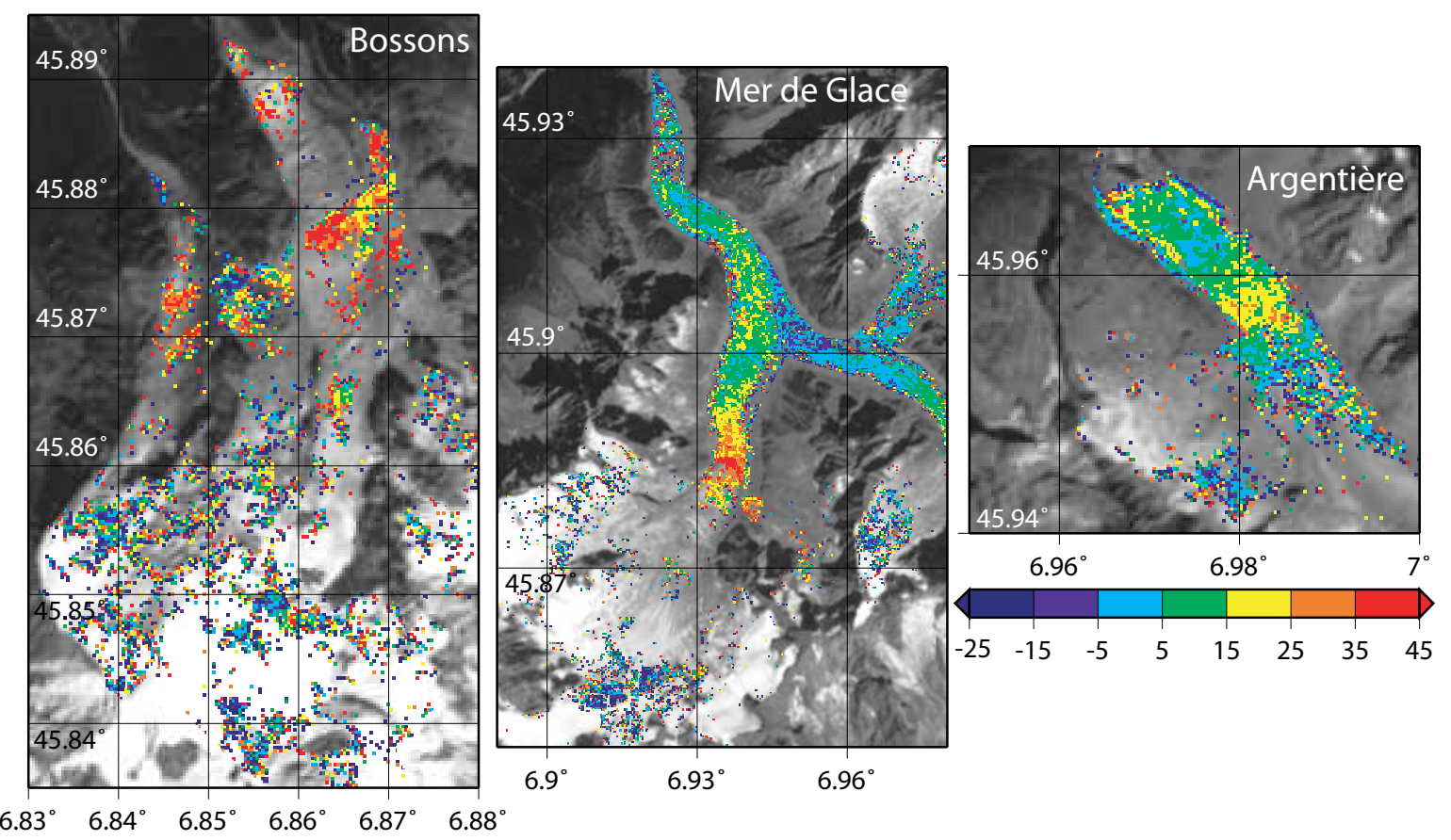

Fig. 4.21 - Cartes des variations de vitesse en ligne obtenues par soustraction des champs de vitesse : \{août/septembre\} - \{juillet/août\} pour le glacier des Bossons et de Taconnaz (à gauche), pour la Mer de Glace (au centre) et pour le glacier d'Argentière (à droite). Les zones rouges (positives) sont des régions où le glacier est plus lent en septembre qu'en juillet.

Les variations de vitesse à haute altitude restent faibles, de l'ordre de $10 \mathrm{~m} / \mathrm{a}$ mais elles peuvent atteindre $20 \mathrm{~m} / \mathrm{a}$ (Mer de Glace). Le plus souvent, c'est une légère accélération du glacier en septembre par rapport à juillet que l'on observe.

Sur le glacier des Bossons (et de Taconnaz juste à l'Ouest), toute la zone sous la ligne de neige montre un ralentissement important atteignant $50 \mathrm{~m} / \mathrm{a}$. Mais, pour ces deux glaciers, fortement crevassés et pentus, la cartographie est loin d'être complète.

Un ralentissement est également observé pour la langue de la Mer de Glace, mais sa distribution spatiale n'est pas homogène. La figure 4.22 permet de faire correspondre les fluctuations de vitesse avec la morphologie supra-glaciaire. C'est pour la zone du Tacul, au pied de la chute de séracs du Géant (zone fortement crevassée) que le ralentissement est le plus important, il atteint $50 \mathrm{~m} / \mathrm{a}$. En descendant le glacier, son amplitude décroît jusqu'à la latitude $45.9^{\circ}$, où la vitesse est presque identique pour les deux périodes. Juste en aval, au niveau d'une nouvelle zone crevassée, le ralentissement redevient significatif. On notera l'absence de variation significative de vitesse sur le glacier de Leschaux et sur la partie la plus basse de la Mer de Glace. 

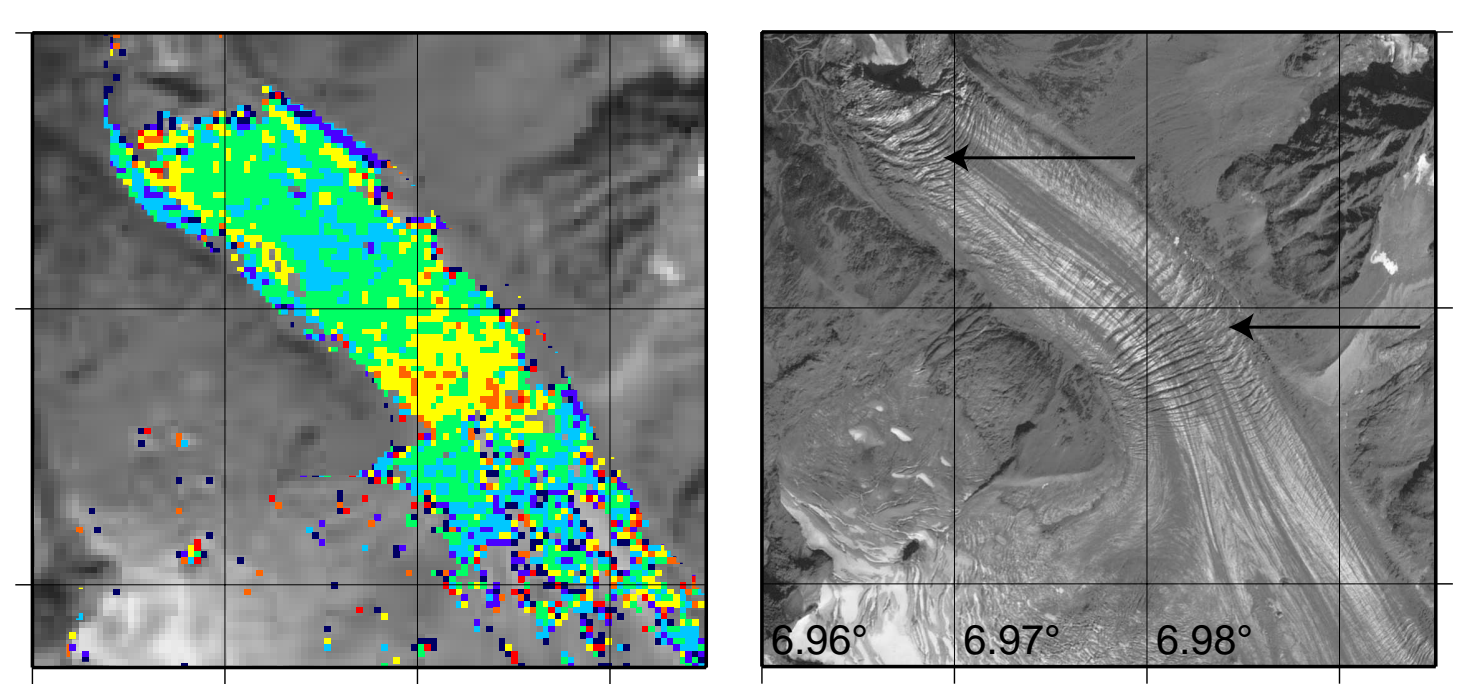

$45.97^{\circ}$

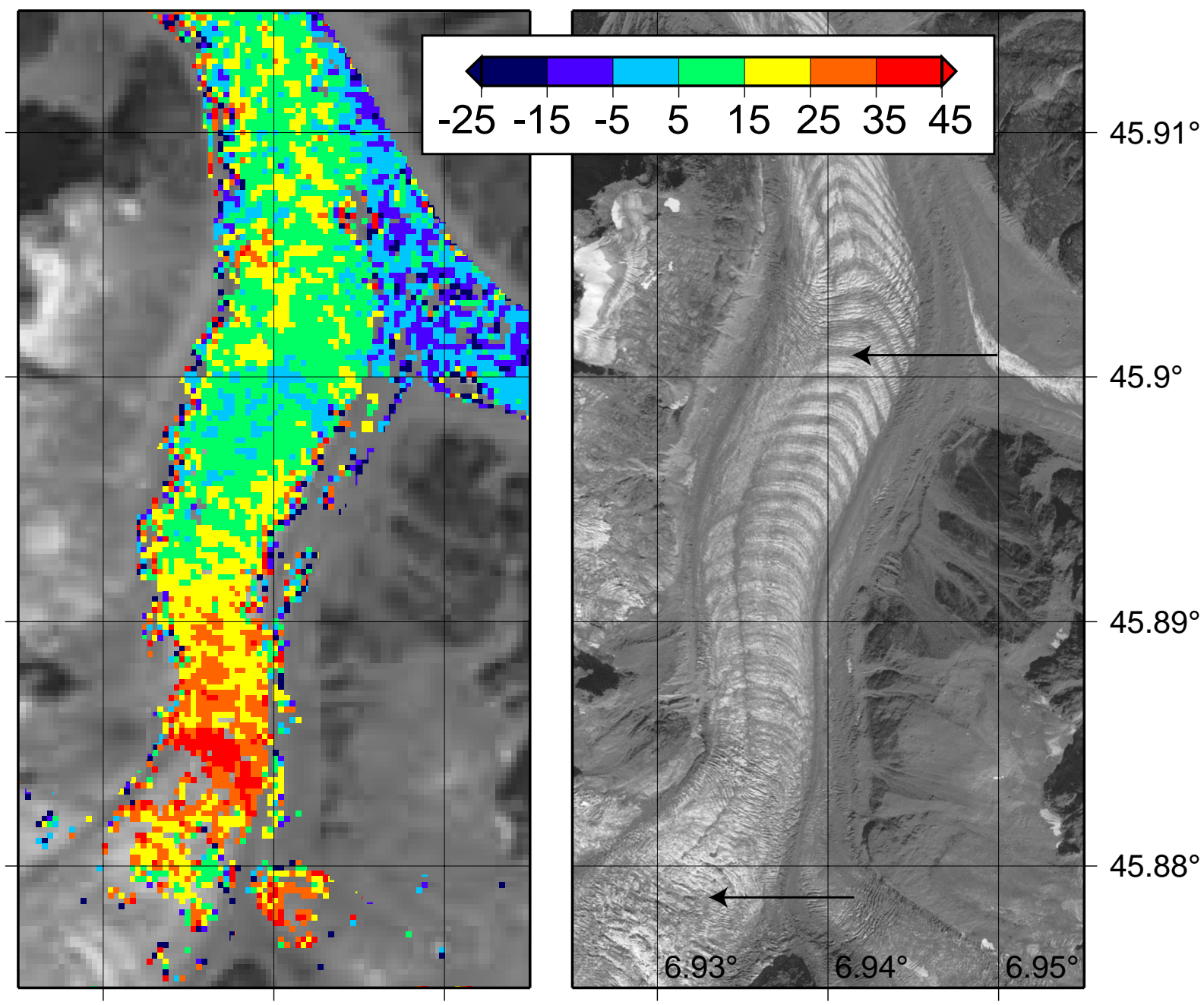

FIG. 4.22 - Comparaison de la carte de variations de vitesse ( $m / a)$ avec les structures de surface (image SPOT5 du 23 août 2003) sur le glacier d'Argentière (en haut) et la Mer de Glace (en bas). Les flèches pointent vers des zones où les crevasses sont nombreuses. 
Pour le glacier d'Argentière, les variations de vitesse sont plus faibles mais toujours reliées à la morphologie de surface. Les zones de ralentissement sont les zones les plus crevassées. Cette localisation précise suggère un rôle de la fusion de surface et de la pénétration de l'eau de fonte via les crevasses jusqu'à la base du glacier.

Nos observations tranchent avec l'assertion de Lliboutry \& Reynaud (1981) que "les fluctuations du glissement basal au cours du temps sont les mêmes sur de longues distances". La partie de la Mer de Glace affectée par des variations du glissement n'est d'ailleurs pas la même : dans notre cas c'est la zone au dessus de la confluence avec Leschaux alors que Lliboutry \& Reynaud (1981) observent essentiellement la zone en aval.

\subsubsection{Une interprétation de ces variations de vitesse}

Les variations sur des échelles de temps de quelques jours des glaciers sont liées à des modifications du glissement basal (Willis, 1995). En effet, la déformation interne ne peut varier significativement sur des échelles de temps aussi courtes pendant lesquelles l'épaisseur et la température du glacier sont relativement constantes. Ceci est corroboré par l'étude de la déformation de trous de forages (Harper et al., 2001). Les événements d'accélération des glaciers sont connus et communs au printemps quand débute la fusion de surface. Les étapes suivantes semblent mises en jeu : pénétration de l'eau de fonte à la base du glacier grâce aux crevasses, augmentation de pression d'eau, soulèvement du glacier et donc accélération car le frottement sur son lit rocheux est réduit (Iken et al., 1983). Si ces événements sont surtout présents au printemps c'est qu'à cette époque de l'année le réseau hydrologique est peu développé. En été, les canaux sous-glaciaires permettent d'évacuer l'eau vers l'aval ce qui limite l'augmentation de pression d'eau. Dans nos observations, la relation étroite entre les champs de crevasses et les zones de variation de vitesse suggère également un rôle de l'eau de fonte.

A partir de la température moyenne à la station de Chamonix et en utilisant un taux de décroissance de $6^{\circ} / \mathrm{km}$, la température à toute altitude peut être estimée. Sur la figure 4.23 est représentée l'altitude de l'isotherme $0^{\circ} \mathrm{C}$, qui indique approximativement l'altitude maximale où la fusion intervient à la surface du glacier. Les périodes couvertes par les couples d'images SPOT5 sont indiquées. Les températures plus élevées fin juillet et début août conduisent à une fusion à beaucoup plus haute altitude. Vers $2500 \mathrm{~m}$ (au cœur des séracs du Géant), l'ablation de surface est deux fois plus forte dans l'intervalle d'acquisition du couple \{juillet/août que pour le couple \{août/septembre\} (lame d'eau de $6.4 \mathrm{~cm} /$ jour contre $3 \mathrm{~cm} /$ jour). Cette figure montre aussi la remontée brutale de l'isotherme $0^{\circ} \mathrm{C}$ entre le $1^{e r}$ juillet et le 19 juillet, date d'acquisition de la première image SPOT5.

A partir de ces observations, le mécanisme suivant peut être proposé pour expliquer l'accélération du glacier du Tacul en juillet/août par rapport à août/septembre. Ce mécanisme est illustré par la figure 4.24 .

Fin juillet et début août, la ligne de neige se situe déjà à haute altitude (vers $2900 \mathrm{~m}$ ). Les températures élevées au pic de la canicule engendrent une fonte intense de la glace qui affleure (faible albédo de la glace par rapport à la neige). Cette eau de fonte pourrait gagner la base du glacier principalement par les crevasses de la chute de séracs du Géant où l'épaisseur du glacier est minimale. Cette zone n'ayant probablement pas subi de périodes d'intense fusion plus tôt dans l'été, le réseau hydrologique sous-glaciaire est peu développé et évacue mal l'eau de fonte. La forte pression d'eau basale expliquerait alors une intensification du glissement basal et l'accélération du glacier. Cette accélération affecte une zone de 1 à $2 \mathrm{~km}$ de long (soit environ 
5 fois l'épaisseur du glacier). La prochaine zone crevassée vers l'aval se situe au niveau de la confluence avec le glacier de Leschaux. En dessous de cette zone, le réseau hydrologique est déjà bien développé mi-juillet et l'eau de fonte excédentaire liée à la canicule engendre, certes, une accélération mais de plus faible amplitude.

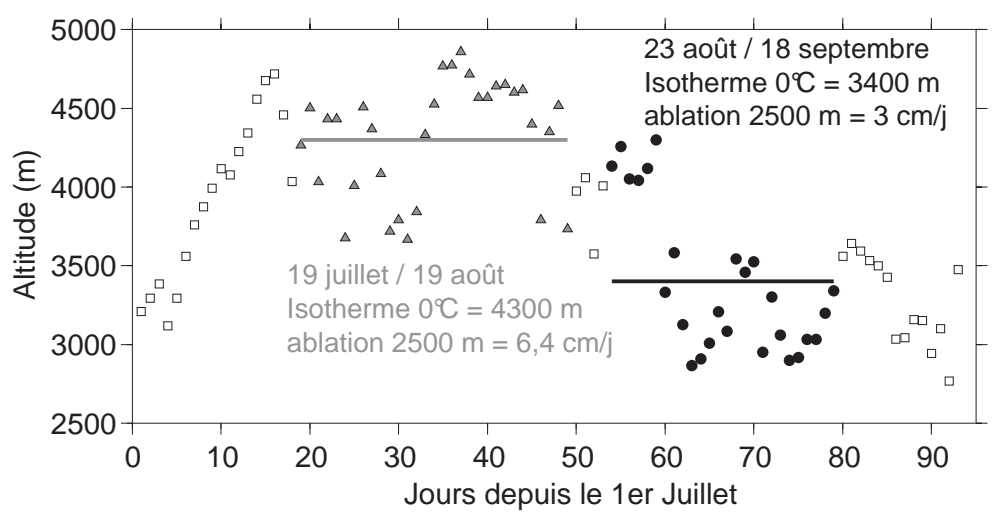

FIG. 4.23 - Altitude (m) de l'isotherme $0^{\circ} \mathrm{C}$ au cours de l'été 2003. La canicule de fin juillet / début août s'accompagne de la remontée de cet isotherme à haute altitude. Ceci explique les fortes différences de fusion de surface pour les deux couples d'images SPOT5.

Fin août et début septembre, la fusion au niveau des séracs du Géant est deux fois plus faible que début août. Le réseau hydrologique, bien développé après le fort apport en eau début août, peut évacuer vers l'aval cette eau de fonte : la pression d'eau revient à son niveau normal, le glissement basal aussi.

Pour le glacier d'Argentière, les zones d'accélération en juillet/août par rapport à août/septembre sont plus localisées, juste au niveau de deux zones crevassées. Une interprétation similaire peut être proposée. La saturation du réseau hydrologique au moment du pic d'ablation début août engendre une augmentation locale du glissement du glacier.

La différence d'extension des zones affectées par une accélération estivale sur la Mer de Glace (large) et le glacier d'Argentière (plus réduite) est plus difficile à comprendre. Le développement du réseau hydrologique ou la transmission des contraintes longitudinales vers l'aval semblent des pistes à explorer pour expliquer ces différences.

\subsubsection{Discussion de ces variations de vitesse}

La répétition de la mesure des déplacements de surface des glaciers du Mont-Blanc permet donc d'étudier des variations à court terme de l'écoulement. La large couverture spatiale de l'imagerie satellitaire permet une cartographie d'ensemble du phénomène et de le mettre en relation avec la morphologie supra-glaciaire. Une intense fusion de surface n'est pas suffisante pour expliquer l'augmentation du glissement basal, la présence de crevasses (ou de moulins) est indispensable pour véhiculer cette eau de fonte vers la base du glacier. La distribution des crevasses va donc en partie contrôler les zones affectées par un glissement basal intensifié. Notre étude suggère un rôle important du développement du réseau hydrologique (la "plomberie" du glacier d'après (Paterson, 1994)) et donc de l'avancement dans la période d'ablation. Un fort événement de fonte aura d'autant plus d'influence qu'il interviendra tôt dans l'année. 


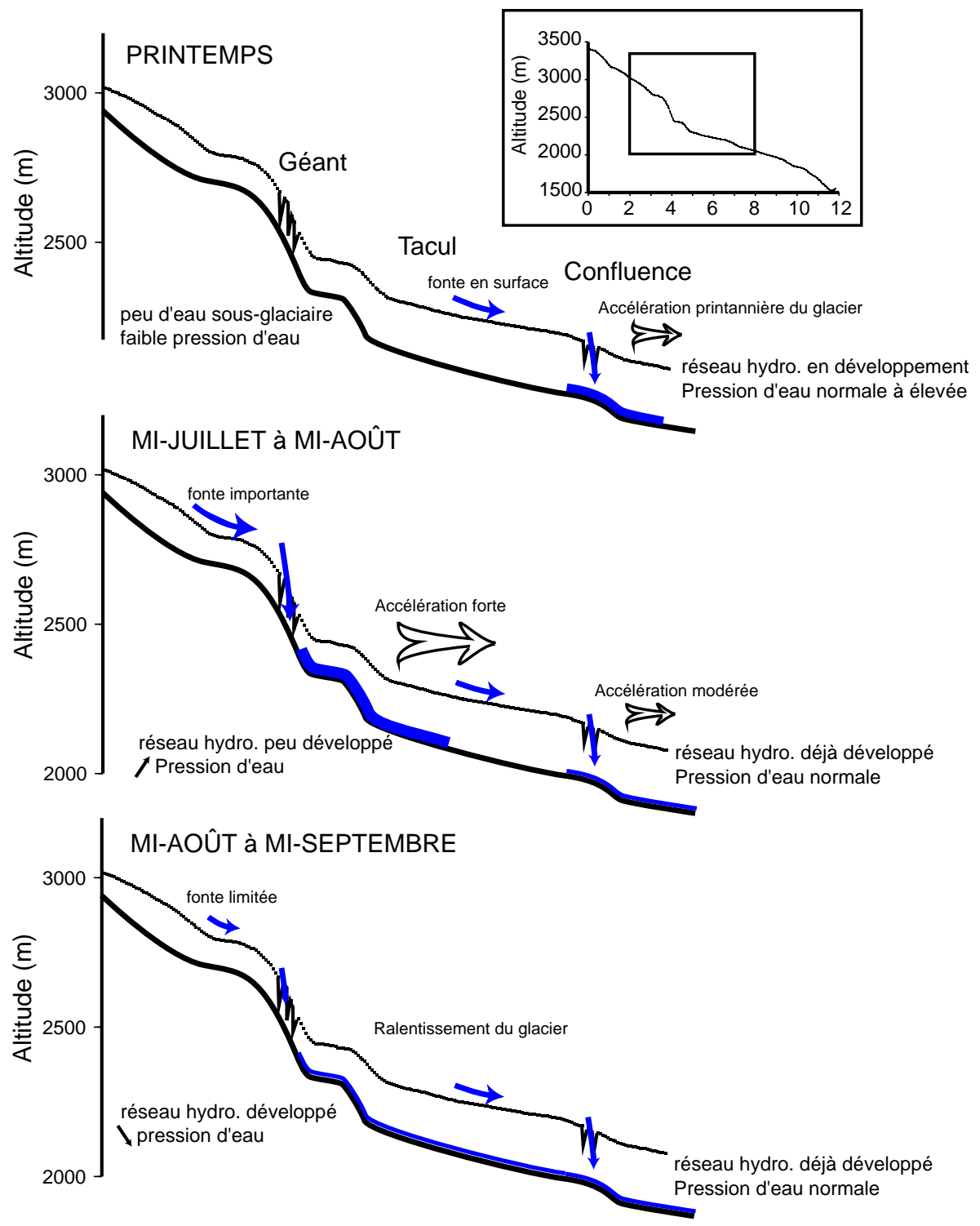

FIG. 4.24 - Une interprétation possible des variations de vitesse observées pour la Mer de Glace au cours de l'été 2003. La topographie de surface est réelle alors que la topographie basale est hypothétique. L'afflux massif d'eau de fonte à haute altitude par les séracs du Géant au moment de la canicule explique une augmentation importante des vitesses dans la zone du Tacul. Le développement du réseau hydrologique et une fonte limitée expliquent le ralentissement début septembre.

Nos conclusions sur le rôle de la fusion de surface rejoignent celles de Zwally et al. (2002a) pour le Groenland. Leur étude, qui couple également observations météorologiques et mesures des vitesses de surface (en quelques points seulement grâce au DGPS), met en évidence une corrélation entre les périodes de fonte et les accélérations du glacier. Dans un contexte de réchauffement climatique, ce mécanisme est important pour comprendre et prédire l'évolution des calottes glaciaires ou des glaciers de montagne dans les prochaines années. Une forte fusion de surface, couplée à la présence de crevasses, accélère le glacier ce qui intensifie le flux de glace vers l'aval. Ce processus transfère donc de la masse vers les zones où la fonte est la plus forte. Il tend 
ainsi à accélérer les pertes de masse des glaciers.

Nos travaux sont originaux grâce à une large couverture spatiale du phénomène, inégalée à notre connaissance. Notre étude est donc complémentaire de travaux de terrains sur des zones localisées qui permettent une étude fine des processus mis en jeu ou de travaux théoriques qui permettent de tester et représenter ces processus dans des modèles. Outre l'absence de mesure de vitesse au printemps, la principale limite de notre étude est la résolution temporelle. Nous pouvons simplement discuter les variations de vitesse entre deux périodes d'environ 1 mois, qui cachent très certainement des évènements beaucoup plus rapides de quelques heures à quelques jours. Le couplage entre la mesure satellitaire et des mesures continues de terrain (GPS permanent) pourrait permettre d'allier les avantages des deux méthodes pour parvenir à une description spatio-temporelle détaillée de ces variations de vitesse.

\subsection{Variabilité interannuelle de l'écoulement}

Dans la partie précédente, l'utilisation de deux couples d'images SPOT5 acquis au cours de l'été 2003 nous a permis d'étudier des variations de l'écoulement sur de courtes périodes de temps. L'archive d'images SPOT étant riche sur les Alpes du Nord, nous avons pu utiliser notre chaîne de traitement pour étudier la variabilité interannuelle de la vitesse. "ME DECOIS PAS" a donc du être adaptée aux images SPOT1-4 notamment pour éliminer les discontinuités au niveau des inter-barrettes (Van Puymbroeck et al., 2000). Dans le tableau 4.3, sont recensés les différents couples d'images à notre disposition. Ces couples d'images ont été choisis pour minimiser le rapport $\mathrm{B} / \mathrm{H}$ tout en étant aussi proches que possible de la fin de la période d'ablation quand le contraste radiométrique est le plus important sur les glaciers. Ce choix permet de palier (partiellement) le gain élevé des images qui n'ont pas été acquises à but "glaciologique".

\subsubsection{Comparaison des vitesses estivales}

Parmi les couples d'images dans la tableau 4.3, certains conduisent à des mesures de vitesse aberrantes et ne sont pas utilisés par la suite. La séparation temporelle (donc le rapport signal/bruit) est trop faible pour le couple $\{19$ août/1 sept. 1990\}. Les vitesses déduites du couple $\{29$ août/24 sept. 2000$\}$ sont également bruitées semble-t-il à cause d'une chute de neige intervenue peu de temps avant le 24 septembre. Les mesures estivales pour les couples de 1988, 1993 et 2003 sont donc comparées par la suite.

Sur la figure 4.25 sont représentées les cartes des vitesses pour les glaciers de Bossons, de la Mer de Glace s.l. et d'Argentière. Tous les champs de vitesses ont été filtrés en utilisant la méthode présentée au §4.4.4.2. Ces cartes soulignent que des mesures cohérentes sont aussi possibles avec les satellites SPOT1-4. Le faible gain des images de 2003 explique que la zone d'accumulation soit mieux couverte. La même échelle de couleur est utilisée pour les 3 années et suggère des vitesses plus élevées en 1988 et 1993 par rapport à 2003. Des profils longitudinaux (figure 4.26) de chacun des 3 glaciers permettent de mieux caractériser ces écarts.

Le fait le plus marquant est le net ralentissement en 2003 des glaciers au niveau des chutes de séracs où leur vitesse est maximale. Ce type d'observation est difficile sur le terrain car ces zones sont inaccessibles. Sur la Mer de Glace, la vitesse passe de $700 \mathrm{~m} / \mathrm{a}$ (1988 \& 1993) à 600 m/a en 2003. Reynaud (1973) a utilisé des photographies aériennes également en fin d'été (30 septembre, 18 et 31 octobre 1969) pour observer les vitesses de cette chute de séracs. Ses mesures 
mettent en évidence la grande hétérogénéité des vitesses de ces zones. La mesure satellitaire, qui s'appuie sur des fenêtres de corrélation de plusieurs dizaines de mètres, lisse ces hétérogénéités. La vitesse maximale était voisine de $850 \mathrm{~m} / \mathrm{a}$ en 1969 (et légèrement supérieure, $950 \mathrm{~m} / \mathrm{a}$, en 1962). L'ensemble de ces données suggère un ralentissement progressif mais marqué (-30 \%) de cette zone du glacier entre 1960 et 2000. La même observation s'applique à la chute de sérac du glacier d'Argentière. Pour les Bossons, un ralentissement est aussi visible à haute altitude (à $1.5 \mathrm{~km}$ du début du profil) alors que le maximum de l'écoulement (à $3 \mathrm{~km}$ du début du profil) reste presque inchangé pour les différents étés.

TAB. 4.3 - Les couples d'images SPOT utilisés pour étudier la variabilité interannuelle des vitesses. La première partie du tableau correspond aux images acquises le même été alors que la seconde partie décrit des couples acquis à un an d'intervalle. Pour les couples estivaux, la moyenne des températures à la station de Chamonix pendant l'intervalle de temps considéré est fournie.

\begin{tabular}{lccccc}
\hline Couples d'images & Satellite & angle d'incidence & $\mathrm{B} / \mathrm{H}$ & $\delta \mathrm{t}$ (jours) & $\mathrm{T}_{\text {moy }}$ \\
\hline 27 août/22 sept. 1988 & SPOT1/SPOT1 & $-23.4^{\circ} /-23.4^{\circ}$ & 0.0001 & 26 & 12.1 \\
19 août/1 sept. 1990 & SPOT1/SPOT2 & $-1.9^{\circ} /-2.3^{\circ}$ & 0.001 & 13 & 15.2 \\
30 juil./2 sept. 1993 & SPOT1/SPOT2 & $+5.6^{\circ} /+5.9^{\circ}$ & 0.0007 & 34 & 16.2 \\
29 août/24 sept. 2000 & SPOT4/SPOT4 & $-28.3^{\circ} /-25.5^{\circ}$ & 0.0001 & 26 & 12.9 \\
23 août/18 sept. 2003 & SPOT5/SPOT5 & $+15.7^{\circ} /+17.3^{\circ}$ & 0.0006 & 26 & 13.8 \\
\hline 2 sept. $1993 / 09$ août 1994 & SPOT2/SPOT3 & $+5.9^{\circ} /+5.9^{\circ}$ & 0.002 & 341 & \\
25 août $2000 / 29$ août 2001 & SPOT1/SPOT1 & $+20.9^{\circ} /+25.4^{\circ}$ & 0.01 & 369 & \\
\hline
\end{tabular}

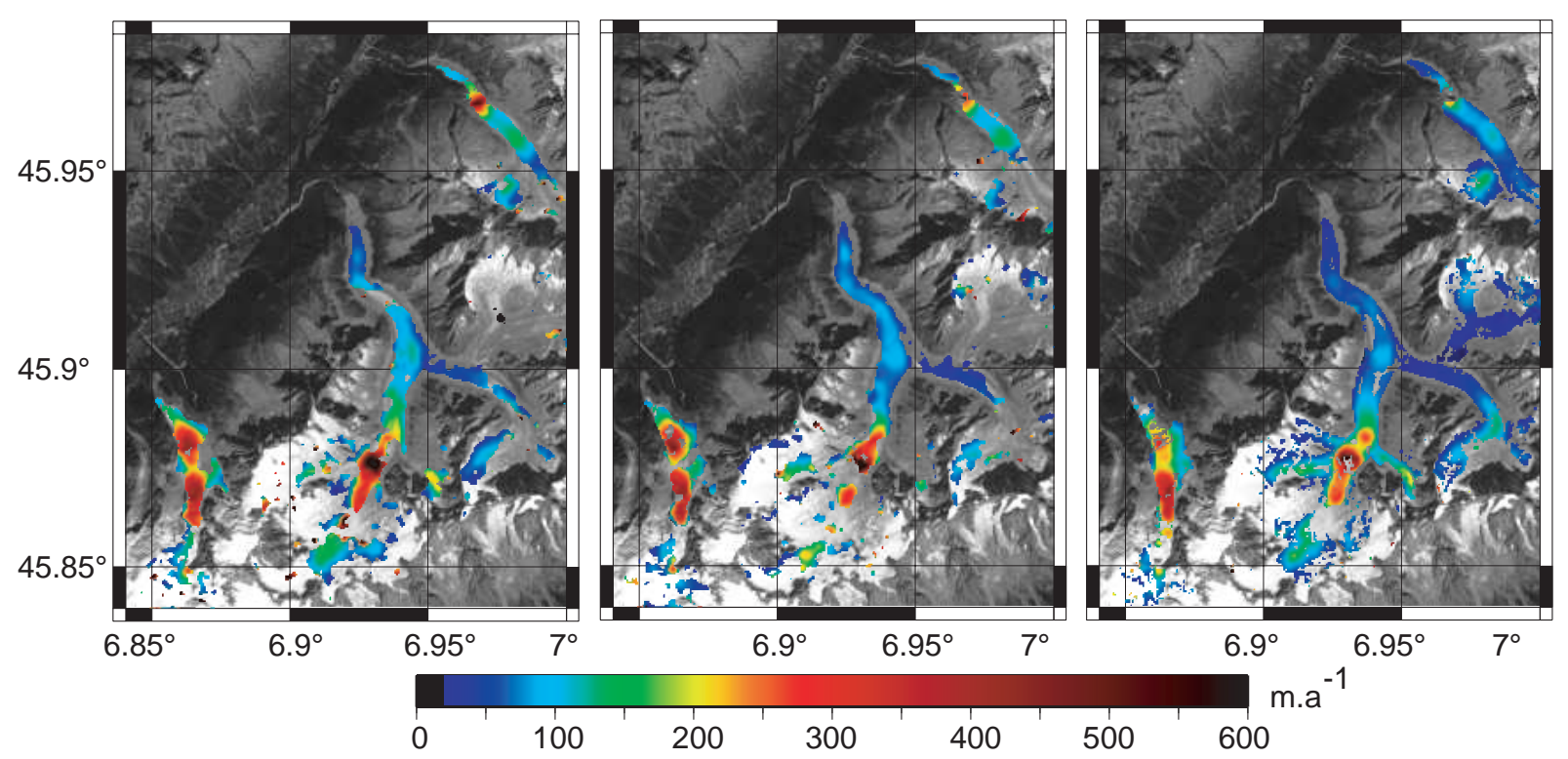

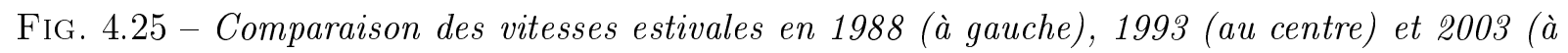
droite) pour les glaciers des Bossons, d'Argentière et la Mer de Glace. 

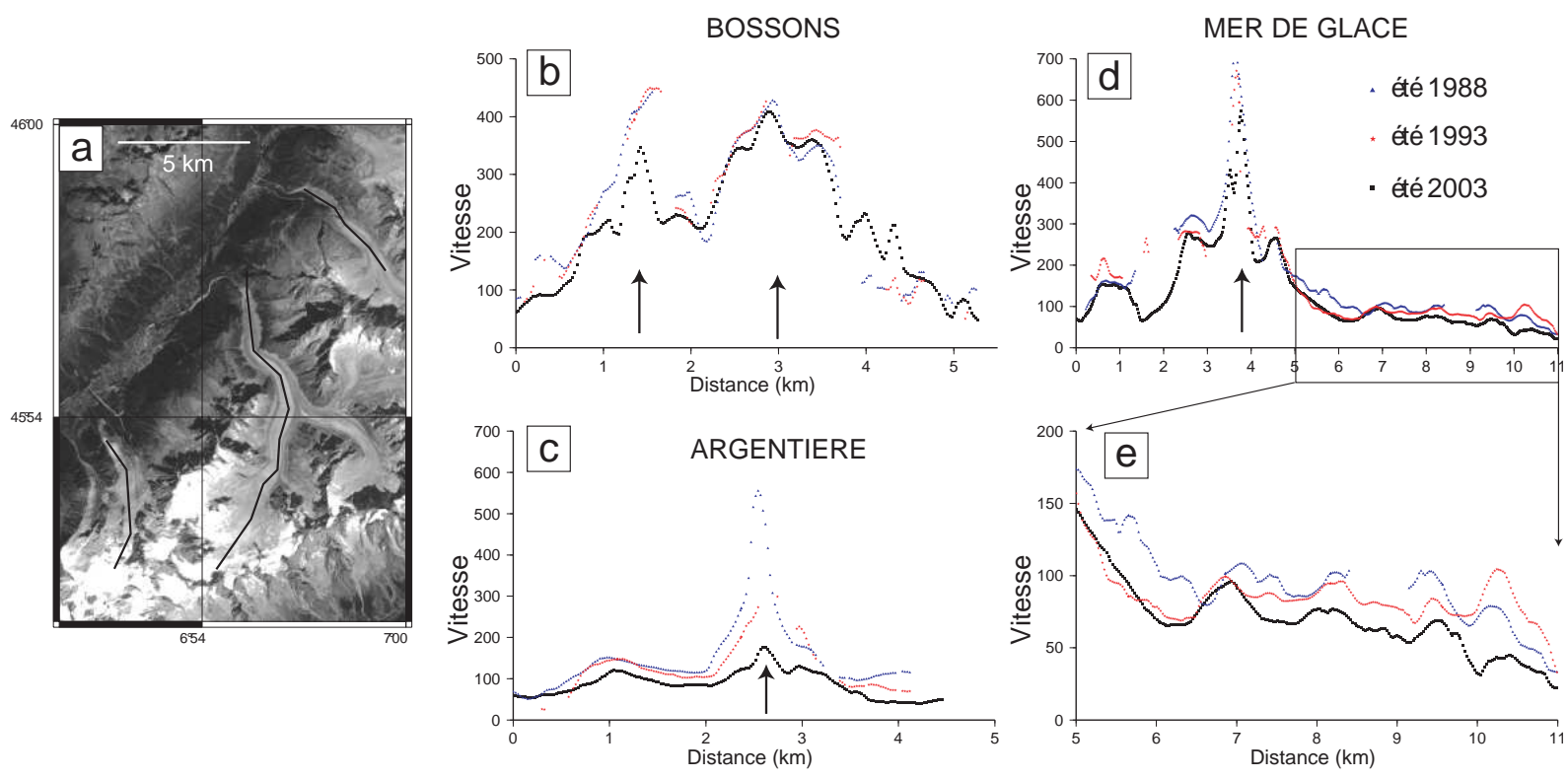

Fig. 4.26 - Profils longitudinaux des vitesses horizontales $(\mathrm{m} / \mathrm{a})$ pour les glaciers des Bossons (b), d'Argentière (c) et de la Mer de Glace (d). Ces profils sont localisés sur l'image SPOT du 23 août 2003 (a). Pour la Mer de glace, en plus du profil général, un zoom sur la partie basse est également présenté (e). Les flèches localisent les chutes de séracs.

Le profil détaillé de la partie basse de la Mer de Glace montre la bonne cohérence entre les différentes années avec des pics relatifs de vitesse localisés aux mêmes endroits. Au delà du point $6.5 \mathrm{~km}$ du profil, on observe, en moyenne, une décroissance de la vitesse au cours du temps, surtout entre 1993 et 2003. Au dessus de $6.5 \mathrm{~km}$, les vitesses sont identiques en 1993 et 2003, alors que le glacier est $30 \mathrm{~m} / \mathrm{a}$ plus rapide en 1988. Il s'agit de la zone où des variations à court terme des vitesse ont été observées au cours de l'été 2003 (figure 4.19). Ceci suggère donc une accélération du glissement du glacier et montre que cette zone au pied de la chute de sérac du Géant joue un rôle clef dans la dynamique de la Mer de Glace. Notons toutefois que les incertitudes sur des vitesses estivales déduites de couples d'images SPOT1-4 sont fortes : \pm 2 à 3 $\mathrm{m}$ sur les déplacements (§4.6.2) soit $\pm 30 \mathrm{~m} / \mathrm{a}$ environ. Les fluctuations de vitesses enregistrées ici sont donc en limite de significativité.

L'interprétation de cette évolution des vitesses estivales avec $\mathrm{V}_{88}>\mathrm{V}_{93}>\mathrm{V}_{03}$ n'est pas simple. Les variations du glissement basal sont une des explications possibles. Toutefois, il n'existe pas de relation évidente entre les changements de vitesse et les températures moyennes (tableau 4.3) à Chamonix $\left(\mathrm{T}_{93}>\mathrm{T}_{03}>\mathrm{T}_{88}\right)$ qui indiquent l'intensité de la fusion donc l'apport en eau à la base du glacier. Nous avons vu, de toute façon, que l'intensité du glissement basal dépend d'autres paramètres comme le développement du réseau hydrologique sous-glaciaire. Une autre interprétation possible de ce ralentissement entre 1988 et 2003 est une diminution de la déformation interne du glacier. Cette hypothèse est renforcée par l'analyse des variations des vitesses annuelles. 


\subsubsection{Comparaison des vitesses annuelles}

Deux paires d'images seulement sont disponibles pour étudier les vitesses annuelles. Ces couples d'images ont été sélectionnés pour :

- augmenter la séparation temporelle donc le déplacement du glacier ce qui améliore la précision (rapport signal/bruit).

- tester si la corrélation est maintenue après une année et, si oui, dans quelles zones du glacier.

- mieux comprendre le ralentissement observé sur les couples estivaux dans une situation (moyenne annuelle) où les variations du glissement basal doivent être faibles.

Sur la figure 4.27 sont représentées les vitesses horizontales sur la Mer de Glace pour la période 1993-94 et 2000-01. Les autres glaciers ne sont pas montrés : la corrélation ne fonctionne que sur la partie médiane du glacier d'Argentière (entre 2400 et $2600 \mathrm{~m}$ ). Aucune mesure n'est obtenue sur les zones fortement crevassées comme le glacier des Bossons, leur morphologie est trop variable. Ceci confirme que, si la séparation temporelle est trop longue, seules les vitesses de certaines parties de la zone d'ablation peuvent être observées par corrélation d'images optiques (Kääb, 2005). Ainsi est souligné l'intérêt d'images espacées de quelques semaines seulement.
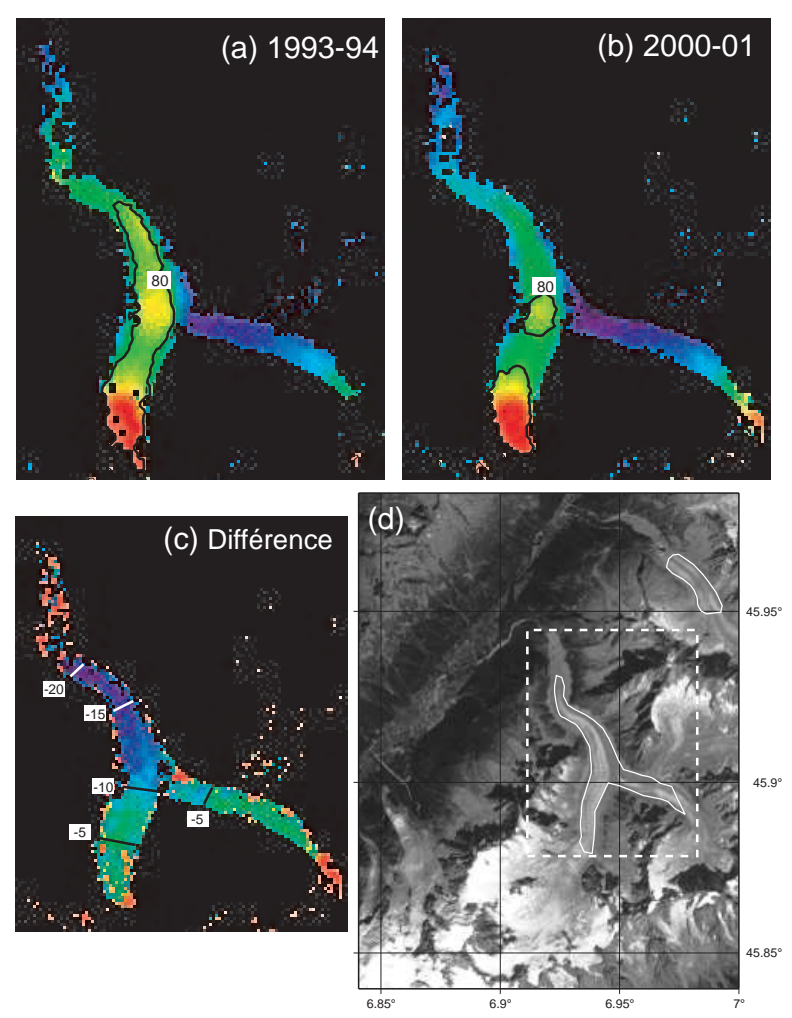

Fig. 4.27 - Vitesses annuelles pour les périodes 1993-94 (V93-94 en a), 2000-01 (V00-01 en b) et différence $V_{00-01}-V_{93-94}(c)$. L'image SPOT (d) permet de localiser la zone couverte par les cartes $a, b$ et $c$ (pointillé blanc) et les zones des glaciers où des mesures de vitesse sont possibles pour des images espacées d'une année (trait blanc continu). Pour les champs de vitesses (a et b), l'isocontour $80 \mathrm{~m} \mathrm{a}^{-1}$ a été dessiné. Pour la carte des différences de vitesse, la position de 5 isocontours est aussi indiquée. 
La comparaison des champs de vitesse pour 1993-94 et 2000-01 indique un net ralentissement de la Mer de Glace (voir la remontée vers l'amont de l'iso-vitesse $80 \mathrm{~m} / \mathrm{a}$ ). Un profil longitudinal des vitesses de la Mer de Glace permet de mieux caractériser ce ralentissement (figure 4.28). Le long de ce profil, le bon accord des vitesses "SPOT", pour les deux périodes, dans la zone du "Tacul" et hors du glacier (vitesses voisines de 0) suggère une bonne précision. La comparaison avec les vitesses in situ annuelles (données LGGE) le confirme. L'écart moyen est de $3 \mathrm{~m} / \mathrm{a}$ en 2000-01 (écart type $1.6 \mathrm{~m} / \mathrm{a}, \mathrm{N}=6$ ) et $1.4 \mathrm{~m} / \mathrm{a}$ (écart type $1.3 \mathrm{~m} / \mathrm{a}, \mathrm{N}=10$ ) en 1993-94. Des angles d'incidence presque verticaux en 1993-94 expliquent sans doute le biais plus faible (peu d'effet des variations d'épaisseur du glacier). L'incertitude des vitesses "SPOT" annuelles est donc de 2 à $3 \mathrm{~m} / \mathrm{a}$ ce qui confirme la précision de 0.2 à 0.3 pixel (le pixel des images SPOT1-4 est de $10 \mathrm{~m}$ ) obtenue pour l'été 2003. Les variations de vitesses observées sont donc significatives dès qu'elles atteignent $5 \mathrm{~m} / \mathrm{a}$. Entre 1993-94 et 2000-01, le ralentissement est plus important à basse altitude. Cette figure montre également que pour l'ensemble de la période 1965-1980, les vitesses étaient assez peu variables (Lliboutry \& Reynaud, 1981). Entre 1980 et 1993-94, la ralentissement est quasi uniforme le long du profil.
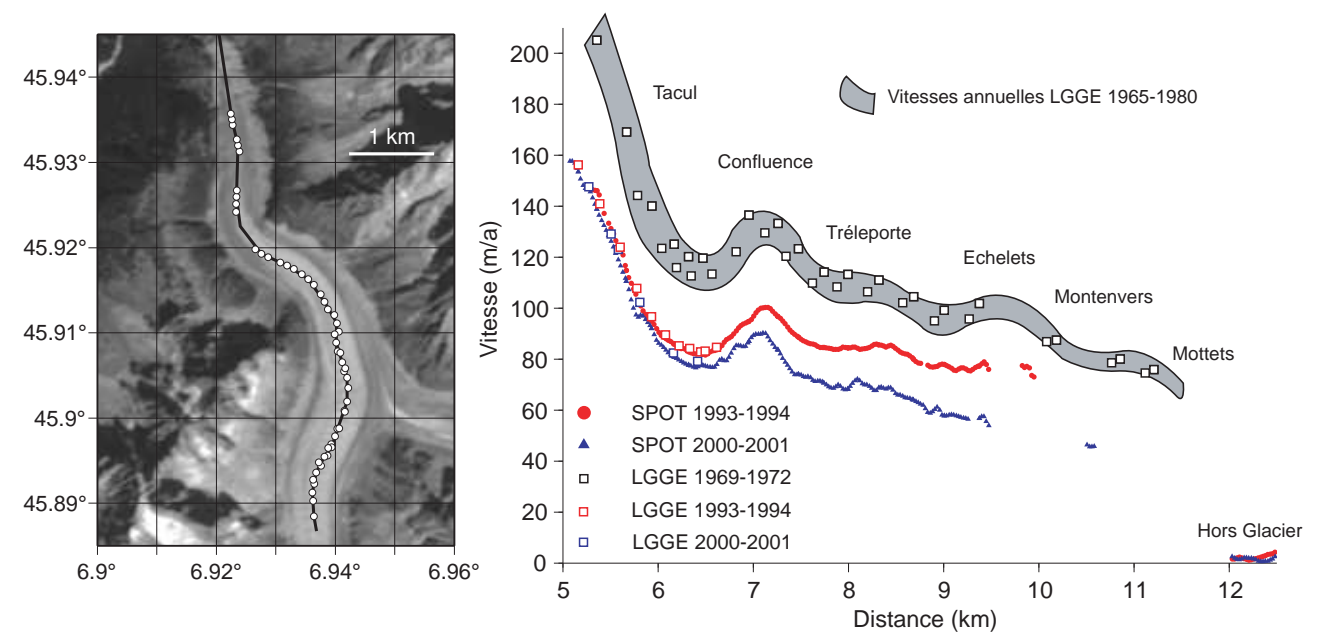

FIG. 4.28 - A gauche : localisation du profil longitudinal de la Mer de Glace passant par les balises (points blanc) de 1969 à 1971. A droite : vitesses annuelles le long de ce profil. Les vitesses déduites des couples d'images SPOT pour 1993-94 et 2000-01 sont comparés avec les vitesses annuelles mesurées par le LGGE et publiées par Lliboutry 6 Reynaud (1981).

Ce ralentissement, mesuré ici à l'échelle annuelle, rejoint celui observé sur les vitesses estivales de 1988, 1993 et 2003, mais peut difficilement s'expliquer par un changement du glissement basal. Ceci est corroboré par l'observation de la zone la plus rapide de notre profil ( $\mathrm{km} 5$ à 6$)$ où la vitesse n'a presque pas varié entre 1993-94 et 2000-01. Or, nous avons montré que cette zone (au pied de la chute de séracs du Géant) est sensible à des changements du glissement basal (cf. figure 4.19 \& figure 4.26). C'est donc en terme de variations de la déformation interne que nous interprétons ce ralentissement de la Mer de Glace. Mais pour tester cette hypothèse, des données complémentaires sur l'amincissement du glacier sont nécessaires et font l'objet du chapitre 5. Ensuite, la relation entre amincissement et ralentissement du glacier sera abordée dans la synthèse de cette thèse $(\S 7.2 .3)$. 


\subsection{Conclusions}

Dans ce chapitre, nous avons donc présenté une chaîne de traitement des images SPOT permettant de déterminer les déplacements en surface des glaciers. La précision de notre méthodologie est 5 fois meilleure $( \pm 0.2$ pixel) que celle atteinte par les études précédentes sur les glaciers. Si la géométrie d'acquisition des images est adaptée, une telle précision peut être obtenue même sans point de contrôle au sol et avec un MNT de basse résolution. Notre étude a également permis de détecter des biais qui peuvent affecter les résultats de la corrélation d'images et qui, à notre connaissance, n'avaient pas été mentionnés auparavant dans la littérature. Les changements de longueur et d'orientation des ombres sont un premier type de biais. Il a peu d'influence sur les glaciers. En revanche, les changements d'altitude de la surface du glacier, liés à l'ablation ou l'accumulation, peuvent biaiser la mesure des déplacements horizontaux.

L'acquisition de deux couples d'images SPOT5 au cours de l'été 2003, nous a permis de cartographier les variations de vitesses à court terme des principaux glaciers du massif du Mont-Blanc. Nous mettons en évidence une relation des zones où la vitesse varie avec la densité des crevasses. L'analyse des conditions météorologiques permet de proposer une interprétation hydrologique à ces variations de vitesse : l'intensité de la fonte en surface et le développement du réseau hydrologique sous-glaciaire agissent de concert pour contrôler le glissement basal du glacier. Une accélération des glaciers de montagne par lubrification basale tend à transporter de la masse vers les zones de fortes ablations, conduisant donc à des pertes de masse supérieures. La compréhension de tels mécanismes est importante car ils conditionnent en partie le bilan de masse de certaines calottes glaciaires (Zwally et al., 2002a). L'échantillonnage temporel de la mesure satellitaire (quelques semaines) reste insuffisant par rapport aux échelles de temps du phénomène (quelques heures à quelques jours). Le couplage de la vision globale obtenue par satellite avec des mesures continues in situ (DGPS ou corrélation de photographies terrestres quotidiennes) est prometteur pour décrire toutes les échelles spatio-temporelles des fluctuations des vitesses glaciaires.

Ces variations du glissement basal expliquent difficilement en revanche l'évolution des vitesses annuelles qui diminuent régulièrement depuis le début des années 1980 (pour la Mer de Glace tout au moins). Nous pensons que c'est la déformation interne qui varie à ces échelles de temps. Nous testerons cette hypothèse dans la synthèse (chapitre 7) après que les variations d'épaisseur des glaciers aient été déterminées par satellite également. Ce ralentissement des glaciers diminue les flux de glace vers les parties basses. Cela accentue les pertes d'épaisseurs des parties basses (rétroaction positive) mais préserve en revanche les parties hautes. On soulignera ici que les relevés de terrains réguliers du LGGE mesurent également (mais en quelques sites seulement) cette évolution à long terme.

Le schéma de la figure 4.1 peut alors être complété en ajoutant les mécanismes observés par l'imagerie satellitaire optique.

Les perspectives seront détaillées dans la conclusion générale mais elles concernent principalement :

1. Le passage à la modélisation pour aller plus loin dans la compréhension des phénomènes détectés dans ce travail. Nos mesures de vitesses constituent en effet une contrainte forte pour un modèle d'écoulement glaciaire (Guðmundsson, communication personnelle). On peut envisager ainsi de mieux contraindre les paramètres rhéologiques d'écoulement des glaciers pour, in fine, modéliser leur évolution future. 
2. Une analyse détaillée de la géométrie satellitaire permet d'espérer la mesure des 3 composantes des déplacements de la surface terrestre. Cette perspective est détaillée en annexe G. Si l'application aux glaciers semble délicate, d'autres déformations géophysiques pourront être cartographiées à 3 dimensions.

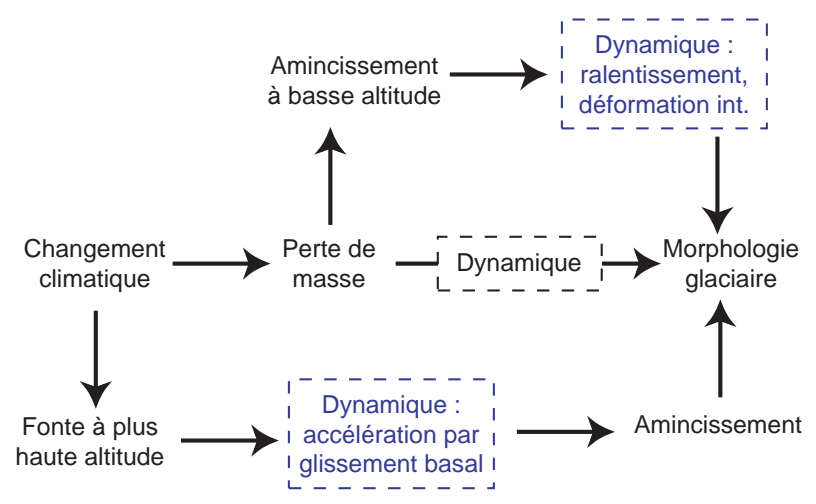

FIG. 4.29 - Le schéma de la figure 4.1 a été complété en ajoutant les processus mis en évidence en partie grâce à nos observations satellitaires. Seule la modélisation permettrait de tester l'importance relative de ces effets et de leurs rétroactions.

Nous l'avons déjà indiqué, notre méthodologie n'est pas seulement applicable à l'écoulement des glaciers. Dans le cadre de cette thèse, elle a aussi permis d'étudier d'autres phénomènes géophysiques "Terre solide". Ces résultats sont présentés dans deux annexes :

- annexe E : application de "ME DECOIS PAS" à la mesure de la déformation co-sismique (Izmit-Turquie, Al Hoceima-Maroc).

- annexe F : application de "ME DECOIS PAS" à la détection automatique de glissements de terrain (Alpes du Sud). 


\section{Chapitre 5}

\section{Satellite \& bilan de masse des glaciers}

\section{Sommaire}

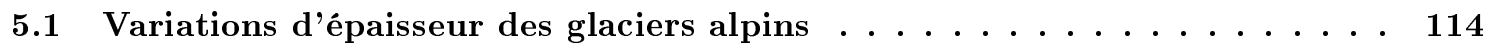

5.1.1 Construction de MNT des glaciers à partir d'images satellites . . . . . . . . . . . . 114

5.1 .2 Ajustements spatio-temporels des MNT . . . . . . . . . . . . . . . 116

5.1 .3 Extraction des variations moyennes d'épaisseur . . . . . . . . . . . . . . . . . . 119

5.1 .4 Précision de la mesure satellitaire . . . . . . . . . . . . . . . . . . . . 120

5.1.5 Evolution des variations d'épaisseur lors des 25 dernières années . . . . . . . . . 123

5.2 Vers l'estimation satellitaire du bilan de masse glaciaire . . . . . . . . . 124

5.2 .1 Comparaison des MNT aux profils topographiques . . . . . . . . . . . . . 124

5.2.2 Variations d'épaisseur des glaciers en 1979-1999 et 1999-2003 . . . . . . . . . . 128

5.2 .3 Bilan de masse des glaciers alpins entre 1979 et $2003 \ldots \ldots$. . . . . . . . . 131

5.3 Bilans de masse de 2 glaciers himalayens . . . . . . . . . . . . . 134

5.3 .1 La zone d'étude $:$ l'Himalaya indienne . . . . . . . . . . . . . . 134

5.3 .2 Génération d'un MNT SPOT5 sans point d'appui . . . . . . . . . . . . . . . . . . 135

5.3 .3 Déclin récent du Bara Shigri et Chhota Shigri . . . . . . . . . . . . . . . . . 136

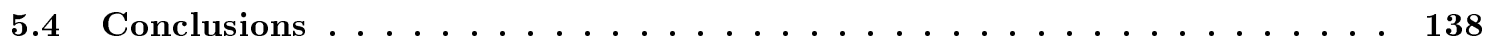

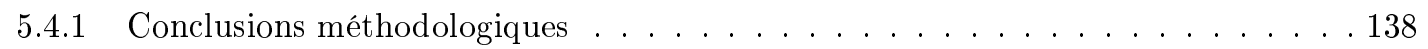

5.4 .2 Conclusions glaciologiques . . . . . . . . . . . . . . . . . . 139

Le chapitre 2 nous a montré que le bilan de masse est le paramètre qui caractérise le mieux l'état de santé du glacier. En effet, les changements annuels des précipitations ou de l'ablation se traduisent directement par une perte ou un gain de masse du glacier (Vincent, 2002). Cela tranche avec les avancées ou reculs des fronts glaciaires qui se font avec un délai, variable selon les glaciers (Jóhannesson et al., 1989). Le bilan de masse est aussi un paramètre clef pour quantifier la contribution des glaciers à l'élévation du niveau marin (Meier, 1984).

Mais les mesures de terrain sont délicates et coûteuses et seulement 300 glaciers environ sont, ou ont vu, leurs bilans mesurés in situ (Dyurgerov, 2003). L'outil "satellite" semble le plus adapté pour étendre la mesure de ce paramètre à des glaciers plus nombreux. L'objectif est donc de démontrer que le bilan de masse des glaciers peut être mesuré depuis l'espace. Une première technique satellitaire consiste à suivre l'altitude de la ligne de neige à la fin de période d'ablation (figure 3.10). Mais, nous avons vu qu'il était difficile d'extrapoler cette mesure sur d'autres glaciers que ceux déjà étudiés in situ. Nous proposons donc d'appliquer à des images satellites la méthode géodétique fondée sur la différence de deux topographies (§2.1.3.4). 
Cette méthode n'a jamais été appliquée à des images satellites. Jusqu'à récemment, cela semblait même impossible à certains auteurs. Ostrem \& Haakensen (1999) affirment "... but for the calculation of change in volume a much higher accuracy is required in surface elevation determination than can be obtained from space observation" et Kääb (2002) estime lui que "glacier thickness change, usually in the order of $m$ year $^{-1}$ (...) can hardly be detected from repeated ASTER DEMs". Nous allons voir comment une méthode originale, appliquée à des images de suffisamment haute résolution permet de mesurer des variations d'épaisseur avec une précision métrique, précision compatible avec un suivi des glaciers de montagnes tous les 3 à 5 ans. Notre étude centrée sur la Mer de Glace a donné suite à une publication présentée en annexe B (Berthier et al., 2004).

Nous détaillerons donc dans ce chapitre notre méthodologie pour extraire les variations d'épaisseur des glaciers alpins (§5.1). Nous discuterons ensuite la conversion des variations de volume en changement de masse glaciaire et proposerons une estimation du bilan de masse d'une partie des glaciers du Mont-Blanc pour la période 1979-2003 (§5.2). Les premiers résultats de notre méthodologie appliquée à des glaciers himalayens seront aussi présentés (§5.3) avant de conclure.

\subsection{Variations d'épaisseur des glaciers alpins}

Le principe de la méthode géodétique est simple : comparer deux topographies suffisamment précises des glaciers pour déterminer les variations d'épaisseur entre deux dates. Nous verrons donc comment ces topographies sont déduites d'images satellites, et comment elles sont ajustées à une topographie de référence. Nos variations d'épaisseur seront testées en les comparant à des profils transversaux mesurés sur le terrain et des photographies aériennes. L'évolution du taux d'amincissement de la Mer de Glace sera ensuite discutée et comparée aux variables météorologiques.

\subsubsection{Construction de MNT des glaciers à partir d'images satellites}

La construction de MNT à partir d'images satellites optiques est classique (Toutin, 2001). Nous avons donc utilisé un logiciel commercial PCI-Géomatica qui génère des MNT à partir de couples d'images. Le choix d'un logiciel existant a permis un gain de temps important (développer une chaîne de production de MNT nécessite plusieurs mois à plusieurs années) même si toutes les étapes ne sont pas contrôlées.

Les principales étapes de réalisation d'un MNT avec PCI-Géomatica à partir d'un couple d'images sont (Toutin \& Cheng, 2002) :

1. Orientation absolue des images (i.e. amélioration du modèle "stéréo" §4.3.1) grâce à des points de contrôle au sol (notés GCP). Des points homologues peuvent également améliorer l'orientation relative des deux images.

2. Transformation des images en géométrie épipolaire ${ }^{1}$

3. Corrélation des deux images épipolaires pour extraire la parallaxe (figure 5.1).

4. Conversion de la parallaxe en altitude à partir du modèle "stéréo".

\footnotetext{
${ }^{1}$ Géométrie identique est celle qui permet la vision stéréoscopique pour les photographies aériennes. Dans cette géométrie, deux détails homologues se trouveront sur la même ligne des images.
} 
5. Filtrage du MNT, notamment pour compléter les zones où la corrélation a échoué. Cette étape n'est pas utilisée car, pour estimer les variations d'épaisseur, nous préférerons l'absence de valeurs plutôt que des valeurs interpolées.

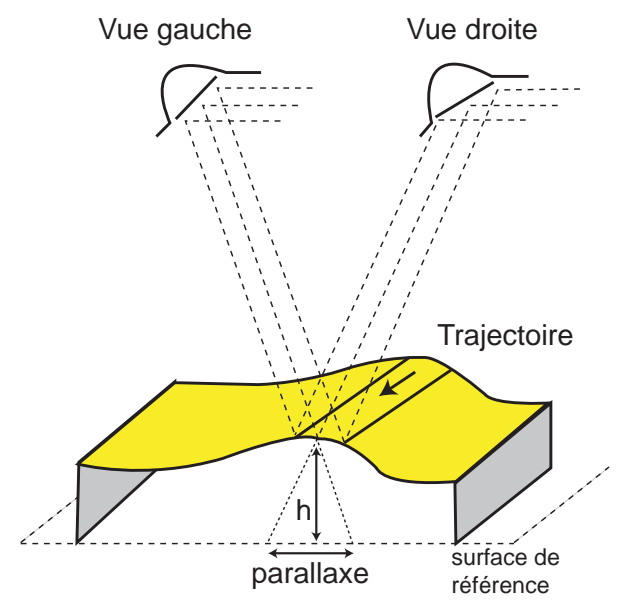

FiG. 5.1 - Observation d'un même point au sol (à une altitude h) depuis deux positions différentes par les satellites SPOT. La parallaxe mesure la distorsion entre les deux images et est directement liée à l'altitude (h) du point considéré. Adapté d'après CNES (1996).

Des erreurs sur le modèle "stéréo" vont affecter la conversion de la parallaxe en altitude et peuvent donc engendrer des biais (erreurs systématiques) dans le MNT. Des erreurs lors de la corrélation des images épipolaires augmentent le bruit du MNT mais ne doivent pas créer d'erreurs systématiques sur la topographie.

Les images de chacun des couples stéréoscopiques doivent répondre à des critères précis :

- Elles doivent être acquises de points de vue différents pour augmenter les distorsions, donc la sensibilité au relief (fort rapport B/H, voir §4.4.2). Dans les zones où le relief est modéré, un $\mathrm{B} / \mathrm{H}$ de 1 permet une bonne précision. Mais lorsque la topographie est abrupte, des distorsions trop fortes peuvent dégrader l'étape de corrélation. Un B/H de 0.6 à 0.8 est préférable en zone montagneuse.

- La séparation temporelle doit être courte. Ainsi, on limite les changements de l'éclairement solaire et de l'état de surface (moins de risque de chutes de neige par exemple). Une faible séparation temporelle limite également l'écoulement du glacier et évite donc un biais dans les MNT. L'idéal est d'utiliser des images acquises simultanément (capteurs HRS à bord de SPOT5 ou ASTER voir §3.2). Mais les images HRS ne sont pas disponibles pour les scientifiques, alors que la résolution et le contrôle orbital d'ASTER ne sont pas suffisants (Kääb, 2002). Ce sont les capacités de dépointage de SPOT1-5 (stéréoscopie latérale depuis des orbites différentes) que nous mettons à profit dans cette thèse.

- Enfin, comme dans le cas de la mesure des vitesses d'écoulement, un gain faible est important pour optimiser le contraste radiométrique sur toutes les zones du glacier.

Le tableau 5.1 décrit les images utilisées dans cette étude. On notera que seul le couple d'images SPOT5 de 2003 présente un gain de 1.

La disponibilité de GCP précis semble a priori indispensable pour la réalisation du MNT (nous verrons au $§ 5.3$ que le bon contrôle orbital de SPOT5 permet de s'en passer). Des campagnes DGPS ont donc été réalisées en août, septembre et octobre 2003 (cf. §4.4.2). Chaque MNT SPOT est réalisé avec le même jeu de GCP pour assurer une bonne cohérence entre les 
différentes topographies.

\begin{tabular}{lccccc}
\hline Date & Capteur & Résolution $(\mathrm{m})$ & Angle d'incidence & $\mathrm{B} / \mathrm{H}$ & Gain \\
\hline $1994-10-16$ & SPOT3 & 10 & -4.6 & 0.5 & 7 \\
$1994-10-17$ & SPOT3 & 10 & -30.3 & & 8 \\
\hline $2000-08-25$ & SPOT2 & 10 & -22.3 & 0.79 & 6 \\
$2000-08-25$ & SPOT1 & 10 & 20.9 & & 7 \\
\hline $2000-08-29$ & SPOT4 & 10 & -28.3 & 0.71 & 3 \\
$2000-08-29$ & SPOT2 & 10 & +9.7 & & 7 \\
\hline $2003-08-19$ & SPOT5 & 2.5 & -15.2 & 0.60 & 1 \\
$2003-08-23$ & SPOT5 & 2.5 & +15.7 & & 1 \\
\hline
\end{tabular}

TAB. 5.1 - Images SPOT utilisées pour construire des topographies multi-dates des glaciers du massif du Mont-Blanc.

La figure 5.2 compare les MNT calculés en 2000 et 2003. La différence la plus frappante concerne les zones à haute altitude, beaucoup mieux couvertes en 2003. Un résultat important est que, si la séparation temporelle et le gain sont faibles, la topographie est calculée dans la zone d'accumulation. Sur cette figure, deux autres MNT sont aussi présentés. Le premier a été obtenu auprès de l'IGN. Il a été établi à partir de couples de photographies aériennes datant de 1979 (Huon, IGN, communication personnelle). Le second, SRTM, a été construit lui par interférométrie SAR appliquée à des images acquises par la navette spatiale en février 2000 (Rabus et al., 2003).

\subsubsection{Ajustements spatio-temporels des MNT}

A ce stade, l'observation attentive des MNT montre des différences systématiques (biais) sur des zones hors des glaciers où aucun mouvement vertical n'est attendu. La correction de ces biais se fait en trois temps :

Pour éviter des décalages horizontaux entre les MNT, un ajustement planimétrique est d'abord réalisé. Lorsque des orthoimages ${ }^{2}$ sont disponibles (cas des MNT déduits d'images SPOT) cet ajustement est obtenu à partir d'une corrélation des orthoimages. Les décalages à appliquer sont toujours très faibles, grâce à un jeu de GCP homogène. Dans le cas des MNT SRTM et IGN, il n'y a pas d'orthoimages associées. On corrèle donc des images générées par ombrage des MNT (images dites shaded relief) avec une orthoimage de référence (image SPOT5 de 2003).

A l'échelle de quelques années, il est raisonnable de supposer que les zones hors des glaciers ne connaissent pas de mouvements verticaux importants (les mouvements tectoniques sont de quelques $\mathrm{mm}$ /a dans cette zone). Leur altitude devrait donc être la même pour les différents MNT. Sur ces zones stables, les MNT sont comparés deux à deux. Les différences sont calculées pixel à pixel et les distributions estimées. Sur la figure 5.3, on compare deux MNT SPOT1-4 générés à 4 jours d'intervalle en août 2000 (tableau 5.1). Le biais global entre les deux MNT est faible $(2.5 \mathrm{~m})$. L'écart type $(23 \mathrm{~m})$ informe sur la précision de la différence. En revanche,

\footnotetext{
${ }^{2}$ Les orthoimages sont des images satellites orthorectifiées i.e. projetées au sol grâce au MNT
} 
lorsque on étudie indépendamment chaque niveau d'altitude (partie droite de la figure 5.3), il existe un biais en fonction de l'altitude. Il est important de corriger ce biais sachant que, par la suite, nous étudierons les variations d'épaisseur des glaciers également en fonction de l'altitude. Ce biais n'est pas définitivement pénalisant puisque ce ne sont pas les altitudes absolues mais les variations relatives d'altitude de la surface des glaciers que l'on recherche. Un ajustement altimétrique est donc nécessaire.

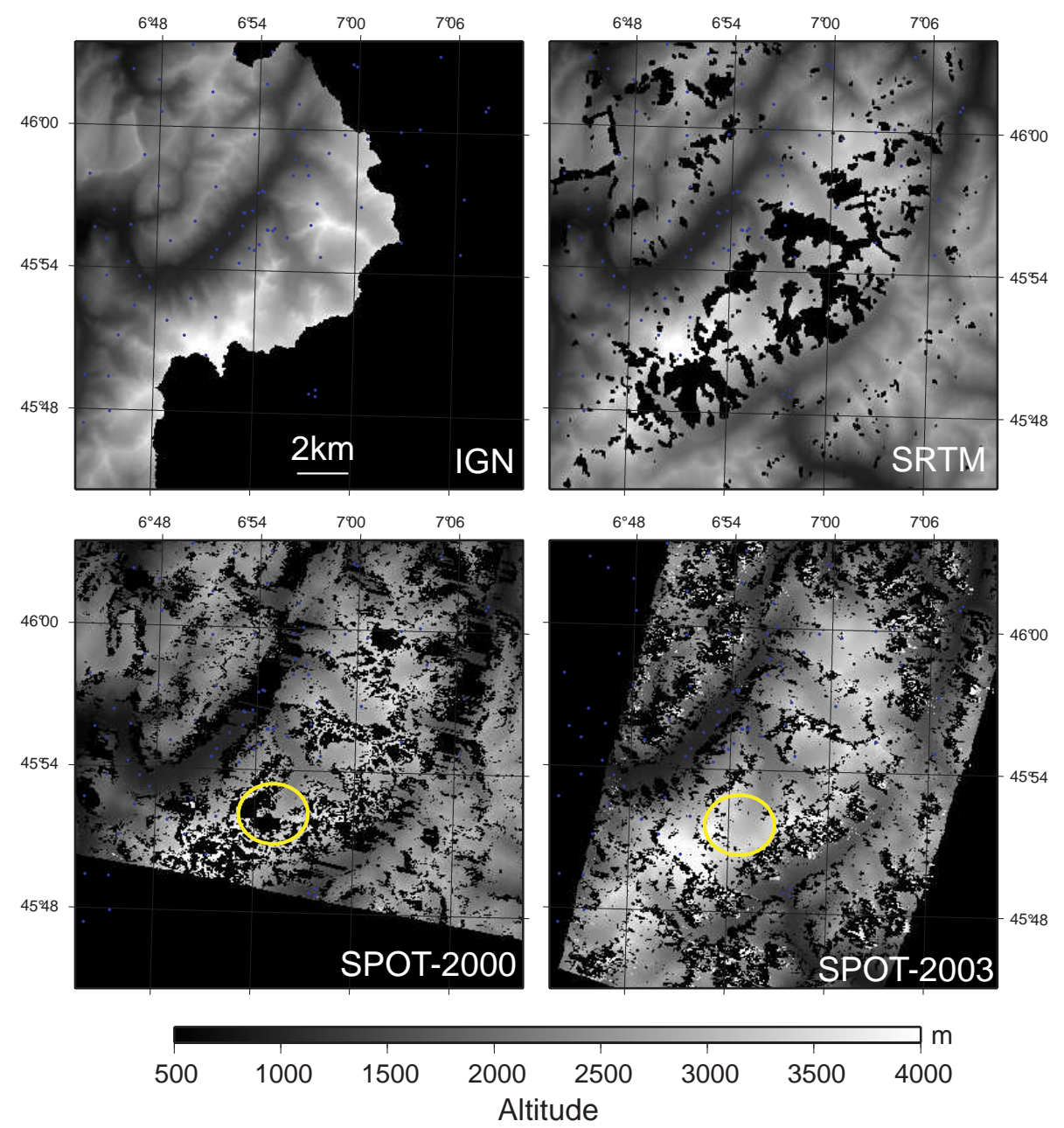

FIG. 5.2 - Quatre des MNT disponibles sur le massif du Mont-Blanc. En noir, les "trous" des MNT. La comparaison des deux MNT déduits d'images SPOT pour août 2000 et 2003 (en bas) met en évidence le rôle du gain faible des images qui permet d'obtenir une cartographie presque complète des zones d'accumulation (cercle jaune entourant le bassin d'accumulation de la Mer de Glace). Le MNT de l'IGN (1979, résolution $80 \mathrm{~m}$ ) ne couvre que la France, alors que le MNT SRTM (février 2000, résolution $90 \mathrm{~m}$ ) présente lui des trous aux hautes altitudes et sur les fortes pentes.

Ce biais altimétrique concerne essentiellement les MNT déduits des images SPOT1-4. Il est quasiment absent du MNT SPOT5 de 2003 (cf. §5.2). Nous n'avons pas d'explication définitive à ce biais qui dépend de l'altitude mais quasiment pas de la position géographique (latitude ou longitude). Il pourrait résulter de la conjonction de GCP qui ne couvrent pas toute la gamme des altitudes (notamment les plus hautes altitudes) avec les orbites de SPOT1-4 qui sont moins 
bien maîtrisées et connues.
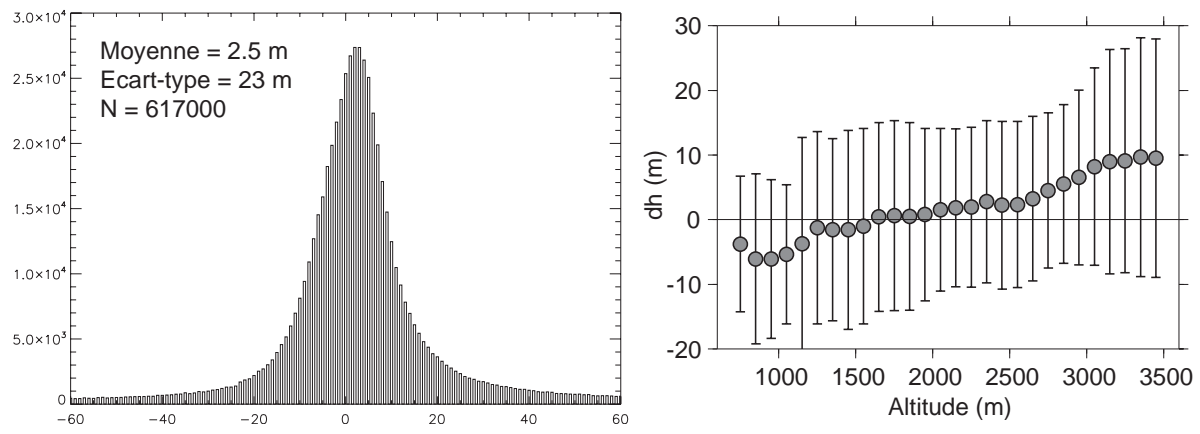

FIG. 5.3 - Différence de deux MNT SPOT1-4 générés à 4 jours d'intervalle fin août 2000. En 4 jours, on n'attend pas de variation significative d'épaisseur sur les glaciers. A gauche, la distribution des différences des deux MNT. A droite, ces différences ont été calculées pour chaque tranche d'altitude et indiquent un biais en fonction de l'altitude.

Afin de corriger ce biais, une première méthode a consisté à extraire des images et du MNT SPOT5 (2003) des GCP plus nombreux et couvrant toute la gamme des altitudes pour générer les autres MNT. Ceci permet par exemple d'extraire des GCP sur les pointements rocheux à haute altitude. Mais les résultats ont été peu probants. La seconde méthodologie (plus empirique) consiste à estimer ce biais hors des glaciers par rapport au MNT de 2003 (choisi comme référence), à le modéliser en utilisant un polynôme (de degré 4 ici) puis à le corriger dans tout le MNT. La figure 5.4 illustre la correction du biais du MNT d'août 2000.

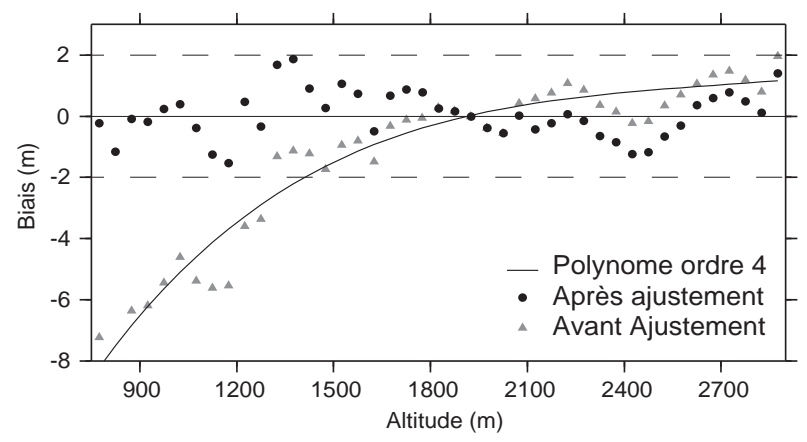

FIG. 5.4 - Biais du MNT du 25 août 2000 par rapport au MNT de 2003 en fonction de l'altitude sur les zones stables autour des glaciers (triangles gris). La courbe montre la modélisation de ce biais par un polynôme de degré 4, les ronds noirs le biais résiduel après correction.

Une dernière correction permet de prendre en compte les différences de date entre les MNT (figure 5.5). Au cours de l'été, l'ablation peut être très forte (4 à $5 \mathrm{~m}$ à basse altitude sur la Mer de Glace par exemple) et biaiser nos comparaisons. Il convient donc de corriger cet effet pour ramener les MNT à une date commune, le 15 septembre (date moyenne des relevés de terrain). On souhaite en effet comparer des cartes topographiques établies au voisinage de la fin de la saison d'ablation (Krimmel, 1999). 

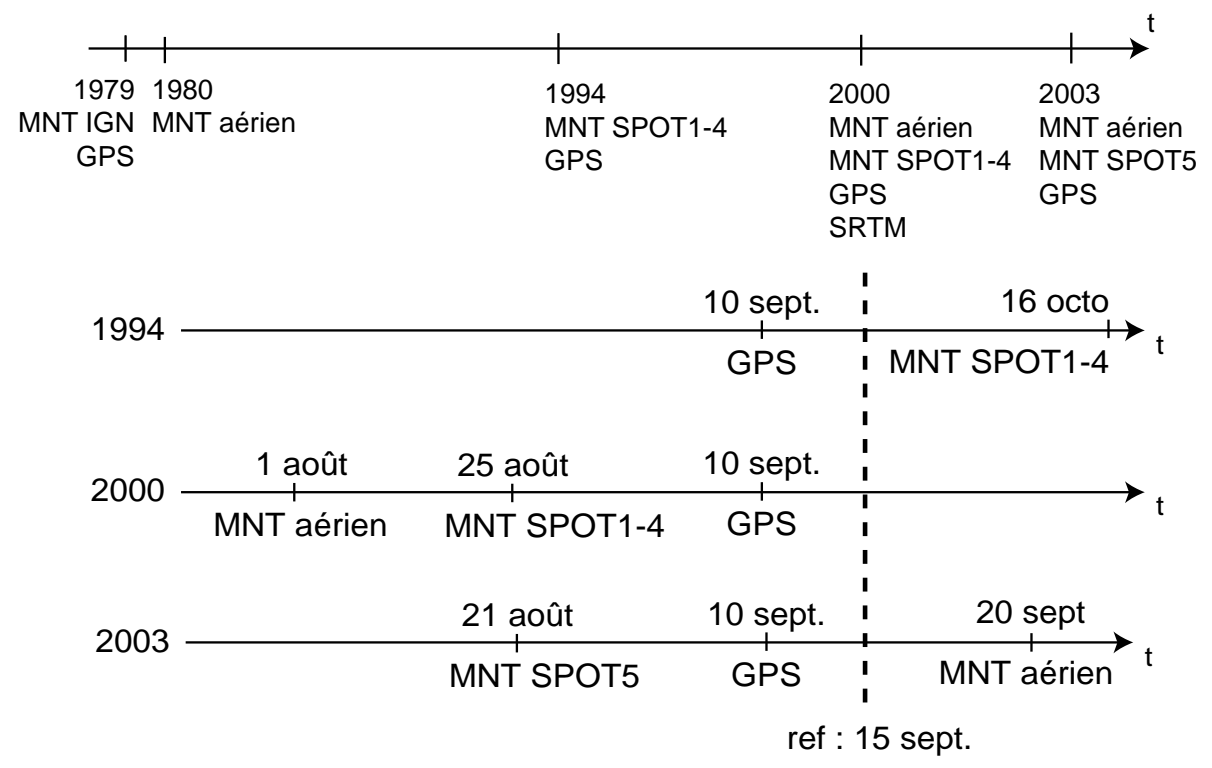

FiG. 5.5 - Comparaison des différentes années (en haut) et dates (en bas) d'acquisition des données satellitaires, aériennes et in situ (notées GPS même si elles incluent des mesures antérieures à l'avènement du GPS) pour le massif du Mont-Blanc. Pour être comparables, toutes les données sont ajustées au 15 septembre de chaque année.

Cet ajustement temporel est réalisé en estimant l'ablation grâce à un modèle degré jour (voir Vincent (2002) et §4.4.4.2). Si les données sont acquises avant (respectivement après) le 15 septembre, l'ablation déduite des températures à Chamonix est soustraite (resp. ajoutée) aux altitudes sur le glacier. Pour éviter les incertitudes liées à cet ajustement (qui restent inférieures à $\pm 0.5 \mathrm{~m}$ ), les images satellites doivent donc être acquises, si possible, à la fin de la période d'ablation (mais avant les premières chutes de neige).

Après ces ajustements planimétrique, altimétrique et temporel, les MNT peuvent être comparés deux à deux sur les glaciers pour extraire les variations d'épaisseur. La différence de deux MNT est nommée par la suite MNT différentiel.

\subsubsection{Extraction des variations moyennes d'épaisseur}

La précision des MNT satellitaires est en général de l'ordre de la taille du pixel, soit $10 \mathrm{~m}$ pour SPOT1-4 et $2.5 \mathrm{~m}$ pour SPOT5. Cette précision n'est pas assez bonne pour mesurer les changements d'épaisseur pour chaque pixel. L'incertitude serait alors de $\sqrt{10^{2}+10^{2}}$ soit $\pm 14 \mathrm{~m}$ (images SPOT1-4). Pour limiter cette incertitude, les changements d'épaisseur sont calculés en faisant l'hypothèse qu'ils sont homogènes au sein d'une tranche d'altitude.

Cette hypothèse semble raisonnable en zone d'ablation où le facteur qui contrôle principalement le bilan de masse, la température, varie essentiellement avec l'altitude. En zone d'accumulation, les hétérogénéités des précipitations, la redistribution de la neige par le vent et l'accumulation par les avalanches rendent cette hypothèse plus discutable. Notons ici que cette même hypothèse est souvent faite lors de l'estimation des bilans de masse par la méthode glaciologique, toutes les observations au sein d'une tranche d'altitude étant moyennées.

Pour chaque tranche d'altitude, un filtrage de type gaussien est appliqué. La partie centrale 
de la figure 5.6 montre ce filtrage dans la cas de la Mer de Glace pour la tranche d'altitude 2000-2050 m. L'histogramme des variations d'épaisseur montre une distribution quasi gaussienne (courbe bleue). La dispersion de notre distribution résulte à la fois du bruit des MNT mais aussi de variations d'épaisseur non nécessairement homogènes au sein d'une tranche d'altitude. Le changement moyen d'épaisseur est déterminé comme la moyenne de toutes les valeurs distantes de moins de trois écart-types du mode de la gaussienne. L'intérêt de ce filtrage est d'éliminer des valeurs aberrantes qui pourraient biaiser notre estimation des changements d'épaisseur.

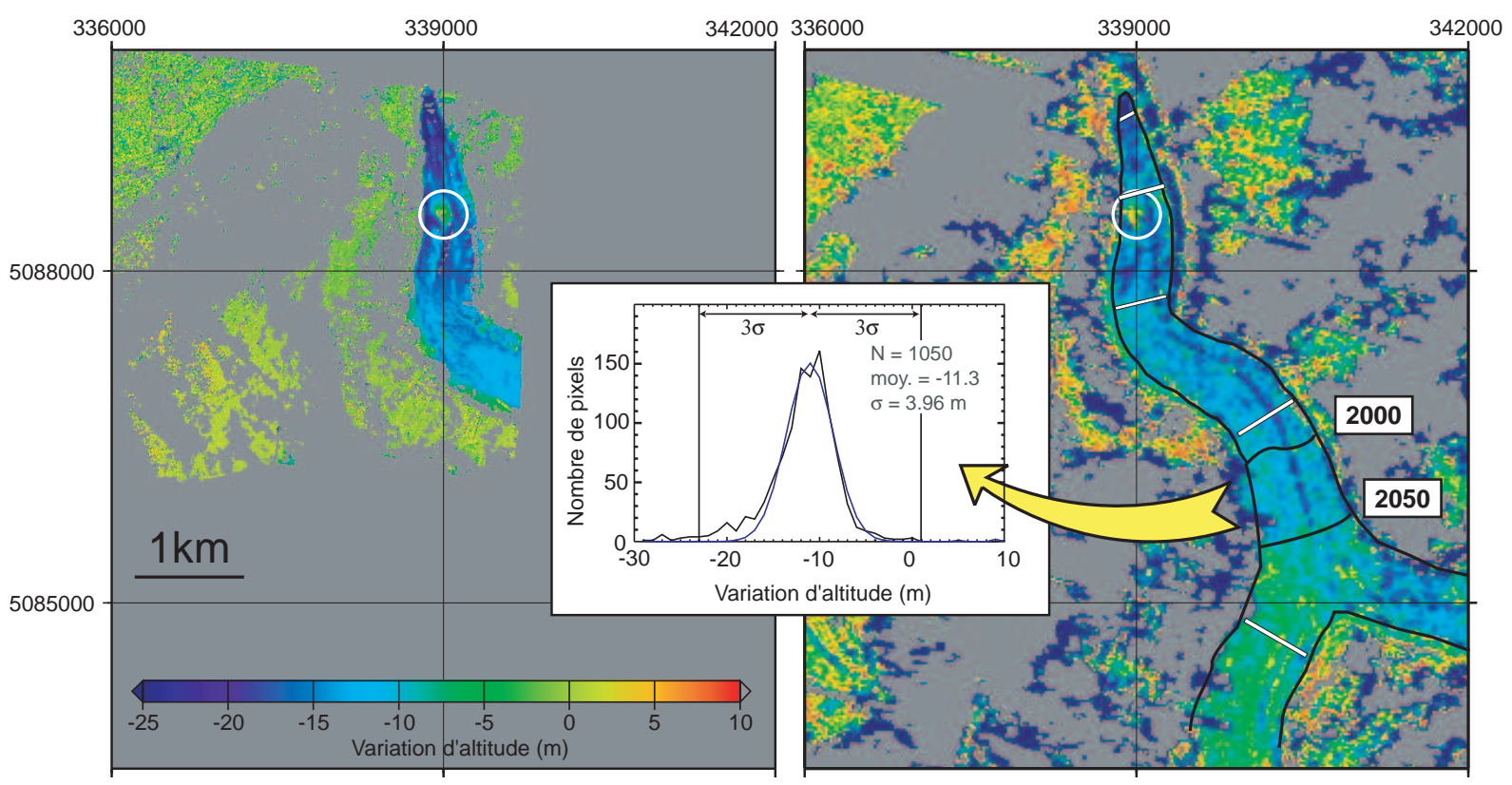

FIG. 5.6 - MNT différentiels pour la période 2000-2003. A gauche, le MNT différentiel est déduit de photographies aériennes, à droite, d'images SPOT. L'échelle de couleur est la même pour les deux MNT. Les profils topographiques, réalisés chaque année sur la Mer de Glace, sont localisés. Un cercle blanc entoure une zone du glacier où des pierres limitent l'ablation. Au centre, l'histogramme des variations d'épaisseur pour une tranche d'altitude et la gaussienne (en bleu) qui s'approche le plus de cet histogramme.

\subsubsection{Précision de la mesure satellitaire}

Les MNT satellitaires étant réputés insuffisamment précis pour déterminer les variations d'épaisseur des glaciers, une attention toute particulière doit être portée à la validation par des mesures indépendantes et plus précises : MNT différentiels aériens et profils topographiques.

\subsubsection{Comparaison aux MNT différentiels aériens}

En 2000 et 2003, des campagnes de l'IGN ont permis l'acquisition de couples stéréoscopiques de photographies aériennes sur la Mer de Glace. Nous avons traité ces couples par photogrammétrie numérique (avec le logiciel PCI-Géomatica également) pour déduire des MNT précis de la partie basse du glacier. On peut comparer ainsi des MNT différentiels aériens et satellitaires pour la période 2000-2003 (figure 5.6). 
Le bruit est plus fort pour les données satellitaires mais, dans les deux MNT différentiels, il existe un signal clair d'amincissement du glacier. L'amplitude est voisine (entre 10 et $20 \mathrm{~m}$ en trois ans selon les zones) et la distribution spatiale similaire. Ainsi, une zone où l'ablation est réduite par la présence de pierres en surface du glacier se distingue clairement sur les deux cartes. La comparaison aux profils topographiques permet de quantifier la précision de la mesure satellitaire.

\subsubsection{Comparaison aux profils topographiques}

Chaque année, lors des mesures de fin d'année hydrologique, le LGGE relève l'altitude de profils transversaux : 5 en surface de la Mer de Glace et 4 pour le glacier d'Argentière (figure 5.7). Le DGPS remplace aujourd'hui les mesures topographiques traditionnelles. Il s'agit là d'un jeu de données précieux pour valider notre mesure satellitaire. L'altitude moyenne d'un profil est précise à $\pm 0.3 \mathrm{~m}$ (Vincent, communication personnelle).

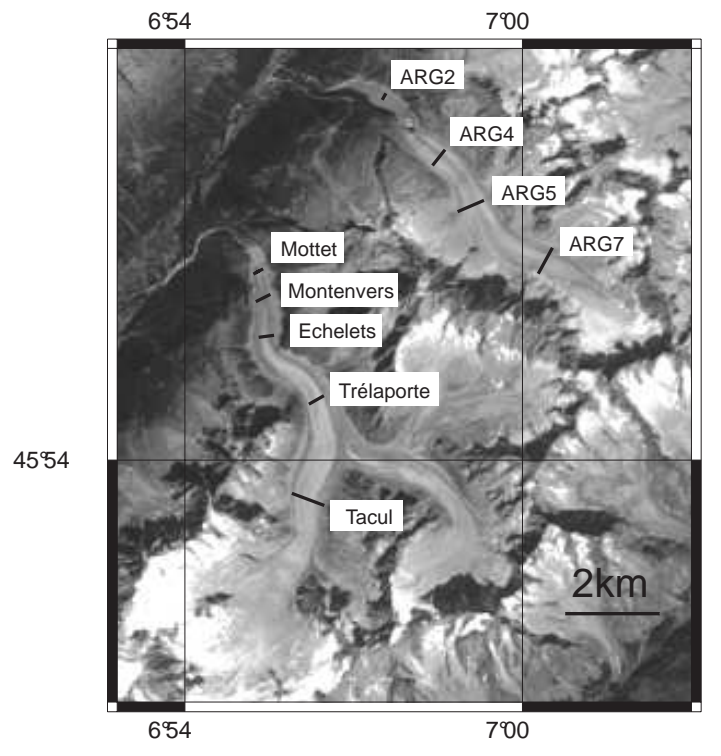

FIG. 5.7 - Localisation et noms des profils transversaux mesurés chaque année par le LGGE sur le glacier d'Argentière et la Mer de Glace.

La figure 5.8 compare ces relevés aux variations d'épaisseur déduites des images satellites par tranche de 50 m d'altitude. Deux périodes sont représentées : 1994-2000 et 2000-2003.

Un trait commun aux deux glaciers, Argentière et Mer de Glace, est le fort écart-type au sein de chaque tranche d'altitude dans les parties hautes du glacier (visible par les barres d'erreur). C'est le bruit important des MNT de 1994 et 2000 dans la zone d'accumulation (où les images sont souvent saturées) qui expliquent cette dispersion.

Pour la Mer de Glace, il y a un très bon accord entre mesure satellitaire et vérité terrain. Lorsqu'on extrait, par interpolation linéaire, la variation d'épaisseur satellitaire à l'altitude de chacun des profils topographiques, la différence moyenne est de $1.1 \mathrm{~m}$ (les pertes d'épaisseur sont légèrement sous-estimées par satellite) avec un écart type de $1.4 \mathrm{~m}(\mathrm{~N}=10)$. Au dessus de $2500 \mathrm{~m}$, il n'existe plus de vérité terrain pour tester nos mesures mais la comparaison des deux périodes montre des tendances opposées et une forte dispersion qui invitent à la prudence. Les 
bons résultats dans la partie basse du glacier où la pente est faible et le signal fort ne peuvent donc pas être extrapolés à la zone haute.
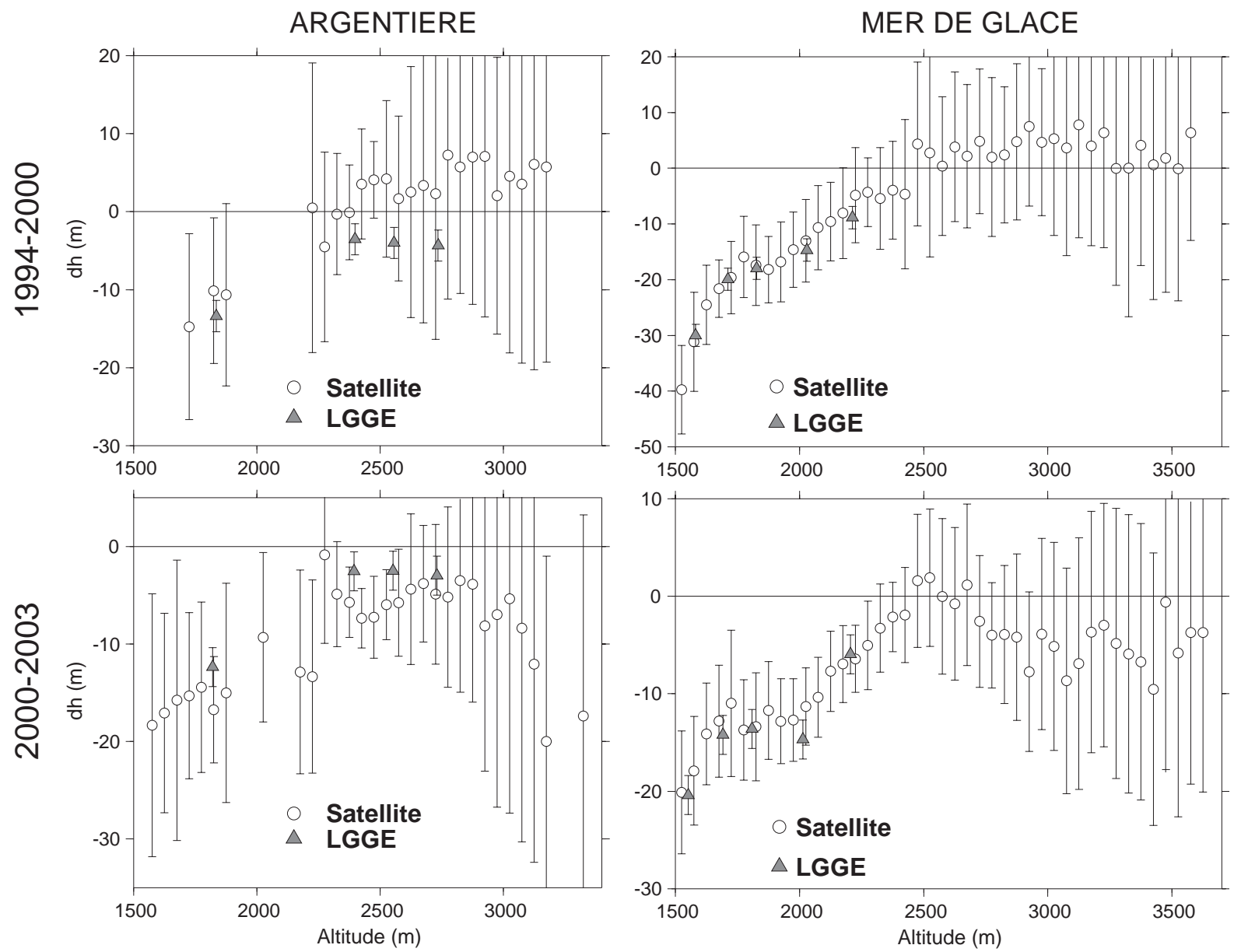

Fig. 5.8 - Variations d'épaisseur de la Mer de Glace et du glacier d'Argentière obtenues par l'imagerie satellitaire (cercle) et in situ (triangles) pour 1994-2000 (en haut) et 2000-2003 (en bas). Les incertitudes sont prises égales à l'écart type au sein de chaque tranche d'altitude pour les mesures satellitaires.

Les résultats sur le glacier d'Argentière confirment cette observation. Le profil à $1800 \mathrm{~m}$ montre des résultats satisfaisants. Entre 1900 et 2400 m, dans la zone de la chute de séracs de Lognan, les mesures satellitaires sont absentes ou affectées par une forte incertitude. Pour les trois profils transversaux entre 2400 et $2700 \mathrm{~m}$, la mesure satellitaire s'écarte de la vérité terrain. En moyenne, la méthode satellitaire sous-estime à nouveau de $1.1 \mathrm{~m}$ les pertes d'épaisseur, l'écart type étant cette fois de $5 \mathrm{~m}(\mathrm{~N}=8)$. A haute altitude (au dessus de $2500 \mathrm{~m}$ environ), les valeurs satellitaires ne semblent pas fiables avec les mêmes tendances que pour la Mer de Glace: épaississement du glacier (de 5 m environ) entre 1994 et 2000, et amincissement (de 5 à $10 \mathrm{~m}$ ) entre 2000 et 2003.

Ces écarts à haute altitude pour les deux glaciers s'expliquent probablement par le principe même de l'ajustement altimétrique. Cet ajustement utilise en effet les zones non englacées pour estimer le biais entre les MNT. Or, ces zones sont absentes ou limitées à quelques pointements rocheux au voisinage des sommets. On est donc contraint à extrapoler le biais évalué à plus basse 
altitude ce qui est loin d'être satisfaisant.

\subsubsection{Evolution des variations d'épaisseur lors des 25 dernières années}

Dans la partie basse de la Mer de Glace, où les mesures satellitaires sont fiables, l'évolution temporelle du taux d'amincissement peut être étudiée (figure 5.9). Aux trois MNT satellitaires de 1994, 2000 et 2003, on ajoute le MNT de 1979 obtenu auprès de l'IGN³ .

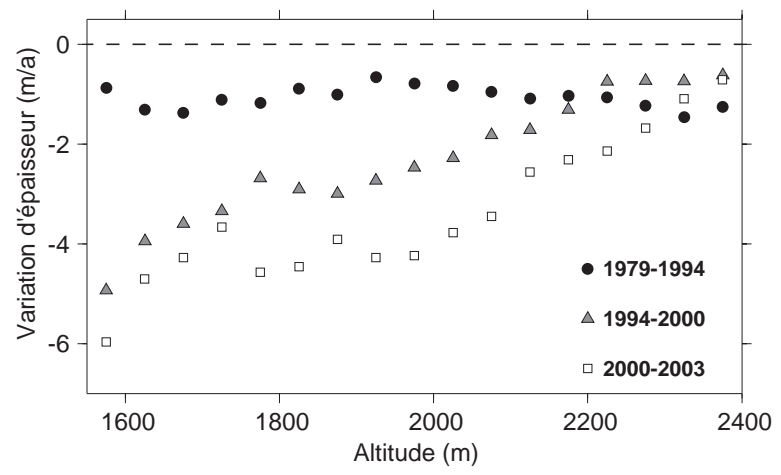

Fig. 5.9 - Taux moyen de variation d'épaisseur de la partie basse de la Mer de Glace pour 3 périodes distinctes : 1979-1994, 1994-2000 et 2000-2003.

Cette figure met clairement en évidence une accélération des pertes d'épaisseur dans cette zone lors des 24 dernières années. Ainsi, entre 1900 et 2200 m, la perte moyenne annuelle d'épaisseur est de $0.9 \mathrm{~m}$ entre 1979-1994, $2.1 \mathrm{~m}$ entre 1994-2000 et $3.4 \mathrm{~m}$ entre 2000-2003. L'augmentation de l'ablation locale peut-elle, à elle seule, expliquer cette évolution?

Une manière simple de tester cette hypothèse est d'utiliser le modèle degré-jour calibré à partir des observations de terrain (Vincent, 2002). Chaque degré positif à une altitude donnée engendre une ablation de $0.0067 \mathrm{~m}$ de glace par jour. Les températures moyennes journalières à Chamonix (à $1100 \mathrm{~m}$ ) permettent ainsi de déduire l'ablation moyenne annuelle entre 1900 et $2200 \mathrm{~m}$. Le tableau 5.2 montre que l'augmentation de l'ablation locale est insuffisante pour expliquer les fortes différences entre les périodes. Certes, notre modèle est rudimentaire mais ceci confirme que les variations locales d'épaisseur ne peuvent pas être reliées simplement aux fluctuations climatiques. Dans la conclusion de cette thèse, nous testerons si des changements de la dynamique peuvent rendre compte de cet amincissement.

\footnotetext{
${ }^{3}$ Après enquête auprès de l'IGN, 1979 serait l'année d'acquisition des photographies aériennes dont est issu ce MNT. Dans un mail daté du 3 Sep 2003, l'IGN nous informait que "le travail a été fait en 1981-82 à partir de photos au 1/60 000 de 1979". Une incertitude subsiste quant à cette date et les résultats pour la période 1979-1994 doivent être observés avec circonspection... Nous en reparlerons. Notons toutefois qu'une erreur d'une année ne change que de $6 \%$ le taux d'amincissement.
} 
TAB. 5.2 - L'augmentation des températures suffit-elle à expliquer l'évolution du taux d'amincissement au bas de la Mer de Glace? Pour trois périodes, les taux d'amincissement ( $\delta h)$ sont comparés à l'ablation annuelle déduite d'un modèle degré jour pour la zone 1900-2200 m. L'augmentation de l'ablation (de $1.2 \mathrm{~m} /$ a sur toute la période) n'explique que la moitié de l'accroissement des pertes d'épaisseur (de $2.5 \mathrm{~m} / \mathrm{a}$ ). Des statistiques complémentaires fournissent le nombre de jours par an où il y a ablation ainsi que l'ablation moyenne lorsqu'elle se produit.

\begin{tabular}{lccccc}
\hline & $1979-1994$ & $\rightarrow$ & $1994-2000$ & $\rightarrow$ & $2000-2003$ \\
\hline$\delta \mathrm{h}$ (m glace/an) & -0.89 & -1.16 & -2.05 & -1.38 & -3.43 \\
Ablation (m glace/an) & 9.35 & +0.28 & 9.63 & +0.92 & 10.55 \\
Jours d'ablation (/an) & 205 & & 218 & & 219 \\
Ablation moyenne (cm glace/jour) & 4.58 & & 4.39 & & 4.8 \\
\hline
\end{tabular}

Dans cette première partie, nous avons démontré qu'il est possible d'extraire d'images satellites des variations d'épaisseur des glaciers pour des zones où la pente n'est pas trop forte et le contraste radiométrique des images suffisant. Ces conditions sont remplies sur la langue de la Mer de Glace où l'amincissement est mesuré avec une précision de l'ordre de 1 à $2 \mathrm{~m}$. Cette précision n'est pas compatible avec un suivi annuel, le signal étant alors du même ordre de grandeur que les barres d'erreur. En revanche, un suivi tous les 3 à 5 ans est souhaitable. Parce que les MNT déduits d'images d'archives SPOT1-4 couvrent mal la zone d'accumulation des glaciers et présentent des biais, il n'est pas possible d'estimer le bilan de masse à partir de données purement satellitaires. En revanche, le MNT SPOT5 de 2003 peut être comparé aux MNT de l'IGN (1979) ou SRTM (février 2000) pour tester la conversion des variations d'épaisseur en bilan de masse et proposer la première mesure du bilan de masse de l'ensemble des glaciers du massif du Mont-Blanc.

\subsection{Vers l'estimation satellitaire du bilan de masse glaciaire}

Dans cette partie, un seul MNT satellitaire est utilisé (celui de 2003) et comparé à des topographies d'autres origines : la navette spatiale pour SRTM et des photographies aériennes pour le MNT de l'IGN. Mais la méthode présentée pourra prochainement être appliquée à des données purement satellitaires dès l'acquisition d'un autre MNT SPOT5 (programmée pour l'été 2005 ou 2006 par l'Observatoire des Glaciers http ://gge.obs.ujf-grenoble.fr/axes/glaciers//ServiceObs/). Comme le montre la figure 5.2, ces trois MNT couvrent (au moins partiellement) la zone d'accumulation. Ils doivent donc permettre de mesurer la variation de volume et le bilan de masse des différents glaciers du Mont-Blanc.

\subsubsection{Comparaison des MNT aux profils topographiques}

Pour évaluer leur précision, ces MNT sont comparés aux 9 profils transversaux mesurés chaque année par le LGGE (figure 5.10 et figure 5.11). 

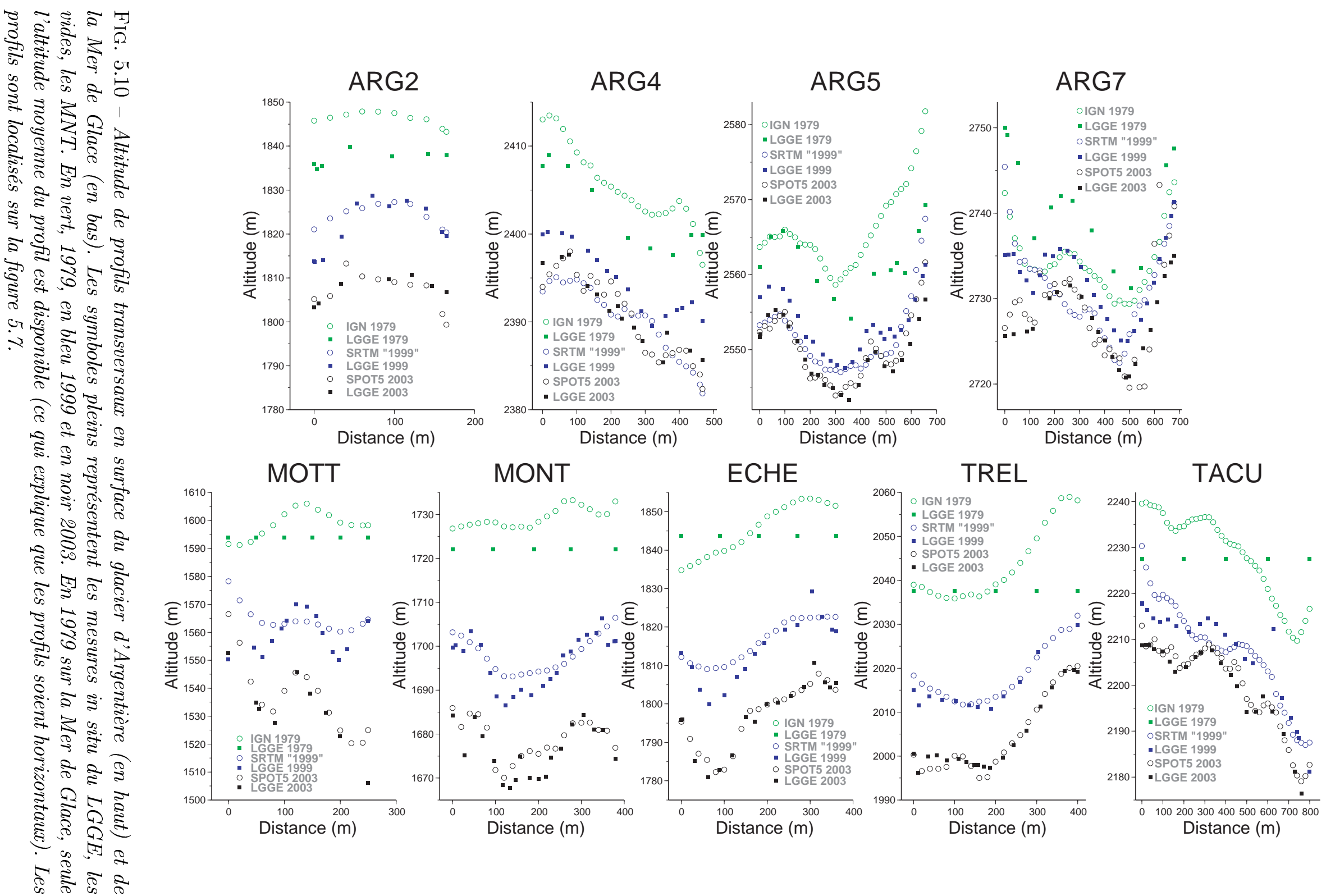


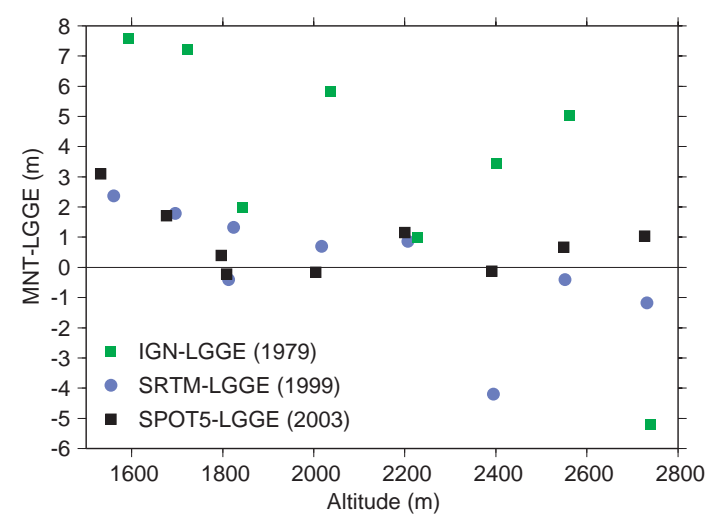

FIG. 5.11 - Différences des altitudes moyennes des profils transversaux sur les glaciers d'Argentière et de la Mer de Glace en fonction de l'altitude. En noir SPOT5 - LGGE [2003], en bleu SRTM - LGGE [1999] et en vert IGN - LGGE [1979].

On remarquera tout d'abord l'asymétrie de certains profils transversaux. La dénivellation peut atteindre $30 \mathrm{~m}$ (profil Tacul) d'un bord à l'autre du glacier. Cette asymétrie peut s'expliquer par la confluence de deux glaciers : ainsi le profil de Trélaporte en aval de la confluence entre les glaciers de Leschaux et du Géant est plus élevé en rive droite, dans la zone où la glace vient de Leschaux. On observera d'ailleurs que les pertes d'épaisseurs entre 2000 et 2003 ne sont pas homogènes le long de ce profil. Supérieures à $15 \mathrm{~m}$ en rive gauche, elle sont inférieures à $10 \mathrm{~m}$ en rive droite. L'explication de ces pertes différentes est double. Tout d'abord, la glace en rive droite, venant de Leschaux, est sale et couverte de débris ce qui la protège du rayonnement solaire. D'autre part, la dynamique différente des deux glaciers qui convergent (Leschaux est presque stagnant alors que le glacier du Géant s'écoule à près de $100 \mathrm{~m} / \mathrm{a}$ ) doit certainement jouer un rôle. Ces zones de convergence de plusieurs glaciers pourraient ainsi se révéler des sites privilégiés pour distinguer les contributions respectives de l'ablation locale et de la dynamique glaciaire aux variations d'épaisseur.

Les différences entre le MNT-IGN (1979) et les profils topographiques sont importantes, le MNT-IGN étant trop haut, principalement à basse altitude. Pour mieux caractériser ces différences, le MNT-IGN a été comparé aux altitudes de points mesurés par photogrammétrie aérienne en septembre 1980 sur le glacier d'Argentière (figure 5.12). La dispersion des valeurs est importante mais il n'existe pas de biais systématique du MNT à basse altitude. Au dessus de $2800 \mathrm{~m}$ (glacier des Améthystes notamment), le MNT-IGN est trop bas (une dizaine de mètres environ) sans que la différence temporelle (une année entre 1979 et 1980) n'explique un tel écart. On sait que la photogrammétrie aérienne fournie des résultats moins précis dans les zones hautes des glaciers. C'est peut-être l'explication de ces différences.

Profils et cartes confirment nos doutes concernant la précision et/ou l'année d'établissement du MNT-IGN. La mise à jour par l'IGN des topographies glaciaires n'est pas réalisée systématiquement et ne concerne parfois que certains glaciers seulement (Vincent, communication personnelle). Nous utiliserons toutefois ce MNT en gardant en mémoire cette incertitude temporelle $^{4}$. Ceci souligne l'importance de bien connaître la date d'établissement d'un MNT dans le

\footnotetext{
${ }^{4}$ Nous n'avons pas réussi à lever cette incertitude en comparant systématiquement ce MNT aux altitudes des profils transversaux mesurées chaque année. Une telle approche fonctionnerait (avec une incertitude de 1 à 2 ans) pour la période la plus récente car l'amincissement est important et monotone. Mais l'année 1980 correspond à un maximum relatif d'altitude des différents profils avec des variations faibles pour les années autour.
} 
cas des glaciers.

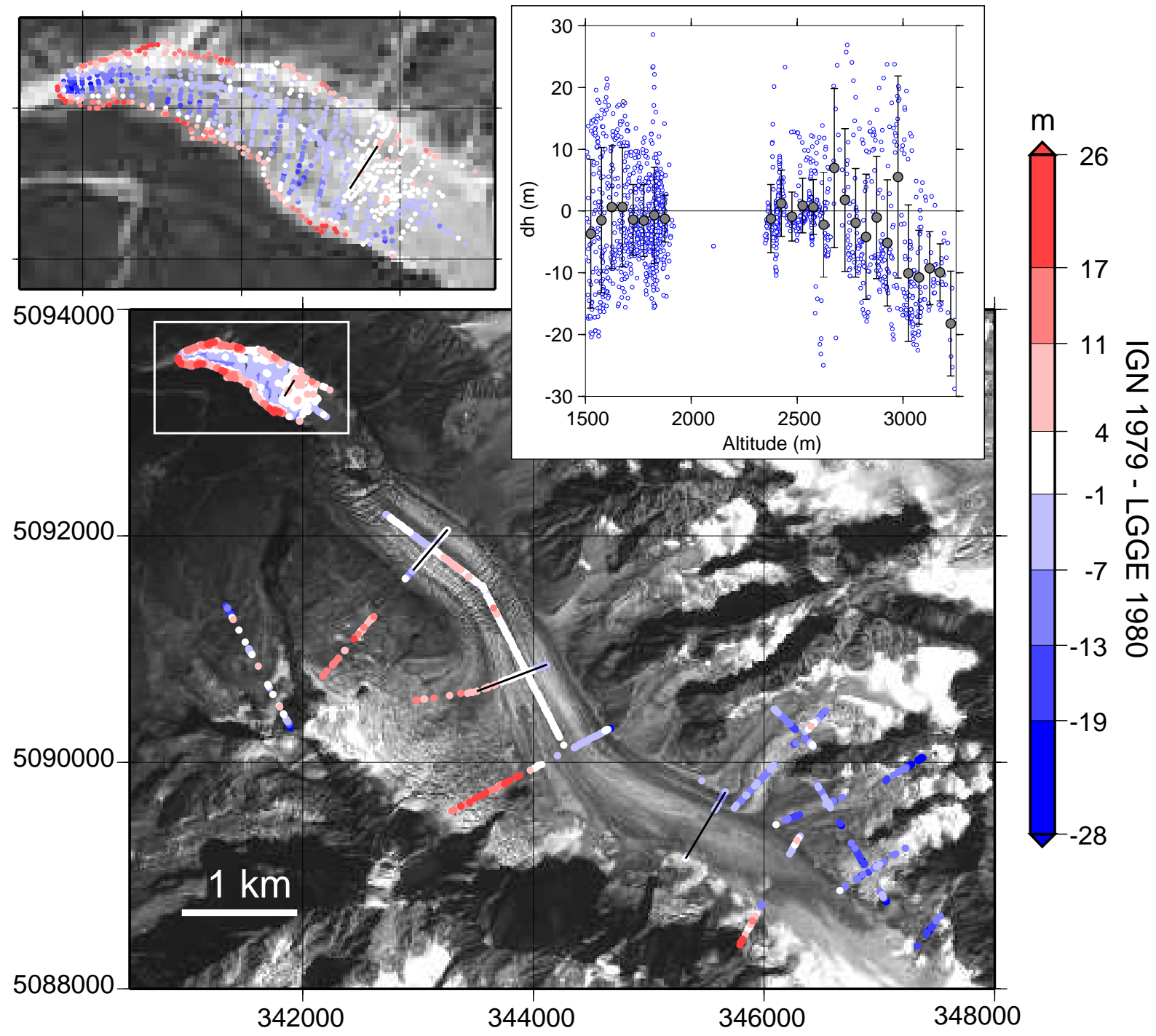

FIG. 5.12 - Différence des altitudes entre le MNT de l'IGN (1979) et des points mesurés sur un couple de photographies aériennes de 1980 (données LGGE). Pour chaque point, le code couleur représente les différences d'altitude. En rouge (respectivement en bleu), les zones où le MNT-IGN est plus haut (respectivement plus bas). Un détail de la partie basse du glacier est aussi montré en haut à gauche. En fond, image SPOT5 du 23 août 2003. En haut à droite, ces écarts sont représentés en fonction de l'altitude, les ronds gris étant les valeurs moyennes par tranche de $50 \mathrm{~m}$ d'altitude.

La précision du MNT SPOT5 est en revanche prometteuse. Lorsqu'on compare l'altitude moyenne des profils, SPOT5 est seulement $0.8 \mathrm{~m}$ plus haut (écart-type $=1.1 \mathrm{~m} ; \mathrm{N}=9$ ). En éliminant le profil "MOTTET" (différence de $3 \mathrm{~m}$ ), l'écart se réduit à $0.5 \mathrm{~m}$ (écart-type $=0.7 \mathrm{~m}$, $\mathrm{N}=8$ ). La figure 5.11 montre aussi que le MNT SPOT5 ne présente pas de biais avec l'altitude (les profils s'échelonnent entre 1800 et $2700 \mathrm{~m}$ ). 
Le MNT-SRTM est construit par InSAR à partir d'images acquises en bande $\mathrm{C}(\lambda=5.6 \mathrm{~cm})$ en février 2000 (Rabus et al., 2003). Ce MNT se situe 3 à $5 \mathrm{~m}$ au-dessus des profils GPS de septembre 2000 (non montré) mais voisin de ceux de septembre 1999 (figure 5.10). Pour comprendre cette observation, il faut s'interroger sur la pénétration de l'onde RADAR dans la couverture neigeuse de février. Weber Hoen \& Zebker (2000) estiment à près de $20 \mathrm{~m}$ cette pénétration pour la calotte groenlandaise. Rignot et al. (2001) ont montré que la pénétration en bande C était faible $(<1-2 \mathrm{~m}$ ) dans la glace mais plus forte dans la neige ou le névé froids (jusqu'à $10 \mathrm{~m}$ ). Cette longueur moyenne de pénétration doit donc être prise en compte lorsque SRTM cartographie des zones enneigées (contrairement à ce que disent Rignot et al. (2003)!). En zone d'ablation, nos comparaisons confirment que SRTM observe la topographie de septembre ou octobre 1999. Par la suite, nous considérerons donc que le MNT-SRTM correspond à la surface d'ablation de 1999. Toutefois, un léger biais avec l'altitude apparait (figure 5.11). Nous l'expliquons par le fait que l'onde RADAR pénètre la couche de neige hivernale à haute altitude (température basse et donc neige seiche) alors qu'à basse altitude l'onde ne pénètre pas (neige humide) donc la topographie SRTM est trop haute (figure 5.13). Ces biais pourraient également s'expliquer par l'utilisation d'une altitude d'ambiguïté ${ }^{5}$ constante lors de la conversion des franges interférométriques en dénivellations (Arnaud, communication personnelle). Ceci reste à vérifier. Une étude approfondie de ces biais de SRTM hors et sur les glaciers semble importante.

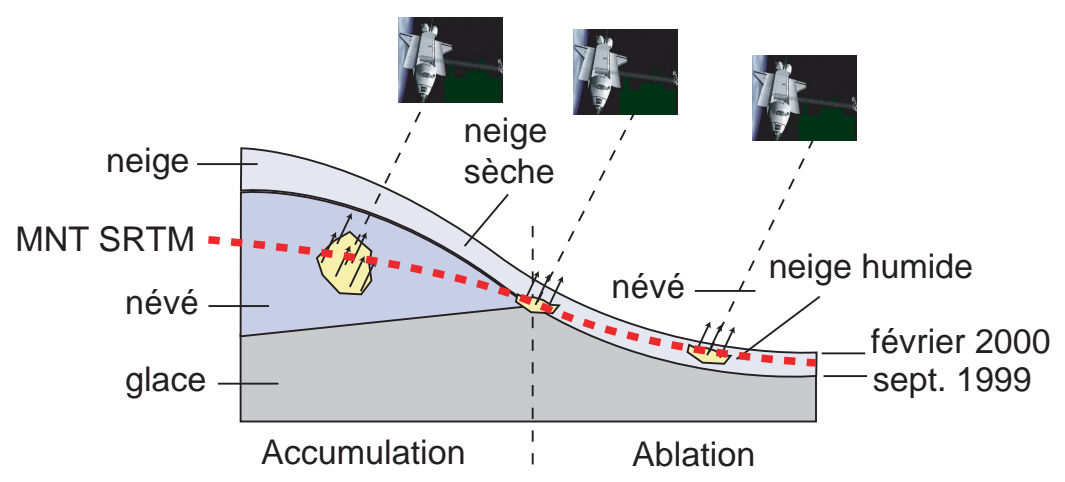

FIG. 5.13 - Pénétration du signal RADAR de SRTM (en bande C) selon les zones du glacier en présence d'une couche de neige frâ̂che. On notera que les transitions neige $\rightarrow$ névé et névé $\rightarrow$ glace ne sont pas aussi brutales en réalité.

\subsubsection{Variations d'épaisseur des glaciers en 1979-1999 et 1999-2003}

La figure 5.14 permet d'étudier les variations d'épaisseur de certains glaciers du massif du Mont-Blanc. Cette figure confirme le fort amincissement de tous ces glaciers à basse altitude, notamment lors de la période récente. A plus haute altitude, les variations d'épaisseur sont plus faibles.

Comme décrit précédemment, un filtre gaussien appliqué à chaque tranche d'altitude permet d'estimer les variations d'épaisseur en fonction de l'altitude en éliminant les valeurs aberrantes (figure 5.15).

\footnotetext{
${ }^{5}$ C'est la différence d'altitude au sol qui engendre un cycle de frange dans un interférogramme (Massonnet \& Feigl, 1998).
} 

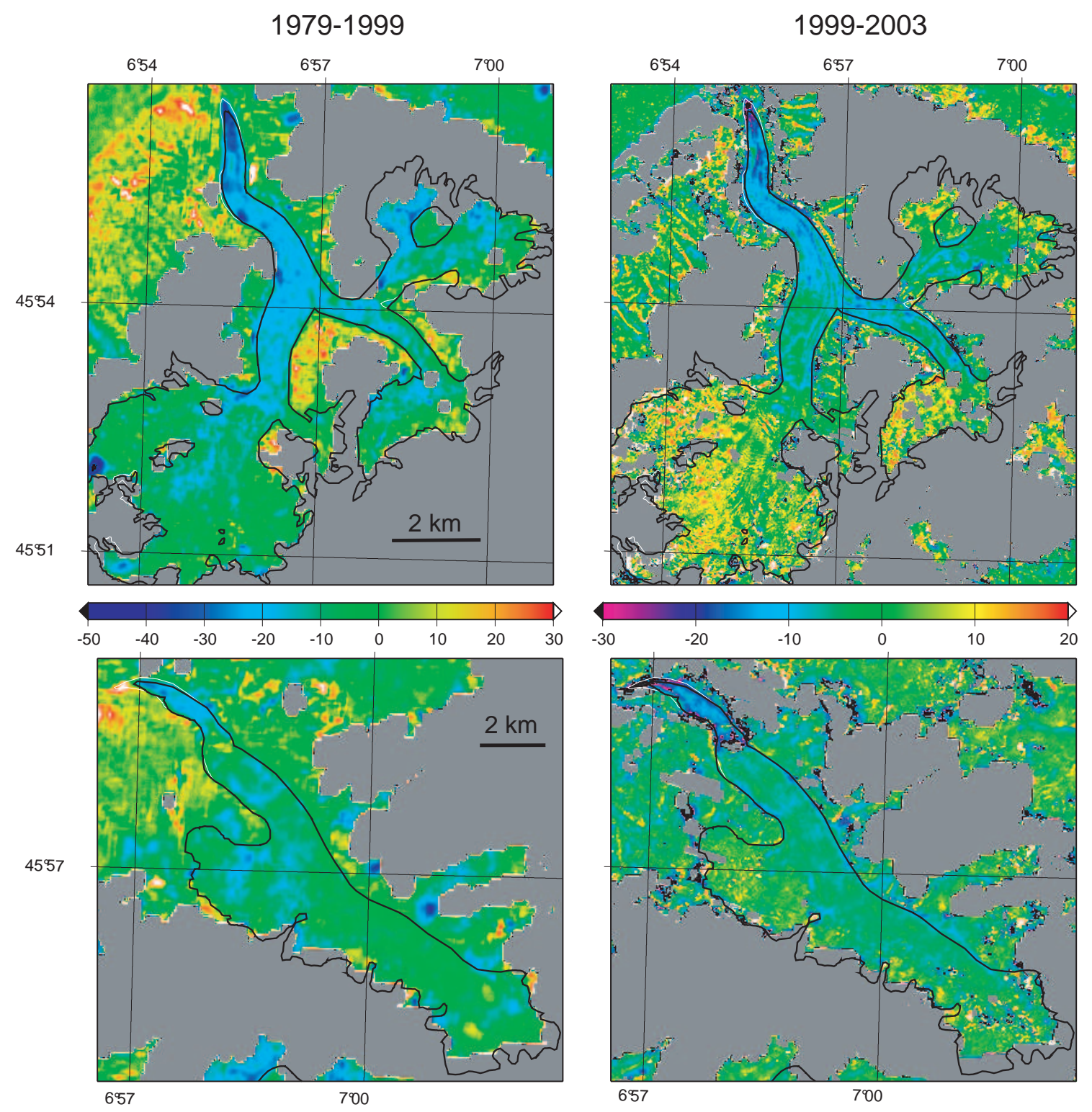

Fig. 5.14 - Cartes des variations d'épaisseur (en mètres) de la Mer de Glace et ses tributaires (en haut) et du glacier d'Argentière (en bas) pour la période 1979-1999 (à gauche) et 1999-2003 (à droite). Les échelles de couleur sont différentes pour les deux périodes. En noir, les limites des glaciers en août 2003 (en blanc les fronts glaciaires en 1988).

Si le signal à basse altitude est cohérent pour les différents glaciers et pour les deux périodes, les variations d'épaisseur au dessus de 2800-3000 m sont plus suspectes. En effet, la tendance est inverse avec un amincissement entre 1979 et 1999 ( 5 à $10 \mathrm{~m}$ ) et un épaississement du même ordre de grandeur entre 1999 et 2003. Les fluctuations des variables climatiques (températures et surtout précipitations) ne peuvent expliquer une telle inversion : une étude climatologique (Météo-France, 2005) soulignant, au contraire, une diminution marquée de l'enneigement des Alpes du Nord depuis la fin des années 1990. Nous suspectons un artefact de notre mesure. Il est logique, alors, de s'interroger sur la précision de la topographie commune aux deux périodes, le MNT SRTM de février 2000. Comme déjà décrit, dans les zones d'accumulation (au dessus de 
$3000 \mathrm{~m}$ ), la pénétration de l'écho radar jusqu'à parfois 10 ou 20 m pourrait expliquer un biais de ce MNT. La topographie SRTM se situerait alors en dessous de la surface réelle (figure 5.13), entraînant une erreur opposée sur les variations d'épaisseur pour les deux périodes (figure 5.16). Des tests montrent également des biais importants de SRTM par rapport aux autres topographies hors des glaciers. Il convient maintenant d'etudier ces biais plus en details. Quelle qu'en soit l'origine, cette observation est importante : les études glaciologiques utilisant SRTM se multiplient et sont probablement affectées par ce biais. Ainsi, l'estimation de la contribution des glaciers patagoniens à l'élévation du niveau marin de $0.1 \mathrm{~mm} /$ a entre 1995 et 2000 proposée par Rignot et al. (2003) pourrait être largement surestimée. Ceci invite à la prudence lors de l'utilisation de ce MNT, notamment en zone d'accumulation.
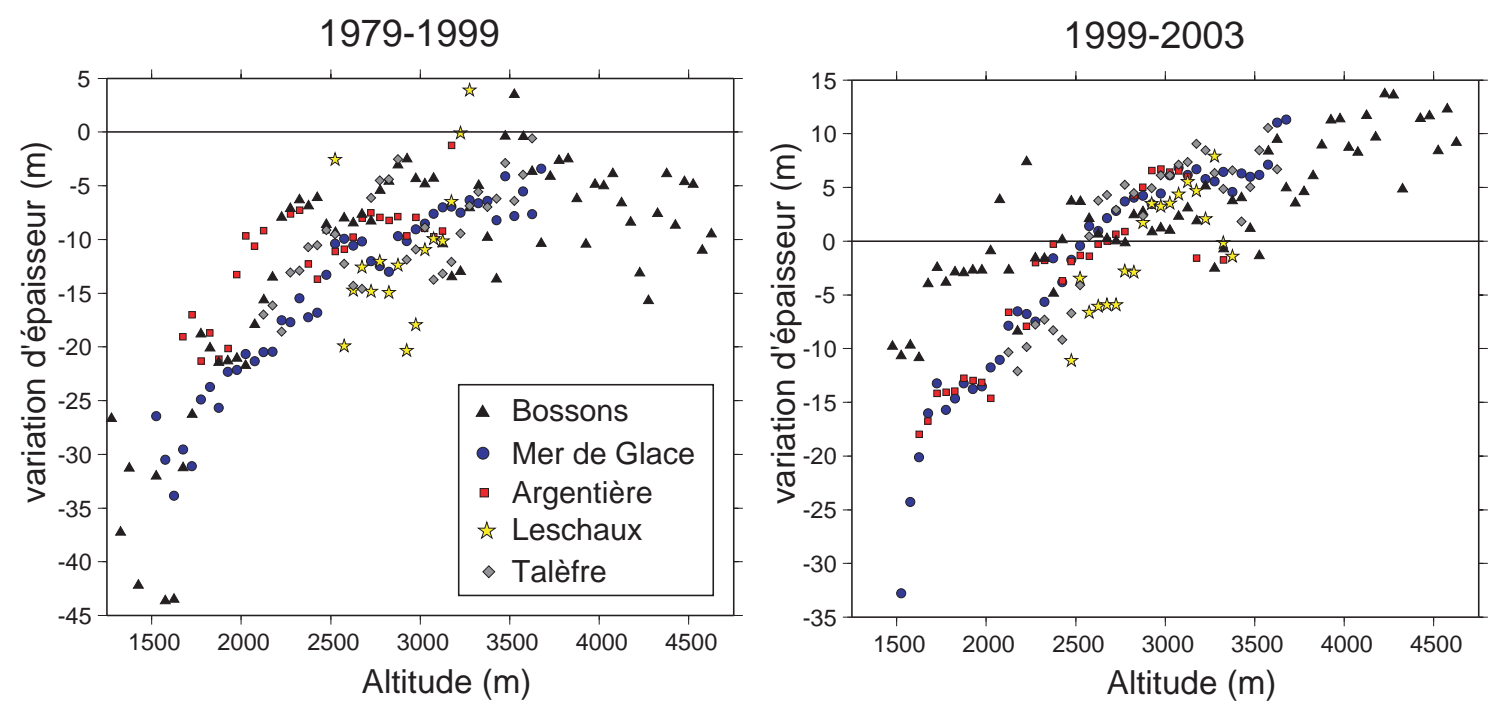

FIG. 5.15 - Variations d'épaisseur de quelques glaciers du massif du Mont-Blanc par tranche d'altitude pour la période 1979-1999 (à gauche) et 1999-2003 (à droite).

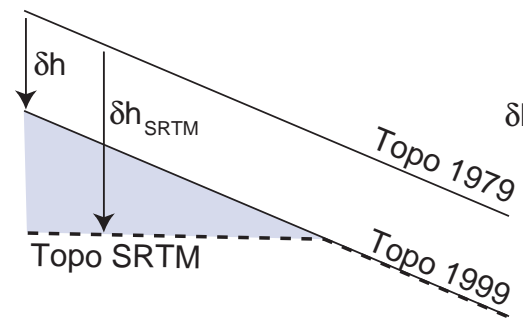

Sur-estimation perte de volume

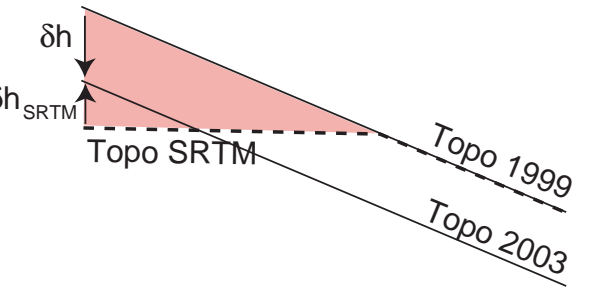

Sousestimation perte de volume

FIG. 5.16 - Implication des biais de MNT-SRTM sur les bilans de masse des glaciers. On se place dans la situation théorique où les pertes d'épaisseur sont homogènes avec l'altitude. A gauche, SRTM est le MNT le plus récent, à droite le plus ancien. La topographie réelle est en trait plein, celle mesurée par SRTM en tireté.

Compte tenu de ce probable biais de SRTM, nous nous contenterons par la suite de comparer le MNT SPOT5 au MNT de l'IGN (couvrant la France uniquement) pour estimer le bilan de masse de quelques glaciers du Mont-Blanc pour la période 1979-2003. 


\subsubsection{Bilan de masse des glaciers alpins entre 1979 et 2003}

La figure 5.17 montre le MNT différentiel pour la période 1979-2003.

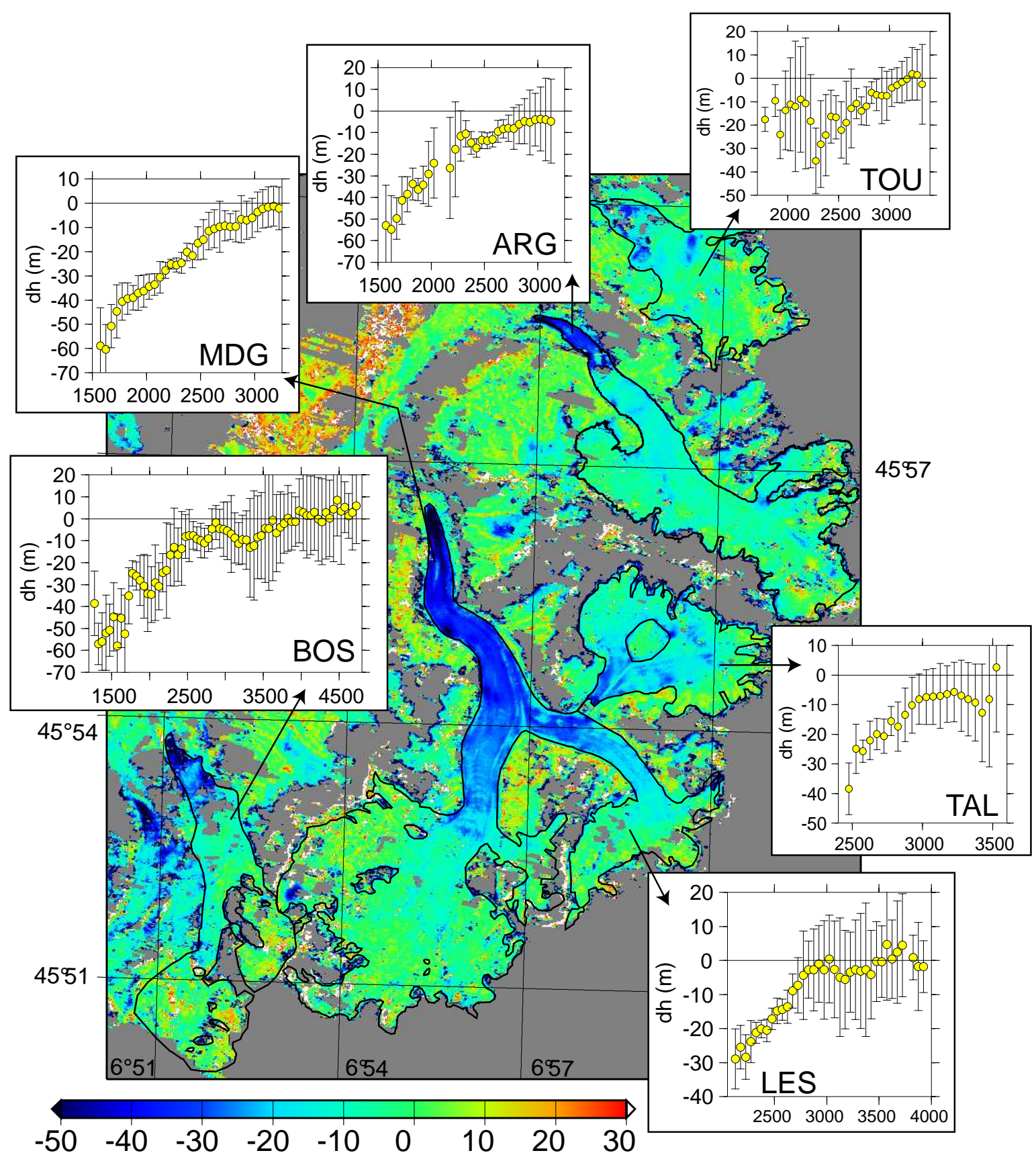

FIG. 5.17 - Carte des variations d'épaisseur de quelques glaciers du massif du Mont-Blanc pour la période 1979-2003. Les variations sont aussi fournies en fonction de d'altitude. Les barres d'erreur correspondent à l'écart type au sein de chaque tranche d'altitude. Du Nord vers le Sud: $T O U=$ Tour $; A R G=$ Argentière $;$ TAL $=$ Talèfre : $M D G=$ Mer de Glace $;$ LES $=$ Leschaux; $B O S=$ Bossons. 
Le signal d'amincissement à basse altitude est commun à tous les glaciers. Deux arguments suggèrent une bonne précision et l'absence de biais systématique. Tout d'abord, l'écart type au sein de chaque tranche d'altitude reste modéré, voire faible sur les zones de faible pente comme la langue de la Mer de glace ou le bas du glacier de Leschaux. D'autre part, à haute altitude et pour tous les glaciers, les variations d'épaisseurs sont proches de zéro : le biais noté dans le paragraphe précédent semble bien un artéfact de la topographie SRTM.

Ces courbes nous permettent d'évaluer la variation de volume de chacun des glaciers : la variation d'épaisseur moyenne d'une tranche d'altitude est multipliée par sa surface (tableau 5.3). Quelques tranches d'altitude n'ont pas de données fiables et sont donc écartées du calcul. Mais leur proportion reste limitée (seconde colonne du tableau). Pour convertir les variations de volume en bilan de masse, une hypothèse doit être faite sur la densité du matériel gagné ou perdu. Deux solutions sont testées. Dans l'hypothèse 1 (Andreassen et al., 2002), on suppose que le profil de densité reste constant dans la zone d'accumulation et donc que le matériel gagné ou perdu est de la glace $(\rho=0.9)$ sur tout le glacier. Dans l'hypothèse 2 (Haag et al., sous presse), au dessus de la ligne d'équilibre c'est du névé $(\rho=0.6)$ qui est gagné ou perdu ce qui limite la perte de masse. Pour cette seconde hypothèse il faut connaître l'altitude de la ligne d'équilibre. En première approximation à partir des données de Rabatel et al. (2005), elle a été prise constante et égale à $2800 \mathrm{~m}$ pour tous les glaciers. Ceci permet un premier test de sensibilité. Le choix de l'une ou l'autre des hypothèses à un impact modéré pour la plupart des glaciers, environ $5 \%$ de différence. Logiquement, l'écart augmente pour les glaciers à large zone d'accumulation. Pour trancher entre ces deux hypothèses, des mesures in situ semblent indispensables. Il serait intéressant par exemple de comparer un profil de densité réalisé dans la zone d'accumulation au début des années 1980 à un profil actuel. A l'avenir, il semble important de réaliser ce type de mesure en parallèle à l'acquisition des MNT SPOT5.

Tous les glaciers présentent un bilan de masse négatif, la moyenne se situant entre - 0.4 et $-0.5 \mathrm{~m} / \mathrm{a}$. C'est sur le glacier des Bossons que la lame d'eau perdue est la plus faible. Cela s'explique par l'hypsométrie favorable de ce glacier puisque $70 \%$ de sa surface se situe au dessus de $3000 \mathrm{~m}$. Le bilan de masse le plus négatif est celui du glacier du Couvercle. Des biais entre les MNT, observés dans cette zone, expliquent partiellement ce bilan de masse très négatif. Mais l'exposition de ce glacier, tourné vers le Sud, joue aussi certainement un rôle. L'absence de données sur les glaciers italiens et suisses ne nous permet pas d'étudier systématiquement ces effets d'exposition. Ceci pourrait être fait en prolongement de cette thèse si des MNT des versants italiens et suisses sont obtenus ou grâce à la construction d'un nouvel MNT SPOT5 dans les mois à années à venir.

Seul le glacier d'Argentière présente un dispositif in situ suffisamment développé pour permettre l'évaluation de son bilan de masse par la méthode glaciologique. Plus précisément, le bilan de ce glacier est obtenu par le LGGE en combinant la méthode géodétique, appliquée à des photographies aériennes, et des relevés de terrain (C. Vincent, communication personnelle). Le bilan cumulé pour la période 1979-2003 atteint $-13.1 \mathrm{~m}$ (w.e.) contre -8.4 m (w.e.) pour notre estimation (hypothèse 1). 
TAB. 5.3 - Superficies, variations de volume et bilans de masse de quelques glaciers du côté français du massif du Mont-Blanc entre $19^{7}$ et 2003. Les bilans de masse sont calculés en distinguant deux hypothèses : $\rho=0.6$ ou $\rho=0.9$ (voir texte). La seconde colonne indique le pourcentage de la surface totale qui a été utilisé pour le calcul du bilan de masse.

\begin{tabular}{lccccc}
\hline Glacier & $\mathrm{S}\left(\mathrm{km}^{2}\right)$ & $\mathrm{S}_{O K}(\%)$ & $\Delta \mathrm{V}\left(\mathrm{km}^{3}\right)$ & $\mathrm{b}_{1}\left(\mathrm{~m} \mathrm{a}^{-1}\right.$ w.e. $)$ & $\mathrm{b}_{2}\left(\mathrm{~m} \mathrm{a}^{-1}\right.$ w.e. $)$ \\
\hline Mer de Glace & 22.7 & 90.5 & -0.239 & -0.4 & -0.38 \\
Argentière* & 13.2 & 97.3 & -0.115 & -0.35 & -0.31 \\
Leschaux & 5.9 & 91.8 & -0.064 & -0.41 & -0.40 \\
Talèfre & 7.4 & 96.9 & -0.089 & -0.45 & -0.38 \\
Bossons & 9.3 & 85.8 & -0.052 & -0.11 & -0.10 \\
Tour & 7.2 & 95.4 & -0.051 & -0.27 & -0.24 \\
Bionnassay & 4.2 & 64.1 & -0.096 & -0.95 & -0.8 \\
Couvercle** & 8.3 & 81.7 & -0.240 & -1.13 & -1.00 \\
\hline
\end{tabular}

* Pour ce glacier, la zone de calcul du bilan de masse est rigoureusement la même que celle utilisée par le LGGE, incluant les glaciers des Améthystes, des Rognons et excluant une zone à haute altitude mal échantillonnée en 2003.

** L'incertitude est forte sur le bilan de masse car dans cette partie Sud du massif du Mont Blanc, il s'est avéré difficile de superposer les MNT au niveau des zones stables autour des glaciers.

Ces différences, à première vue importantes, restent du même ordre de grandeur que celles de la littérature. Un tableau de synthèse compilé par Haag et al. (sous presse) montre en effet des écarts qui peuvent atteindre 100\%. Dans notre cas, plusieurs explications sont possibles :

- L'incertitude temporelle sur le MNT-IGN. Notre bilan de masse satellitaire couvre normalement la période 1979 à 2003 . Compte tenu de bilans de masse en moyenne positifs pour ce glacier avant 1979 et négatifs après, il suffit d'un décalage de quelques années pour que la différence entre les bilans de masse terrain et satellitaire se réduise.

- Un biais du MNT-IGN à haute altitude. Sa comparaison avec les relevés photogrammétriques de 1980 (figure 5.12) indique qu'il est trop bas au dessus de $2800 \mathrm{~m}$. Ceci expliquerait que notre MNT différentiel sous-estime les pertes de volume entre 1979 et 2003 (situation analogue à la partie droite de la figure 5.16).

- un échantillonnage spatial différent des deux méthodes. Notre MNT différentiel couvre $97 \%$ de la surface totale du glacier alors que les mesures terrain ne couvrent que certaines zones seulement.

Il est difficile de trancher entre les différentes hypothèses. C'est sans doute la combinaison des sources d'erreurs qui expliquent les écarts observés. A ce stade, la mesure in situ nous semble encore plus fiable. Seule la construction d'une nouvelle topographie SPOT5 permettra d'éviter une bonne partie des incertitudes (temporelles, biais avec l'altitude) et d'aller plus loin dans la comparaison des différentes méthodes d'établissement du bilan de masse glaciaire.

Nos travaux indiquent des bilans de masse négatifs pour tous les glaciers du massif du Mont- 
Blanc depuis 1979. La perte de masse moyenne est équivalente à une lame d'eau de $0.5 \mathrm{~m}$ par an et semble s'être accélérée à la fin des années 90. Mais les biais du MNT SRTM et l'absence de mesures satellitaires d'archive dans la zone d'accumulation nous empêchent de quantifier précisément cette accélération.

\subsection{Bilans de masse de 2 glaciers himalayens}

L'un des enjeux majeurs de la glaciologie est la mesure des bilans de masse de glaciers inaccessibles et de grande taille. Les glaciers himalayens entrent dans cette catégorie car ils se situent dans des zones à très forte topographie, où l'accès par voie terrestre est difficile. Très peu de glaciers himalayens sont donc instrumentés alors même qu'ils constituent le chateau d'eau d'une des régions les plus peuplées de la planète. On qualifie souvent l'Himalaya de $3^{\text {eme }}$ pôle.

Le suivi du bilan de masse du glacier du Chhota Shigri a débuté en 2002 dans le cadre d'une collaboration entre l'Université de Jawaharlal Nehru en Inde et l'unité de recherche Great Ice de l'IRD $^{6}$. L'échantillonnage spatio-temporel des mesures in situ est limité par les difficultés d'accès, la taille du glacier (14 $\mathrm{km}^{2}$ environ) et sa haute altitude (il s'étage entre 4000 et $6000 \mathrm{~m}$ ). La télédétection semble l'outil le mieux adapté pour confirmer les observations de terrain, les étendre à tout le glacier, puis les régionaliser à l'échelle du massif.

\subsubsection{La zone d'étude : l'Himalaya indienne}

Le Chhota Shigri et son immense voisin, le Bara Shigri, se situent dans la partie indienne de l'Himalaya (figure 5.18).
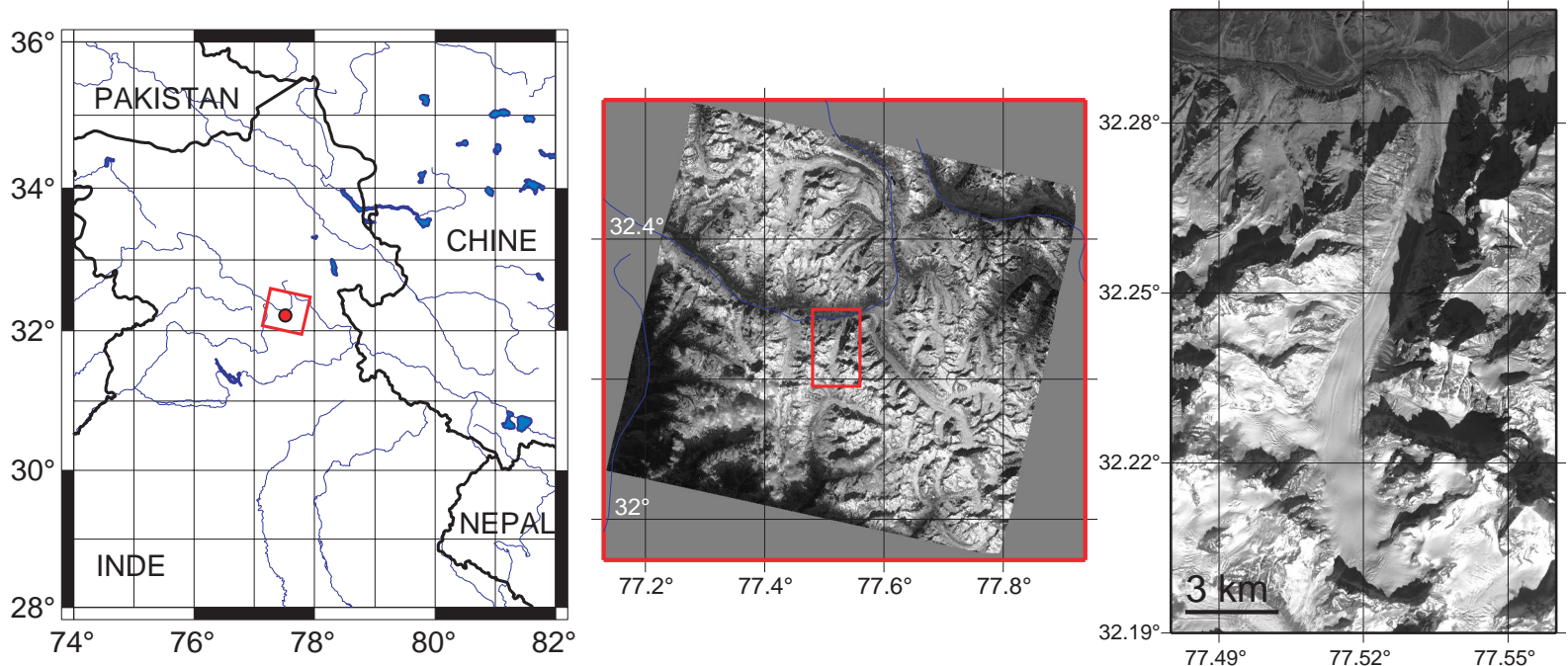

FIG. 5.18 - Zone d'étude. A gauche, localisation des images SPOT5 au cour de l'Himalaya indienne. Au centre, image SPOT5 du 13 novembre 2004. Le cadre rouge localise le glacier du Chhota Shigri qu'on observe plus en détail à droite. On notera l'importance des ombres.

Un couple stéréoscopique d'images SPOT5 a été acquis sur cette zone en novembre 2004

\footnotetext{
${ }^{6}$ Institut de Recherche pour le Développement.
} 
(tableau 5.4) avec une séparation temporelle de 1 jour et un rapport B/H adapté pour ces zones de forte topographie. Les nuages sont quasi absents et le gain est minimum. En revanche, l'acquisition tardive (novembre) a deux désavantages. D'une part, l'inspection des images montre que des chutes de neige se sont produites depuis la fin de l'année hydrologique (septembre 2004). D'autre part, les ombres sont particulièrement importantes. Parce qu'aucun point de contrôle n'est disponible dans cette région, nous avons dû développer une méthodologie qui utilise la précision du modèle stéréo de SPOT5 pour produire des GCP et calculer le MNT.

TAB. 5.4 - Images SPOT5 acquises en Novembre 2004 sur le glacier du Chhota Shigri.

\begin{tabular}{lcccc}
\hline Couple d'images & Angles d'incidence & $\mathrm{B} / \mathrm{H}$ & $\delta \mathrm{t}$ (jours) & Gain \\
\hline 12 nov. / 13 nov. & $+20 /-13.9$ & 0.61 & 1 & 1 \\
\hline
\end{tabular}

\subsubsection{Génération d'un MNT SPOT5 sans point d'appui}

La figure 5.19 synthétise les étapes qui permettent de calculer un MNT à partir de deux images SPOT5 sans points de contrôle au sol. Notre méthode nécessite la disponibilité d'un MNT sur la région (ici SRTM). In fine, la précision du MNT SPOT5 devrait être du même ordre de grandeur que celle du MNT préexistant.

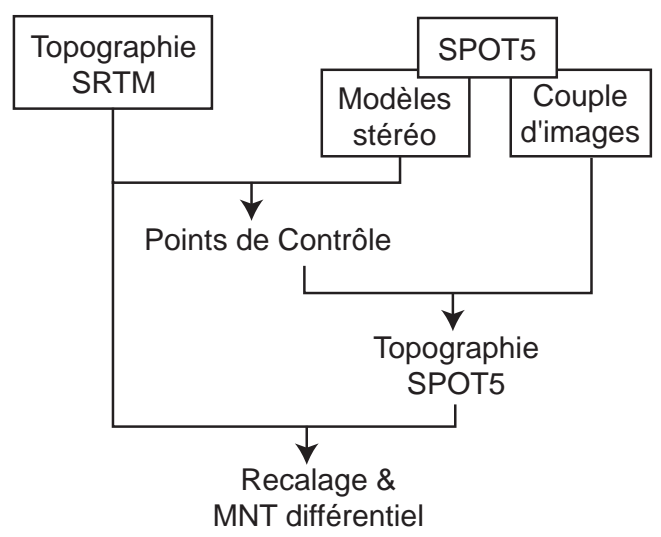

FiG. 5.19 - Réalisation d'un MNT sans point d'appui à partir d'un couple d'images SPOT5.

Le MNT SPOT5 calculé, son positionnement en latitude et longitude est affecté par les mêmes erreurs de localisation que les images SPOT5, i.e. $\pm 30 \mathrm{~m}$ (intervalle de confiance à $66 \%$ ). Le MNT-SRTM peut être utilisé comme référence pour un nécessaire ajustement planimétrique. Différentes techniques ont été explorées pour ce recalage de deux MNT : corrélation des MNT, des images shaded relief qui en dérivent, minimisation de l'écart type de leur différence etc... Mais il est difficile de juger quelle technique est la plus performante dans le contexte himalayen où il n'y a pas de vérité terrain. Aussi, il serait intéressant de tester ces différentes procédures dans le cas alpin (MNT SPOT5 de 2003) où des GCP précis et nombreux sont disponibles pour évaluer les résultats. Dans ce domaine de comparaison et recalage de MNT, des développements méthodologiques semblent nécessaires. Ils pourraient être réalisés dans le cadre du programme d'accompagnement à la mission satellitaire ORFEO (Vadon, com. personnelle). 


\subsubsection{Déclin récent du Bara Shigri et Chhota Shigri}

Un MNT différentiel est construit en comparant le MNT SPOT5 (2004) au MNT SRTM (1999). La différence permet d'étudier les variations cumulées d'épaisseur des glaciers durant 5 années hydrologiques (figure 5.20).
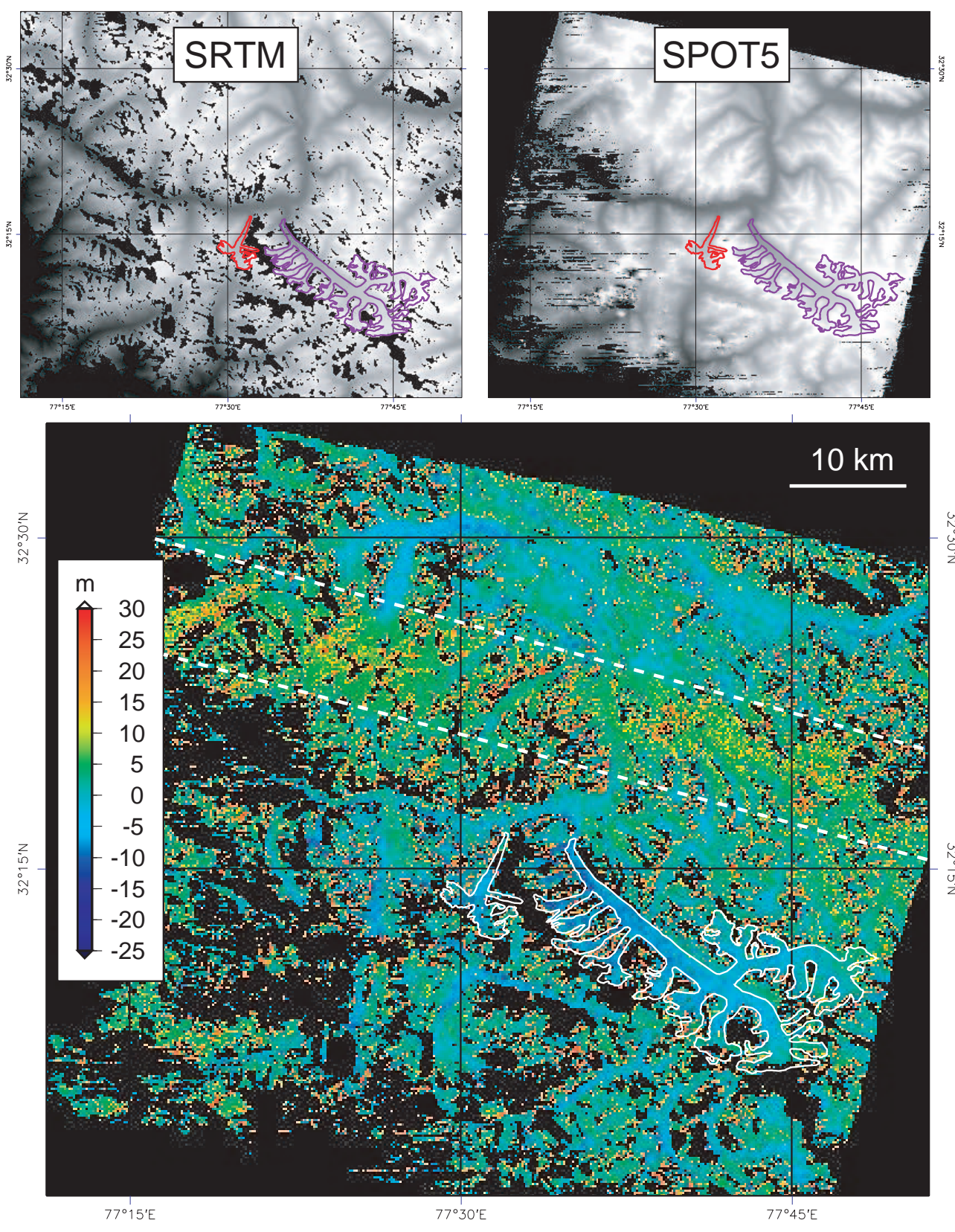

FIG. 5.20 - En haut : topographies SRTM et SPOT5 sur notre zone d'étude. En noir, les zones où les MNT présentent des trous. Les limites des glaciers du Chhota Shigri (en rouge) et Bara Shigri (en bleu) sont localisées. En bas : MNT différentiel pour la période 1999-2004. Comme dans le cas des Alpes, les glaciers se caractérisent par d'importantes pertes d'épaisseurs. Les lignes blanches tiretées délimitent une bande où le biais entre les deux MNT est important. 
Nous devons tout d'abord noter un artefact important dans le MNT différentiel dans sa partie Nord. Sur une bande de $10 \mathrm{~km}$ de large (approximativement parallèle à la direction des colonnes de l'image SPOT5), le MNT SPOT5 est systématiquement trop haut. Nous l'interprétons comme un effet de tangage du satellite le long de sa trajectoire lors de l'acquisition d'une des images du couple SPOT5. Les deux glaciers d'intérêt étant hors de cette zone, cet artefact n'a pas été étudié plus en détail pour le moment ni corrigé.

Un signal clair d'amincissement de tous les glaciers de cette zone est visible. L'amplitude des pertes d'épaisseur est d'une dizaine de mètres, plus dans les zones basses des glaciers. La figure 5.21 permet d'observer pour deux glaciers la distribution de cet amincissement en fonction de l'altitude.

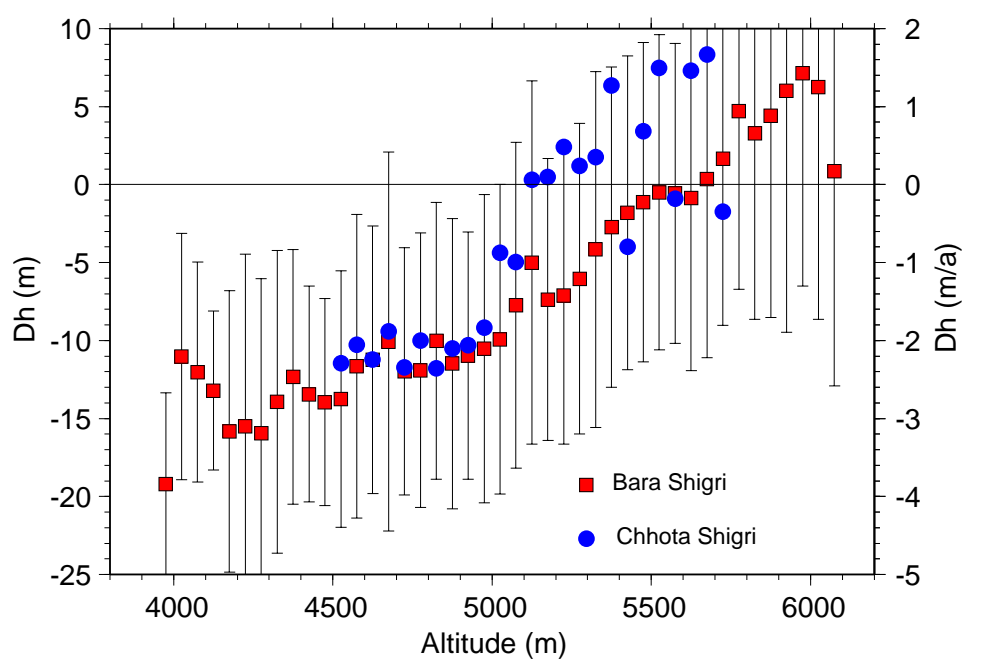

Fig. 5.21 - Variations d'épaisseur des glaciers du Bara et Chhota Shigri en fonction de l'altitude. Pour le Chhota Shigri, les ombres importantes (figure 5.18) empêchent une estimation des pertes d'épaisseur à "basse" altitude, entre 4000 et $4500 \mathrm{~m}$. Les barres d'erreurs indiquent l'écart type des variations d'épaisseur pour chaque tranche d'altitude du Bara Shigri.

Les courbes de la figure 5.21, combinées aux hypsométries des deux glaciers, permettent d'évaluer les pertes de volume et les bilans de masse de ces deux glaciers (tableau 5.5).

TAB. 5.5 - Superficies, variations de volume et bilans de masse de deux glaciers himalayens entre 1999 et 2004. Pour le calcul des bilans de masse, seule l'hypothèse $1(\rho=0.9)$ du tableau 5.3 a été retenue.

\begin{tabular}{lccc}
\hline Glacier & $\mathrm{S}\left(\mathrm{km}^{2}\right)$ & $\Delta \mathrm{V}\left(\mathrm{km}^{3}\right)$ & $\mathrm{b}_{1}\left(\mathrm{~m} \mathrm{a}^{-1}\right.$ w.e. $)$ \\
\hline Chhota Shigri & 14.3 & -0.07 & -0.86 \\
Bara Shigri & 114.7 & -0.66 & -0.95 \\
\hline
\end{tabular}

Avant de discuter ces résultats, nous souhaitons attirer l'attention sur les fortes incertitudes sur ces valeurs. Elles s'expliquent surtout par le biais probable de SRTM dans les zones de haute 
altitude. Ces biais devraient conduire à une sous-estimation des pertes de masse (figure 5.16, partie de droite). Et pourtant, les deux glaciers présentent des bilans de masse très largement négatifs, proches de $-1 \mathrm{~m} / \mathrm{a}$ (w.e.). Ces résultats sont consistants avec les premiers bilans de masse mesurés in situ, qui sont voisins de $-1 \mathrm{~m} / \mathrm{a}$ (w.e.) pour le Chhota Shigri (Rajesh et al., 2005).

Ainsi, en 5 ans, le Bara Shigri a perdu de $0.66 \mathrm{~km}^{3}$ de glace, c'est considérable. Ces bilans sont encore plus négatifs que ceux des glaciers alpins (mais la période de mesure est différente puisque dans les Alpes les bilans de masse sont calculés entre 1979 et 2003). Nous reviendrons sur cette comparaison en conclusion de cette thèse.

Suite à ces résultats préliminaires encourageants, il convient maintenant d'optimiser notre estimation du bilan de masse. L'acquisition de 4 ou 5 points d'appuis précis suffirait à affiner le modèle géométrique des images SPOT5 et donc améliorer le MNT. Une comparaison plus systématique des deux topographies sur les zones entourant les glaciers devrait permettre d'estimer l'incertitude de nos pertes d'épaisseur. Quantifier le biais de SRTM en zone d'accumulation (pénétration de l'écho radar) semble aussi important.

Étendre nos calculs à tous les glaciers présents sur nos images SPOT5 permettrait de confirmer si le déclin rapide observé sur le Bara Shigri et le Chhota Shigri concerne tous les glaciers de ce massif. Un couple d'images SPOT5 a également été acquis en novembre 2004 sur la région du Gangotri (à environ $200 \mathrm{~km}$ au Sud-Est) et pourrait permettre d'étudier la variabilité des pertes de masse glaciaires à l'échelle régionale cette fois.

En parallèle à cette étude glaciologique, une étude climatique de toute la région de l'Himalaya de l'Ouest semble nécessaire. (Yadav et al., 2004) montrent en effet que, depuis 20 ans, cette zone est l'une des rares de la planète où l'on assiste à une chute des températures moyennes. C'est contradictoire avec le signal climatique contenu dans des bilans de masse très négatifs. Confirmer cette baisse des températures, observer l'évolution récente des précipitations sont des travaux qu'il convient maintenant d'entreprendre.

\subsection{Conclusions}

\subsubsection{Conclusions méthodologiques}

Nos résultats suggèrent que nous sommes tout près de la mesure satellitaire du bilan de masse glaciaire. D'une part, nous démontrons que des variations d'épaisseur des glaciers alpins peuvent être déduites de l'imagerie satellitaire optique. D'autre part, le MNT déduit d'images SPOT5 (2003) couvre toutes les zones du glacier et ne présente pas de biais marqué en fonction de l'altitude. L'acquisition d'un second MNT SPOT5 sur les Alpes dans les mois (ou années) à venir devrait confirmer cette conclusion.

La précision actuelle des MNT (même SPOT5) semble interdire le travail au pas de temps annuel. Il nous semble plus judicieux d'utiliser les MNT différentiels satellitaires pour des intervalles temporels de 3 à 5 ans. Il devient urgent de constituer une véritable base de données satellitaires sur des zones diversifiées pour pouvoir, dans trois à cinq ans, mesurer les bilans de masses glaciaires. En effet, nous avons démontré ici que la topographie SRTM est affectée par des biais dans la zone d'accumulation des glaciers et doit être utilisée avec précaution.

L'application à une zone retirée du globe (Himalaya) pour laquelle aucun point d'appui n'est 
disponible démontre la robustesse de notre méthodologie. C'est un spectre large de glaciers qui peut ainsi être étudié.

\subsubsection{Conclusions glaciologiques}

Notre étude indique une accélération des pertes de masse à basse altitude alors que les zones hautes semblent en équilibre. Cela se matérialise par un amincissement fort de la langue de la Mer de Glace. Mais l'augmentation des températures n'explique qu'une partie de ces pertes d'épaisseur. L'observation simultanée d'un ralentissement significatif de cette zone (§4.6.2) confirme le lien fort entre dynamique glaciaire et variations d'épaisseur. Ce lien sera repris au chapitre 7 et nous chercherons à quantifier si le ralentissement du glacier est responsable de la part non expliquée de l'amincissement.

Des résultats préliminaires sur l'Himalaya indiquent des bilans de masse glaciaires encore plus négatifs que dans les Alpes mais il faut modérer cette observation par des périodes d'étude différentes pour les deux massifs. Il convient maintenant de déterminer si les autres glaciers himalayens suivent la même évolution. Il est délicat d'extrapoler à toute une région les résultats obtenus sur quelques glaciers seulement. Mais, si tous les glaciers himalayens, couvrant $35000 \mathrm{~km}^{2}$ (WWF, 2005), subissaient les mêmes pertes, 0.8 à $1 \mathrm{~m} \mathrm{a}^{-1}$ (w.e.), ils libéreraient 28 à $35 \mathrm{~km}^{3}$ d'eau vers la mer chaque année. Cela correspond à une élévation du niveau moyen des mers de 0.08 à $0.1 \mathrm{~mm} / \mathrm{an}^{7}$. C'est moins que les glaciers d'Alaska (Arendt et al., 2002), mais au moins autant que les glaciers patagoniens (Rignot et al., 2003).

\footnotetext{
${ }^{7} 360 \mathrm{~km}^{3}$ d'eau correspondent à $1 \mathrm{~mm}$ de niveau marin
} 



\section{Chapitre 6}

\section{Dynamique de la calotte glaciaire du Vatnajökull.}

\section{Sommaire}

6.1 La calotte glaciaire du Vatnajökull . . . . . . . . . . . . . . . . . . 141

6.2 Soulèvement de la plateforme de Grímsvötn . . . . . . . . . . . . . . . 143

6.2.1 Plateforme, lac sous-glaciaire et volcan de Grímsvötn . . . . . . . . . . . . . . . 144

6.2.2 Cartographie du soulèvement de la plateforme de Grímsvötn . . . . . . . . . . . 146

6.2.3 Crue potentielle, bilan énergétique et activité géothermique . . . . . . . . . . . . 149

6.2 .4 Perspectives et implications de cette mesure . . . . . . . . . . . . . . . . 150

6.3 Variations volumétriques du Vatnajökull . . . . . . . . . . . . 151

6.3.1 Les topographies disponibles sur la calotte (figure 6.8) . . . . . . . . . . 151

6.3 .2 Observation et origine des variations d'épaisseur . . . . . . . . . . . 153

6.4 Conclusions . . . . . . . . . . . . . . . . . . 157

Par sa morphologie, ses caractéristiques thermiques et dynamiques, la calotte glaciaire du Vatnajökull est intermédiaire entre les glaciers de montagne et les calotte polaires. L'étude de ce glacier, de la taille de la Corse, se déroule dans le cadre d'une collaboration avec l'équipe du Science Institute (University of Iceland, Reykjavik). Deux missions en Islande (une troisième suivra cette thèse) ont permis de mieux connaître l'équipe islandaise et le Vatnajökull à travers des campagnes de terrain dans des conditions climatiques parfois extrêmes. La désinstallation d'une station météorologique située au centre du Vatnajökull, à 3 heures du matin dans la tempête, restera un souvenir marquant. Certains des travaux que nous présentons sur cette calotte glaciaire sont en cours de réalisation $(\S 6.3)$ et demandent à être poursuivis.

\subsection{La calotte glaciaire du Vatnajökull}

Comme le suggère son nom anglais (Iceland : pays de glace), les glaciers jouent un rôle important dans la morphologie de cette île de $103000 \mathrm{~km}^{2}$. Près de $11 \%$ de la surface de l'Islande est couverte par les glaces. Le plus grand glacier de l'île est la calotte du Vatnajökull couvrant $8200 \mathrm{~km}^{2}(120 \times 90 \mathrm{~km}$ environ figure 6.1) : "Vatna" est l'eau en Islandais alors que "Jökull" désigne un glacier. Cette petite calotte glaciaire ${ }^{1}$ a attiré et passionné les glaciologues depuis

\footnotetext{
${ }^{1}$ ice cap en anglais
} 
de nombreuses années (Ahlmann, 1948), car elle condense et rend accessible à l'observation une grande diversité de processus glaciaires (Fischer, 2002) :

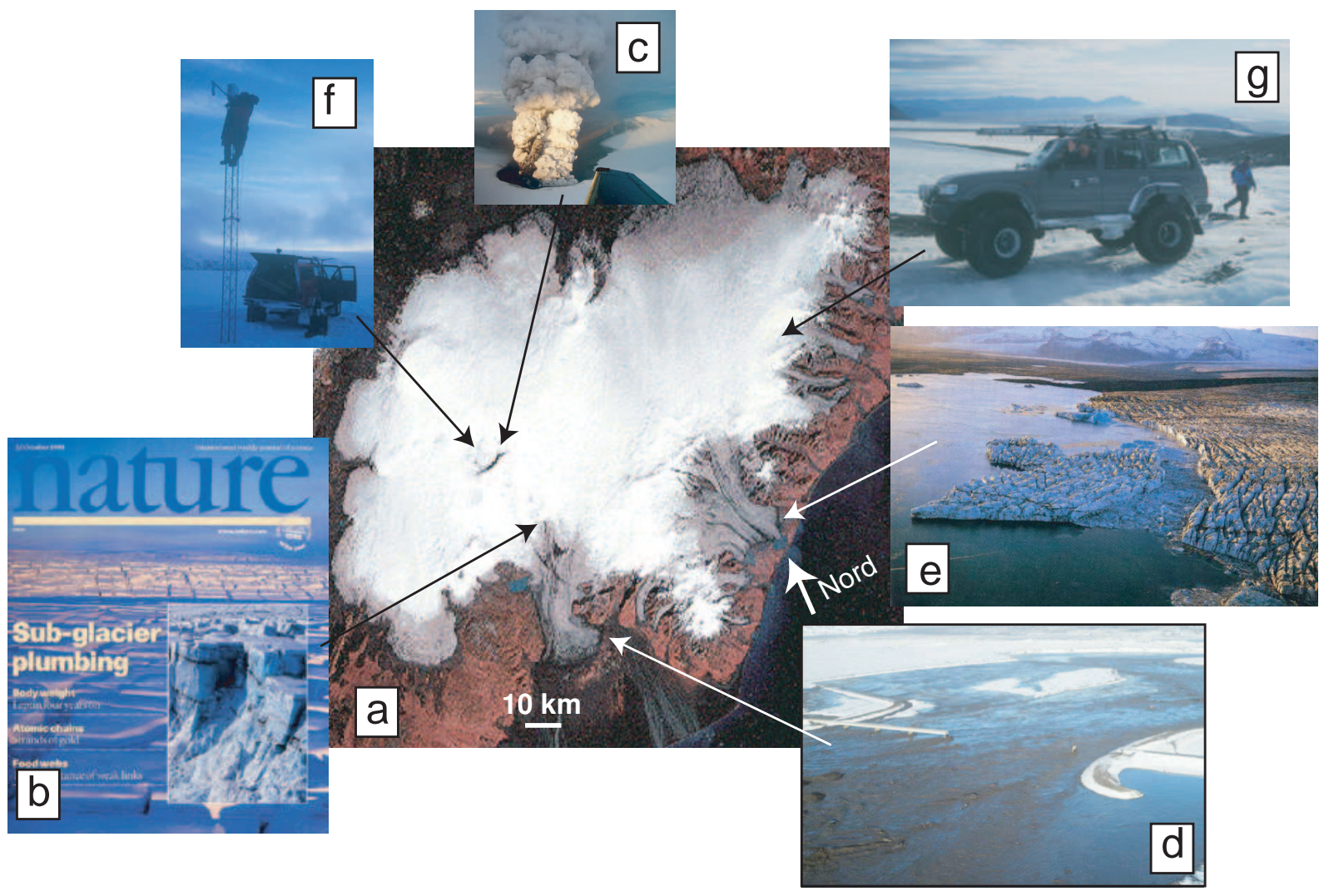

FIG. 6.1 - (a) Image LANDSAT MSS de la calotte du Vatnajökull. La diversité des processus glaciaires est suggérée par les photos : (b) surges (voir texte), (c) éruptions volcaniques, (d) crues dévastatrices et (e) vêlage d'icebergs se marient pour conférer à la calotte islandaise l'une des dynamiques glaciaires les plus passionnantes de notre planète. L'observation in situ est difficile: (f) installation de la station MAST sur la plateforme de Grimsvötn, (g) départ en $4 \times 4$ pour une compagne de terrain (septembre 2003).

- Il s'agit de la plus grande calotte glaciaire tempérée du globe. Elle couvre une large gamme d'altitudes, depuis le niveau de la mer jusqu'à $2200 \mathrm{~m}$. Son volume atteint $3150 \mathrm{~km}^{3}$, équivalent à près de $10 \mathrm{~mm}$ du niveau marin.

- Cette calotte est sujette à des climats contrastés avec des précipitations annuelles qui s'échelonnent entre $400 \mathrm{~mm}$ au Nord et $4000 \mathrm{~mm}$ sur sa marge Sud. Ce contraste climatique s'accompagne de disparités morphologiques. Les glaciers du Sud et de l'Est évacuent les fortes précipitations par des langues glaciaires rapides et relativement étroites car confinées par des parois abruptes. Chacune de ces langues reste plus large que la Mer de Glace. Les glaciers du Nord et de l'Ouest s'écoulent plus lentement sur des pentes faibles et ils drainent mal la neige et la glace qui s'accumulent alors dans les régions hautes. Quand l'excès de masse dépasse un seuil, il est évacué par de brutales crues glaciaires. Lors de ces crues, nommées surges en anglais ${ }^{2}$, les vitesses de surface du glacier peuvent être multipliées par

\footnotetext{
${ }^{2}$ Le terme français exact est le "foirage" mais il est très peu usité. Le terme de crue pouvant lui entraîner une confusion avec la crue hydrologique, c'est le terme anglais de surge qui sera utilisé dans la suite de ce chapitre
} 
un facteur 100 (Björnsson et al., 2003; Fischer et al., 2003).

- La "magie" du Jokulsárlón (le lagon glaciaire figure 6.1 (e)) n'a pas seulement attiré les équipes de tournage de deux des "James Bond". Ce site est également un lieu privilégié pour comprendre la dynamique du vêlage des icebergs (Björnsson et al., 2001a), phénomène important pour prévoir l'évolution du bilan de masse des calottes polaires.

- Enfin, le Vatnajökull est situé à l'aplomb de la dorsale médio-Atlantique, à l'endroit même où cette dorsale interagit avec un point chaud. Périodiquement, les volcans de Grímsvötn et de Bárðarbunga se réveillent, engendrant de violentes éruptions sous-glaciaires (Guðmundsson et al., 1997) de type phréatomagmatiques ${ }^{3}$. Ce jeu combiné de la glace et du feu est l'un des symboles de l'Islande.

Ces différents phénomènes et leurs interactions rendent complexes la dynamique de la calotte glaciaire mais permettent aussi de révéler des processus jusqu'alors mal compris. Par exemple, l'étude détaillée de l'impact de l'éruption volcanique de Gjálp et du jökulhlaup ${ }^{4}$ consécutif sur l'écoulement du Skeiðarárjokull alors en surge a permis à Björnsson (1998) de préciser les mécanismes contrôlant les surges, notamment le rôle du réseau hydrologique sous-glaciaire.

La logistique et l'énergie dépensée par l'équipe islandaise du Science Institute pour instrumenter le Vatnajökull est à la hauteur du gigantisme de ce glacier. Les mesures de terrain sont réalisées en moto-neige ou grâce à d'énormes véhicules tout-terrains. Dans un tel contexte, la télédétection en général et l'imagerie satellitaire en particulier peuvent apporter leur large couverture. L'interférométrie radar a ainsi permis d'étudier la réponse du glacier à la fonte brutale lors des éruptions volcaniques sous-glaciaires et de décrire l'évolution des vitesses au cours d'un surge (Björnsson et al., 2001b; Guðmundsson et al., 2002a; Fischer, 2002; Fischer et al., 2003). Mais, pour cette calotte soumise à des conditions météorologiques très variables, la cohérence entre images SAR n'est maintenue que si la séparation temporelle n'est que d'une journée et, même dans ces conditions, 25\% seulement de la surface des interférogrammes est utilisable (Fischer, 2002). Pour les mêmes raisons que dans les Alpes (§3.3.3.1), cette technique n'est plus utilisable actuellement.

C'est dans ce contexte que nous proposons d'appliquer les méthodologies développées sur les glaciers alpins pour étudier différents aspects de la dynamique du Vatnajökull. Une application originale de la corrélation d'images optiques nous permet d'étudier le mouvement vertical d'une plateforme de glace flottant sur le lac de Grímsvötn. L'extension et le bilan d'énergie du lac sousglaciaire peuvent ainsi être évalués (§6.2). Un MNT différentiel satellitaire permet de mesurer les changements géométriques de la calotte entre 1998 et $2004(\S 6.3)$.

\subsection{Soulèvement de la plateforme de Grímsvötn}

A notre connaissance, la corrélation d'images optiques a toujours été utilisée pour mesurer des déplacements horizontaux des nuages, de la surface de l'océan ou de la surface terrestre (chapitre 4). L'originalité méthodologique de notre étude est la mesure d'un mouvement purement vertical. Nos travaux sont détaillés dans un article (annexe D) soumis à Earth and Planetary Science Letters (Berthier et al., soumis).

Après avoir présenté le fonctionnement de notre zone d'étude, le lac de Grímsvötn, nous détaillerons comment la corrélation d'images SPOT5 mesure le remplissage de ce lac entre les 11

\footnotetext{
${ }^{3}$ Éruptions volcaniques violente pendants lesquelles le magma interagit avec de l'eau au cours de sa remontée

${ }^{4}$ crue hydrologique brutale liee à la vidange d'une poche d'eau sous-glaciaire
} 
et 16 août 2004 ce qui permet de déterminer l'extension du lac sous-glaciaire, la taille de la crue potentielle et d'estimer le flux géothermique de cette zone.

\subsubsection{Plateforme, lac sous-glaciaire et volcan de Grímsvötn}

La figure 6.2 permet de localiser la dépression de Grímsvötn au cœur de la calotte glaciaire du Vatnajökull, au sud-est de l'Islande.

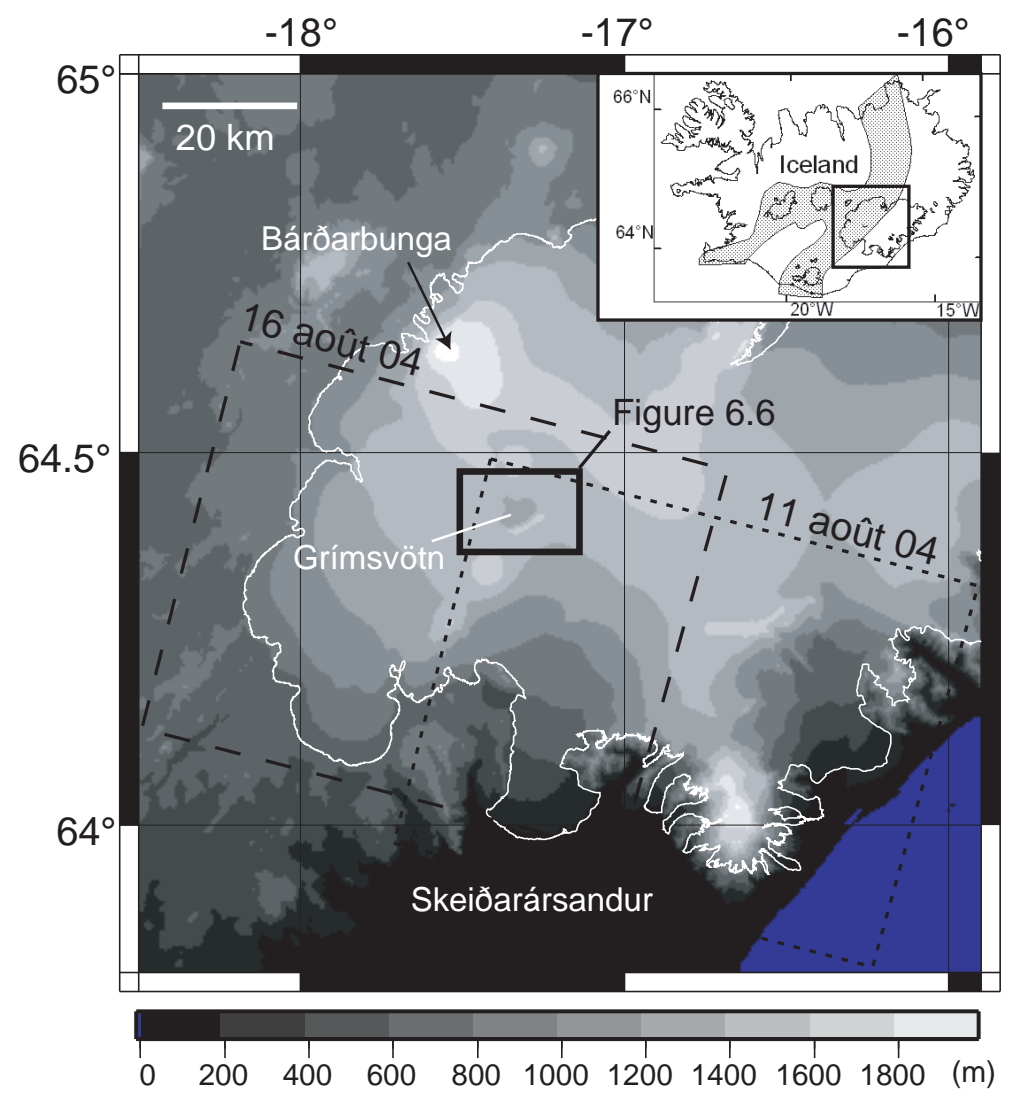

FIG. 6.2 - Topographie de la calotte glaciaire du Vatnajökull (données Science Institute). La dépression au niveau de la plateforme de glace de Grímsvötn est visible. Les limites des images SPOT5 ont été reportées. L'encart montre que la calotte se trouve à l'aplomb de la zone volcanique du Sud-Est l'une des bifurcations de la dorsale médio-Atlantique.

Cette dépression correspond à une plateforme ${ }^{5}$ de glace flottant sur un lac, lui-même audessus d'un volcan, le volcan de Grímsvötn (figure 6.3). La continuelle activité géothermique au niveau du volcan fait fondre la glace et alimente le lac sous-glaciaire. Ce lac est également rempli par la fonte en surface du glacier sur un bassin versant de plus de $200 \mathrm{~km}^{2}$, l'eau gagnant le lac par les crevasses (Björnsson \& Guðmundsson, 1993). Dans sa partie Est et Sud, le lac est délimité par les flancs abrupts de la caldeira (montagne de Grímsfjall), mais il peut s'étendre vers le Nord et l'Ouest où la topographie du lit rocheux est moins marquée. Lorsque le niveau du lac varie c'est l'ensemble de la plateforme (épaisse d'environ $250 \mathrm{~m}$ ) qui s'élève ou subside.

\footnotetext{
${ }^{5}$ Une plateforme (ice shelf en anglais) est une épaisse couche de glace flottante. Les plus grosses plateformes bordent l'Antarctique (Ross, Ronne-Filchner, Amery).
} 


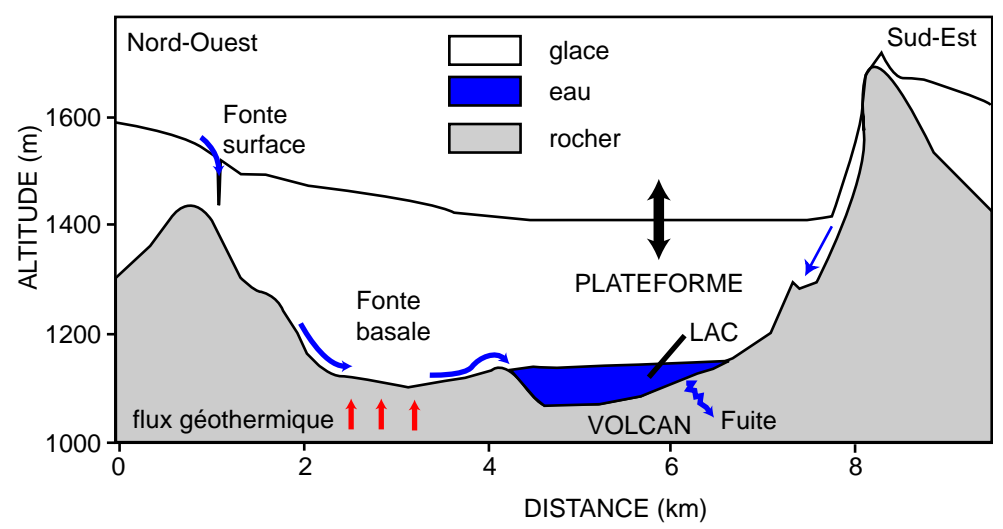

Fig. 6.3 - Coupe schématique Nord-Ouest-Sud-Est du lac de Grímsvötn. Adapté de Björnsson \& Guðmundsson (1993).

Ce lac sous-glaciaire présente un fonctionnement cyclique, puisque remplissages et vidanges alternent. Les variations d'altitude de la plateforme de glace reflètent le niveau du lac et illustrent son fonctionnement (figure 6.4).
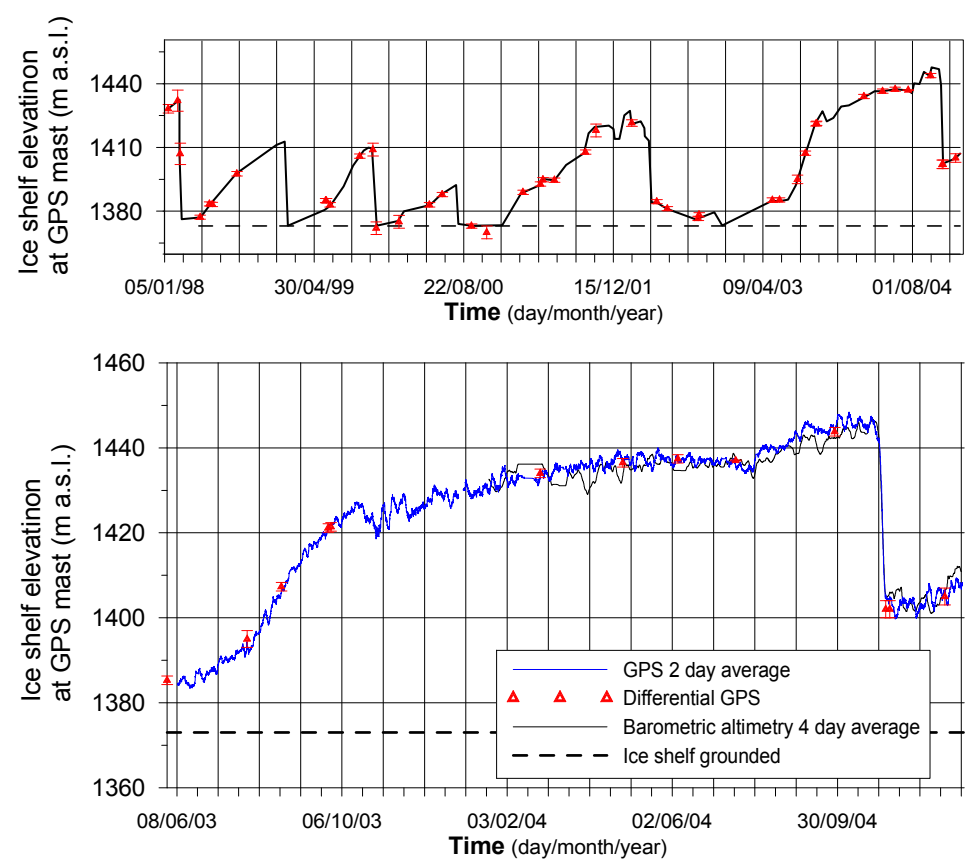

FIG. 6.4 - Altitude de la plateforme de Grímsvötn déduite de relevés GPS standards, de mesures de pression atmosphérique et de quelques positionnements DGPS (données Science Institute). La figure du haut montre la période 1998-2004, celle du bas détaille la période juin 2003-décembre 2004. La ligne tiretée horizontale indique l'altitude de la plateforme de glace lorsqu'elle repose sur le lit rocheux (lac vide).

Ces courbes montrent un remplissage progressif, lié à l'apport d'eau de fonte. Le niveau du lac monte plus rapidement en été à cause de la fonte plus intense en surface. A l'est, la vidange du lac est empêchée par un barrage de glace. Lorsque le niveau d'eau atteint une valeur critique, 
la glace du barrage se soulève, le lac se vidange et engendre une crue brutale nommée jökulhlaup (Björnsson, 2002; Robert, 2005). Les jökulhlaups de Grímsvötn sont dévastateurs, détruisant les ponts de l'unique route circulaire d'Islande et isolant ainsi une partie de la population.

La dernière vidange du lac de Grímsvötn s'est produite fin octobre 2004. Quelques jours après, le volcan est entré en éruption. La vidange du lac et la chute brutale de la charge exercée sur les roches sous-jacentes auraient provoqué l'éruption volcanique (Sigmundsson et al., 2004). Dans d'autres cas, l'éruption du volcan est au contraire précurseur, entraînant successivement une fonte massive de la glace, le remplissage rapide du lac puis son déversement. Ce scénario s'est déroulé en 1996 lors de l'éruption de Gjálp (Guðmundsson et al., 1997). Ces deux séquences d'évènements illustrent la relation étroite entre le lac et le volcan de Grímsvötn. Il est important de suivre le niveau du lac mais également son extension géographique pour estimer la taille de la crue potentielle et caractériser l'activité géothermique du volcan. Ceci peut être fait, indirectement, en suivant les changements d'altitude de la plateforme de glace. C'est ce que nous proposons ici, en corrélant des images SPOT5 acquises au cours de l'été 2004.

\subsubsection{Cartographie du soulèvement de la plateforme de Grímsvötn}

Comme décrit dans le chapitre 4, la corrélation de deux images optiques peut être sensible à un déplacement vertical si elles sont acquises du même point de vue mais avec des angles d'incidence importants (au maximum $30^{\circ}$ pour les satellites SPOT). Le point de vue similaire permet de limiter les distorsions entre les images alors que l'incidence non verticale engendre une sensibilité aux déplacements verticaux. La figure 6.5 présente une géométrie simplifiée dans le cas d'un mouvement purement vertical ( $\alpha$ désigne l'azimut de la direction des lignes et $\theta$ l'angle d'incidence).

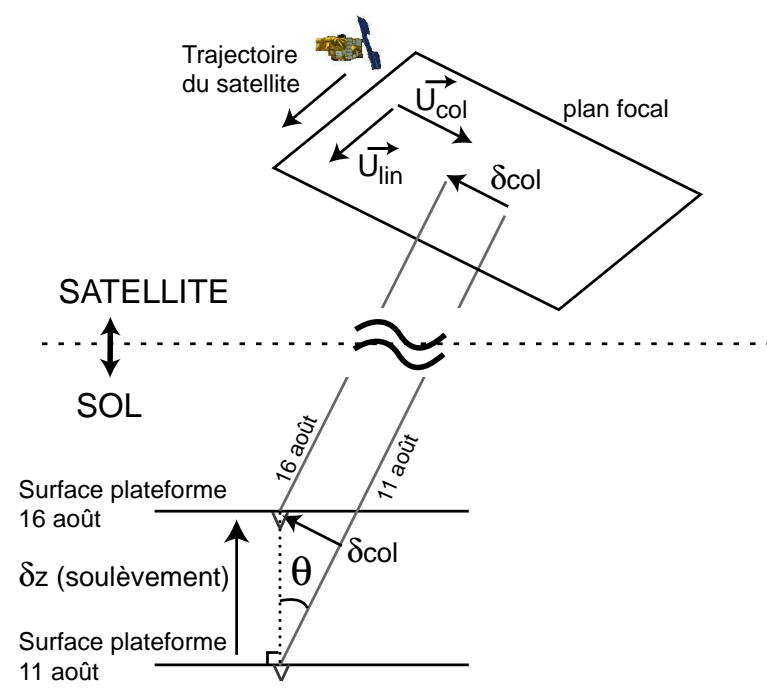

FIG. 6.5 - Effet d'un mouvement purement vertical de la surface terrestre sur les décalages en colonnes entre deux images acquises avec un angle d'incidence non vertical.

La glace de la zone de Grímsvötn a un mouvement horizontal faible (10 à $20 \mathrm{~m} / \mathrm{a}$ ), orienté Nord-Sud : $\delta_{\lambda}$ est donc négligeable. L'équation 4.10 permet de convertir les décalages en colonnes $\left(\delta_{C O L}\right)$ et lignes $\left(\delta_{L I G}\right)$ en déplacements verticaux $\left(\delta_{z}\right)$ : 


$$
\delta_{z}=\frac{\delta_{C O L}-\delta_{L I G} \times \cos \theta \times \frac{\sin \alpha}{\cos \alpha}}{\sin \theta}
$$

Le tableau 6.1 liste les images SPOT5 acquises au cours de l'été 2004 sur la zone de Grímsvötn. Les empreintes au sol de ces images sont reportées sur la figure 6.2. Les scènes du 11 et du 16 août sont adaptées à la mesure des déplacements, celles de début octobre à l'élaboration d'un MNT.

TAB. 6.1 - Caractéristiques des images SPOT5 utilisées pour mesurer le soulèvement de la plateforme de Grímsvötn.

\begin{tabular}{lccrr}
\hline Couples d'images & Résolution & Angles d'incidence & $B / H$ & $\delta \mathrm{t}$ \\
\hline $11 \& 16$ Aug. 04 & $2.5 \mathrm{~m}$ & $-27 /-26.7$ & 0.064 & 5 \\
$07 \& 09$ Oct. 04 & $5 \mathrm{~m}$ & $-26.6 / 24.6$ & 0.95 & 2 \\
\hline
\end{tabular}

Avec des angles d'incidence de $27^{\circ}$, la sensibilité au mouvement vertical est de : $\sin 27=0.45$. Autrement dit, un déplacement vertical d'un mètre se traduira par un décalage en colonne de $0.45 \mathrm{~m}$.

Parce que les images ne sont pas acquises exactement du même point de vue (cf. rapport $\mathrm{B} / \mathrm{H}$ du tableau 6.1), les erreurs du MNT perturbent la mesure du déplacement. Une erreur topographique de $10 \mathrm{~m}$ engendre en effet un déplacement horizontal de $0.64 \mathrm{~m}$ dans la direction des colonnes soit une erreur de $0.64 / 0.45=1.4 \mathrm{~m}$ sur le déplacement vertical. C'est l'ordre de grandeur du signal recherché. Comme la zone étudiée connaît d'importants changements morphologiques liés à l'ablation en surface, aux éruptions volcaniques et à la dynamique glaciaire (Magnússon et al., 2005), un MNT contemporain est indispensable. Il est construit à partir d'images SPOT5 acquises à deux jours d'intervalle, début octobre 2004.

L'image du 16 août est projetée dans la géométrie de l'image du 11 août en utilisant la méthodologie décrite dans le chapitre 4. Elles sont ensuite corrélées pour mesurer les déplacements dans la direction des colonnes et des lignes. En ligne, le déplacement moyen sur la plateforme de glace est faible $(14 \mathrm{~m} / \mathrm{a})$ et dirigé vers le Sud ce qui est en accord avec les mesures de terrain. En colonne, un biais à grande longueur d'onde existe entre les images. Les régions non englacées communes aux deux images (d'où sont extraits les points homologues) sont réduites et limitées à la partie Sud ce qui peut expliquer une superposition qui n'est pas parfaite. Pour éliminer ce biais, nous supposons que, durant les 5 jours séparant les deux images SPOT5, le déplacement en colonne pour les zones qui entourent la plateforme est faible. Un polynôme de $1^{\text {er }}$ ordre (de la forme $\mathrm{a} \times \mathrm{COL}+\mathrm{b} \times \mathrm{LIG}+\mathrm{c}$ ) modélise correctement ce biais et permet de le supprimer.

La figure 6.6 montre les déplacements verticaux du lac de Grímsvötn durant 5 jours. Cette carte est bruitée car le signal mesuré (déplacement en colonne) est de l'ordre de $0.75 \mathrm{~m}$, à peine plus que la précision de $0.5 \mathrm{~m}$ déduite des mesures sur les glaciers alpins (chapitre 4). A partir de cette carte, la zone affectée par le soulèvement est délimitée et refléte la superficie du lac sousglaciaire, $10.9 \mathrm{~km}^{2}$. Le soulèvement moyen de la plateforme est de $1.71 \pm 0.6 \mathrm{~m}$ soit $0.34 \mathrm{~m} /$ jour. Ceci traduit l'addition de $0.018 \mathrm{~km}^{3}$ d'eau dans le lac sous-glaciaire. 

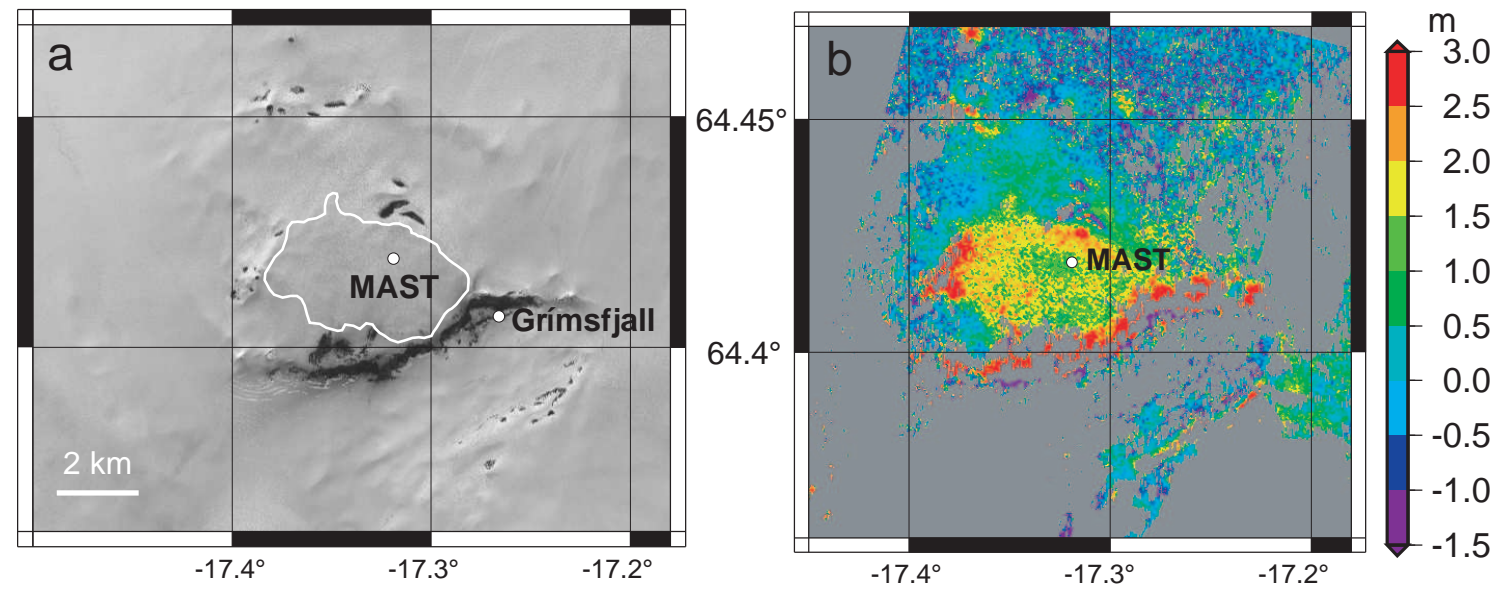

FIG. 6.6 - Limites du lac de Grímsvötn reportées sur l'image SPOT du 11 août 2004 (à gauche) et carte des déplacements verticaux (en mètres) pour la période du 11 au 16 août (à droite).

Les relevés de terrain du Science Institute devraient permettre de tester l'exactitude de la mesure satellitaire. Une station (nommée MAST par les islandais voir figure 6.1) est installée sur la plateforme de glace. Cette station (haute de 6 à $9 \mathrm{~m}$ pour éviter l'ensevelissement sous la neige!) est équipée d'un GPS standard ${ }^{6}$ et d'un ensemble thermomètre/baromètre. La différence des pressions entre MAST et la station stable de Grímsfjall (à $3 \mathrm{~km}$ au sud-est) est convertie en différence d'altitude. Ces deux mesures (GPS et barométrique) sont affectées par de fortes incertitudes à cause, notamment, des conditions atmosphériques extrêmes (figure 6.7).

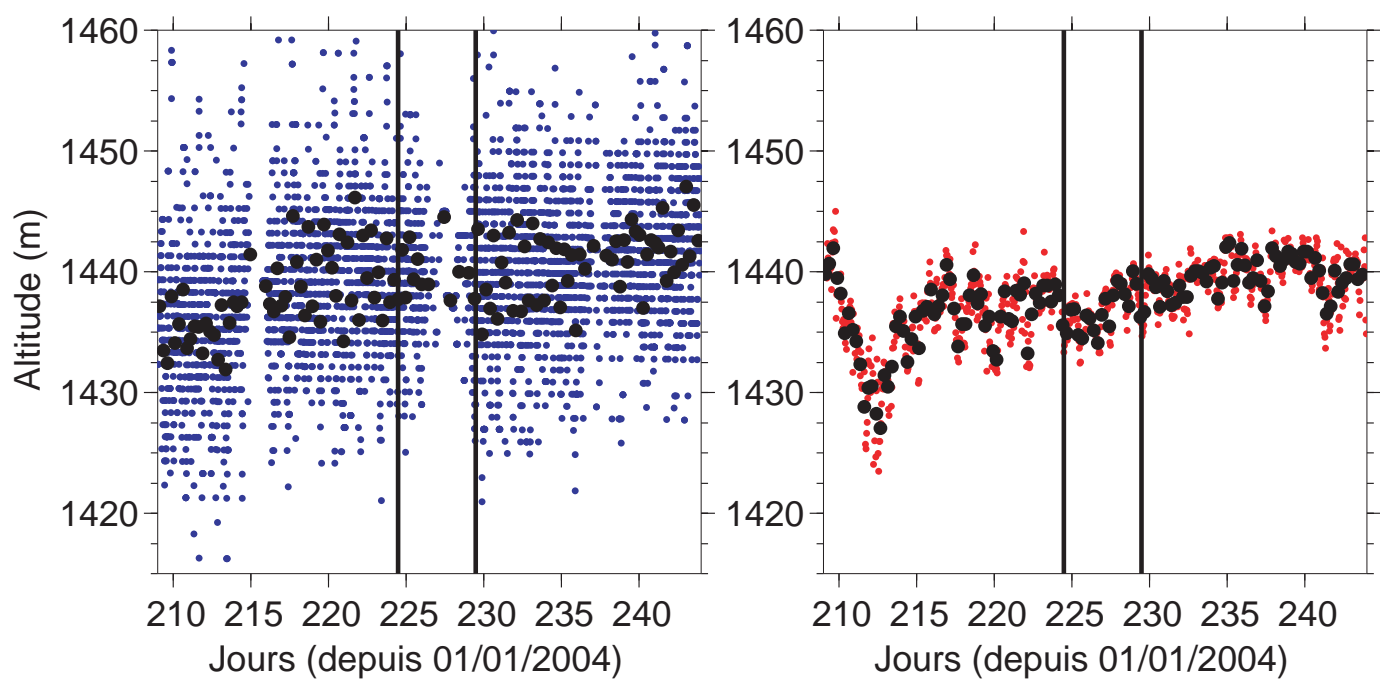

FIG. 6.7 - Altitude de la station MAST (sur la plateforme de Grímsvötn) entre le 27 Juillet et le 31 août 2004 mesurée par un GPS standard (à gauche) et déduite de mesures de pression atmosphérique (à droite). Les ronds noirs indiquent les moyennes toutes les 6 heures. La même échelle verticale est utilisée pour les deux courbes pour faciliter la comparaison. Les traits verticaux indiquent les dates d'acquisition des 2 images SPOT5.

\footnotetext{
${ }^{6}$ c'est à dire non différentiel
} 
Pour les deux séries (même en moyenne toutes les 6 heures), la dispersion des valeurs est forte. Pour la période du 11 au 16 août, le soulèvement est de $+0.78 \mathrm{~m} / \mathrm{j}$ d'après les mesures barométriques, $-0.25 \mathrm{~m} / \mathrm{j}$ d'après le GPS alors qu'à cet endroit précis la mesure satellitaire indique un soulèvement de $+0.23 \mathrm{~m} / \mathrm{j}$. Ces divergences entre les mesures in situ confirment qu'elles sont peu précises pour de courtes périodes de temps. C'est seulement en observant une période suffisamment longue (35 jours) que les 3 mesures (GPS, barométrique et satellitaire) deviennent statistiquement non différentes (tableau 6.2). Sur une durée de quelques jours, la mesure satellitaire s'avère donc plus précise que les mesures in situ.

TAB. 6.2 - Comparaison des taux de soulèvement ( $m / j$ jour) à la station MAST pour différents intervalles de temps. La mesure SPOT5 conduit à $+0.23 \pm 0.1 \mathrm{~m} /$ jour entre le 11 et le 16 août. Les taux de soulèvement mesurés par les différents instruments étant indépendants, l'incertitude sur la différence de deux taux ( $x$ et $y$ ) vaut : $\sigma_{x-y}=\sqrt{\sigma_{x}{ }^{2}+\sigma_{y}{ }^{2}}$. A l'intervalle de confiance de $66 \%$ (respectivement 90\%), deux taux ne sont pas significativement différents si leur différence est plus petite que $\sigma_{x-y}$ (respectivement ${ }^{2}{ }^{*} \sigma_{x-y}$ ). Ces cas apparaissent en gras.

\begin{tabular}{lcccc}
\hline & 11-16 Août & 6-21 Août & 1-26 Août & 27 Juil-31 Août \\
\hline$\delta$ t (jour) & 5 & 15 & 25 & 35 \\
\hline SPOT5 & $0.23 \pm 0.1$ & & & \\
\hline Baro & $0.78 \pm 0.32$ & $0.24 \pm 0.06$ & $0.19 \pm 0.03$ & $0.19 \pm 0.02$ \\
\hline GPS & $-0.25 \pm 0.6$ & $-0.02 \pm 0.1$ & $0.05 \pm 0.05$ & $0.18 \pm 0.03$ \\
\hline Baro - GPS & $1.03 \pm 0.68$ & $0.26 \pm 0.12$ & $0.14 \pm 0.06$ & $\mathbf{0 . 0 1} \pm \mathbf{0 . 0 3}$ \\
\hline SPOT5 - Baro & $-0.55 \pm 0.33$ & $\mathbf{0 . 0 1} \pm \mathbf{0 . 1 2}$ & $\mathbf{0 . 0 4} \pm \mathbf{0 . 1}$ & $\mathbf{0 . 0 3} \pm \mathbf{0 . 1}$ \\
\hline SPOT5 - GPS & $0.48 \pm 0.61$ & $0.25 \pm 0.14$ & $0.18 \pm 0.11$ & $\mathbf{0 . 0 5} \pm \mathbf{0 . 1}$ \\
\hline
\end{tabular}

Des données in situ trop imprécises ne permettent pas d'évaluer l'exactitude de notre mesure satellitaire. Nous pouvons simplement conclure à un bon accord entre les différents taux de soulèvement lorsque les données de terrain sont analysées sur une période suffisamment longue.

\subsubsection{Crue potentielle, bilan énergétique et activité géothermique}

Notre mesure de l'extension du lac et de son remplissage durant ces 5 jours est intéressante à double titre.

Tout d'abord, elle permet d'estimer le volume maximal du jökulhlaup potentiel. Ces crues engendrent des destructions importantes et il est utile de prévoir leur ampleur (Björnsson, 2002). Une limite supérieure du volume du lac $\left(\mathcal{V}_{l a c}\right)$ est obtenue en faisant l'hypothèse que ses bords sont verticaux :

$$
\mathcal{V}_{\text {lac }}=\left(\mathcal{Z}_{\text {plateforme }}-\mathcal{Z}_{\text {pose }}\right) \times \mathcal{S}_{\text {SPOT5 }}
$$

où $\mathcal{Z}_{\text {plateforme }}$ est l'altitude de la plateforme, $\mathcal{Z}_{\text {pose }}$ l'altitude de la plateforme quand elle est posée (ligne tiretée sur la figure 6.4) et $\mathcal{S}_{S P O T 5}$ la surface du lac estimée par la corrélation d'images 
SPOT5. Mi août 2004, ce volume s'élevait à $0.74 \mathrm{~km}^{3}$. Cette estimation pourrait être affinée en prenant en compte la géométrie complexe du lit rocheux.

D'autre part, notre mesure du soulèvement permet de dresser un bilan énergétique du lac et d'estimer le flux de chaleur moyen du volcan de Grímsvötn quelques semaines seulement avant son éruption. En 5 jours, $0.018 \mathrm{~km}^{3}$ d'eau se sont donc accumulés. L'ablation en surface pour cette période estivale est estimée à $1.3 \mathrm{~cm} /$ jour (données Science Institute). Le lac de Grímsvötn draine les eaux d'un bassin versant de $200 \mathrm{~km}^{2}$. Ainsi, environ $0.013 \mathrm{~km}^{3}$ d'eau de fonte en surface ont contribué au remplissage du lac. Les $0.005 \mathrm{~km}^{3}$ restant s'expliquent par la fonte de la base du glacier. Si la glace basale de la plateforme fondait, c'est une subsidence que l'on devrait observer $\left(\rho_{\text {glace }}<\rho_{\text {eau }}\right)$. C'est donc la base du glacier autour du lac qui fond. Cette zone de fonte couvre 50 à $60 \mathrm{~km}^{2}$ (Björnsson \& Guðmundsson, 1993).

La calotte du Vatnajökull étant tempérée (glace au point de fusion), la puissance $\mathcal{P}$ nécessaire pour faire fondre une masse $\mathcal{M}_{\text {glace }}$ de glace est :

$$
\mathcal{P}=\frac{\mathcal{M}_{\text {glace }} \times \mathcal{C}_{p}}{\delta t}
$$

où $\mathcal{C}_{p}$ est la chaleur latente de fusion de la glace $\left(334 \mathrm{~J} \mathrm{~kg}^{-1}\right.$ d'après Paterson $(1994, \mathrm{p}$ 58)) Cette puissance atteint près de $4000 \mathrm{MW}$ ce qui correspond à un flux moyen de chaleur ${ }^{7}$ de 65 à $80 \mathrm{~W} \cdot \mathrm{m}^{-2}$ (Ragnarsson \& Helgason, 2004). Cette valeur est supérieure au flux moyen calculé par Björnsson \& Guðmundsson (1993) sur la période 1960-91 : $40 \mathrm{~W} \cdot \mathrm{m}^{-2}$. Ce flux géothermique presque doublé pourrait s'expliquer par la reprise de l'activité du volcan de Grímsvötn, quelques semaines avant son éruption. Toutefois, notre estimation est entachée de fortes incertitudes puisqu'elle cumule les erreurs sur l'estimation du soulèvement, de l'ablation de surface et de l'extension de la zone responsable de la fusion basale.

\subsubsection{Perspectives et implications de cette mesure}

La corrélation d'images SPOT5 à $2.5 \mathrm{~m}$ de résolution permet donc de mesurer des mouvements verticaux avec une précision de l'ordre de $0.5 \mathrm{~m}$.

Cette technique offre des perspectives intéressantes pour l'étude des phénomènes hydrologiques sous-glaciaires. La plateforme de Grímsvötn constitue un site privilégié à cause de la rapidité des mouvements qui l'affectent. Après 5 jours seulement, le soulèvement est suffisant pour être mesurable. Notre méthode pourrait être utilisée pour détecter de nouvelles zones d'activité géothermique sous-glaciaire ou quantifier l'intensité du flux de chaleur de zones déjà connues (comme le glacier du Mýrdalsjökull, au Sud de l'Islande, lui aussi installé sur un volcan actif [Björnsson, communication personnelle]). Actuellement, la précision semble insuffisante pour mesurer les mouvements verticaux de quelques dizaines de centimètres d'amplitude qui accompagnent les variations du contenu et les transferts d'eau à la base des glaciers de montagne ou émissaires des calottes polaires (Iken et al., 1983; Gray et al., 2005). Ceci devrait par contre être possible avec des missions futures : les capteurs optiques sur ORFEO-PLEIADE auront par exemple une résolution de $0.7 \mathrm{~m}$. Si une simple règle de trois s'applique, la résolution 4 fois plus haute que pour SPOT5 devrait abaisser à environ $\pm 10 \mathrm{~cm}$ la précision sur la mesure des mouvements verticaux.

\footnotetext{
${ }^{7}$ Pour fixer les idées, le flux de chaleur moyen à la surface de la Terre est de $82 \mathrm{~mW} \mathrm{~m}^{-2}$ alors que celui de l'Islande est de $290 \mathrm{~mW} \mathrm{~m}^{-2}$
} 
Notre étude confirme également que la corrélation d'images optiques fonctionne sur les zones enneigées. La plateforme de Grímsvötn se situe en effet au-dessus de la ligne de neige même au mois d'août. Le principal facteur limitant est l'écart de temps entre les images : la probabilité d'une chute de neige, de modifications de la surface par le vent ou la fonte augmente avec la séparation temporelle. Ainsi, suite à des chutes de neige, la corrélation a échoué sur cette zone entre l'image du 16 août et une image acquise le 26 septembre, 40 jours plus tard. A cause de cette décorrélation, c'est la technique des MNT différentiels (chapitre 5) qui est la plus adaptée pour étudier des mouvements verticaux qui se produisent sur le long terme. Dans la partie suivante, elle est appliquée à la partie ouest du Vatnajökull.

\subsection{Variations volumétriques du Vatnajökull}

L'équipe islandaise du Science Institute a déjà à sa disposition plusieurs topographies du Vatnajökull. En les comparant, Magnússon et al. (2005) ont pu quantifier les changements d'épaisseur de la partie Ouest de la calotte glaciaire pour la période allant du milieu des années 1980 à 1998. Les pertes moyennes d'épaisseur sont de $1 \mathrm{~m} /$ a mais leur distribution spatiale est complexe, résultat de l'activité géothermique, des surges et des hétérogénéités du bilan de masse. Nous souhaitons ici prolonger cette étude afin d'étudier la réponse topographique à long terme du glacier à ces multiples perturbations. Pour cela, nous comparons un MNT construit à partir d'images SPOT5 de 2004 avec des topographies de 1997 et 1998.

\subsubsection{Les topographies disponibles sur la calotte (figure 6.8)}

\subsubsection{La topographie de 1997 : MNT-AERIEN}

Cette topographie a été réalisée à partir de photographies aériennes acquises en août 1997 (Münzer et al., 1999). Elle couvre la majorité du glacier de Skeiðarárjökull et remonte au Nord jusqu'aux zones de Grímsvötn et de Gjálp. Sa précision est de $\pm 1 \mathrm{~m}$ dans les zones basses. A plus haute altitude, des nuages masquent la plateforme de Grímsvötn et le faible contraste des photographies aériennes altère la précision. Pour notre étude, nous n'avions à notre disposition que la partie basse du Skeiðarárjökull.

\subsubsection{La topographie de 1998 : MNT-EMISAR}

En août 1998, des images SAR aéroportées (capteur EMISAR danois) ont été acquises sur la partie Ouest du Vatnajökull. Leur traitement interférométrique a permis la réalisation d'un MNT à $5 \mathrm{~m}$ de résolution (Magnússon, 2003). Le bruit de ce MNT est assez important sur les zones de haute altitude. La comparaison avec des mesures GPS cinématique in situ indique également des erreurs systématiques, atteignant jusqu'à $10 \mathrm{~m}$. Ces biais ont été corrigées (grâce aux relevés GPS) et les biais résiduels ne doivent pas excéder $1 \mathrm{~m}$ (Magnússon et al., 2004).

\subsubsection{La topographie de 2004 : MNT-SPOT5}

Les deux images SPOT5 acquises début octobre 2004 (tableau 6.1) sont utilisées pour construire un MNT en suivant la même méthodologie que pour les Alpes et l'Himalaya (chapitre 5). Seules 
des images de résolution $5 \mathrm{~m}$ (au lieu de $2.5 \mathrm{~m}$ ) ont été acquises (problème de programmation par Spotimage). Des nuages expliquent l'absence de valeurs dans la partie Sud-Ouest, sur la langue terminale du Síðujökull.

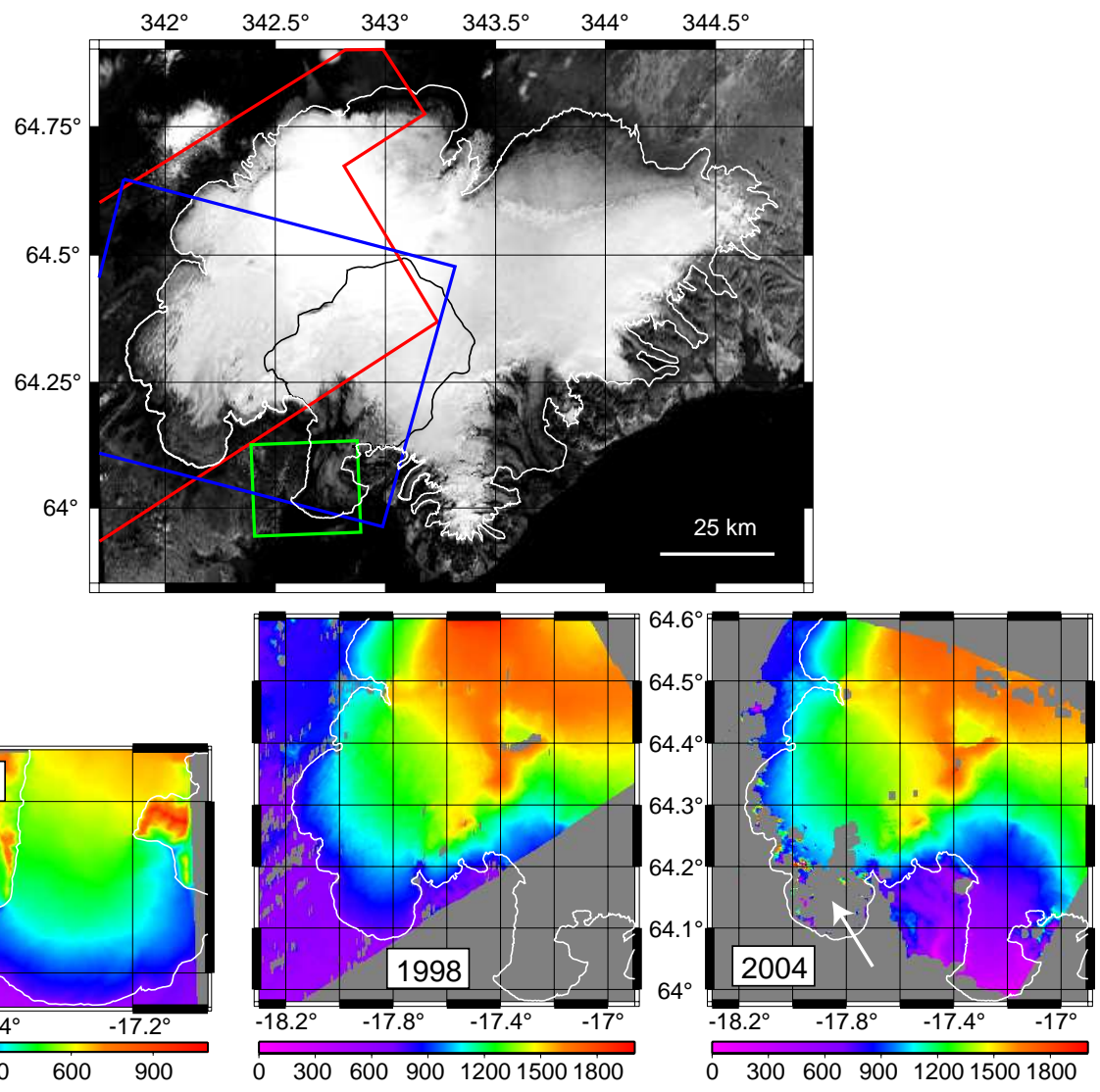

FIG. 6.8 - Les trois topographies de la calotte du Vatnajökull à notre disposition : 1997, 1998 et 2004. Leur limites ont été localisées sur une image Landsat : en vert 1997, en rouge 1998 et en bleu 2004. Les limites de la calotte (trait blanc) et du bassin versant du Skeiðarárjökull (trait noir) ont été ajoutées. Pour 2004, une flèche pointe vers la partie basse du Siðujökull où des nuages ont empêché le calcul du MNT.

Seuls quelques points de contrôle (GCP) sont disponibles. De plus, leur répartition n'est pas homogène car le glacier occupe la grande majorité des images. Ceci explique un biais dans le MNT (le modèle stéréo des images n'est pas bien contraint). Ce biais est visible lorsqu'on compare le MNT SPOT5 à des mesures de GPS cinématique réalisées fin septembre 2004 à bord de voitures parcourant le glacier. La topographie SPOT5 est trop basse au Nord, trop haute au Sud.

Pour le moment, ce biais a été corrigé en le modélisant par un polynôme de premier ordre. Mais cette approche n'est pas satisfaisante notamment parce qu'il n'y a pas de mesure GPS cinématique dans la partie la plus sud du glacier. Pour éviter les incertitudes liées à cette correction, nous espérons recalculer un MNT non biaisé en mesurant, sur le terrain, des points de contrôle mieux répartis dans les images.

Une autre source d'erreur dans le MNT est le changement des conditions d'illumination entre les deux images. Le 7 octobre, l'image est acquise à $10 \mathrm{~h} 23$ (heure locale). Le soleil se situe $19.7^{\circ}$ 
au dessus de l'horizon (azimut $=\mathbf{1 7 0 . 9}^{\circ}$ ). Le 9 octobre (acquisition à $11 \mathrm{~h} 25$ ), le soleil se situe $19^{\circ}$ au dessus de l'horizon (azimut $=\mathbf{1 8 7}^{\circ}$ ). Ces rayons solaires rasant aux azimuts différents créent des ombres importantes et d'orientations différentes sur les deux images, donc des erreurs dans la topographie. Notons que si les images étaient acquises à la même heure, les ombres seraient au contraire un avantage accroissant le contraste radiométrique sur le glacier.

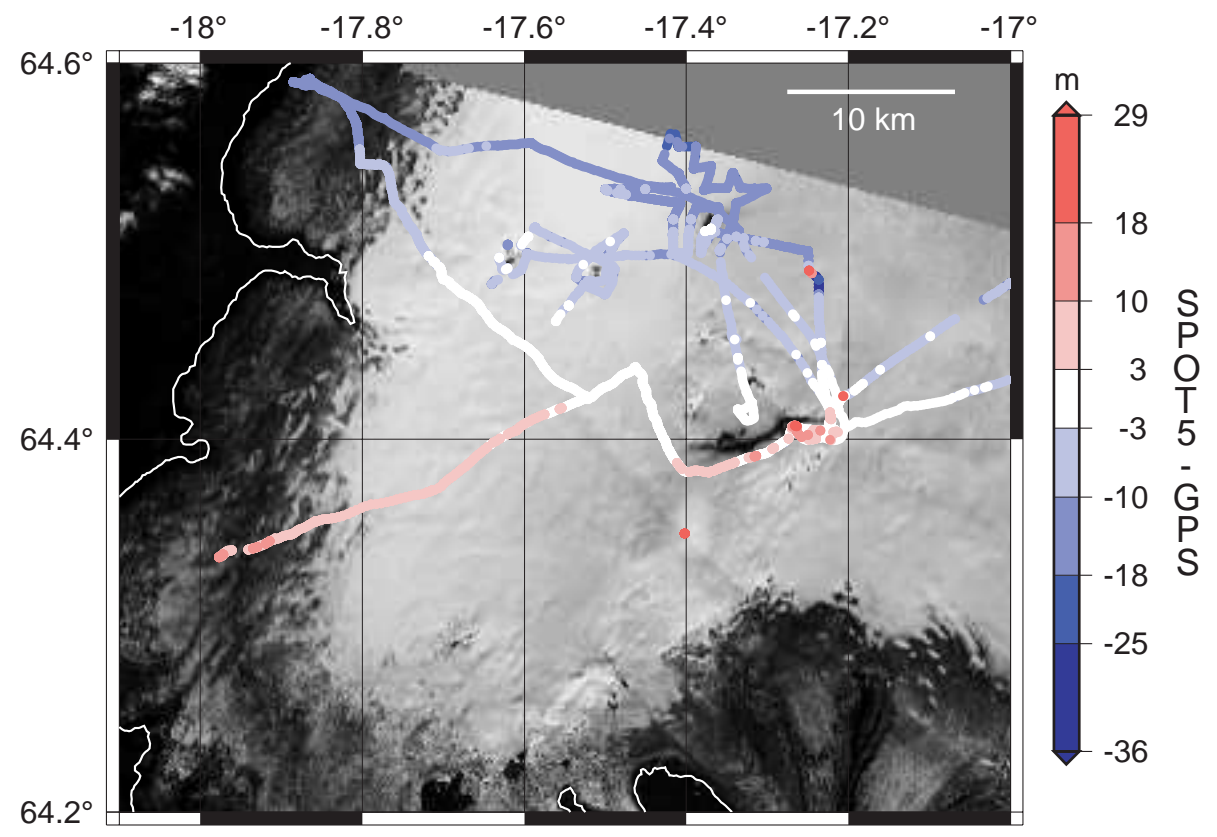

Fig. 6.9 - Différence entre le MNT-SPOT5 et des relevés GPS cinématiques en voiture. En arrière plan, une image SPOT5 d'août 2004, en blanc les limites de la calotte.

Lorsqu'on compare le MNT SPOT5 aux 13000 mesures de GPS cinématique, il est $4 \mathrm{~m}$ trop bas (écart type $=10.9 \mathrm{~m}$ ). Ceci doit être gardé à l'esprit lors de la comparaison du MNT SPOT5 à des topographies plus anciennes : les gains d'épaisseur sont sous-estimés et les pertes surestimées.

\subsubsection{Observation et origine des variations d'épaisseur}

La figure 6.10 compare les variations d'épaisseur pour les périodes 1985 à 1998 (à gauche) et 1998 à 2004 (à droite).

\subsubsection{Effets climatique et dynamique}

Pour la période 1985 à 1998, les glaciers émissaires de l'Ouest de la calotte s'amincissent à haute altitude et s'épaississent sur leurs bords. La particularité de ces glaciers est d'avoir connu des surges dans les années 1990 : 1994 pour Síðujökull, 1994 pour Tungnaárjökull et 1996 pour Sylgjujökull. Ces brutales accélérations des glaciers s'accompagnent d'un important transfert de masse vers les zones basses expliquant les variations d'épaisseur observées (Magnússon et al., 2005). Pour le glacier de Köldukvíslarjökull (surge en 1992), il est surprenant de ne pas observer d'amincissement à haute altitude. 

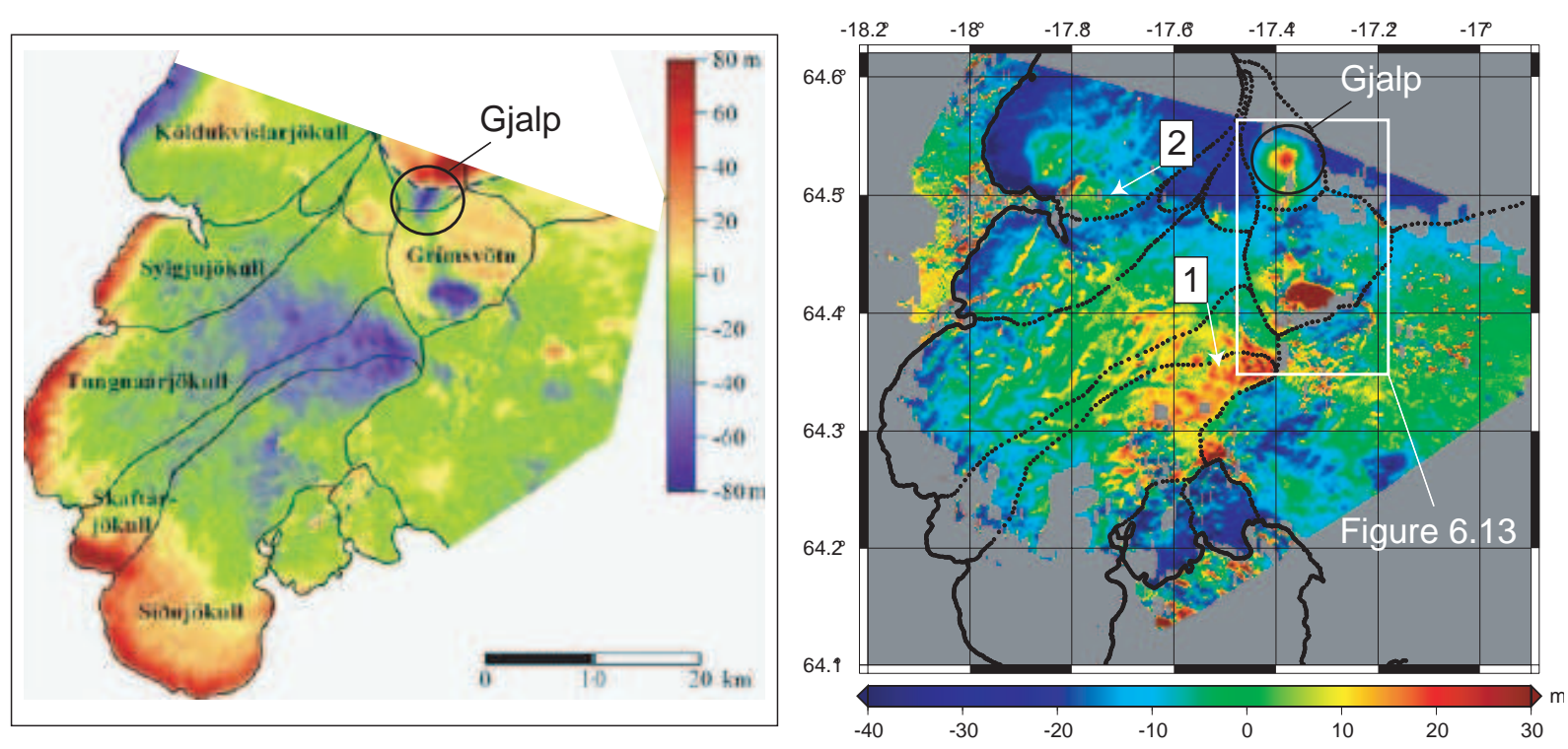

FIG. 6.10 - Cartographie des variations d'épaisseur de la partie Ouest du Vatnajökull pour 1985 à 1998 (à gauche, extrait de Magnússon et al. (2005)) et 1998 à 2004 (à droite).
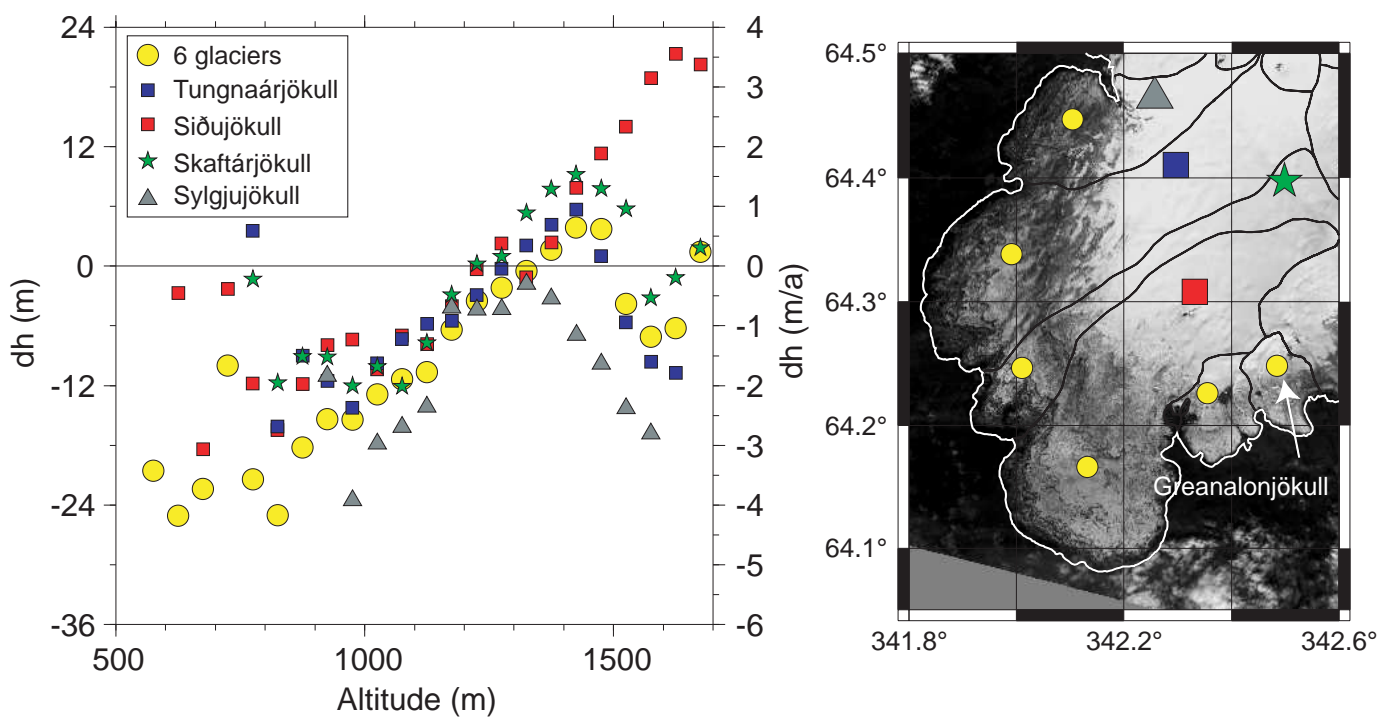

FIG. 6.11 - Variations d'épaisseur en fonction de l'altitude pour différents glaciers émissaires de l'Ouest de la calotte du Vatnajökull. Les ronds jaunes correspondent aux valeurs moyennes pour 6 glaciers : Grcenalónsjökull et le glacier juste à l'Est, Siðujökull, Skaftárjökull, Tungnaárjökull, Sylgjujökull. Les zones basses (moins de $500 \mathrm{~m}$ ) du Siðujökull sont mal échantillonnées à cause des nuages. L'image SPOT5 de droite permet de localiser les différents glaciers.

Pour la période récente, l'évolution est opposée : les pertes d'épaisseur sont importantes (20 à $30 \mathrm{~m}$ ) dans les zones marginales de la calotte alors que dans le bassin d'accumulation (zone notée 1), l'épaississement atteint une vingtaine de mètres. C'est une évolution post-surge logique, la glace véhiculée à basse altitude est sujette à une forte ablation alors que dans les zones hautes, le ralentissement du glacier combiné à l'accumulation conduisent à son épaississement. Ce sont 
donc à la fois la dynamique et les facteurs climatiques (affectant l'ablation et l'accumulation) qui conditionnent l'évolution géométrique de la calotte. Pour le glacier de Köldukvíslarjökull, on remarquera une zone à l'équilibre, voire qui s'épaissit (zone notée 2), effet retardé probable du surge de 1992.

Pour ces différents glaciers, les variations d'épaisseur en fonction de l'altitude ont également été calculées (figure 6.11). Le biais du MNT SPOT5 étant surtout fort dans la partie Nord du MNT, on se contente d'étudier les glaciers les plus au Sud. Ces courbes confirment l'amincissement à basse altitude et l'épaississement en zone d'accumulation. Logiquement, c'est le glacier qui s'était le plus aminci (le Síðujökull) entre 1985 et 1998 qui connaît le plus fort épaississement.

Un MNT différentiel est aussi construit pour la période 1997-2004. A cause de la faible extension du MNT de 1997 (figure 6.8), seule la langue terminale du Skeiðarárjökull peut être étudiée (figure 6.12).

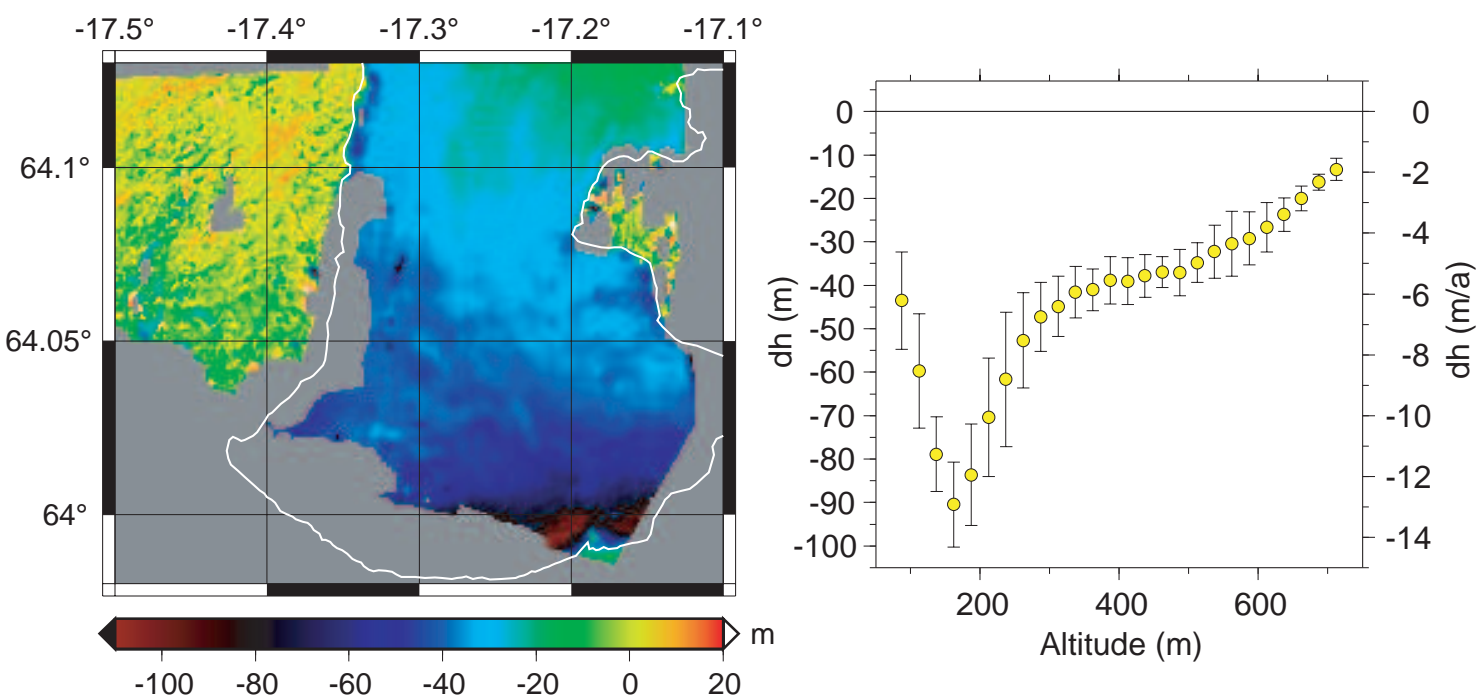

FIG. 6.12 - Pertes d'épaisseur sur la langue du Skeiðarárjökull entre 1997 et 2004. A gauche, carte des variations d'épaisseur avec en blanc les limites du glacier. A droite, les variations d'épaisseur sont représentées en fonction de l'altitude.

Notons tout d'abord que nous n'échantillonnons que $10 \%\left(150 \mathrm{~km}^{2}\right)$ de la surface totale de cet immense glacier (environ $1400 \mathrm{~km}^{2}$, voir son bassin versant sur la figure 6.8). Le bilan de masse ne peut donc pas être estimé. Le MNT différentiel couvre, en revanche, $70 \%$ de la surface entre 75 et $725 \mathrm{~m}$ d'altitude. L'observation des zones hors du glacier souligne la bonne superposition des deux MNT et l'absence de biais importants. L'amincissement est fort avec des taux moyens de $6 \mathrm{~m} / \mathrm{a}$ et des taux maximums qui approchent $15 \mathrm{~m} / \mathrm{a}$. Vers $600 \mathrm{~m}$ d'altitude, les pertes d'épaisseurs sont de $4 \mathrm{~m} /$ a environ ce qui est du même ordre de grandeur que pour les glaciers plus à l'Ouest (figure 6.11). Malgré des effets importants de la dynamique, il existe une cohérence régionale des pertes d'épaisseur glaciaire en réponse à un signal climatique identique.

En faisant l'hypothèse que toute la calotte du Vatnajökull subit les mêmes pertes d'épaisseur (ce qui reste à démontrer), il est possible d'estimer les pertes annuelles de glace. Pour cela, on combine la courbe moyenne d'amincissement en fonction de l'altitude avec la courbe hypsométrique de tout le glacier. $3 \%$ de la surface totale du glacier $\left(275 \mathrm{~km}^{2}\right)$ se trouve au dessus de $1700 \mathrm{~m}$, où nous n'avons pas d'estimation des variations d'épaisseur. Cette zone n'est donc pas 
prise en compte. Au total, nous estimons à $10,7 \mathrm{~km}^{3}$ les pertes de masse annuelles de la calotte. Cela représente $0.03 \mathrm{~mm} / \mathrm{a}$ d'élévation du niveau marin.

\subsubsection{Effet de l'activité géothermique sous-glaciaire}

Un détail des variations d'épaisseur entre 1998 et 2004 dans la partie centrale de la calotte permet d'observer l'effet des activités géothermique et volcanique sous-glaciaires (figure 6.13). La zone de la plateforme de Grímsvötn (étudiée dans le §6.2) est clairement visible. Entre août 1998 et octobre 2004, elle s'est soulevée d'une cinquantaine de mètres. Cette évolution masque en fait plusieurs cycles de remplissage et vidange du lac de Grímsvötn (figure 6.4).
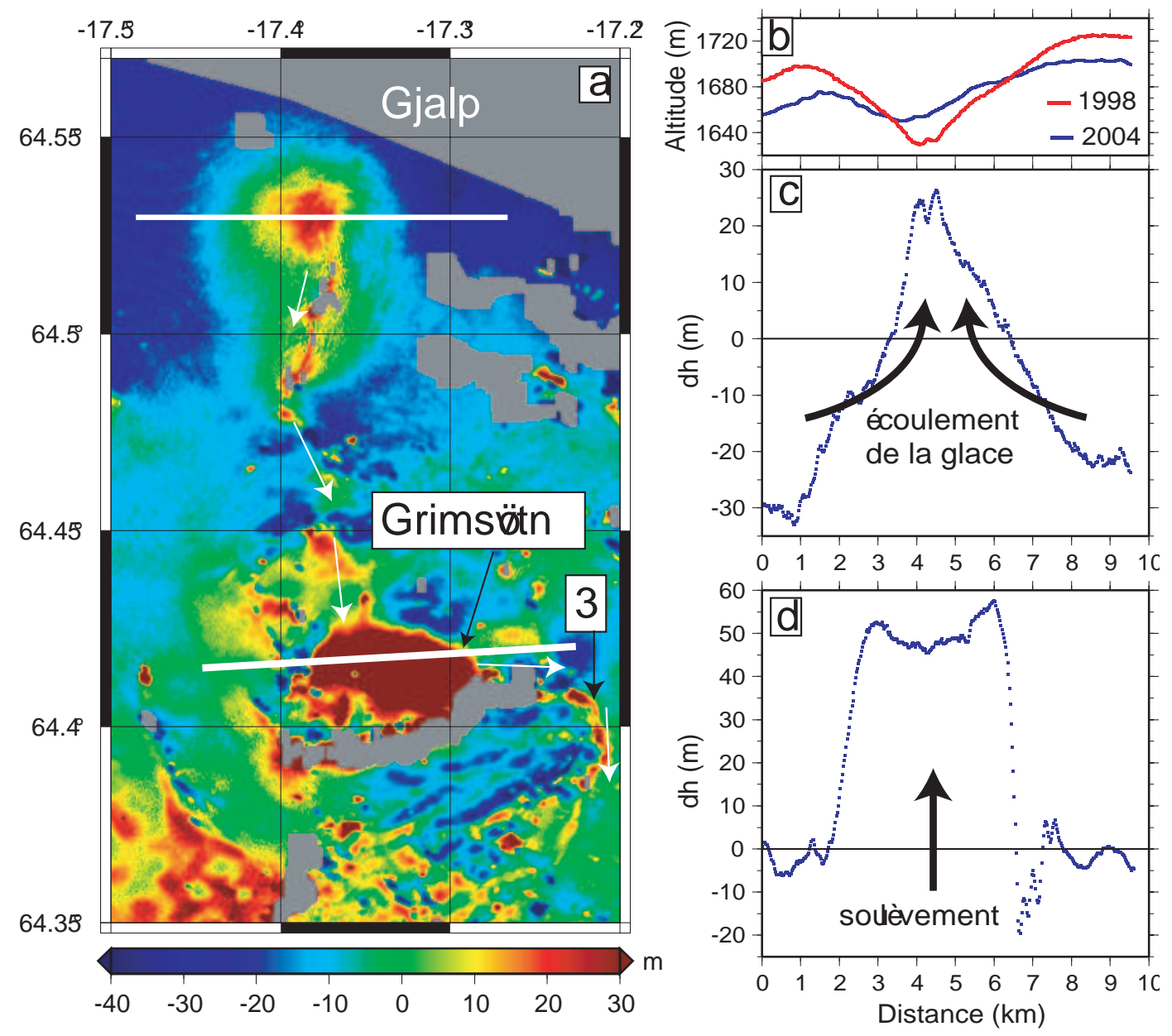

Fig. 6.13 - Variations d'épaisseur dans les zones de Gjálp et Grímsvötn. Les flèches blanches indiquent le trajet des eaux de fonte lors de l'éruption de Gjálp en 1996 (a). Profils Ouest-Est de la topographie (b) et des variations d'épaisseur (c) pour la zone de Gjálp et des changements d'altitude de la plateforme de Grímsvötn (d).

Certaines structures de cette carte sont liées à l'éruption sous-glaciaire de Gjálp (Octobre 1996). Cette éruption a creusé une large dépression au Nord de Grímsvötn (zone d'amincissement en bleu sur la partie droite de la figure 6.10). Pour la période 1998-2004, c'est un épaississement (25 mètres au maximum) qui est observé. Ceci traduit l'écoulement de la glace vers la dépression 
(Björnsson et al., 2001b; Guðmundsson et al., 2002a) qui se comble progressivement. Le fort amincissement observé en périphérie de cette zone s'explique en partie par cet écoulement du glacier. Mais le biais du MNT SPOT5 est également fort dans cette région, la plus éloignée de tous nos points d'appui.

Plus au Sud on observe également le remplissage (par la glace) d'un canyon (zone notée 3) qui avait été creusé par les eaux de fonte lors de l'éruption de 1996 (Guðmundsson et al., 1997; Aðalgeirsdottír et al., 2000).

Le principal intérêt des mesures que nous ajoutons (MNT SPOT5 de 2004) à celles de Magnússon et al. (2005) est qu'elles permettent d'étudier la réponse à long terme du glacier après des perturbations importantes. Nous mesurons ainsi l'épaississement progressif de la zone d'accumulation dans la phase post-surge. Le remplissage par la glace de dépressions créées lors des éruptions volcaniques est aussi clairement identifiable sur le MNT différentiel.

En moyenne, pour les différents glaciers émissaires du versant Ouest de la calotte (couvrant plus de $1100 \mathrm{~km}^{2}$ ), le bilan de masse entre 1998 et 2004 est de $-1.2 \mathrm{~m} / \mathrm{a}$. Nous comparerons en conclusion (chapitre 7), ces bilans de masse islandais à ceux des glaciers alpins et himalayens.

\subsection{Conclusions}

Une limitation forte à l'utilisation des images optiques sur l'Islande est la fréquente couverture nuageuse et le faible éclairement d'octobre à mars. La couverture neigeuse persistante jusqu'en juin (au moins) limite finalement la fenêtre d'acquisition à la période juillet/mi-octobre. Cette fenêtre est restreinte mais, au bout du compte, nous avons toujours obtenu des images satisfaisantes à une exception près. Nous aurions en effet souhaité étudier l'impact du Jökulhlaup de fin octobre 2004 (vidange du lac de Grímsvötn) sur l'écoulement du Skeiðarárjökull en obtenant une image supplémentaire courant novembre 2004. Mais l'éclairement solaire s'est révélé trop faible (Spotimage, communication personnelle). La corrélation d'images d'amplitude SAR ou la technique du speckle tracking pourraient permettre à l'avenir de mener cette étude (Michel \& Rignot, 1999; Strozzi et al., 2002).

Pour cette calotte glaciaire, la difficulté principale dans le traitement des images n'est pas une topographie abrupte comme dans les Alpes et l'Himalaya. C'est surtout la faible proportion des zones rocheuses stables par rapport aux zones englacées qui engendrent des biais dans les MNT et dans les cartes de vitesses de surface. Les données du Science Institute ont été importantes pour mettre en évidence et, lorsque c'était possible, corriger ces biais. Une fois de plus, les approches in situ et satellitaires sont complémentaires.

La diversité de nos outils utilisant les images SPOT5 révèle des aspects variés, encore mal connus de la dynamique de cette calotte. L'extension et le bilan énergétique d'un lac sousglaciaire ont ainsi pu être déterminés. L'effet morphologique des surges avait déjà été observé par Magnússon et al. (2005). Nous ajoutons ici la réaction sur le long terme qui montre que les zones de haute altitude s'épaississent rapidement dans la phase post-surge.

Un travail important et passionnant reste à faire dans l'interprétation conjointe des évolutions géométrique et dynamique de la calotte. La construction de champs de vitesse des glaciers

émissaires par corrélation d'images SPOT5 (mais aussi LANDSAT et ASTER) et leur utilisation pour tester et contraindre des modèles d'écoulement ont déjà débuté (Björnsson et al., 2005). 



\section{Chapitre 7}

\section{Synthèse et perspectives}

\section{Sommaire}

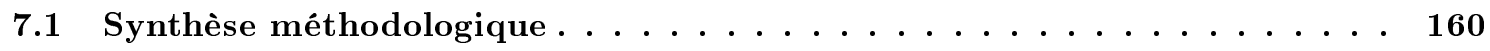

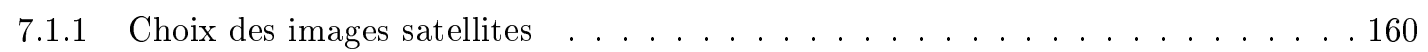

7.1 .2 Mouvements horizontaux des glaciers . . . . . . . . . . . . . . . . . 160

7.1 .3 Variations d'altitude de la surface des glaciers . . . . . . . . . . . . . . . . 161

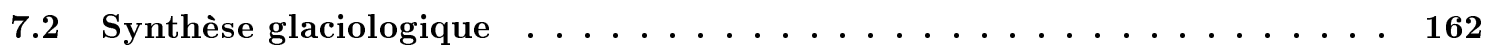

7.2 .1 Importance de l'hydrologie sous-glaciaire . . . . . . . . . . . . . . . . . 162

7.2 .2 Adaptation dynamique des glaciers aux changements climatiques . . . . . . . . . . . . . . 164

7.2 .3 Évolution comparée des glaciers alpins, himalayens et islandais . . . . . . . . . . 167

7.3 Perspectives ........................ 168

7.3.1 Relation entre l'écoulement et l'hydrologie sous-glaciaire . . . . . . . . . . . . 169

7.3.2 Réponse dynamique des glaciers au changement climatique . . . . . . . . . . 169

7.3.3 Bilan de masse et contribution des glaciers à l'élévation du niveau marin . . . . . 170

Dans un contexte de changement climatique, les glaciers de montagne subissent des évolutions rapides de leur volume et de leur dynamique. Il est important de mieux caractériser ces évolutions car :

- les glaciers sont de bons indicateurs climatiques (IPCC, 2001);

- leur contribution à l'élévation du niveau marin est importante $(0.4 \mathrm{~mm} / \mathrm{a}$ d'après (Dyurgerov, 2003)) mais incertaine $( \pm 0.5 \mathrm{~mm} / \mathrm{a})$;

- ils jouent un rôle clef pour la ressource en eau de nombreuses régions du globe;

- leur évolution, en réponse aux changements climatiques futurs, reste incertaine.

Mais les glaciers de montagne se situent dans des régions à la topographie abrupte, au climat sévère et sont donc difficiles d'accès. Un petit nombre seulement est étudié in situ, et les plus gros glaciers sont rarement observés. Si l'observation par satellite est également compliquée par cet environnement extrême, elle peut toutefois permettre un suivi homogène et régulier à l'échelle régionale, voire globale.

L'objectif de cette thèse était donc d'utiliser l'imagerie satellitaire optique pour caractériser la réponse des glaciers de montagne aux changements climatiques. Pour cela, des avancées méthodologiques ont permis la mesure satellitaire de deux paramètres clefs : les vitesses d'écoulement et les variations d'épaisseur des glaciers. 
Nous synthétisons ici les principaux résultats obtenus en distinguant les aspects méthodologiques et glaciologiques. Nous reprendrons ensuite quelques unes des perspectives déjà évoquées dans le manuscrit en regard des missions satellitaires futures.

\subsection{Synthèse méthodologique}

Avant de détailler les principaux développements méthodologiques de cette thèse, nous souhaitons souligner le rôle des relevés in situ et leur complémentarité avec les mesures de télédétection spatiale. La haute résolution des images satellites actuelles (et futures) et des méthodologies adaptées permettent désormais le suivi des glaciers de montagne. Mais des sites de validation, bien instrumentés comme les glaciers du massif du Mont-Blanc, sont nécessaires. Ils permettent d'associer une précision voire de détecter d'éventuels biais de la mesure satellitaire. Cette étape est indispensable pour envisager l'étude de régions difficiles d'accès voire inexplorées.

\subsubsection{Choix des images satellites}

Le choix des images satellites est crucial pour de bonnes observations. Pour l'application et le développement de nos méthodologies, un préliminaire indispensable a été de comprendre les propriétés radiométriques et surtout géométriques des images SPOT. Ceci a notamment permis la sélection ou la programmation d'images acquises dans des conditions bien précises.

Pour la construction de MNT comme pour la mesure des mouvements, les couples d'images doivent présenter :

- un gain faible pour éviter la saturation radiométrique sur la surface des glaciers à forte réflectance;

- un état de surface peu changeant (saison sèche);

- des conditions d'éclairement solaire similaires pour éviter des ombres trop différentes.

Ensuite, selon l'objectif visé, les principales différences sont :

- une séparation temporelle de quelques semaines (voire une année) pour la mesure des vitesses, la plus faible possible pour l'élaboration des MNT;

- des points de vue proches pour la mesure des vitesse (l'idéal étant deux images séparées par un multiple du cycle orbital), opposés pour la construction des MNT;

- dans le cas de la mesure des vitesses, l'angle d'incidence sera préféré faible pour les mouvements horizontaux, fort pour les mouvements verticaux.

La résolution des images est également un paramètre critique. Pour la corrélation d'images par exemple, elle permet, à précision égale, de limiter l'écart temporel entre deux couples d'images et donc d'étudier des variations à plus haute fréquence de l'écoulement des glaciers. SPOT5 constitue probablement le meilleur compromis actuel entre une haute résolution $(2.5 \mathrm{~m})$ et une couverture spatiale de $60 \times 60 \mathrm{~km}^{2}$ qui permet d'embrasser tout un massif de montagne en un "clin d'oeil".

\subsubsection{Mouvements horizontaux des glaciers}

L'écoulement horizontal des glaciers, de plus forte amplitude, est le plus "facile" à mesurer. Idéalement, il s'obtient directement en corrélant deux images acquises au nadir du satellite et 
parfaitement recalées. Toutefois, si les images présentent des angles d'incidence forts (mais voisins), une information extérieure, permettant d'estimer les variations d'épaisseur du glacier, est nécessaire. Dans cette thèse, elle a été obtenue par un modèle degré jour, fondé sur les températures à Chamonix et calibré sur des observations in situ réalisées par le LGGE. Ce biais sur les vitesses horizontales, lié aux variations d'épaisseur, pourrait aussi affecter la corrélation d'images d'amplitude SAR (Strozzi et al., 2002), acquises également avec des angles d'incidence importants. A notre connaissance, il n'a jamais été pris en compte.

Si les images sont acquises du même point de vue, des mesures précises sont obtenues sans aucun point de contrôle au sol même avec un MNT de très basse résolution. "ME DECOIS $P A S^{\prime \prime}{ }^{1}$ conduit à une précision de \pm 0.2 pixel (images SPOT1-5), 5 fois meilleure que les résultats obtenus précédemment sur les glaciers. C'est notre bonne prise en compte de la géométrie SPOT par des outils adaptés (librairie SP5LIB) qui permet une telle précision. De tels outils devraient donc être pérennisés pour les missions spatiales futures (ORFEO-PLEIADE).

La corrélation d'images multi-capteurs (SPOT, LANDSAT, ASTER) est possible mais moins précise à cause des différences géométriques entre les images. Nos résultats sur la calotte islandaise (non présentés dans la thèse) conduisent à une précision de \pm 1 pixel.

"ME DECOIS PAS" ne se limite pas à l'observation des mouvements glaciaires. Les glissements de terrain peuvent également être détectés et cartographiés (annexe F) ainsi que la rupture (ou non) de surface et les déformations induites par les séismes (annexe E). Pour ces applications "Terre Solide", l'interférométrie SAR (InSAR), lorsqu'elle est applicable, reste un outil de prédilection mais la corrélation d'images apporte des informations complémentaires. Plus exotiquement, on peut même envisager de corréler des images de la calotte glaciaire martienne (capteur HRS-C) pour détecter d'éventuels mouvements de la glace extra-terrestre...

\subsubsection{Variations d'altitude de la surface des glaciers}

Les variations d'altitude de la surface des glaciers s'expliquent soit par un mouvement vertical en masse de tout le glacier soit par un changement d'épaisseur (effet combiné du bilan de masse en surface et de la dynamique). Deux méthodes différentes nous ont permis d'étudier ces processus.

La corrélation de deux images optiques aux angles d'incidence forts permet (pour la première fois à notre connaissance) de mesurer les déplacements verticaux du glacier (plateforme de Grímsvötn) qui se produisent en l'espace de quelques jours. La réalisation d'une cartographie à 3 dimensions des mouvements géophysiques est envisageable même si le cas des glaciers s'avère complexe comme décrit dans l'annexe G.

La mesure des variations d'épaisseur (donc du bilan de masse glaciaire si la densité est connue) se fonde, elle, sur la comparaison de topographies acquises à quelques années d'intervalle. Deux couples d'images stéréoscopiques sont nécessaires. De longue date, elle a été appliquée aux photographies aériennes. L'originalité de notre approche est l'utilisation d'images satellites optiques, couvrant une surface de 1 à 2 ordres de grandeur plus importante que les données aériennes. Les variations d'épaisseur glaciaire sont ainsi mesurées avec une précision de $\mathbf{1}$ à $\mathbf{2} \mathbf{~ m}$ sur les zones d'ablation peu pentues comme la langue de la Mer de Glace. Les résultats sont moins satisfaisants dans les zones d'accumulation. Toutefois, la topographie SPOT5 de 2003 reste précise à toutes les altitudes ce qui est prometteur pour l'avenir. Compte tenu des taux

\footnotetext{
${ }^{1}$ Acronyme désignant notre chaîne de "MEsure des DEplacements par COrrélation d'Images Spot avec une Précision Assez Sympa"
} 
d'amincissements récents des glaciers, la précision actuelle de notre méthode permet un suivi des bilans de masse glaciaires tous les 3 à 5 ans.

Des topographies d'origines différentes peuvent également être utilisées. A plusieurs reprises, les MNT dérivés de l'imagerie optique ont ainsi été comparées à ceux construits par InSAR (SRTM, EMISAR). Mais l'utilisation de ces derniers impose la prise en compte de la pénétration de l'onde radar dans la neige et le névé des parties hautes et froides des glaciers. Notre étude sur les glaciers alpins montre que la topographie SRTM est, systématiquement, 5 à $10 \mathrm{~m}$ trop basse dans les bassins d'accumulation. C'est un résultat important, à l'heure où cette topographie quasi mondiale est utilisée par de nombreux glaciologues. Quand SRTM est la topographie la plus ancienne dans le MNT différentiel (cas de notre étude sur l'Himalaya), on minimise les pertes d'épaisseurs. C'est donc une borne supérieure du bilan de masse que l'on obtient. C'est l'inverse lorsque SRTM est la topographie la plus récente. Une des conséquences est que la contribution des glaciers patagoniens à l'élévation de niveau de la mer $(0.1 \mathrm{~mm} / \mathrm{a}$ pour la période $1995-2000$ d'après Rignot et al. (2003)) pourrait être surestimée. Mais ceci reste à démontrer.

Notre méthodologie a conduit à des résultats encourageants pour des massifs glaciaires aux morphologies différentes, sous diverses influences climatiques (Alpes, Himalaya, Islande). Un résultat fort est la possibilité de construire, à partir d'images SPOT5, des topographies précises sans aucun point d'appui si un MNT antérieur est disponible. C'est la bonne connaissance des orbites de SPOT5 qui permet un tel résultat. Toutefois, des biais sont apparus sur les glaciers (comme le Vatnajökull) où la proportion des zones non englacées est faible. Pour ces zones, il reste à déterminer si quelques points de contrôle précis suffisent à limiter ces biais. Ce travail sera réalisé dans les prochains mois.

Si l'estimation des variations volumétriques est en progrès, sa conversion en changement de masse reste problématique. La densité du matériel gagné ou perdu (0.6 à 0.9 selon qu'il s'agit de névé ou de glace) est un sujet débattu. Seuls des profils de densité en zone d'accumulation, mesurés in situ à quelques années d'intervalle, pourraient trancher entre ces deux hypothèses. Le choix d'une ou l'autre de ces hypothèses modifie le bilan de masse de 10 à $20 \%$. La complémentarité entre mesures satellitaires et in situ est à nouveau soulignée.

\subsection{Synthèse glaciologique}

$\mathrm{Si}$, après la partie bibliographique, la thèse a été organisée sous forme de trois "chantiers" (1/ vitesse d'écoulement des glaciers alpins, 2 / bilan de masse puis 3/ application à la calotte glaciaire islandaise du Vatnajökull), nous pouvons maintenant synthétiser nos résultats scientifiques en trois grands volets thématiques.

\subsubsection{Importance de l'hydrologie sous-glaciaire}

Dans deux contextes différents, nous avons montré que l'eau sous-glaciaire exerçait un contrôle fort sur la dynamique du glacier.

Ce lien est évident dans le cas du lac de Grimsvötn sous la calotte glaciaire du Vatnajökull. Les remplissages et les vidanges du lac conditionnent les mouvements de la plateforme de glace qui le surplombe. L'observation par SPOT5 des mouvements verticaux de la glace permet de

contraindre l'extension du lac et fournit une limite supérieure à son volume donc de la crue 
potentielle liée à sa vidange. En ajoutant des mesures in situ d'ablation, l'activité géothermique de cette zone est aussi estimée.

Le rôle de l'hydrologie sous-glaciaire est plus indirect pour expliquer les variations de vitesse à court terme des glaciers alpins. La mesure satellitaire des vitesses de surface pour deux périodes de l'été 2003 permet de cartographier les fluctuations de l'écoulement à l'échelle du glacier. C'est un progrès par rapport à des mesures in situ en quelques sites. L'amplitude de ces variations est de l'ordre de 10 à $20 \%$ de la vitesse totale. Les zones affectées sont corrélées avec l'intensité du crevassement du glacier ce qui suggère un rôle de la pénétration de l'eau de fonte en surface vers le lit rocheux. Un schéma interprétatif est donc proposé qui prend en compte la température (donc la fusion), la localisation des crevasses et le développement du réseau hydrologique sous-glaciaire.

Ce rôle de l'eau sous-glaciaire n'est pas limité aux glaciers tempérés. En étudiant la courbure de la topographie de l'Antarctique, Rémy \& Legrésy (2004) ont pu cartographier la présence d'un dense réseau hydrologique sous la glace. Pour l'un des émissaires de la calotte, le glacier Mertz, une zone d'écoulement intense se localise dans l'axe d'une probable "rivière" sous glaciaire (figure 7.1).
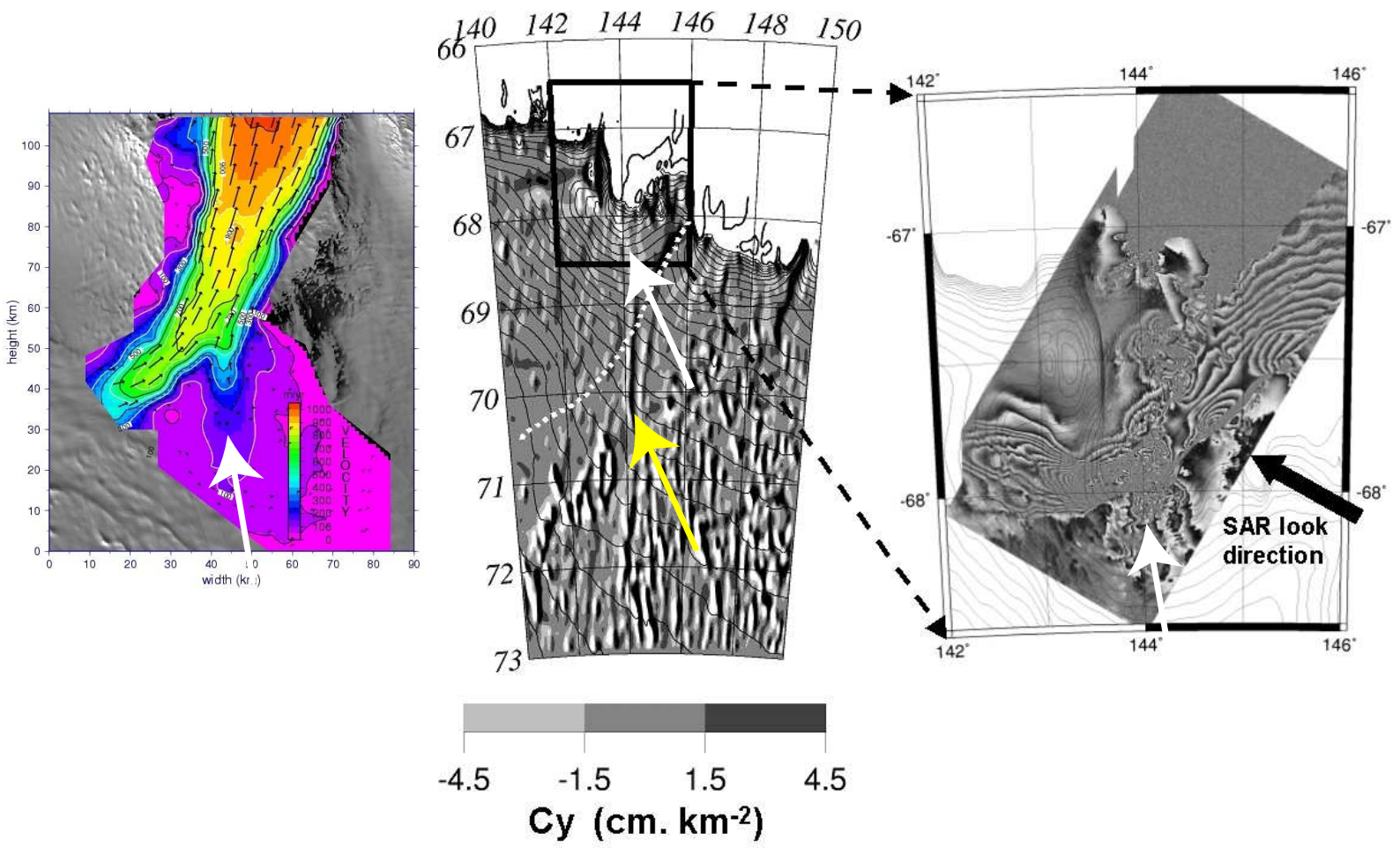

Fig. 7.1 - Relation entre le réseau hydrologique et les vitesses d'écoulement du glacier Mertz. A gauche : carte des vitesses de surface obtenue par corrélation d'images LANDSAT acquises en février 2000 et décembre 2001 (Berthier et al., 2003). A droite, interférogramme déduit d'un couple d'images ERS espacées d'un jour (Legrésy et al., sous presse). Ces deux cartes montrent qu'en plus de la zone d'écoulement majeure venant de l'Ouest, il existe un tributaire secondaire venant du Sud (flèches blanches). Au centre : Courbure de la surface de l'Antarctique dans la région du Glacier Mertz. Rémy \&ٔ Legrésy (2004) ont montré que ce paramètre était relié aux réseaux hydrologiques sous-glaciaires. Une flèche jaune pointe vers une structure linéaire et allongée qui se termine au niveau du tributaire secondaire du Glacier Mertz. 
Nous confirmons ainsi l'importance de l'hydrologie sous-glaciaire dans deux contextes différents et la complémentarité de différentes mesures (topographie, vitesses d'écoulement déduites d'images radar ou optiques). Cet aspect mérite donc d'être étudié à l'avenir, d'autant plus que la nature même de ce réseau hydrologique reste débattue (Fountain et al., 2005).

\subsubsection{Adaptation dynamique des glaciers aux changements climatiques}

Depuis le début des années 80, la Mer de Glace connaît un ralentissement significatif, atteignant $40 \%$ en 25 ans par endroit (chapitre 4, figure 4.28). En parallèle, nous avons vu que les pertes d'épaisseur sur la langue de ce glacier étaient importantes (chapitre 5, figure 5.17 par exemple). Nous distinguons deux périodes, 1979-1994 et 1994-2000, et comparons sur la figure 7.2 les variations d'épaisseur et de vitesse.
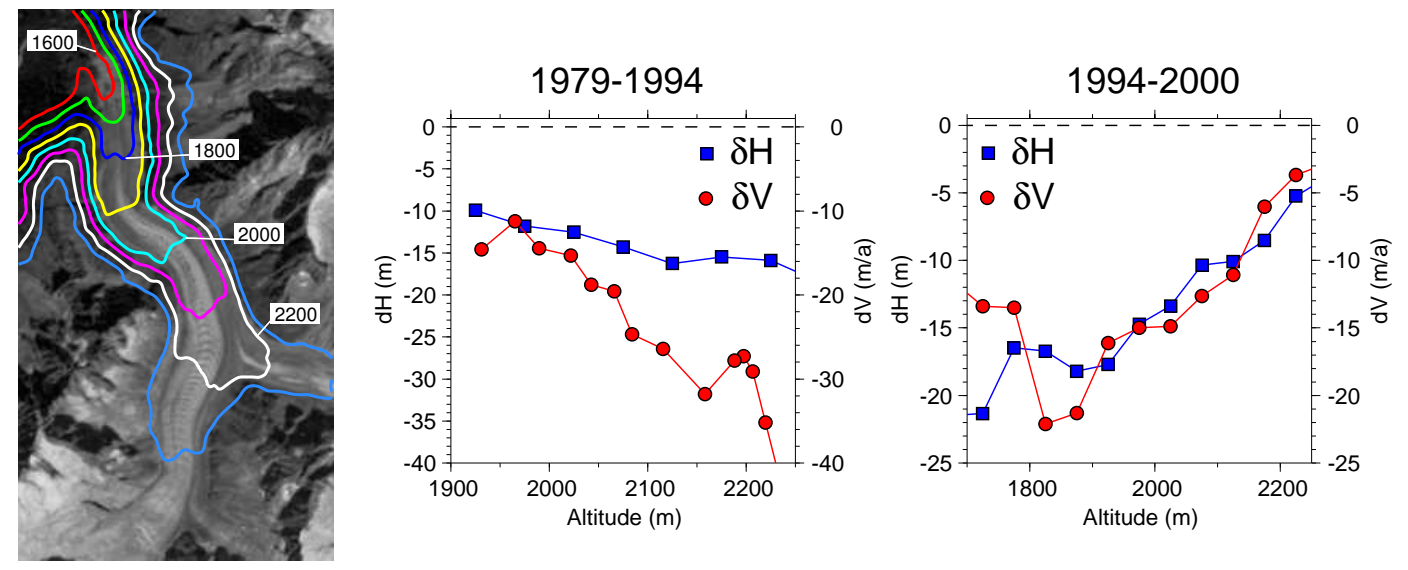

FIG. 7.2 - Variations conjointes d'épaisseur $(\delta H)$ et de vitesse $(\delta V)$ pour la zone 1900-2200 m de la Mer de Glace. Au centre, pour la période 1979-1994 à droite pour la période 1994-2000. Les fluctuations de vitesse et d'épaisseur sont des valeurs moyennes pour chaque tranche d'altitude à l'exception des variations de vitesse pour la période 1979-1994 qui sont estimées ponctuellement au niveau de quelques balises. La même échelle est utilisée pour les variations de vitesse et d'épaisseur. A gauche, image SPOT5 de la Mer de Glace avec les iso-contours d'altitude.

Pour les deux périodes, il existe une relation quasi linéaire entre fluctuations de vitesse et d'épaisseur. Mais, pour 1979-1994, le ralentissement (exprimé en m/a) est deux fois plus fort que l'amincissement (exprimé en m) alors que pour 1994-2000, les deux variations sont du même ordre de grandeur. Une autre différence est qu'en 1979-1994, les fluctuations sont plus fortes pour les zones hautes, c'est l'inverse entre 1994 et 2000 où vers $2200 \mathrm{~m}$, les variations sont quasi nulles. Ces courbes montrent la complexité de la relation entre les changements d'épaisseur et de vitesse du glacier. Notons ici que nos mesures ne couvrent que la zone d'ablation de la Mer de Glace.

Pour expliquer ces variations jointes, on peut revenir à l'équation de continuité (équation 2.4) appliquée à une tranche d'altitude :

$$
\mathcal{S}_{i} \frac{\partial h_{i}}{\partial t}=F_{i}-F_{i+1}+\mathcal{S}_{i} \times \frac{b_{i}}{\rho_{i}}
$$

Dans cette équation, les flux de glace $\left(\mathrm{F}_{i}\right)$ dépendent de la vitesse d'écoulement, elle même 
reliée (tout au moins sa composante de déformation interne) à l'épaisseur du glacier (équation 2.12 que nous rappelons ici) :

$$
u_{s}-u_{b}=\frac{2 \mathcal{A}}{n+1}(\rho g \sin \alpha)^{n} h^{n+1}
$$

Si on combine ces deux équations, le système n'est pas linéaire. Nous ne chercherons pas ici à le résoudre. Nous adoptons plutôt une approche très simplifiée, en étudiant deux situations bien distinctes. Dans un premier temps, nous verrons quelle est la part de l'amincissement du glacier qui s'explique par la diminution des flux de glace (équation 7.1). Dans un second temps, nous quantifierons l'ordre de grandeur du changement de vitesse d'un glacier qui s'amincit (équation 7.2 ).

\subsubsection{Réduction des flux de glace $\rightarrow$ amincissement}

Nous avons vu au chapitre 5 que la simple augmentation de l'ablation locale ne suffisait pas à expliquer l'ampleur des pertes d'épaisseur récente de la Mer de Glace. Le ralentissement du glacier s'accompagne d'une diminution des flux vers l'aval et peut donc contribuer, lui aussi, à l'amincissement. Si dans l'équation 7.1, le bilan de masse d'une portion (de surface $\mathcal{S}_{i}$ ) du glacier est supposé nul, sa variation d'épaisseur au cours du temps dépendra des flux amonts $\left(F_{i}\right)$ et avals $\left(F_{i+1}\right)$ :

$$
\frac{\Delta h_{i}}{\Delta t}=\frac{F_{i}-F_{i+1}}{\mathcal{S}_{i}}
$$

Pour estimer ces flux à travers des sections transversales de la Mer de Glace nous utilisons :

- la topographie du lit rocheux présentée par Lliboutry \& Reynaud (1981, figure 2) et obtenue par des études antérieures (Süsstrunk, 1951; Vallon, 1961; Gluck, 1967). Nous avons scanné, numérisé puis interpolé la carte des iso-altitudes du lit rocheux fournie par le LGGE.

- l'hypothèse, proposée par Nye (1965) et utilisée par Reynaud et al. (1986), que la vitesse moyenne pour une section transversale du glacier est égale à la vitesse moyenne en surface (notée $<u_{s, i}>$ ).

Le flux à travers une section transversale de surface $A_{i}$ s'obtient alors par :

$$
F_{i}=A_{i} \times<u_{s, i}>
$$

Notons ici une hypothèse forte dans notre calcul de l'amincissement. Nous disposons des vitesses annuelles pour trois périodes différentes que nous convertissons en (et comparons avec l') amincissement moyen pour l'intervalle de temps considéré. Cela suppose que les vitesses ont varié linéairement dans l'intervalle de temps. Un calcul rigoureux devrait se baser sur les vitesses moyennes pour toute la période.

En comparant les flux de glace pour 1993-94 et 2000-01 entre 1900 et $2200 \mathrm{~m}$ d'altitude, nous estimons à $0.2 \mathrm{~m} / \mathrm{a}$ l'accélération de l'amincissement du glacier liée aux évolutions des flux. Pour la même période, l'accélération de l'amincissement mesurée par MNT différentiel est de $1.5 \mathrm{~m} / \mathrm{a}$ (valeur déduite du tableau 5.2). L'évolution de la dynamique glaciaire explique donc de 10 à $15 \%$ de l'accélération de l'amincissement de cette zone. 
Pour la période 1979-1994, l'estimation des flux est plus incertaine car seules les vitesses pour quelques balises au centre du glacier sont disponibles. Nos calculs indiquent que le ralentissement du glacier (plus fort à haute qu'à basse altitude) expliquerait un amincissement dynamique de $0.35 \mathrm{~m} / \mathrm{a}$ du glacier.

En comparant maintenant les deux périodes (1979-1994 et 1994-2000), nous avions noté une accélération des pertes d'épaisseur (de 0.89 à $2.05 \mathrm{~m} / \mathrm{a}$ soit $1.16 \mathrm{~m} / \mathrm{a}$, tableau 5.2). L'augmentation des températures, donc de l'ablation locale, n'expliquerait que $0.28 \mathrm{~m} / \mathrm{a}$. Les effets dynamiques ne semblent pas suffisants pour expliquer les $0.88 \mathrm{~m} /$ a restant. La question reste donc ouverte quand à l'accélération des pertes d'épaisseur à basse altitude sur la Mer de Glace.

Implicitement, nous supposons ci-dessus que l'épaisseur du glacier réagit à des changements de sa dynamique. On peut également prendre le problème à l'envers en étudiant comment la dynamique glaciaire répond à des fluctuations de son épaisseur.

\subsubsection{Interprétation en terme de variation de la déformation interne}

Nous utilisons ici l'hypothèse (forte) qu'aux échelles de temps annuelles, le glissement basal ne varie pas. Pour la période 1994-2000, ceci est suggéré par l'observation d'une vitesse constante dans une zone (km 5 à 6 du profil longitudinal de la figure 4.28) sensible justement à des fluctuations du glissement basal ( $\mathrm{km} 0$ à 1 du profil longitudinal de la figure 4.19). Les fluctuations de la vitesse en surface du glacier sont alors liées à des variations de la déformation interne de la glace. Un glacier qui s'amincit doit voir sa vitesse diminuer. A partir de l'équation 7.2 (qui dérive de la loi de Glen), si on néglige le changement de la pente du glacier, on peut estimer qu'elle est la variation de la vitesse de surface $\delta u_{s}$ liée à une variation $\delta h$ de son épaisseur :

$$
\delta u_{s}=2 \mathcal{A}(f \rho g \sin \alpha h)^{n} \times \delta h
$$

Paterson (1994) recommande les valeurs suivantes pour un glacier de vallée : $\mathrm{f}=0.5, \mathcal{A}=2 \times$ $10^{-16}$ et $\mathrm{n}=3$. Pour la période $1994-2000$, avec ce jeu de paramètres, le ralentissement calculé est de $6.1 \mathrm{~m} /$ a soit la moitié de celui mesuré $(12.6 \mathrm{~m} / \mathrm{a})$. On notera ici la sensibilité à la valeur des différents paramètres. Il suffit que $\mathrm{n}=3.06$ ou que $\mathcal{A}=4.1 \times 10^{-16}$ pour expliquer l'intégralité du ralentissement. Ces valeurs restent largement cohérentes avec la littérature (Paterson, 1994; Marshall et al., 2002). Notons également que négliger le terme de variation de la pente du glacier lorsqu'on différencie l'équation 7.2 est probablement injustifié en particulier au front du glacier où l'amincissement est fort.

Les mesures dont nous disposons pourraient aussi permettre de mieux contraindre la valeur de l'exposant $\mathrm{n}$. La différentielle logarithmique de l'équation 7.2 conduit à la relation (tracée sur la figure 7.3$)$ :

$$
\frac{\delta u_{s}}{u_{s}}=(n+1) \frac{\delta h}{h}+\text { constante }
$$

Faire passer une droite par le nuage de points de la partie gauche de la figure 7.3 n'a pas de sens. En revanche, pour les altitudes entre 2000 et $2300 \mathrm{~m}$, la relation est quasi linéaire (équation de la droite : $\left.\frac{\delta u_{s}}{u_{s}}=4.25 \frac{\delta h}{h}+0.047 ; \mathrm{r}^{2}=0.97\right)$ indiquant une valeur de 3.25 pour l'exposant de la loi de Glen, valeur cohérente avec la littérature (Marshall et al., 2002). A plus basse altitude, si on tentait de faire passer une droite pas les observations, la pente serait plus faible. Mais, pour 
cette partie basse du glacier, une simple droite ne rend pas compte des observations. Une des raisons est que, pour ces zones en fort amincissement, on ne peut pas négliger les variations de la pente (terme $\sin \alpha)$.

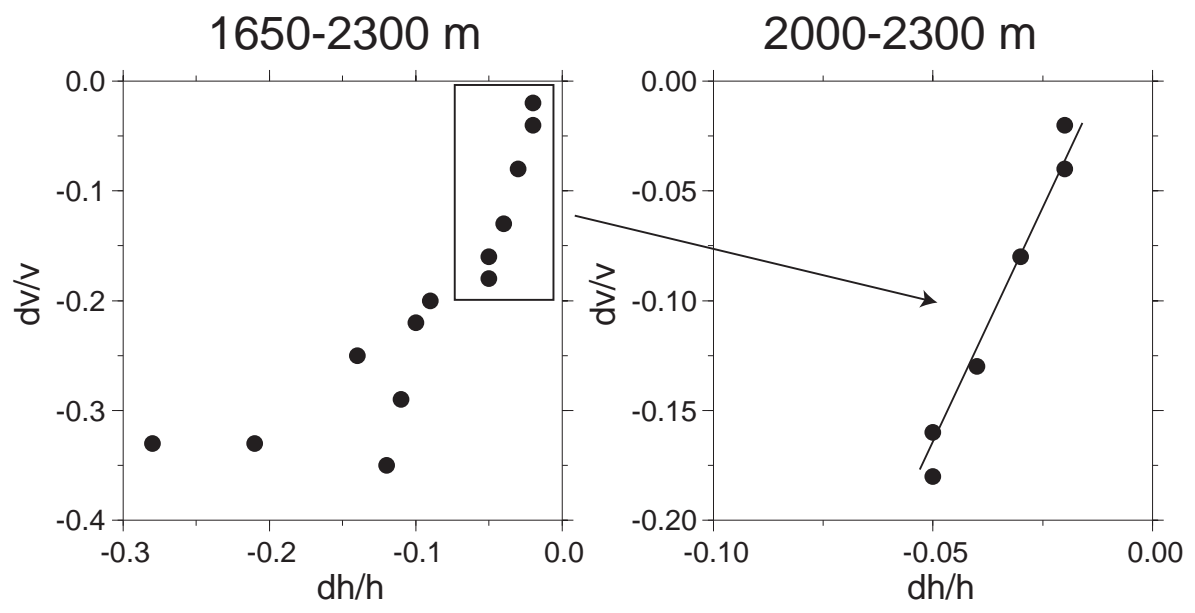

Fig. 7.3 - Relation entre $\frac{\delta u_{s}}{u_{s}}$ et $\frac{\delta h}{h}$ pour la zone 1650 à $2300 \mathrm{~m}$ de la Mer de Glace entre 1994 et 2000. A droite, un détail pour la zone entre 2000 et $2300 \mathrm{~m}$.

Il reste beaucoup à faire pour comprendre l'ajustement dynamique d'un glacier de montagne à des pertes rapides de masse. Ci-dessus nous avons distingué deux situations "stéréotypées". Dans un premier cas, la diminution des flux de glace permet d'expliquer une partie de l'amincissement du glacier. Dans un second cas, les pertes d'épaisseur donc la diminution de la déformation interne expliquent une partie du ralentissement du glacier. En réalité, épaisseur glaciaire et écoulement fluctuent de concert. C'est donc vers un modèle plus complexe qu'il faut se tourner pour comprendre les variations conjointes de ces deux paramètres. Nous suggérons aussi (figure 7.3) qu'une telle étude pourrait permettre de préciser les paramètres encore mal contraints (Rémy \& Testut, 2005) de "La" loi de Glen, loi d'écoulement de la glace.

Certes, les rétroactions liées à la dynamique glaciaire ne peuvent pas être négligées pour anticiper la réponse des glaciers aux changements climatiques futurs. Ainsi, le ralentissement de la Mer de Glace sous $2400 \mathrm{~m}$ préserve les zones à plus haute altitude et "condamne" la partie basse du glacier. Mais pour les régions basses de ce glacier, il semble que les effets dynamiques restent faibles comparés à l'augmentation de l'ablation. Nous abondons ainsi dans le sens de Paul et al. (2004) qui affirment que c'est le "downwasting", i.e. la désintégration sur place sans ajustement dynamique notable qui régit le déclin récent des glaciers alpins.

\subsection{3 Évolution comparée des glaciers alpins, himalayens et islandais}

Dans trois massifs de montagne différents nous avons pu appliquer la technique des MNT différentiels : massif du Mont-Blanc dans les Alpes, massif du Bara Shigri dans l'Himalaya de l'Ouest et Ouest-Vatnajökull en Islande. Même si les périodes étudiées ne sont pas exactement les mêmes et en dépit des incertitudes sur certains des MNT différentiels, il est intéressant de comparer l'évolution des glaciers dans trois massif séparés par plusieurs milliers de kilomètres (figure 7.4).

Il existe un signal commun à tous ces glaciers : l'amincissement est fort à basse altitude 
(plusieurs mètres par an) et les variations plus faibles dans les zones hautes. Ce signal commun est modulé par des facteurs dynamiques, propres à chaque région. L'épaississement dans les zones d'accumulation islandaises s'explique ainsi par un contexte post-surge.

Il est encore trop tôt pour traduire ce recul glaciaire en terme d'élévation du niveau marin. Il convient maintenant d'étendre nos bilans à un plus grand nombre de glaciers (en Himalaya notamment) pour étudier la représentativité régionale des mesures obtenues lors de cette thèse. Si les autres glaciers ont des comportements similaires, la contribution cumulée de l'Himalaya et de la calotte du Vatnajökull pourrait être de 0.1 à $0.15 \mathrm{~mm} / \mathrm{a}$, soit près de $5 \%$ de l'augmentation actuelle du niveau moyen des mers.

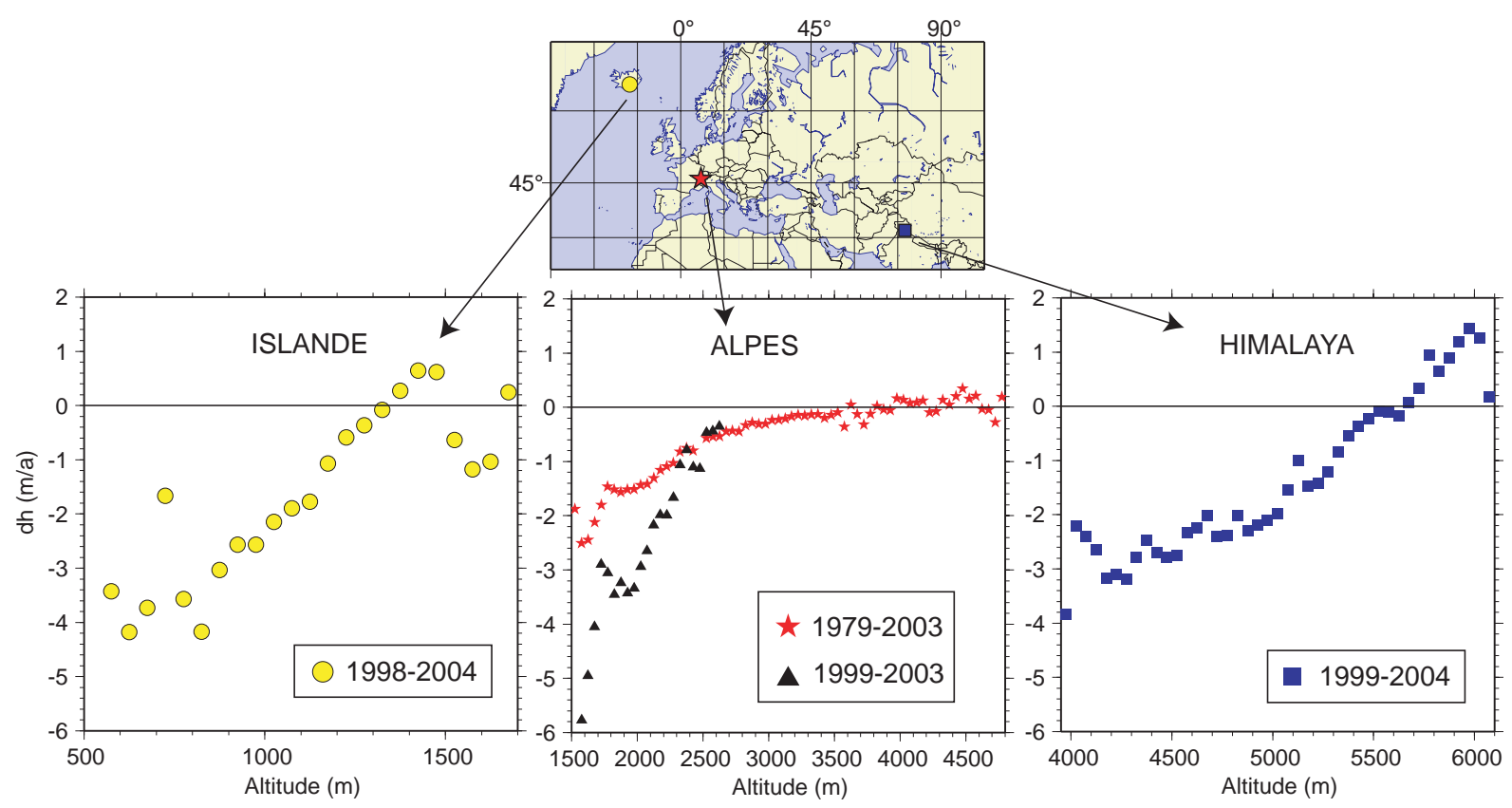

FIG. 7.4 - Variations d'épaisseur en fonction de l'altitude pour quelques glaciers islandais (à gauche), alpins (au centre) et himalayens (à gauche). Pour les glaciers alpins, deux périodes sont distinguées dans la zone basse.

On ne peut pas généraliser à toute la cryosphère cette observation de bilans de masse très négatifs dans ces trois régions du globe. Ainsi, une étude (terminée au début de cette thèse), nous a permis de montrer que le glacier Mertz en Antarctique avait un bilan de masse légèrement excédentaire (Berthier et al., 2003). Ce résultat est concordant avec l'observation récente d'un bilan de masse positif à l'échelle de l'Antarctique de L'Est (Davis et al., 2005). Cela souligne la nécessité d'étudier un spectre large de glaciers dans des régions climatiques variées pour caractériser objectivement la réponse glaciaire aux changements climatiques.

\subsection{Perspectives}

Pour les trois volets détaillés ci-dessus les missions satellitaires actuelles ou futures offrent des perspectives passionnantes. 


\subsubsection{Relation entre l'écoulement et l'hydrologie sous-glaciaire}

La principale limite de notre étude des variations de vitesse des glaciers alpins pour l'été 2003 tient à un échantillonnage temporel insuffisant. Les fluctuations de vitesse, enregistrées en un mois, moyennent des fluctuations à plus haute fréquence.

Pour aller plus loin dans l'étude de la relation entre l'écoulement glaciaire et l'hydrologie, il faut donc améliorer la résolution temporelle. Il semble prometteur de coupler des relevés in situ continus (DGPS par exemple) en quelques points clefs du glacier à la vision d'ensemble du satellite. La corrélation de photographies terrestres quotidiennes ou hebdomadaires est aussi une voie à explorer (Evans, 2000). De telles images devraient être acquises prochainement sur le glacier d'Argentière par l'ORE GLACIOCLIM² et sont déjà disponibles sur des glaciers andins.

Une meilleure résolution satellitaire se traduira par une précision accrue et permettra de diminuer la séparation temporelle entre deux images à corréler. On peut ainsi espérer cartographier les vitesses glaciaires tous les 15 jours entre mai et octobre puis relier leurs variations à la fonte et au développement du réseau hydrologique comme nous l'avons esquissé pour l'été 2003. Ceci pourrait se faire dans les prochaines années grâce au programme franco-italien ORFEO-PLEIADE (http ://smsc.cnes.fr/PLEIADES/Fr/). Pour sa partie optique, cette constellation comprendra deux satellites de résolution $0.7 \mathrm{~m}, 4$ fois meilleure que SPOT5 en mode THR.

Des mouvements verticaux d'amplitude métrique peuvent actuellement être mesurés par corrélation d'images acquises avec des angles d'incidence forts. Une meilleure résolution permet d'espérer la détection de tels mouvements dans le cas des glaciers de montagne (amplitude décimétrique) et de les relier à l'ablation en surface et à la variation de leur contenu basal en eau. L'idéal pour une telle étude serait évidemment de combiner l'interférométrie SAR et la corrélation d'images optiques. Mais ceci suppose une mission satellitaire SAR spécifique avec un court temps de revisite (1 jour idéalement) qui n'est pas envisagée actuellement.

\subsubsection{Réponse dynamique des glaciers au changement climatique}

L'étude des variations concomitantes d'épaisseur et d'écoulement de la Mer de Glace montre que la dynamique des glaciers est importante pour comprendre leur évolution actuelle et future. C'est aussi un moyen de mieux contraindre certains paramètres mal connus des lois d'écoulement des glaciers. Une physique trop simplifiée semble être la principale limite pour comprendre pleinement nos observations et c'est vers une modélisation plus détaillée qu'il faut maintenant se diriger. Ceci pourra être fait dans le cadre d'une collaboration avec l'équipe de Garry Clarke de l'Université de British Columbia (Vancouver, Canada).

La calotte glaciaire du Vatnajökull semble un site adapté pour poursuivre notre étude de la réponse glaciaire à des fluctuations climatiques. Du fait de sa taille et de sa géométrie, intermédiaires entre glaciers de montagne et calotte polaire, le Vatnajökull peut être observé par un nombre important de capteurs satellitaires. Notre collaboration avec l'équipe islandaise devrait se poursuivre dans les années à venir notamment grâce au programme OASIS $^{3}$ qui permettra l'acquisition d'images SPOT par les islandais. Des mesures altimétriques précises pourraient compléter les informations extraites des images SPOT. Nous prévoyons de travailler dans les prochains mois sur les données du laser altimétrique de ICEsat. Le satellite CRYOsat, dans son mode interférométrique, devrait également permettre des observations topographiques précises.

\footnotetext{
${ }^{2}$ Observatoire de Recherche en Environnement : les GLACIers, un Observatoire du CLIMat

${ }^{3}$ Optimising Access to Spot Infrastructure for Science http ://medias.obs-mip.fr/oasis/
} 
Les archives ERS-1, ERS-2 et Topex-Poséidon pourraient enfin être exploitées pour suivre l'évolution depuis 1991 des zones à faible pente au centre de la calotte. Ces nombreuses données pourront contraindre des modèles dynamiques d'écoulement à l'échelle d'un glacier émissaire (Björnsson et al., 2005) ou de l'ensemble de la calotte (Marshall et al., en preparation) et ainsi, simuler leur évolution future.

\subsubsection{Bilan de masse et contribution des glaciers à l'élévation du niveau marin}

Concernant le bilan de masse glaciaire, trois aspects méritent d'être développés dans les années à venir et pourraient bénéficier d'approches novatrices ou des futures missions satellitaires.

A l'échelle du glacier, il reste à démontrer qu'un bilan de masse purement satellitaire fiable puisse être déterminé. Cela devrait être fait dans les prochains mois grâce à l'acquisition d'un nouveau couple stéréoscopique SPOT5 sur le massif du Mont-Blanc. Nous pourrons alors étudier de manière systématique les effets d'exposition en comparant les bilans de masse des versants français, italiens et suisses. Actuellement, sur les glaciers alpins, il semble difficile de travailler au pas de temps annuel étant donné la précision métrique des MNT SPOT5, mais des missions futures (PLEIADES) devraient le permettre. Le fait que des topographies précises aient été obtenues même après des chutes de neige (cas du MNT himalayen) permet même d'entrevoir la mesure du bilan de masse saisonnier. Pour cela, en plus des topographies "classiques" de fin de saison d'ablation (septembre dans les Alpes), un MNT en fin de période d'accumulation (avril dans les Alpes) sera nécessaire. Une telle approche reste largement hypothétique (notamment à cause des nuages) mais permettrait aussi de suivre l'évolution de l'épaisseur du manteau neigeux au cours de l'hiver.

A l'échelle de quelques glaciers, la mesure satellitaire pourrait être complétée par l'installation de gravimètres et/ou inclinomètres sur les roches à proximité des glaciers. Ces méthodes intégratrices devraient permettre de détecter les (très) faibles variations de la gravité (intensité et direction) qui sont engendrées par les pertes de masse glaciaires. Des mesures DGPS simultanées doivent permettre de quantifier la contribution du soulèvement local lié au rebond élastique de la lithosphère. Un tel projet est porté par Muriel Llubes (LEGOS) et laisse espérer un suivi annuel, voire saisonnier, des gains et des pertes du glacier.

A l'échelle de massifs entiers, il faut poursuivre l'estimation des bilans de masse pour des périodes de 5 à 10 ans afin d'estimer leur contribution à l'élévation du niveau marin. Si la couverture satellitaire est un progrès certain par rapport aux rares mesures in situ, il ne semble pas encore possible d'échantillonner l'intégralité des glaciers d'une région. On pourrait s'en approcher si les images de capteurs comme SPOT5-HRS étaient disponibles... Pour le moment, des techniques d'extrapolation de quelques bilans de masse, fondées sur des inventaires comme ceux de GLIMS, restent nécessaires. Trois sites nous semblent importants car ils pourraient contribuer significativement à l'élévation du niveau marin. Pour les champs de glace Nord et Sud de la Patagonie (collaboration Great Ice, IRD), l'estimation de Rignot et al. (2003) pour la période 1995-2000 pourrait être biaisée par l'utilisation du MNT SRTM. Pour le démontrer, deux ou trois couples stéréoscopiques d'images SPOT5 seraient suffisants. Pour le Vatnajökull (collaboration Science Institute), nous mesurons un déclin important dans la partie Ouest. En est-il de même pour l'ensemble de la calotte, étant donné les fortes hétérogénéités du forçage climatique? La même question se pose pour les glaciers himalayens (collaboration Great Ice, IRD). Elle prend alors une autre dimension car c'est la ressource en eau d'une des régions les plus peuplées de la planète qui en dépend. 


\section{Annexe A}

\section{Vitesse et Bilan de Masse du Glacier MERTZ (Antarctique de l'Est)}

Cette étude a été réalisée lors d'un stage "pré-doc" au sein du National Snow and Ice Data Center (Etats-Unis) et terminée au début de cette thèse. Elle a permis de :

- mesurer le champs de vitesse du Glacier Mertz;

- conclure à la stabilité de sa dynamique à l'échelle d'une dizaine d'années ;

- proposer une nouvelle estimation du bilan de masse par la méthode des flux qui suggère un bilan de masse légèrement positif mais soulève la question de la fiabilité des cartes d'accumulation dans cette région.

- revoir à la baisse (-40\%) la fusion basale de la partie flottante de ce glacier par rapport à une estimation antérieure Rignot (2002).

Ces résultats ont donné suite à une publication présentée dans les pages qui suivent :

Berthier, E., Raup, B., and Scambos, T. A., 2003. New velocity map and mass-balance estimate of Mertz Glacier, East Antarctica, derived from Landsat sequential imagery. J. Glaciol., 49 (167). 


\section{New velocity map and mass-balance estimate of Mertz Glacier, East Antarctica, derived from Landsat sequential imagery}

Etienne Berthier, ${ }^{1}$ Bruce RAup,${ }^{2}$ Ted SCAmbos, ${ }^{2}$

\begin{abstract}
Automatic feature tracking on two Landsat images (acquired in January 2000 and December 2001) generates a complete and accurate velocity field of the Mertz Glacier, East Antarctica. This velocity field shows two main tributaries to the ice stream. Between the tributaries, a likely obstruction feature in the bedrock results in a slow down of the flow. A third Landsat image, acquired in 1989 and combined with the 2000 image, permits the determination of the glacier mean velocity during the nineties. Although some portions of the Mertz Glacier system show evidence of slight speed increase, we conclude that the Mertz flow speed is constant within our uncertainty $\left(35.2 \mathrm{~m} \mathrm{a}^{-1}\right)$. Using this complete velocity field, new estimates of the ice discharge flux, $17.8 \mathrm{~km}^{3} \mathrm{a}^{-1}\left(16.4 \mathrm{Gt} \mathrm{a}^{-1}\right)$, and of the basal melting of the tongue, $11 \mathrm{~m}$ of ice per year, are given. Our results lead to an apparent imbalance of the drainage basin (ice discharge $3.5 \mathrm{~km}^{3} \mathrm{a}^{-1}$ lower than the accumulation). Considering previous studies in the Mertz Glacier area, we then discuss the uncertainty of this imbalance and the problems with accumulation mapping for this region.
\end{abstract}

Citation: Berthier E., B. Raup and T. Scambos. New velocity map and mass-balance estimate of Mertz Glacier, East Antarctica, derived from Landsat sequential imagery. Journal of Glaciology. Vol. 49, No 167. 2003.

\section{INTRODUCTION}

Ice streams are fast-flowing currents of ice feeding ice shelves or reaching the sea at their terminus [Hughes, 1977]. These ice streams and their complex extensions inland, detected by balance velocity mapping [Bamber and others, 2000], drain most of the accumulation of the Antarctic ice sheet and consequently are critical for its mass balance: they control the output (or discharge fluxes) of the ice sheet. Mass balance is a key parameter to estimate the evolution of the Antarctic ice sheet and its contribution to sea level rise.

Fast moving areas of the ice sheet are, with ice shelves, regions where rapid changes can occur. They are an important system to monitor for effects of climate change. Indeed, a rapid evolution in surface velocity and thickness of a few West Antarctic ice streams has been measured recently. The Pine Island Glacier accelerated by $18 \pm 2 \%$ between 1992 and 2000 [Rignot, 2001; Rignot and others, 2002]. Altimetry [Wingham and others, 1998] showed that

${ }^{1}$ LEGOS, Toulouse, FRANCE

${ }^{2}$ NSIDC, Boulder, UNITED STATES

MS received 13 January 2003 and accepted in revised form 28 July 2003. both the Thwaites and Pine Island drainage basins experienced a drop in elevation of $11 \mathrm{~cm} / \mathrm{a}$ between 1992 and 1996 . Whillans Ice Stream has seen a number of changes in extent, thickness, speed and even direction of flow in its catchment [Bindschadler and Vornberger, 1998; Joughin and Tulaczyk, 2002; Conway and others, 2002].

The continuous interaction of ice streams with the sea also makes them sensitive to changes in sea level and temperature. The fact that the regional pattern of sea level and temperature changes is far from homogeneous [Cabanes and others, 2001] combined with the evidence that sea temperature has an impact on ice streams [Rignot and Jacobs, 2002] implies that an independent study of the different drainage basins is necessary.

Accurate velocity fields must be calculated to 1) detect any velocity change in the past or in the future, 2) estimate the discharge fluxes, the basal melting and the mass balance of each individual drainage basin. Different methodologies have been developed to produce velocity fields on these fast moving glaciers. Repeat visible or near infrared images of the same area can be used to track the displacement of features moving with the ice [Lucchita and Ferguson, 1986]. Development of automatic feature tracking algorithms [Scambos and others, 1992] significantly increased the accuracy and the efficiency of this approach. A velocity field can also be produced from radar imagery. Using two synthetic aperture radar (SAR) images of the Rutford Ice Stream with short time separation (6 days), Goldstein and others [1993] showed the efficiency of interferometric SAR (InSAR) to accurately measure the one dimensional displacements of the fast moving glaciers. Combining ascending and descending passes of the satellite and assuming that ice flows parallel to the surface, one can estimate quasi-three-dimensional velocities [Joughin and others, 1998; Mohr and others, 1998]. However, coverage of the ice sheet with ascending and descending InSAR pairs is limited. Recently, "speckle tracking", cross correlation tracking of SAR coherence speckle, has been combined with SAR interferometry to produce two dimensional velocity fields [Gray and others, 2001].

The goal of the present study is to determine a complete and accurate velocity field for the Mertz glacier, the derived strain rate maps, and to compare our estimates of the discharge fluxes, the basal melting and the mass balance with the previous estimates. In the first section, we present a description of the Mertz Glacier. Then, in the second and third sections, we describe our methodology and the resulting velocity and strain-rate maps. The fourth section details the calculation of the discharges fluxes, basal melting and mass balance.

\section{STUDY AREA}

Located in the King George V land (Fig. 1, East Antarctica), the Mertz Glacier has a prominent ice tongue, extending over $100 \mathrm{~km}$ into the ocean, and drains an area of 83080 
$\mathrm{km}^{2}$ [Rignot, 2002], representing only $0.8 \%$ of the grounded East Antarctic ice sheet. This glacier received its name after a tragic expedition led by the Australian Sir Douglas Mawson [Mawson, 1998]. Dr X. Mertz was a companion of Mawson who died in January 1913 during the trek back, having already lost B. Ninnis and much of their supplies in a crevasse. To avoid starvation, they were forced to eat their sled dogs. The livers of the dogs, according to recent investigation, concentrated vitamin $\mathrm{A}$ in amounts toxic to humans; vitamin A poisoning was a key factor in Mertz's death.

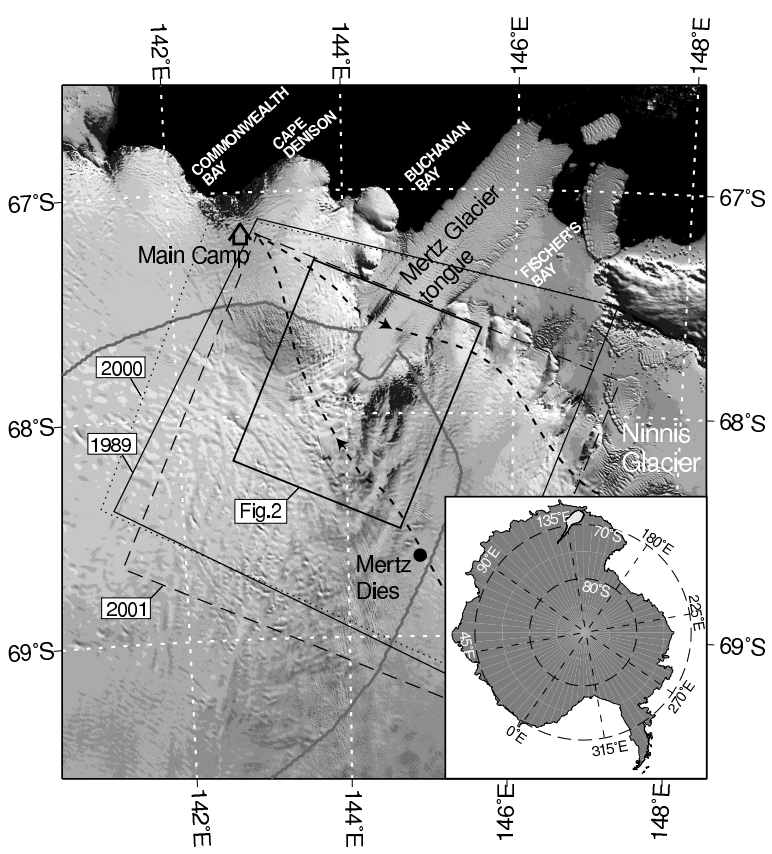

Figure 1. Location of the Mertz Glacier, East Antarctica. The catchment area of the Mertz is shown in white in the inset. On this MODIS image, we show footprints of the three Landsat scenes and the subscene where velocity measurements were computed. The dashed black line represents the trip followed by Mawson and his fellows during their tragic expedition.

One of the scientific goals of the expedition was to map the coast and the extent of the Mertz Glacier tongue. With these early century data, Wendler and others [1996] calculated an increase of over $110 \%$ of the area of this tongue in 80 years. Between 1962 and 1993, the tongue advanced about $26 \mathrm{~km}$, i.e. $840 \mathrm{~m} \mathrm{a}^{-1}$. Since the tongue is floating and in the absence of calving, this value can be considered as the approximate velocity of the ice when crossing the grounding line plus an additional increment of velocity. Wendler and others [1996] also used a SAR image pair separated by 19 months to determine the short term velocity of the tongue. A mean value of $1020 \mathrm{~m} \mathrm{a}^{-1}$ was calculated suggesting that no significant difference exists between the long and short term trend. Yet, this study was limited to the tongue area. Their surface velocity estimation also allowed a first estimation of the ice discharge flux of the Mertz Glacier: $14 \mathrm{~km}^{3} \mathrm{a}^{-1}$ was proposed. Indeed, a recent study by Rignot [2002] showed that this estimation was performed kilometers downstream of the grounding line. Thus, their discharge flux is probably underestimated due to basal melting of the floating tongue. Frezzotti and others [1998] showed that the evolution of the length of the tongue is far from linear: from $150 \mathrm{~km}$ in 1912, it reduced to $113 \mathrm{~km}$ in 1958 and increased again to $155 \mathrm{~km}$ in 1996 . They concluded that at least one major calving event occurred between 1912 and 1956.

Rignot [2002] focused on nine glaciers of East Antarctica using InSAR data. A second estimation of the mass balance of the Mertz Glacier was made but poor accuracy of the velocity field (due to a bad relative orientation of the SAR line of sight and the ice flow) increased the uncertainty on the mass balance. The ice discharge flux of the ice stream was estimated to be $19.8 \mathrm{~km}^{3} \mathrm{a}^{-1}$ at the grounding line and the author proposed a basal melting of $18 \pm 6 \mathrm{~m} \mathrm{a}^{-1}$ of ice just downstream of the grounding line.

\section{DATA AND METHODOLOGY}

\section{The 3 Landsat Images and their pre-processing}

Three Landsat images, with a $30 \mathrm{~m}$ pixel size, are used to determine surface velocities (see Table 1 ). The main velocity field is inferred from a pair of Landsat 7 ETM+ images acquired 21.5 months apart: 26 February 2000 and 13 December 2001. Thus, the main velocity field can be regarded as a two years velocity mean centered in mid-January 2001. The last scene, available from Landsat $5 \mathrm{TM}$, was acquired 2 January 1989. Correlated with the 2000 image, it gives us the 11-year mean velocity of the ice stream centered in late July 1994. Comparison of the two sets of velocity could help to detect possible changes of the Mertz Glacier over the last decade.

Table 1. Landsat Images used to study the Mertz Glacier

\begin{tabular}{lll}
\hline Date & Sensor & Scene ID \\
\hline 1989-01-02 & Landsat 5, TM & LT5082108008900210 \\
2000-02-26 & Landsat 7, ETM+ & L71082108_10820000226 \\
2001-12-13 & Landsat 7, ETM+ & LE7082108000134750 \\
\hline
\end{tabular}

Pre-processing of the images is done to remove the noise and to enhance the small ice features (mostly crevasses) known to move with the flow [Lucchita and Ferguson, 1986]. First, we compute the first principal component of the visible and the near-infrared bands. This step yields low-noise images and enhances the topography. Scan-line striping was removed from the 1989 image using a destriping algorithm, described in Crippen [1989]. A spatial filter is then applied to create two sets of images: the low-pass filtered set is used for the coregistration, the high-pass filtered set for the determination of the displacements. The scale of the filter is chosen to separate the small scale features, moving with the flow, from the fixed long-wavelength features due to the response of ice flowing over topographic features of the bedrock [Paterson, 1994]. This is obtained with a scale of the filter roughly equal to the thickness of the ice [Budd and Carter, 1971], about $1 \mathrm{~km}$ in the case of Mertz Glacier.

The coregistration of the images is one of the key points of the pre-processing because it will highly affects the accuracy of the velocities, especially when the time separation is short. First, "visual" coregistration is performed shifting one of the two images with an offset deduced from comparing the bedrock features in each pair of images. Then, a cross correlation method (described below), is applied between each low-pass filtered pair. The largest windows are 
used for this correlation to encompass the maximum of the low-frequency undulations. Using this methodology, Scambos and others [1992] assumed an accuracy of \pm 1 pixel for the coregistration. Our results (mostly the standard deviation) indicate that $\mathrm{a} \pm 2$ pixels accuracy is more reasonable in our study. If $P_{s}$ is the pixel size $(30 \mathrm{~m})$ and $\Delta t$ the time separation between the two images, this leads to an error for the velocities of $2 \mathrm{P}_{s} / \Delta$ t. This error will be referred later in the article as the "systematic error" and noted $\sigma_{v_{\text {syst }}}$. It equals $\pm 33 \mathrm{ma}^{-1}$ for the $2000-2001$ pair and $\pm 5.3 \mathrm{ma}^{-1}$ for the 1989-2000 pair.

\section{Determination of the velocity field}

A complete description of the methodology followed to generate the velocity field is found in Scambos and others [1992]. Briefly, two high-pass filtered images are used to track the motion of small features by cross-correlating their brightness pattern. Generally, a $32 \times 32$ pixel area is used as a correlation window, but it is changed to $16 \times 16$ when tracking sharp features or to $64 \times 64$ for larger, diffuse ones. The cross correlation algorithm determines the displacement of the features to sub-pixel precision as well as the strength of the correlation and an estimation of the error in the displacement. The displacement is then assigned to the center of the segment linking the two successive positions of the moving feature.

For the 2000-2001 pair, the 21.5-month time separation results in very little change in surface features and reasonable displacements. Even in the fastest part of the ice stream, the maximum displacement is 60 pixels. Thus, features do not show strong deformation and are easy to track. The accuracy of the cross-correlation is estimated to be \pm 0.5 pixel for this pair, which corresponds to $\pm 8 \mathrm{ma}^{-1}$. This error, inherent to the cross correlation, will be referred below as the "random error" and noted $\sigma_{v_{\text {rand }}}$. Computed using Equation (4), the total uncertainty for the 2000-2001 velocities reaches:

$$
\sigma_{v_{00-01}}=\sqrt{\sigma_{v_{\text {rand }}}^{2}+\sigma_{v_{\text {syst }}}^{2}}= \pm 34.4 \mathrm{ma}^{-1}
$$

Note that the uncertainty is independent of the absolute value of the velocity. The relative error would be $100 \%$ in an area moving at $34 \mathrm{~m} \mathrm{a}^{-1}$ and only $3.4 \%$ if the ice speed reaches $1000 \mathrm{ma}^{-1}$.

For the 1989-2000 pair, the situation is more complicated because of the 11-year time separation. Displacements are much larger, reaching 350 pixels in the center of the ice stream. Moreover, changes due to the wind modification of features and strong distortion of the crevasses make difficult their tracking either visually or with the cross-correlation algorithm. In this case, the accuracy is estimated to be around \pm 2 pixels for the displacements. Due to the long time separation, the precision on the velocities remains high: the total uncertainty for the $1989-2000$ velocity $\left(\sigma_{v_{89-00}}\right)$ equals $\pm 7.6 \mathrm{ma}^{-1}$.

\section{RESULTS}

\section{Velocity field of the Mertz}

The main velocity field is obtained from the 2000-2001 pair and a contour map is shown in Figure 2. More than 16700 velocity points were used to produce this map. Each of these velocity points results from the cross correlation algorithm. Automatic selection criteria remove the matches with low correlation strength or large errors. A vector median-filter is also applied to remove inconsistent vectors in regions of high density of velocity points [Astola and others, 1990]. Eventually, each match is reviewed visually on the computer screen. The contour map is then produced in two steps. Velocity points are resampled to a $2 \mathrm{~km}$ grid, keeping in each grid cell a mean velocity value at the mean location of all the velocity points inside the cell. Then, a surface is fitted to all these values using a $1 \mathrm{~km}$ grid size. This dataset of velocity is available at http://nsidc.org/data/velmap/.

To better represent the ice velocity gradients and the shear on the margin of the ice stream, two longitudinal profiles (panels a and b) and one transversal profile (panel c) are shown in Figure 3. To encompass enough velocity points, these profiles are created from the projection of all the velocity measurements within a certain distance of the profile. The width of the swath of the projection must be wide enough to include much velocity points and narrow enough to well represent the region where the velocity gradient is high. A width of 100 pixels, i.e. $3 \mathrm{~km}$, is found to be a good compromise. Also in Figure 3, for the 2000-2001 pair, we added the velocities (plain line) and their error envelopes (dotted line) extracted from a high resolution gridded velocity field.

In Figures 2 and 3, we plot the grounding line (labeled "GL") determined by Rignot [2002] using tidal movement of the tongue as visible with InSAR. Using an original combination of interferograms from the descending pass, Pötzsch and others [2000] also located the grounding line of the Mertz. The two maps are consistent even if some differences exist. In Figure 2, we located the gate $20 \mathrm{~km}$ downstream of the grounding line (labeled "20k") used to calculate basal melting.

Velocities range from a few meters per year to more than $1000 \mathrm{ma}^{-1}$. The contour map shows a well defined main tributary feeding the ice stream from the lower left corner, i.e. southwest, of the map. In this main tributary speed increases rapidly from $500 \mathrm{ma}^{-1}$ to $750 \mathrm{ma}^{-1}$ in $8 \mathrm{~km}$ (A to A' in Figure 3a). This velocity gradient is mainly due to an increase of the surface slope. In the new topography derived from multiple SAR interferograms by Pötzsch and others [2000], it coincides with altitudes decreasing from 500 to $100 \mathrm{~m}$. Below this area, the speed increases still further from $750 \mathrm{~m} \mathrm{a}^{-1}$ to $1000 \mathrm{~m} \mathrm{a}^{-1}$ in $75 \mathrm{~km}$.

Upstream mapping of the velocity in this main tributary is impossible due to a lack of trackable features. In these regions, where crevasses are absent, cross correlation on optical imagery reaches its limit. Moreover, with our uncertainty of $\pm 34.4 \mathrm{ma}^{-1}$, velocity measurements in the catchment area would be highly uncertain.

This south-western location of the main tributary is in agreement with the catchment area derived from topographic data by Rignot [2002]. The drainage basin is mostly located in the western and south-western part of the ice stream (Fig. 1). In the eastern and southern parts, the ice is drained by the Ninnis Glacier system, the neighboring ice stream to the east.

The velocity map also highlights a second tributary located east of the first one. A longitudinal profile along the tributary (B to B' in Figure $3 \mathrm{~b}$ ) also shows a great velocity increase when approaching the grounding line. In this zone, few kilometers upstream from the grounding line, a $550 \mathrm{~m} \mathrm{a}^{-1}$ increase of velocity is observed in only $17 \mathrm{~km}$. Note that these velocity gradients (and consequently the 


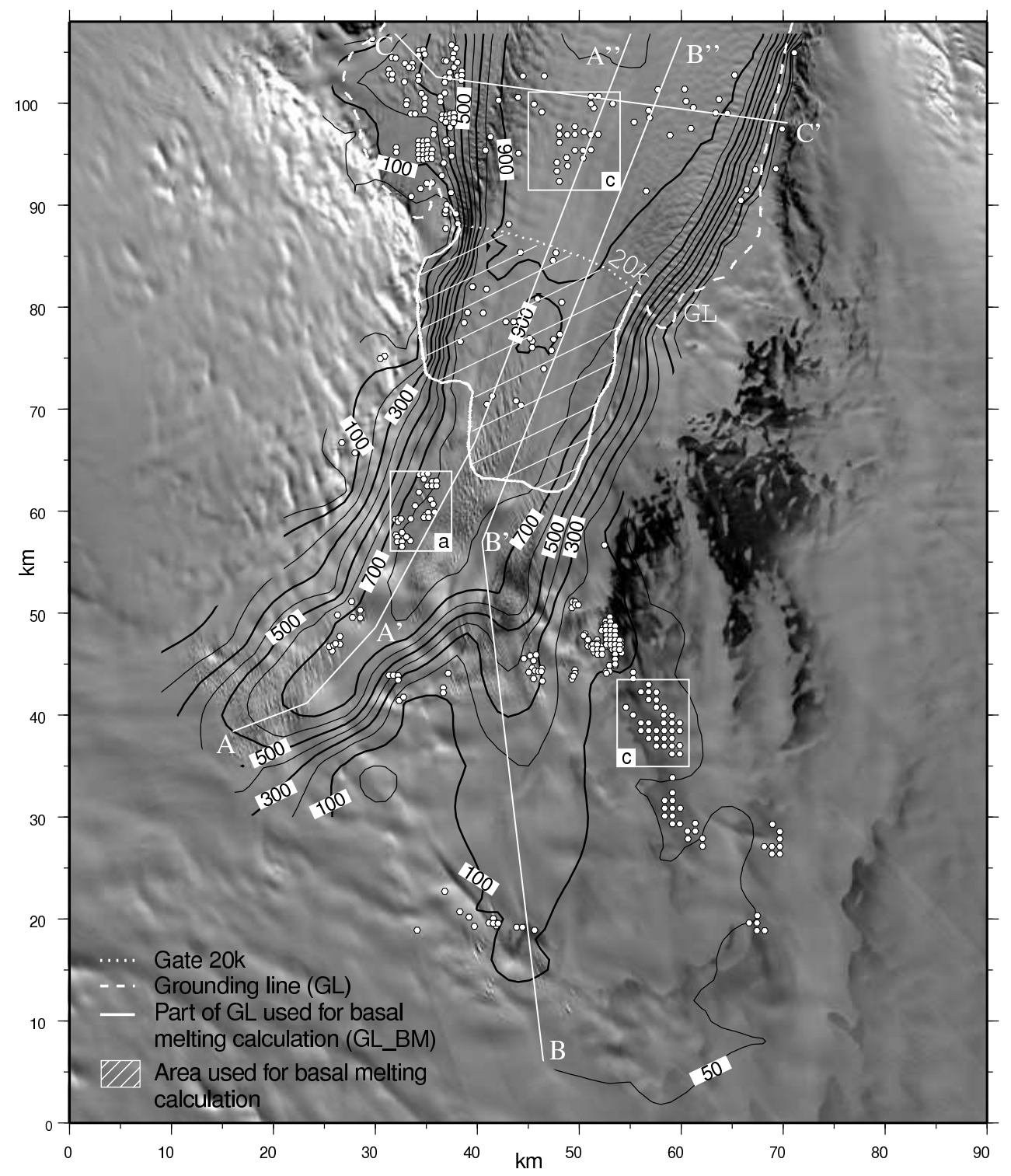

Figure 2. Contour map of the velocity field of the Mertz Glacier deduced from the 2000-2001 pair of images. Contours are drawn every $100 \mathrm{~m} \mathrm{a}^{-1}$ except for the $50 \mathrm{~m} \mathrm{a}^{-1}$ contour. Contours are plotted only where velocity data are available. White circles represent the velocity points deduced from the 89-00 pair of images. Ice flows from the lower part to the upper part of the map. White lines locate the three velocity profiles of Figure 3, white boxes (named a, b and c) the subscenes detailed in Figure 4.The grounding line and the gate $20 \mathrm{~km}$ downstream are also plotted.

strain rate determined below) have small errors because they result from relative differences in velocity: the systematic error due to the misfit in the coregistration vanishes, only the random error inherent in the automatic feature tracking remains.

\section{Comparison to the $1989-2000$ velocities}

The second pair of images (1989 and 2000) does not produce a complete velocity field: surface features were greatly deformed, so matches are sparse and many of them not reliable. 433 displacement vectors are obtained : not enough to create a reasonable velocity contour map for the whole ice stream. However, in a few regions where the density and quality of the matches are good enough, we can make a comparison with the 2000-2001 velocities. Given the uncertainty in the two data sets, a change in velocity is significant if greater than $\sqrt{\sigma_{v_{00-01}^{2}}^{2}+\sigma_{v_{89-00}}^{2}}$, i.e. $35.2 \mathrm{ma}^{-1}$. Thus, even for the fastest part of the glacier the change in velocity must be greater than $3.5 \%$ to be detectable.

On the longitudinal profile $A-A^{\prime}-A^{\prime \prime}$ and the transverse profile $C-C^{\prime}$ in Figure 3, we observe that most of the 1989-2000 velocity points are similar in magnitude to the slowest points for the 2000-2001 pair but not different enough to conclude that a major change in the flow speed occurred. Our plot of the velocity profile, i.e. a projection 
of all the velocity points within a certain distance of the profile, affects the quality of the comparison. It artificially creates a noise in the velocity due to the spatial variation of the velocity within this distance.
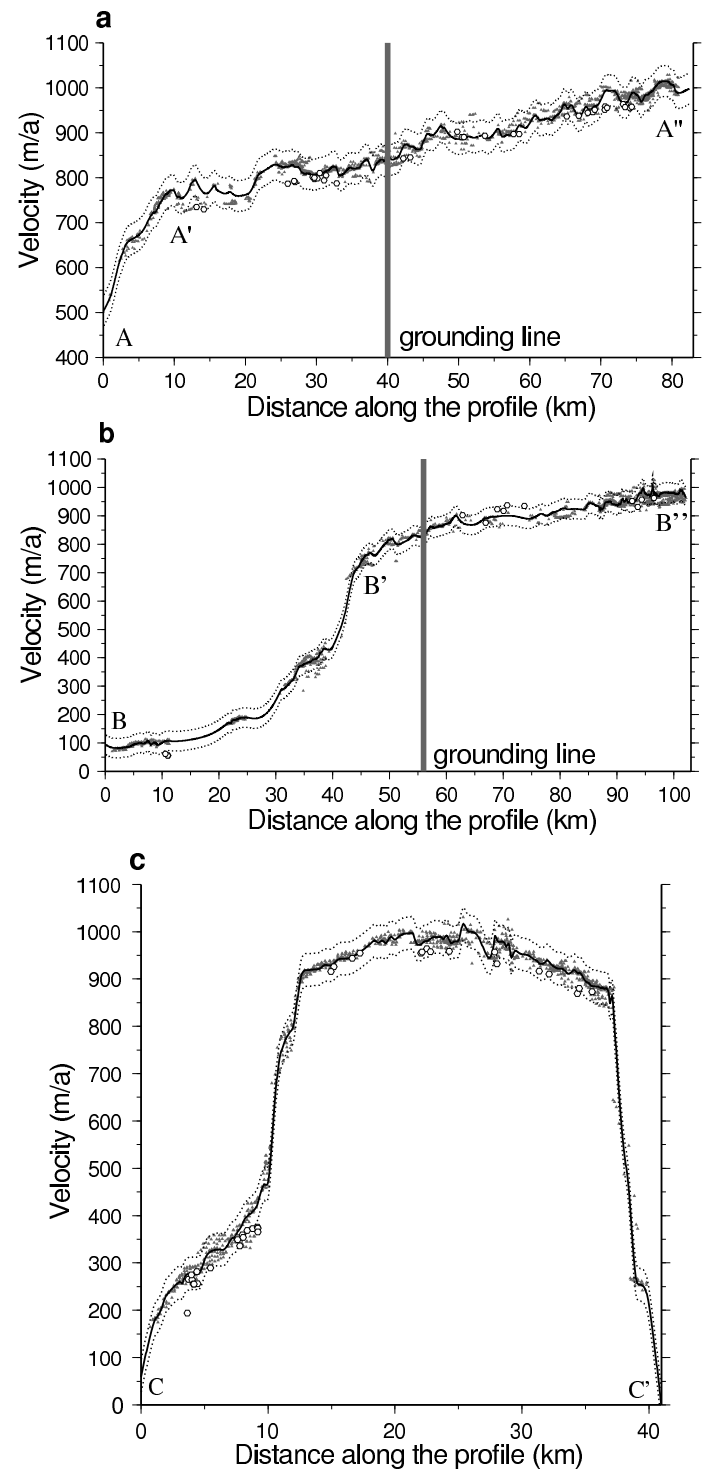

Figure 3. Longitudinal and transversal velocity profiles of the ice stream: (a) along the main tributary ; (b) along the secondary tributary ; (c) transverse in the downstream part of the ice stream. The gray triangles represent velocity measurements from the 2000-2001 pair, the white circles measurements from the 1989-2000 pair. The black line represents the gridded velocity for the 2000-2001 pair and the dotted lines the error envelopes obtained by adding and subtracting the uncertainty (34.4 $\left.\mathrm{ma}^{-1}\right)$.

Comparing directly the velocities in a few regions is more precise: three regions are located in Figure 2 and are shown in more detail in Figure 4. On these regions, we plotted the velocity contours for the 2000-2001 pair and wrote the velocity values obtained from the 1989-2000 pair. For the two subscenes located along the main flow (Fig. 4a and 4b), a consistent velocity change of $30 \mathrm{~m} \mathrm{a}^{-1}$ is observed. This change is under our sensitivity limit of about $35.2 \mathrm{ma}^{-1}$. However, when looking at the third subscene located in a region of slow moving ice (Fig. 4c), we can see that this sensitivity limit may be overestimated: the two sets of velocities are in good agreement and differ only by $10 \mathrm{ma}^{-1}$ in the worst case. This observation holds for all the slow moving areas. Thus, the $35.2 \mathrm{~m} \mathrm{a}^{-1}$ value (proved above to be independent from the speed), is quite conservative.

We calculated the mean difference between the two sets of velocities. Where measurements are available for the oldest pair, we extracted from the gridded data set the velocities for the 2000-2001 pair. The mean velocity increase reaches $15 \mathrm{~m} \mathrm{a}^{-1}$, but the standard deviation (r.m.s.) on this difference $\left(45 \mathrm{ma}^{-1}\right)$, reflects its large spatial variability and the uncertainties.

The Mertz ice stream may have experienced a slight increase in speed during the last decade but we can not prove it. So, we conclude that the flow of the Mertz Glacier is stationary within our uncertainty. The main limitation is the lack of velocity measurements for the old pair, which prevents us from carrying out a statistical study. A velocity change, if existing, could be better detected with a supplementary image from 1991 or 1992. Thus, we would produce a complete velocity field for the early nineties and increase the time separation between the two pairs.

\section{Strain rate of the ice stream}

In the previous section, we touched on the topic of longitudinal strain rate. Indeed, an increase in the velocity of $250 \mathrm{ma}^{-1}$ in $8 \mathrm{~km}$ along the main flow is similar to a longitudinal strain rate of $0.03 \mathrm{a}^{-1}$. This value is in good agreement with the longitudinal strain-rate map (Fig. 5a). This map was produced using the method described in Nye [1959]. This calculation includes a rotation to a local coordinate system parallel (x axis) and perpendicular (y axis) to the flow direction. Starting from a $1 \mathrm{~km}$ gridded velocity field, we calculated the longitudinal, transverse and shear strain rates using the equations shown in appendix of Bindschadler and others [1996]. Then, these results were resampled to a $6 \mathrm{~km}$ grid, a scale which proved to be a good compromise between enhancing the signal to noise ratio (by filtering the small scale dynamics effect) and preserving the significant spatial features.

The longitudinal strain-rate map (Fig. 5a) underscores the two main tributaries of the ice stream where a significant increase in velocity creates a positive strain rate (over $0.03 \mathrm{a}^{-1}$ ), i.e. a horizontal stretching. Increase in the density of crevasses with a direction perpendicular to the flow can be observed in these regions. Between the two tributaries a region of negative longitudinal strain rate reflects the effects of a probable obstruction feature in the bedrock. The flow slows down and compression appears upstream of this feature. After crossing the grounding line, the center part of the ice stream experiences low longitudinal strain rate in comparison to the margins. For the whole ice stream, a positive mean longitudinal strain rate $\left(0.6 \times 10^{-3} \mathrm{a}^{-1}\right)$ shows that a horizontal stretching regime prevails. The margins are affected by a large shear strain rate exceeding $0.04 \mathrm{a}^{-1}$ (Fig. 5b). The ice stream is divided into two symmetric regions affected by a shear of similar amplitude but opposite signs. This pattern underscores the highly symmetric 


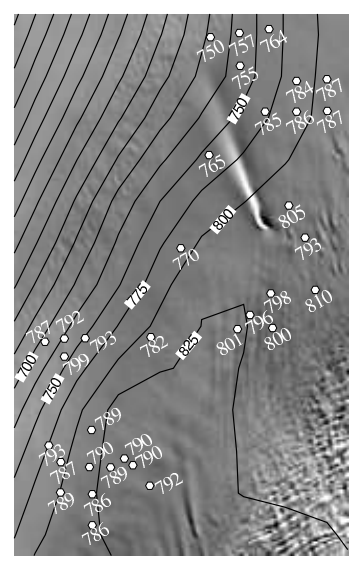

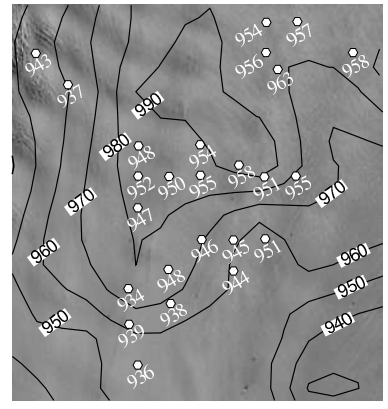

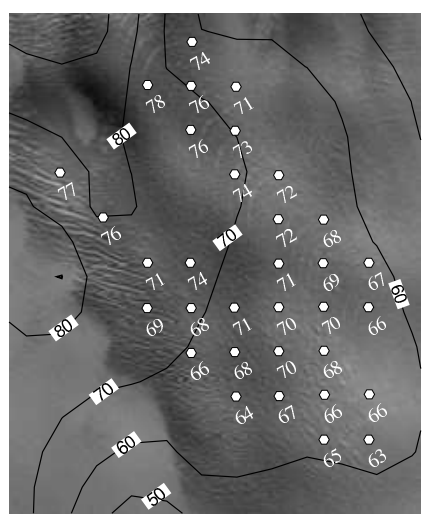

c

Figure 4. Three regions of the ice stream where enough matches for the old pair of images (1989-2000, white circles) allow a significant comparison with the velocity contours from the recent pair (2000-2001). The two first subscenes (a) and (b), along the main tributary, show an increase in velocity around $30 \mathrm{ma}^{-1}$ and the third (c), in a slow moving part of the glacier, shows no change in velocity.

a

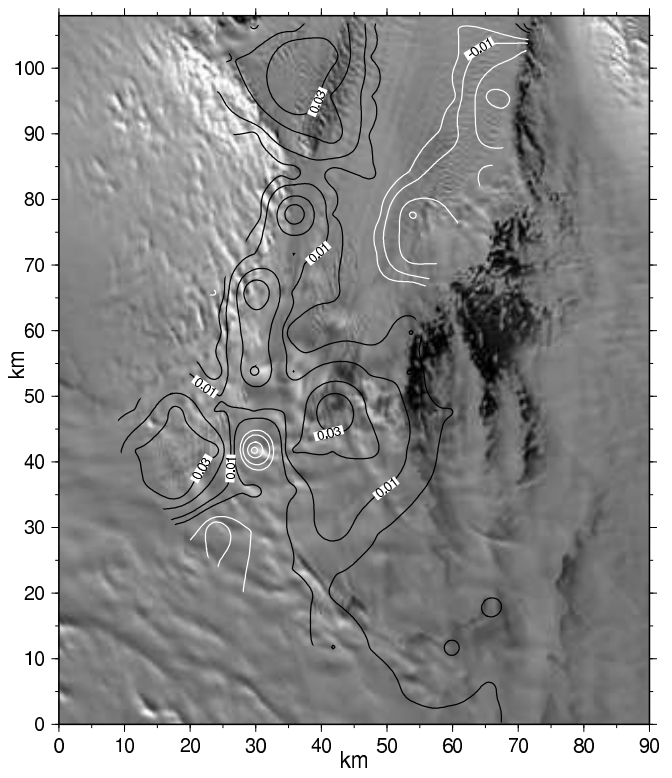

\section{b}

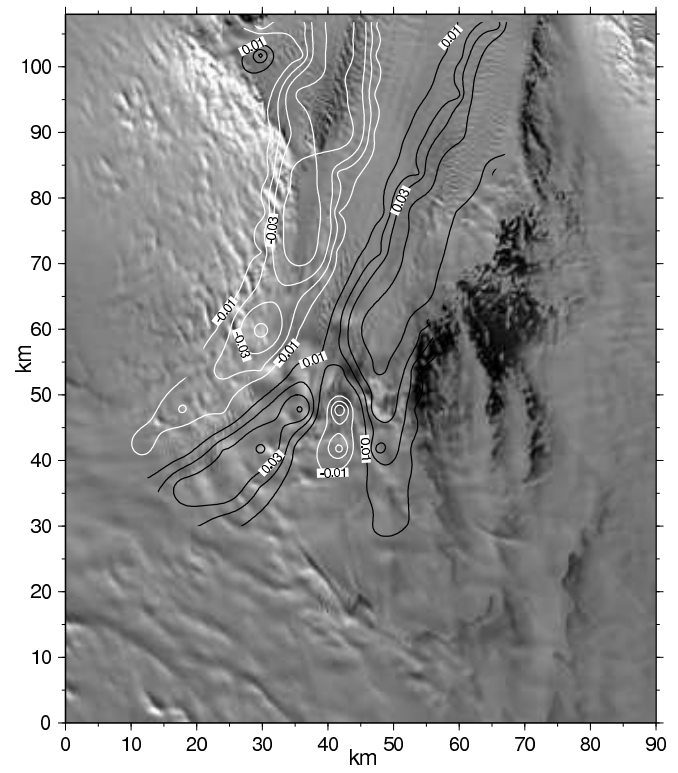

Figure 5. Strain-rate maps of the Mertz Glacier deduced from the 2000-2001 velocity field: (a) the longitudinal strain rate and (b) the shear strain rate.

flow in the Mertz Glacier. Transverse strain rate (not shown here) is generally opposite in sign of the longitudinal one.

From the continuity equation we can also derive the vertical strain rate for the grounded part of the ice stream. For an incompressible medium (constant density) that is not changing state (ignoring accumulation, ablation, and bottom melting), the divergence of the velocity is zero:

$$
\vec{\nabla} \cdot \vec{v}=0
$$

Replacing the velocity vector with its components in each direction leads to:

$$
\frac{\partial v_{x}}{\partial x}+\frac{\partial v_{y}}{\partial y}+\frac{\partial v_{z}}{\partial z}=0
$$

By definition of the strain rates:

$$
\dot{\epsilon_{z}}=-\left(\dot{\epsilon_{x}}+\dot{\epsilon_{y}}\right)
$$

We can thus derive the surface vertical strain rate from the longitudinal and transversal strain rates. The values of the vertical strain rate are mainly negative. A mean value of $-0.7 \times 10^{-3} \mathrm{a}^{-1}$ for the whole grounded ice stream is the result of the thinning of the ice while flowing to the sea [Bindschadler and others, 1996]. 


\section{MASS BALANCE OF THE MERTZ GLACIER}

\section{Discharge fluxes}

Getting accurate and well-distributed velocity measurements allows the calculation of the discharge fluxes of the ice stream at different "gates" transverse to the flow and crossing the whole ice stream. Each gate is broken into a number of small segments or "individual gates", through each of which the discharge flux is calculated and summed. The number of gates must be high enough to take into account each change in the magnitude or the direction of the velocity. The mass flux through a given gate, $\Phi_{\text {gate }}$, is calculated according to:

$$
\Phi_{\text {gate }}=\sum_{i} v_{\perp i} \cdot H_{i} \cdot \delta w_{i}
$$

with:

$v_{\perp i}$ the velocity perpendicular to the $i^{t h}$ segment,

$H_{i}$ the mean ice thickness of the $i^{t h}$ segment,

$\delta w_{i}$ the width of the $i^{t h}$ segment.

For the ice thickness, due to the lack of reliable data in the region of the Mertz Glacier (the BEDMAP database includes only 3 points over the Mertz Glacier tongue), we used the assumption proposed by Rignot [2002] of hydrostatic equilibrium of the floating part of the ice stream. Following Rignot [2002], the ice thickness is deduced from the ERS Digital Elevation Model (DEM) by multiplying the elevation by 8.05 . This factor includes both the hydrostatic equilibrium of ice floating over sea water and an offset in geoidal height. Restricted to this assumption, we were only able to calculate discharge fluxes downstream of the grounding line. According to Rignot [2002], the uncertainty on these estimates of ice thickness, $\sigma_{H}$, can be considered as a constant equal to $100 \mathrm{~m}$.

Another key assumption in Equation (3) is constant velocity with depth. Bindschadler and others [1996] showed that this is already a good approximation for the grounded ice stream. For the floating ice, for which no basal drag is present, the bottom of the ice is a free surface and this assumption is all the more realistic.

At the grounding line of the Mertz Glacier (noted GL in Figure 2), we calculated an ice discharge flux of $17.8 \mathrm{~km}^{3} \mathrm{a}^{-1}$.

The uncertainty on this value has to be calculated carefully. As detailed previously, the uncertainty for the main velocity field, $\sigma_{v_{00-01}}$ is $34.4 \mathrm{ma}^{-1}$. This is also the uncertainty on the velocity perpendicular to each segment: in the worst case, this velocity error will be perpendicular to the segment. We assume that the uncertainty on the width of each gate is negligible, supposing that the pixel size of $30 \mathrm{~m}$ provided by Landsat is accurate. The calculation of uncertainty is made using the following formula extracted from Bevington [1969]. If $\Phi$ is our final result, composed of a functional relation $\mathrm{f}$ between independent parameters $\mathrm{x}$, $\mathrm{y}, \mathrm{z}, \ldots$ and if $\sigma_{x}, \sigma_{y}, \sigma_{z}, \ldots$ are the uncertainties in $\mathrm{x}, \mathrm{y}, \mathrm{z}$, ... and are uncorrelated, then the uncertainty in $\Phi$ is:

$$
\sigma_{\Phi}^{2}=\sigma_{x}^{2}\left(\frac{\partial f}{\partial x}\right)^{2}+\sigma_{y}^{2}\left(\frac{\partial f}{\partial y}\right)^{2}+\sigma_{z}^{2}\left(\frac{\partial f}{\partial z}\right)^{2}+\ldots
$$

Applying this equation to the discharge fluxes leads to an uncertainty of:

$$
\sigma_{\Phi}^{2}=\sum_{i}\left(\sigma_{v_{00-01}}^{2} \cdot H_{i}^{2}+\sigma_{H}^{2} \cdot v_{\perp i}^{2}\right) \cdot \delta w_{i}^{2}
$$

The uncertainty on the ice discharge flux at the grounding line is $1.2 \mathrm{~km}^{3} \mathrm{a}^{-1}$ and is equally due to the uncertainty on the velocity and on the ice thickness.

\section{Basal melting}

As proposed by Rignot [1996], the melting at the base of the floating part of the ice stream can be calculated by using at least two transverse gates. To calculate the basal melting of the Mertz, we used a closed contour including part of the grounding line and another gate located $20 \mathrm{~km}$ downstream (noted GL_BM and 20k respectively in Figure 2). The area delimited by this contour is $375 \mathrm{~km}^{2}$. The basal melting $\dot{\mathcal{B}}$ is calculated by:

$$
\dot{\mathcal{B}}=\frac{\Phi_{G L_{\mathcal{B M}}}-\Phi_{20 k}}{\mathcal{S}}+\dot{\mathcal{A}}
$$

with:

$\Phi_{G L_{\mathcal{B} M}}$ the discharge flux ( $\mathrm{m}^{3} \mathrm{a}^{-1}$ of ice) at (part of) the grounding line,

$\Phi_{20 k}$ the discharge flux ( $\mathrm{m}^{3} \mathrm{a}^{-1}$ of ice) $20 \mathrm{~km}$ downstream of the grounding line,

$\mathcal{S}$ the area $\left(\mathrm{m}^{2}\right)$ between the two gates,

$\dot{\mathcal{A}}$ the accumulation rate $\left(\mathrm{m} \mathrm{a}^{-1}\right.$ of ice) between the two gates.

Note the difference between $\Phi_{G L_{\mathcal{B} M}}$ and $\Phi_{G L}: \Phi_{G L}$ is the discharge flux through the whole grounding line, whereas $\Phi_{G L_{\mathcal{B M}}}$ is the discharge flux through the part of the grounding line forming a closed contour with the gate $20 \mathrm{~km}$ downstream (see Figure 2 to visualize this difference). Thus, $\Phi_{G L_{\mathcal{B} \mathcal{M}}}<\Phi_{G L}$.

With $\Phi_{G L_{\mathcal{B} \mathcal{M}}}=16.6 \pm 1.14 \mathrm{~km}^{3} \mathrm{a}^{-1}$ and $\Phi_{20 k}=12.6$ $\pm 0.91 \mathrm{~km}^{3} \mathrm{a}^{-1}$ of ice, and an accumulation rate around $0.35 \mathrm{~m} \mathrm{a}^{-1}$ of ice [Vaughan and others, 1999], we estimate the basal melting to be $11 \mathrm{ma}^{-1}$ of ice.

Assuming no error in $\mathcal{S}$ and an accumulation accurate at $\pm 0.1 \mathrm{ma}^{-1}\left(\sigma_{\dot{\mathcal{A}}}\right)$, the uncertainty $\sigma_{\dot{\mathcal{B}}}$ on the basal melting is:

$$
\sigma_{\dot{\mathcal{B}}}^{2}=\left(\frac{\sigma_{\Phi_{G L, \mathcal{B M}}}}{\mathcal{S}}\right)^{2}+\left(\frac{\sigma_{\Phi_{20 k}}}{\mathcal{S}}\right)^{2}+\sigma_{\dot{\mathcal{A}}}^{2}
$$

We estimate $\sigma_{\dot{\mathcal{B}}}$ to be $\pm 3.9 \mathrm{ma}^{-1}$ of ice.

$11 \mathrm{ma}^{-1}$ of ice is nearly $40 \%$ lower than the value of $18 \mathrm{~m} \mathrm{a}^{-1}$ of ice calculated by Rignot [2002]. This difference is mostly due to our slower flow speed when crossing the grounding line: at the grounding line, the difference between our ice discharge flux $\left(16.6 \pm 1.14 \mathrm{~km}^{3} \mathrm{a}^{-1}\right)$ and that from Rignot [2002] $\left(19.8 \pm 1.14 \mathrm{~km}^{3} \mathrm{a}^{-1}\right)$ is $3.2 \mathrm{~km}^{3} \mathrm{a}^{-1}$ whereas it is only $0.5 \mathrm{~km}^{3} \mathrm{a}^{-1}$ at the gate $20 \mathrm{~km}$ downstream (12.6 versus $13.1 \pm 0.91 \mathrm{~km}^{3} \mathrm{a}^{-1}$ ). We demonstrated previously that the flow of the Mertz Glacier is nearly constant and thus, we attribute these difference to errors in the velocities of Rignot [2002]. These errors could be due to the fact that "both [Ninnis and Mertz] glaciers flow nearly parallel to the satellite track direction" [Rignot, 2002] which affects 
the accuracy of the InSAR measurement of the ice velocities. Still, basal melting remains the main factor of ablation for the tongue of the Mertz Glacier. Due to the limited northern extension of the Landsat scenes we used, the calculation could not be extended in the seaward part of the tongue. Our value of the basal melting is only representative of the first kilometers of the Mertz Glacier tongue. Frezzotti and others [2000], studying outlet glaciers from the Dome $\mathrm{C}$ area, showed that basal melting (and freezing rate) can significantly change along the floating tongue.

\section{Mass Balance}

The mass balance of the drainage basin can then be estimated using the mass budget method as detailed in Rignot [2002]. The total input was calculated by Rignot [2002]. Using a DEM of Antarctica, the drainage basin of the Mertz is delimited and has an area of $83080 \mathrm{~km}^{2}$. On this area, the digital accumulation map of Vaughan and others [1999] and Giovinetto and Zwally [2000] are integrated to estimate the total annual accumulation: $21.3 \mathrm{~km}^{3} \mathrm{a}^{-1}$ of ice. The mean accumulation for the whole catchment area is thus $256 \mathrm{~mm} \mathrm{a}^{-1}$ of ice. This value is lower than the $272 \mathrm{~mm} \mathrm{a}^{-1}$ of ice previously proposed by Wendler and others [1996].

Thus, the Mertz Glacier seems not to be in balance with an annual input $3.5 \mathrm{~km}^{3}$ of ice higher than the output. If existing, such an imbalance would result in a rate of ice thickening of $4.2 \mathrm{~cm} \mathrm{a}^{-1}$ over the catchment area. Using altimeter data from SEASAT and ERS-1, Rémy and Legrésy [1998] mapped the changes in the height of the Antarctic ice sheet between 1978 and 1992. The only area showing a significant rise of the surface is located between $140^{\circ} \mathrm{E}$ to $150^{\circ} \mathrm{E}$ and between $70^{\circ} \mathrm{S}$ to $72^{\circ} \mathrm{S}$ and includes part of the Mertz drainage basin. The rise (20-25\% of the accumulation rate) is not very different from our imbalance of $3.5 \mathrm{~km}^{3}$ of ice, which represents $16.5 \%$ of the accumulation rate on the Mertz drainage basin.

On the other hand, the map proposed by Wingham and others [1998] does not show any significant elevation change in the catchment area of the Mertz Glacier. We are quite confident in our estimation of the velocity at the grounding line. More uncertainties remain in the ice thickness which results from an assumption. We also wonder whether the accumulation rate on the drainage basin is accurate in an area subject to the strongest wind in the world [Wendler and others, 1994] and where blue ice areas are widespread. In the accumulation rate map of Vaughan and others [1999], they are assumed to be areas of nil accumulation although they undergo ablation. Despite the assertion by Bintanja and van den Broeke [1995] that blue ice areas are "too small to play an important role in the total surface mass balance regime in Antarctica", we believe that, in some catchments such as Mertz, they could be significant. Another uncertainty results from the very coarse grid in the accumulation rate map of Vaughan and others [1999]: the grid spacing of one degree is probably too coarse to represent the steep gradient in precipitation near the coast. We suspect that the export of snow by wind [Loewe, 1972] is not well taken into account in the accumulation map. The low density of measurements used by Vaughan and others [1999] in the Mertz area could affect the extrapolation process to a gridded data set of accumulation: the extrapolation of points with less wind erosion to areas of much greater wind erosion (such as the grounding line of the Mertz Glacier) could lead to an overestimation of the accumulation rate in those windy areas. If we assume no imbalance for the Mertz glacier, our measurements suggest that the wind could export $16.5 \%$ of the total precipitation on the drainage basin. This is close to the value of $15 \%$ proposed by Wendler and others [1996]. Blowing winds not only export snow: they also increase sublimation. Bintanja [1998] quantified this effect using a one-dimensional atmospheric surface layer model based on observations from automatic weather stations on a transect from Dumont d'Urville to Dome C. Extrapolated to the whole East Antarctica, the model show that snowdrift sublimation removes $19 \mathrm{~mm}$ of ice par year. It represents $7.5 \%$ of the total precipitation on the Mertz drainage basin.

\section{CONCLUSION}

From two Landsat images with a $30 \mathrm{~m}$ ground resolution, automatic feature tracking allows the determination of the velocity map of the Mertz Glacier between February 2000 and December 2001, with an accuracy of $34.4 \mathrm{ma}^{-1}$. The Mertz Glacier presents a highly symmetric flow, with a strong increase in velocity when reaching the grounding line. An older image, acquired in 1989, permits a few measurements of the mean velocity during the nineties. Comparing this second velocity data set with the main velocity field, we conclude that the flow of the Mertz is stationary within our sensitivity limit of $35.2 \mathrm{~m} \mathrm{a}^{-1}$. The quality of the main velocity field allows a new assessment of the discharge flux, the basal melting and the mass balance of the drainage basin of the Mertz Glacier. The ice discharge flux at the grounding line is $17.8 \pm 1.2 \mathrm{~km}^{3} \mathrm{a}^{-1}$. Comparing with the annual accumulation of $21.3 \mathrm{~km}^{3}$ of ice leads to an imbalance, the input being $20 \%$ higher than the output.

From the published studies, it is not clear whether the Mertz Glacier accumulation area experienced any elevation change. Our results agree with the positive imbalance of $20 \%$ observed by Rémy and Legrésy [1998]. On the other hand, if we assume [as do Vaughan and others, 1999], that no elevation change occurs in the area, our imbalance could result from errors in the measurements and gridding of the accumulation. In this part of Antarctica, subjected to the strongest winds in the world, the importance of blue ice and negative local net surface balance should be reassessed. Getting thickness profiles transverse to the ice stream would also increase the reliability of the discharge fluxes. The basal melting on the floating ice tongue, $11 \pm 3.9 \mathrm{~m} \mathrm{a}^{-1}$ of ice, is lower that the previous estimate $\left(18 \pm 6 \mathrm{~m} \mathrm{a}^{-1}\right.$ of ice) by Rignot [2002].

Acknowledgments. The first author would like to thank Roger Barry for welcoming him at NSIDC (National Snow and Ice Data Center) and IRD (Institut de Recherche pour le Développement) for contributing to the funding of the internship. This study was supported by NASA Grant NAG5-3438. We thank Eric Rignot (Jet Propulsion Laboratory) for providing data of elevation, accumulation and location of the grounding line of the Mertz area. We sincerely thank Dr. Massimo Frezzotti, another anonymous reviewer and Dr. Jacques Meyssonnier (Scientific Editor) for making useful comments on the earlier versions of the manuscript. Paul Wessel and Walter Smith's GMT software [Wessel and Smith, 1998] illustrated the article.

\section{References}

Astola, J., P. Haavisto and N. Yrjö. 1990. Vector median filters. Proceedings of the IEEE, 78(4), 678-689.

Bamber, L. J., D. G. Vaughan and I. Joughin. 2000. Widespread complex flow in the interior of the Antarctic ice sheet. Science, 287(5456), 1248-1250.

Bevington, P. R. 1969. Data reduction and error analysis for the physical sciences. McGraw-Hill, Inc. 
Bindschadler, R. A. and P. L. Vornberger. 1998. Changes in the West Antarctic ice sheet since 1963 from declassified satellite photography. Science, 279(5351), 689-692.

Bindschadler, R. A., P. L. Vornberger, D. D. Blankenship, T. A. Scambos and R. Jacobel. 1996. Surface velocity and mass balance of Ice Streams D and E, West Antarctica. J. Glaciol., 42(142), 461-475.

Bintanja, R. 1998. The contribution of snowdrift sublimation to the surface mass balance of Antarctica. Ann. Glaciol., 27, 251-259.

Bintanja, R. and M. R. van den Broeke. 1995. The surface energy balance of Antarctic snow and blue ice. J. Appl. Meteor., 34(4), 902-926.

Budd, W. F. and D. B. Carter. 1971. An analysis of the relation between the surface and bedrock profiles of ice caps. $J$. Glaciol., 10(59), 197-209.

Cabanes, C., A. Cazenave and C. Le Provost. 2001. Sea level rise during past 40 years determined from satellite and in situ observations. Science, 294(5543), 840-842.

Conway, H., G. Catania, C. F. Raymond, A. M. Gades, T. A. Scambos and H. Engelhardt. 2002. Switch of flow direction in an Antarctic ice stream. Nature, 419(6906), 465-467.

Crippen, R. E. 1989. A simple filtering routine for the cosmetic removal of scan-line noise from Landsat TM P-tape imagery. Photogramm. Eng. Remote Sens., 55(3), 327-331.

Frezzotti, M., A. Cimbelli and J. G. Ferrigno. 1998. Ice-front change and iceberg behaviour along Oates and George V Coasts, Antarctica, 1912-96. Ann. Glaciol., 27, 643-650.

Frezzotti, M., I. E. Tabacco and A. Zirizzotti. 2000. Ice discharge of eastern Dome C drainage area, Antarctica, inferred from airborne radar survey and satellite image analysis. J. Glaciol., 46(153), 253-264.

Giovinetto, M. and J. Zwally. 2000. Spatial distribution of net surface accumulation on the Antarctic ice. Ann. Glaciol., 31, $171-178$.

Goldstein, R. M., H. Engelhardt, B. Kamb and R. M. Frolich. 1993. Satellite radar interferometry for monitoring ice sheet motion: application to an Antartic ice stream. Science, 262(5139), 1525-1530.

Gray, A. L., N. Short, K. E. Mattar and K. C. Jezek. 2001. Velocities and flux of the Filchner Ice Shelf and its tributaries determined from speckle tracking interferometry. Can. J. Remote Sensing, 27(3), 193-206.

Hughes, T. 1977. West Antarctic ice streams. Rev. Geophys. Space Phys., 15(1), 1-46.

Joughin, I. and S. Tulaczyk. 2002. Positive mass balance of the Ross ice streams, West Antarctica. Science, 295(5554), 476480.

Joughin, I. R., R. Kwok and M. A. Fahnestock. 1998. Interferometric estimation of the three-dimensional ice-flow velocity vector using ascending and descending passes. IEEE $T$. Geosci. Remote, 36(1), 25-37.

Loewe, F. 1972. The land of storms. Weather, 27(3), 110-121.

Lucchita, B. K. and H. M. Ferguson. 1986. Antarctica: measuring glacier velocity from satellite images. Science, 234(4780), 1105-1108.
Mawson, D. 1998. The home of the blizzard, a true story of Antarctic survival. First edition. St. Martin's Press, New York.

Mohr, J. J., N. Reeh and S. N. Madsen. 1998. Three dimensional glacial flow and surface elevation measured with radar interferometry. Nature, 391(6664), 273-276.

Nye, J. F. 1959. A method of determining the strain-rate tensor at the surface of a glacier. J. Glaciol., 3(25), 409-419.

Paterson, W. S. B. 1994. The physics of glaciers. $3^{\text {rd }}$ edition. Pergamon, New York.

Pötzsch, A., B. Legresy, W. Korth and R. Dietrich. 2000. Glaciological investigation of Mertz Glacier, East Antarctica, using SAR interferometry and field observations. Proceedings of ERS - ENVISAT Symposium in Gothenburg.

Rémy, F. and B. Legrésy. 1998. Antarctic non-stationary signals derived from Seasat-ERS-1 alitmetry comparison. Ann. Glaciol., 27, 81-85.

Rignot, E. 1996. Tidal motion, ice velocity and melt rate of Petermann Gletscher, Greenland, measured from interferometry. J. Glaciol., 42(142), 476-485.

Rignot, E. 2001. Evidence for rapid retreat and mass loss of Thwaites Glacier, West Antarctica. J. Glaciol., 47(157), 213222.

Rignot, E. 2002. Mass balance of East Antarctic glaciers and ice shelves from satellite data. Ann. Glaciol., 34, 217-227.

Rignot, E. and S. S. Jacobs. 2002. Rapid bottom melting widespread near Antarctic ice sheet grounding lines. Science, 296(5575), 2020-2023.

Rignot, E., D. G. Vaughan, M. Schmeltz, T. Dupont and D. MacAyeal. 2002. Acceleration of Pine Island and Thwaites Glaciers, West Antartica. Ann. Glaciol., 34, 189-194.

Scambos, T. A., M. J. Dutkiewicz, J. C. Wilsoni and R. A. Bindschadler. 1992. Application of image cross-correlation to the measurement of glacier velocity using satellite image data. Remote Sensing Environ., 42(3), 177-186.

Vaughan, D. G., J. L. Bamber, J. Russell and P. R. Cooper. 1999. Reassessment of net surface mass balance in Antarctica. J. Climate, 12(4), 933-946.

Wendler, G., K. Ahlnäs and G. S. Lingle. 1996. On Mertz and Ninnis glaciers, East Antarctica. J. Glaciol., 42(142), 447-453.

Wendler, G., J. C. André, P. Pettré, J. Gosink and T. Parish. 1994. Katabatic winds in Adélie Land. In D. Bromwich and C. Stearns, eds., Antarctic meteorology and climatology: studies based on automatic weather stations, Antarctic Research Series 61., 23-45. American Geophysical Union, Washington DC.

Wessel, P. and W. H. F. Smith. 1998. New, improved version of generic mapping tools released. EOS T. Am. Geophy. Un., $\mathbf{7 9}(47), 579$.

Wingham, D. J., A. J. Ridout, R. Scharroo, R. J. Arthern and C. K. Shum. 1998. Antarctic elevation change from 1992 to 1996. Science, 282(5388), 456-458.

Etienne Berthier, OMP-LEGOS, 18 av. E. Belin, 31401 Toulouse ; etienne.berthier@cnes.fr

Bruce Raup \& Ted Scambos, NSIDC, University of Colorado, Boulder, USA ; braup@nsidc.org ; teds@colorado.edu 


\section{Annexe B}

\section{Amincissement de la Mer de Glace déduit d'Images Satellitaires}

Berthier, E., Arnaud, Y., Baratoux, D., Vincent, C., \& Rémy, F., 2004. Recent rapid thinning of the "Mer de glace" glacier derived from satellite optical images. Geophys. Res. Lett., 31 (17), L17401. 


\title{
Recent rapid thinning of the "Mer de Glace" glacier derived from satellite optical images
}

\author{
E. Berthier, ${ }^{1}$ Y. Arnaud, ${ }^{2}$ D. Baratoux,${ }^{3}$ C. Vincent, ${ }^{4}$ and F. Rémy ${ }^{1}$ \\ Received 8 June 2004; revised 27 July 2004; accepted 6 August 2004; published 3 September 2004.
}

[1] The rapid wastage of mountain glaciers and their contribution to sea level rise require worldwide monitoring of their mass balance. In this paper, we show that changes in glacier thickness can be accurately measured from satellite images. We use SPOT image pairs to build Digital Elevation Models (DEMs) of the Mont Blanc area (French Alps) for different years. To register the DEMs, we adjust their longitude, latitude and altitude over motionless areas. The uncertainty of the thickness change measurement is greatly reduced by averaging over areas covering altitude intervals of $50 \mathrm{~m}$. Comparisons with topographic profiles and a differential DEM from aerial photographs obtained on the Mer de Glace indicate an overall accuracy of $1 \mathrm{~m}$ for the thickness change measurement. Below $2100 \mathrm{~m}$, satellite DEMs show an evolution of the thinning rate from $1 \pm$ $0.4 \mathrm{~m} \cdot \mathrm{a}^{-1}$ (years 1979-1994) to $4.1 \pm 1.7 \mathrm{~m} \cdot \mathrm{a}^{-1}$ (20002003). INDEX TERMS: 1224 Geodesy and Gravity: Photogrammetry; 1640 Global Change: Remote sensing; 1827 Hydrology: Glaciology (1863); 1884 Hydrology: Water supply. Citation: Berthier, E., Y. Arnaud, D. Baratoux, C. Vincent, and F. Rémy (2004), Recent rapid thinning of the "Mer de Glace" glacier derived from satellite optical images, Geophys. Res. Lett., 31, L17401, doi:10.1029/2004GL020706.

\section{Introduction}

[2] Mountain glaciers are considered to be a reliable indicator of climate change [Intergovernmental Panel on Climate Change, 2001]. Even if they only represent $4 \%$ of the cryosphere, they contribute to $27 \%$ of the sea level rise observed between 1988 and 1998 [Dyurgerov, 2003].

[3] Measuring mass balance is a straightforward approach for quantifying the temporal evolution of glaciers. It is directly related to local climate variability. Yearly changes in precipitation and/or energy balance affect the accumulation or ablation on the glacier [Vincent, 2002]. Mass balance is also a direct measurement of the contribution of glaciers to sea level rise [Rignot et al., 2003]. Although the length of glaciers can be used as a climate indicator [Oerlemans, 1994], the low correlation between terminus position and volume change at short time scale [Arendt et al., 2002] reinforces the need of mass balance measurements.

[4] The traditional way to measure mass balance, known as the glaciological method [Paterson, 1994], is time-consuming and difficult in harsh environment. Accord-

\footnotetext{
${ }^{1}$ LEGOS (UPS-CNES-CNRS-IRD), Toulouse, France.

${ }^{2}$ GREAT ICE (IRD), St. Martin d'Hères, France.

${ }^{3}$ LDTP (UPS-CNES-CNRS-IRD), Toulouse, France.

${ }^{4}$ LGGE (UJF-CNRS), St. Martin d'Hères, France.
}

Copyright 2004 by the American Geophysical Union. 0094-8276/04/2004GL020706\$05.00 ing to Braithwaite [2002], only 246 mountain glaciers are (or have been) monitored with this method, representing less than $2 \%$ of their global area. There is a regional bias in these measurements: two thirds of the monitored glaciers are located in North America and Europe.

[5] Remote sensing provides a suitable way to increase the number of monitored glaciers, especially in remote areas. Most remote sensing approaches are based on the "geodetic" method [Finsterwalder, 1954], of comparing two maps or DEMs of the same area established during different years. If both maps cover the whole glacier, their difference, the volume change, can be converted to mass balance assuming a constant density of $900 \mathrm{~kg} \cdot \mathrm{m}^{-3}$ [Andreassen et al., 2002]. In this study, we address only the problem of measuring thickness change and not its conversion to mass balance.

[6] Differential DEMs derived from aerial photographs are considered as the most reliable measurement of mass balance over one or several decades [Andreassen et al., 2002] and, thus, are used to check the quality of cumulative mass balance determined by the glaciological method [Krimmel, 1999]. This approach is limited by the small coverage of the photographs ( 30 to $40 \mathrm{~km}^{2}$ ) and is generally restricted to glaciers already monitored on the field. Thus, it is not a suitable way to extend mass balance measurement to a large number of glaciers. Recently, airborne laser altimetry has also proved to be an efficient tool to estimate the volume change of glaciers [Echelmeyer et al., 1996; Baltsavias et al., 1999]. Its accuracy in the accumulation area and the possibility to extract thickness changes rapidly for large glaciers [Arendt et al., 2002] are the advantages of this method. Yet, the cost and the limited sampling are important drawbacks.

[7] Applying the geodetic method to satellite images is a third way to measure mass balance by remote sensing. In this paper, we derived for the first time from satellite DEMs an accurate measurement of thickness change for a mountain glacier. We validate our results by comparison with topographic and aerial photographs measurements. The $\mathrm{Mer}$ de Glace, the largest glacier in the French Alps, has been chosen as the test area because good satellite image pairs are available and ground surveys are performed yearly.

\section{Methodology}

[8] Our approach adapts the geodetic method to satellite images. The first step calculates the DEMs. Some adjustments and corrections are needed to reduce the biases between the DEMs. The mean thickness change is then extracted for each altitude intervals on the glacier.

\subsection{Construction of Satellite DEMs}

[9] DEMs are calculated from pairs of SPOT satellite images with opposite incidence angles and short time 
Table 1. Satellite Images Used in This Study

\begin{tabular}{cccc}
\hline Date & Sensor & Incidence Angle & Gain \\
\hline $1994-10-16$ & SPOT3 & -4.6 & 7 \\
$1994-10-17$ & SPOT3 & -30.3 & 8 \\
$2000-08-25$ & SPOT2 & -22.3 & 6 \\
$2000-08-25$ & SPOT1 & 20.9 & 7 \\
$2000-08-29$ & SPOT4 & -28.3 & 3 \\
$2000-08-29$ & SPOT2 & +9.7 & 7 \\
$2003-08-19$ & SPOT5 & -15.2 & 1 \\
$2003-08-23$ & SPOT5 & +15.7 & 1 \\
\hline
\end{tabular}

separation using the PCI Geomatica software as described by Toutin and Cheng [2002]. The main steps are the orientation of level 1A images from ground control points (GCPs), transformation to epipolar geometry, parallax-matching, and parallax-to-DEM conversion. The same set of 30 precise GCPs, measured in 2003 during a differential GPS (DGPS) field campaign to within $\pm 0.5 \mathrm{~m}$, is used for all the images.

[10] Following this method, we calculate satellite DEMs for 1994, 2000 and 2003 (Table 1). In 1994 and 2000, SPOT panchromatic images with $10 \mathrm{~m}$ ground resolution are used. For 2003, the DEM is deduced from SPOT5-THR images with a $2.5 \mathrm{~m}$ ground resolution. The same software is used to calculate DEMs from aerial photographs for 2000 and 2003.

\subsection{Adjustment of the Different DEMs}

[11] Even with a consistent set of GCPs, we observe some biases between the different DEMs off the glaciers, where no elevation change is expected. We attribute these biases to the non-uniform distribution of the GCPs in altitudes. To correct these biases, the 2003 DEM, the most accurate, is chosen as reference and the other DEMs are adjusted to this reference in two steps.

[12] The first adjustment corrects the shifts in latitude and longitude. This step is important because a small horizontal offset between two DEMs can produce a large elevation error where the topographic slope is steep. For each DEM, the corresponding orthorectified image is calculated. Where no movement is expected, off the glaciers, a precise cross correlation of each image with the 2003 reference image leads to a map of horizontal offsets in latitude and longitude. We then apply these shifts to correct the DEMs. The shifts are all less than $3 \mathrm{~m}$ and their standard deviation is of the order of $1 \mathrm{~m}$. Combined with a mean slope of $18^{\circ}$ for the $\mathrm{Mer}$ de Glace, it leads to an uncertainty of $0.4 \mathrm{~m}$ in elevation.

[13] After this adjustment, the difference between the DEMs does not show any spatial pattern and is corrected with an altimetric adjustment. For each altitude intervals on the unglaciated area, we compute the mean difference between each DEM and the reference 2003 DEM. Figure 1 illustrates how this bias is corrected in the case of the 2000 DEM. A fourth order polynomial curve provides a good approximation of this bias: it is used to correct all the altitudes in the 2000 DEM. After adjustment, the bias is never greater than $2 \mathrm{~m}$, the value we interpret as the uncertainty introduced by this correction. This is a mean value but, locally, due to shadowing effects or steep slopes the bias can be larger.

\subsection{Ablation Correction}

[14] A thickness change on the glacier can be interpreted if the measurements are performed each year close to the end of the ablation season [Krimmel, 1999], near the 15th September in the French Alps. Since our different data sets (satellite images, aerial photographs and GPS measurements) do not meet this condition, we have to take into account the ablation occurring between the date of survey and the 15th September. Ablation is estimated from an empirical degree-day model tuned with field measurements [Vincent, 2002]. Each positive degree at a given altitude on the glacier is converted to an ablation of $6.6 \mathrm{~mm}$ of ice. The daily temperature is calculated from the temperature of the nearby Chamonix weather station assuming a constant lapse rate of $6 \% \mathrm{~km}$. The uncertainty induced by this correction is proportional to the time difference between the survey and the 15th September. It is never greater than $0.5 \mathrm{~m}$. Future studies should plan to acquire data very near to the end of the ablation season, but before the first snowfall. They could also use an ablation model taking into account varying solar orientation.

\subsection{Extraction of the Mean Thickness Change}

[15] At each position, we now compute the difference between the younger and the older DEM, such that thickening is reckoned positive. The right panel in Figure 2 shows the satellite differential DEM for the 2000-2003 period. A vertical accuracy of the order of the pixel size is assumed for DEMs derived from optical images [Toutin and Cheng, 2002], i.e., $10 \mathrm{~m}$ for our DEMs, except the one derived from SPOT5 images. Although this is not precise enough to measure the thickness change for each individual pixel on the glacier, we can reduce the uncertainty assuming that all points at a given altitude experience the same thickness change. For each $50 \mathrm{~m}$ altitude interval, we compute the histogram of the thickness change. The distribution is well-approximated by a Gaussian curve (Figure 2), which permits the calculation of the mean thickness change as the mean of all the values less than $3 \sigma$ from the mean. The scatter is explained by two reasons. First, shadowing and debris coverage on the glacier make the assumption of a similar behaviour for all points at a given altitude only partly true. The scatter also reflects the random errors in the two DEMs. Accordingly, this standard deviation is a reasonable first assessment of the uncertainty in the thickness change measurement.

[16] The total uncertainty can be estimated by the quadratic sum of the different independent errors in the processing: errors in the two compared DEMs, errors introduced by the planimetric, altimetric adjustments and due to our correction of the ablation. This equation yields an overall uncertainty of $14 \mathrm{~m}$, mainly due to the errors in the

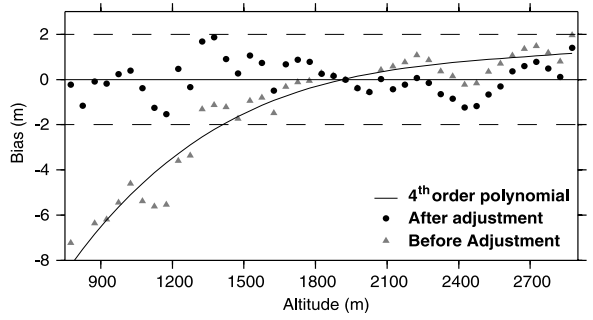

Figure 1. Bias between the 2000 and 2003 DEM as a function of altitude on the unglaciated areas before (gray triangles) and after adjustment (black circles). 


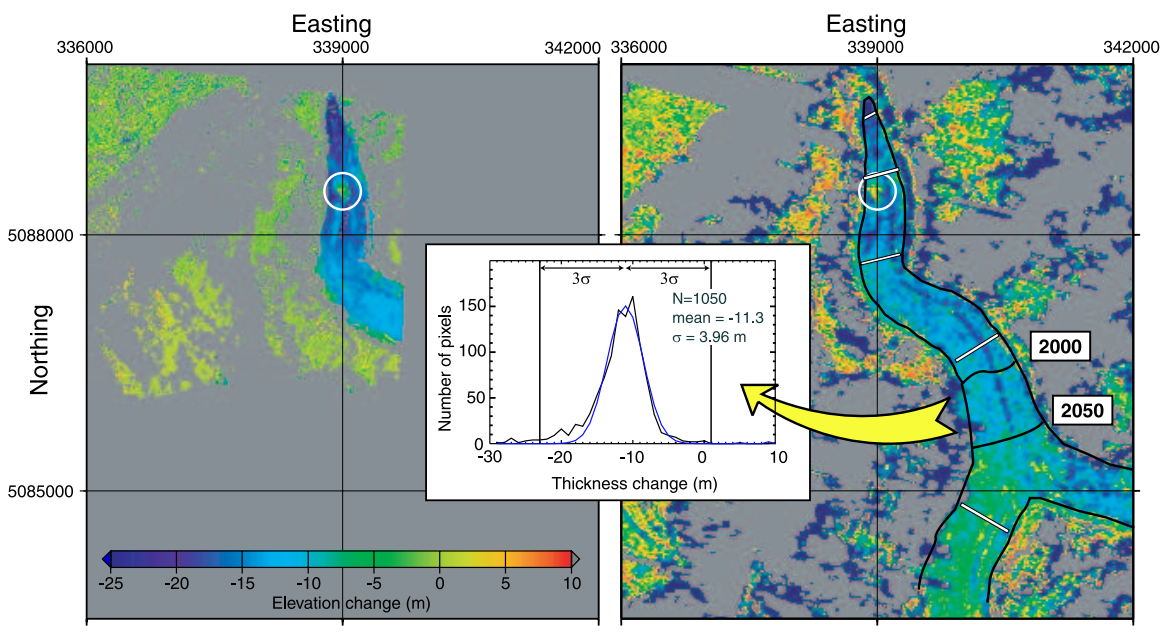

Figure 2. Differential DEMs for the 2000-2003 period derived from aerial photographs (left panel) and satellite images (right panel). A debris-covered area on the glacier is surrounded by a white circle. The white lines (right panel) locate the topographic profiles. The histogram of the thickness change for the 2000-2050 m altitude interval is drawn in black in the center of the figure. The distribution is well approximated by a Gaussian curve (blue line).

satellite DEMs. This uncertainty is large compared to the thickness change, that we expect to be of the order of a few meters. But it is reduced by averaging. If the scatter observed in the histogram in Figure 2 was purely due to Gaussian noise, this uncertainty would be divided by the square root of the number of pixels within an altitude interval (around 500), and would vary from 0.5 to $1 \mathrm{~m}$. To account for non-Gaussian (systematic) effects such as local thickness change variations, the true validation is obtained by comparison with ground truth.

\section{Validation of Our Methodology}

[17] In 2000, DEMs were calculated from satellite image pairs acquired the 25th and the 29th of August. Since August 29th DEM includes only the northern part of the Mer de Glace, the differential DEM covers the lower part of the glacier from 1500 to $2050 \mathrm{~m}$. Using altitude intervals of $50 \mathrm{~m}$, we obtain 11 measurements of thickness change. The surface of the glacier is $1.3 \mathrm{~m}(\sigma=1.28 \mathrm{~m}, \mathrm{~N}=11)$ lower on the 29th DEM than on the 25th. Of this, ablation deduced from the degree-day model, explains around $0.34 \mathrm{~m}(0.3 \mathrm{~m}$ at $2050 \mathrm{~m}$ altitude and $0.39 \mathrm{~m}$ at $1500 \mathrm{~m})$. The remaining $0.96 \mathrm{~m}$ is the uncertainty on our measurement.

[18] In Figure 3, we compare the thickness change derived from satellite differential DEMs to topographic transverse surveys performed each year on the glacier, with a typical accuracy of $0.3 \mathrm{~m}$. Five profiles are measured each year on the lower part of Mer de Glace at locations shown in Figure 2. For the 1994-2000 time interval, the agreement is excellent. For the 2000-2003 time interval, the agreement is also good except for the measurement at $1690 \mathrm{~m}$ where the topographic profile gives a thinning of $16.7 \mathrm{~m}$ but the satellite measurement $12 \mathrm{~m}$. This difference is explained by the fact that the satellite measurement includes an area where the glacier is covered by debris (Figure 2) which slows the ablation between 1690 and $1760 \mathrm{~m}$. The topographic profile, performed on bare ice just downstream of the debris-covered area, is not affected by this limited ablation. For the nine remaining measurements, we interpolate the satellite measurements at the altitude of the topographic profile to find a mean difference of $0.65 \mathrm{~m}$ $(\sigma=1.57 \mathrm{~m}, \mathrm{~N}=9)$.

[19] In Figure 2, we compare the spatial distribution of thickness change for the 2000-2003 period deduced from aerial photographs and satellite images. Despite its coarser spatial resolution, the satellite differential DEM is able to capture the small-scale features of the thickness change map. For example, the debris-covered area has the same extent in both differential DEMs. Between 1500 and $1950 \mathrm{~m}$ ( 9 altitude intervals of $50 \mathrm{~m}$ ), the mean difference between the two differential DEMs is $1.14 \mathrm{~m}(\sigma=1.67 \mathrm{~m}$, $\mathrm{N}=9$ ).

\section{Evolution During the Last 25 Years}

[20] From satellite DEMs for 1994, 2000, 2003 and an aerial DEM for 1979 (obtained from the French National Geographic Institute), the rate of thickness change for each

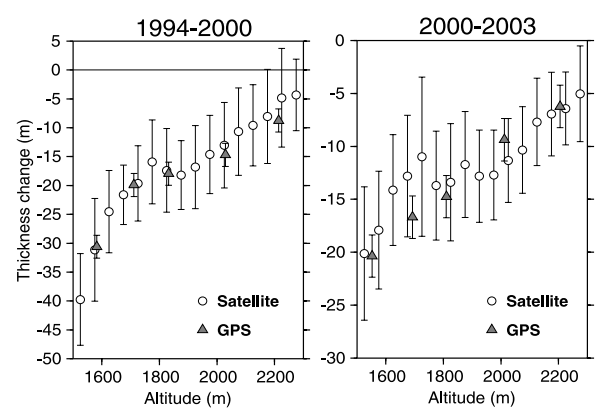

Figure 3. Comparison of the thickness change extracted from satellite images (circles) and deduced from topographic (traditional or DGPS) measurements (triangles). The left panel show the thickness change for the 19942000 time interval, the right panel for 2000-2003. 


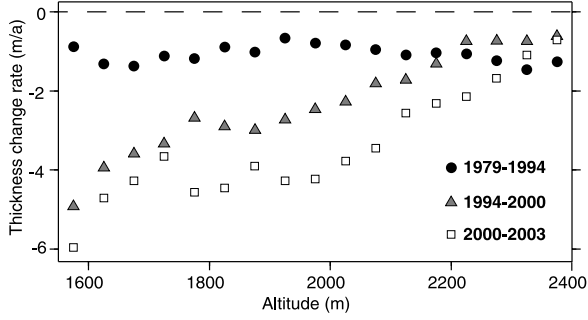

Figure 4. Rate of thickness change on the lower Mer de Glace for the last 25 years. The errors bars were not added to preserve the clarity of the figure.

period is calculated. At altitudes over $2500 \mathrm{~m}$, our data does not permit any definitive conclusion. Indeed, in the snowcovered accumulation area, the weak contrast of the images reduces the accuracy of the DEMs. At lower altitude (Figure 4), the thinning rate is strong during the last 10 years. Between 1600 and $2100 \mathrm{~m}$, it increased markedly from $1 \pm 0.4{\mathrm{~m} . \mathrm{a}^{-1}}_{(1979-1994)}$ to $4.1 \pm 1.7 \mathrm{~m} \cdot \mathrm{a}^{-1}(2000-$

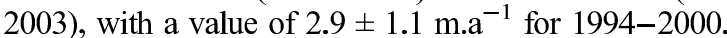

[21] It is beyond the scope of this paper to explain this rapid thinning at low altitude. It is probably the delayed response of the glacier to a series of negative mass balances during the period 1982-2002. An enhanced and longer summer ablation is responsible for this loss of mass and is related to the regional increase of the air temperature [Vincent et al., 2004]. Only modelling of the ice fluxes based on a good knowledge of the ice flow could lead to an explanation of this rapid thinning.

\section{Conclusion}

[22] In this study, we have demonstrated that accurate thickness changes of mountain glaciers can be derived from satellite data. We used some simple, but precise, adjustments and statistical procedures to reach an overall accuracy of $1 \mathrm{~m}$ for the thickness change. Moreover, the satellite differential DEM is also able to capture the spatial pattern of the thickness change map. Our data show a rapid thinning of the Mer de Glace during the last 10 years below $2500 \mathrm{~m}$.

[23] To validate our methodology, we have focused on the Mer de Glace. We plan now to extend this work to other glaciers of the Mont Blanc area, included in our images, to study the effect of slope and orientation on their wasting. Here, we have concentrated on the glacier thickness changes at low elevations and could not calculate the total mass budget of the glacier. Indeed, this budget can only be determined if the two compared DEM covers the whole glacier. Only the 2003 DEM, derived from SPOT5 scenes, meets this requirement because the images were acquired with the low gain needed for contrast in the snow-covered accumulation area. In the near future, another accurate SPOT5 DEM over the Mont Blanc glaciers could allow us to calculate their total mass balance.
[24] Now that accurate thickness change can be derived from satellite images, glaciologists can take advantage of their large footprints (3600 $\mathrm{km}^{2}$ for SPOT images), 100 times larger than an aerial photograph. Satellite images also enable monitoring of remote area at regular intervals. The accuracy of our thickness change measurements is of the order of $1 \mathrm{~m}$, i.e., 1/10th of the pixel size. In the near future, applying our method to satellite optical images with sub-meter resolution could monitor the thickness change of remote glaciers with a precision of the order of $10 \mathrm{~cm}$.

[25] Acknowledgments. SPOT images were acquired thanks to the ISIS program (copyright CNES). This work was supported by the French national program ACI-OT Glaciers. GMT software [Wessel and Smith, 1998] illustrated the article. Kurt Feigl provided an invaluable help during the redaction of the manuscript. The first author gratefully acknowledges a thesis fellowship from the French government. The comments of James E. Saiers (scientific editor), Bruce H. Raup and an anonymous reviewer led to significant improvements of the manuscript.

\section{References}

Andreassen, L. M., H. Elvehy, and B. Kjllmoen (2002), Using aerial photography to study glacier changes in Norway, Ann. Glaciol., 34, 343-348.

Arendt, A. A., K. A. Echelmeyer, W. D. Harrison et al. (2002), Rapid wastage of Alaska glaciers and their contribution to rising sea level, Science, 297, 382-386.

Baltsavias, E. P., E. Favey, A. Bauder et al. (1999), Digital surface modelling by airborne laser scanning and digital photogrammetry for glacier monitoring, Photogramm. Rec., 17, 243-273.

Braithwaite, R. J. (2002), Glacier mass balance: The first 50 years of international monitoring, Prog. Phys. Geogr, 26, 76-95.

Dyurgerov, M. B. (2003), Mountain and subpolar glaciers show an increase in sensitivity to climate warming and intensification of the water cycle, J. Hydrol., 282, 164-176.

Echelmeyer, K., W. Harrison, C. Larsen et al. (1996), Airborne surface profiling of glaciers: A case-study in Alaska, J. Glaciol., 42, 538-547.

Finsterwalder, R. (1954), Photogrammetry and glacier research with special reference to glacier retreat in the eastern Alps, J. Glaciol., 2, 306-315. Intergovernmental Panel on Climate Change (2001), Climate Change 2001.

The Scientific Basis, edited by J. T. Houghton et al., Cambridge Univ. Press, New York

Krimmel, R. M. (1999), Analysis of difference between direct and geodetic mass balance measurements at South Cascade glacier, Washington, Geogr. Ann., 81A, 653-658.

Oerlemans, J. (1994), Quantifying global warming from the retreat of glaciers, Science, 264, 243-245.

Paterson, W. S. B. (1994), The Physics of Glaciers, 3rd ed., Pergamon, New York.

Rignot, E., A. Rivera, and G. Casassa (2003), Contribution of the Patagonia icefields of South America to sea level rise, Science, 302, 434-437.

Toutin, T., and P. Cheng (2002), A comparison of automated DEM extraction results using along-track ASTER and across-track SPOT stereo images, Opt. Eng., 41, 2102-2106.

Vincent, C. (2002), Influence of climate change over the 20th century on four French glacier mass balances, J. Geophys. Res., 107(D19), 4375, doi:10.1029/2001JD000832.

Vincent, C., G. Kappenberger, F. Valla et al. (2004), Ice ablation as evidence of climate change in the Alps over the 20th century, J. Geophys. Res., 109, D10104, doi:10.1029/2003JD003857.

Wessel, P., and W. H. F. Smith (1998), New, improved version of Generic Mapping Tools released, Eos Trans. $A G U, 79(47), 579$. [version 3.1]

Y. Arnaud, GREAT ICE (IRD), St. Martin d'Hères, France.

D. Baratoux, LDTP (UPS-CNES-CNRS-IRD), Toulouse, France.

E. Berthier and F. Rémy, LEGOS, 18 av. E. Belin, F-31401 Toulouse, France. (etienne.berthier@cnes.fr)

C. Vincent, LGGE (UJF-CNRS), St. Martin d'Hères, France. 



\section{Annexe C}

\section{SPOT5 mesure l'Ecoulement des Glaciers de Montagne (Alpes)}

Berthier, E., Vadon, H., Baratoux, D., Arnaud, Y., Vincent, C., Feigl, K. L., Rémy, F., \& Legrésy, B., 2005. Mountain glacier surface motion derived from satellite optical imagery. Remote Sensing Environ., 95 (1), 14-28. 


\title{
Surface motion of mountain glaciers derived from satellite optical imagery
}

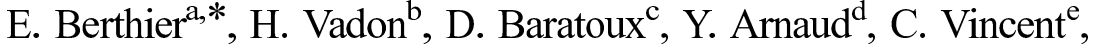 \\ K. L. Feigl ${ }^{\mathrm{c}}$, F. Rémy ${ }^{\mathrm{a}}$, B. Legrésy ${ }^{\mathrm{a}}$ \\ ${ }^{\mathrm{a}} L E G O S / C N R S / U P S, 18$ av. Ed. Belin, 31401, Toulouse Cedex 9, France \\ ${ }^{\mathrm{b}}$ CNES, 18 av. Ed. Belin, 31401, Toulouse Cedex 9, France \\ ${ }^{\mathrm{c}} \mathrm{LDTP} / \mathrm{CNRS} / \mathrm{UPS}, 14$ av. Ed. Belin, 31400, Toulouse, France \\ ${ }^{\mathrm{d} G R E A T ~ I C E}$ (IRD) 54 Rue Moliére, BP 96, 38402 St Martin d'Héres, France \\ ${ }^{\circ} L G G E$ (UJF-CNRS) 54 Rue Moliére, BP 96, 38402 St Martin d'Héres, France
}

Received 21 June 2004; received in revised form 21 September 2004; accepted 3 November 2004

\begin{abstract}
A complete and detailed map of the ice-velocity field on mountain glaciers is obtained by cross-correlating SPOT5 optical images. This approach offers an alternative to SAR interferometry, because no present or planned RADAR satellite mission provides data with a temporal separation short enough to derive the displacements of glaciers. The methodology presented in this study does not require ground control points (GCPs). The key step is a precise relative orientation of the two images obtained by adjusting the stereo model of one "slave" image assuming that the other "master" image is well georeferenced. It is performed with numerous precisely-located homologous points extracted automatically. The strong ablation occurring during summer time on the glaciers requires a correction to obtain unbiased displacements. The accuracy of our measurement is assessed based on a comparison with nearly simultaneous differential GPS surveys performed on two glaciers of the Mont Blanc area (Alps). If the images have similar incidence angles and correlate well, the accuracy is on the order of $0.5 \mathrm{~m}$, or 1/5 of the pixel size. Similar results are also obtained without GCPs. An acceleration event, observed in early August for the Mer de Glace glacier, is interpreted in term of an increase in basal sliding. Our methodology, applied to SPOT5 images, can potentially be used to derive the displacements of the Earth's surface caused by landslides, earthquakes, and volcanoes.
\end{abstract}

(C) 2004 Elsevier Inc. All rights reserved.

PACS: 92.40.V; 07.87; 06.30.B

Keywords: Mountain glaciers; Surface displacement; SPOT5; Cross-correlation; Satellite optical images

\section{Introduction}

Accurate displacement measurements are needed to understand the dynamics of glaciers. Such measurements contribute to a better knowledge of the rheological parameters controlling the flow of glaciers. They are important to monitor icefalls, glacier surges (Fischer et al., 2003), and glacier hazards (Kääb et al., 2003). They can also detect ice-velocity changes caused by global warming (Rignot et al., 2002). Differential Global Positioning System (DGPS) ground surveys, synthetic aperture radar interferometry (InSAR), and optical image cross-correlation are the

\footnotetext{
* Corresponding author. Tel.: +33 561333017.

E-mail address: etienne.berthier@cnes.fr (E. Berthier).
}

0034-4257/\$ - see front matter (C) 2004 Elsevier Inc. All rights reserved. doi:10.1016/j.rse.2004.11.005 main ways to determine glacier displacements. The first two methods are the most accurate but present some severe limitations for the monitoring of mountain glaciers, i.e. all glaciers except large ice caps, ice fields, and the Greenland and Antarctic ice sheets. This study applies cross-correlation to well coregistered SPOT5 optical images to measure mountain glacier surface velocities.

Even with the advent of the DGPS, it remains difficult and time-consuming to perform regular ground-based surveys of glacier flow. Among the 86 regularly monitored glaciers with time series longer than 10 years (Braithwaite, 2002), only a few stakes on the flat parts can reasonably be surveyed, excluding icefalls and remote glaciers.

The 1990s brought some great improvements in the measurement of the surface motion of glaciers from satellite 
data. Two new techniques have been extensively investigated, especially on the rapid and large ice streams draining the Antarctic and Greenland ice sheets: InSAR and feature-tracking on optical images.

Combining two SAR images of the Rutford Ice Stream with short time separation (6 days), Goldstein et al. (1993) used InSAR to measure the flow of ice streams. Basic InSAR only measures the projection of the displacement vector onto the satellite line of sight. However, combining ascending and descending passes of the satellite and adding constraints on the ice flow yields all three components of the displacement vector (Joughin et al., 1998; Mohr et al., 1998). Recently, intensity-tracking and coherence-tracking, two cross-correlation techniques applied to SAR data, have been combined with InSAR to produce two-dimensional velocity fields (Gray et al., 2001; Strozzi et al., 2002).

If the correlation between the two radar images is good and the tropospheric, orbital, and topographic contributions can be modelled, the precision of InSAR is on the order of a centimeter (Massonnet \& Feigl, 1998). However, additional errors may arise in resolving the phase ambiguity through unwrapping, especially in areas where the displacement gradient (i.e. strain) approaches the threshold of about $10^{-3}$ for ERS (Massonnet \& Feigl, 1998). This condition often occurs in icefalls or marginal shear zones of glaciers (Goldstein et al., 1993).

On mountain glaciers, only a few studies (Mattar et al., 1998; Rabus \& Fatland, 2000; Strozzi et al., 2002) have succeeded in measuring motion with InSAR. The steep topography, the strong tropospheric contribution, and the small size of the glaciers are obstacles to overcome. But the major problem is the time between two successive images. If it exceeds 1 or 3 days (Strozzi et al., 2002), the displacement gradient is larger than the threshold $\left(10^{-3}\right.$ for ERS), destroying the interferometric fringes. Furthermore, after a few days, the correlation is low due to rapid changes on the glacier surface. Only the ERS-1 ice phase (3day orbital cycle) and the ERS-1 and ERS-2 Tandem Mission (1 day separating the passes of the satellites) can be used to derive velocity fields on glaciers. Consequently, no present or planned satellite mission can measure the motion of mountain glaciers using InSAR.

Repeated visible or near infrared images of the same area can be used to track the displacement of features such as crevasses or surficial debris moving with the ice (Lucchitta \& Ferguson, 1986). Development of automatic featuretracking algorithms has substantially increased the accuracy and the efficiency of this approach (Scambos et al., 1992).

The aim of our study is to demonstrate that highresolution and accurate surface displacement maps can be routinely obtained on mountain glaciers using optical images. The goal is to provide an alternative to InSAR for the measurement of the glacier flow.

Correlation of optical images provides the two horizontal components of the displacement vector contrary to InSAR. Furthermore, the measurement is unambiguous: absolute displacements can be referenced to motionless areas which are always available for mountain glaciers. This approach can be applied to images with a large time separation. For some outlet glaciers of Greenland or Antarctic ice sheets, the persistence of the surface features permits velocity measurements from images separated by as much as 11 years (Berthier et al., 2003). Some velocity fields have also been derived from optical images separated by more than a year on mountain glaciers (e.g., Kääb, 2002). Previous studies on Antarctic ice streams (Scambos et al., 1992; Frezzotti et al., 1998) or mountain glaciers (Kääb, 2002) generally reached an accuracy of \pm 1 pixel. A smaller uncertainty and a methodology adapted to mountain glaciers are the focus of our study.

In the next section, we describe a procedure to extract displacements of the ground surface from two SPOT5 images. The images and the different data needed to apply and validate our methodology are presented for the Mont Blanc area in Section 3. Maps of the satellite-derived displacements and accuracy of our measurements are presented in Section 4. An acceleration event of the $\mathrm{Mer}$ de Glace glacier is also discussed before presenting conclusions.

\section{Methodology}

Even slightly different incidence angles can create a relative distortion between two satellite images of the same area. If the two images are correlated, the resulting offsets in the image lines and columns are the sums of the contributions from misregistration, topography, orbits, and attitude as well as the glacier-dynamics signal. To obtain a valid measurement, we must remove all the contributions except the glacier flow. The principle of our method is to resample one of the images (called the "slave" image) in the native (1A) geometry of the other, "master" image just as for InSAR (Massonnet \& Feigl, 1998). To do this, we use an accurate stereoscopic model (called stereo model in the following), a digital elevation model (DEM), and an interpolator that respects the Shannon criteria. The two images can then be correlated to estimate the deformation of the glaciers. Fig. 1 presents all the steps required to measure glacier-surface displacement from two satellite images. All the algorithms describing the SPOT5 stereo model and used in this study are the property of the Centre National d'Etude Spatial (CNES). The information needed to perform these calculations are available in a handbook (SPOT-Image, 2002), available upon request to the SPOT-Image company.

\subsection{Characteristics of mountain glaciers and required accuracy}

The time separation between images is one of the critical factors for a reliable measurement. It must be long enough to increase the signal (the flow of the glaciers) but short 


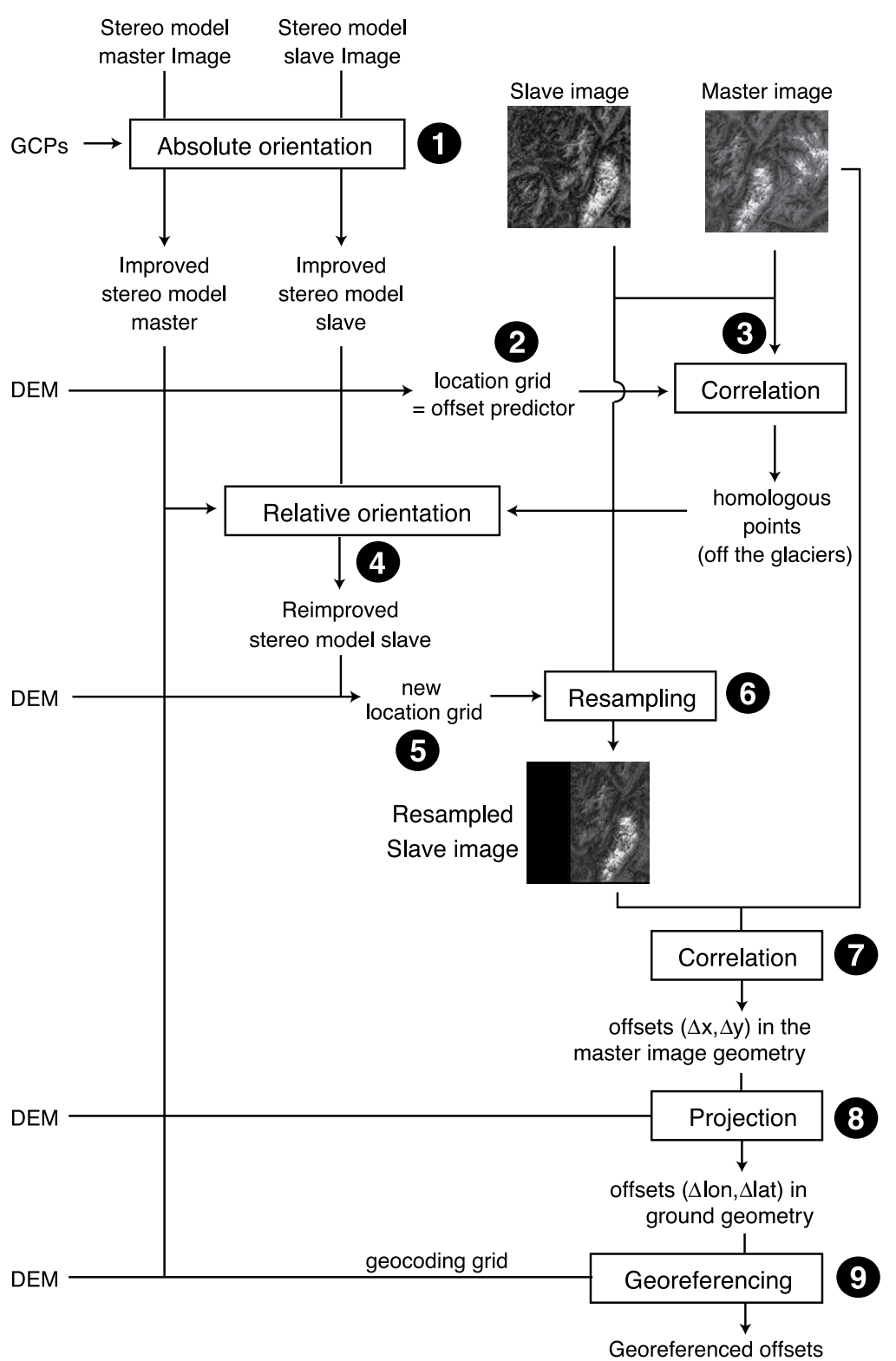

Fig. 1. Flowchart of the methodology followed to measure the surface displacements from two SPOT5 images. On the left side, GCPs (ground control points) and DEM (digital elevation model) represent external informations.

enough to preserve the features for tracking. Indeed, for many mountain glaciers, a long time separation will reduce the efficacy of the cross-correlation for two reasons.

First, the surface features of the glaciers may change due to melting of snow cover or ice, snowfall or changes caused by windblown snow. Correlation between the two images breaks down and only sparse measurements can be obtained.

Second, good features for the correlation such as the crevasses and the Forbes bands, appear from year to year at the same places with similar shapes, like river eddies. For example, crevasses open regularly upstream of an icefall. We tested that correlating two images 1 year apart on the Mer de Glace glacier can lead to an (incorrect) null displacement in some parts of the glacier. For shorter times, the spatial continuity of the displacement field prevents this problem.

Studying a glacier flowing at $75 \mathrm{~m} \mathrm{a}^{-1}$ with images separated by 1 month, we seek to detect a displacement of $6.25 \mathrm{~m}$. A reasonable accuracy is obtained if the error does not exceed $0.62 \mathrm{~m}(10 \%)$. Using SPOT5 images with a pixel size of $2.5 \mathrm{~m}$, we will achieve this goal if the uncertainty is \pm 0.25 pixels, four times better than previously reported. In 
previous studies, the uncertainties resulted mainly from the difficulty of coregistering and removing the topographic distortion between the two images. These two issues are addressed thoroughly in our methodology.

\subsection{Absolute orientation (step 1 in Fig. 1)}

Ground control points (GCPs) are identifiable with an accuracy of \pm 1 pixel on both images. They also have known geographic coordinates. They improve the stereo model and consequently the georeferencing of both master and slave images. The stereo model, including the position and the attitude of the satellite, determines the correct ground position of each point in the image (Toutin \& Cheng, 2002). The stereo model is computed with an iterative leastsquares bundle block adjustment. Estimates of the attitude (roll, pitch, and yaw) and the focal length of the sensor are provided with the images. From $n$ GCPs, we estimate a new attitude and focal length which minimizes the function:

$$
\sum_{i=1}^{n}\left\|\overrightarrow{G_{i} G_{i}^{\prime}}\right\|^{2}
$$

where $G_{i}=(\lambda, \phi, z)_{i}$ is the exact position of the $i$ th GCP and $G^{\prime}{ }_{i}=\left(\lambda^{\prime}, \phi, z^{\prime}\right)_{i}$ its ground position deduced from its image coordinates using the stereo model. $\lambda, \phi, z$ represent the latitude, longitude, and ellipsoidal height. A rigorous satellite stereo model is more accurate than a simple, polynomial-based model (Toutin \& Cheng, 2002). For areas with rugged topography, a polynomial does not suffice to accurately model the stereoscopic distortions between images. A rigorous model also takes advantage of the SPOT5 satellite products which include a precise description of the orbit and attitude.

After this step, each point on the images can be located on the ground with an accuracy of $\pm 2.5 \mathrm{~m}$ (one pixel) or slightly better.

\subsection{Relative orientation (step 2 to 4 in Fig. 1)}

Without GCPs, this relative orientation is the only way to obtain well coregistered images. Even if GCPs are available, this step will improve the coregistration because it uses numerous and accurate homologous points. It yields two images coregistered within a few tenths of a pixel. It improves the stereo model of the slave image only, by assuming that the master image is now perfectly georeferenced.

The motionless homologous points are identified by cross-correlating the two images. The automatic extraction of homologous points is difficult directly because the same feature can be separated by as many as a thousand pixels between the two images. Instead, we first compute a location grid using the stereo models of the two images and a DEM. This grid provides the rough offset separating a pixel of the slave image from its corresponding pixel in the master image. It is used as a first estimate (or an offset predictor) to correlate the master and the slave image. Using a mask which excludes all the glaciers, we then compute the correlation only on the motionless areas of the images (step 3 ). Retaining only the points with the best correlation, we create a set of accurate homologous points distributed throughout the images.

The homologous points are used to improve the stereo model of the slave image only (step 4). It yields new values of the attitude (roll, pitch, and yaw) and focal length.

\subsection{Resampling of the slave image (steps 5 and 6 in Fig. 1)}

The objective of this step is to project the slave image into the geometry of the master image so that the remaining offsets represent only the deformation of the Earth's surface.

The stereo models of the two images are combined with a DEM to create a new, more precise, location grid in step 5. This grid contains the orbital, attitude, and topographic contributions to the total offsets between the two images. It is used to create a resampled slave image in step 6 , as if it had been acquired from exactly the same viewpoint as the master image. We use a resampling method which respects the Shannon criteria to preserve the radiometry and to minimize aliasing. As in Vadon and Massonnet (2000), we apply an apodized cardinal sine (cardinal sine multiplied by a Gaussian function) for interpolation. It is the best compromise between the Shannon criteria requirement and the filter length. It also leads to lower bias than other interpolators (Van Puymbroeck et al., 2000).

\subsection{Cross correlation (step 7 in Fig. 1)}

Now that they share the same geometry, the two images are correlated to estimate the offsets (step 7). We use the MEDICIS correlator software, developed at CNES and commercially available (Centre National d'Etude Spatiale, 2002). For SPOT5 images, the accuracy of the correlation itself is expected to be on the order of a few hundredths of a pixel. Surface changes and radiometric differences caused by different incidence and/or solar illumination angles are the limiting factors for the correlation.

At each grid point, the offsets between the two images are deduced from the position of the maximum of the correlation. This maximum is found in an iterative process. On subscenes of 21 by 21 pixels (or 52.5 by $52.5 \mathrm{~m}$ for SPOT5 images), we compute the correlation coefficient between the master and a shifted slave image. The sub-pixel shift is applied to the slave image with a cardinal sine interpolator. It is more accurate than simple interpolation of the correlation coefficient grid calculated for every pixel (Vadon \& Massonnet, 2000).

With correlation windows of $21 \times 21$ pixels, a posting of 10 pixels $(25 \mathrm{~m})$ captures the fine details of the ice flow. The correlation leads to two offset fields: offsets in the image 
column direction $(\Delta x)$ and line direction $(\Delta y)$. The correlation coefficient helps to assess the quality of the measurement.

\subsection{Extracting ground displacements (steps 8 and 9 in Fig. 1)}

The offsets measured between the two images represent the projection of the displacement vector onto the plane of the master image. These offsets can be used either in the native image geometry or transformed to displacements in latitude and longitude. Usually, offsets in pixels are simply converted to displacements in meters multiplying by a constant value for the pixel size (2.5 m for SPOT5). This is an approximation in areas with rugged topography. In the glacial areas in our images, the ground distance contained within a pixel ranges from 2.5 to $3 \mathrm{~m}$ in the line direction and from 2.5 to over $10 \mathrm{~m}$ in the column direction. It is even larger on the steep slopes surrounding the glaciers.

Fig. 2 illustrates the conversion from image offsets to ground displacements. The master image stereo model and the DEM are used to compute the corresponding location on the ground $\left(\lambda_{\mathrm{m}}, \phi_{\mathrm{m}}, z_{\mathrm{m}}\right)$ for each pixel of the master image $(x, y)$. When the slave image is acquired, the pixel has moved to a new position $(x+\Delta x, y+\Delta y)$ measured by the cross-correlation. Using the same transformation, we compute the new location $\left(\lambda_{\mathrm{s}}, \phi_{\mathrm{s}}, z_{\mathrm{s}}\right)$ of this point on the ground. We then deduce the displacements in latitude $\Delta \phi$ and longitude $\Delta \lambda$ and the total horizontal displacement. A value of the change in altitude $(\Delta z)$ of the point is also obtained. This is not a measurement of the vertical component of the displacement vector because of ice ablation and the ice flow direction that is not exactly parallel to the surface (Paterson, 1994).

Now, we know the displacements in latitude and longitude every 10 pixels of the master image. In step 9, we map them into a geographic reference system using a georeferencing grid calculated with the master image stereo

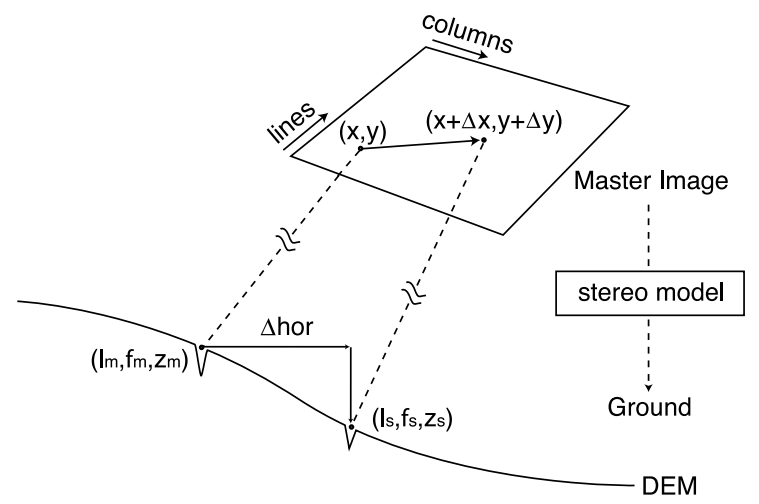

Fig. 2. Conversion of offsets in master image geometry to georeferenced ground displacements.
Table 1

Characteristics of the four SPOT5 images used in this study

\begin{tabular}{lll}
\hline Date (2003) & ID & Incid. angle \\
\hline 19 Jul & $50512570307191024221 \mathrm{~B} 0$ & -23.6 \\
19 Aug & $50522570308191028122 \mathrm{~B} 4$ & -15.2 \\
$23 \mathrm{Aug}$ & $50512570308231051191 \mathrm{~B} 0$ & +15.7 \\
$18 \mathrm{Sep}$ & $50522570309181051081 \mathrm{~B} 3$ & +17.3 \\
\hline
\end{tabular}

The image pairs (19 Jul/19 Aug 2003) and (23 Aug/18 Sep 2003) are used to measure the displacements of the glaciers in 31 and 26 days, respectively. The 19 Aug/23 Aug 2003 pair is used to compute a DEM of the Mont Blanc area. Note that the incidence angle refers to the center of the images.

model and the DEM. This grid is then used to project the offsets. The resampling is also performed using an apodized cardinal sine.

\section{Study area and available data}

In this section, we describe the data used to test and validate our methodology on glaciers of the Mont Blanc area. The two largest glaciers of this mountain range, the Mer de Glace and Argentière glaciers have been studied for more than a century (Reynaud, 1980). Their accessibility facilitates the field campaigns for verification of satellitederived measurements.

\subsection{SPOT-5 images of the Mont Blanc area}

The SPOT5 satellite was launched on 4 May 2002 with a repeat-orbital cycle of 26 days. The ground resolution has been improved with a pixel size of $2.5 \mathrm{~m}$ in THR mode (compared to $10 \mathrm{~m}$ for SPOT1-4), while retaining an area footprint on the ground of $60 \times 0 \mathrm{~km}$. Precise orbital ephemeris and attitude descriptions are provided with the images. Without any ground control points, an image is located on the ground with a precision of $30 \mathrm{~m} \mathrm{rms}$. A detailed description of the SPOT5 mission can be found in Fratter et al. (2001).

Four SPOT5 images of the Mont Blanc area were acquired during the summer of 2003 (Table 1). The time of acquisition of the images is crucial. Acquiring images during the dry season (July to September in the French Alps), 1 or 2 months apart seems the best compromise. Before July, the snowline is still at a low elevation and the snow cover from the previous winter still masks the surface features. After September, snowfalls can occur at low elevation and dramatically increase the albedo of the glacier. The main displacement field is derived from the images acquired on 23 August and 18 September 2003 (named image pair \#2 in the following). In Fig. 3, the outlines of these two images are plotted on a DEM of the area. Separated by one orbital cycle, they have similar viewpoints. The small difference in the incidence angles at the center of the images (Table 1) is explained by their different footprints. The images acquired on 19 July and 

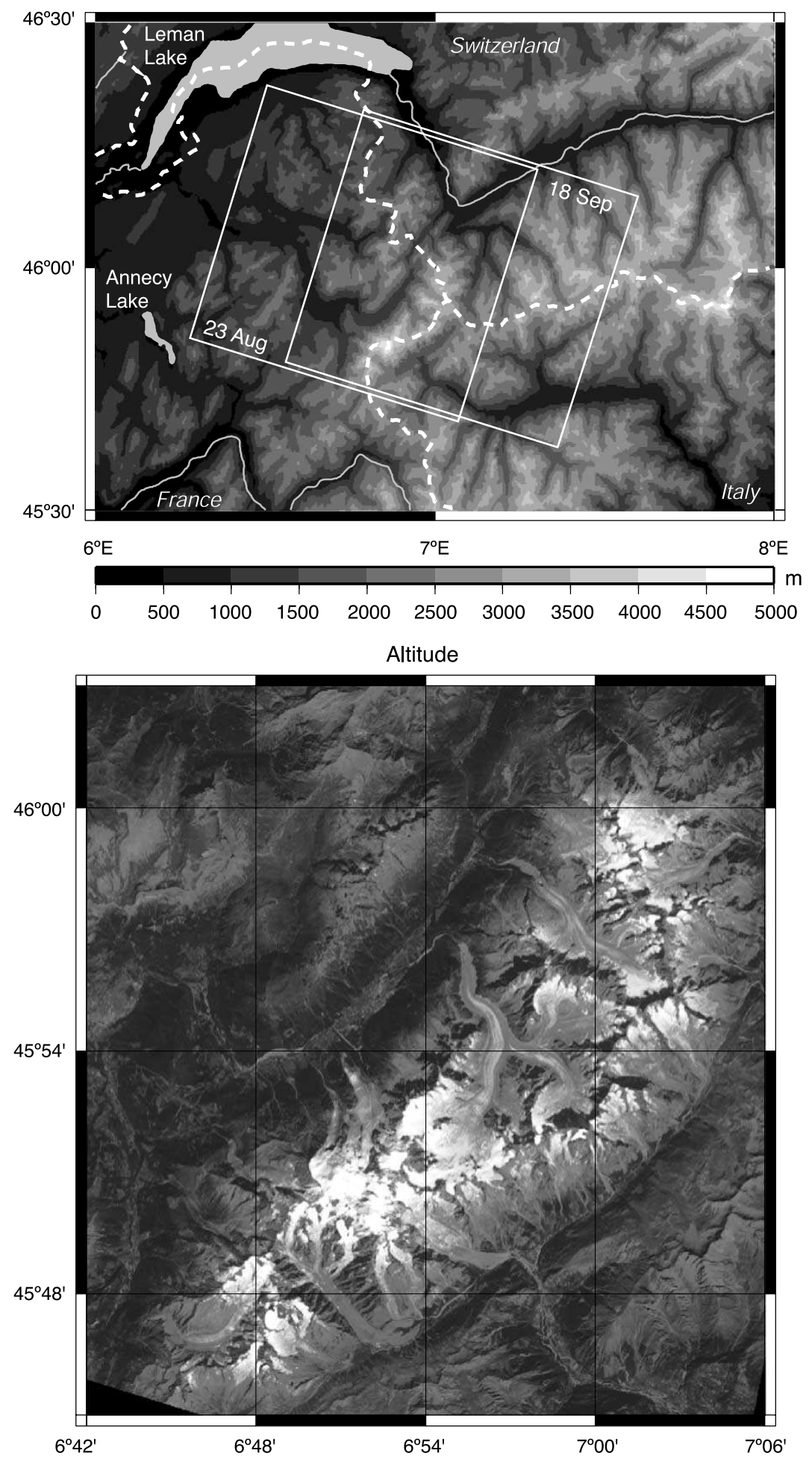

Fig. 3. Outlines of the SPOT5 scenes (23 August 2003 and 18 September 2003) used to derive the main velocity field (upper panel). The background image is the high-resolution DEM used in this study. The lower panel is a subscene of the 23 August 2003 image showing the Mont Blanc area. 
19 August 2003 (image pair \#1) are also combined to estimate the displacement during 31 days. This pair is used to test a less favourable satellite configuration (with a difference of $8.5^{\circ}$ in the incidence angles) and to determine whether the glaciers can experience short-term velocity change.

All these images were acquired with the lowest possible gain. A low gain avoids radiometric saturation on the glacier, especially in the bright snow-covered accumulation area (Ferrigno \& Williams, 1983). Level 1A imagery is used to avoid any radiometric and geometric resampling of the data. This level ensures better control of the image geometry (Al-Rousan et al., 1997).

\subsection{Digital elevation model (DEM)}

To model and remove the stereoscopic distortions between two images, a DEM is needed. Because mountain glaciers in the Mont Blanc area are experiencing rapid thinning (Berthier et al., 2004), a DEM referring to an epoch as near in time as possible to the acquisition date of the satellite images seems preferable. The SPOT5 images acquired on 19 and 23 August 2003 with opposite incidence angles and a short time separation (Table 1) were combined to compute a fine resolution DEM of the area, with a posting of $20 \mathrm{~m}$. The DEM was produced using the Orthoengine module of the PCI-Geomatica software (Toutin \& Cheng, 2002). The accuracy of this DEM was tested with 48 independent check-points on the slopes surroundings the glaciers. The mean difference between the SPOT5 DEM and the check-points is $2.5 \mathrm{~m}(\sigma=10 \mathrm{~m})$, the DEM being slightly lower. On the flat surface of the Mer de Glace and Argentière glaciers, this difference is only $0.15 \mathrm{~m}(\sigma=1.1$ $\mathrm{m})$. Because of shadowing, clouds and the difference in areal coverage, this DEM does not encompass the entire area of interest. A complete DEM is needed and obtained using the SRTM DEM (Rabus et al., 2003) and another SPOT DEM available for 2000. The coarse GTOPO30 DEM (USGS, 1996) was also used to prove that accurate displacements can be derived without any precise, contemporaneous DEM.

\subsection{Ground control points (GCPs)}

Some GCPs are preferable to improve the absolute georeferencing of the four SPOT5 scenes. They have been acquired in September and October 2003 with an ASHTECH single-frequency differential GPS (DGPS) system. The accuracy of these GCP positions is on the order of 0.5 $\mathrm{m}$ horizontally and $1 \mathrm{~m}$ vertically. They are used in the absolute orientation of the images (step 1). During the computation of the stereo model, the least reliable GCPs are excluded. Table 2 provides some statistics on the GCPs retained. For the different SPOT5 images, the same set of GCPs leads to slightly different residuals ranging from 1.55 to $2.55 \mathrm{~m}$. Table 2 also shows the results of the relative orientation for the different pairs of images (last column). For image pair \#2, the orientation is greatly improved. For this pair, the relative orientation is also accurate without GCPs, even when using the less precise GTOPO30 DEM. For image pair \#1, the relative orientation does not lead to any improvement because of the less favourable satellite configuration. The significant $8.5^{\circ}$ difference in the incidence angles of the two images in pair \#1 also explains why the relative orientation is inaccurate without GCPs, even with a precise DEM.

\subsection{DGPS measurements of the glacier displacement}

Measurement of glacier displacements from satellite images are rarely compared to field measurements because it is difficult to be on the glaciers during the exact time of acquisition of satellite images. Two DGPS field campaigns were planned on the Mer de Glace and Argentière glaciers in August and September 2003 to measure the surface displacements. Because of possible cloud cover and the satellite schedule, the acquisition dates of the SPOT5 images could not be known precisely in advance. Con-

Table 2

Standard deviation of the residuals (meters) for the absolute and relative orientation of the different SPOT5 scenes

\begin{tabular}{|c|c|c|c|c|c|}
\hline \multirow[t]{2}{*}{ Image pair } & \multirow[t]{2}{*}{ Date (2003) } & \multicolumn{2}{|c|}{ Abs. orient. } & \multicolumn{2}{|c|}{ Rel. orient. } \\
\hline & & $\sigma$ & Max. residual & $\sigma$ & Max, residual \\
\hline \multirow[t]{2}{*}{ \#1 with GCPs; HR DEM } & $19 \mathrm{Jul}$ & 2.55 & 4.3 & \multirow[t]{2}{*}{2.84} & \multirow[t]{2}{*}{11.1} \\
\hline & 19 Aug & 1.89 & 3.59 & & \\
\hline \#1 no GCPs; HR DEM & $19 \mathrm{Jul} / 19$ Aug & - & - & 5.45 & 31.7 \\
\hline \#1 no GCPs; LR DEM & $19 \mathrm{Jul} / 19 \mathrm{Aug}$ & - & - & 14.5 & 38.8 \\
\hline \multirow[t]{2}{*}{ \#2 with GCPs; HR DEM } & $23 \mathrm{Aug}$ & 1.55 & 2.66 & \multirow[t]{2}{*}{0.63} & \multirow[t]{2}{*}{1.45} \\
\hline & 18 Sep & 1.82 & 2.97 & & \\
\hline \#2 no GCPs; HR DEM & $23 \mathrm{Aug} / 18 \mathrm{Sep}$ & - & - & 0.67 & 1.68 \\
\hline \#2 no GCPs; LR DEM & $23 \mathrm{Aug} / 18 \mathrm{Sep}$ & - & - & 0.67 & 1.68 \\
\hline
\end{tabular}

For each image, the absolute orientation is computed from ground control points (GCPs). The relative orientation is based on homologous points extracted by cross-correlation of the two images and does not necessary require an absolute orientation beforehand. The maximum residual for the GCPs (respectively homologous points) retained in the absolute (resp. relative) orientation is also given. HR refers to the high resolution ( $20 \mathrm{~m}) \mathrm{DEM}$ and LR to the low resolution (1 km) GTOPO30 DEM. 


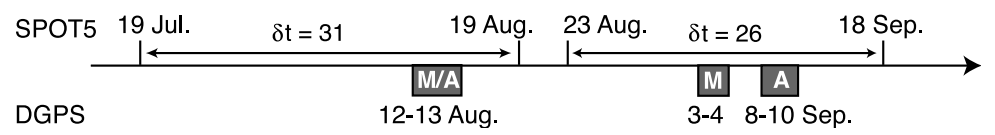

Fig. 4. Comparison between the acquisition times of the SPOT5 images and the DGPS surveys of the glaciers displacements. For the DGPS survey, M refers to the Mer de Glace glacier, A to the Argentière Glacier.

sequently, the ground surveys were not performed exactly at the same time (Fig. 4). A Leica dual-frequency GPS was used to survey the two successive positions of 29 ablation stakes and painted stones on the glaciers. The precision of the positioning itself is on the order of a few centimeters. The main uncertainty results from the difficulty in placing the GPS antenna exactly at the same position relative to the stakes (a puddle of water usually surrounds each stake in summertime). Consequently, the uncertainty is $15 \mathrm{~cm}$ for a single measurement, leading to a displacement accuracy of $\pm 21 \mathrm{~cm}$. For painted stones, the uncertainty is larger, but difficult to estimate because they can move or roll independently of the underlying ice.

\section{Results}

\subsection{Map of the displacements in the Mont Blanc area}

Fig. 5 shows the horizontal displacement of the ground surface in the Mont Blanc area derived from image pair \#2. On these glaciers, no overall velocity measurements had ever been performed. The highest speed occurs on the steep icefalls of the Mer de Glace, Bosson and Brenva glaciers, with velocities over $500 \mathrm{~m} \mathrm{a}^{-1}$. Some small-scale features of the displacement field also appear clearly. For example, the increase in velocity of the Mer de Glace glacier near the confluence with the Leschaux glacier is visible in Fig. 5d. Some problems also appear. No displacements can be measured for areas in shadows; e.g., on the upper part of the Leschaux glacier under the steep north face of the Grandes Jorasses. At high elevation on the accumulation zone, even with a low gain, the noise remains noticeable.

\subsection{Residuals in the unglaciarized area}

The accuracy of the velocities can be first assessed by a null test over motionless, ice-free areas of the images. We retain only the points with a correlation coefficient over 0.7 . Fig. 6 shows the histograms of the residuals (in meters) in the image line direction for image pair \#2. As expected, the stronger the correlation coefficient, the smaller the scatter. If the correlation coefficient is greater than 0.95 , the uncertainty (noted $\sigma$ ) is $1.8 \mathrm{~m}$ whereas $\sigma=6.6 \mathrm{~m}$ if the correlation coefficient is smaller than 0.75 . Unexpectedly, the histograms are not exactly centered on 0 . Furthermore, the weaker the correlation coefficient, the more negative the average residual (bias) in the line direction.
We suggest that the change in solar illumination angle explains this bias (Fig. 7). between the two acquisition dates, the Sun has moved, changing the orientation and length of its shadows. The apparent displacement of the shadows is negative and oriented mainly parallel to image lines. The strength of the correlation is weakened by a secondary correlation peak due to the shifted shadows. The larger the shadow, the larger the shift between the two dates and the weaker the correlation coefficient. We verify this hypothesis by comparing the residuals off the glaciers in the image line direction for two different slopes. The southeastfacing slopes, oriented toward the Sun at 9:00 AM local solar time (when the satellite images were acquired), present short shadows and, consequently, have high correlation coefficients and small residuals. The northwest-facing slopes, where the shadows are large, present low correlation coefficient and strong, negative residuals in the line direction.

However, on the glaciers, this "shadowing effect" does not cause a systematic error in our measurements. Shadows are only created by the surficial debris and the surrounding mountains. The best way to ensure a limited bias is to retain only the measurements with the highest correlation coefficient. The effect could be larger for other applications such as landslides, especially if images are acquired during different seasons.

Table 3 gives the offsets off the glaciers for both image pairs in column and line directions. The "shadowing effect" also affects the residuals in lines for image pair $\# 1$, but the effect is small because the illumination angle of the Sun did not change much between the two images. In both directions, the standard deviation is larger for pair \#1 than for pair \#2 because of the difference in the incidence angles of the two images (Table 1). Even with a precise DEM, the distortions between the images could not be perfectly modelled and suppressed.

Our images were acquired for glaciological purposes with a low gain: digital numbers are ranging from 10 to 30 off the glaciers and from 30 to 200 on the glaciers. Consequently, the strength of the correlation peak is much larger on the glaciers. It is thus difficult to transpose readily the uncertainties estimated on the steep slopes surrounding the glaciers to the glaciers. Only DGPS observations can provide a precise estimation of the accuracy of our method.

\subsection{Comparison with DGPS displacements}

To cover the same length of time, the satellite-derived displacements (occurring in 26 or 31 days) and the DGPS 


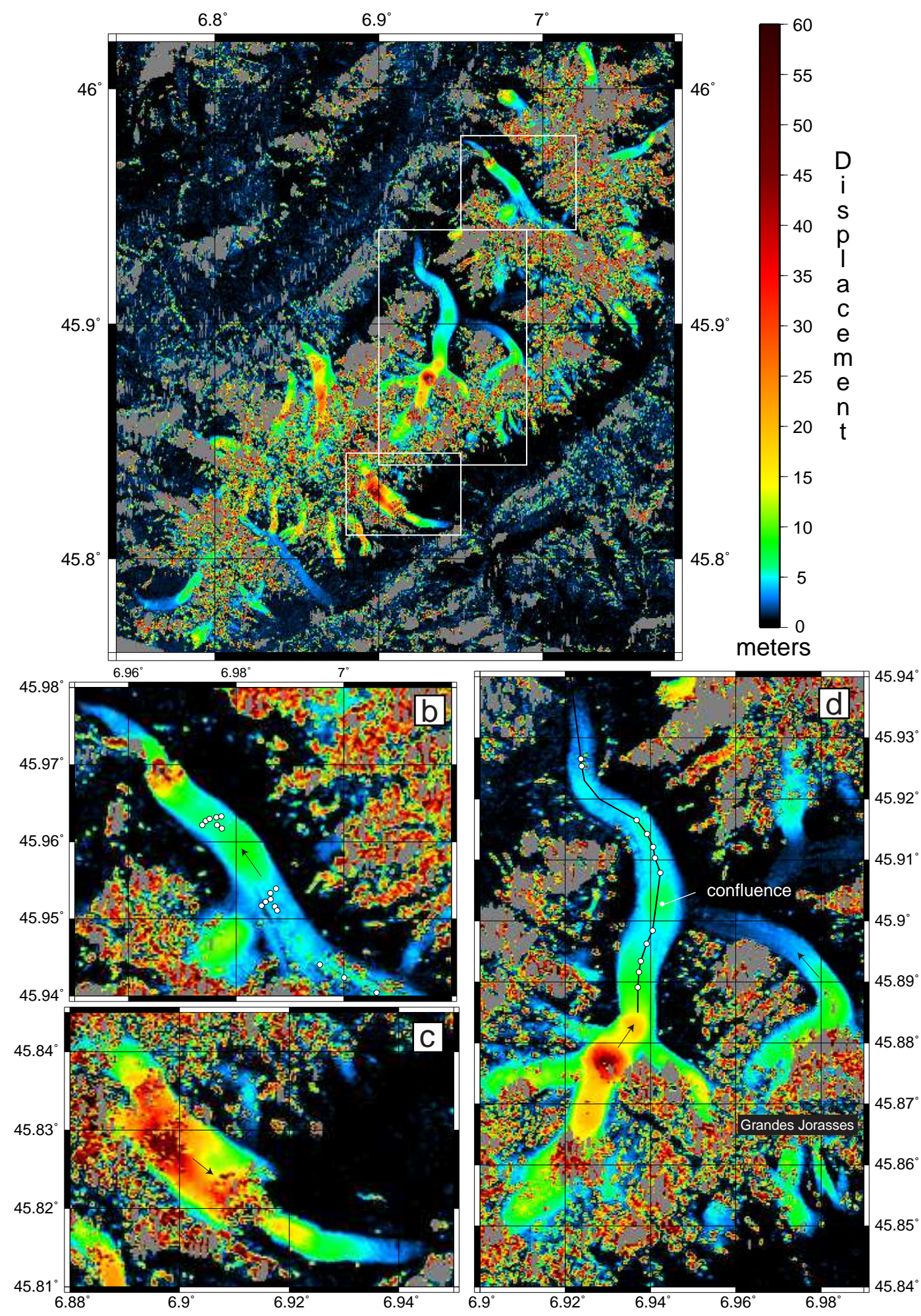

Fig. 5. Horizontal displacement of glaciers of the Mont Blanc area between the 23 August and 18 September 2003 (26 days). The upper panel (a) shows the entire Mont Blanc area. The white outlines encompass the Argentière, Brenva and Mer de Glace glaciers shown in the three lower panels (b, c, and d), respectively. Where the correlation coefficient is too weak, pixels appear in grey. The white dots are the ablation stakes measured by DGPS. The black line is the longitudinal profile on the Mer de Glace glacier shown in Fig. 8. The black arrows in the three lower panels indicate the direction of flow. 

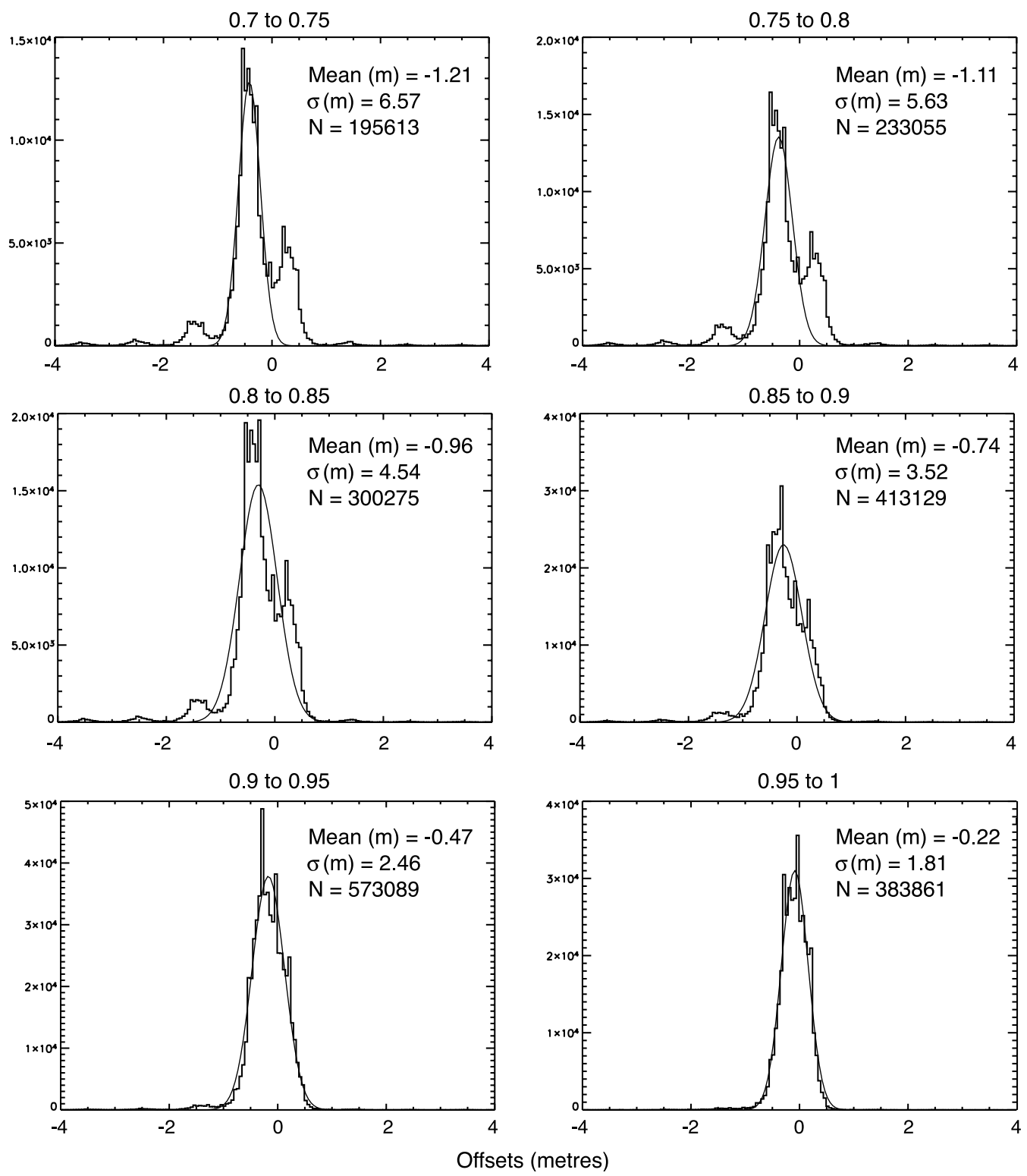

Fig. 6. Histograms of the residuals in the image line direction off the glaciers as a function of the correlation coefficient for image pair \#2. The mean, the standard deviation $(\sigma)$, and the number of points $(\mathrm{N})$ of our distribution are indicated on each plot.

measured displacements (occurring in 21 to 28 days depending on the date of survey) are rescaled to the displacement (in meters) occurring during 26 days. This time interval was chosen because it is the duration of the SPOT orbital-repeat cycle. This rescaling implicitly assumes that the ice-velocity is constant.

\subsubsection{The influence of changes in glacier elevation}

In Fig. 8, we compare, along a longitudinal profile of the Mer de Glace glacier, the displacements in the column direction of the 19 July 2003 image derived from image pair \#1 and from DGPS. Satellite measurements along a single profile and the mean of five parallel profiles separated by 25 $\mathrm{m}$ are displayed. The small difference between these two data sets indicates that the short-wavelength noise is small. There is a clear systematic bias between the satellite and DGPS displacements. This bias, on the order of $1.5 \mathrm{~m}$, is not observed in the direction of image lines.

This shift is the consequence of the non-vertical incidence angle of the satellite images (Table 1) combined with the strong ablation lowering the glacier surface during summer time. Fig. 9 illustrates this difference between measured and real displacements. Elevation changes, assumed to be equal to surface ablation for a short summer period, are estimated 


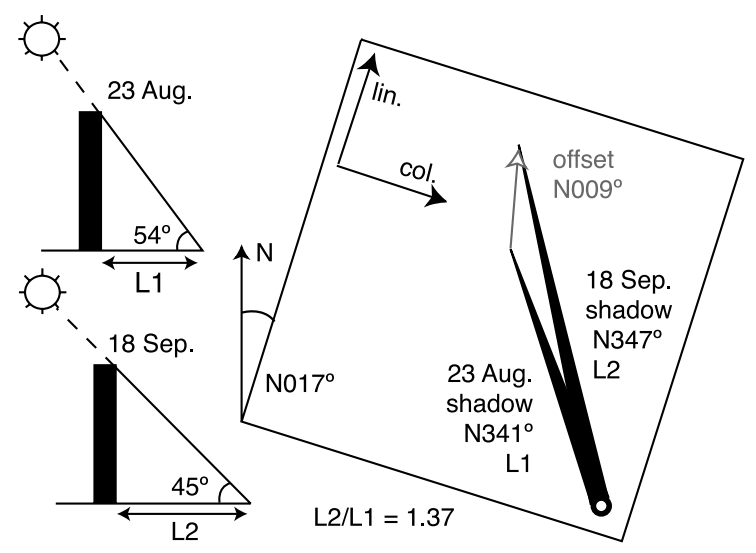

Fig. 7. Effect of the Sun's illumination angle on 23 August and 18 September 2003 on the length and orientation of the shadows. This effect explains why some unglaciarized areas present, mostly in the image line direction, some negative residuals.

from a simple empirical degree-day model tuned with field measurements (Vincent, 2002). Each positive degree at a given altitude on the glacier yields an ablation of $6.6 \mathrm{~mm}$ of ice. The temperature is calculated from the records of the nearby Chamonix weather station assuming a constant adiabatic lapse rate of $6 \%$ km. Between 19 July and 19 August 2003, the estimated ablation is on the order of $3 \mathrm{~m}$ of ice at $1900 \mathrm{~m}$ on the glacier. Combined with the $-24^{\circ}$ incidence angle of the 19 July 2003 image, we calculate that our method overestimates the column offset by $1.3 \mathrm{~m}$. This value is the same as the shift observed in Fig. 8 . We account for this effect by modeling the ablation of the ice.

The correction is made by lowering, in the DEM, the surface of the glaciers with the estimated ablation. The correct ground position $\left(\lambda_{i}, \phi_{\mathrm{s}}, z_{\mathrm{s}}\right.$ in Fig. 9) of the image point $(x+\Delta x, y+\Delta y)$ is deduced from this corrected DEM. New values of the column displacements are obtained and added to Fig. 8 (grey triangles). This simple modelling corrects most of the systematic shift in the column displacements. The uncertainty of the estimation of the elevation change from the degree-day model is large, on the order of $30 \%$. At $1900 \mathrm{~m}$, it contributes $\pm 0.4 \mathrm{~m}$ of uncertainty to the column displacement measurement for image pair \#1 and $\pm 0.13 \mathrm{~m}$ for image pair \#2.

Pairs of images acquired with similar and large incidence angles could be used to measure precisely elevation change on glaciers, or more generally, on the Earth's surface. If the magnitude or the direction of the ice flow is known accurately, the differences between the real and the satellite-derived column displacements could be converted to changes of elevation.

\subsubsection{Accuracy of the satellite-derived displacements}

Finally, we compare the DGPS and SPOT5-derived displacements. At the location of each stake, we extract the value of the displacements in column, line, and the correlation coefficient for each satellite image pair. Of the 29 stakes surveyed, 12 are located on the Mer de Glace glacier, and 17 on the Argentière Glacier. In Table 4 , we present the results of the comparison. Some of the differences could result from the temporal mismatch between the dates of surveys (Fig. 4) and from the uncertainties in the DGPS measurements $( \pm 21 \mathrm{~cm})$. This table underlines the high accuracy obtained for both image pairs. As expected, the rms discrepancy is generally smaller for image pair \#2 (on the order of $0.5 \mathrm{~m}$ in both image directions) than for image pair \#1 (around $1 \mathrm{~m}$ ). For all 29 stakes, the absolute differences between SPOT and DGPS displacements are on the order of $1 / 5$ of the pixel size $(0.5 \mathrm{~m})$ except in the line direction for image pair \#2. When considering each glacier independently, the results are slightly different.

For the Mer de Glace glacier, the most important differences appear in the line direction for image pair \#2. We believe that this difference cannot be explained by an error in the satellite measurement and is, therefore, a real velocity change of the glacier. First, the accuracy is two times better for pair \#2 than for pair \#1, as shown by the standard deviations in Table 4. We also expect the displacements in the line direction to be the most accurate because the stereoscopic effect and errors due to ablation affect the column direction only. The "shadowing effect" described previously could explain a systematic error in the image line direction. But shadows are limited on glaciers and, if they were present, would lead to an apparent acceleration of the Mer de Glace glacier. These differences could result from a short-term velocity change of the Mer de Glace glacier discussed below.

\subsection{Accuracy without GCPs and with a coarse DEM}

As explained previously, our methodology can be applied without GCPs by skipping the step of the

Table 3

Mean and standard deviation (in parentheses), in meters, of the residuals off the glaciers for different correlation coefficients intervals

\begin{tabular}{lrlrrrrr}
\hline Image pair & & $0.7-0.75$ & $0.75-0.8$ & \multicolumn{1}{c}{$0.8-0.85$} & \multicolumn{1}{c}{$0.85-0.9$} & \multicolumn{1}{l}{$0.9-0.95$} & $0.95-1$ \\
\hline$\# 1$ & Col. & $0.20(12.34)$ & $0.16(10.46)$ & $0.16(8.27)$ & $0.05(6.32)$ & $-0.10(4.74)$ & $-0.20(4.24)$ \\
& Lin. & $-0.48(14.04)$ & $-0.41(11.44)$ & $-0.34(8.40)$ & $-0.21(5.60)$ & $-0.10(3.28)$ & $-0.02(2.35)$ \\
$\# 2$ & Col. & $-0.30(5.33)$ & $-0.31(4.58)$ & $-0.28(3.68)$ & $-0.23(2.84)$ & $-0.14(1.96)$ & $-0.06(1.47)$ \\
& Lin. & $-1.21(6.57)$ & $-1.11(5.63)$ & $-0.96(4.54)$ & $-0.74(3.52)$ & $-0.47(2.46)$ & $-0.22(1.81)$ \\
\hline
\end{tabular}

Residuals in column (Col.) and line (Lin.) directions for the two pairs of images are presented. Note that the standard deviations are roughly twice as small for image pair \#2. Also, note the relation between the residuals in lines and the correlation coefficient caused by the "shadowing effect" (see text). 


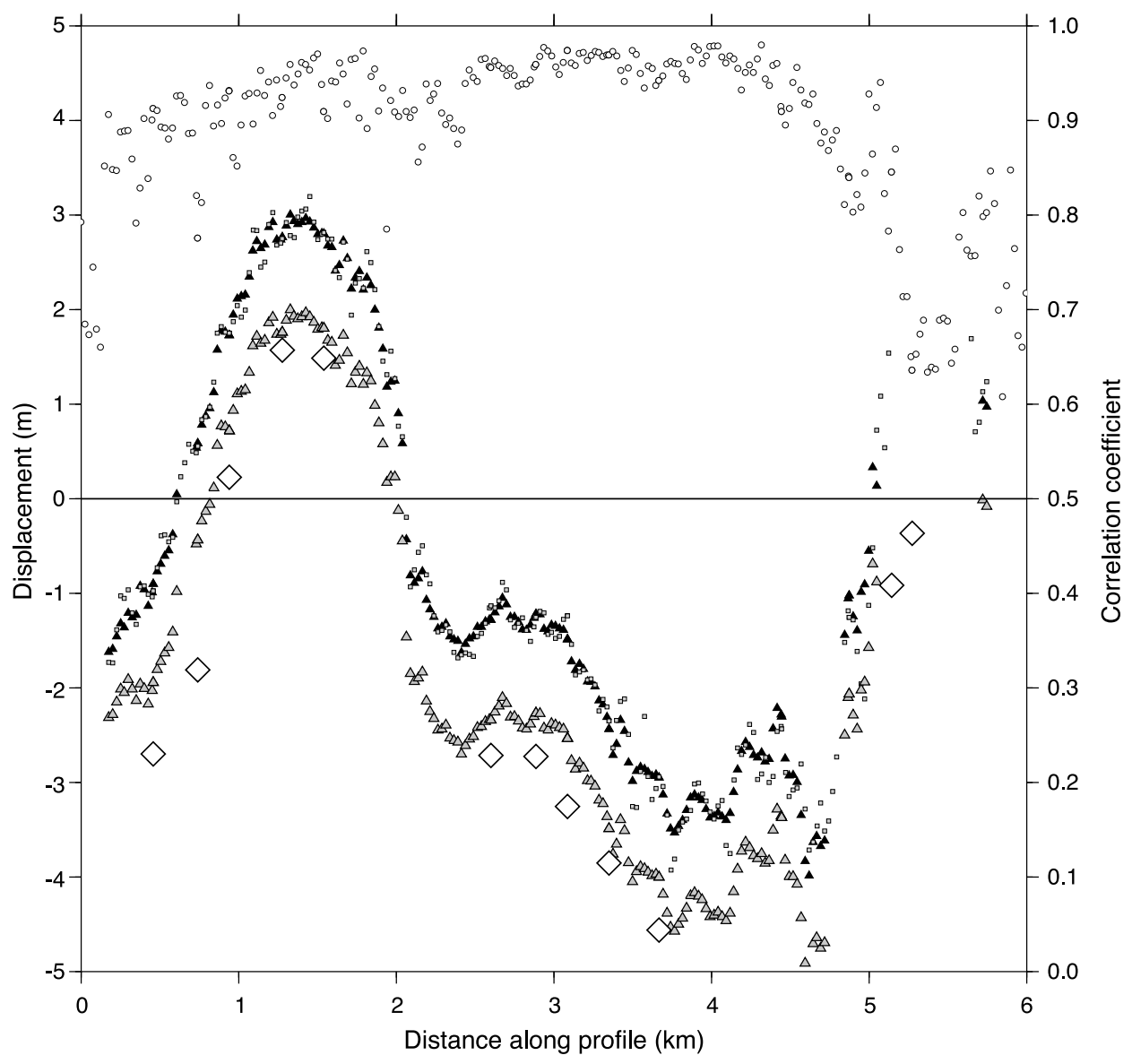

Fig. 8. Displacements along a longitudinal profile of the Mer de Glace glacier in the column image direction deduced from the 19 July and 19 August 2003 image pair. All displacements were rescaled to 26 days. The small light grey squares are the displacements from SPOT images along a single profile, whereas the black triangles represent the mean of 5 parallel profiles separated by $25 \mathrm{~m}$. The white diamonds are the DGPS displacements. The black circles (filled in white) represent the correlation coefficient of the satellite measurement (right axis). The grey triangles represent the satellite displacements after correction for ablation.

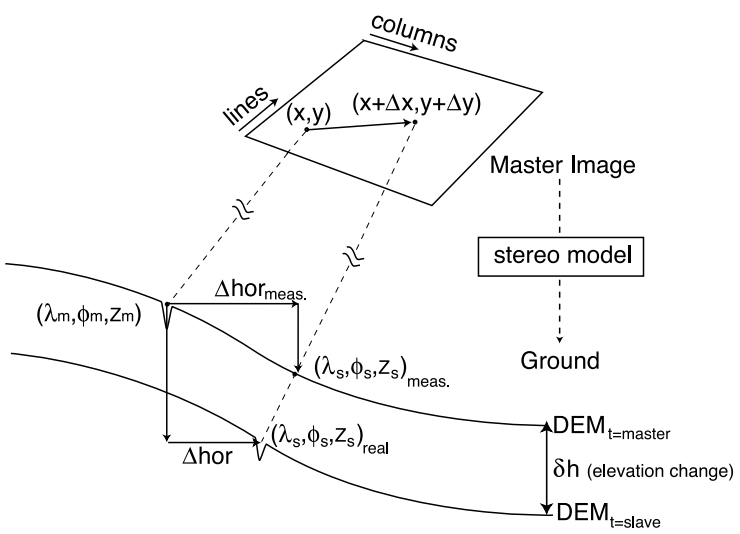

Fig. 9. Difference between the real and measured displacements caused by lowering of the glacier surface. This systematic error only affects measurements in the column direction. absolute orientation. Consequently, we obtain two well coregistered images but with a shift relative to the DEM. The stereoscopic effect is less well modelled so that some distortions remain between the two images. We choose image pair \#2 to test the accuracy obtained without GCPs because this pair presents a weak stereoscopic effect. The relative orientation of the two images is not significantly better with GCPs as shown in Table 2. The comparison with the DGPS survey (Table 4) confirms that accurate displacements can be obtained without GCPs. It is interesting to note that the difference is even smaller in some locations. We obtain a similar accuracy when using the GTOPO30 DEM (also without GCPs) instead of the high resolution DEM. It indicates that, with a good image pair, our methodology can be applied to remote areas where no high resolution DEM is available. The main limitation in using a coarse DEM is that elevation errors in the DEM, combined with non- 
Table 4

Mean and standard deviation (in parentheses), in meters, of the difference between the displacements derived from SPOT5 images and measured during a differential GPS field campaign

\begin{tabular}{llccc}
\hline Image pair & \multicolumn{2}{c}{ Argentière } & Mer de Glace & \multicolumn{1}{c}{ All data } \\
\hline$\# 1$ & Col. & $0.38(0.71)$ & $0.6(0.34)$ & $0.49(0.56)$ \\
& Lin. & $0.65(0.93)$ & $0.39(0.38)$ & $0.53(0.72)$ \\
$\# 2$ & & & & \\
& Col. & $0.22(0.47)$ & $0(0.4)$ & $0.12(0.45)$ \\
& Lin. & $0.47(0.53)$ & $1.33(0.46)$ & $0.85(0.66)$ \\
$\# 2$ no GCPs & Col. & $0.28(0.46)$ & $0.01(0.47)$ & $0.16(0.45)$ \\
& Lin. & $0.52(0.6)$ & $1.2(0.33)$ & $0.84(0.6)$ \\
\#2 no GCPs & Col. & $0.07(0.43)$ & $-0.35(0.42)$ & $-0.12(0.47)$ \\
GTOPO30 & Lin. & $0.54(0.65)$ & $1.29(0.44)$ & $0.88(0.67)$ \\
\hline
\end{tabular}

The offsets in columns (Col.) and lines (Lin.) for the two image pairs and also image pair \#2 without GCPs (with the high resolution DEM and the GTOPO30 DEM) are presented.

vertical incidence angles, will result in georeferencing errors. They can lead to significant displacement errors in areas where the velocity gradient is large, which is not the case near our DGPS surveys, located close to the glacier centerline.

\subsection{A likely ice-acceleration event on the Mer de Glace glacier}

Fig. 10 shows that a velocity change may affect that part of the Mer de Glace glacier located between the Géant icefall and its confluence with the Leschaux glacier. The mean velocity change between image pair \#1 and \#2 for this part of the profile is $12.6 \mathrm{~m} \mathrm{a}^{-1}$ or $11.6 \%$ in 30 days. The DGPS velocities are even slightly greater than the one deduced from image pair $\# 1$, dating the ice-acceleration event in mid-August 2003. Such rapid summer velocity changes of glaciers have been reported previously. They are usually explained by higher sliding velocities due to higher basal water pressures (e.g., Mair et al., 2001). In early August 2003, a pronounced heat wave baked Europe, increasing the surface melting on glaciers in the Alps. This rapid water input could explain the increase in basal water pressure and sliding on part of the Mer de Glace glacier. In

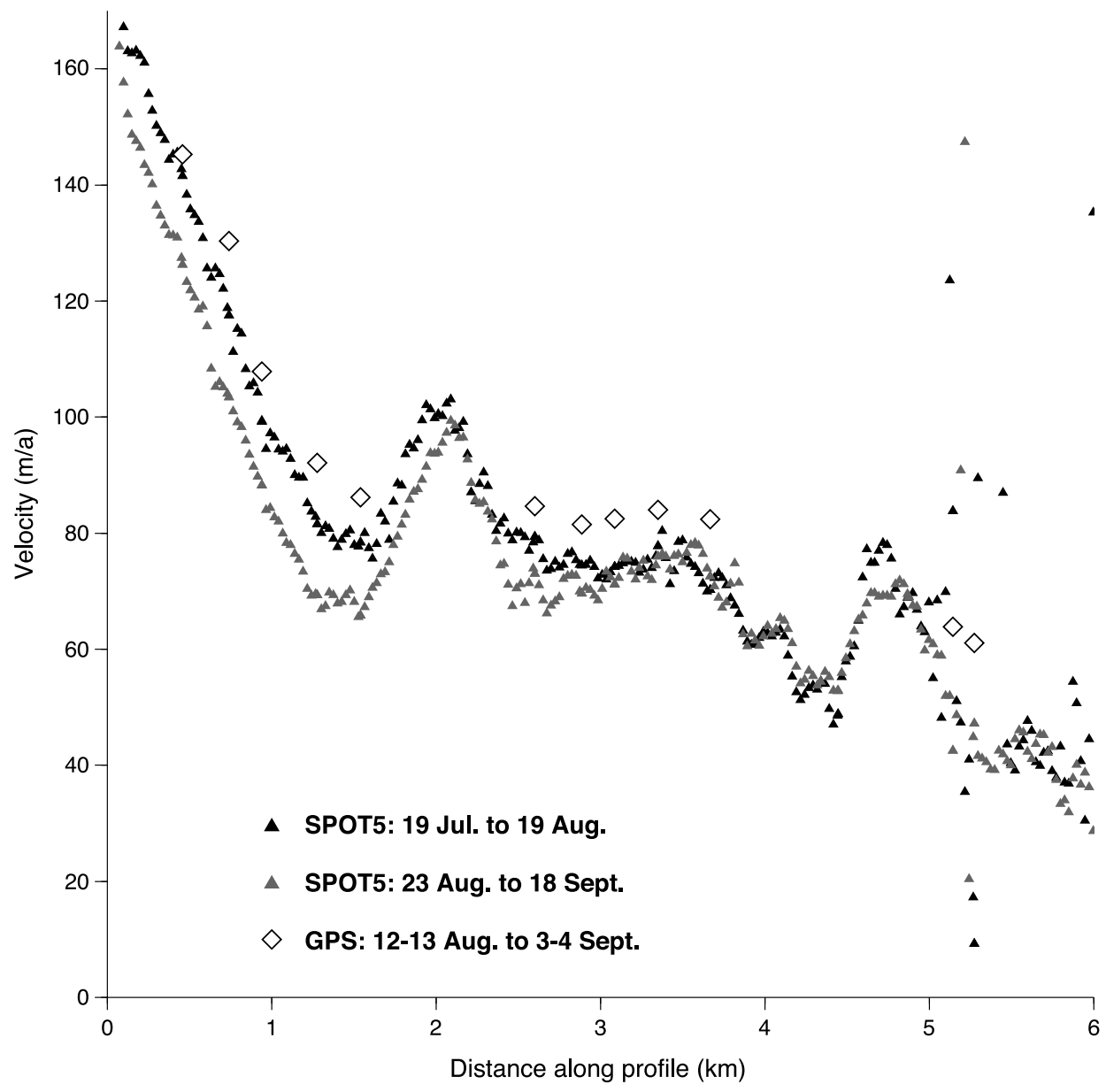

Fig. 10. Horizontal surface velocities along a longitudinal profile of the Mer de Glace glacier determined from satellite images and DGPS surveys. 
future work, a more detailed study of the spatial pattern of the velocity change will help better understand the causes of this ice-acceleration event.

\section{Conclusions}

The goal of measuring displacements on mountain glaciers with an accuracy of one fourth the pixel size (0.62 m with SPOT5 images) has been achieved. The uncertainty in the DGPS survey $(0.21 \mathrm{~cm})$, the temporal mismatch between the ground surveys, and the acquisition dates of the SPOT5 images, combined with a sudden increase in ice-velocity prevent us from confirming definitively the accuracy from field observations. Yet, an uncertainty of $0.5 \mathrm{~m}$ in each image direction seems reasonable if satellite images are acquired from a similar point of view. This uncertainty of $0.5 \mathrm{~m}$ over 26 days is equivalent to $2 \mathrm{~cm}$ over 1 day, very close to the accuracy obtained from InSAR using ERS-1/ERS-2 tandem pairs. Our approach is accurate enough to measure a $10 \%$ velocity increase on the Mer de Glace glacier during and just after the pronounced heat wave in Europe in early August 2003.

Because our approach does not require GCPs, our methodology can be routinely and rapidly applied to new pairs of images. The high accuracy obtained without GCPs using the GTOPO30 DEM suggests the possibility of monitoring numerous glaciers, even in remote areas and without a contemporaneous DEM. The key step is the relative orientation of the two correlated images using precise homologous points. The accuracy of the measurement is controlled mainly by the radiometry (low gain is better for glaciers to avoid sensor saturation) and the geometry of the images. The "shadowing" effect, highlighted on the motionless area surrounding the glaciers, could be a problem for the applications other than glaciology. The rapidly changing elevation of mountain glaciers in summertime, because of ablation, can create a systematic error in the displacements along the image column direction. Addressing this issue, however, requires external information. This systematic bias could be used to monitor the elevation change of glaciers throughout the ablation season.

Images acquired from a similar point of view, with incidence angles differing only by a few degrees, are best. Vertical incidence eliminates the systematic errors caused by ablation but such a constraint also reduces the likelihood of obtaining two good images.

Among the limitations of our approach is that a velocity measurement during the summer may not be representative of the annual dynamics of a glacier. Thus, annual surveys of ablation stakes on a few glaciers are needed and complement the satellite-derived measurements. Another limitation is the difficulty in acquiring two cloud-free images with similar incidence angles.

Other geological phenomena that deforms the Earth's surface, such as earthquakes, landslides, and volcanoes could also be surveyed using the cross-correlation of SPOT5 images. Depending on the expected direction of displacements, the incidence angles could be chosen for optimum accuracy. For mostly horizontal (respectively vertical) displacements, images with vertical (respectively oblique) incidence angles are preferable. For these applications other than glaciology, one interesting and promising topic to explore would be the fusion of displacements obtained by InSAR and optical image cross-correlation to obtain a 3dimensional surface-displacement map.

\section{Acknowledgments}

Aurélie Bouilllon provided useful help concerning the SPOT5 algorithms. The comments of R. S. Williams Jr. and an anonymous reviewer led to significant improvements of the manuscript. We thank M. Bauer who provided valuable guidance as Editor-in-Chief. SPOT5 images were acquired thanks to the ISIS program (copyright CNES). This work was supported by the French national program ACI-OT Glaciers, the GDR STRAINSAR and the French GLACIOCLIM program. The LGGE and IRD Great Ice Unit provided support for the field experiment. GMT software (Wessel \& Smith, 1998) illustrated the article. The first author gratefully acknowledges a thesis fellowship from the French government.

\section{References}

Al-Rousan, N., Cheng, P., Petrie, G., Toutin, T., \& Valadan Zoej, M. (1997) Automated DEM extraction and orthoimage generation from SPOT level 1B imagery. Photogrammetric Engineering and Remote Sensing, 63, 965-974.

Berthier, E., Amaud, Y., Baratoux, D., Vincent, C., \& Rémy, F. (2004). Recent rapid thinning of the "Mer de glace" glacier derived from satellite optical images. Geophysical Research Letters, 31(17) doi10.1029/2004GLO20706.

Berthier, E., Raup, B., \& Scambos, T. A. (2003). New velocity map and mass-balance estimate of Mertz Glacier, East Antarctica, derived from Landsat sequential imagery. Journal of Glaciology, 49(167).

Braithwaite, R. J. (2002). Glacier mass balance: the first 50 years of international monitoring. Progress in Physical Geography, 26(1), 76-95.

Centre National d'Etude Spatiale, (2002). MEDICIS software, distributed by CSSI.

Ferrigno, J. G., \& Williams, R. S., Jr. (1983). Limitations in the use of Landsat images for mapping and others purposes in snow- and icecovered regions: Antarctica, Iceland and Cape Code, Massachusetts. International Symposium on Remote Sensing of Environment 17th, vol. 1. (pp. 335-355) Ann Arbor, Michigan: Environmental Research Institute of Michigan.

Fischer, A., Rott, H., \& Björnsson, H. (2003). Observation of recent surges of Vatnajökull, Iceland, by means of ERS SAR interferometry. Annals of Glaciology, 37, 69-76.

Fratter, D., Moulin, M., Ruiz, H., \& Charvet, P. D. Z. (2001). The SPOT-5 mission. 52nd International Astronautical Congress. Toulouse, France.

Frezzotti, M., Capra, A., \& Vittuari, L. (1998). Comparison between glacier ice velocities inferred from GPS and sequential satellite images. Annals of Glaciology, 27, 54-60. 
Goldstein, R. M., Engelhardt, H., Kamb, B., \& Frolich, R. M. (1993). Satellite radar interferometry for monitoring ice sheet motion: application to an Antartic ice stream. Science, 262(5139), $1525-1530$.

Gray, A. L., Short, N., Mattar, K. E., \& Jezek, K. C. (2001). Velocities and flux of the Filchner Ice Shelf and its tributaries determined from speckle tracking interferometry. Canadian Journal of Remote Sensing, 27(3), 193-206.

Joughin, I. R., Kwok, R., \& Fahnestock, M. A. (1998). Interferometric estimation of the three-dimensional ice-flow velocity vector using ascending and descending passes. IEEE Transactions on Geoscience and Remote Sensing, 36(1), 25-37.

Kääb, A. (2002). Monitoring high-mountain terrain deformation from repeated air- and spaceborne optical data: examples using digital aerial imagery and ASTER data. ISPRS Journal of Photogrammetry and Remote Sensing, 57(1-2), 39-52.

Kääb, A., Wessels, R., Haeberli, W., Huggel, C., Kargel, J., \& Khalsa, S. (2003). Rapid ASTER imaging facilitates timely assessment of glacier hazards and disasters. EOS, Transactions, Am. Geophy. Un., 84(13), $117-121$.

Lucchitta, B. K., \& Ferguson, H. M. (1986). Antarctica: measuring glacier velocity from satellite images. Science, 234(4780), 1105-1108.

Mair, D., Nienow, P., Willis, I., \& Sharp, M. (2001). Spatial patterns of glacier motion during a high velocity event: Haut Glacier d'Arolla, Switzerland. Journal of Glaciology, 47(156), 9-20.

Massonnet, D., \& Feigl, K. (1998). Radar interferometry and its application to changes in the Earth's surface. Reviews of Geophysics, 36(4), 441-500.

Mattar, K. E., Vachon, P. W., Geudtner, D., Gray, A. L., Cumming, I. G., \& Brugman, M. (1998). Validation of alpine glacier velocity measurements using ERS tandem-mission SAR data. IEEE Transactions on Geoscience and Remote Sensing, 36(3), 973-984.

Mohr, J. J., Reeh, N., \& Madsen, S. N. (1998). Three dimensional glacial flow and surface elevation measured with radar interferometry. Nature, 391(6664), 273-276.

Paterson, W. S. B. (1994). The physics of glaciers (3rd edition). New York: Pergamon.

Rabus, B., Eineder, M., Roth, A., \& Bamler, R. (2003). The shuttle radar topography mission-a new class of digital elevation models acquired by spaceborne radar. ISPRS Journal of Photogrammetry and Remote Sensing, 57, 241-262.

Rabus, R. T., \& Fatland, D. R. (2000). Comparison of SAR-interferometric and surveyed velocities on a mountain glacier: Black Rapids Glacier, Alaska, U.S.A. Journal of Glaciology, 46(152), 119-128.

Reynaud, L. (1980). Reconstruction of past velocities using Forbes bands (Mer de Glace). Zeitschrift fur Gletscherkunde und Glazialgeologie, 15(2), 149-163.

Rignot, E., Vaughan, D. G., Schmeltz, M., Dupont, T., \& MacAyeal, D. (2002). Acceleration of Pine Island and Thwaites Glaciers, West Antartica. Annals of Glaciology, 34, 189-194.

Scambos, T. A., Dutkiewicz, M. J., Wilsoni, J. C., \& Bindschadler, R. A. (1992). Application of image cross-correlation to the measurement of glacier velocity using satellite image data. Remote Sensing of Environment, 42(3), 177-186.

SPOT-Image, (2002). SPOT Satellite Geometry Handbook, SNT-73_12-SI, Edition 1, Revision 0.

Strozzi, T., Luckman, A., Murray, T., Wegmüller, U., \& Werner, C. (2002). Glacier motion estimation using SAR offset-tracking procedures. IEEE Transactions on Geoscience and Remote Sensing, 40(11), 2384-2391.

Toutin, T., \& Cheng, P. (2002). A comparison of automated DEM extraction results using along-track ASTER and across-track SPOT stereo images. Optical Engineering, 41(9), 2102-2106.

USGS, (1996). The GTOPO30 DEM. http://edcdaac.usgs.gov/gtopo30/ gtopo30.asp

Vadon, H., \& Massonnet, D. (2000, 24-28 July). Earthquake displacement fields mapped by very precise correlation: complementary with radar interferometry. In I. Periodicals (Ed.), IEEE International Geoscience Remote Sensing Symposium. New Jersey (pp. 2700-2702).

Van Puymbroeck, N., Michel, R., Binet, R., Avouac, J. P., \& Taboury, J. (2000). Measuring earthquakes from optical satellite images. Applied Optics, 39(20), 3486-3494.

Vincent, C. (2002). Influence of climate change over the 20th century on four French glacier mass balances. Journal of Geophysical Research, 107(D19), 4375.

Wessel, P., \& Smith, W. H. F. (1998). New, improved version of generic mapping tools released. EOS, Transactions, Am. Geophy. Un., 79(47), 579. 


\section{Annexe D}

\section{Soulèvement de la Plateforme Glaciaire de Grimsvötn (Islande)}

L'article présenté ici a été soumis le 24 avril 2004 :

Berthier, E., Björnsson, H., Pálsson, F., Llubes, M., Feigl, K. L., \& Rémy, F., soumis. The level of the Grímsvötn subglacial lake, Vatnajökull, Iceland, monitored with SPOT5 images. Earth Planet. Sc. Lett.. 


\title{
The level of the Grímsvötn subglacial lake, Vatnajökull, Iceland, monitored with SPOT5 images
}

\author{
E. Berthier ${ }^{\mathrm{a}, *}$ H. Björnsson ${ }^{\mathrm{b}}$ F. Pálsson ${ }^{\mathrm{b}}$ M. Llubes ${ }^{\mathrm{a}}$ K. L. Feigl ${ }^{\mathrm{c}}$ F. Rémy ${ }^{\mathrm{a}}$ \\ ${ }^{a}$ LEGOS/CNRS/UPS, 18 av. Ed. Belin, 31401, Toulouse Cedex 9, FRANCE \\ ${ }^{\mathrm{b}}$ Institute of Earth Sciences, University of Iceland, Reykjavik, ICELAND \\ ${ }^{\mathrm{c}}$ DTP/CNRS/UPS, 14 av. Ed. Belin, 31400, Toulouse, FRANCE
}

\begin{abstract}
Using the correlation of two satellite optical images, we monitor the inflow of water in the Grímsvötn lake, located underneath the Vatnajökull ice cap (Iceland). The vertical displacement field of the ice shelf floating on the lake is estimated from two SPOT5 images acquired 5 days apart with similar, non-vertical incidence. The uplift map shows that $10.9 \mathrm{~km}^{2}$ of ice is floating and permit us to estimate the water and energy balance of the subglacial lake. Between 11 and 16 August 2004, the ice shelf rose by $1.71 \mathrm{~m}$ indicating that the volume of liquid water in the lake increased by $0.019 \mathrm{~km}^{3}$. Surface melting contributed $70 \%$ of the accumulated water and the rest came from ice melted by the subglacial geothermal activity. The power required to melt $0.019 \mathrm{~km}^{3}$ (water equivalent) of basal ice in 5 days is $4000 \mathrm{MW}$, corresponding to an average geothermal flux of $70 \mathrm{~W} \mathrm{~m}^{-2}$. Our technique is suitable for mapping short-term elevation changes of glaciers. If the surface features are preserved, vertical displacements are measured every $25 \mathrm{~m}$ with an accuracy of about $0.5 \mathrm{~m}$. Applied to two pairs of images, it could solve for the 3-dimensional displacements of the Earth's surface.
\end{abstract}

Key words: Geothermal activity, subglacial hydrology, SPOT5, Iceland, image correlation PACS:

\section{Introduction}

Exploration of subglacial hydrology on a regional scale by monitoring glacier surface displacements from space is a challenging task. The displacements of the ice surface may be due to accumulation and release of water in basal storage or water merely lubricating the bed and aiding glacier sliding. Such variations have been

\footnotetext{
* Corresponding author

Email address:

etienne.berthier@cnes.fr(E. Berthier).
}

monitored in situ at single ground-based points and along profiles by geodetic and DGPS surveying and aerial photography $(1 ; 2 ; 3 ; 4)$. During the last 15 years, satellite and airborne remote sensing techniques have captured variations in topography over large areas. These techniques include repeated profiling by laser or radar altimetry $(5 ; 6 ; 7)$, but in terms of accuracy (within few centimetres) and spatial coverage $(100 \times 100 \mathrm{~km}$ for a SAR scene $)$ SAR interferometry (InSAR) is the most efficient technique for mapping elevation changes $(8 ; 9)$. However, with the exception of cold, high, polar areas of low accumulation rate (10), conventional In- 
SAR cannot be applied if images are separated by more than 1 to 3 days (11). Speckle tracking alleviates this shortening in certain cases (12). In our case, on the temperate Vatnajökull ice cap, only C-Band SAR images separated by one day correlate well enough for interferometric comparison (13). On the other hand, optical images correlate over longer intervals, typically from a few days in the accumulation area to a few years in the ablation zone of glaciers. Such optical images have previously been used to describe horizontal motion of glaciers $(14 ; 15)$, horizontal displacements caused by earthquakes $(16 ; 17)$, and landslides (18). Here, we present the first measurement of vertical motion obtained by correlating optical images. We apply our method to determine the extent and magnitude of the uplift of a floating ice shelf during the filling of a subglacial lake (Grímsvötn) located in the interior of the Vatnajökull ice cap $\left(8200 \mathrm{~km}^{2}\right)$ in southeast Iceland.

\section{Site description}

The Grímsvötn subglacial lake is situated in an ice-filled caldera at the Grímsvötn volcanic centre (Figure 1, N 64.41 ${ }^{\circ}, \mathrm{W} 17.33^{\circ}$ ). The intense geothermal activity continuously melts the ice and meltwater accumulates in the subglacial lake. Its level rises until the lake drains subglacially (at an interval of 1 to 10 years) in large floods (named jökulhlaups in Iceland) to the outwash plain Skeidarársandur (4). A 270-m-thick ice shelf floats on the lake. To the south and the west, the lake is confined by the steep caldera walls but can expand to the north and northeast as the water level rises $(4 ; 19)$. Moreover, ice is occasionally melted by volcanic eruptions in the Grímsvötn volcano. Its mean eruption frequency has been close to once per decade during the eight past centuries (20). The most recent eruptions took place in 1934,

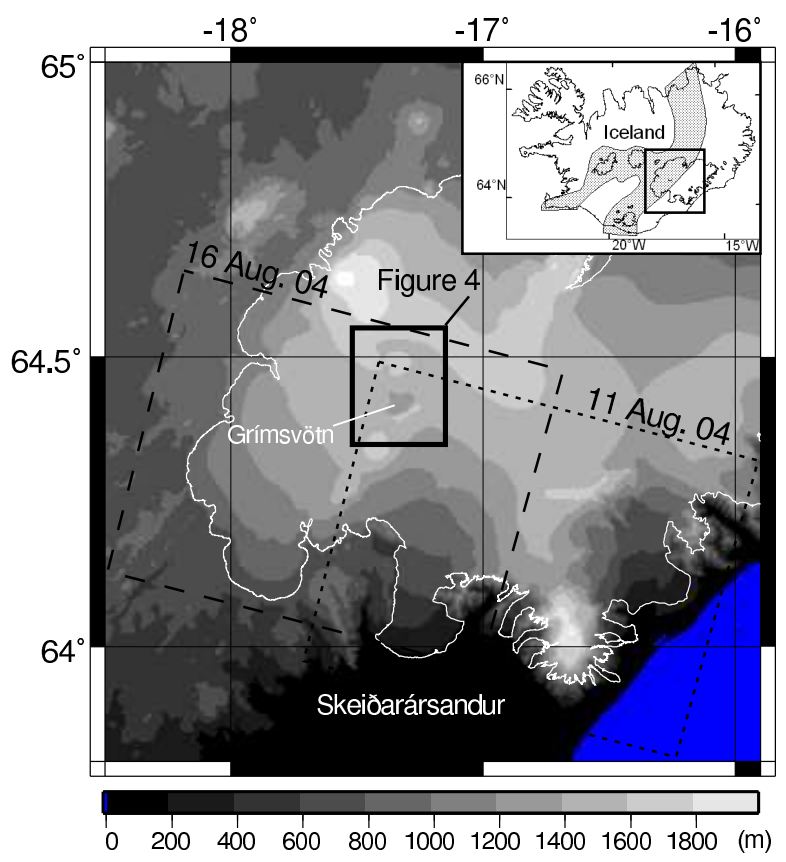

Fig. 1. Study area of Grímsvötn in Vatnajökull ice cap. The footprints of the SPOT5 images of 11 and 16 August 2004 are located on a digital elevation model of the ice cap whose margins are drawn in white. The thick black box shows the area displayed in the Figure 4. The inset locates the volcanic zones and the major ice caps in Iceland.

1983, 1998 and November 2004.

Monitoring the lake level of Grímsvötn is essential for forecasting the timing of jökulhlaups. A GPS receiver, located at the centre of the floating ice shelf, monitors its elevation continuously (Figure 2). Precise DGPS surveys are also performed about 6 times a year. From June 2003 to late October 2004, the floating ice shelf rose by $60 \mathrm{~m}$. Then, it dropped suddenly due to an abrupt drainage of the lake, leading to a jökulhlaup. An eruption of the Grímsvötn volcano started a few days later, presumably triggered by the 15-m-drop in the lake level (21). The volcano geothermal flux fills gradually the lake, whose abrupt subsidence release the overburden pressure and triggers an eruption.

Observations of the area of the floating ice cover 

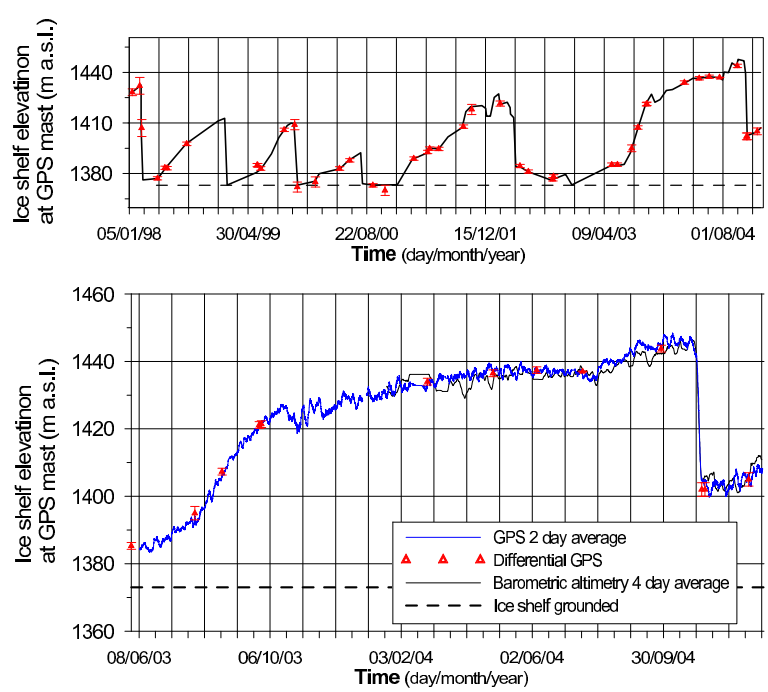

Fig. 2. Elevation of the Grímsvötn floating ice shelf deduced from standard GPS, barometric pressure and sparse DGPS measurements at a single location (MAST). The upper panel displays the 1998-2004 period, the lower panel the June 2003-December 2004 period. The horizontal dashed line indicates the elevation (about $1370 \mathrm{~m}$ ) where the ice shelf is grounded. A longer time series (years 1930 to 2001) of ice shelf elevations can be found in (4).

are required for estimating the lake volume and, consequently, the size of the outburst floods. After the lake drains in a jökulhlaup, the areal extent of the ice shelf can be delineated by the outermost crevasses which are formed when the ice shelf abruptly subsides (19). Yet, this a posteriori approach cannot forecast the amount of water to be released. A previous study has used InSAR from the ERS satellites tandem mission to map the extent and rate of the uplift during the period 1996-1999 (4). Since the tandem mission ended, we propose a new methodology to monitor glacier surface elevation changes from satellite optical images.

\section{Methodology}

Our method uses the correlation of two optical SPOT5 images to measure their offsets in the line and column directions and then derive the vertical component of the displacement field. This technique measures the projection of a ground displacement $\vec{\delta}\left[\delta_{\lambda}, \delta_{\phi}, \delta_{z}\right]$ in the focal plane of the sensor (with $\lambda$ the longitude, $\phi$ the latitude, and $\mathrm{z}$ the local vertical axis). This focal plane can be defined by two unit vectors $\overrightarrow{U_{\text {lin }}}$ and $\overrightarrow{U_{\text {col }}}$ indicating the line and column directions respectively.

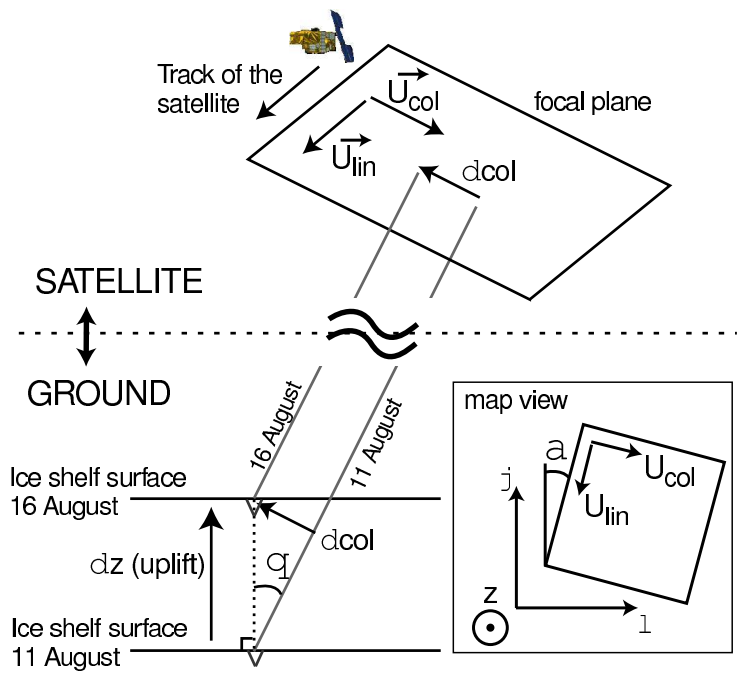

Fig. 3. Schematic vertical cross section showing how a vertical displacement between the acquisition dates of the satellite images produces an offset in the column direction. The sensitivity to the vertical displacement is proportional to the sine of the incidence angle $\theta$. The inset map view defines the azimuth $\alpha$, angle between the North and the line direction.

If $\theta$ is the incidence angle (varying by $\pm 2^{\circ}$ throughout the images) and $\alpha$ the (azimuth) angle between the line direction and the North (varying by $\pm 0.01^{\circ}$ and thus considered as constant), the coordinates of $\overrightarrow{U_{\text {lin }}}$ and $\overrightarrow{U_{\text {col }}}$ are

$\overrightarrow{U_{\text {lin }}}=[-\sin \alpha,-\cos \alpha, 0]$ 
$\overrightarrow{U_{\text {col }}}=[\cos \theta \cos \alpha,-\cos \theta \sin \alpha, \sin \theta]$

The correlation of the satellite images measures the displacements $\delta_{c o l}$ and $\delta_{l i n}$

$\delta_{\text {col }}=\vec{\delta} \cdot \overrightarrow{U_{c o l}}$

$\delta_{\text {lin }}=\vec{\delta} \cdot \overrightarrow{U_{\text {lin }}}$

If the horizontal displacements $\delta_{\lambda}$ and $\delta_{\phi}$ can be neglected, Equation 3 simply reduces to

$\delta_{z}=\frac{\delta_{c o l}}{\sin \theta}$

A purely vertical movement will thus induce an offset in the column direction (Figure 3). Furthermore, Equation 5 shows that large incidence angles increase the sensitivity to vertical motion. For the images used in this study, the $27^{\circ}$ incidence angles lead to a sensitivity of the order of 0.45 , i.e. $45 \%$ of the vertical motion should be visible in the column-offset field.

In the case of the Grímsvötn ice shelf the horizontal glacier flow is slow and mainly oriented southward such that $\delta_{\lambda}$ can be neglected, but not $\delta_{\phi}$. With these assumptions, combining Equation 3 and 4, we deduce the vertical displacement:

$\delta_{z}=\frac{\delta_{c o l}-\delta_{\text {lin }} \times \cos \theta \times \frac{\sin \alpha}{\cos \alpha}}{\sin \theta}$

The accuracy of the ground displacements obtained by correlating two optical images is controlled mainly by the distortions between the images (22). The distortions are proportional to the ratio of the baseline (the distance between the two satellite positions) to the altitude of the satellite. When correlating two images, the $B / H$ ratio measures the apparent horizontal displacement that will be induced by an error in the digital elevation model (DEM) of the area (16). In an optimal satellite configuration, the two images are acquired from exactly the same viewpoint, the value of $B / H$ is small and the offsets in column only reflect the surface elevation changes. Favorable situation occurs when the time separation between the image dates is a multiple of the orbital cycle duration, i.e. 26 days in the case of the SPOT5 satellite.

\section{Measurements. Spatial extent of the up- lift}

We have mapped the uplift of the Grímsvötn ice shelf by correlating two SPOT5 images acquired on 11 and 16 August 2004 (Table 1) with a ground resolution of $2.5 \mathrm{~m}$. Their footprints are drawn in Figure 1. They have the lowest possible gain (1 for SPOT5) which is crucial to avoid radiometric saturation of the sensor on the reflective surface of the glacier. For our study, we could not obtain satellite image pairs separated by exactly one 26-day SPOT5 orbital cycle due to cloud coverage and satellite scheduling. Furthermore, in 26 days, changes on the glacier surface could degrade the image correlation, especially in the upper accumulation area. Consequently, we apply our methodology to images acquired 5 days apart from slightly different viewpoints, with a $B / H$ ratio of 0.064 . In this case, an error of $10 \mathrm{~m}$ in the DEM would create an apparent horizontal displacement (in the image column direction) of $0.64 \mathrm{~m}$, equivalent to a vertical displacement of $1.42 \mathrm{~m}$. An accurate DEM of the ice cap is thus required.

We have calculated a DEM from a second independent pair of SPOT5 images acquired on 7 and 9 October 2004 (Table 1). These images have a similar footprint as the 16 August image (Figure 1). Only a $5 \mathrm{~m}$ resolution is available due to sensor problems. The two days time separation ensures a good correlation between the images even in the snow-covered region. The 
Table 1

Characteristics of the 2004 SPOT5 image pairs used in this study. $B / H$ is the ratio of the baseline (distance separating the two successive positions of the satellite) to the altitude of the satellite. High $B / H$ values are recommended for DEM computation whereas low $B / H$ values are best for motion mapping.

\begin{tabular}{lccrc}
\hline Image Pair & Pix. size & Incid. angle & $B / H$ & $\delta \mathrm{t}$ \\
\hline $11 \& 16$ Aug. & $2.5 \mathrm{~m}$ & $-27 /-26.7$ & 0.064 & 5 \\
07 \& 09 Oct. & $5 \mathrm{~m}$ & $-26.6 / 24.6$ & 0.95 & 2 \\
\hline
\end{tabular}

glacier flow in the slow moving area surrounding the Grímsvötn caldera during two days is small enough to avoid a systematic bias in the DEM. Errors could also result from the change in the length of the shadows due to the different acquisition times of the two images (at 13:25 on 7 October and 12:23 on 9 October). The $B / H$ is close to 1 and optimal for elevation mapping of the smooth topography of the ice cap. The accuracy of our DEM is assessed by comparing its elevation with a kinematic GPS survey in a vehicle driving on the glacier in late September 2004. The accuracy of the kinematic GPS measurement is of the order of $\pm 0.15 \mathrm{~m}$. For the 13800 comparison points, the SPOT5 DEM is $0.75 \mathrm{~m}$ lower than the GPS data, with a RMS scatter of $7.8 \mathrm{~m}$. On the Grímsvötn ice shelf itself, our DEM has been corrected for the vertical displacement occurring between midAugust (date of the images used to map the uplift) and early October. The permanent GPS station on the ice shelf indicates an uplift of $4.5 \mathrm{~m}$ during this time. An error of $10 \%$ in this correction, which relies on the extrapolating the GPS measurement to the entire ice shelf, would alter the vertical displacement measurement by less than a decimeter.

Figure 4 depicts the surface elevation changes measured by comparing the 2004 DEM with an older DEM, produced by airborne InSAR images acquired in August 1998 (23). The accu-

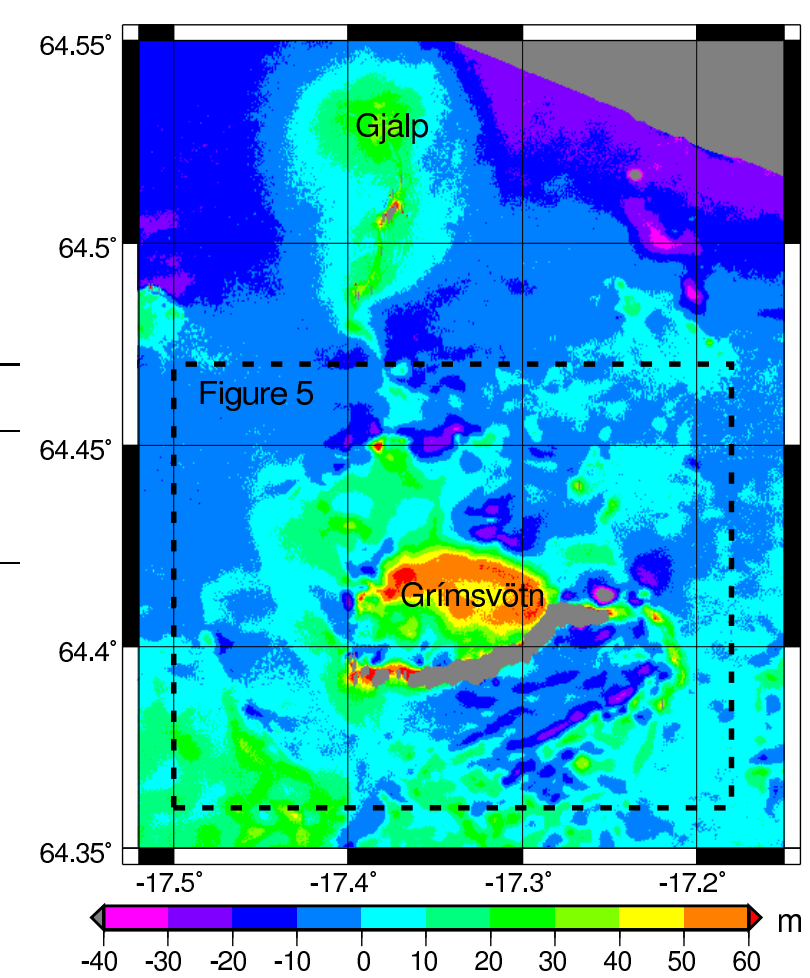

Fig. 4. Elevation changes of the Grímsvötn and Gjálp areas for the 1998-2004 period. The differential DEM is obtained by subtracting the 1998 EMISAR DEM from the 2004 SPOT5 DEM. Positive values (red) correspond to an uplift (e.g., Grímsvötn ice shelf) or an ice thickening (e.g., the Gjálp area). Gray areas correspond to gap either in the EMISAR or in the SPOT5 DEM. The dashed black rectangle locates the area covered by Figure 5 .

racy of the $1998 \mathrm{DEM}$ is 2 to $3 \mathrm{~m}$ over the glacier surface. The differential DEM provides a measurement of the long term elevation change of the Grímsvötn ice shelf. The ice shelf was $55 \mathrm{~m}$ higher in 2004 than in 1998, albeit several drainage events during the 6 years (see the upper panel in Figure 2). Our map shows glacier thickening of 30 to $40 \mathrm{~m}$ in the northern Grímsvötn and Gjálp areas due to inflow of ice to the depression created by the Gjálp eruption in 1996 (24). Farther from the depression, thinning of the glacier is detected. This inflow has been monitored and modelled with $\operatorname{InSAR}(9 ; 25)$. 
Using the 2004 DEM and following the methodology presented by Berthier et al. (22), we coregister and correlate the satellite images acquired in August. From homologous points extracted automatically on the stable (off glacier) area, the 16 August image is resampled in the geometry of the 11 August image. The area covered by both SPOT5 images (Figure 1) contains very few stationnary points. The homologous points are extracted far away from our area of interest, which slightly degrades the coregistration of the two images.

The correlation of the 11 August image with the resampled 16 August image is performed every 10 pixels using windows of 21 by 21 pixels. Conversion of offsets in image geometry to ground displacements takes into account the varying pixel size across the image. Noise in the displacement maps is due to noise in the DEM and changes at the glacier surface. Around the Grímsvötn ice shelf, the mean offsets in the image line direction are small $(0.1$ pixel) and slightly positive (not shown). They indicate a mean southward velocity of $14 \mathrm{~m} / \mathrm{a}$, slightly smaller than the $20 \mathrm{~m} / \mathrm{a}$ measured at the ground-based DGPS station. Offsets in the column direction have to be corrected for a long wavelength bias. We attribute this bias to the difficulty of co-registering the two satellite images from homologous points located on the edges of the images, far away from our area of interest. This bias could also result from errors in the DEM. Because we are only interested in the relative displacement of the floating ice shelf compared to its surroundings, we account for this bias by removing from the column offset field a first-order polynomial which approximates the column offsets outside the ice shelf (17). The displacements in the column direction are then converted into vertical displacement using Equation 6. The result maps the uplift of the ice shelf between 11 and 16 August (Figure 5).

This map delimits the extent of the uplift zone.
The boundaries are displayed on the SPOT5 images in the left panel of Figure 5. The area affected by the uplift covers $10.9 \mathrm{~km}^{2}\left( \pm 1 \mathrm{~km}^{2}\right)$. The uncertainties concern the southeast part of the ice shelf where errors in the DEM (due to the shadows of the $300 \mathrm{~m}$ high caldera walls) create a bias in the uplift map. Over this area, the mean uplift is $1.71 \mathrm{~m}$ with a standard deviation of $\pm 0.6 \mathrm{~m}$. This standard deviation includes both measurement uncertainty and uplift variability over the ice shelf. The mean uplift corresponds to a displacement in the column direction of 0.3 pixels. This signal is only slightly larger than the (conservative) accuracy of \pm 0.2 pixels found in a previous application of our technique (22). This low signal-to-noise ratio explains the noise in the uplift map and the uncertainty in delineating the extent of the uplift zone. The main source of uncertainty in our map is the errors in the DEM. Errors could also originate from subtracting the first-order polynomial, neglecting horizontal ice flow in longitude $\left(\delta_{\lambda}\right)$ or from the biases introduced by the image correlation itself.

A permanent survey station located on the ice shelf (named MAST) provides two groundbased elevation measurements. A standard GPS receiver measures the elevation every $10 \mathrm{~min}$ utes. The error of an individual measurement is \pm 5 to $20 \mathrm{~m}$. Comparison with DGPS measurements indicates an uncertainty of $\pm 2 \mathrm{~m}$ for the daily average. Another estimate is provided by standard barometric altimetry between one station on the ice shelf (MAST) and another on the mountain Grímsfjall, $3 \mathrm{~km}$ south-east of MAST (Figure 5). Barometric pressure and temperature are recorded every hour at both stations. The sites are affected by high winds, and inverse temperature gradients. The uncertainty in this estimation of the elevation difference between the two stations is therefore high, \pm 2 to $5 \mathrm{~m}$ depending on atmospheric conditions. 

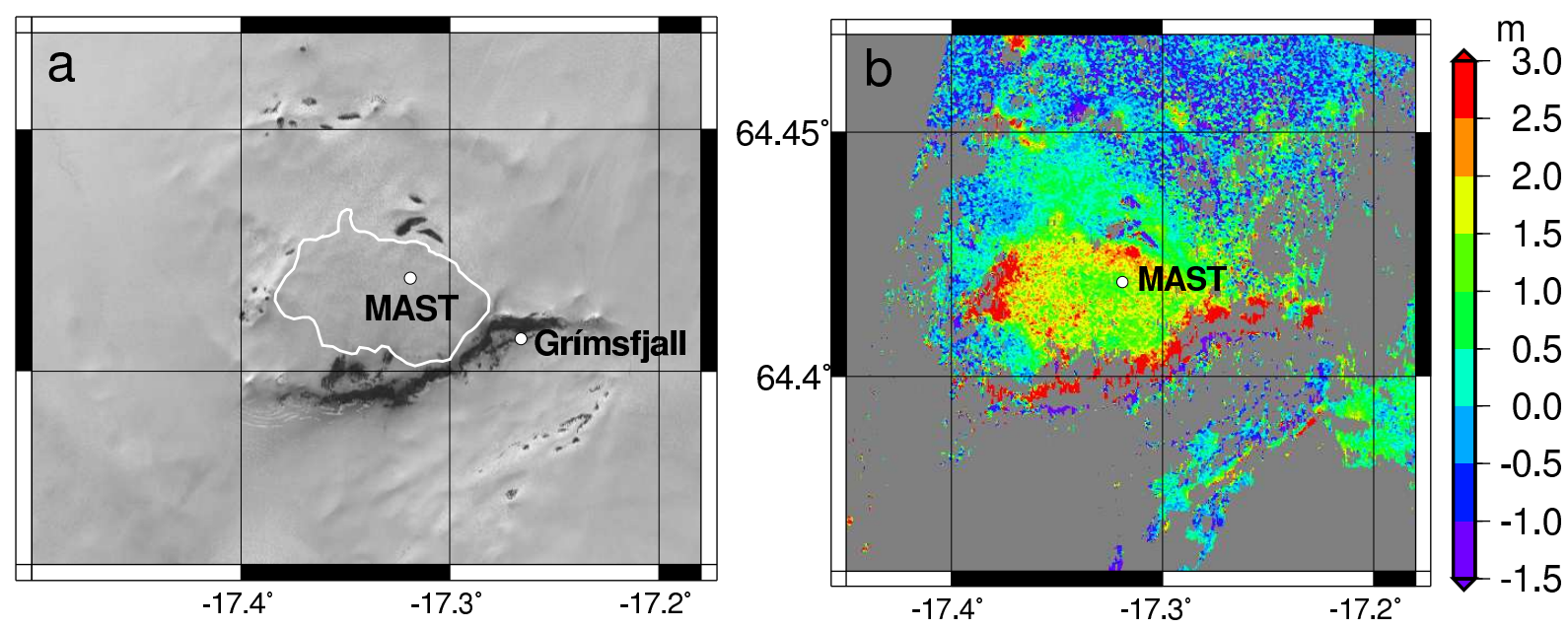

Fig. 5. (a) SPOT5 image of the Grímsvötn caldera acquired the 16 August 2004. The extent of the subglacial lake (white line) has been derived from the map of the uplift. The GPS station (MAST) on the floating ice shelf and the Grímsfjall mountain are located. (b) Map of the uplift of the Grímvötn ice shelf between the 11 and 16 August 2004. In grey, pixels were the correlation failed.

Table 2

Comparison of the uplift rates (m/day) at MAST for different time intervals with the $0.23 \pm 0.1 \mathrm{~m} /$ day derived from SPOT5 images for 11-16 August. The uncertainty on the difference of two rates is $\sigma_{x-y}=\sqrt{\sigma_{x}^{2}+\sigma_{y}^{2}}$ because the compared rates are all independant. At the 66\% confidence interval (respectively 95\%), two uplift rates are not significantly different when their difference is smaller then $\sigma_{x-y}$ (respectively $2 * \sigma_{x-y}$ ). These cases appear in bold.

\begin{tabular}{lcccc}
\hline & 11-16 Aug. & 6-21 Aug. & 1-26 Aug. & 27 Jul.-31 Aug. \\
\hline$\delta \mathrm{t}$ (days) & 5 & 15 & 25 & 35 \\
\hline Baro & $0.78 \pm 0.32$ & $0.24 \pm 0.06$ & $0.19 \pm 0.03$ & $0.19 \pm 0.02$ \\
\hline GPS & $-0.25 \pm 0.6$ & $-0.02 \pm 0.1$ & $0.05 \pm 0.05$ & $0.18 \pm 0.03$ \\
\hline Baro - GPS & $1.03 \pm 0.68$ & $0.26 \pm 0.12$ & $0.14 \pm 0.06$ & $\mathbf{0 . 0 1} \pm \mathbf{0 . 0 3}$ \\
\hline SPOT5 - Baro & $-0.55 \pm 0.33$ & $\mathbf{0 . 0 1} \pm \mathbf{0 . 1 2}$ & $\mathbf{0 . 0 4} \pm \mathbf{0 . 1}$ & $\mathbf{0 . 0 3} \pm \mathbf{0 . 1}$ \\
\hline SPOT5 - GPS & $0.48 \pm 0.61$ & $0.25 \pm 0.14$ & $0.18 \pm 0.11$ & $\mathbf{0 . 0 5} \pm \mathbf{0 . 1}$ \\
\hline
\end{tabular}


In Table 2, we compare the different uplift rates and determine if their difference is statiscally significant. Different time intervals are investigated. The difference between GPS and barometric altimetry measurements show that estimating a mean uplift rate for a short-time interval from our ground data is not robust. The two ground-based uplift rates agree only when the time interval is as much as 35 days. In this case, at the $95 \%$ confidence interval, the ground uplift rates $0.18 \pm 0.03$ and $0.19 \pm 0.02$ $\mathrm{m} /$ day are not significantly smaller than the $0.23 \pm 0.1 \mathrm{~m} /$ day obtained from satellite data. Thus, assuming a constant uplift rate over one month, a good agreement is found between satellite and ground measurements.

The satellite-derived uplift rate $(0.23 \pm 0.1 \mathrm{~m} /$ day $)$ is also in good agreement with the maximum rates measured with InSAR during the period 1996-1999 (9). The extent and the shape of the subglacial lake determined by correlating SPOT5 images agree well with the one determined previously $(9 ; 19)$.

\section{Discussion}

Our results allow a discussion of the water and energy balance of the subglacial lake. During our 5-day observation interval in August 2004, the mean lake level rose by $1.71 \mathrm{~m}$ over a $10.9 \mathrm{~km}^{2}$ area indicating that $0.018 \mathrm{~km}^{3}$ of water was added to the subglacial lake. Surface melting contributed about $0.013 \mathrm{~km}^{3}$ of water to the lake from a water drainage basin of $200 \mathrm{~km}^{2}$ with mean surface ablation of about $1.3 \mathrm{~cm}$ (water equivalent)/day (Science Institute data). The remaining $0.005 \mathrm{~km}^{3}$ is due to melting by geothermal heat of basal ice in the 50 to $60 \mathrm{~km}^{2}$ surrounding the lake $(19 ; 26)$. The power required to melt this much ice in 5 days is $4000 \mathrm{MW}$, yielding an average geothermal flux of the order of $70 \mathrm{~W} \cdot \mathrm{m}^{-2}$. These estimates of the total power and the geothermal flux are a factor of 1.5 to 2 higher than values over the period 1960-1991 (19). Although our short-term estimates are uncertain, they might suggest that basal meltwater from the Gjálp eruption site of 1996 drains to the lake, leading to an overestimate of the ice melted into the Grímsvötn area.

Assuming a vertical wall bordering the lake we can assess an upper limit of the total water volume $\left(\mathcal{V}_{\text {lake }}\right)$ contained in Grímsvötn

$\mathcal{V}_{\text {lake }}=\left(\mathcal{Z}_{\text {iceshelf }}-\mathcal{Z}_{\text {grounded }}\right) \times \mathcal{S}_{\text {SPOT5 }}$

where $\mathcal{Z}_{\text {iceshelf }}$ stands for the elevation of the ice shelf, $\mathcal{Z}_{\text {grounded }}$ the elevation of the ice shelf when it is grounded (dashed horizontal line in Figure 2) and $\mathcal{S}_{\text {SPOT5 }}$ the surface area of the lake estimated by SPOT5 correlation. $\mathcal{V}_{\text {lake }}$ amounted to $0.74 \mathrm{~km}^{3}$ in mid-August 2004.

From a methodological point of view, this study permits discussion of different ways to measure elevation changes of glacier surfaces. First, long-term elevation changes can be monitored using differential DEM (Figure 4). The 
compared DEM can be from various origins (optical satellite images, SAR images, aerial photographs) and the accuracy of the elevation change measurement will be directly controlled by the accuracy of each individual DEM. With DEM derived from SPOT satellite optical images, an accuracy of $\pm 2 \mathrm{~m}$ can be reached (27). On the other hand, we obtain a better accuracy by correlating SPOT5 images acquired with similar, large incidence angles. The similar viewpoint limits the impact of topographic errors, whereas the large incidence angles increase the sensitivity to the vertical motion occurring between the two acquisition dates.

The main limitation of this new methodology is the need for good correlation between the two images which requires limited changes of the surface features. Over glacier surfaces, good correlations have been reported in the lower ablation zone for summer images separated by as much as one year (15). In the upper accumulation zone, typically, correlation breaks down if the images are separated by more than a few weeks due to snowdrift and snowfall. For example, over the Grímsvötn ice shelf, no results could be obtained by correlating the 11 August image with another SPOT5 image acquired 6 weeks later, on 26 September.

In our case, the slightly different incidence angles of the correlated images implied the use of a precise contemporous DEM. In other cases, this solution may not be possible. If the correlation remains high after at least 26 days (one orbital cycle of SPOT5), this disadvantage could be avoided by using images acquired from the same viewpoint. In such a case, even a very coarse DEM such as GTOPO-30 would suffice.

This technique could also provide a full 3dimensional description of the displacement field. By correlating two optical images, we measure two of the three components of the displacement vector. Equations 3 and 4 consti- tute thus a system of two equations with three unknowns $\left[\delta_{\lambda}, \delta_{\phi}, \delta_{z}\right]$. In this study, the system could be solved because $\delta_{\lambda}$ can be neglected. In general, an independent measurement of at least one non-parallel component of the same displacement field is required to solve the system (e.g. Gray et al. (10)). This additional measurement could be provided by InSAR, but the SAR images need to span the same time interval and this technique is not applicable to temperate glaciers. We propose in Figure 6 an optical satellite configuration that would increase the sensitivity to vertical motion and resolve the $3 \mathrm{D}$ component of the surface motion. The image correlation is applied to two image pairs acquired from front and back looking position of the satellite. Some sensors, like HRS on board the SPOT5 satellite, could perform this measurement but, to our knowledge, their raw data are not available to the public. The ASTER sensor on board TERRA also presents some stereoscopic capabilities but its resolution and orbital control are not good enough to monitor vertical motion rarely exceeding a few meters.

In this satellite configuration, the displacements in lines and columns are measured twice from two different viewpoints. Four measurements are available to determine the three components of the displacement vector; no assumption on the horizontal flow is needed.

In the case of purely vertical motion, the vertical displacement is given by

$\delta h=\frac{\delta \operatorname{lin}_{\text {front }}-\delta \operatorname{lin}_{\text {back }}}{2 \sin \theta}$

If the incidence angle ( $\theta$ in Figure 6$)$ is $20^{\circ}$ (as for the HRS system), $70 \%$ of the vertical displacement would be measured by the image correlation versus $45 \%$ for our measurement of the Grímsvötn ice shelf uplift. 


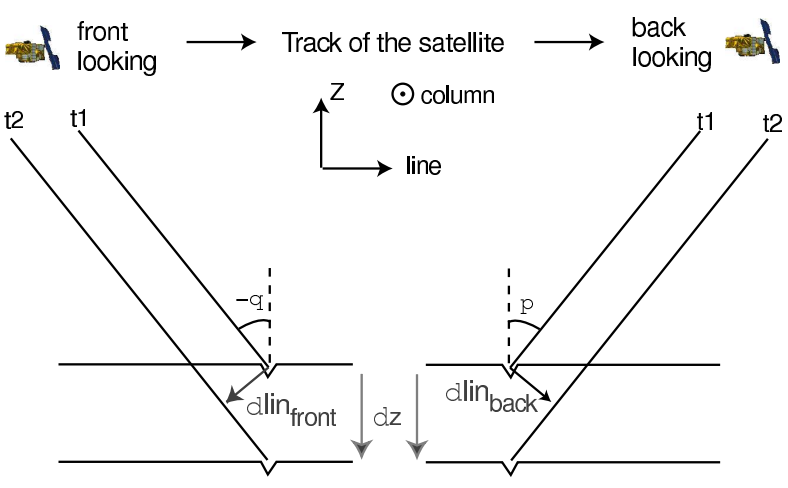

Fig. 6. A potential satellite configuration for mapping the three-dimensional displacement field of the Earth's surface. We assume an along-track stereo capability similar to the HRS system on-board the SPOT5 satellite. During the first pass of the satellite $\left(\mathrm{t}_{1}\right)$, two images are acquired along the track of the satellite from fore- and aft- looking sensors. One orbital cycle later during the second pass $\left(\mathrm{t}_{2}\right)$, two more scenes are acquired. By correlating the images acquired from a similar point of view, one can deduce all three components of the displacement field (here the displacement of a crevasse on the surface of a glacier). Note that this figure is drawn in a vertical plane that contains the track of the satellite and lies perpendicular to the plane used in Figure 3 because we consider the case of a sensor with along-track stereo capabilities (such as ASTER or HRS or HRS-C on-board Mars Express (29)). The results presented in this article are based on the HRG sensor on board SPOT5 with across-track stereo capabilities.

\section{Conclusions}

We have measured ice rising at an average rate of $23 \mathrm{~cm} /$ day above a subglacial lake in southeast Iceland. We conclude that correlation of SPOT5 images is a useful method to observe vertical motions of glaciers. Application of the technique has given motivating results for studies of the mass and energy balance of a subglacial lake over a geothermal area. The technique has the potential to detect new areas of subglacial geothermal activity. Such areas are important for the assessment of natural hazards in volcanic regions. As a supplement to others technique (10), correlation of satellite optical images may contribute to regional studies of subglacial hydrology and its relation to glacier dynamics.

The accuracy of the measurement cannot be definitively assessed here due to inaccurate ground-based measurements but appears to be of the order of $\pm 0.5 \mathrm{~m}$, one fifth of the pixel size. This accuracy is mainly controlled by the ground resolution and remains one order of magnitude worse than that obtained with SAR interferometry. Better accuracy could be obtained in the near future thanks to the sub-meter ground resolution planned for satellite missions following the SPOT5 program. The main limitation of our technique is the difficulty of obtaining two cloud-free images from a similar point of view. Our scalar measurement of the Grímsvötn elevation changes is based on one pair of images. If two image pairs from a satellite with along-track stereo capabilities were available, it would be possible be to solve for the 3-dimensional vector displacements.

\section{Acknowledgements}

Hélène Vadon provided useful help concerning the SPOT5 geometry. We thank Jørgen Dall (EMI, Technical University of Denmark) and Eyjólfur Magnússon for processing and providing the EMISAR 1998 DEM. SPOT5 images were acquired thanks to the ISIS program (copyright CNES). This work was supported by the French embassy in Iceland, the University of Iceland Research Fund, the Science Fund of Iceland (Rannís) and the Icelandic Road Authority Research Fund. GMT software (28) illustrated the article. The first author gratefully acknowledges a thesis fellowship from the French Government. 


\section{References}

[1] A. Iken, H. Röthlisberger, A. Flotron, W. Haeberli, The uplift of Unteraargletscher at the beginning of the melt season - a consequence of water storage at the bed?, J. Glaciol. 29 (101) (1983) 28-47.

[2] B. Kamb, H. Engelhardt, Waves of accelerated motion in a glacier approaching surge: The mini-surges of Variegated glacier, Alaska, U.S.A., J. Glaciol. 33 (113) (1987) 27-46.

[3] W. D. Harrison, K. A. Echelmeyer, H. Engelhardt, Short-period observations of speed, strain and seismicity on Ice Stream B, Antarctica., J. Glaciol. 39 (133) (1993) 463-470.

[4] H. Björnsson, Subglacial lakes and jökulhlaups in Iceland, Global Planet. Change 35 (2002) 255-271.

[5] K. A. Echelmeyer, W. D. Harrison, C. F. Larsen, J. Sapiano, J. E. Mitchell, J. DeMallie, B. Rabus, G. Adalgeirsdòttir, L. Sombardier, Airborne surface profiling of glaciers: a case-study in Alaska, J. Glaciol. 42 (142) (1996) 538-547.

[6] F. Rémy, P. Shaeffer, B. Legrésy, Ice flow physical processes derived from the ERS1 high-resolution map of the Antarctica and Greenland ice sheets, Geophys. J. Int. 139 (3) (1999) 645-656.

[7] J. Zwally, B. Schutz, et al., ICESat's laser measurements of polar ice, atmosphere, ocean, and land, J. Geodyn. 34 (2002) 405-445.

[8] D. Massonnet, K. Feigl, Radar interferometry and its application to changes in the Earth's surface, Rev. Geophys. 36 (4) (1998) 441-500.

[9] H. Björnsson, H. Rott, S. Gudmundsson, A. Fischer, A. Siegel, M. Gudmundsson, Glacier-volcano interactions deduced by SAR interferometry, J. Glaciol. 47 (156) (2001) 58-70.

[10] A. L. Gray, I. Joughin, S. Tulaczyk, V. B.
Spikes, R. Bindschadler, K. C. Jezek, Evidence for subglacial water transport in the West Antarctic Ice Sheet through three-dimensional satellite radar interferometry, Geophys. Res. Lett. 32 (L03501), doi:10.1029/2004GL021387.

[11] T. Strozzi, A. Luckman, T. Murray, U. Wegmller, C. Werner, Glacier motion estimation using SAR offset-tracking procedures, IEEE T. Geosci. Remote 40 (11) (2002) 2384-2391.

[12] I. Joughin, Ice-sheet velocity mapping: A combined interferometric and speckle tracking approach, Ann. Glaciol. 34 (2002) 195-201.

[13] A. Fischer, H. Rott, H. Björnsson, Observation of recent surges of Vatnajökull, Iceland, by means of ERS SAR interferometry, Ann. Glaciol. 37 (2003) 69-76.

[14] T. A. Scambos, M. J. Dutkiewicz, J. C. Wilsoni, R. A. Bindschadler, Application of image cross-correlation to the measurement of glacier velocity using satellite image data, Remote Sensing Environ. 42 (3) (1992) 177-186.

[15] A. Kääb, Combination of SRTM3 and repeat ASTER data for deriving alpine glacier flow velocities in the Bhutan Himalaya, Remote Sensing Environ. 94 (4) (2005) 463-474.

[16] N. Van Puymbroeck, R. Michel, R. Binet, J. P. Avouac, J. Taboury, Measuring earthquakes from optical satellite images, Appl. Opt. 39 (20) (2000) 3486-3494.

[17] H. Vadon, D. Massonnet, Earthquake displacement fields mapped by very precise correlation: complementary with radar interferometry, in: I. Periodicals (Ed.), IEEE International Geoscience Remote Sensing Symposium, New Jersey, 2000, pp. 27002702.

[18] C. Delacourt, P. Allemand, B. Casson, H. Vadon, Velocity field of the "La Clapière" landslide measured by the correlation of aerial and QuickBird satellite im- 
ages, Geophys. Res. Lett. 31 (15) (2004) L15619.

[19] H. Björnsson, T. M. Gudmundsson, Variations in the thermal output of the subglacial Grimsvtön caldera, Iceland, Geophys. Res. Lett. 20 (19) (1993) 21272130.

[20] G. Larsen, M. T. Gudmundsson, H. Björnsson, Eight centuries of periodic volcanism at the center of the Iceland hot spot revealed by glacier tephrastratigraphy, Geology 26 (10) (1998) 943-946.

[21] F. Sigmundsson, E. Sturkell, V. Pinel, P. Einarsson, R. Pedersen, H. Geirsson, M. T. Gudmundsson, H. Bjornsson, C. Pagli, Deformation and eruption forecasting at volcanoes under retreating ice caps: discriminating signs of magma inflow and ice unloading at Grimsvotn and Katla volcanoes, EOS Trans. AGU 85 (47), fall Meet. Suppl.

[22] E. Berthier, H. Vadon, D. Baratoux, Y. Arnaud, C. Vincent, K. L. Feigl, F. Rémy, B. Legrésy, Mountain glacier surface motion derived from satellite optical imagery, Remote Sensing Environ. 95 (1) (2005) 14-28.

[23] E. Magnússon, H. Björnsson, F. Pálsson, J. Dall, Glaciological application of InSAR topography data of W-Vatnajökull acquired in 1998, Jökull 54 (2004) 17-36.

[24] M. T. Gudmunsson, F. Sigmundsson, $\mathrm{H}$. Björnsson, Ice-volcano interaction of the 1996 Gjálp subglacial eruption, Vatnajökull, Iceland, Nature 389 (1997) 954957.

[25] E. Magnússon, H. Björnsson, J. Dall, F. Pálsson, Volume changes of Vatnajökull ice cap, Iceland, due to surface mass balance, ice flow, and sub-glacial melting at geothermal areas, Geophys. Res. Lett. 32 (5) (2005) L05504.

[26] H. Björnsson, Hydrology of ice caps in volcanic regions, 45, Soc. Sci. Isl., Reykjavík, 1988.
[27] E. Berthier, Y. Arnaud, D. Baratoux, C. Vincent, F. Rémy, Recent rapid thinning of the "Mer de glace" glacier derived from satellite optical images, Geophys. Res. Lett. 31 (17) (2004) L17401.

[28] P. Wessel, W. H. F. Smith, New, improved version of generic mapping tools released, EOS T. Am. Geophy. Un. 79 (47) (1998) 579.

[29] G. Neukum, R. Jaumann, the HRSC Co-Investigator, the Experiment Team, HRSC: the High Resolution Stereo Camera of Mars Express, ESA Special Paper 1240, available at: http://www.esa.int/esapub/pi/scientificPI.htm. 



\section{Annexe E}

\section{"ME DECOIS PAS" \& déformation co-sismique}

L'étude du cycle sismique requiert des mesures précises des déformations de la surface terrestre. Ces déformations sont de quelques millimètres ou centimètres par an durant la phase inter-sismique. Le GPS différentiel est l'instrument le plus adapté à l'estimation de ces très faibles mouvements. Les déplacements induits par le séisme lui même (dits co-sismiques) sont décimétriques à métriques. L'InSAR est la technique de prédilection pour les cartographier (Massonnet \& Feigl, 1998). Elle montre toutefois ces limites au voisinage de la faille où le gradient de déformation est trop important. C'est dans cette zone que la corrélation d'images est complémentaire.

La corrélation d'images SPOT a donc été appliquée a plusieurs reprises à la mesure de la déformation co-sismique. Elle localise précisément la rupture de surface (si elle se produit) et mesure le jeu le long de cette rupture. Les séismes d'Izmit en Turquie (Vadon \& Massonnet, 2000; Van Puymbroeck et al., 2000; Michel \& Avouac, 2002; Feigl et al., 2002), de Chi Chi à Taïwan (Dominguez et al., 2003), de Kokoxili en Chine (Klinger et al., 2004) et de Bam en Iran (Binet \& Bollinger, 2005) ont été observés par cette méthode.

Le séisme d'Izmit, parce qu'il a déjà été bien étudié par cette technique, nous a servi de site test pour adapter notre chaîne de traitement. Nous verrons que nos résultats sont cohérents avec les travaux précédents et qu'ils permettent d'affiner l'estimation des paramètres d'un modèle simple de séisme. Ce modèle amélioré est comparé à des mesures indépendantes par GPS décrites par ailleurs (Reilinger \& Co-auteurs, 2000; Feigl et al., 2002).

Le séisme d'Al-Hoceima au Maroc est beaucoup plus récent et moins bien documenté. Ce séisme a engendré des déformations beaucoup plus faibles (décimétriques). L'objectif de la corrélation d'images SPOT5 est de déterminer si la rupture a atteint la surface. Répondre à cette question est important pour contraindre un modèle de faille fondé essentiellement sur des données InSAR.

\section{E.1 Séisme d'Izmit : déformation co-sismique}

Le séisme d'Izmit s'est produit le 17 août 1999. De magnitude 7.4, il est lié à la rupture d'un segment de près de $120 \mathrm{~km}$ de long de la faille Nord Anatolienne. L'épicentre se situe près de 
la ville d'Izmit, située entre la Mer de Marmara à l'ouest et le lac Sapanca à l'Est (figure E.1). C'est d'ailleurs cette zone uniquement qui est incluse dans les deux images SPOT du 9 juillet et du 16 septembre 1999 que nous utilisons. Le rapport B/H pour ce couple d'image est faible, voisin de 0.01 .

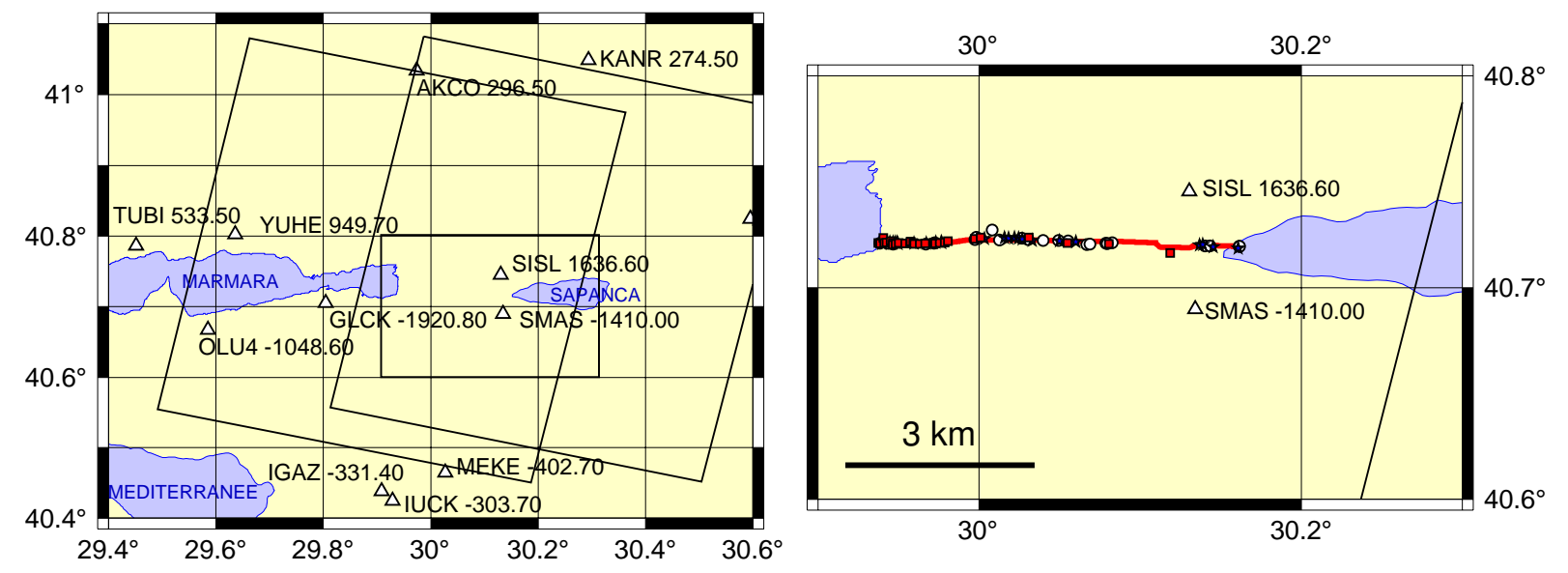

FIG. E.1 - A gauche : Localisation des images SPOT1-4 dont la partie commune se limite à la région entre la Mer de Marmara et le Lac Sapanca. Les triangles indiquent les mesures GPS (en millimètres) de la déformation co-sismique. A droite : détail de la zone de faille. Les lieux où la rupture de surface a été cartographiée sont localisés (les différents figurés correspondent à différents auteurs) ainsi que sa position (trait rouge) déduite de la corrélation des images SPOT.

\section{E.1.1 Particularités méthodologiques}

Par rapport aux glaciers, pour lesquels des zones stables sont facilement identifiables afin d'extraire des points homologues, un séisme de forte magnitude va déformer l'ensemble d'une scène SPOT. Pour remédier à cette difficulté, lors de l'extraction de ces points homologues, un modèle a priori de déformation induite par la faille est utilisé. C'est la principale originalité de notre étude sur Izmit.

La déformation (nommée par la suite a priori et notée $\mathrm{U}$ en mètres) a une distance $\mathrm{X}(\mathrm{km})$ de la faille est approchée par la relation :

$$
U=\frac{S}{2}\left[1 \times \operatorname{signe}(X)-\frac{2}{\pi} \arctan \left(\frac{X}{D}\right)\right]
$$

où S (mètres) désigne le glissement (Slip en anglais) le long de la faille et $\mathrm{D}$ (Depth) la profondeur $(\mathrm{km}) \mathrm{du}$ foyer du séisme. Il existe une discontinuité en $\mathrm{X}=0$ tel que $\mathrm{u}_{X \rightarrow 0^{+}}-\mathrm{u}_{X \rightarrow 0^{-}}=\mathrm{S}$. Les paramètres choisis sont (Feigl, communication personnelle) : $\mathrm{S}=3 \mathrm{~m}$ et $\mathrm{D}=25 \mathrm{~km}$ (figure E.2). Pour appliquer un tel modèle, la géométrie de la faille doit être connue. Nous la déduisons d'une première corrélation des images SPOT utilisant des points homologues extraits uniquement dans la partie Nord des images.

Les points homologues sont donc extraits par corrélation des deux images (comme pour les glaciers) mais leurs coordonnées dans l'image secondaire (post-sismique) sont corrigées des décalages induits par la faille (tels que modélisés par l'équation E.1). Ces points permettent alors une orientation relative précise des deux images. 

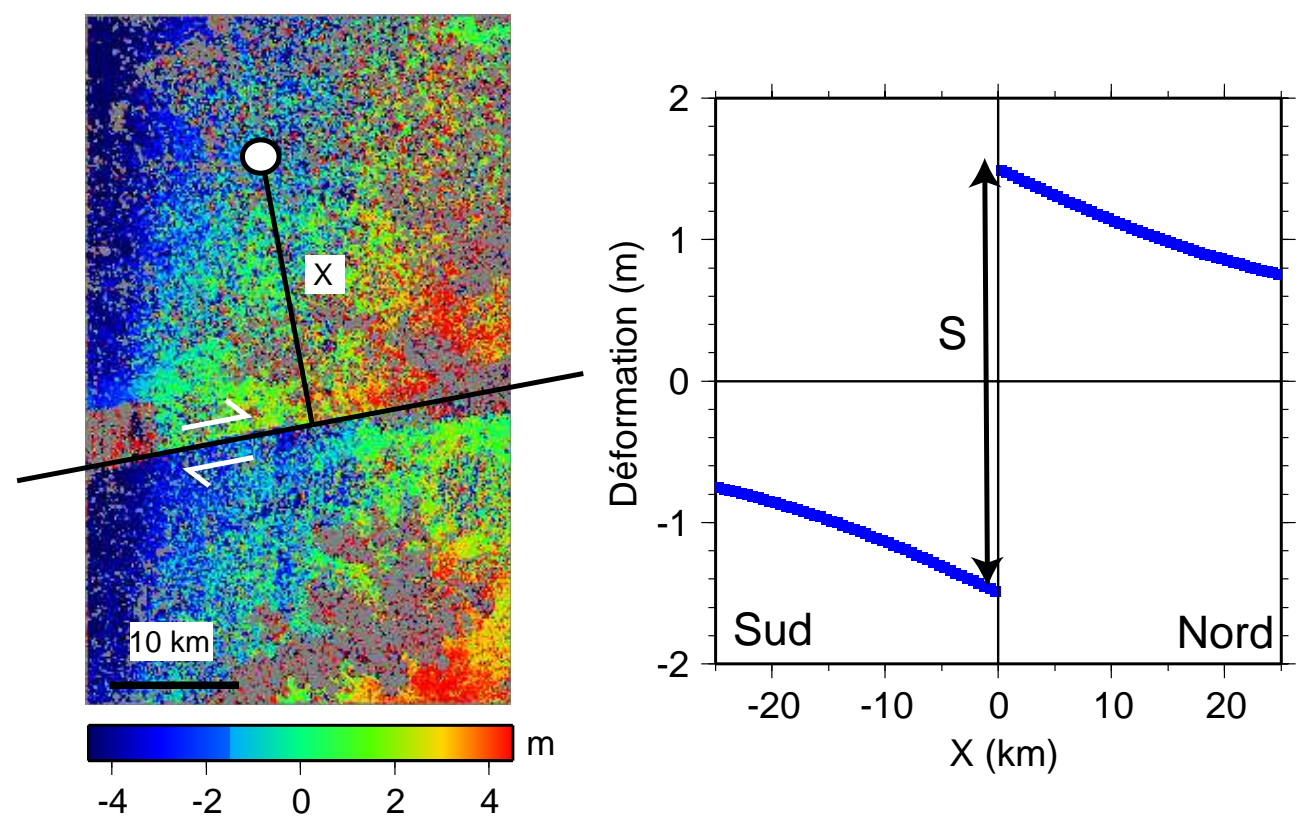

FIG. E.2 - A gauche : définition de la distance $X(\mathrm{~km})$ à la faille. Une première corrélation des images SPOT indique que la faille peut être assimilée à une droite. A droite : modèle a priori de déformation en fonction de la distance à la faille. S désigne le glissement le long de la faille.

Après ré-échantillonnage de l'image secondaire puis corrélation, il reste deux types de biais à corriger dans le champ de déformation (figure E.3) :

- Un biais à grande longueur d'onde qui avait déjà été noté par Vadon \& Massonnet (2000). Ce biais est probablement lié à un effet orbital : les orbites de SPOT1-4 sont moins bien connues que celle de SPOT5. Il est modélisé par un polynôme de $1^{e r}$ ordre après avoir soustrait la déformation a priori de la faille.

- des biais liés au système optique de SPOT1-4 et qui se traduisent par des sauts brutaux dans les cartes de décalage aux limites des inter-barrettes (Van Puymbroeck et al., 2000). Ces biais sont corrigés en estimant l'amplitude de chacun des sauts.

Après élimination des différents biais, on ajoute à la carte finale des résidus (notée RESIDU3 sur la figure E.3) le modèle de déformation a priori pour obtenir la carte des déformations présentée en figure E.4. 

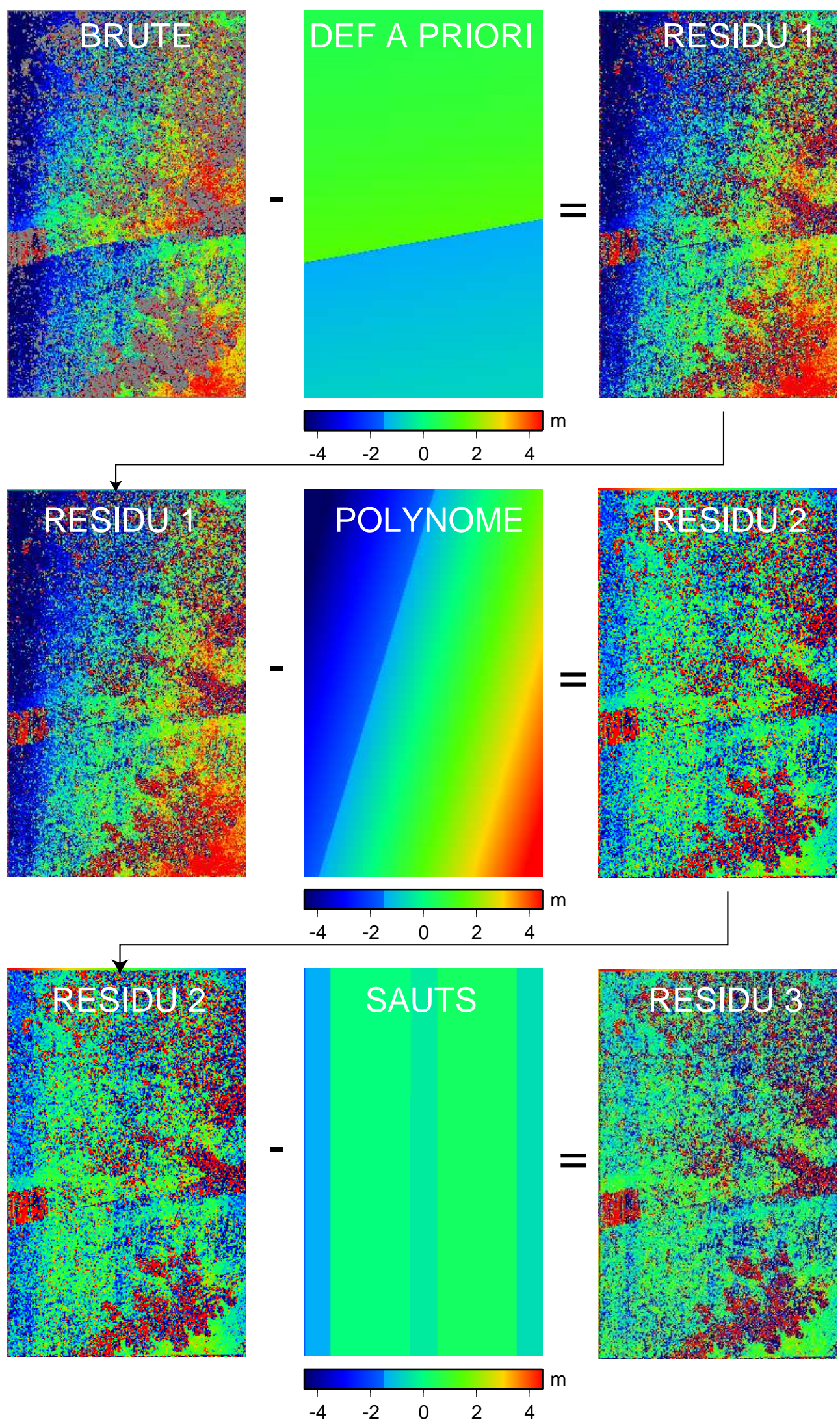

FIG. E.3 - Suite des étapes permettant la correction des différents biais dans les décalages en longitude. L'échelle de couleur est la même pour toutes les cartes. 


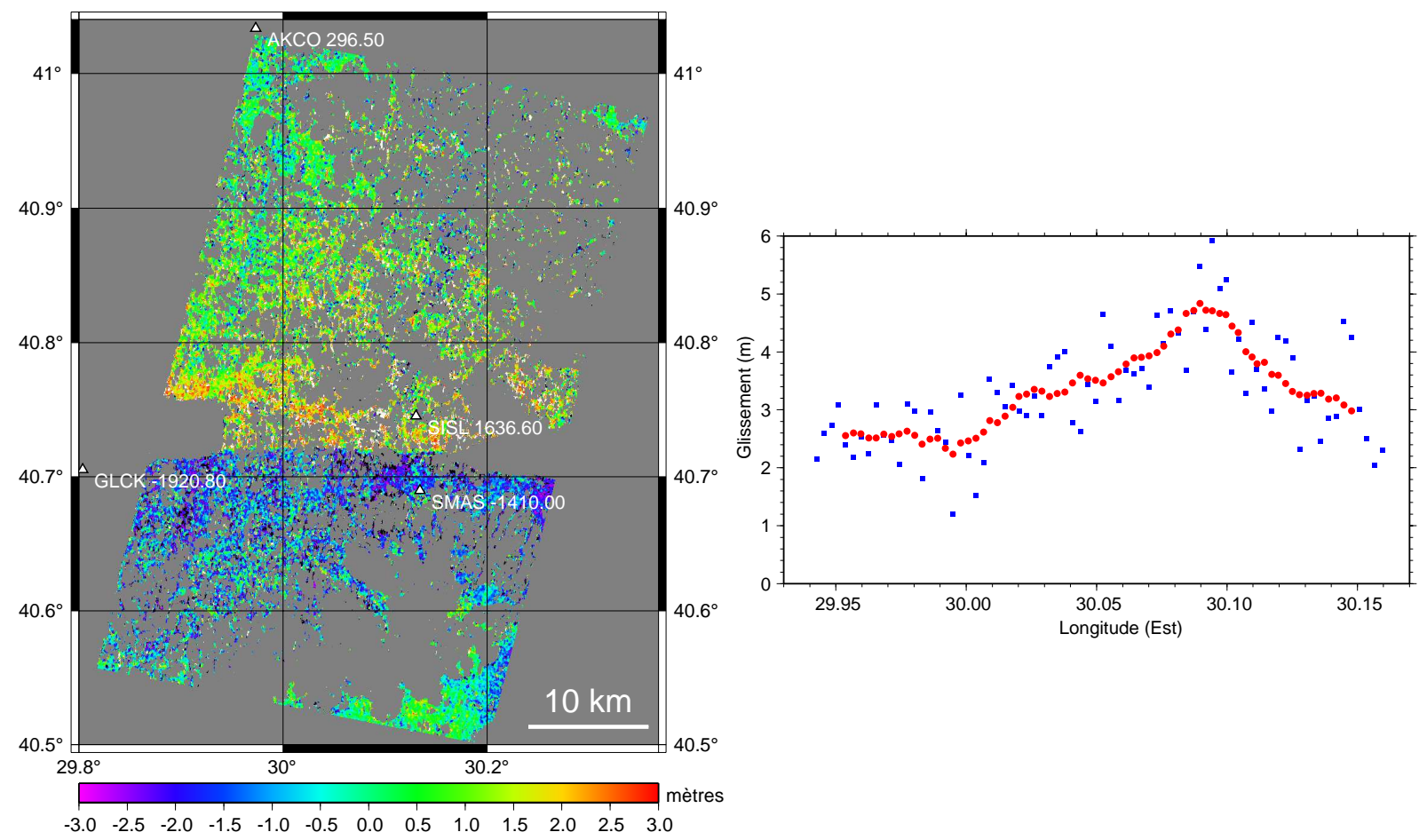

Fig. E.4 - A gauche : carte des déformations en longitude induites par le séisme d'Izmit. Les déplacements (en millimètres) mesurés à des stations GPS ont été reportés. En gris les zones où la corrélation a échoué. A droite : valeur du glissement à la surface le long du segment de faille entre la Mer de Marmara et le lac Sapanca. Le glissement est estimé tous les $300 \mathrm{~m}$ le long de la faille (carrés bleus). Les ronds rouges correspondent à une moyenne mobile sur $3 \mathrm{~km}$.

\section{E.1.2 Cartographie de la rupture de surface et valeur du glissement}

Parce que la faille d'Izmit est orientée Est-Ouest et a surtout un jeu décrochant, la déformation est essentiellement dans la direction des longitudes. C'est donc cette carte (figure E.4) que l'on utilise pour localiser la faille. Pour tester la qualité de cette localisation, on mesure la distance entre la faille déduite de la corrélation SPOT et les mesures in situ de la rupture (figure E.1). Pour 73 points, l'écart moyen est de $12 \mathrm{~m}$ (écart-type $=76 \mathrm{~m}$ ). Ceci démontre la bonne précision de la cartographie obtenue par corrélation.

Le glissement le long de cette faille est déterminé en moyennant des profils perpendiculaires à la faille. Il est en moyenne de $3.5 \mathrm{~m}$ (en accord avec les résultat de Michel \& Avouac (2002) pour cette portion de faille) et n'est pas identique tout le long du segment (figure E.4 à droite).

\section{E.1.3 Amélioration du modèle de rupture de surface}

Lorsqu'on compare les résultats de la corrélation SPOT avec le modèle a priori de déformation, des écarts importants apparaissent (figure E.5). Les 2 paramètres de ce modèle (D, profondeur du séisme et $\mathrm{S}$, glissement) sont donc ajustés pour minimiser la différence entre les déformations modélisées et celles mesurées par SPOT. Une inversion nous conduit aux valeurs $\mathrm{D}=9.5 \mathrm{~km}$ et $\mathrm{S}=3.6 \mathrm{~m}$. 
Le modèle amélioré s'avère en très bon accord avec des mesures indépendantes de la déformation co-sismique obtenues par GPS. Lorsqu'on le compare aux 4 mesures GPS dans la région étudiée, le biais du modèle amélioré est de seulement $0.03 \mathrm{~m}$ avec un écart-type de $0.09 \mathrm{~m}$.

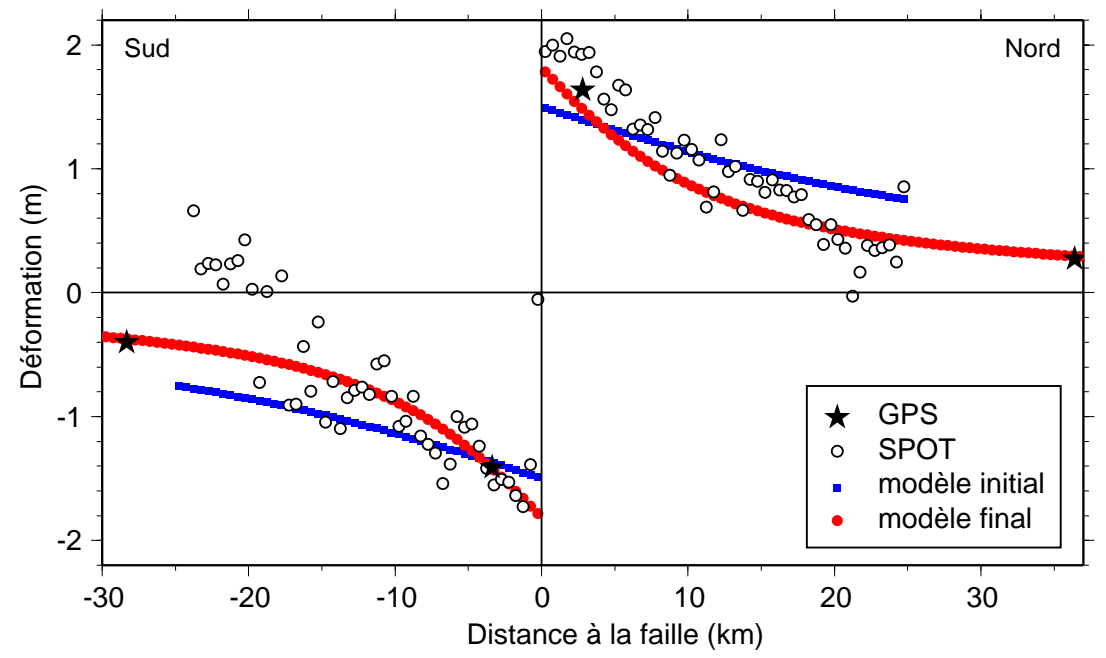

FIG. E.5 - Déformation en fonction de la distance à la faille pour l'ensemble du bloc entre la Mer de Marmara et le bloc Sapanca. On compare les mesures SPOT et GPS, aux déformations modélisées a priori et après ajustement des paramètres du modèle. Les déformations positives dans les données SPOT vers -20 km sont probablement un artefact de la mesure puisque de tels valeurs n'existent pas dans les données GPS.

Nos résultats confirment que la corrélation d'images est un outil adapté à la mesure de la déformation co-sismique en particulier au voisinage de la rupture. Une comparaison systématique à des mesures in situ montre que la localisation de la faille n'est presque pas biaisé et précise à \pm 150 m (intervalle de confiance à $90 \%$ ). Le champ de déformation déduit de la corrélation peut être utilisé pour contraindre un modèle de faille cherchant les valeurs optimales des paramètres sources du séisme. Ce travail devrait être poursuivi dans les mois à venir (Feigl et al., en preparation).

\section{E.2 Séisme d'Al-Hoceima : rupture de surface?}

Le séisme d'Al-Hoceima ( $\mathrm{Mw}=6.4)$ s'est produit le 24 février 2004 dans le Nord du Maroc (figure E.6). Une description du séisme et de ces effets pourra être trouvée dans Jabour et al. (2004). Les travaux présentés ici, utilisant la corrélation d'images SPOT5, s'intègrent dans un plus vaste chantier dans lequel diverses méthodes géodésiques (GPS, InSAR, corrélation d’images) sont combinées pour mieux caractériser la déformation inter- et co- sismique dans cette région. Ce travail, au centre de la thèse d'Abdelilah TAHAYT, devrait se conclure par deux articles (Fadil et al., en preparation; Tahayt et al., en preparation).

Ce séisme est moins bien documenté que celui d'Izmit. Ainsi, il existe des incertitudes fortes sur la localisation du foyer du séisme. Les campagnes de terrain n'ont pas détecté de rupture primaire de la surface terrestre et ne permettent pas de trancher facilement sur la direction de la faille (problème du choix d'un des 2 plans nodals du mécanisme au foyer). Seules deux stations 
GPS ont enregistré la déformation liée au séisme. C'est l'InSAR qui caractérise le mieux la déformation co-sismique. En contraignant un modèle de rupture par les données interférométriques, Tahayt et al. (2005) proposent une faille est orientée NE-SW. Cette faille modélisée est nommée faille "InSAR". Par une méthode similaire mais en utilisant deux interférogrammes, Biggs et al. (2005) privilégient l'autre plan nodal soit une orientation NW-SE. L'orientation de la faille et sa rupture ou non de la surface terrestre sont parmi les questions importantes restant ouvertes. La corrélation d'images SPOT5 apporte-t-elle des informations complémentaires?

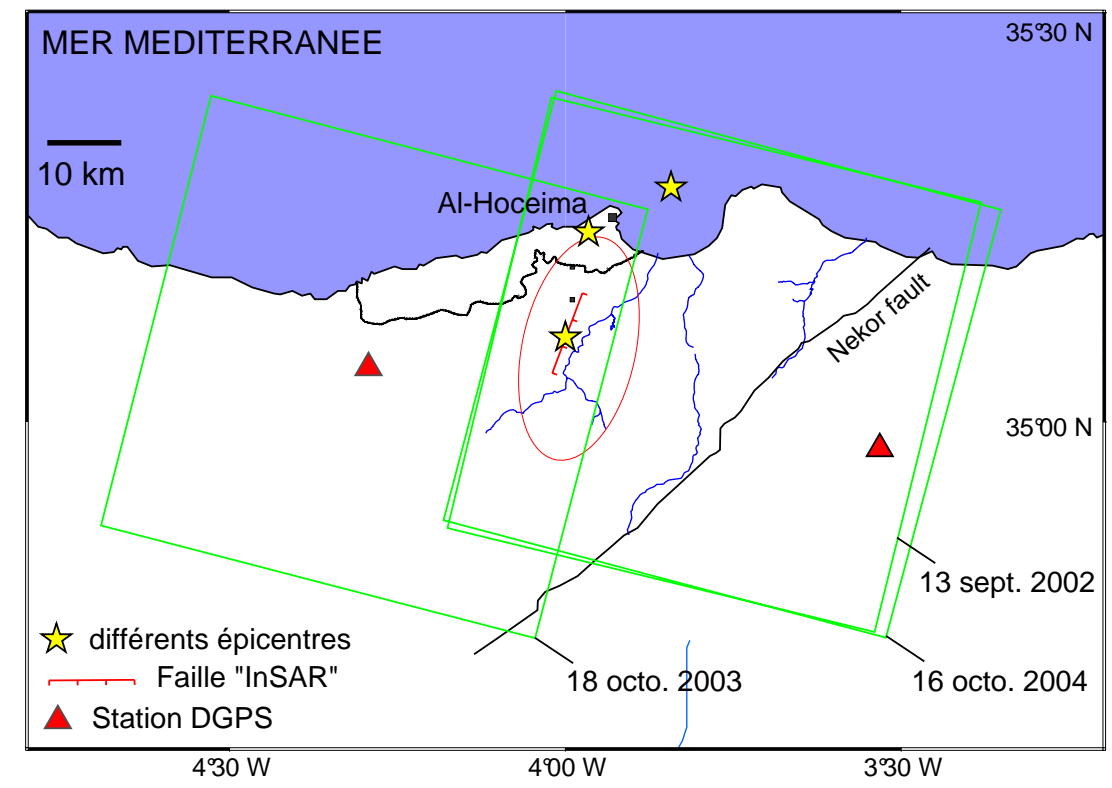

Fig. E.6 - Zone géographique où s'est produit le séisme d'Al-Hoceima. Les empreintes au sol des images SPOT sont positionnées ainsi que la faille "InSAR". Adapté d'une figure d'A. TAHAYT.

\section{E.2.1 Déformations mesurées par SPOT5}

Deux images SPOT5 pré-sismiques ont été acquises les 13 septembre 2002 et 18 octobre 2003 (tableau E.1), Une image post-sismique le 16 octobre 2004. Cette image a la même empreinte au sol que celle du 13 septembre 2002 mais est acquise d'un point de vue différent (rapport B/H de 0.24). Une erreur de $10 \mathrm{~m}$ du MNT (précision du MNT SRTM utilisé dans cette étude) entraînera une distorsion (surtout en colonnes) de $2.4 \mathrm{~m}$ entre les deux images, largement supérieure au signal recherché. Cette paire n'est donc pas adaptée à la mesure de déformations d'amplitude décimétrique.

En revanche, les images du 18 octobre 2003 et du 16 octobre 2004 sont acquises avec exactement 14 cycles orbitaux (soit 364 jours) d'écart donc du même point de vue. Ceci se traduit par un $\mathrm{B} / \mathrm{H}$ très faible ${ }^{1}$. La sensibilité à des erreurs du MNT est quasi nulle. Le fait que les images soient acquises à un an d'écart assure des conditions d'illumination quasi identiques. C'est donc ce couple qui est utilisé par la suite. Il est dommage que la région commune à ces deux images soit aussi réduite. Cela souligne la difficulté de programmer les images dans les conditions optimales.

\footnotetext{
${ }^{1}$ Si les angles d'incidence ne sont pas égaux c'est parce qu'ils correspondent au centre des images qui sont différents pour les deux scènes. Réciproquement, des angles d'incidence proches ne sont pas une condition suffisante pour que les distorsions entre les images soient faibles...
} 
TAB. E.1 - Couples d'images SPOT5 sur la zone d'Al-Hoceima.

\begin{tabular}{lcrr}
\hline Couple d'images & Angle d'incidence & $\mathrm{B} / \mathrm{H}$ & $\delta \mathrm{t}$ \\
\hline 13 Sept. $2002 / 16$ Octo. 2004 & $-1.8 /+12.0$ & 0.24 & 764 \\
18 Octo. $2003 / 16$ Octo. 2004 & $+8.5 /+12.0$ & 0.0007 & 364 \\
\hline
\end{tabular}

Puisque ce n'est pas le champ de déformation mais uniquement la zone où la faille aurait pu rompre la surface qui nous intéresse, un traitement plus simple que pour Izmit est utilisé. De toute façon, au moment où la corrélation a été réalisée, la connaissance a priori du séisme (localisation et paramètres sources) était trop limitée pour reproduire la méthodologie "Izmit". On fait simplement l'hypothèse que le bloc à l'Ouest de la faille est stable, c'est donc dans cette zone uniquement que sont extraits les points homologues. Après corrélation, on cartographie les déplacements dans la direction des longitudes et latitudes (figure E.7).

Tout d'abord, on notera que les déformations restent faibles, comprises essentiellement entre -0.5 et $0.5 \mathrm{~m}$ (soit entre -0.2 et 0.2 pixel d'une image SPOT5 THR). Comme déjà remarqué par Binet \& Bollinger (2005), il reste des distorsions entre les deux images très probablement d'origine orbitale (méconnaissance de l'attitude du satellite). Cela se traduit par des ondulations $(\lambda=4$ à $5 \mathrm{~km}$ ) dans les champs de déplacements (surtout dans les déplacements en longitudes, ce qui suggère un effet de roulis).

On ne voit pas de discontinuité nette dans les champs de déplacements au niveau de la faille "InSAR". On observe simplement, dans la partie Sud du champs de déplacement en latitude, une discontinuité orientée $\mathrm{N} 110^{\circ}$, perpendiculairement à la faille "InSAR" (figure E.7). La direction de cette discontinuité est en accord avec celle de la faille privilégiée par Biggs et al. (2005). 


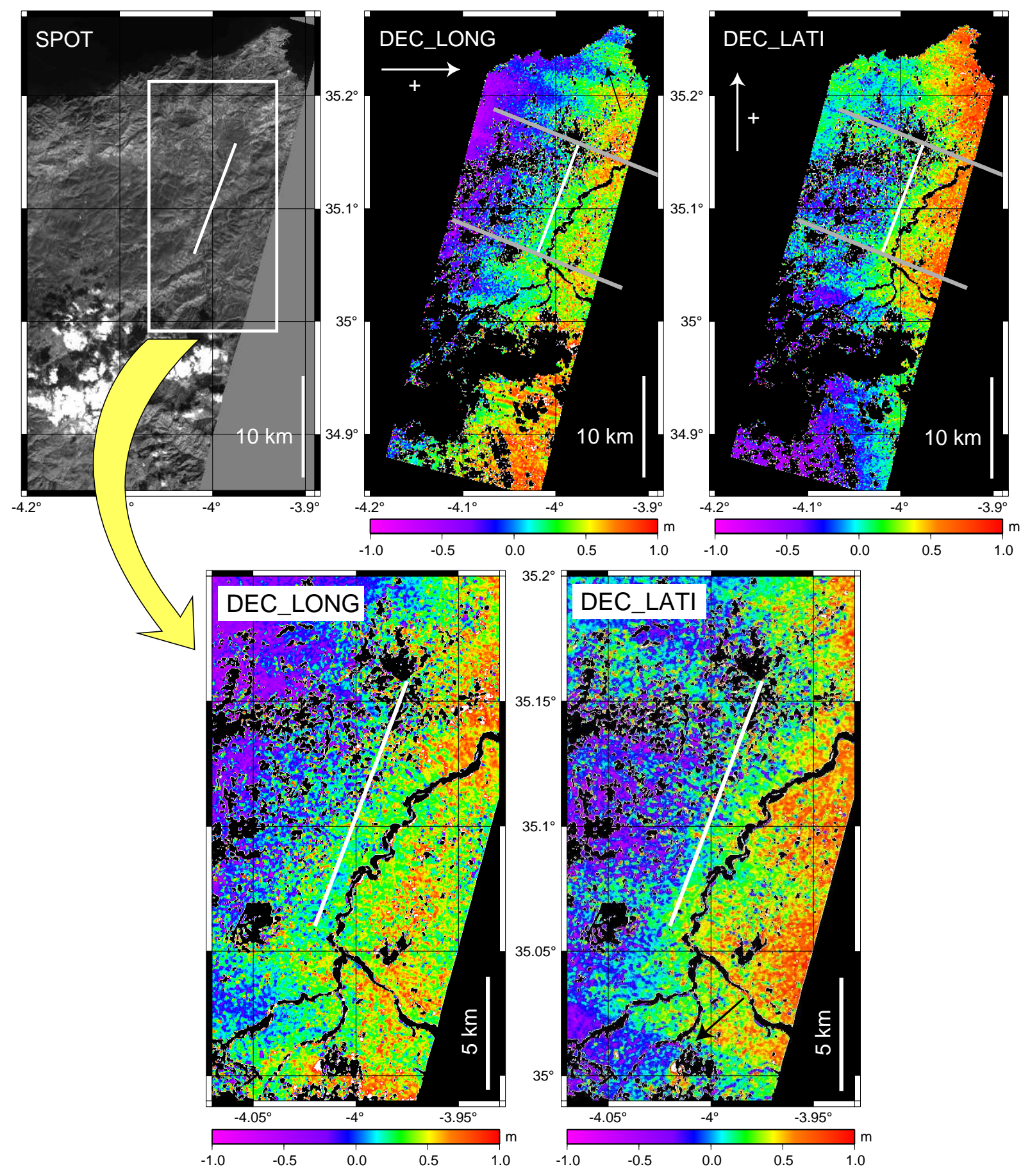

FiG. E.7 - En haut : carte des déplacements en longitude (au centre) et latitude (à droite) entre les images du 18 octobre 2003 et 16 octobre 2004. En noir, les zones où la corrélation a échoué. A gauche, la zone correspondante de l'image SPOT5 du 18 octobre 2003. La faille "InSAR" a été reportée (en blanc) ainsi que 2 profils qui lui sont perpendiculaires (en gris). La flèche indique des ondulations dans les champs de déplacements. En bas : détail de la zone de faille. Une flèche noire pointe sur une zone (en bas) où il existe une discontinuité dans les déplacements en latitude. 


\section{E.2.2 Y'a-t-il eu rupture de surface?}

Pour répondre à cette question, nous avons extraits et moyennés des profils perpendiculaires à la faille "InSAR" (figure E.8). Les deux profils extrêmes ont été localisés sur la figure E.7 (traits gris).
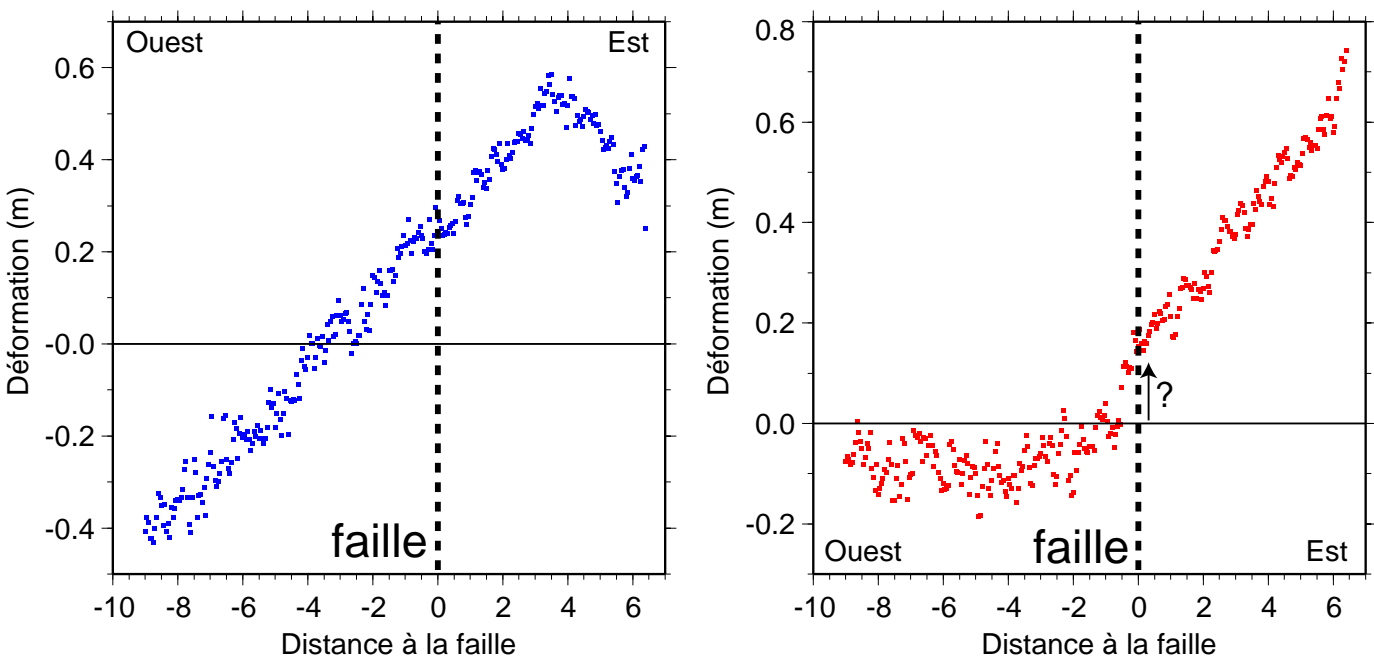

FiG. E.8 - Moyenne de profils perpendiculaires à la faille "InSAR". A gauche, pour les déplacements en longitude. A droite pour ceux en latitude. La flèche et le "?" indiquent une faible discontinuité qui traduirait un léger (environ $10 \mathrm{~cm}$ ) jeu sénestre.

L'interprétation de la forme de ces profils n'est pas simple. Elle traduit à la fois les déformations induites par le séisme et (surtout) les distorsions géométriques résiduelles. Mais la continuité de ces profils, notamment au niveau de la faille "InSAR", permet de conclure que, s'il y a eu rupture de surface, elle n'a pas entraîné de glissements supérieurs à $50 \mathrm{~cm}$ (et vraisemblablement $25 \mathrm{~cm})$.

La corrélation d'images est donc adaptée à la cartographie de la rupture (ou son absence) lors d'un séisme et à l'estimation du jeu le long de cette rupture. Elle est plus délicate à utiliser pour étudier la déformation loin de la faille à cause des distorsions à basse fréquence entre les images, le plus souvent d'origines orbitales. Nous avons montré qu'un modèle a priori de déformation co-sismique est utile à la corrélation d'images dont les résultats, peuvent, en retour, affiner les paramètres d'un tel modèle. 


\section{Annexe F}

\section{"ME DECOIS PAS" \& glissements de terrain}

\section{F.1 Introduction}

Les glissements de terrain (parfois nommés mouvements gravitaires) correspondent au déplacement d'une masse de terrains meubles ou rocheux le long d'une surface de rupture par cisaillement (http ://www.brgm.fr/). Leur cinématique est contrôlée à la fois par des facteurs internes à la masse glissée (rhéologie, hydraulique, géométrie) et des paramètres extérieurs (précipitations, anthropisation végétation, tectonique), qui agissent à différentes échelles de temps et d'espace (Delacourt, 2004). Leur dynamique est régie par la mécanique des sols (éboulements rocheux, glissements de terrain) ou par la mécanique des fluides (laves torrentielles, écoulements hyperconcentrés, crues).

Du point de vue de leur déformation, les glissements de terrains se positionnent de manière intermédiaire entre les glaciers et les séismes. Comme les glaciers, leurs mouvements sont (plus ou moins) continus mais l'amplitude des déplacements est, en général, plus faible. Il est important d'opérer un suivi régulier des glissements de terrain car ces mouvements sont loin d'être stables et prévisibles. Ils touchent parfois directement les activités humaines.

Le suivi des mouvements de terrain peut se faire à partir de mesure in situ : GPS différentiel, tachéométrie, visée laser. L'interférométrie radar (InSAR) permet la mesure des déplacements avec une précision centimétrique et une résolution décamétrique (Fruneau et al., 1996; Rott et al., 1999). La différence des phases n'est possible que si les deux images sont cohérentes ce qui suppose une séparation temporelle de quelques jours à quelques mois selon le site étudié.

La corrélation d'images optiques à très haute résolution (taille du pixel inférieure à $1 \mathrm{~m}$ ) fournie elle aussi une large couverture spatiale. Les scènes corrélées peuvent être des photographies aériennes ou des images satellites. L'intérêt des photographies aériennes, outre leur résolution (50 $\mathrm{cm}$ ), réside dans l'existence d'une archive importante (tout au moins en France) permettant de remonter plusieurs décennies dans la passé (Delacourt et al., 2004). La très haute résolution permet également de proposer une zonation des glissements de terrain et de la relier aux paramètres lithologiques ou hydrométéorologiques (Casson et al., 2003).

La corrélation d'image satellitaires haute résolution (2.5 mètres pour SPOT5) ne conduira évidemment pas une meilleure précision. En revanche, sa très large couverture spatiale $(60 \times$ 
$60 \mathrm{~km}$ pour SPOT) permet d'envisager la détection et le suivi des mouvements gravitaires à l'échelle régionale. Cette technique peut par exemple être utile pour suivre en continu des glissements dont l'activité faible ne justifie pas l'investissement de régulières campagnes de terrain.

Par rapport aux glaciers, la lenteur des mouvements gravitaires est en partie compensée par une persistance des structures de surface sur une plus longue durée. Des images espacées de une voire plusieurs années (contre quelques semaines pour les glaciers) peuvent donc être corrélées ce qui présente trois avantages :

1. le signal à détecter augmente avec l'écart temporel;

2. le choix des images à corréler est plus large;

3. il devient ainsi possible (et conseillé) d'utiliser des images acquises aux même dates chaque année ce qui limite les effets de changements d'orientation des ombres.

L'objectif de notre étude est la détection des glissements de terrain dans les Alpes du Sud. Ces glissements sont connus et bien instrumentés mais le "jeu" consiste ici à les localiser sans connaissance a priori de leur position.

Ces travaux sur les mouvements gravitaires ont été menés dans le cadre d'une sympathique collaboration avec Christophe Delacourt de l'Université de Lyon (puis Brest en septembre 2005). C'est grâce à David Baratoux que cette collaboration s'est initié.

\section{F.2 Zones d'étude et images SPOT5}

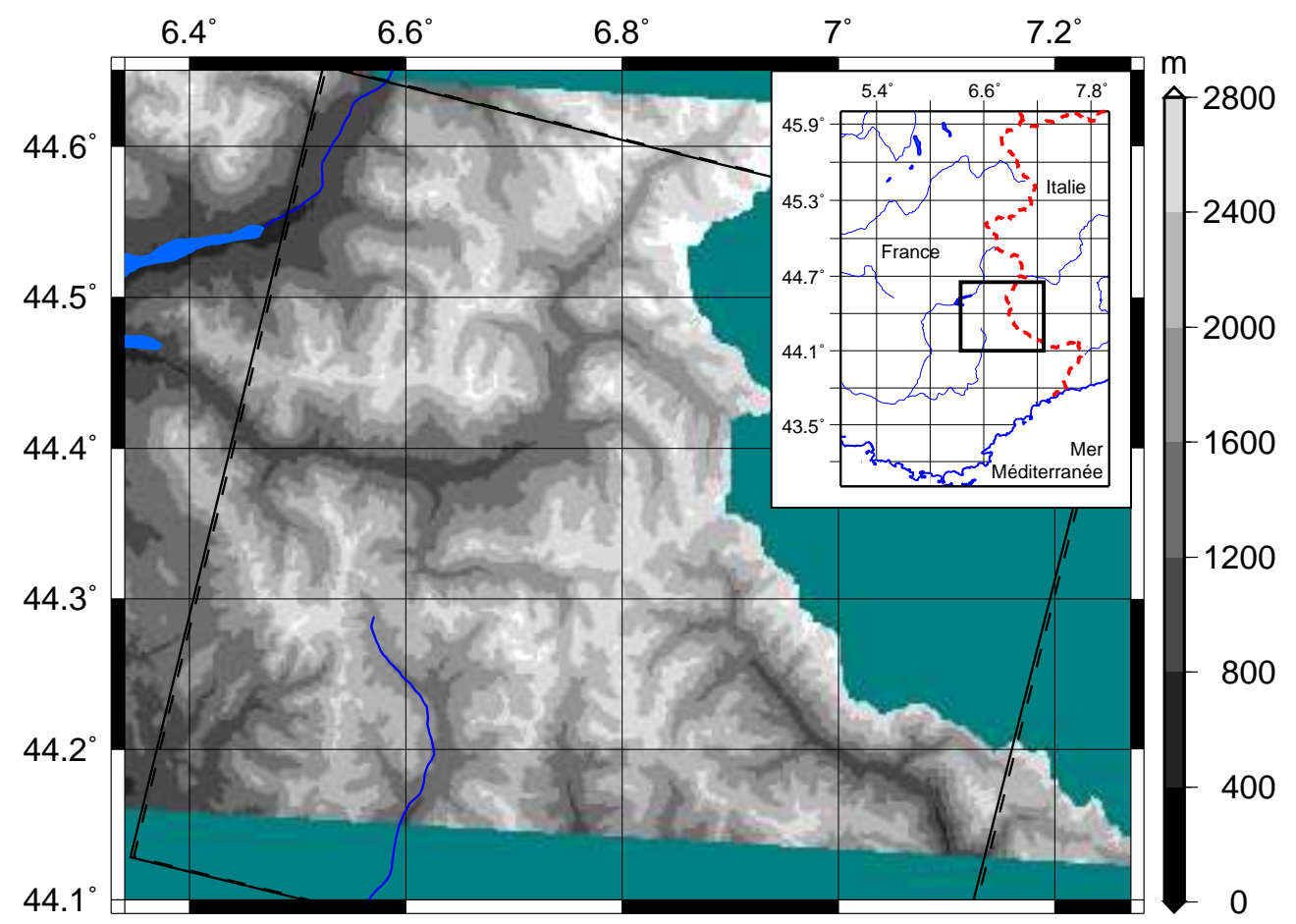

Fig. F.1 - Topographie de la zone étudiée dans les Alpes du Sud et localisation des empreintes au sol des images SPOT5. Un MNT de résolution $50 \mathrm{~m}$ est utilisé sur la partie française des images. L'encart localise la zone d'étude dans le Sud-Est de la France. 
Les caractéristiques des images utilisées pour cette étude sont listées dans le tableau F.1, leurs empreintes au sol (quasi confondues) sont positionnées sur la figure F.1. Entre l'acquisition des deux images, 338 jours soit 13 cycles orbitaux de 26 jours se sont écoulé. Les deux images sont acquises depuis des positions quasi identiques : le rapport B/H est faible (tableau F.1). Cette configuration, qui minimise les distorsions, est optimale pour notre étude. Les angles d'incidence assez faibles font que la contribution des mouvements verticaux aux décalages dans la direction des colonnes peut être négligée.

TAB. F.1 - Images SPOT5 permettant pour la détection des glissements de terrain sur les Alpes du Sud. Sol.= solaire; Azi. = azimut.

\begin{tabular}{lcccccc}
\hline Site d'étude & date & Angle d'inc. & Azi. Sol. & Elev. Sol. & B/H & $\delta$ t (jours) \\
\hline Alpes du Sud & 19 sept. 2003 & -8.67 & 160.3 & 45.8 & \multirow{2}{*}{0.0059} & 338 \\
\cline { 2 - 5 } & 22 août 2004 & -8.97 & 152.2 & 54.8 & & \\
\hline
\end{tabular}

\section{F.3 Orientation absolue et relative des images}

L'orientation absolue (§4.3.1) est réalisée à partir de points de contrôle essentiellement situés à proximité des glissements de terrain (figure F.2). Leur répartition n'est pas homogène mais permet une orientation satisfaisante de l'image du 19 septembre 2003. L'écart type moyen des résidus de cette orientation pour les 7 points de contrôle valides est de $2.04 \mathrm{~m}$ en ligne et $2.71 \mathrm{~m}$ en colonne.
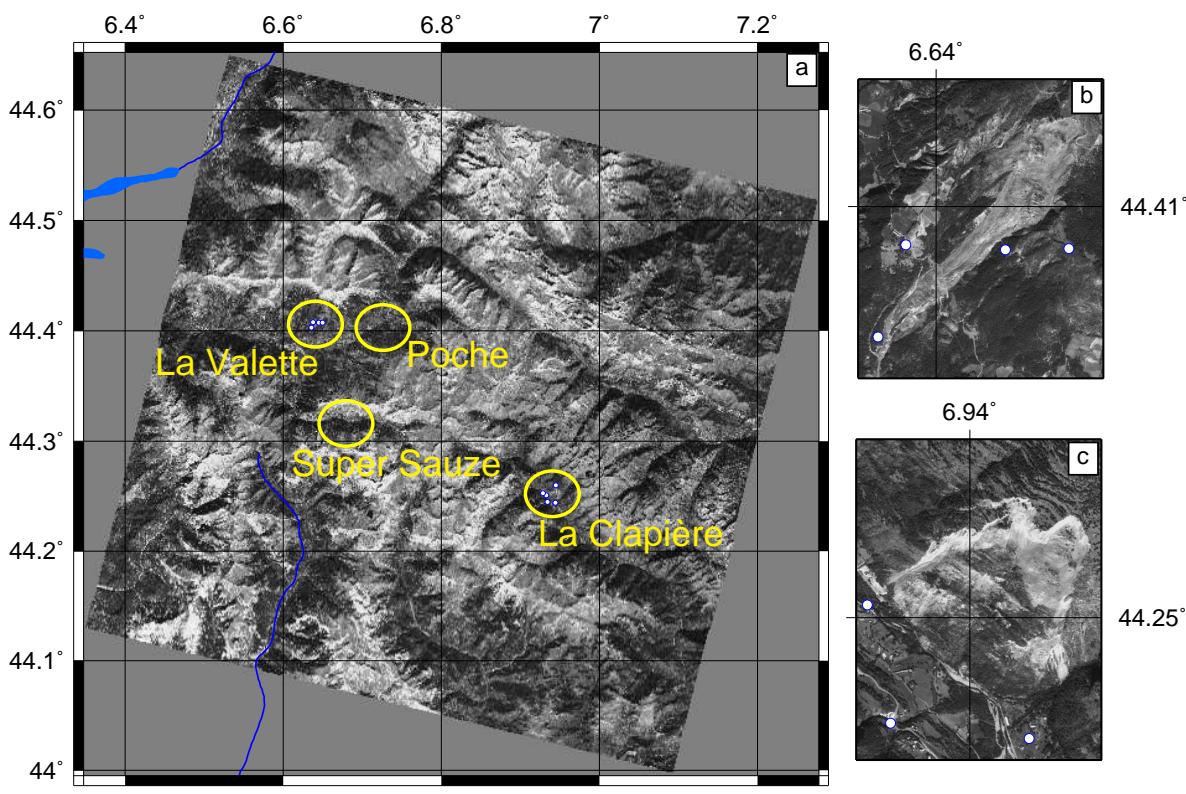

FIG. F.2 - Localisation des points de contrôle (en blanc) utilisés pour l'orientation absolue de l'image SPOT5 du 19 septembre 2003 (a). Les principaux glissements de terrain de cette zone sont identifiés et des zooms sur La Clapière (b) et La Valette (c) sont présentés. 
Faisant l'hypothèse que l'image du 19 septembre 2003 est maintenant bien géoréférencée, l'orientation relative va permettre de lui superposer l'image du 22 août 2004 sur les zones sans mouvements. Elle est réalisée en deux étapes ici :

- dans une première étape, les points homologues (PH) sont extraits "manuellement", en identifiant à l'écran des pixels identiques. Les écart-types moyens des résidus de cette orientation sont : $\sigma_{\text {col }}=0.85 \mathrm{~m} ; \sigma_{\text {lig }}=1.29 \mathrm{~m} ; \mathrm{N}=33$.

- dans une seconde étape, les PH sont extraits automatiquement par corrélation des images sur les zones stables (ici tout l'image est utilisée car on ne connait pas a priori les zones sans mouvements). Les écarts types moyens des résidus de cette orientation sont : $\sigma_{c o l}=0.29 \mathrm{~m}$; $\sigma_{\text {lig }}=0.46 \mathrm{~m} ; \mathrm{N}=259$.

Après cette orientation relative, l'image de 2004 est ré-échantillonnée dans la géométrie de celle de 2003. Les deux images sont alors corrélées tous les 5 pixels en utilisant des fenêtres de 21 par 21 pixels. Les distributions des décalages en colonne et en ligne sont présentées en figure F.3. Pour les décalages en colonne, la distribution est bien centrée sur zéro avec un écart type de 0.37 pixels (soit $0.92 \mathrm{~m}$ ) alors qu'en ligne, la moyenne de tous les décalages n'est pas nulle (biais de $0.3 \mathrm{~m}$ ) et l'écart type est légèrement plus fort.

Décalage en Lignes

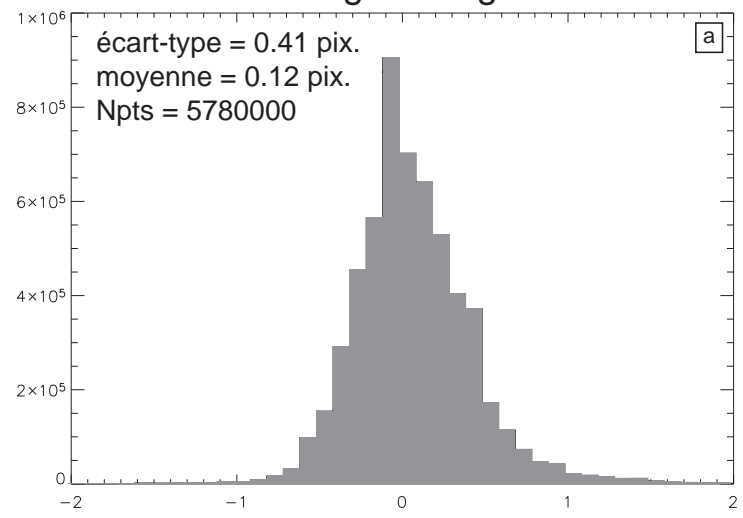

Décalage en Colonnes

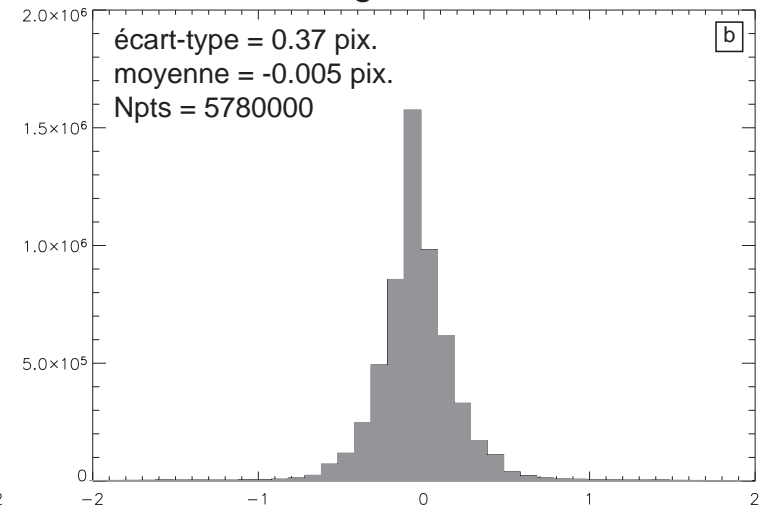

FIG. F.3 - Distribution des décalages en lignes (a) et colonnes (b) pour l'ensemble des zones où la corrélation est considérée comme valide. On trouve une distribution symétrique pour les décalages en colonne alors qu'il existe un léger biais sur les décalages en ligne.

Comme démontré au §4.4.4.1, la distribution non centrée des écarts en ligne ne peut pas s'expliquer par un effet topographique, le rapport B/H étant plus faible d'un facteur 20 dans la direction des lignes que dans la direction des colonnes. C'est à nouveau le résultat du changement de longueur et d'orientation des ombres (figure F.4) qui crée un décalage apparent des ombres du Sud vers le Nord et donc affecte essentiellement les décalages en ligne.

Cet effet n'affecte pas (ou peu) la mesure de l'écoulement glaciaire car les ombres sont limités à la surface des glaciers (chapitre 4). Il pourrait toutefois créer un biais dans le cas des glissements de terrain si des ombres sont présentent. On préconisera donc la corrélation d'images acquises dans les mêmes conditions d'éclairement pour minimiser cet effet. Si une étude plus poussée de cet effet était entreprise, on peut indiquer deux pistes de travail :

- Cet effet dépendant de paramètres connus comme les conditions d'éclairements, les orientations des pentes, il semble possible de le modéliser à partir d'une hypothèse sur la hauteur moyenne des structures (arbres, bâtiments...) créant les ombres. 
- On pourrait aller plus loin et utiliser cet effet pour mesurer par inversion la hauteur moyenne d'une végétation clairsemée ou de bâtiments dont les ombres sont bien identifiables.

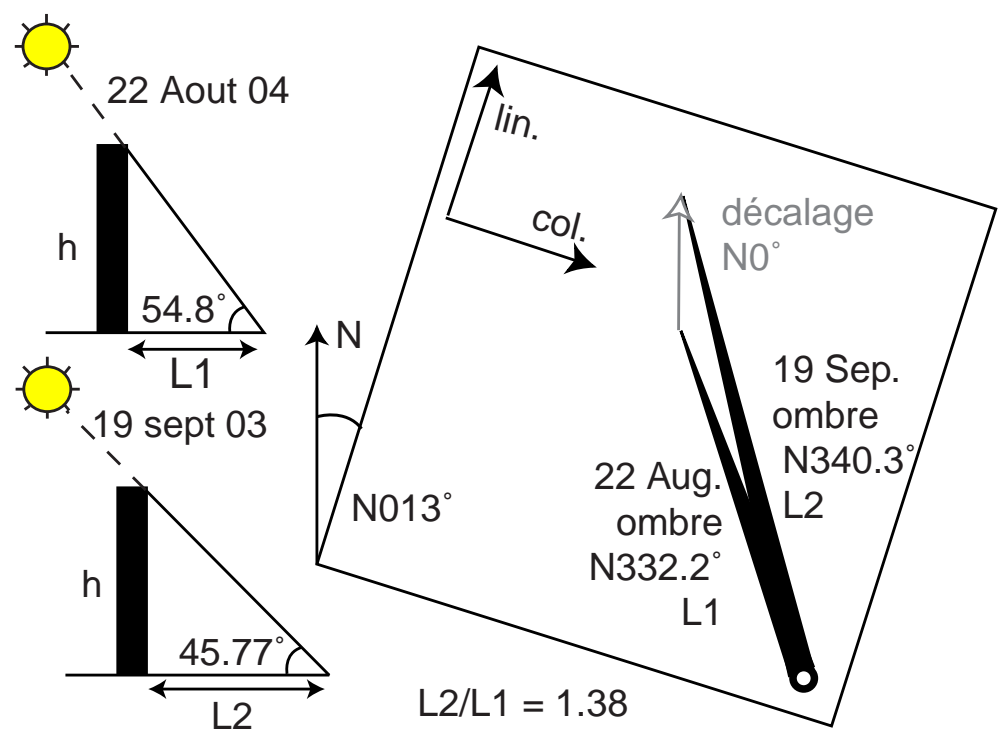

FiG. F.4 - Schéma expliquant comment le changement des conditions d'éclairement solaire entre les deux dates d'acquisition des images génère des décalages artificiels principalement dans la direction des lignes.

\section{F.4 Détection des glissements de terrain}

Pour chercher les glissements sur notre zone, nous n'avons pas développé d'algorithme de détection automatique. De tels algorithmes pourraient se fonder sur la recherche de plusieurs pixels voisins pour lesquels les décalages en ligne et/ou en colonne seraient homogènes et significativement supérieurs à un seuil de détection (par exemple 2 fois l'écart type de la figure F.3). Ici, nous avons opéré cette recherche visuellement en modifiant à l'écran la dynamique des champs de déplacements. De cette manière, trois glissements ont été détectés : La Valette, Super Sauze et le glissement de Poche. En revanche, le glissement de La Clapière n'a pas été trouvé. La figure F.5 montre les champs de vitesse horizontale pour les 4 glissements mentionnés ci-dessus. Les zones où le déplacement est inférieure à $0.5 \mathrm{~m} \cdot \mathrm{a}^{-1}$ (soit $\sim 0.2$ pixel de décalage) apparaissent en noir. Pour les 3 glissements détectés les vitesses maximums atteignent entre 10 et $15 \mathrm{~m} \cdot \mathrm{a}^{-1}$. Si le glissement de La Clapière n'a pas été détecté, c'est simplement car, durant cette année, son activité a été faible. 

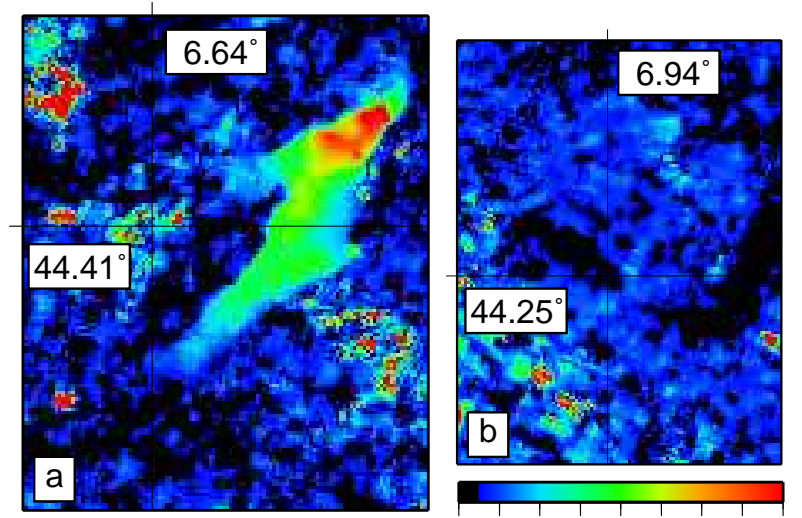

$\begin{array}{llllllllll}0 & 1 & 2 & 3 & 4 & 5 & 6 & 7 & 8\end{array}$
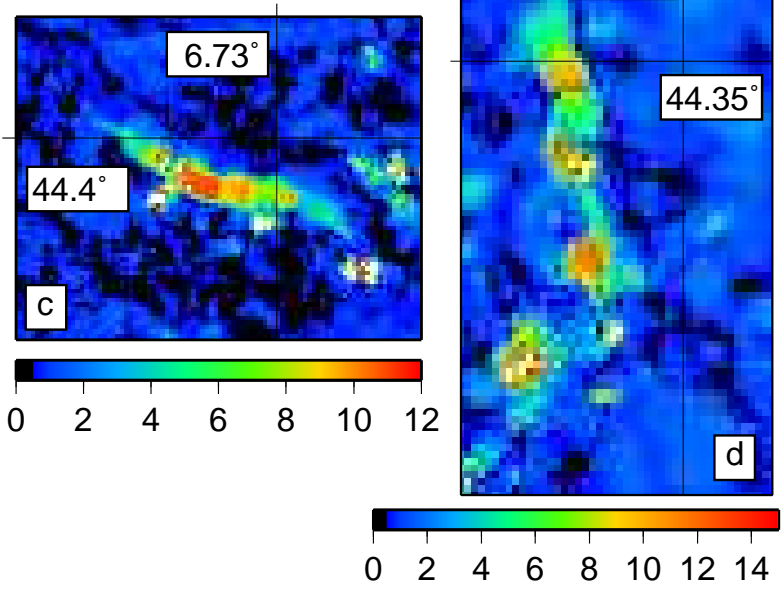

FIG. F.5 - Vitesse horizontale $\left(m . a^{-1}\right)$ des différents glissements inclus dans les images SPOT5. Les glissements sont (a) La Valette (b) La Clapière (c) Poche (d) Super Sauze. On notera l'absence de déplacement significatif sur la Clapière.

Nous n'irons pas plus loin dans l'interprétation de ces résultats qui est en cours (Delacourt et al., en preparation). On peut simplement noter que nos mesures satellitaires détectent :

- un ralentissement très sensible du glissement de La Clapière;

- une reprise d'activité du glissement de La Valette. Le suivi in situ de ce glissement a été très fortement allégé car les mouvements étaient faibles ces dernières années. Ici, notre mesure dépasse les enjeux scientifiques puisqu'une route qui traverse la partie basse du glissement est menacée.

- La corrélation SPOT5 permet pour la première fois par télédétection de suivre l'amplitude de la déformation de la coulée de Super Sauze (même si notre champ de déformation n'est pas continu car la corrélation ne marche pas sur certaines zones). Les photos aériennes (une mission tous les 4 à 5 ans) étaient en effet trop espacées dans le temps pour que les structures de surface persistent.

Ces quelques points révèlent l'intérêt de notre méthode satellitaire qui peut, de plus, être appliquée très rapidement (48h de traitement et temps de calcul) à une nouvelle zone, notamment en situation quasi-opérationnelle. 


\section{Annexe G}

\section{Et l'écoulement 3D des glaciers?}

Suite à la mesure de mouvements horizontaux (chapitre 4) et verticaux (chapitre 6), il est logique de s'interroger sur la possibilité de mesurer les 3 composantes du champ de déplacement à la surface terrestre. En prolongement de nos travaux sur la corrélation d'images, nous développons ici deux méthodes pour atteindre cet objectif. Si ces méthodes semblent adaptées à la terre solide (volcan, séisme, etc...), leur application aux glaciers pose certains problèmes.

La corrélation d'images optiques fournie deux mesures alors que trois sont nécessaires pour caractériser un vecteur déplacement à la surface de la Terre. Pour obtenir ce vecteur, des hypothèses peuvent être faites sur la direction de l'écoulement (§3.3.3.1). Mais ces hypothèses sont rarement valides pour les glaciers de montagne où les fortes accumulations et ablations induisent des vitesses d'enfouissement et d'émergence.

Gray et al. (2005) ont combiné récemment la technique du speckle-tracking (sensible surtout aux déplacements horizontaux) avec l'InSAR (sensible aux mouvements verticaux) pour obtenir un champs de vitesse 3D sur une zone de l'Antarctique où la cohérence est maintenue même après 24 jours (durée du cycle orbital de RADARSAT). Mais le speckle-tracking fourni des résultats décevants sur les glaciers du massif du Mont-Blanc (Arnaud, communication personnelle). Pour obtenir la nécessaire $3^{\text {eme }}$ donnée, différentes approches sont possibles. Les deux que nous décrivons s'appuient sur des systèmes satellitaires qui fournissent une large couverture spatiale. Quelques mesures GPS peuvent aussi être utilisées (Guðmundsson et al., 2002b), mais on perd alors la large couverture satellitaire.

\section{G.1 Fusion InSAR / corrélation d'images optiques}

L'InSAR mesure la projection du vecteur déplacement $\vec{\delta}\left(\delta_{\lambda}, \delta_{\phi}, \delta_{z}\right)$ dans la ligne de visée ${ }^{1}$ du satellite radar. Cette composante est notée $\delta_{L O S}$. La sensibilité de l'InSAR aux mouvements de surface est indiquée par le vecteur unitaire $\overrightarrow{U_{L O S}}=\left[\lambda_{L O S}, \phi_{L O S}, z_{L O S}\right]$ dirigé de la surface terrestre vers le satellite. Dans le cas des satellites ERS, un exemple des coordonnées de ce vecteur (Guðmundsson et al., 2002a) est [-0.39, -0.11, 0.91]. L'InSAR est donc particulièrement sensible aux mouvements verticaux. La corrélation d'images optiques étant elle plus sensible aux mouvements horizontaux, il est logique de combiner les deux techniques.

Si le vecteur unitaire $\overrightarrow{U_{L O S}}$ est relativement constant, les vecteurs unitaires en optique sont

\footnotetext{
${ }^{1}$ Line Of Sight -LOS- en anglais
} 
eux beaucoup plus variables d'une image à l'autre du fait du dépointage des satellites SPOT.

Dans le cas du couple acquis au cours de l'été 2003 entre le 23 août et le 18 septembre $\left(\theta=16.5^{\circ}\right.$ au niveau du massif du Mont Blanc et $\left.\alpha=17.06^{\circ}\right)$, ces vecteurs unitaires ont pour coordonnées : $\overrightarrow{U_{L I G}}=[-0.29,-0.96,0]$ et $\overrightarrow{U_{C O L}}=[0.91,-0.28,0.28]$

Chacune des mesures est donc particulièrement sensible à l'une des composantes du vecteur déplacement. Le problème consiste à résoudre le système de 3 équations à 3 inconnues :

$$
\left\{\begin{aligned}
\delta_{L O S} & =\overrightarrow{U_{L O S}} \cdot \vec{\delta} \\
\delta_{L I G} & =\overrightarrow{U_{L I G}} \cdot \vec{\delta} \\
\delta_{C O L} & =\overrightarrow{U_{C O L}} \cdot \vec{\delta}
\end{aligned}\right.
$$

En remplaçant par les valeurs numériques :

$$
\left\{\begin{array}{l}
\delta_{L O S}=-0.39 \delta_{\lambda}-0.11 \delta_{\phi}+0.91 \delta_{z} \\
\delta_{L I G}=-0.29 \delta_{\lambda}-0.96 \delta_{\phi} \\
\delta_{C O L}=0.91 \delta_{\lambda}-0.28 \delta_{\phi}+0.28 \delta_{z}
\end{array}\right.
$$

La résolution de système de 3 équations à 3 inconnues $\left(\delta_{\lambda}, \delta_{\phi}, \delta_{z}\right)$ revient à inverser la matrice A définit par :

$$
A=\left[\begin{array}{ccc}
-0.39 & -0.11 & 0.91 \\
-0.29 & 0.96 & 0 \\
0.91 & -0.28 & 0.28
\end{array}\right]
$$

L'inversion conduit à :

$$
A^{-1}=\left[\begin{array}{ccc}
-0.28 & 0.23 & 0.9 \\
0.08 & -0.97 & -0.27 \\
0.99 & -0.21 & 0.35
\end{array}\right]
$$

On obtient donc les 3 composantes du vecteur déplacement $\vec{\delta}$. Cette combinaison InSARoptique pourrait être utilisée pour des mouvements géophysiques autres que ceux des glaciers. L'InSAR n'est plus possible actuellement sur ces derniers, une alternative est donc proposée qui utilise une double corrélation d'images optiques.

\section{G.2 Corrélation de deux couples d'images optiques}

Pour obtenir deux couples d'images optiques avec chacun des angles d'incidence semblables et non verticaux, les capteurs à capacité stéréoscopiques le long de la trajectoire (HRS sur SPOT5 ou ASTER sur TERRA) semblent les plus adaptés. La figure G.1 illustre le cas d'un satellite comme HRS avec deux capteurs, l'un tourné vers l'avant, l'autre vers l'arrière. 


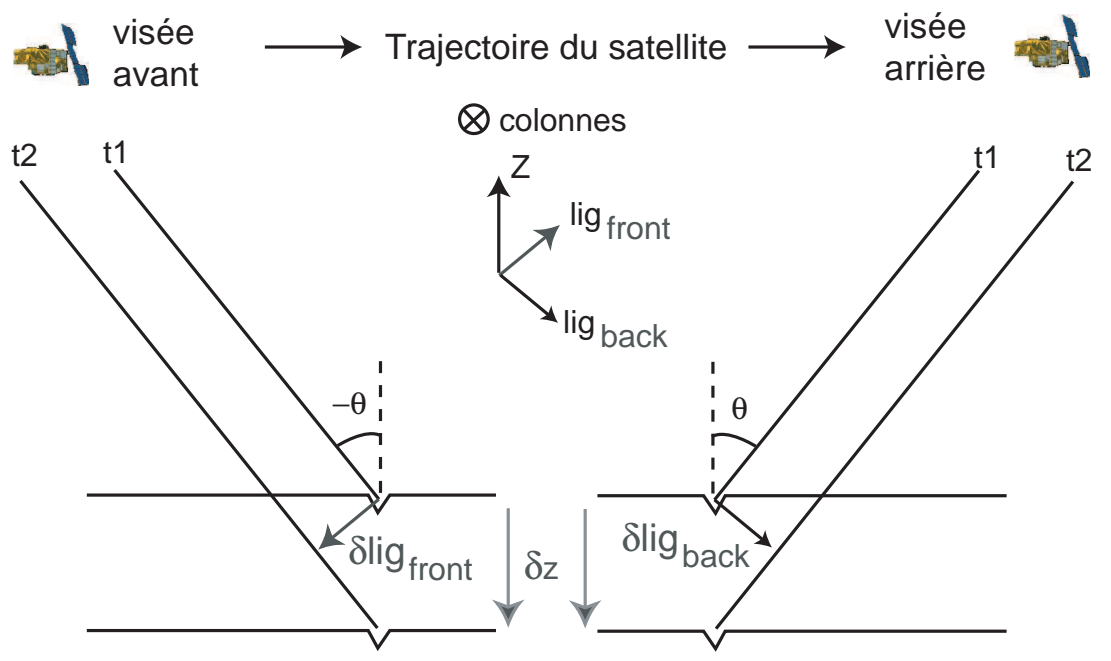

FiG. G.1 - Une configuration satellitaire qui permettrait la mesure des 3 composantes du vecteur déplacement de la surface terrestre. Cette configuration permet également d'accroître la sensibilité de la corrélation d'images aux mouvements verticaux.

Si les indices $f$ et $b$ indiquent la visée vers l'avant (front) et vers l'arrière (back), la corrélation mesure :

$$
\left\{\begin{array}{l}
\overrightarrow{U_{L I G_{f}}} \cdot \vec{\delta}=\delta_{L I G_{f}} \\
\overrightarrow{U_{C O L_{f}}} \cdot \vec{\delta}=\delta_{C O L_{f}} \\
\overrightarrow{U_{L I G_{b}}} \cdot \vec{\delta}=\delta_{L I G_{b}} \\
\overrightarrow{U_{C O L_{b}}} \cdot \vec{\delta}=\delta_{C O L_{b}}
\end{array}\right.
$$

Ce système donne l'impression d'être surdéterminé avec 4 équations pour seulement 3 inconnues, les coordonnées de $\vec{\delta}$. Dans la pratique, les vecteurs de sensibilité $\overrightarrow{U_{C O L_{f}}}$ et $\overrightarrow{U_{C O L_{b}}}$ sont quasiment identiques car ils sont horizontaux et perpendiculaires à la trace du satellite. En revanche, $\overrightarrow{U_{L I G_{f}}}$ et $\overrightarrow{U_{L I G_{b}}}$ sont différents : ils sont parallèles à la trace mais orientés vers l'avant et l'arrière. On dispose donc de 3 mesures indépendantes et non parallèles du même déplacement, le système peut être résolu. Si un mouvement purement vertical est étudiée $\vec{\delta}\left[0,0, \delta_{z}\right]$, son amplitude peut être déduite de :

$$
\delta_{z}=\frac{\delta_{L I G_{f}}-\delta_{L I G_{b}}}{2 \times \sin \theta}
$$

Le facteur 2 au dénominateur montre que cette configuration permet une sensibilité 2 fois plus forte aux mouvements verticaux (On comparera l'équation G.4 à l'équation 4.8). Avec ce système, il n'est pas nécessaire de supposer que le mouvement est constant au cours du temps comme c'est le cas en couplant l'InSAR (typiquement mesure du déplacement en 1 jour) et la corrélation optique (déplacement en quelques semaines).

Nous n'avons pas pu tester la faisabilité et la précision d'une telle mesure au cours de cette thèse, faute d'images adaptées. Les images du satellite ASTER ont une résolution trop faible $(15 \mathrm{~m})$ et une orbite mal contrôlée, alors que les images de SPOT5-HRS ne sont pas, malheureusement, disponibles pour la communauté scientifique. 


\section{G.3 Ces applications sont-elles possibles sur les glaciers? Autre- ment dit... que mesure-t-on?}

L'application des méthodes décrites précédemment ne pose pas de problèmes pour suivre des phénomènes géophysiques à volume constant (glissement de terrain, déformation co-sismique, gonflement et subsidence des volcans). En revanche, dans le cas des glaciers l'ablation et l'accumulation vont perturber les mesures satellitaires des vecteurs déplacements (figure G.2). Il faut revenir à la nature même de la mesure.
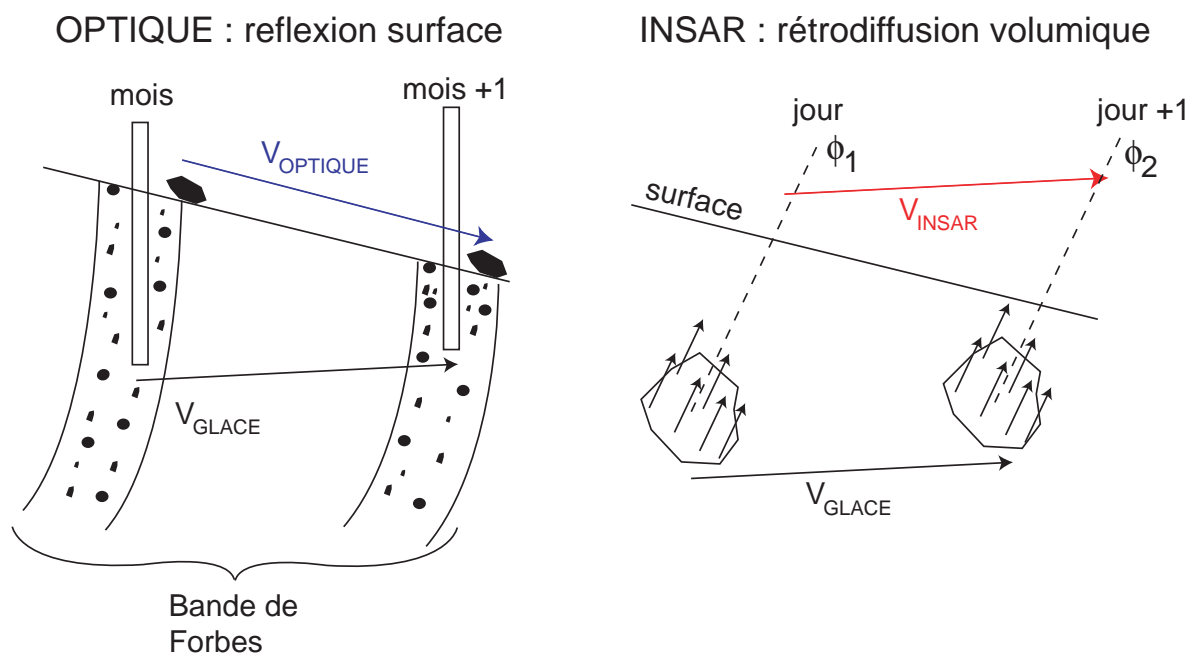

FIG. G.2 - Comparaison des vitesses réelles du glacier en zone d'ablation avec celles mesurées par corrélation de deux images optiques (espacées d'un mois en été) et par InSAR (images acquises à 1 jour d'intervalle).

Dans le cas des images optiques, c'est la réflectance de la surface (il n'y a pas de pénétration du rayonnement solaire) que l'on corrèle entre les images. Les impuretés, les pierres ou les crevasses qui engendrent le contraste radiométrique ne suivent pas le mouvement des particules de glace. Même en résolvant les 3 composantes du vecteur vitesse, la corrélation optique mesurera toujours un déplacement parallèle à la surface du glacier. L'InSAR est plus performant de ce point de vue puisque le signal radar pénètre dans le neige (plusieurs dizaines de mètres) ou dans le glace (quelques décimètres à mètres) et donc l'interférométrie permet de suivre le déplacement d'un ensemble de cibles (qui forment un pixel) dont on peut penser (?) qu'elles se déplacent avec les particules de glace.

La combinaison InSAR-optique serait donc la plus prometteuse mais supposerait, pour l'instant, de combiner des mesures pour des intervalles de temps très différents (1 jour en InSAR, 1 à 2 mois en optique) et uniquement pour quelques périodes des années 1996 à 1999 où ERS1 et ERS2 étaient en tandem. 


\section{Bibliographie}

Aðalgeirsdottír, G., Guðmundsson, G. H., \& Björnsson, H., 2000. The response of a glacier to a surface disturbance, a case study on the Vatnajökull ice cap. Ann. Glaciol., 31, 104-110.

Abdalati, W., Krabill, W. B., Frederick, E. B., Manizade, S., Martin, C., Sonntag, J., Swift, R., Thomas, R. H., Yungel, J., \& Koerner, R., 2004. Elevation changes of ice caps in the Canadian Arctic Archipelago. J. Geophys. Res., 109 (F4).

Agassiz, L., 1840. Etudes sur les glaciers. Jent et Gassman, Neutchatel, Switzerland.

Ahlmann, W., 1948. Glaciological research on the North Atlantic coasts. Royal Geographical Society, London.

Andreassen, L. M., Elvehøy, H., \& Kjøllmoen, B., 2002. Using aerial photography to study glacier changes in Norway. Ann. Glaciol., 34, 343-348.

Arendt, A. A., Echelmeyer, K. A., Harrison, W. D., Lingle, C. S., \& Valentine, V. B., 2002. Rapid wastage of Alaska glaciers and their contribution to rising sea level. Science, 297, 382-386.

Arnaud, Y., Muller, F., Vuille, M., \& Ribstein, P., 2001. El Niño-Southern Oscillation (ENSO) influence on a Sajama volcano glacier (Bolivia) from 1963 to 1998 as seen from Landsat data and aerial photography. J. Geophys. Res., 106 (D16), 17,773.

Baltsavias, E. P., Favey, E., Bauder, A., Bösch, H., \& Pateraki, M., 1999. Digital surface modelling by airborne laser scanning and digital photogrammetry for glacier monitoring. Photogramm. Rec., 17 (98), 243-273.

Bard, E., 2004. Greenhouse effect and ice ages : historical perspective. C. R. Geoscience, 336, $603-638$.

Berger, A., 1988. Milankovitch theory and climate. Rev. Geophys., 26, 624-657.

Berthier, E., Arnaud, Y., Baratoux, D., Vincent, C., \& Rémy, F., 2004. Recent rapid thinning of the "Mer de glace" glacier derived from satellite optical images. Geophys. Res. Lett., 31 (17), L17401.

Berthier, E., Björnsson, H., Pálsson, F., Llubes, M., Feigl, K. L., \& Rémy, F., soumis. The level of the Grímsvötn subglacial lake, Vatnajökull, Iceland, monitored with SPOT5 images. Earth Planet. Sc. Lett..

Berthier, E., Gallaire, R., Fuertes, R., Chazarin, J.-P., Wagnon, P., Sicart, J.-E., Francou, B., Ribstein, P., \& Baldivieso, H., 2001. Mesures météorologiques, hydrologiques et glaciologiques sur le glacier Zongo, année hydrologique 1999-2000. Rapport Technique 3, IRD-Bolivie. 94pp. 
Berthier, E., Raup, B., \& Scambos, T. A., 2003. New velocity map and mass-balance estimate of Mertz Glacier, East Antarctica, derived from Landsat sequential imagery. J. Glaciol., 49 (167).

Berthier, E., Vadon, H., Baratoux, D., Arnaud, Y., Vincent, C., Feigl, K. L., Rémy, F., \& Legrésy, B., 2005. Mountain glacier surface motion derived from satellite optical imagery. Remote Sensing Environ., 95 (1), 14-28.

Biggs, J., Wright, T., Parsons, B., Jachson, J., Funning, G., Emmerson, B., \& Bergman, E., 2005. The $2004 \mathrm{Al}$ Hoceima, Marocco earthquake : observations from InSAR and seismology. In EGU Conference, Vienna.

Bindschadler, R., Dowdeswell, J., Hall, D., \& Winther, J.-D., 2001. Glaciological applications with Landsat-7 imagery : early assessments. Remote Sensing Environ., 78, 163-179.

Bindschadler, R. A., 1998. Monitoring ice sheet behavior from space. Rev. Geophys., 36 (1), 79-104.

Bindschadler, R. A. \& Scambos, T. A., 1991. Satellite-image-derived velocity field for an Antarctic ice stream. Science, 252 (5003), 242-246.

Binet, R. \& Bollinger, L., 2005. Horizontal coseismic deformation of the 2003 Bam (Iran) earthquake measured from SPOT-5 THR satellite imagery. Geophys. Res. Lett., 32 (L02307). Doi :10.1029/2004GL021897.

Björnsson, H., 1998. Hydrological characteristics of the drainage system beneath a surging glacier. Nature, 395, 771-774.

Björnsson, H., 2002. Subglacial lakes and jökulhlaups in Iceland. Global Planet. Change, 35, $255-271$.

Björnsson, H., Guðmundsson, S., Pálsson, F., \& Berthier, E., 2005. Rapid evolution of a proglacial coastal lake. 20th century changes in Jökulsárlón at Breiðamerkursandur, Vatnajökull, Iceland. In Second International Costal Symposium in Iceland. Höfn.

Björnsson, H. \& Guðmundsson, T. M., 1993. Variations in the thermal output of the subglacial Grimsvtön caldera, Iceland. Geophys. Res. Lett., 20 (19), 2127-2130.

Björnsson, H., Pálsson, F., \& Guðmundsson, S., 2001a. Jökulsárlon at Breiðamerkursandur, Vatnajökull, Iceland : 20th century changes and future outlook. Jökull, 50, 1-18.

Björnsson, H., Pálsson, F., Sigurdsson, O., \& Flowers, G. E., 2003. Surges of glaciers in Iceland. Ann. Glaciol., 36, 82-90.

Björnsson, H., Rott, H., Guðmundsson, S., Fischer, A., Siegel, A., \& Guðmundsson, M. T., 2001b. Glacier-volcano interactions deduced by SAR interferometry. J. Glaciol., 47 (156), 58-70.

Bordier, A. C., 1773. Voyage pittoresque aux glacières de Savoye fait en 1772. L. A. Caille, Genève.

Braithwaite, R. J., 2002. Glacier mass balance : the first 50 years of international monitoring. Prog. Phys. Geog., 26 (1), 76-95.

Braithwaite, R. J. \& Raper, S. C. B., 2002. Glaciers and their contribution to sea level change. Phys. Chem. Earth., 27, 1445-1454. 
Buisson, A., Guirado, F., Lacroix, F., \& Valla, F., 1999. Glaciers à risques. Alpes françaises. Inventaire et suivi. Rapport technique, CEMAGREF, CNRS, RTM, Ministère de l'aménagement du territoire et des transports.

Cabanes, C., Cazenave, A., \& Le Provost, C., 2001. Sea level rise during past 40 years determined from satellite and in situ observations. Science, 294 (5543), 840-842.

Casson, B., Delacourt, C., Baratoux, D., \& Allemand, P., 2003. Seventeen years of the "la Clapiere" landslide evolution analysed from ortho-rectified aerial photographs. Eng. Geology, 68, 123-139.

Cazenave, A. \& Nerem, R. S., 2004. Present-day sea level change : observations and causes. Rev. Geophys., 42 (3001).

Centre National d'Etude Spatiale, 2002. programme MEDICIS, distribué par CSSI.

Chinn, T. J., 1999. New Zealand glacier response to climate change of the past 2 decades. Global Planet. Change, 22, 155-168.

Church, J. A., White, N. J., Coleman, R., Lambeck, K., \& Mitrovica, J. X., 2004. Estimates of the regional distribution of sea level rise over the 1950-2000 period. J. Climate, 17 (13), 2609-2625.

CNES, 1996. Guide des utilisateurs de donnés SPOT, Vol. 1 "Manuel de référence; Vol. 2, "Guide de l'utilisateur" ; Vol. 3, "Guide pratique". Centre National d'Etude Spatiale, Toulouse, France.

Cook, A. J., Fox, A. J., Vaughan, D. G., \& Ferrigno, J. G., 2005. Retreating glacier fronts on the Antarctic Peninsula over the past half-century. Science, 308, 541-544.

Crippen, R. E., 1992. Measurement of subresolution terrain displacements using SPOT panchromatic imagery. Episodes, 15 (1), 56-61.

Davis, C. H., Li, Y., McConnell, J. R., Frey, M. M., \& Hanna, E., 2005. Snowfall-driven growth in East Antarctic ice sheet mitigates recent sea-level rise. Science, 308 (5730), 1898-1900.

De Ruyter de Wildt, M. S., Oerlemans, J., \& Björnsson, H., 2002. A method for monitoring glacier mass balance using satellite albedo measurements : application to Vatnajökull (Iceland). J. Glaciol., 48 (161), 267-278.

de Saussure, H. B., 1803. Voyage dans les Alpes. Julie Boch, Neuchatel.

Delacourt, C., 2004. Flux de matière à la surface des planètes : mesures et conséquences géodynamiques. Diplôme d'habilitation à diriger les recherches. Université C. Bernard - Lyon I.

Delacourt, C., Allemand, P., Casson, B., \& Vadon, H., 2004. Velocity field of the "La Clapière" landslide measured by the correlation of aerial and QuickBird satellite images. Geophys. Res. Lett., 31 (15), L15619.

Delacourt, C., Berthier, E., \& Allemand, P., en preparation. Detection of landslides in Alpine area by SPOT5 optical correlation. J. Geodyn..

Dominguez, S., Avouac, J.-P., \& Michel, R., 2003. Horizontal coseismic deformation of the 1999 Chi-Chi earthquake measured from SPOT satellite images : implications for the seismic cycle along the western foothills of central Taiwan. J. Geophys. Res., 108 (B2). 
Dowdeswell, J. A., Unwin, B., Nuttall, A.-M., \& Wingham, D. J., 1999. Velocity structure, flow instability and mass flux on a large arctic ice cap from satellite radar interferometry. Earth Planet. Sc. Lett., 167, 131-140.

Dyurgerov, M. B., 2002. Glacier mass balance and regime : data of measurements and analysis. Occasional Paper \# 55. http ://instaar.colorado.edu/other/download/OP55_glaciers.pdf.

Dyurgerov, M. B., 2003. Mountain and subpolar glaciers show an increase in sensitivity to climate warming and intensification of the water cycle. J. Hydrol., 282, 164-176.

Echelmeyer, K. A., Harrison, W. D., Larsen, C. F., Sapiano, J., Mitchell, J. E., DeMallie, J., Rabus, B., Adalgeirsdòttir, G., \& Sombardier, L., 1996. Airborne surface profiling of glaciers : a case-study in Alaska. J. Glaciol., 42 (142), 538-547.

Emery, W. J., Thomas, A. C., Collins, M., Crawford, W. J., \& Mackas, D. L., 1986. An objective method for computing advective surface velocities from sequential infrared satellite images. $J$. Geophys. Res., 91 (C11), 12865-12878.

Evans, A. N., 2000. Full field glacier surface motion computation from digital image sequences. IEEE T. Geosci. Remote, 38 (2), 1064-1072.

Fadil, A., Tahayt, A., et al., en preparation. Present-day deformation in Marocco.

Feigl, K. L., Berthier, E., Akarvardar, S., Sarti, F., McClusky, S., Ergintav, S., \& Reilinger, R., en preparation. An improved estimate of slip distribution for the Izmit mainshock from coseismic GPS, ERS-1, RADARSAT and SPOT measurements. J. Seismology.

Feigl, K. L., Sarti, F., Vadon, H., McClusky, S., Ergintav, S., Durand, P., Bürgmann, R., Rigo, A., Massonnet, D., \& Reilinger, R., 2002. Estimating slip distribution for the Izmit mainshock from coseismic GPS, ERS-1, RADARSAT, and SPOT measurements. Bulletin of the Seismological Society of America, 92 (1), 138-160.

Fily, M., Dedieu, J.-P., \& Surdyk, S., 1995. A SAR image study of a snow-covered area in the French Alps. Remote Sensing Environ., 51, 253-262.

Fischer, A., 2002. Icedynamics of Vatnajökull, Iceland, investigated by means of ERS SAR Interferometry. Thèse de Doctorat, Leopold Franzens Universität, Innsbruck.

Fischer, A., Rott, H., \& Björnsson, H., 2003. Observation of recent surges of Vatnajökull, Iceland, by means of ERS SAR interferometry. Ann. Glaciol., 37, 69-76.

Forbes, J. D., 1845. Travels through the Alps of Savoy and other parts of the Pennin chain with observations on the phenomena of Glaciers. Adam \& Charles Black, Edinburgh, 2nd edition.

Forel, F. A., 1895. Les variations périodiques des glaciers. Discours préliminaire. Archives des sciences physiques et naturelles, 34, 209-229.

Fountain, A. G., Jacobel, R. W., Schlichting, R., \& Jansson, P., 2005. Fractures as the main pathways of water flow in temperate glaciers. Nature, 433, 618-621.

Francou, B., Vuille, M., Favier, V., \& Caceres, B., 2004. New evidence for an ENSO impact on low-latitude glaciers : Antizana 15, Andes of Ecuador, 0²8'S. J. Geophys. Res., 109 (D18106). 
Francou, B., Vuille, M., Wagnon, P., Mendoza, J., \& Sicart, J.-E., 2003. Tropical climate change recorded by a glacier in the central Andes during the last decades of the twentieth century : Chacaltaya, Bolivia, 16 ${ }^{\circ}$ S. J. Geophys. Res., 108 (D5), 4154.

Fratter, D., Moulin, M., Ruiz, H., Charvet, P., \& Zobler, D., 2001. The SPOT-5 Mission. In 52 ${ }^{\text {nd }}$ International Astronautical Congress. Toulouse, France.

Fruneau, B., Achache, J., \& Delacourt, C., 1996. Observation and modelling of the Saint-Etiennede-Tinée landslide using SAR interferometry. Tectonophysics, 265, 181-190.

Funk, M., Morelli, R., \& Stahel, W., 1997. Mass balance of Griesgletscher 1961-1994 : different methods of determination. Zeitschrift für Gletscherkunde und Glazialgeologie, 33 (1), 41-55.

Glen, J. W., 1955. The creep of polycrystalline ice. Proceedings of the Royal Society, 228, $519-538$.

Gluck, S., 1967. Détermination du lit rocheux sous la Mer de Glace par séismique-réflexion. $C R$ Acad. Sci. II A, 264 (19), 2272-2275.

Goldstein, R. M., Engelhardt, H., Kamb, B., \& Frolich, R. M., 1993. Satellite RADAR interferometry for monitoring ice sheet motion : application to an Antartic ice stream. Science, 262 (5139), 1525-1530.

Gray, A. L., Joughin, I., Tulaczyk, S., Spikes, V. B., Bindschadler, R., \& Jezek, K. C., 2005. Evidence for subglacial water transport in the West Antarctic Ice Sheet through three-dimensional satellite radar interferometry. Geophys. Res. Lett., 32 (L03501). Doi :10.1029/2004GL021387.

Gray, A. L., Short, N., Mattar, K. E., \& Jezek, K. C., 2001. Velocities and flux of the Filchner Ice Shelf and its tributaries determined from speckle tracking interferometry. Can. J. Remote Sensing, 27 (3), 193-206.

Guðmundsson, G. H. \& Bauder, A., 1999. Towards an indirect determination of the mass-balance distribution of glaciers using the kinematic boundary condition. Geogr. Ann., 81A (4), 575583.

Guðmundsson, M. T., Sigmundsson, F., \& Björnsson, H., 1997. Ice-volcano interaction of the 1996 Gjálp subglacial eruption, Vatnajökull, Iceland. Nature, 389, 954-957.

Guðmundsson, S., Guðmundsson, M. T., Björnsson, H., Sigmundsson, F., Rott, H., \& Carstensen, J. M., 2002a. Three-dimensional glacier surface motion maps at the Gjálp eruption site, Iceland, inferred from combining InSAR and other ice-displacement data. Ann. Glaciol., 34, 315-322.

Guðmundsson, S., Sigmundsson, F., \& Carstensen, J. M., 2002b. Three-dimensional surface motion maps estimated from combined InSAR and GPS data. J. Geophys. Res., 107 (B10). Doi : 10.1029/2001JB000283.

Guneriussen, T., Høgda, K. A., Johnson, H., \& Lauknes, I., 2001. InSAR for estimating changes in snow water equivalent of dry snow. IEEE T. Geosci. Remote, 39 (10), 2101-2108.

Haag, W., Braun, L. N., Uvarov, V. N., \& Makarevich, K. G., sous presse. A comparison of three methods of mass balance determination in the Tuyuksu glacier region, Tien Shan. J. Glaciol.. 
Haeberli, W., Holze, M., \& Suter, P. C., 1998. Into the second century of worldwide glacier monitoring : prospects and strategies. In Studies and reports in hydrology, edited by UNESCO, volume 217. Paris.

Hall, D. K., Bayr, K. J., Shöner, W., Bindschadler, R. A., \& Chien, J., 2003. Consideration of the errors inherent in mapping historical glacier positions in Austria from the ground and space (1893-2001). Remote Sensing Environ., 86, 566-577.

Hall, D. K., Tait, A. B., Foster, J. L., Chang, A. T. C., \& Allen, M., 2000. Intercomparison of satellite-derived snow-cover maps. Ann. Glaciol., 31, 369-376.

Hanson, B., Hooken, R. L., \& Grace, E. M. J., 1998. Short-term velocity and water-pressure variations down-glacier from a riegel, Storglaciären, Sweden. J. Glaciol., 44 (147), 359-367.

Harper, J. T., Humphrey, N. F., \& Pfeffer, W. T., 1998. Three-dimensional deformation measured in an Alaskan Glacier. Science, 281 (5381), 1340-1342.

Harper, J. T., Humphrey, N. F., Pfeffer, W. T., Huzurbazar, S. V., Bahr, D. B., \& Welch, B. C., 2001. Spatial variability in the flow of a valley glacier : deformation of a large array of boreholes. J. Geophys. Res., 106 (B5), 8547-8562.

Hoelzle, M., Haeberli, W., Dischl, M., \& Peschke, W., 2003. Secular glacier mass balances derived from cumulative glacier length changes. Global Planet. Change, 36, 295-306.

Hoelzle, M., Vonder Mühll, D., Bauder, A., \& Guðmundsson, G. H., 1998. Les glaciers des Alpes suisses en 1996/1997. Rapport de la Commission glaciologique de l'Académie suisse des sciences naturelles, $\mathbf{1 1 8 .}$

Hubbard, A., Willis, I., Sharp, M., Mair, D., Nienow, P., Hubbard, B., \& Blatter, H., 2000. Glacier mass-balance determination by remote sensing and high-resolution modelling. J. Glaciol., 46 (154), 491-498.

Iken, A., Röthlisberger, H., Flotron, A., \& Haeberli, W., 1983. The uplift of Unteraargletscher at the beginning of the melt season - a consequence of water storage at the bed? J. Glaciol., 29 (101), 28-47.

IPCC, 2001. Climate change 2001 : the scientific basis. Intergovernmental Panel on Climate Change. http ://www.grida.no/climate/ipcc_tar/wg1/.

Jabour, N., Kasmi, M., Menzhi, M., Hni, L., Timoulali, Y., \& Badrane, S., 2004. The February $24^{\text {th }}, 2004$ AL Hoceima earthquake. Newsletter of the European-Mediterranean Seismological Centre.

Jóhannesson, T., Raymond, C. F., \& Waddington, E. W., 1989. Time-scale for adjustment of glaciers to changes in mass balance. J. Glaciol., 35 (121), 355-369.

Joughin, I., 2002. Ice-sheet velocity mapping : A combined interferometric and speckle tracking approach. Ann. Glaciol., 34, 195-201.

Joughin, I., Winebrenner, D., Fahnestock, M., Kwok, R., \& Krabill, W., 1996. Measurement of icesheet topography using satellite radar interferometry. J. Glaciol., 42 (140), 10-22. 
Joughin, I. R., Kwok, R., \& Fahnestock, M. A., 1998. Interferometric estimation of the threedimensional ice-flow velocity vector using ascending and descending passes. IEEE T. Geosci. Remote, 36 (1), 25-37.

Jouzel, J., Hoffmann, G., Parrenin, F., \& Waelbroeck, C., 2002. Atmospheric oxygen 18 and sea-level changes. Quaternary Sci. Rev., 21 (13), 307-314.

Kääb, A., 2002. Monitoring high-mountain terrain deformation from repeated air- and spaceborne optical data : examples using digital aerial imagery and ASTER data. ISPRS J. Photogramm., 57 (1-2), 39-52.

Kääb, A., 2005. Combination of SRTM3 and repeat ASTER data for deriving alpine glacier flow velocities in the Bhutan Himalaya. Remote Sensing Environ., 94 (4), 463-474.

Khromova, T. E., Dyurgerov, M. B., \& Barry, R. G., 2003. Late-twentieth century changes in glacier extent in the Ak-shirak Range, Central Asia, determined from historical data and ASTER imagery. Geophys. Res. Lett., 30 (1863).

Klinger, Y., Michel, R., Van der Woerd, J., Xu, X., \& Tapponnier, P., 2004. Slip-distribution and rupture pattern of the 14 November 2001, Mw 7.8 Kokoxili earthquake (China). EOS Trans. $A G U, 85$ (47). Fall Meet. Suppl.

König, M., Winther, J.-G., \& Isaksson, E., 2001a. Measuring snow and glacier properties from satellite. Rev. Geophys., 39 (1).

König, M., Winther, J.-G., Knudsen, N. T., \& Guneriussen, T., 2001b. Firn-line detection on Austre Okstindbreen, Norway, with airborne multipolarization SAR. J. Glaciol., 47 (157), 251-257.

Krabill, W., Hanna, E., Huybrechts, P., Abdalati, W., Cappelen, J., Csatho, B., Frederick, E., Manizade, S., Martin, C., Sonntag, J., Swift, R., Thomas, R., \& Yungel, J., 2004. Greenland ice sheet : increased coastal thinning. Geophys. Res. Lett., 31 (24402).

Krimmel, R. M., 1999. Analysis of difference between direct and geodetic mass balance measurements at South Cascade Glacier, Washington. Geogr. Ann., 81A (4), 653-658.

Kuhn, M., 1993. Possible future contribution to sea level change from small glaciers. In Climate and Sea Level Change : Observations, Projections and Implications, edited by R. W. et al. Cambridge University Press, Cambridge, pages 134-143.

Kwok, R. \& Fahnestock, M., 1996. Ice sheet motion and topography from radar interferometry. IEEE T. Geosci. Remote, 34 (1), 189-200.

Kääb, A. \& Funk, M., 1999. Modelling mass balance using photogrammetric and geophysical data. A pilot study at Gries glacier, Swiss Alps. J. Glaciol., 45 (151), 575-583.

Latry, C. \& Rougé, B., 2002. Les yeux de lynx de SPOT5. La Recherche, 359, 98-99.

Leese, J. A., Novak, C. S., \& Clarke, B. B., 1971. An automated technique for obtaining cloud motion from geosynchronous satellite data using cross correlation. J. Appl. Meteor., 10 (1), $118-132$. 
Legrésy, B., Berthier, E., Vincent, C., Gay, M., Arnaud, Y., \& Rémy, F., 2002. Satellite imagery for investigation of small alpine glacier. In International Glaciological Society Meeting. Chamonix.

Legrésy, B., Potzsch, A., Tabacco, I., Rémy, F., \& Dietrich, R., sous presse. Influence of tides and tidal current on the Mertz Glacier, Antarctica. J. Glaciol..

Lemarchand, F., 2002. La dangereuse vidange des lacs glaciaires. La Recherche, 356, 16-17.

Lliboutry, L., 1964. Traité de Glaciologie. Masson, Paris.

Lliboutry, L., 1971. La catastrophe du Yungay (Pérou). In UGGI - IAHS - ICSI Symposium Moskow, volume 104. IAHS Publ.

Lliboutry, L., 1993. Glaciers. Encyclopédie Universalis, Corpus 10, 495-503. Paris.

Lliboutry, L. \& Reynaud, L., 1981. "Global dynamics" of a temperate valley glacier, Mer de Glace, and past velocities deduced from Forbes bands. J. Glaciol., 27 (96), 207-226.

Lombard, A., Cazenave, A., DoMinh, K., Cabanes, C., \& Nerem, R. S., 2005. Thermosteric sea level rise for the past 50 years; comparison with tide gauges and inference on water mass contribution. Global Planet. Change. In press.

Lucchitta, B. K. \& Ferguson, H. M., 1986. Antarctica : measuring glacier velocity from satellite images. Science, 234 (4780), 1105-1108.

Luckman, A., Murray, T., \& Strozzi, T., 2002. Three-dimensional surface flow evolution throughout a glacier surge measured by satellite radar interferometry. Geophys. Res. Lett., 29 (23).

Magnússon, E., 2003. Airborne SAR data from S-Iceland : Analyses, DEM improvements and glaciological application. Master's thesis, Univ. of Iceland, Reykjavík. Available at http ://www.raunvis.hi.is/ eyjolfm/Master.pdf.

Magnússon, E., Björnsson, H., Dall, J., \& Pálsson, F., 2005. Volume changes of Vatnajökull ice cap, Iceland, due to surface mass balance, ice flow, and sub-glacial melting at geothermal areas. Geophys. Res. Lett., 32 (5), L05504.

Magnússon, E., Björnsson, H., Pálsson, F., \& Dall, J., 2004. Glaciological application of InSAR topography data of W-Vatnajökull acquired in 1998. Jökull, 54, 17-36.

Marshall, H. P., Harper, J. T., Pfeffer, W. T., \& Humphrey, N. F., 2002. Depth-varying constitutive properties observed in an isothermal glacier. Geophys. Res. Lett..

Marshall, S. J., Björnsson, H., Flowers, G. E., \& Clarke, G. K. C., en preparation. Simulation of Vatnajökull Ice Cap Dynamics. J. Geophys. Res..

Massonnet, D. \& Feigl, K., 1998. RADAR interferometry and its application to changes in the Earth's surface. Rev. Geophys., 36 (4), 441-500.

Massonnet, D., Rossi, M., Carmona, C., Adragna, F., Peltzer, G., Feigl, K. L., \& Rabaute, T., 1993. The displacement field of the Landers earthquake mapped by radar interferometry. Nature, 364, 138-142. 
Mattar, K. E., Vachon, P. W., Geudtner, D., Gray, A. L., Cumming, I. G., \& Brugman, M., 1998. Validation of alpine glacier velocity measurements using ERS tandem-mission SAR data. IEEE T. Geosci. Remote, 36 (3), 973-984.

Meier, M., 1962. Proposed definitions for glacier mass budget terms. J. Glaciol., 4 (33), 252-263.

Meier, M. F., 1984. Contribution of small glaciers to global sea level. Science, 226, 1418-1421.

Météo-France, 2005. Une étude climatologique des Alpes apporte un nouveau signe du réchauffement du climat. http ://www.meteo-france.com/FR/actus/dossier/article.jsp ?docid=11700. Consulté le 28 mai 2005.

Michel, R. \& Avouac, J.-P., 2002. Deformation due to the 17 August 1999 Izmit, Turkey, earthquake measured from SPOT images. J. Geophys. Res., 107 (B4), 2062.

Michel, R., Avouac, J. P., \& Taboury, J., 1999. Measuring ground displacements from SAR amplitude images: Application to the Landers earthquake. Geophys. Res. Lett., 26 (7).

Michel, R. \& Rignot, E., 1999. Flow of Glaciar Moreno, Argentina, from repeat-pass Shuttle Imaging Radar images : a comparison of the phase correlation method with RADAR interferometry. J. Glaciol., 45 (149), 93-100.

Milankovitch, M. M., 1941. Canon of Insolation and the Ice-Age Problem. Royal Serbian Academy, Belgrade, Yugoslavia.

Miller, L. \& Douglas, B. C., 2004. Mass and volume contributions to 20th century global sea level rise. Nature, 406-409.

Mohr, J. J., Reeh, N., \& Madsen, S. N., 1998. Three dimensional glacial flow and surface elevation measured with RADAR interferometry. Nature, 391 (6664), 273-276.

Moreau, L. \& Vivian, R., 2000. Dans le secrêt des glaciers de Mont-Blanc. Glénat, Grenoble.

Mougin, P., 1934. Etudes Glaciologiques. Tom. 5. Direction des Eaux et du Génie Rural, Service des forces hydrauliques. Imprimerie Nationale.

Münzer, U., Bacher, U., \& Guðmundsson, A., 1999. Precision aerial survey of Vatnajökull, Iceland by digital photogrammetry. In Third Turkish German Joint Geodetic Days - Towards a Digital Age. Istanbul Tech. Univ.

Murray, T., Strozzi, T., Luckman, A., Jiskoot, H., \& Christakos, P., 2003. Is there a single surge mechanism? Contrasts in dynamics between glacier surges in Svalbard and other regions. J. Geophys. Res., 108 (B5). Doi :10.1029/2002JB001906.

Ninnis, R. M., Emery, W. J., \& Collins, M. J., 1986. Automated extraction of pack ice motion from Advanced Very High Resolution Radiometer imagery. J. Geophys. Res., 91 (C9), 1072510734.

Nye, J. F., 1965. The flow of a glacier in a channel of rectangular, elliptic or parabolic crosssection. J. Glaciol., 5 (41), 661-690.

Oerlemans, J., 1994. Quantifying global warming from the retreat of glaciers. Science, 264, 243-245. 
Oerlemans, J., 2001. Glaciers and Climate Change. A. A. Balkema, Lisse / Abingdon / Exton / Tokyo.

Oerlemans, J., 2005. Extracting a climate signal from 169 glacier records. Science, 308 (5722), 675-677. doi : 10.1126/science.1107046.

Oerlemans, J., Anderson, B., Hubbard, A., Huybrechts, P., Johannesson, T., Knap, W. H., Schmeits, M., Stroeven, A. P., van de Wal, R. S. W., Wallinga, J., \& Zuo, Z., 1998. Modelling the response of glaciers to climate warming. Climate Dyn., 14 (4), 267-274.

Ostrem, G. \& Haakensen, N., 1999. Map comparison or traditional mass-balance measurements : which method is better? Geogr. Ann., 81A (4), 703-711.

Paterson, W. S. B., 1994. The physics of glaciers. Pergamon, New York, 3rd edition.

Paul, F., Kääb, A., Maisch, M., Kellenberger, T., \& Haeberli, W., 2001. The new remote-sensingderived Swiss glacier inventory : I.Methods. Ann. Glaciol., 34, 355-361.

Paul, F., Kääb, A., Maisch, M., Kellenberger, T., \& Haeberli, W., 2004. Rapid Desintegration of alpine glaciers observed with satellite data. Geophys. Res. Lett., 31 (L21402).

Penck, A. \& Brückner, E., 1909. Die Alpen im Eiszeitälter. Chr. Herm. Tauchnitz, Leipzig (Germany).

Rabatel, A., Dedieu, J.-P., \& Vincent, C., 2005. The use of remote sensing data to determine equilibrium line altitude and mass balance time series. Validation on three French glaciers for the 1994-2002 period. J. Glaciol.. Sous presse.

Rabus, B., Eineder, M., Roth, A., \& Bamler, R., 2003. The shuttle RADAR topography mission-a new class of digital elevation models acquired by spaceborne RADAR. ISPRS J. Photogramm., 57, 241-262.

Rabus, R. T. \& Fatland, D. R., 2000. Comparison of SAR-interferometric and surveyed velocities on a mountain glacier : Black Rapids Glacier, Alaska, U.S.A. J. Glaciol., 46 (152), 119-128.

Ragnarsson, A. \& Helgason, P., 2004. Energy in Iceland : Historical Perspective, Present Status, Future Outlook. Rapport technique, Natl. Energy Auth. of Iceland, Reykjavík.

Rajesh, K., Linda, A., Wagnon, P., Sharma, P., \& Arnaud, Y., 2005. Chhota Shigri glacier mass balance monitoring of september 13-September 28. Rapport technique, Jawaharlal Nehru University \& Institut de Recherche pour le Développement.

Raymond, C. F., 1971. Flow in a transverse section of Athabasca Glacier, Alberta, Canada. J. Glaciol., 10, 55-84.

Reid, F. H., 1896. The mechanics of glaciers. J. of Geology, 4, 912-928.

Reilinger, R. E. \& Co-auteurs, 2000. Coseismic and postseismic fault slip for the 17 August 1999, $\mathrm{M}=7.4$, Izmit, Turkey earthquake. Science, 289, 1519-1524.

Rémy, F., 2003. L'Antarctique. La mémoire de la Terre vue de l'espace. CNRS Editions.

Rémy, F. \& Legrésy, B., 1998. Antarctic non-stationary signals derived from Seasat-ERS-1 alitmetry comparison. Ann. Glaciol., 27, 81-85. 
Rémy, F. \& Legrésy, B., 2004. Subglacial hydrological networks in Antarctica and their impact on ice flow. Ann. Glaciol., 39. Sous presse.

Rémy, F. \& Testut, L., 2005. Un solide qui coule! C. R. Geoscience. Soumis.

Rémy, F., Testut, L., \& Legrésy, B., 2002. Random fluctuations of snow accumulation over Antarctica and their relation to sea level change. Climate Dyn., 19, 267-276.

Reynaud, L., 1973. Etude de la dynamique des séracs du Géant (Massif du Mont Blanc). Thèse de Doctorat, Université scientifique et médicale de Grenoble.

Reynaud, L., 1979. Reconstruction of past velocities using Forbes Bands (Mer de Glace). $Z$. Gletscherk. Glazialgeol., 15 (2), 149-163.

Reynaud, L., Vallon, M., \& Letreguilly, A., 1986. Mass balance measurements : problems and two new methods of determining variations. J. Glaciol., 32 (112), 446-454.

Ribstein, P., Tiriau, E., Francou, B., \& Saravia, R., 1995. Tropical climate and glacier hydrology : A case study in Bolivia. J. Hydrol., 221-234.

Rignot, E., 2002. Mass balance of East Antarctic glaciers and ice shelves from satellite data. Ann. Glaciol., 34, 217-227.

Rignot, E., Casassa, G., Gogineni, P., Krabill, W., Rivera, A., \& Thomas, R., 2004. Accelerated ice discharge from the Antarctic Peninsula following the collapse of Larsen B ice shelf. Geophys. Res. Lett., 31 (L18401).

Rignot, E., Casassa, G., Gogineni, S., Kanagaratnam, P., Krabill, W., Pritchard, H., Rivera, A., Thomas, R., Turner, J., \& Vaughan, D., 2005. Recent ice loss from the Fleming and other glaciers, Wordie Bay, West Antarctic Peninsula. Geophys. Res. Lett., 32 (L07502). Doi :10.1029/2004GL021947.

Rignot, E., Echelmeyer, K., \& Krabill, W., 2001. Penetration depth of interferometric syntheticaperture radar signals in snow and ice. Geophys. Res. Lett., 28 (18), 3501-3504.

Rignot, E., Rivera, A., \& Casassa, G., 2003. Contribution of the Patagonia Icefields of South America to Sea Level Rise. Science, 302, 434-437.

Rignot, E. \& Thomas, R. H., 2002. Mass Balance of Polar Ice Sheets. Science, 297 (5586), 1502-1506.

Robert, M. J., 2005. Jökulhlaups : a reassessment of floodwater flow through glaciers. Rev. Geophys., 43 (RG1002). Doi :10.1029/2003RG000147.

Rott, H., Nagler, T., \& Scheiber, R., 2003. Snow mass retrieval by means of SAR interferometry. In Fringe 2003, edited by ESA-ESRIN. http ://earth.esa.int/fringe03/proceedings/.

Rott, H., Scheuchl, B., Siegel, A., \& Grasemann, B., 1999. Monitoring very slow slope motion by means of SAR interferometry : a case study from a mass waste above a reservoir in the Otztal Alps, Austria. Geophys. Res. Lett., 26 (11), 1629-1632.

Sauber, J. M. \& Molnia, B. F., 2004. Glacier ice mass fluctuations and fault instability in tectonically active Southern Alaska. Global Planet. Change, 42, 279-294. 
Savage, J. C. \& Paterson, W. S. B., 1963. Borehole measurements in the Athabasca glacier. J. Geophys. Res., 68, 4521-4536.

Scambos, T. A., Bohlander, J. A., C. A. Shuman, C. A., \& Skvarca, P., 2004. Glacier acceleration and thinning after ice shelf collapse in the Larsen B embayment, Antarctica. Geophys. Res. Lett., 31 (L18402).

Scambos, T. A., Dutkiewicz, M. J., Wilsoni, J. C., \& Bindschadler, R. A., 1992. Application of image cross-correlation to the measurement of glacier velocity using satellite image data. Remote Sensing Environ., 42 (3), 177-186.

Scambos, T. A., Hulbe, C., Fahnestock, M., \& Bohlander, J., 2000. The link between climate warming and break-up of ice shelves in the Antarctic Peninsula. J. Glaciol., 46 (154), 516-530.

Shahgedanova, M., Stokes, C. R., Gurney, S. D., \& Popovnin, V., 2005. Interactions between mass balance, atmospheric circulation, and recent climate change on the Djankuat Glacier, Caucasus Mountains, Russia. J. Geophys. Res., 110 (D04108).

Sicart, J.-E., 2002. Contribution à l'étude des flux d'énergie, du bilan de masse et du débit de fonte d'un glacier tropical : le Zongo, Bolivie. Thèse de Doctorat, Université Paris VI.

Sicart, J.-E., Ribstein, P., Francou, B., Pouyaud, B., \& Condom, T., sous presse. Hydrological and glaciological mass balances of a tropical glacier : Zongo, Bolivia. Global Planet. Change.

Sigmundsson, F., Sturkell, E., Pinel, V., Einarsson, P., Pedersen, R., Geirsson, H., Guðmundsson, M. T., Björnsson, H., \& Pagli, C., 2004. Deformation and eruption forecasting at volcanoes under retreating ice caps : discriminating signs of magma inflow and ice unloading at Grímsvötn and Katla volcanoes. EOS Trans. AGU, 85 (47). Fall Meet. Suppl.

Silverio, W. \& Jaquet, J.-M., 2005. Glacial cover mapping (1987-1996) of the Cordillera Blanca (Peru) using satellite imagery. Remote Sensing Environ., 95, 342-350.

Singh, P. \& Singh, V. P., 2001. Snow and glacier hydrology. Kluwer Academic Publishers, Dordrecht / Boston / London.

Six, D., 2000. Analyse statistique des distributions des séries de bilans de masse des glaciers alpins et des calottes polaires de l'hémisphère nord. Thèse de Doctorat, Université Joseph Fourier - Grenoble I.

Stroeve, J. C., Serreze, M. C., Fetterer, F., Arbetter, T., Meier, W., Maslanik, J., \& K., K., 2005. Tracking the Arctic's shrinking ice cover : Another extreme September minimum in 2004. Geophys. Res. Lett., 32 (L04501).

Strozzi, T., Luckman, A., Murray, T., Wegmüller, U., \& Werner, C., 2002. Glacier motion estimation using SAR offset-tracking procedures. IEEE T. Geosci. Remote, 40 (11), 23842391.

Sugiyama, S. \& Guðmundsson, G. H., 2003. Diurnal variations in vertical strain observed in a temperate calley glacier. Geophys. Res. Lett., 30 (2).

Sugiyama, S. \& Guðmundsson, G. H., sous presse. Short-term variations in glacier flow controlled by subglacial water pressure at Lauteraargletscher, Bernese Alps, Switzerland. J. Glaciol.. 
Süsstrunk, A., 1951. Sondage du glacier par la méthode sismique. La Houille Blanche, A, 309-319.

Tahayt, A., Fadil, A., Berthier, E., Feigl, K. L., Mourabit, T., McClusky, S., Reilinger, R., Serroukh, M., Gomez, F., \& Ben Sari, D., 2005. Estimation of the source parameters of Al-Hoceima earthquake of February 24th, 2004 from field observations and data of geodetic satellite systems : ENVISAT, GPS, and SPOT5. In Séisme d'Al Hoceima : bilan et perspectives.

Tahayt, A., Fadil, A., Reilinger, R., Feigl, K. L., \& Berthier, E., en preparation. Source parameters of Al-Hoceima earthquake of February 24th, 2004 from field observations and geodetic satellite systems : ENVISAT, GPS, and SPOT5.

Testut, L., 2000. Apport de la topographie à l'étude des calottes polaires. Thèse de Doctorat, Université Toulouse III - Paul Sabatier.

Thomas, R., Rignot, E., Casassa, G., Kanagaratnam, P., Acuña, C., Akins, T., Brecher, H., Frederick, E., Gogineni, P., Krabill, W., Manizade, S., Ramamoorthy, H., Rivera, A., Russell, R., Sonntag, J., Swift, R., Yungel, J., \& Zwally, J., 2004. Accelerated Sea-Level Rise from West Antarctica. Science, 306, 255-258.

Toutin, T., 2001. Elevation modelling from satellite VIR data: a review. Int. J. Remote Sens., 22 (6), 1097-1225.

Toutin, T., 2002. Three-dimensional topographic mapping with ASTER stereo data in rugged topography. IEEE T. Geosci. Remote, 40 (10), 2241-2247.

Toutin, T. \& Cheng, P., 2002. A comparison of automated DEM extraction results using alongtrack ASTER and across-track SPOT stereo images. Opt. Eng., 41 (9), 2102-2106.

Trigo, R. M., Garciá-Herrera, R., Diáz, J., Trigo, I. F., \& Valente, M. A., 2005. How exceptional was the early August 2003 heatwave in France? Geophys. Res. Lett., 32 (L10701). Doi : 10.1029/2005GL022410.

Tvede, A. \& Laumann, T., 1997. Glacial variations on a meso-scale example from glaciers in the Aurland Mountains, southern Norway. Ann. Glaciol., 24, 130-134.

USGS, 1996. The GTOPO30 DEM. http ://edcdaac.usgs.gov/gtopo30/gtopo30.asp.

Vadon, H. \& Berthier, E., 2004. Measurement of glacier velocity fields using very precise multitemporal correlation on high resolution SPOT5 images. In ISPRS meeting. Istanbul.

Vadon, H. \& Massonnet, D., 2000. Earthquake displacement fields mapped by very precise correlation : complementary with RADAR interferometry. In IEEE International Geoscience Remote Sensing Symposium, edited by I. Periodicals. New Jersey.

Vallon, M., 1961. Epaisseur du glacier du Tacul (Massif du Mont-Blanc). CR Acad. Sci. II A, 252 (12), 1815-1817.

Vallon, M., Petit, J.-R., \& Fabre, B., 1976. Study of an ice core to the bedrock in the accumulation area of an alpine glacier. J. Glaciol., 17, 13-28.

Vallot, J., 1900. Expérience sur la marche et la variation de la Mer de Glace. Annales de l'Observatoire Météorologique, Physique et Glaciaire de Mont-Blanc, Tom. 4, 35-157. 
Van der Veen, C. J., 1999. Fundamentals of glacier dynamics. A. A. Balkema, Netherlands.

Van Puymbroeck, N., Michel, R., Binet, R., Avouac, J. P., \& Taboury, J., 2000. Measuring earthquakes from optical satellite images. Appl. Opt., 39 (20), 3486-3494.

Vincent, C., 2002. Influence of climate change over the 20th Century on four French glacier mass balances. J. Geophys. Res., 107 (D18).

Vincent, C., Kappenberger, G., Valla, F., Bauder, A., Funk, M., \& Le Meur, E., 2004. Ice ablation as evidence of climate change in the Alps over the 20th century. J. Geophys. Res., 109 (D10).

Vincent, C., Ribstein, P., Favier, V., Wagnon, P., Francou, B., Le Meur, E., \& Six, D., $2005 a$. Glaciers fluctuations in the Alps and in the tropical Andes. C. R. Geoscience, 337, 97-106.

Vincent, C. Le Meur, E., Six, D., \& Funk, M., 2005b. Solving the paradox of the end of the Little Ice Age in the Alps. Geophys. Res. Lett., 32 (9), doi : 10.1029/2005GL022552.

Vivian, R., 2001. Glaciers du Mont-Blanc. La Fontaine de Siloé, Montmélian.

Wagnon, P., Sicart, J., Berthier, E., \& Chazarin, J., 2003. Wintertime high altitude surface energy balance of a Bolivian glacier, Illimani, $6340 \mathrm{~m}$ above sea level (a.s.l.). J. Geophys. Res., 108 (D6), 10.1029.

Weber Hoen, E. \& Zebker, H. A., 2000. Penetration Depths Inferred from Interferometric Volume Decorrelation Observed over the Greenland Ice Sheet. IEEE T. Geosci. Remote, 38 (6), 25712583 .

Weertman, J., 1957. On the sliding of glaciers. J. Glaciol., 3, 33-38.

Wessel, P. \& Smith, W. H. F., 1998. New, improved version of generic mapping tools released. EOS T. Am. Geophy. Un., 79 (47), 579.

Wessels, R. L., Kargel, J. S., \& Kieffer, H. H., 2002. ASTER measurment of supraglacial lakes in the Mount Everest region of the Himalaya. Ann. Glaciol., 34, 399-408.

Williams, R. S., J. \& Hall, D. K., 1993. Glaciers. In Atlas of satellite observations related to global change, edited by R. J. Gurey, J. L. Foster, \& C. L. Parkinson. Cambridge University Press, Cambridge, pages 401-421.

Willis, I. C., 1995. Intra-annual variations in glacier motion : a review. Prog. Phys. Geog., 19 (1), 61-106.

WWF, 2005. An overview of glaciers, glacier retreat, and subsequent impacts in Nepal, India and China. http ://www.panda.org/about_wwf/what_we_do/climate_change/publications/.

Yadav, R. R., Won-Kyu, P., Singh, J., \& Dubey, B., 2004. Do the western Himalaya defy global warming? Geophys. Res. Lett., 31 (L17201). Doi :10.1029/2004GL020201.

Zwally, J., Abdalati, W., Herring, T., Larson, K., Saba, J., \& Steffen, K., 2002a. Surface melt-induced acceleration of Greenland ice-sheet flow. Science, 297 (5579), 218-222. Doi : $10.1126 /$ science. 1072708 .

Zwally, J., Schutz, B., et al., 2002b. ICESat's laser measurements of polar ice, atmosphere, ocean, and land. J. Geodyn., 34, 405-445. 